In cooperation with the Texas Water Development Board

\title{
Results of Streamflow Gain-Loss Studies in Texas, With Emphasis on Gains From and Losses to Major and Minor Aquifers
}

Open-File Report 02-068

U.S. Department of the Interior U.S. Geological Survey 
U.S. Department of the Interior

U.S. Geological Survey

\section{Results of Streamflow Gain-Loss Studies in Texas, With Emphasis on Gains From and Losses to Major and Minor Aquifers}

By Raymond M. Slade, Jr., J. Taylor Bentley, and Dana Michaud

U.S. GEOLOGICAL SURVEY

Open-File Report 02-068

In cooperation with the Texas Water Development Board

Austin, Texas

2002 


\section{U.S. DEPARTMENT OF THE INTERIOR}

Gale A. Norton, Secretary

\section{U.S. GEOLOGICAL SURVEY}

Charles G. Groat, Director

Any use of trade, product, or firm names is for descriptive purposes only and does not imply endorsement by the U.S. Government.

For additional information write to

\section{District Chief}

U.S. Geological Survey

8027 Exchange Dr.

Austin, TX 78754-4733

E-mail: dc_tx@usgs.gov

Copies of this report can be purchased from

U.S. Geological Survey

Branch of Information Services

Box 25286

Denver, CO 80225-0286

E-mail: infoservices@usgs.gov 


\section{CONTENTS}

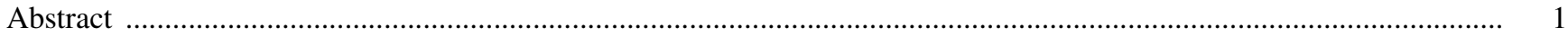

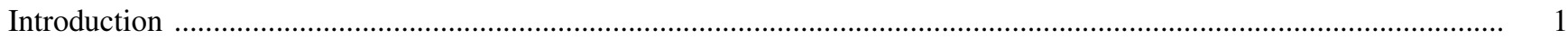

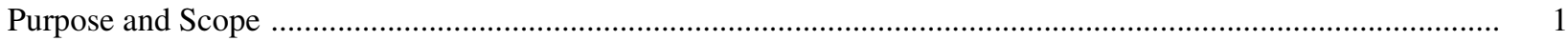

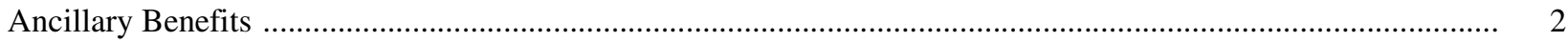

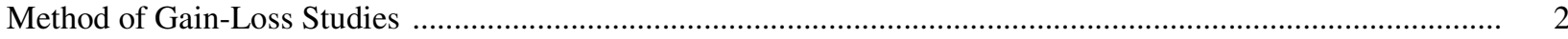

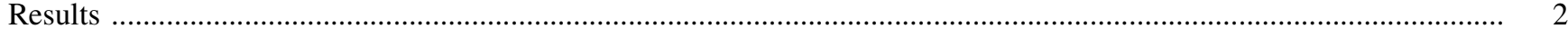

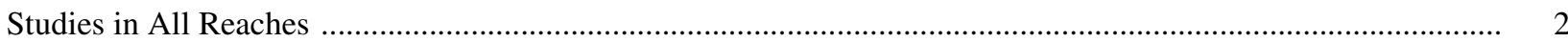

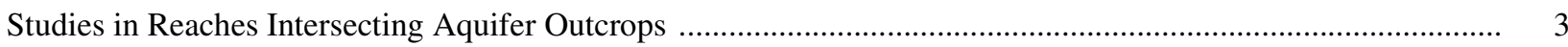

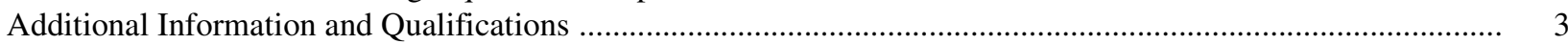

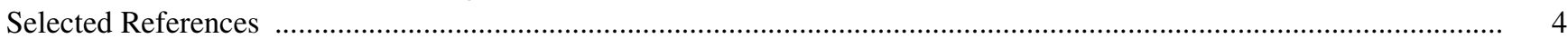

\section{PLATES}

1-2. Maps showing:

1. Locations of streamflow gain-loss sites and outcrops of major aquifers in Texas

2. Locations of streamflow gain-loss sites and outcrops of minor aquifers in Texas

\section{TABLES}

[at end of report]

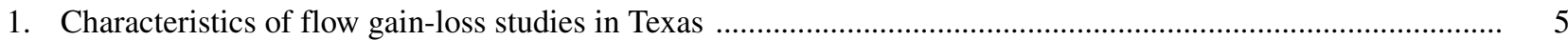

2. Inventory of daily mean streamflows for active and discontinued streamflow-gaging stations in Texas ........... 23

3. Median streamflow and associated hydraulic characteristics for streamflow-gaging stations in Texas ............. 45

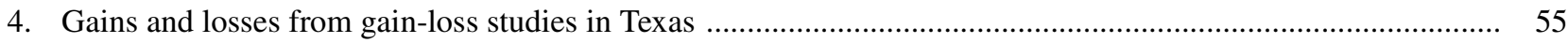




\title{
Results of Streamflow Gain-Loss Studies in Texas, With Emphasis on Gains From and Losses to Major and Minor Aquifers
}

\author{
By Raymond M. Slade, Jr., J. Taylor Bentley, and Dana Michaud
}

\begin{abstract}
Data for all 366 known streamflow gain-loss studies conducted by the U.S. Geological Survey in Texas were aggregated. A water-budget equation that includes discharges for main channels, tributaries, return flows, and withdrawals was used to document the channel gain or loss for each of 2,872 subreaches for the studies. The channel gain or loss represents discharge from or recharge to aquifers crossed by the streams. Where applicable, the major or minor aquifer outcrop traversed by each subreach was identified, as was the length and location for each subreach. These data will be used to estimate recharge or discharge for major and minor aquifers in Texas, as needed by the Ground-Water Availability Modeling Program being conducted by the Texas Water Development Board. The data also can be used, along with current flow rates for streamflow-gaging stations, to estimate streamflow at sites remote from gaging stations, including sites where streamflow availability is needed for permitted withdrawals.
\end{abstract}

\section{INTRODUCTION}

As part of the Ground-Water Availability Modeling (GAM) Program currently (2001) being conducted by the Texas Water Development Board (TWDB), data are needed to quantify the interaction of surface water and ground water for the nine major aquifers (Ashworth and Hopkins, 1995) and most of the 20 minor aquifers in Texas. Where streams flow across aquifer outcrops, channel gains and losses constitute aquifer discharge and recharge, respectively. To make this aquifer discharge and recharge information available for the GAM Program, the U.S. Geological Survey (USGS), in cooperation with the TWDB, compiled data and computed streamflow gains and losses from all available records of gain-loss studies done by the USGS in Texas.

Since 1918, the USGS has conducted streamflow gain-loss studies on streams throughout much of Texas. The usual objective of the gain-loss studies was to obtain data that could be used to estimate discharge from or recharge to shallow aquifers. Most gain-loss studies were done during low-flow conditions because low flows are more likely to be steady (not changing with time) than other flows (except in reaches downstream from major springs or reaches downstream from reservoirs where sustained releases account for most of the flow).

In 1958, the data for all known streamflow gainloss studies were compiled and published in a report by the Texas Board of Water Engineers (currently the TWDB) and the USGS (Texas Board of Water Engineers, 1960). The data for most of the studies done since 1958 have been published in annual data reports and other reports by the USGS. This study carries the documentation of gain-loss studies a step farther: The gains and losses in stream subreaches (channel segments between flow-measuring sites in a reach) were related to major and minor aquifer outcrops in digital and geographic information system (GIS) databases.

\section{Purpose and Scope}

The purpose of this report is to summarize the results of 366 gain-loss studies involving 249 unique reaches of streams throughout Texas since 1918. The locations of subreaches for which gains and losses were computed are indicated by streamflow-measurement sites on maps of major and minor aquifer outcrops. The gain-loss studies are tabulated by sequential number, major river basin, stream name, and reach identification, and the total gain or loss for each reach is given. The gains and losses for each subreach are tabulated by sequential number for the gain-loss study and located by latitude and longitude of the upstream end of the 
subreach. Where applicable, the major or minor aquifer outcrop traversed by a subreach is identified.

\section{Ancillary Benefits}

The compilation of streamflow gain-loss data could be beneficial to the Water Uses and Availability Section of the Water Resources Management Division of the Texas Natural Resource Conservation Commission (TNRCC). That section is responsible for permitting surface-water withdrawals in Texas. Most of the recently issued permits represent contingency permits, which authorize surface-water withdrawals only when the streamflow exceeds a threshold rate. The threshold streamflow rate for each contingency permit generally represents the total discharge needed to sustain permitted withdrawals downstream from the withdrawal point for the contingency permit plus any streamflow required as inflow to receiving bays or estuaries. Contingency permits are used to protect the existing water rights of users downstream from newer users.

The TNRCC and others associated with surfacewater usage often use USGS current streamflow data available on the World Wide Web to verify existing streamflow conditions pertinent to contingency permits. However, there are only about 350 existing streamflowgaging stations, and the location of withdrawal points for contingency permits often are many miles from the nearest streamflow-gaging station. Stream-channel gain and loss data can be used, along with the current streamflow rates for gaging stations, to estimate the current streamflow for sites remote from the gaging stations, including sites that represent surface-water withdrawals for contingency permits.

Reservoir owners also could benefit from the compilation of streamflow gain-loss data. Many reservoir owners are required to release sufficient water to sustain the permitted withdrawal rate for downstream water rights. The permitted users are guaranteed a specific withdrawal rate. The gains and losses of channel flow can be used by reservoir owners to help determine reservoir release rates needed to sustain permitted downstream withdrawal rates.

\section{Method of Gain-Loss Studies}

The usual method of gain-loss studies is to identify a stream reach and obtain streamflow measurements along the main channel of the reach. The location of each main-channel measurement site is referenced and documented as a distance on the stream channel, usually upstream from its mouth. The channel gain or channel loss can be computed for the subreach between each main-channel measurement site by equating inflows to outflows plus flow gain or loss in the subreach:

$$
Q_{u}+Q_{t}+Q_{r}=Q_{d}+Q_{w}+Q_{e}+Q_{g}
$$

where

$\mathrm{Q}_{\mathrm{u}}=$ streamflow in at upstream end of subreach;

$\mathrm{Q}_{\mathrm{t}}=$ streamflow from tributaries into subreach;

$\mathrm{Q}_{\mathrm{r}}=$ return flows to subreach;

$\mathrm{Q}_{\mathrm{d}}=$ streamflow out at downstream end of subreach;

$\mathrm{Q}_{\mathrm{W}}=$ withdrawals from subreach;

$\mathrm{Q}_{\mathrm{e}}=$ evapotranspiration from subreach; and

$\mathrm{Q}_{\mathrm{g}}=$ gain (positive) or loss (negative) in subreach.

Thus,

$$
\mathrm{Q}_{\mathrm{g}}=\mathrm{Q}_{\mathrm{u}}+\mathrm{Q}_{\mathrm{t}}+\mathrm{Q}_{\mathrm{r}}-\mathrm{Q}_{\mathrm{d}}-\mathrm{Q}_{\mathrm{w}}-\mathrm{Q}_{\mathrm{e}}
$$

For most streams, underflow (flow parallel to stream through shallow channel-bed deposits) and bank storage are considered negligible or minimal.

Many of the studies were done during winter to minimize evapotranspiration. Also, the short length of most subreaches and minimal width of the streams during low-flow conditions would allow only minimal evapotranspiration losses. Therefore, $\mathrm{Q}_{\mathrm{e}}$ is assumed to be zero in the computations for this report. In each gainloss study, attempts were made to identify and measure the discharge for all flowing tributaries, return flows, and withdrawals. If these discharges could not be measured, attempts were made to obtain the discharges from other sources such as the TNRCC. However, the USGS cannot verify that all inflow or outflow sources for the reaches were accounted for.

\section{RESULTS}

\section{Studies in All Reaches}

Three-hundred sixty-six streamflow gain-loss studies in 249 unique reaches were identified and included in this investigation. More than one study has been done at many of the reaches. The locations of streamflow-measurement sites for the studies are shown on plate 1 . The studies included about 4,941 measurements of which 3,238 were made at sites on the main channels of the study reaches; the remaining measurements were made on tributaries to the main channels or represent withdrawals. A tabular summary of the flowloss studies (table 1) includes for each study the major 
river basin, stream name, study reach identification, date of study, reach length (in river miles), total number of measurement sites, number of sites on the main channel, major aquifer outcrop(s) intersected by the reach, total streamflow gain or loss in the reach, streamflow gain or loss per mile of reach length, and reference for the data. The reaches for many studies are identified in table 1 by eight-digit numbers for streamflow-gaging stations. Station numbers and associated station names for Texas streamflow-gaging stations with daily streamflow data are listed in table 2.

Table 3 presents selected streamflow characteristics for all streamflow-gaging stations with computerstored discharge measurements and daily mean streamflows in Texas (346 sites). These data include the station number and name, latitude and longitude, contributing drainage area, and the following data pertinent to median flow conditions: the streamflow, gage height, stream width, stream cross-sectional area, mean velocity, and mean stream depth. Also presented is the elevation of the datum of the gage, which can be added to the gage height to obtain the water-surface elevation above sea level for the median streamflow. The streamflow at the gaging station during a gain-loss study can be compared to the median streamflow to assess the flow conditions during the study.

Equation 2 was used to compute the streamflow gain or loss for each subreach. The data and information for the gains or losses in each of 2,872 subreaches (table 4) include the latitude and longitude at the upstream end of the subreach, the underlying major or minor aquifer outcrop, the streamflow gain or loss, the stream subreach length, the location (river mile) of the upstream end of the subreach, and a descriptive location for selected upstream ends.

The data for most of the studies have been published. The references (table 1) can be consulted for additional information about a study. Detailed descriptions for most of the measuring-site locations are in the referenced reports.

\section{Studies in Reaches Intersecting Aquifer Outcrops}

Most of the gain-loss studies involved reaches that intersect at least one major aquifer outcrop, and many intersect more than one major aquifer outcrop (pl. 1). Relatively few studies have been done involving reaches that intersect the outcrops of the Hueco-Mesilla Bolson and Ogallala aquifers and none involving the
Seymour aquifer (see table below); however, gain-loss studies have been done for many of the major streams that intersect the other six major aquifer outcrops.

Numbers of gain-loss studies associated with the nine major aquifer outcrops in Texas

\begin{tabular}{lc}
\hline \multicolumn{1}{c}{ Major aquifer outcrop } & Number of gain-loss studies \\
\hline Carrizo-Wilcox & 47 \\
Cenozoic Pecos Alluvium & 53 \\
Edwards (Balcones fault zone) & 126 \\
Edwards-Trinity (Plateau) & 82 \\
Gulf Coast & 28 \\
Hueco-Mesilla Bolson & 6 \\
Ogallala & 5 \\
Seymour & 0 \\
Trinity & 92 \\
\hline
\end{tabular}

Plate 2 shows the locations of gain-loss studies in reaches that intersect outcrops of minor aquifers in Texas. Not surprisingly, fewer studies involved reaches intersecting minor aquifer outcrops because the areal extent of minor aquifer outcrops is appreciably less than that of major aquifers.

\section{Additional Information and Qualifications}

Streamflow studies were conducted more than once for many of the reaches. For most reaches with multiple studies, the gains or losses differ between the studies. Much of the difference in gains or losses probably is caused by varying water levels in aquifers adjacent to the reaches. For example, periods of high recharge preceding a study could result in large flow gains, and "dry" conditions preceding a study could result in smaller gains or result in losses. Also, varying bank storage could cause differences in gains or losses. As previously stated, the studies summarized in this report considered bank storage to be minimal or negligible. However, bank storage could represent a substantial source of inflow to main channels of some streams, particularly streams in alluvial outcrops. Streamflow data from nearby gaging stations, if available, can indicate whether bank flow is more than a minimal source of water. Large daily streamflows for dates preceding a 
gain-loss study could imply high recharge conditions and substantial bank flow.

Streamflow data from gaging stations can be used to supplement or augment data from channel gain-loss studies. For example, many streams have more than one gaging station. Daily flow data at proximate gaging sites on the same stream can be used to estimate gains or losses in channel flow for the reach between the stations. Current and historic streamflow data for Texas streamflow-gaging stations are available on the World Wide Web at http://tx.usgs.gov

Streamflow gains and losses are subject to error because of potential error in the streamflow measurements. The potential error in each streamflow measurement typically is about 5 to 8 percent; however, the potential error for most streamflow gains or losses is larger than that, and the potential error increases as the ratio of the gain (or loss) to the measured flow increases. For example, the potential error in a measured flow of 10 cubic feet per second $\left(\mathrm{ft}^{3} / \mathrm{s}\right)$ for a main-channel site would be about $0.5 \mathrm{ft}^{3} / \mathrm{s}$, and the potential error for $11 \mathrm{ft}^{3} / \mathrm{s}$ measured at a downstream main channel site would be about $0.5 \mathrm{ft}^{3} / \mathrm{s}$. The total potential error therefore would be about $1.0 \mathrm{ft}^{3} / \mathrm{s}$ for the two measured flows, which is comparable to the computed gain of $1.0 \mathrm{ft}^{3} / \mathrm{s}$ for the reach between the sites; thus the actual gain could range from 0 to $2.0 \mathrm{ft}^{3} / \mathrm{s}$.

\section{SELECTED REFERENCES}

Ashworth, J.B., and Hopkins, Janie, 1995, Major and minor aquifers of Texas: Texas Water Development Board Report 345, 69 p.

Baker, E.T., Jr., Slade, R.M., Jr., Dorsey, M.E., and Ruiz, L.M., 1986, Geohydrology of the Edwards aquifer in the
Austin area, Texas: Texas Water Development Board Report 293, 216 p.

Land, L.F., Boning, C.W., Harmsen, Lynn, and Reeves, R.D., 1983, Streamflow losses along the Balcones fault zone, Nueces River Basin, Texas: U.S. Geological Survey Water-Resources Investigations Report 83-4168, 72 p.

Slade, R.M., Jr., Gaylord, J.L., Dorsey, M.E., Mitchell, R.N., and Gordon, J.D., 1982, Hydrologic data for urban studies in the Austin, Texas, metropolitan area, 1980: U.S. Geological Survey Open-File Report 82-506, 264 p.

Texas Board of Water Engineers, 1960, Channel gain and loss investigations, Texas streams, 1918-1958: Texas Board of Water Engineers Bulletin 5807-D, 270 p.

U.S. Geological Survey, 1963-65, Surface water records of Texas 1962-64: U.S. Geological Survey [variously paged].

1966-70, 1972, 1975, Water resources data for Texas, water years 1965-69, 1971, 1974-Part 1. Surface water records: U.S. Geological Survey [variously paged].

1976-77, 1981-82a, 1986, Water resources data for Texas, water years 1975-76, 1980-81, 1985Volume 3. Colorado River Basin, Lavaca River Basin, Guadalupe River Basin, Nueces River Basin, Rio Grande Basin, and intervening coastal basins: U.S. Geological Survey Water-Data Reports TX-75-3, 510 p.; TX-76-3, 557 p.; TX-80-3, 583 p.; TX-81-3, 599 p.; TX-85-3, 447 p.

1980, Water resources data for Texas, water year 1979-Volume 2. San Jacinto River Basin, Brazos River Basin, San Bernard River Basin, and intervening coastal basins: U.S. Geological Survey Water-Data Report TX-79-2, $511 \mathrm{p}$.

1982b, Water resources data for Texas, water year 1981 - Volume 1. Arkansas River Basin, Red River Basin, Sabine River Basin, Neches River Basin, Trinity River Basin, and intervening coastal basins: U.S. Geological Survey Water-Data Report TX-81-1, 597 p. 
Table 1. Characteristics of flow gain-loss studies in Texas

[mi, miles; $\mathrm{ft}^{3} / \mathrm{s}$, cubic feet per second; $\mathrm{ft}^{3} / \mathrm{s}$-mi, cubic feet per second per mile; Cr, Creek; USGS, U.S. Geological Survey; --, not applicable; R, River; TBWE, Texas Board of Water Engineers; Fk, Fork; ft, feet; PCWID, Pecos County Water Improvement District; IBWC, International Boundary and Water Commission; RCWID, Reeves County Water Improvement District]

Note: Eight digit streamflow-gaging stations used to identify some reaches

\begin{tabular}{|c|c|c|c|c|c|c|c|c|c|c|c|}
\hline $\begin{array}{l}\text { Stream- } \\
\text { flow } \\
\text { study } \\
\text { no. }\end{array}$ & $\begin{array}{l}\text { Major } \\
\text { river } \\
\text { basin }\end{array}$ & $\begin{array}{l}\text { Stream } \\
\text { name }\end{array}$ & $\begin{array}{c}\text { Reach } \\
\text { identification }\end{array}$ & $\begin{array}{l}\text { Date of } \\
\text { study }\end{array}$ & $\begin{array}{c}\text { Reach } \\
\text { length } \\
\text { (river } \\
\text { mi) }\end{array}$ & $\begin{array}{c}\text { Total } \\
\text { no. of } \\
\text { measure- } \\
\text { ment } \\
\text { sites }\end{array}$ & $\begin{array}{c}\text { No. of } \\
\text { measure- } \\
\text { ment sites } \\
\text { on main } \\
\text { channel }\end{array}$ & $\begin{array}{c}\text { Major aquifer } \\
\text { outcrop(s) } \\
\text { intersected } \\
\text { by reach }\end{array}$ & $\begin{array}{c}\text { Total } \\
\text { gain or } \\
\text { loss }(-) \\
\text { in reach } \\
\left(\mathrm{ft}^{3} / \mathrm{s}\right)\end{array}$ & $\begin{array}{c}\text { Gain or } \\
\text { loss per } \\
\text { mile of } \\
\text { reach } \\
\left(\mathrm{ft}^{3} / \mathrm{s}-\mathrm{mi}\right)\end{array}$ & $\begin{array}{c}\text { Reference } \\
\text { for data }\end{array}$ \\
\hline 1 & Brazos & Berry $\mathrm{Cr}$ & $\begin{array}{l}\text { Briggs to mouth (northeast of } \\
\text { Georgetown) }\end{array}$ & $4 / 21-24 / 1978$ & 30.1 & 17 & 11 & Edwards & 2.25 & 0.075 & USGS (1980) \\
\hline 2 & Brazos & Berry $\mathrm{Cr}$ & $\begin{array}{l}\text { Briggs to mouth (northeast of } \\
\text { Georgetown) }\end{array}$ & $8 / 15 / 1978$ & 30.1 & 17 & 11 & Edwards & -.21 & -.007 & USGS (1980) \\
\hline 3 & Brazos & Berry Cr & $\begin{array}{l}\text { Briggs to mouth (northeast of } \\
\text { Georgetown) }\end{array}$ & $2 / 15 / 1979$ & 30.1 & 17 & 11 & Edwards & 31.29 & 1.04 & USGS (1980) \\
\hline 4 & Brazos & Berry $\mathrm{Cr}$ & $\begin{array}{l}\text { Briggs to mouth (northeast of } \\
\text { Georgetown) }\end{array}$ & 8/14-15/1979 & 30.1 & 17 & 11 & Edwards & 21.75 & .723 & USGS (1980) \\
\hline 5 & Brazos & Brushy $\mathrm{Cr}$ & $\begin{array}{l}\text { northwest of Leander to } 4 \text { mi east of } \\
\text { Round Rock }\end{array}$ & 4/17-18/1978 & 20.8 & 21 & 8 & Edwards & 6.05 & .291 & USGS (1980) \\
\hline 6 & Brazos & Brushy Cr & $\begin{array}{l}\text { northwest of Leander to } 4 \text { mi east of } \\
\text { Round Rock }\end{array}$ & 8/17-18/1978 & 20.8 & 21 & 8 & Edwards & .07 & .003 & USGS (1980) \\
\hline 7 & Brazos & Brushy $\mathrm{Cr}$ & $\begin{array}{l}\text { northwest of Leander to } 4 \text { mi east of } \\
\text { Round Rock }\end{array}$ & 2/13-14/1979 & 20.8 & 21 & 8 & Edwards & 48.05 & 2.31 & USGS (1980) \\
\hline 8 & Brazos & Brushy $\mathrm{Cr}$ & $\begin{array}{l}\text { northwest of Leander to } 4 \text { mi east of } \\
\text { Round Rock }\end{array}$ & 8/13-14/1979 & 20.8 & 21 & 8 & Edwards & 6.57 & .316 & USGS (1980) \\
\hline 9 & Brazos & Hubbard $\mathrm{Cr}$ & $\begin{array}{l}\text { county road } 2.5 \text { mi north of } \\
\text { Sedwick to Breckenridge } \\
(08086500)\end{array}$ & $1 / 25-26 / 1962$ & 24.1 & 14 & 5 & -- & -3.18 & -.132 & USGS (1963) \\
\hline 10 & Brazos & Lampasas R & northeast of Lampasas to mouth & $6 / 3-6 / 1963$ & 79.8 & 22 & 12 & Edwards, Trinity & -5.5 & -.069 & USGS (1964) \\
\hline 11 & Brazos & Lampasas R & $\begin{array}{l}\text { Stillhouse Hollow Dam site to } \\
\text { confluence with Little R }\end{array}$ & $1 / 16 / 1968$ & 15.7 & 6 & 4 & Edwards & 15.88 & 1.011 & USGS (1969) \\
\hline 12 & Brazos & Leon $\mathrm{R}$ & Belton Dam to Little River & 1/16-17/1968 & 9 & 11 & 4 & -- & 2.48 & .276 & USGS (1969) \\
\hline 13 & Brazos & Leon $\mathrm{R}$ & southeast of Eastland to near Hasse & $3 / 13-14 / 1951$ & 45.5 & 18 & 11 & Trinity & .26 & .006 & TBWE (1960) \\
\hline 14 & Brazos & $\begin{array}{l}\text { North Fk San } \\
\quad \text { Gabriel R }\end{array}$ & $\begin{array}{l}\text { above US } 183 \text { to Country Club Rd } \\
\text { at Georgetown }\end{array}$ & $4 / 26-27 / 1978$ & 17 & 15 & 8 & Edwards, Trinity & 3.65 & .215 & USGS (1980) \\
\hline 15 & Brazos & $\begin{array}{l}\text { North Fk San } \\
\quad \text { Gabriel R }\end{array}$ & $\begin{array}{l}\text { above US } 183 \text { to Country Club Rd } \\
\text { at Georgetown }\end{array}$ & $8 / 16-26 / 1978$ & 17 & 15 & 8 & Edwards, Trinity & .07 & .004 & USGS (1980) \\
\hline 16 & Brazos & $\begin{array}{l}\text { North Fk San } \\
\quad \text { Gabriel R }\end{array}$ & $\begin{array}{l}\text { above US } 183 \text { to Country Club Rd } \\
\text { at Georgetown }\end{array}$ & $2 / 13-15 / 1979$ & 17 & 15 & 8 & Edwards, Trinity & 33.74 & 1.985 & USGS (1980) \\
\hline 17 & Brazos & $\begin{array}{l}\text { North Fk San } \\
\quad \text { Gabriel R }\end{array}$ & $\begin{array}{l}\text { above US } 183 \text { to Country Club Rd } \\
\text { at Georgetown }\end{array}$ & $8 / 13-15 / 1979$ & 17 & 15 & 8 & Edwards, Trinity & 9.4 & .553 & USGS (1980) \\
\hline 18 & Brazos & $\begin{array}{l}\text { North Fk San } \\
\quad \text { Gabriel R }\end{array}$ & north of Leander to mouth & $3 / 16-18 / 1964$ & 14.4 & 13 & 6 & Edwards, Trinity & 1.98 & .138 & USGS (1965) \\
\hline
\end{tabular}


Table 1. Characteristics of flow gain-loss studies in Texas-Continued

\begin{tabular}{|c|c|c|c|c|c|c|c|c|c|c|c|}
\hline $\begin{array}{l}\text { Stream- } \\
\text { flow } \\
\text { study } \\
\text { no. }\end{array}$ & $\begin{array}{c}\text { Major } \\
\text { river } \\
\text { basin }\end{array}$ & $\begin{array}{l}\text { Stream } \\
\text { name }\end{array}$ & $\begin{array}{l}\text { Reach } \\
\text { identification }\end{array}$ & $\begin{array}{l}\text { Date of } \\
\text { study }\end{array}$ & $\begin{array}{c}\text { Reach } \\
\text { length } \\
\text { (river } \\
\text { mi) }\end{array}$ & $\begin{array}{c}\text { Total } \\
\text { no. of } \\
\text { measure- } \\
\text { ment } \\
\text { sites }\end{array}$ & $\begin{array}{l}\text { No. of } \\
\text { measure- } \\
\text { ment sites } \\
\text { on main } \\
\text { channel }\end{array}$ & $\begin{array}{c}\text { Major aquifer } \\
\text { outcrop(s) } \\
\text { intersected } \\
\text { by reach }\end{array}$ & $\begin{array}{c}\text { Total } \\
\text { gain or } \\
\text { loss }(-) \\
\text { in reach } \\
\left(\mathrm{ft}^{3} / \mathrm{s}\right)\end{array}$ & $\begin{array}{c}\text { Gain or } \\
\text { loss per } \\
\text { mile of } \\
\text { reach } \\
\left(\mathrm{ft}^{3} / \mathrm{s}-\mathrm{mi}\right)\end{array}$ & $\begin{array}{l}\text { Reference } \\
\text { for data }\end{array}$ \\
\hline 19 & Brazos & Salado $\mathrm{Cr}$ & northwest of Florence to Salado & $4 / 24 / 1978$ & 26.2 & 12 & 7 & Edwards & 15.29 & 0.584 & USGS (1980) \\
\hline 20 & Brazos & Salado $\mathrm{Cr}$ & northwest of Florence to Salado & $8 / 14 / 1978$ & 26.2 & 12 & 7 & Edwards & 8.96 & .342 & USGS (1980) \\
\hline 21 & Brazos & Salado $\mathrm{Cr}$ & northwest of Florence to Salado & $2 / 16 / 1979$ & 26.2 & 12 & 7 & Edwards & 63.0 & 2.405 & USGS (1980) \\
\hline 22 & Brazos & Salado $\mathrm{Cr}$ & northwest of Florence to Salado & $8 / 15 / 1979$ & 26.2 & 12 & 7 & Edwards & 49.09 & 1.874 & USGS (1980) \\
\hline 23 & Brazos & $\begin{array}{l}\text { Salt Prong Hubbard } \\
\quad \mathrm{Cr}\end{array}$ & $\begin{array}{l}8.3 \text { mi above mouth to } 0.5 \mathrm{mi} \text { above } \\
\text { mouth }\end{array}$ & $1 / 25 / 1962$ & 7.8 & 3 & 3 & -- & .84 & .108 & USGS (1963) \\
\hline 24 & Brazos & San Gabriel R & Georgetown (08105000) to mouth & $3 / 17-18 / 1964$ & 49 & 25 & 11 & -- & 10.54 & .215 & USGS (1965) \\
\hline 25 & Brazos & $\begin{array}{l}\text { South Fk San } \\
\quad \text { Gabriel R }\end{array}$ & US 183 to SH 29 at Georgetown & $3 / 16 / 1964$ & 12.8 & 6 & 4 & Edwards & 1.38 & .108 & USGS (1965) \\
\hline 26 & Brazos & $\begin{array}{l}\text { South Fk San } \\
\text { Gabriel R }\end{array}$ & near Bertram to Georgetown & $4 / 19-21 / 1978$ & 29.7 & 21 & 13 & Edwards, Trinity & 5.09 & .171 & USGS (1980) \\
\hline 27 & Brazos & $\begin{array}{l}\text { South Fk San } \\
\quad \text { Gabriel R }\end{array}$ & near Bertram to Georgetown & $8 / 17 / 1978$ & 29.7 & 21 & 13 & Edwards, Trinity & .01 & .0 & USGS (1980) \\
\hline 28 & Brazos & $\begin{array}{l}\text { South Fk San } \\
\text { Gabriel R }\end{array}$ & near Bertram to Georgetown & $2 / 13-15 / 1979$ & 29.7 & 21 & 13 & Edwards, Trinity & 33.22 & 1.119 & USGS (1980) \\
\hline 29 & Brazos & $\begin{array}{l}\text { South Fk San } \\
\text { Gabriel R }\end{array}$ & near Bertram to Georgetown & $8 / 13-15 / 1979$ & 29.7 & 21 & 14 & Edwards, Trinity & 8.59 & .289 & USGS (1980) \\
\hline 30 & Brazos & Sulphur Cr & $\begin{array}{l}\text { Lampasas to } 1.5 \mathrm{mi} \text { downstream } \\
\text { from Burleson } \mathrm{Cr}\end{array}$ & $6 / 30 / 1942$ & 3.67 & 9 & 6 & Trinity & 15.2 & 4.142 & TBWE (1960) \\
\hline 31 & Brazos & Sulphur Cr & $\begin{array}{l}\text { Lampasas to } 1.5 \mathrm{mi} \text { downstream } \\
\text { from Burleson } \mathrm{Cr}\end{array}$ & $8 / 10 / 1942$ & 3.67 & 9 & 6 & Trinity & 11.7 & 3.188 & TBWE (1960) \\
\hline 32 & Colorado & Barton $\mathrm{Cr}$ & $\begin{array}{l}\text { Loop } 360 \text { (08155300) to above } \\
\text { Barton Springs }\end{array}$ & 2/9/1981 & 3.5 & 5 & 5 & Edwards & -17.0 & -4.857 & $\begin{array}{l}\text { Slade and others } \\
\quad \text { (1982) }\end{array}$ \\
\hline 33 & Colorado & Barton $\mathrm{Cr}$ & $\begin{array}{l}\text { Loop } 360(08155300) \text { to above } \\
\text { Barton Springs }\end{array}$ & $4 / 28 / 1981$ & 3.5 & 5 & 5 & Edwards & -5.43 & -1.551 & $\begin{array}{l}\text { Slade and others } \\
\text { (1982) }\end{array}$ \\
\hline 34 & Colorado & Barton $\mathrm{Cr}$ & $\begin{array}{l}\text { northeast of Henly to Barton } \\
\text { Springs }\end{array}$ & $7 / 6-8 / 1970$ & 49.5 & 27 & 24 & Edwards, Trinity & 99.07 & 2.001 & USGS (1972) \\
\hline 35 & Colorado & Barton $\mathrm{Cr}$ & $\begin{array}{l}\text { northeast of Henly to Barton } \\
\text { Springs }\end{array}$ & $10 / 1-3 / 1970$ & 49.5 & 27 & 24 & Edwards, Trinity & 82.44 & 1.665 & USGS (1972) \\
\hline 36 & Colorado & Barton $\mathrm{Cr}$ & $\begin{array}{l}\text { SH } 71(08155200) \text { to Barton } \\
\quad \text { Springs }(08155500)\end{array}$ & $5 / 29 / 1980$ & 21 & 17 & 16 & Edwards, Trinity & 11.02 & .525 & $\begin{array}{l}\text { Slade and others } \\
\text { (1982) }\end{array}$ \\
\hline 37 & Colorado & Beals Cr & FM 87 to $0.5 \mathrm{mi}$ above mouth & 2/24/1986 & 58.7 & 9 & 8 & Ogallala & -.04 & -.001 & unpublished \\
\hline 38 & Colorado & Beals Cr & FM 87 to $0.5 \mathrm{mi}$ above mouth & $12 / 9-10 / 1986$ & 58.7 & 9 & 8 & Ogallala & 2.85 & .049 & unpublished \\
\hline 39 & Colorado & Beals Cr & US 87 to $0.5 \mathrm{mi}$ above mouth & $2 / 27-3 / 1 / 1989$ & 58.7 & 8 & 7 & Ogallala & 3.88 & .066 & unpublished \\
\hline 40 & Colorado & Bear Cr & $\begin{array}{l}\text { southeast of FM } 1826(08158810) \\
\text { to above MoPac RR }\end{array}$ & $5 / 23 / 1980$ & 10.1 & 10 & 8 & Edwards, Trinity & -3.8 & -.376 & $\begin{array}{l}\text { Slade and others } \\
\quad(1982)\end{array}$ \\
\hline 41 & Colorado & Brady $\mathrm{Cr}$ & Brady to mouth & $3 / 29 / 1918$ & 28 & 3 & 3 & -- & 1.7 & .061 & TBWE (1960) \\
\hline 42 & Colorado & Colorado R & $\begin{array}{l}\text { below Lake J.B. Thomas dam to FM } \\
503(08136700)\end{array}$ & $2 / 24-26 / 1986$ & 239.1 & 60 & 28 & -- & 1.89 & .008 & unpublished \\
\hline
\end{tabular}


Table 1. Characteristics of flow gain-loss studies in Texas-Continued

\begin{tabular}{|c|c|c|c|c|c|c|c|c|c|c|c|}
\hline $\begin{array}{l}\text { Stream- } \\
\text { flow } \\
\text { study } \\
\text { no. }\end{array}$ & $\begin{array}{l}\text { Major } \\
\text { river } \\
\text { basin }\end{array}$ & $\begin{array}{c}\text { Stream } \\
\text { name }\end{array}$ & $\begin{array}{c}\text { Reach } \\
\text { identification }\end{array}$ & $\begin{array}{l}\text { Date of } \\
\text { study }\end{array}$ & $\begin{array}{c}\text { Reach } \\
\text { length } \\
\text { (river } \\
\text { mi) }\end{array}$ & $\begin{array}{c}\text { Total } \\
\text { no. of } \\
\text { measure- } \\
\text { ment } \\
\text { sites }\end{array}$ & $\begin{array}{c}\text { No. of } \\
\text { measure- } \\
\text { ment sites } \\
\text { on main } \\
\text { channel }\end{array}$ & $\begin{array}{c}\text { Major aquifer } \\
\text { outcrop(s) } \\
\text { intersected } \\
\text { by reach }\end{array}$ & $\begin{array}{c}\text { Total } \\
\text { gain or } \\
\text { loss }(-) \\
\text { in reach } \\
\left(\mathrm{ft}^{3} / \mathbf{s}\right)\end{array}$ & $\begin{array}{c}\text { Gain or } \\
\text { loss per } \\
\text { mile of } \\
\text { reach } \\
\left(\mathrm{ft}^{3} / \mathrm{s}-\mathrm{mi}\right)\end{array}$ & $\begin{array}{l}\text { Reference } \\
\text { for data }\end{array}$ \\
\hline 43 & Colorado & Colorado $\mathrm{R}$ & $\begin{array}{l}\text { below Lake J.B. Thomas dam to FM } \\
503(08136700)\end{array}$ & $1 / 6-9 / 1987$ & 239.1 & 60 & 28 & -- & -23.64 & -0.099 & unpublished \\
\hline 44 & Colorado & Colorado R & $\begin{array}{l}\text { below Lake J.B. Thomas dam to FM } \\
503(08136700)\end{array}$ & 2/27-3/1/1989 & 239.1 & 60 & 28 & -- & -5.41 & -.023 & unpublished \\
\hline 45 & Colorado & Colorado R & $\begin{array}{l}\text { Bull Cr to Colorado City } \\
\quad(08121000)\end{array}$ & $2 / 14 / 1975$ & 35.5 & 19 & 12 & -- & 7.98 & .225 & USGS (1976) \\
\hline 46 & Colorado & Colorado R & $\begin{array}{l}\text { Bull Cr to Colorado City } \\
\quad(08121000)\end{array}$ & $11 / 13 / 1975$ & 35.5 & 19 & 12 & -- & 4.96 & .14 & USGS (1977) \\
\hline 47 & Colorado & Colorado R & $\begin{array}{l}\text { Bull Cr to Colorado City } \\
\quad(08121000)\end{array}$ & $1 / 20 / 1976$ & 35.5 & 19 & 12 & -- & -2.24 & -.063 & USGS (1977) \\
\hline 48 & Colorado & Colorado R & $\begin{array}{l}\text { Bull Cr to Colorado City } \\
\quad(08121000)\end{array}$ & $3 / 2 / 1976$ & 35.5 & 19 & 12 & -- & -2.25 & -.063 & USGS (1977) \\
\hline 49 & Colorado & Colorado R & $\begin{array}{l}\text { Austin (08158000) to near Bay City } \\
\quad(08162500)\end{array}$ & $8 / 19-21 / 1985$ & 257.6 & 19 & 12 & $\begin{array}{l}\text { Carrizo-Wilcox, Gulf } \\
\text { Coast }\end{array}$ & $-1,634.24$ & -6.344 & USGS (1986) \\
\hline 50 & Colorado & Colorado R & $\begin{array}{l}\text { near Silver }(08123900) \text { to Robert } \\
\text { Lee }(08124000)\end{array}$ & $12 / 14 / 1966$ & 34.7 & 4 & 4 & -- & -2.97 & -.086 & USGS (1968) \\
\hline 51 & Colorado & Colorado R & $\begin{array}{l}\text { near Silver }(08123900) \text { to Robert } \\
\text { Lee }(08124000)\end{array}$ & $3 / 21 / 1967$ & 34.7 & 10 & 10 & -- & -2.69 & -.078 & USGS (1968) \\
\hline 52 & Colorado & Colorado R & $\begin{array}{l}\text { near Vincent }(08118000) \text { to near } \\
\quad \text { Silver }(08123900)\end{array}$ & $4 / 8 / 1968$ & 103.2 & 32 & 22 & -- & -.28 & -.003 & USGS (1969) \\
\hline 53 & Colorado & Colorado R & $\begin{array}{l}\text { Robert Lee to Barton Springs } \\
\quad(08155500)\end{array}$ & $4 / 7-24 / 1925$ & 365 & 106 & 37 & Edwards, Trinity & 96.53 & .264 & TBWE (1960) \\
\hline 54 & Colorado & Colorado R & Robert Lee to mouth & $8 / 7-14 / 1918$ & 593 & 117 & 43 & $\begin{array}{l}\text { Carrizo-Wilcox, } \\
\text { Edwards, Gulf } \\
\text { Coast, Trinity }\end{array}$ & 340.6 & .574 & TBWE (1960) \\
\hline 55 & Colorado & Concho R & $\begin{array}{l}\text { confluence of North and South } \\
\text { Concho (08136000) to FM } 1929\end{array}$ & $2 / 25-26 / 1986$ & 54.7 & 10 & 6 & -- & 7.25 & .133 & unpublished \\
\hline 56 & Colorado & Concho R & $\begin{array}{l}\text { confluence of North and South } \\
\text { Concho (08136000) to FM } 1929\end{array}$ & $1 / 6-7 / 1987$ & 54.7 & 10 & 6 & -- & 36.23 & .662 & unpublished \\
\hline 57 & Colorado & Concho R & $\begin{array}{l}\text { confluence of North and South } \\
\text { Concho (08136000) to FM } 1929\end{array}$ & 2/28-3/1/1989 & 54.7 & 10 & 6 & -- & 37.8 & .691 & unpublished \\
\hline 58 & Colorado & Concho R & $\begin{array}{l}\text { confluence of North and South } \\
\text { Concho to mouth }\end{array}$ & $3 / 27-28 / 1918$ & 51.5 & 14 & 7 & -- & 4.6 & .089 & TBWE (1960) \\
\hline 59 & Colorado & Concho R & $\begin{array}{l}\text { confluence of North and South } \\
\text { Concho to mouth }\end{array}$ & $3 / 17-20 / 1925$ & 54.5 & 28 & 12 & -- & 6.6 & .121 & TBWE (1960) \\
\hline 60 & Colorado & Dove $\mathrm{Cr}$ & $9 \mathrm{mi}$ above Knickerbocker to mouth & $3 / 4-5 / 1925$ & 11.8 & 8 & 5 & $\begin{array}{l}\text { Edwards-Trinity } \\
\quad \text { (Plateau) }\end{array}$ & 15.7 & 1.331 & TBWE (1960) \\
\hline 61 & Colorado & Elm Cr & $\begin{array}{l}\text { FM } 1770 \text { to north of Ballinger } \\
\quad(08127000)\end{array}$ & $2 / 26 / 1986$ & 18.4 & 9 & 7 & -- & -.53 & -.029 & unpublished \\
\hline 62 & Colorado & Elm Cr & $\begin{array}{l}\text { FM } 1770 \text { to north of Ballinger } \\
(08127000)\end{array}$ & $1 / 7 / 1987$ & 18.4 & 9 & 7 & -- & 12.7 & .69 & unpublished \\
\hline
\end{tabular}


Table 1. Characteristics of flow gain-loss studies in Texas-Continued

\begin{tabular}{|c|c|c|c|c|c|c|c|c|c|c|c|}
\hline $\begin{array}{l}\text { Stream- } \\
\text { flow } \\
\text { study } \\
\text { no. }\end{array}$ & $\begin{array}{l}\text { Major } \\
\text { river } \\
\text { basin }\end{array}$ & $\begin{array}{l}\text { Stream } \\
\text { name }\end{array}$ & $\begin{array}{c}\text { Reach } \\
\text { identification }\end{array}$ & $\begin{array}{l}\text { Date of } \\
\text { study }\end{array}$ & $\begin{array}{c}\text { Reach } \\
\text { length } \\
\text { (river } \\
\text { mi) }\end{array}$ & $\begin{array}{c}\text { Total } \\
\text { no. of } \\
\text { measure- } \\
\text { ment } \\
\text { sites }\end{array}$ & $\begin{array}{l}\text { No. of } \\
\text { measure- } \\
\text { ment sites } \\
\text { on main } \\
\text { channel }\end{array}$ & $\begin{array}{c}\text { Major aquifer } \\
\text { outcrop(s) } \\
\text { intersected } \\
\text { by reach }\end{array}$ & $\begin{array}{c}\text { Total } \\
\text { gain or } \\
\text { loss }(-) \\
\text { in reach } \\
\left(\mathrm{ft}^{3} / \mathrm{s}\right)\end{array}$ & $\begin{array}{c}\text { Gain or } \\
\text { loss per } \\
\text { mile of } \\
\text { reach } \\
\left(\mathrm{ft}^{3} / \mathrm{s}-\mathrm{mi}\right)\end{array}$ & $\begin{array}{l}\text { Reference } \\
\text { for data }\end{array}$ \\
\hline 63 & Colorado & $\mathrm{Elm} \mathrm{Cr}$ & $\begin{array}{l}\text { FM } 1770 \text { to north of Ballinger } \\
(08127000)\end{array}$ & $3 / 1 / 1989$ & 18.4 & 9 & 7 & -- & 0.68 & 0.037 & unpublished \\
\hline 64 & Colorado & Johnson Fk Llano R & headwater springs to mouth & $2 / 6-15 / 1925$ & 16.8 & 9 & 8 & $\begin{array}{l}\text { Edwards-Trinity } \\
\quad \text { (Plateau) }\end{array}$ & 8.9 & .53 & TBWE (1960) \\
\hline 65 & Colorado & Llano R & $\begin{array}{l}\text { confluence of North and South } \\
\text { Llano to Llano (08151500) }\end{array}$ & $1 / 17-24 / 1962$ & 83.5 & 56 & 20 & $\begin{array}{l}\text { Edwards-Trinity } \\
\quad \text { (Plateau) }\end{array}$ & 7.84 & .094 & USGS (1963) \\
\hline 66 & Colorado & Llano R & $\begin{array}{l}\text { Junction gaging station to Llano } \\
(08151500)\end{array}$ & $9 / 3-4 / 1952$ & 79 & 12 & 10 & $\begin{array}{l}\text { Edwards-Trinity } \\
\quad \text { (Plateau) }\end{array}$ & -18.83 & -.238 & TBWE (1960) \\
\hline 67 & Colorado & Llano R & Junction to mouth & $3 / 31-4 / 3 / 1918$ & 106 & 26 & 13 & $\begin{array}{l}\text { Edwards-Trinity } \\
\quad \text { (Plateau) }\end{array}$ & 22.3 & .21 & TBWE (1960) \\
\hline 68 & Colorado & Llano R & $\begin{array}{l}\text { confluence of North and South } \\
\text { Llano to mouth }\end{array}$ & $2 / 14-21 / 1925$ & 105 & 23 & 17 & $\begin{array}{l}\text { Edwards-Trinity } \\
\quad \text { (Plateau) }\end{array}$ & 5.7 & .054 & TBWE (1960) \\
\hline 69 & Colorado & Middle Concho R & Kiowa $\mathrm{Cr}$ to mouth & $3 / 10-11 / 1925$ & 47.7 & 14 & 13 & -- & -.8 & -.017 & TBWE (1960) \\
\hline 70 & Colorado & Middle Concho R & San Angelo-Mertzon Rd to mouth & $3 / 27-28 / 1918$ & 22 & 2 & 2 & -- & 3.9 & .177 & TBWE (1960) \\
\hline 71 & Colorado & North Concho R & $\begin{array}{l}13 \text { mi above Sterling City to } \\
\text { confluence with South Concho R }\end{array}$ & $5 / 25-26 / 1918$ & 78 & 40 & 29 & -- & 5.1 & .065 & TBWE (1960) \\
\hline 72 & Colorado & North Concho R & $\begin{array}{l}\text { above Sterling City to confluence } \\
\text { with South Concho R }\end{array}$ & $2 / 28-3 / 17 / 1925$ & 55.3 & 13 & 12 & -- & 2.3 & .042 & TBWE (1960) \\
\hline 73 & Colorado & North Concho R & $\begin{array}{l}\text { Sterling City to confluence with } \\
\text { South Concho R }\end{array}$ & $3 / 27 / 1918$ & 48 & 9 & 7 & -- & -1.1 & -.023 & TBWE (1960) \\
\hline 74 & Colorado & North Llano R & $10 \mathrm{mi}$ above Roosevelt to Junction & $2 / 7-14 / 1925$ & 31.8 & 14 & 10 & $\begin{array}{l}\text { Edwards-Trinity } \\
\quad \text { (Plateau) }\end{array}$ & 6.9 & .217 & TBWE (1960) \\
\hline 75 & Colorado & Onion $\mathrm{Cr}$ & FM 12 to mouth & $4 / 23-24 / 1958$ & 59.2 & 21 & 9 & Edwards, Trinity & -24.9 & -.421 & TBWE (1960) \\
\hline 76 & Colorado & Onion $\mathrm{Cr}$ & $\begin{array}{l}\text { FM } 150(08158700) \text { to US } 183 \\
\quad(08159000)\end{array}$ & $5 / 28 / 1980$ & 35.4 & 19 & 14 & Edwards, Trinity & -82.7 & -2.336 & $\begin{array}{l}\text { Slade and others } \\
\text { (1982) }\end{array}$ \\
\hline 77 & Colorado & Pedernales R & $\begin{array}{l}\text { below Harper to near Johnson City } \\
\quad(08153500)\end{array}$ & $1 / 9-17 / 1956$ & 70 & 69 & 39 & $\begin{array}{l}\text { Edwards-Trinity } \\
\text { (Plateau), Trinity }\end{array}$ & 4.76 & .068 & TBWE (1960) \\
\hline 78 & Colorado & Pedernales R & Harper to Hamilton Pool & $4 / 3-4 / 1962$ & 18 & 12 & 8 & $\begin{array}{l}\text { Edwards-Trinity } \\
\quad \text { (Plateau), Trinity }\end{array}$ & .46 & .026 & USGS (1963) \\
\hline 79 & Colorado & Pedernales R & Harper to Hamilton Pool & $5 / 15-21 / 1962$ & 88.7 & 43 & 23 & Trinity & 12.31 & .139 & USGS (1963) \\
\hline 80 & Colorado & San Saba R & above Fort McKavett to Brady $\mathrm{Cr}$ & $7 / 27-29 / 1933$ & 80.1 & 32 & 18 & $\begin{array}{l}\text { Edwards-Trinity } \\
\quad \text { (Plateau) }\end{array}$ & 7.7 & .096 & TBWE (1960) \\
\hline 81 & Colorado & San Saba R & $\begin{array}{l}\text { below Fort McKavett to Menard } \\
\quad(08144500)\end{array}$ & $2 / 20-21 / 1940$ & 20.9 & 13 & 6 & $\begin{array}{l}\text { Edwards-Trinity } \\
\quad \text { (Plateau) }\end{array}$ & 4.0 & .191 & TBWE (1960) \\
\hline 82 & Colorado & San Saba R & $\begin{array}{l}\text { near Dorans Ranch to San Saba } \\
\quad(08146000)\end{array}$ & $11 / 17-18 / 1921$ & 14 & 6 & 2 & -- & -.9 & -.064 & TBWE (1960) \\
\hline 83 & Colorado & San Saba R & near Fort McKavett to mouth & $3 / 29-31 / 1918$ & 105 & 29 & 11 & $\begin{array}{l}\text { Edwards-Trinity } \\
\quad \text { (Plateau) }\end{array}$ & -2.5 & -.024 & TBWE (1960) \\
\hline 84 & Colorado & Slaughter $\mathrm{Cr}$ & $\begin{array}{l}\text { Bear Cr below FM } 1826 \\
(08158810) \text { to FM } 2304 \\
(08158860)\end{array}$ & $3 / 5 / 1981$ & 7.4 & 6 & 6 & Edwards, Trinity & -54.7 & -7.392 & $\begin{array}{l}\text { Slade and others } \\
\text { (1982) }\end{array}$ \\
\hline
\end{tabular}


Table 1. Characteristics of flow gain-loss studies in Texas-Continued

\begin{tabular}{|c|c|c|c|c|c|c|c|c|c|c|c|}
\hline $\begin{array}{l}\text { Stream- } \\
\text { flow } \\
\text { study } \\
\text { no. }\end{array}$ & $\begin{array}{l}\text { Major } \\
\text { river } \\
\text { basin }\end{array}$ & $\begin{array}{c}\text { Stream } \\
\text { name }\end{array}$ & $\begin{array}{c}\text { Reach } \\
\text { identification }\end{array}$ & $\begin{array}{l}\text { Date of } \\
\text { study }\end{array}$ & $\begin{array}{c}\text { Reach } \\
\text { length } \\
\text { (river } \\
\text { mi) }\end{array}$ & $\begin{array}{c}\text { Total } \\
\text { no. of } \\
\text { measure- } \\
\text { ment } \\
\text { sites }\end{array}$ & $\begin{array}{c}\text { No. of } \\
\text { measure- } \\
\text { ment sites } \\
\text { on main } \\
\text { channel }\end{array}$ & $\begin{array}{c}\text { Major aquifer } \\
\text { outcrop(s) } \\
\text { intersected } \\
\text { by reach }\end{array}$ & $\begin{array}{c}\text { Total } \\
\text { gain or } \\
\text { loss }(-) \\
\text { in reach } \\
\left(\mathrm{ft}^{3} / \mathrm{s}\right)\end{array}$ & $\begin{array}{c}\text { Gain or } \\
\text { loss per } \\
\text { mile of } \\
\text { reach } \\
\left(\mathrm{ft}^{3} / \mathrm{s}-\mathrm{mi}\right)\end{array}$ & $\begin{array}{l}\text { Reference } \\
\text { for data }\end{array}$ \\
\hline 85 & Colorado & Slaughter $\mathrm{Cr}$ & $\begin{array}{l}\text { FM } 1826(08158840) \text { to } \\
\text { downstream from Chappell Ln }\end{array}$ & $5 / 22 / 1980$ & 8.8 & 11 & 10 & Edwards, Trinity & -10.45 & -1.188 & $\begin{array}{l}\text { Slade and others } \\
\text { (1982) }\end{array}$ \\
\hline 86 & Colorado & South Concho R & above Christoval to mouth & $3 / 12-16 / 1925$ & 24.8 & 12 & 5 & $\begin{array}{l}\text { Edwards-Trinity } \\
\quad \text { (Plateau) }\end{array}$ & 19.6 & .79 & TBWE (1960) \\
\hline 87 & Colorado & South Concho R & $\begin{array}{l}\text { above Middle Concho to } 3.5 \mathrm{mi} \\
\text { below Middle Concho }\end{array}$ & $4 / 27 / 1918$ & 1 & 3 & 2 & -- & 2.2 & 2.2 & TBWE (1960) \\
\hline 88 & Colorado & South Concho R & $\begin{array}{l}\text { Christoval to confluence with North } \\
\text { Concho R }\end{array}$ & $3 / 27-28 / 1918$ & 19.8 & 13 & 7 & $\begin{array}{l}\text { Edwards-Trinity } \\
\quad \text { (Plateau) }\end{array}$ & 13.3 & 672 & TBWE (1960) \\
\hline 89 & Colorado & South Concho R & $\begin{array}{l}\text { south of Christoval to above Lake } \\
\text { Nasworthy }\end{array}$ & $6 / 18 / 1953$ & 12 & 6 & 5 & $\begin{array}{l}\text { Edwards-Trinity } \\
\quad \text { (Plateau) }\end{array}$ & -4.64 & -.387 & TBWE (1960) \\
\hline 90 & Colorado & South Llano R & $\begin{array}{l}\text { above Telegraph to confluence with } \\
\text { North Llano R }\end{array}$ & $4 / 1 / 1918$ & 18.5 & 5 & 2 & $\begin{array}{l}\text { Edwards-Trinity } \\
\quad \text { (Plateau) }\end{array}$ & 2.3 & .124 & TBWE (1960) \\
\hline 91 & Colorado & South Llano R & $\begin{array}{l}\text { below confluence of West and South } \\
\text { Fk to mouth }\end{array}$ & $2 / 10-14 / 1925$ & 25.9 & 12 & 9 & $\begin{array}{l}\text { Edwards-Trinity } \\
\quad \text { (Plateau) }\end{array}$ & 26.7 & 1.031 & TBWE (1960) \\
\hline 92 & Colorado & Spring $\mathrm{Cr}$ & above Mertzon to mouth & $3 / 6-11 / 1925$ & 26.5 & 17 & 12 & $\begin{array}{l}\text { Edwards-Trinity } \\
\quad \text { (Plateau) }\end{array}$ & 25.6 & .966 & TBWE (1960) \\
\hline 93 & Colorado & Spring $\mathrm{Cr}$ & above Seven Springs to mouth & $3 / 27 / 1918$ & 27 & 15 & 10 & $\begin{array}{l}\text { Edwards-Trinity } \\
\quad \text { (Plateau) }\end{array}$ & 10.5 & .389 & TBWE (1960) \\
\hline 94 & Colorado & Williamson $\mathrm{Cr}$ & $\begin{array}{l}\text { east of the "Y" (US 290/SH 71) in } \\
\text { Oak Hill (08158920) to FM } 2304 \\
\text { (08158930) }\end{array}$ & $3 / 5 / 1981$ & 5.4 & 6 & 5 & -- & -12.23 & -2.265 & $\begin{array}{l}\text { Slade and others } \\
\text { (1982) }\end{array}$ \\
\hline 95 & Colorado & Williamson $\mathrm{Cr}$ & $\begin{array}{l}\text { Old Oak Hill-Bee Cave Rd to } \\
\text { Jimmy Clay Rd (08158970) }\end{array}$ & $5 / 20 / 1980$ & 13.5 & 16 & 14 & Edwards, Trinity & -4.88 & -.361 & $\begin{array}{l}\text { Slade and others } \\
\text { (1982) }\end{array}$ \\
\hline 96 & Colorado & Wolf $\mathrm{Cr}$ & $12.1 \mathrm{mi}$ above mouth to mouth & $1 / 16 / 1956$ & 12.1 & 10 & 5 & Trinity & 1.9 & .157 & TBWE (1960) \\
\hline 97 & Guadalupe & Blanco R & $\begin{array}{l}10 \text { mi upstream of Wimberley to } \\
\text { northeast of San Marcos }\end{array}$ & $3 / 15-16 / 1955$ & 33.3 & 7 & 6 & Edwards, Trinity & -10.3 & -.309 & TBWE (1960) \\
\hline 98 & Guadalupe & Blanco R & $\begin{array}{l}9 \text { mi below Blanco to Wimberley } \\
(08171000)\end{array}$ & $2 / 25-3 / 4 / 1963$ & 27.1 & 28 & 20 & Trinity & 25.9 & .956 & USGS (1964) \\
\hline 99 & Guadalupe & Blanco R & above Halifax Cr to near Kyle & $7 / 22 / 1924$ & 4.2 & 3 & 3 & Edwards & -13.1 & -3.119 & TBWE (1960) \\
\hline 100 & Guadalupe & Blanco R & $\begin{array}{l}\text { upstream from Little Blanco R to } \\
\text { northeast of San Marcos }\end{array}$ & $1 / 24-28 / 1955$ & 49.6 & 33 & 30 & Edwards, Trinity & -2.65 & -.053 & TBWE (1960) \\
\hline 101 & Guadalupe & Blanco R & $\begin{array}{l}\text { Wimberley }(08171000) \text { to Kyle } \\
(08171300)\end{array}$ & $7 / 10-14 / 1957$ & 16.2 & 8 & 7 & Edwards, Trinity & -13.9 & -.858 & TBWE (1960) \\
\hline 102 & Guadalupe & Blanco R & Wimberley to near Kyle & $6 / 12 / 1924$ & 19.4 & 3 & 3 & Edwards, Trinity & 14.0 & .722 & TBWE (1960) \\
\hline 103 & Guadalupe & Blanco R & Wimberley to near Kyle & $7 / 15-16 / 1924$ & 19.4 & 4 & 4 & Edwards, Trinity & -12.8 & -.66 & TBWE (1960) \\
\hline 104 & Guadalupe & Cibolo $\mathrm{Cr}$ & $\begin{array}{l}\text { Schertz to San Antonio R near Falls } \\
\text { City }(08183500)\end{array}$ & $9 / 12-13 / 1949$ & 62 & 10 & 7 & Carrizo-Wilcox & 13.95 & .225 & TBWE (1960) \\
\hline 105 & Guadalupe & Cypress Cr & FM 1341 to mouth & $3 / 25 / 1965$ & 10 & 4 & 4 & Trinity & 3.35 & .335 & USGS (1966) \\
\hline 106 & Guadalupe & Guadalupe R & $\begin{array}{l}2.5 \text { mi below Spring Branch } \\
(08167500) \text { to below Cranes Mill }\end{array}$ & $5 / 11 / 1955$ & 16.5 & 6 & 3 & Trinity & 63.5 & 3.848 & TBWE (1960) \\
\hline
\end{tabular}


Table 1. Characteristics of flow gain-loss studies in Texas-Continued

\begin{tabular}{|c|c|c|c|c|c|c|c|c|c|c|c|}
\hline $\begin{array}{l}\text { Stream- } \\
\text { flow } \\
\text { study } \\
\text { no. }\end{array}$ & $\begin{array}{l}\text { Major } \\
\text { river } \\
\text { basin }\end{array}$ & $\begin{array}{c}\text { Stream } \\
\text { name }\end{array}$ & $\begin{array}{c}\text { Reach } \\
\text { identification }\end{array}$ & $\begin{array}{l}\text { Date of } \\
\text { study }\end{array}$ & $\begin{array}{c}\text { Reach } \\
\text { length } \\
\text { (river } \\
\text { mi) }\end{array}$ & $\begin{array}{c}\text { Total } \\
\text { no. of } \\
\text { measure- } \\
\text { ment } \\
\text { sites }\end{array}$ & $\begin{array}{c}\text { No. of } \\
\text { measure- } \\
\text { ment sites } \\
\text { on main } \\
\text { channel }\end{array}$ & $\begin{array}{l}\text { Major aquifer } \\
\text { outcrop(s) } \\
\text { intersected } \\
\text { by reach }\end{array}$ & $\begin{array}{c}\text { Total } \\
\text { gain or } \\
\text { loss }(-) \\
\text { in reach } \\
\left(\mathrm{ft}^{3} / \mathrm{s}\right)\end{array}$ & $\begin{array}{l}\text { Gain or } \\
\text { loss per } \\
\text { mile of } \\
\text { reach } \\
\left(\mathrm{ft}^{3} / \mathrm{s}-\mathrm{mi}\right)\end{array}$ & $\begin{array}{l}\text { Reference } \\
\text { for data }\end{array}$ \\
\hline 107 & Guadalupe & Guadalupe R & Canyon Dam to 3.6 mi downstream & $4 / 9 / 1965$ & 3.5 & 9 & 6 & Trinity & -10.57 & -3.02 & USGS (1966) \\
\hline 108 & Guadalupe & Guadalupe R & Canyon Dam to $3.6 \mathrm{mi}$ downstream & $4 / 16 / 1965$ & 3.5 & 8 & 5 & Trinity & .3 & .086 & USGS (1966) \\
\hline 109 & Guadalupe & Guadalupe R & Canyon Dam to 3.6 mi downstream & $4 / 22 / 1965$ & 3.5 & 8 & 5 & Trinity & 2.81 & .803 & USGS (1966) \\
\hline 110 & Guadalupe & Guadalupe $\mathrm{R}$ & Comfort to New Braunfels & $1 / 16-19 / 1928$ & 108.7 & 25 & 13 & Edwards, Trinity & 13.2 & .121 & TBWE (1960) \\
\hline 111 & Guadalupe & Guadalupe $\mathrm{R}$ & Comfort to New Braunfels & $2 / 18-22 / 1929$ & 108.7 & 29 & 16 & Edwards, Trinity & 10.9 & .1 & TBWE (1960) \\
\hline 112 & Guadalupe & Guadalupe $\mathrm{R}$ & $\begin{array}{l}\text { confluence of North and South Fk to } \\
\text { Comfort (08167000) }\end{array}$ & $3 / 16 / 1965$ & .8 & 4 & 4 & Trinity & 22.2 & 27.75 & USGS (1966) \\
\hline 113 & Guadalupe & Guadalupe R & $\begin{array}{l}\text { confluence of North and South Fk to } \\
\text { Comfort }(08167000)\end{array}$ & $3 / 24-25 / 1965$ & 34 & 37 & 12 & Trinity & 47.42 & 1.395 & USGS (1966) \\
\hline 114 & Guadalupe & Guadalupe R & $\begin{array}{l}\text { near Spring Branch to New } \\
\text { Braunfels }\end{array}$ & $1 / 24-31 / 1955$ & 57 & 41 & 33 & Edwards, Trinity & -43.7 & -.767 & TBWE (1960) \\
\hline 115 & Guadalupe & Guadalupe $\mathrm{R}$ & $\begin{array}{l}\text { near Spring Branch to New } \\
\text { Braunfels }\end{array}$ & $2 / 28-3 / 2 / 1955$ & 57 & 15 & 13 & Edwards, Trinity & -1.0 & -.018 & TBWE (1960) \\
\hline 116 & Guadalupe & Guadalupe R & $\begin{array}{l}\text { near Spring Branch to New } \\
\text { Braunfels }\end{array}$ & $3 / 14-15 / 1955$ & 57 & 9 & 8 & Edwards, Trinity & 11.0 & .193 & TBWE (1960) \\
\hline 117 & Guadalupe & Guadalupe R & $\begin{array}{l}\text { near Spring Branch to New } \\
\text { Braunfels }\end{array}$ & 3/29-30/1955 & 67.3 & 10 & 9 & Edwards, Trinity & -1.4 & -.021 & TBWE (1960) \\
\hline 118 & Guadalupe & Guadalupe R & $\begin{array}{l}\text { near Spring Branch to New } \\
\text { Braunfels }\end{array}$ & $4 / 12-13 / 1955$ & 57 & 9 & 8 & Edwards, Trinity & -.3 & -.005 & TBWE (1960) \\
\hline 119 & Guadalupe & Guadalupe R & $\begin{array}{l}\text { near Spring Branch to New } \\
\text { Braunfels }\end{array}$ & 4/26-27/1955 & 57 & 9 & 8 & Edwards, Trinity & .8 & .014 & TBWE (1960) \\
\hline 120 & Guadalupe & Guadalupe R & $\begin{array}{l}\text { near Spring Branch to New } \\
\text { Braunfels }\end{array}$ & 5/9-10/1955 & 51.3 & 8 & 5 & Edwards, Trinity & -499.04 & -9.728 & TBWE (1960) \\
\hline 121 & Guadalupe & Guadalupe R & $\begin{array}{l}\text { near Spring Branch to New } \\
\text { Braunfels }\end{array}$ & $3 / 7-8 / 1962$ & 57 & 14 & 12 & Edwards, Trinity & 23.1 & .405 & USGS (1963) \\
\hline 122 & Guadalupe & Guadalupe R & $\begin{array}{l}\text { near Spring Branch to Wunderlich } \\
\text { Ranch }\end{array}$ & $1 / 17 / 1955$ & 15.1 & 7 & 6 & Trinity & -39.4 & -2.609 & TBWE (1960) \\
\hline 123 & Guadalupe & Johnson Cr & $\begin{array}{l}4.9 \text { mi northwest of Mountain } \\
\text { Home to near mouth }\end{array}$ & $3 / 15-16 / 1965$ & 19 & 16 & 6 & $\begin{array}{l}\text { Edwards-Trinity } \\
\text { (Plateau), Trinity }\end{array}$ & 9.33 & .491 & USGS (1966) \\
\hline 124 & Guadalupe & Medina Canal & $\begin{array}{l}\text { head of canal (08180000) to first } \\
\text { diversion lateral }\end{array}$ & $8 / 15 / 1969$ & 23.6 & 5 & 5 & Edwards & -4.0 & -.169 & USGS (1970) \\
\hline 125 & Guadalupe & Medina R & $\begin{array}{l}5.8 \text { mi above Medina to Turks Head } \\
\text { Mountain in Medina Lake }\end{array}$ & $9 / 6-7 / 1955$ & 45.7 & 26 & 16 & Trinity & -4.84 & -.106 & TBWE (1960) \\
\hline 126 & Guadalupe & Medina R & $\begin{array}{l}5 \text { mi above Lima to } 4 \text { mi below Pipe } \\
\text { Cr }\end{array}$ & $6 / 3-5 / 1925$ & 43.8 & 19 & 12 & Trinity & 2.2 & .05 & TBWE (1960) \\
\hline 127 & Guadalupe & Medina R & $\begin{array}{l}8.3 \mathrm{mi} \text { above Medina to } 1.5 \mathrm{mi} \\
\text { below Bandera-Medina Co line }\end{array}$ & $1 / 3-7 / 1955$ & 47.1 & 28 & 19 & Trinity & -.067 & -.014 & TBWE (1960) \\
\hline 128 & Guadalupe & Medina R & $\begin{array}{l}\text { Bandera to Turks Head Mountain in } \\
\text { Medina Lake }\end{array}$ & $2 / 17-18 / 1955$ & 20.4 & 12 & 7 & Trinity & -4.62 & -.226 & TBWE (1960) \\
\hline 129 & Guadalupe & Medina R & Main Dam to near Riomedina & $12 / 9 / 1924$ & 10.9 & 5 & 5 & Edwards, Trinity & 6.6 & .606 & TBWE (1960) \\
\hline
\end{tabular}


Table 1. Characteristics of flow gain-loss studies in Texas-Continued

\begin{tabular}{|c|c|c|c|c|c|c|c|c|c|c|c|}
\hline $\begin{array}{l}\text { Stream- } \\
\text { flow } \\
\text { study } \\
\text { no. }\end{array}$ & $\begin{array}{l}\text { Major } \\
\text { river } \\
\text { basin }\end{array}$ & $\begin{array}{c}\text { Stream } \\
\text { name }\end{array}$ & $\begin{array}{c}\text { Reach } \\
\text { identification }\end{array}$ & $\begin{array}{l}\text { Date of } \\
\text { study }\end{array}$ & $\begin{array}{c}\text { Reach } \\
\text { length } \\
\text { (river } \\
\text { mi) }\end{array}$ & $\begin{array}{c}\text { Total } \\
\text { no. of } \\
\text { measure- } \\
\text { ment } \\
\text { sites }\end{array}$ & $\begin{array}{l}\text { No. of } \\
\text { measure- } \\
\text { ment sites } \\
\text { on main } \\
\text { channel }\end{array}$ & $\begin{array}{l}\text { Major aquifer } \\
\text { outcrop(s) } \\
\text { intersected } \\
\text { by reach }\end{array}$ & $\begin{array}{c}\text { Total } \\
\text { gain or } \\
\text { loss }(-) \\
\text { in reach } \\
\left(\mathrm{ft}^{3} / \mathrm{s}\right)\end{array}$ & $\begin{array}{l}\text { Gain or } \\
\text { loss per } \\
\text { mile of } \\
\text { reach } \\
\left(\mathrm{ft}^{3} / \mathrm{s}-\mathrm{mi}\right)\end{array}$ & $\begin{array}{l}\text { Reference } \\
\text { for data }\end{array}$ \\
\hline 130 & Guadalupe & Medina $\mathrm{R}$ & $\begin{array}{l}\text { Medina Co Irrigation Co. diversion } \\
\text { dam to Losoya }\end{array}$ & $5 / 26-28 / 1925$ & 55.1 & 19 & 14 & $\begin{array}{l}\text { Carrizo-Wilcox, } \\
\text { Edwards }\end{array}$ & -3.2 & -0.058 & TBWE (1960) \\
\hline 131 & Guadalupe & $\begin{array}{l}\text { North Fk Guadalupe } \\
\quad \mathrm{R}\end{array}$ & $\begin{array}{l}\text { Kerr Wildlife Management Area to } \\
\text { confluence with South Fk } \\
\text { Guadalupe R }\end{array}$ & $3 / 15-16 / 1965$ & 20 & 18 & 10 & $\begin{array}{l}\text { Edwards-Trinity } \\
\text { (Plateau), Trinity }\end{array}$ & 15.32 & .766 & USGS (1966) \\
\hline 132 & Guadalupe & $\begin{array}{l}\text { South Fk Guadalupe } \\
\quad \mathrm{R}\end{array}$ & $\begin{array}{l}\text { Lynxhaven resort to confluence } \\
\text { with North Fk Guadalupe R }\end{array}$ & $3 / 15-16 / 1965$ & 12.2 & 15 & 9 & $\begin{array}{l}\text { Edwards-Trinity } \\
\text { (Plateau), Trinity }\end{array}$ & 11.86 & .972 & USGS (1966) \\
\hline 133 & Guadalupe & Turtle Cr & FM 1273 to FM 692 & $3 / 24-25 / 1965$ & 9.2 & 4 & 3 & Trinity & 11.96 & 1.3 & USGS (1966) \\
\hline 134 & Guadalupe & Verde $\mathrm{Cr}$ & 7.7 mi southwest of Center Point & $3 / 25 / 1965$ & 10.4 & 6 & 3 & Trinity & 6.93 & .666 & USGS (1966) \\
\hline 135 & Lavaca & Lavaca $\mathrm{R}$ & $\begin{array}{l}\text { east of Hallettsville }(08163500) \text { to } \\
\text { southeast of Edna }\end{array}$ & $12 / 14-17 / 1970$ & 65.4 & 18 & 14 & Gulf Coast & 18.23 & .279 & USGS (1972) \\
\hline 136 & Lavaca & Lavaca $\mathrm{R}$ & near Edna & $11 / 4 / 1947$ & 9.5 & 7 & 5 & Gulf Coast & 5.7 & .6 & TBWE (1960) \\
\hline 137 & Lavaca & Lavaca R & near Edna & $8 / 5-6 / 1948$ & 9.5 & 7 & 5 & Gulf Coast & 4.48 & .472 & TBWE (1960) \\
\hline 138 & Lavaca & Navidad R & $\begin{array}{l}\text { east of Hallettsville ( } 08164300) \text { to } \\
\text { SH } 111 \text { bridge }\end{array}$ & $12 / 14-16 / 1970$ & 64.4 & 18 & 12 & Gulf Coast & 11.1 & .172 & USGS (1972) \\
\hline 139 & Neches & $\begin{array}{l}\text { West Fk Bowles } \\
\qquad \mathrm{Cr} \text {-[Bowles } \mathrm{Cr}]\end{array}$ & west of Old London to near Carlisle & $10 / 28 / 1942$ & 6.5 & 11 & 6 & Carrizo-Wilcox & 3.0 & .462 & TBWE (1960) \\
\hline 140 & Nueces & $\begin{array}{l}\text { Atascosa, Frio, and } \\
\text { Nueces R }\end{array}$ & $\begin{array}{l}3 \text { mi southwest of Poteet to near } \\
\text { Mathis }\end{array}$ & $1 / 23-26 / 1951$ & 103.8 & 29 & 14 & Gulf Coast & 4.83 & .047 & TBWE (1960) \\
\hline 141 & Nueces & $\begin{array}{l}\text { Atascosa, Frio, and } \\
\text { Nueces R }\end{array}$ & Campbellton to near Mathis & 4/19-21/1951 & 60.2 & 30 & 15 & Gulf Coast & 1.13 & .019 & TBWE (1960) \\
\hline 142 & Nueces & $\begin{array}{l}\text { Atascosa, Frio, and } \\
\text { Nueces R }\end{array}$ & Campbellton to near Mathis & $4 / 27-5 / 1 / 1951$ & 58.3 & 23 & 13 & Gulf Coast & -.22 & -.004 & TBWE (1960) \\
\hline 143 & Nueces & Dry Frio R & $\begin{array}{l}\text { above Real-Uvalde Co line to below } \\
\text { Reagan Wells (08196000) }\end{array}$ & $12 / 16-20 / 1954$ & 20 & 24 & 15 & $\begin{array}{l}\text { Edwards, Edwards- } \\
\text { Trinity (Plateau) }\end{array}$ & -.75 & -.038 & TBWE (1960) \\
\hline 144 & Nueces & Dry Frio R & $\begin{array}{l}\text { above Real-Uvalde Co line to below } \\
\text { Reagan Wells (08196000) }\end{array}$ & 9/9-13/1955 & 28 & 26 & 20 & $\begin{array}{l}\text { Edwards, Edwards- } \\
\text { Trinity (Plateau) }\end{array}$ & -2.18 & -.078 & TBWE (1960) \\
\hline 145 & Nueces & Dry Frio $\mathrm{R}$ & $\begin{array}{l}\text { above Real-Uvalde Co line to } \\
\text { Reagan Wells }(08196000)\end{array}$ & $1 / 15-20 / 1958$ & 26.1 & 35 & 13 & $\begin{array}{l}\text { Edwards, Edwards- } \\
\text { Trinity (Plateau) }\end{array}$ & 10.01 & .384 & TBWE (1960) \\
\hline 146 & Nueces & Dry Frio $\mathrm{R}$ & near Reagan Wells & $6 / 28 / 1925$ & 15.5 & 7 & 6 & $\begin{array}{l}\text { Edwards, Edwards- } \\
\text { Trinity (Plateau) }\end{array}$ & -1.0 & -.065 & TBWE (1960) \\
\hline 147 & Nueces & Dry Frio R & $\begin{array}{l}\text { Reagan Wells }(08196000) \text { to } 0.9 \mathrm{mi} \\
\quad \text { north of US } 90\end{array}$ & $6 / 18 / 1981$ & 24.6 & 6 & 6 & Edwards & -425.61 & -17.301 & $\begin{array}{l}\text { Land and others } \\
\quad \text { (1983) }\end{array}$ \\
\hline 148 & Nueces & East Frio R & $11 \mathrm{mi}$ above mouth to mouth & $1 / 5-6 / 1955$ & 11 & 12 & 9 & $\begin{array}{l}\text { Edwards-Trinity } \\
\quad \text { (Plateau) }\end{array}$ & -7.01 & -.637 & TBWE (1960) \\
\hline 149 & Nueces & East Frio R & $11 \mathrm{mi}$ above mouth to mouth & 9/7-9/1955 & 11 & 12 & 9 & $\begin{array}{l}\text { Edwards-Trinity } \\
\quad \text { (Plateau) }\end{array}$ & -4.11 & -.374 & TBWE (1960) \\
\hline 150 & Nueces & East Frio R & $5.2 \mathrm{mi}$ above mouth to mouth & $2 / 14 / 1955$ & 5.2 & 5 & 4 & $\begin{array}{l}\text { Edwards-Trinity } \\
\quad \text { (Plateau) }\end{array}$ & -6.95 & -1.337 & TBWE (1960) \\
\hline 151 & Nueces & East Frio R & $5.2 \mathrm{mi}$ above mouth to mouth & $7 / 9 / 1957$ & 5.2 & 5 & 4 & $\begin{array}{l}\text { Edwards-Trinity } \\
\quad \text { (Plateau) }\end{array}$ & -8.49 & -1.633 & TBWE (1960) \\
\hline
\end{tabular}


Table 1. Characteristics of flow gain-loss studies in Texas-Continued

\begin{tabular}{|c|c|c|c|c|c|c|c|c|c|c|c|}
\hline $\begin{array}{c}\text { Stream- } \\
\text { flow } \\
\text { study } \\
\text { no. }\end{array}$ & $\begin{array}{c}\text { Major } \\
\text { river } \\
\text { basin }\end{array}$ & $\begin{array}{l}\text { Stream } \\
\text { name }\end{array}$ & $\begin{array}{c}\text { Reach } \\
\text { identification }\end{array}$ & $\begin{array}{l}\text { Date of } \\
\text { study }\end{array}$ & $\begin{array}{l}\text { Reach } \\
\text { length } \\
\text { (river } \\
\text { mi) }\end{array}$ & $\begin{array}{c}\text { Total } \\
\text { no. of } \\
\text { measure- } \\
\text { ment } \\
\text { sites }\end{array}$ & $\begin{array}{c}\text { No. of } \\
\text { measure- } \\
\text { ment sites } \\
\text { on main } \\
\text { channel }\end{array}$ & $\begin{array}{c}\text { Major aquifer } \\
\text { outcrop(s) } \\
\text { intersected } \\
\text { by reach }\end{array}$ & $\begin{array}{c}\text { Total } \\
\text { gain or } \\
\text { loss }(-) \\
\text { in reach } \\
\left(\mathrm{ft}^{3} / \mathrm{s}\right)\end{array}$ & $\begin{array}{l}\text { Gain or } \\
\text { loss per } \\
\text { mile of } \\
\text { reach } \\
\left(\mathrm{ft}^{3} / \mathrm{s}-\mathrm{mi}\right)\end{array}$ & $\begin{array}{l}\text { Reference } \\
\text { for data }\end{array}$ \\
\hline 152 & Nueces & $\begin{array}{l}\text { East Frio R—[Frio } \\
\text { R] }\end{array}$ & $\begin{array}{l}11.8 \mathrm{mi} \text { above Leakey to } 7 \mathrm{mi} \text { below } \\
\text { Concan }\end{array}$ & $6 / 26-28 / 1925$ & 38.3 & 24 & 16 & $\begin{array}{l}\text { Edwards, Edwards- } \\
\text { Trinity (Plateau) }\end{array}$ & -17.71 & -0.462 & TBWE (1960) \\
\hline 153 & Nueces & $\begin{array}{l}\text { East Frio } \mathrm{R}-[\text { Frio } \\
\mathrm{R}]\end{array}$ & $\begin{array}{l}11 \mathrm{mi} \text { above Leakey to } 3.7 \mathrm{mi} \text { below } \\
\text { Concan }(08195000)\end{array}$ & $5 / 17-23 / 1954$ & 38.5 & 16 & 14 & $\begin{array}{l}\text { Edwards, Edwards- } \\
\text { Trinity (Plateau) }\end{array}$ & 1.57 & .041 & TBWE (1960) \\
\hline 154 & Nueces & Frio $\mathrm{R}$ & $\begin{array}{l}\text { Choke Canyon Reservoir to } \\
\text { Shamrock Refinery }\end{array}$ & $11 / 12-13 / 1991$ & 8.59 & 5 & 4 & Gulf Coast & .12 & .014 & unpublished \\
\hline 155 & Nueces & Frio $\mathrm{R}$ & $\begin{array}{l}\text { Concan }(08195000) \text { to near Uvalde } \\
\quad(08197500)\end{array}$ & $9 / 11 / 1980$ & 28.4 & 7 & 7 & Edwards & -200.14 & -7.047 & $\begin{array}{l}\text { Land and others } \\
\text { (1983) }\end{array}$ \\
\hline 156 & Nueces & Frio $\mathrm{R}$ & Concan (08195000) to near Knippa & $11 / 5-6 / 1932$ & 18.5 & 5 & 5 & Edwards & -233.0 & -12.595 & TBWE (1960) \\
\hline 157 & Nueces & Frio $\mathrm{R}$ & Concan (08195000) to near Knippa & $12 / 20 / 1932$ & 18.5 & 5 & 5 & Edwards & -124.0 & -6.703 & TBWE (1960) \\
\hline 158 & Nueces & Frio $\mathrm{R}$ & near Concan & 7/1/1931 & 6 & 5 & 5 & Edwards & -25.7 & -4.283 & TBWE (1960) \\
\hline 159 & Nueces & Frio $\mathrm{R}$ & near Fowlerton to mouth & $12 / 18-21 / 1967$ & 62.9 & 13 & 6 & Gulf Coast & 10.63 & .169 & USGS (1969) \\
\hline 160 & Nueces & Frio R & $\begin{array}{l}\text { Reagan Wells (08196000) to Uvalde } \\
\quad(08197500)\end{array}$ & $4 / 2 / 1981$ & 28.4 & 7 & 7 & Edwards & -259.18 & -9.126 & $\begin{array}{l}\text { Land and others } \\
\quad \text { (1983) }\end{array}$ \\
\hline 161 & Nueces & Frio $\mathrm{R}$ & $\begin{array}{l}\text { Reagan Wells (08196000) to Uvalde } \\
\quad(08197500)\end{array}$ & $5 / 6 / 1981$ & 28.4 & 7 & 7 & Edwards & -229.0 & -8.063 & $\begin{array}{l}\text { Land and others } \\
\text { (1983) }\end{array}$ \\
\hline 162 & Nueces & Hondo $\mathrm{Cr}$ & near headwaters to US 90 & $4 / 5-8 / 1958$ & 37.2 & 25 & 15 & Edwards, Trinity & -27.78 & -.747 & TBWE (1960) \\
\hline 163 & Nueces & Hondo $\mathrm{Cr}$ & $\begin{array}{l}\text { Tarpley }(08200000) \text { to } 3 \mathrm{mi} \\
\text { downstream from US } 90\end{array}$ & $3 / 27-28 / 1968$ & 29.5 & 13 & 12 & Edwards, Trinity & -98.0 & -3.322 & USGS (1969) \\
\hline 164 & Nueces & Hondo $\mathrm{Cr}$ & $\begin{array}{l}\text { Tarpley }(08200000) \text { to Hondo } \\
\quad(08200700)\end{array}$ & $5 / 27 / 1981$ & 18.9 & 6 & 6 & Edwards & -158.1 & -8.365 & $\begin{array}{l}\text { Land and others } \\
\text { (1983) }\end{array}$ \\
\hline 165 & Nueces & Leona $\mathrm{R}$ & $\begin{array}{l}1.7 \text { mi southeast of Uvalde to } 0.2 \mathrm{mi} \\
\text { east of Zavalla-Frio Co line }\end{array}$ & $2 / 5-8 / 1946$ & 49.4 & 35 & 32 & Carrizo-Wilcox & 2.0 & .04 & TBWE (1960) \\
\hline 166 & Nueces & Leona $\mathrm{R}$ & $\begin{array}{l}1.7 \mathrm{mi} \text { southeast of Uvalde to } 35 \mathrm{mi} \\
\text { southeast of Uvalde }\end{array}$ & $6 / 11-12 / 1931$ & 37.5 & 15 & 12 & Carrizo-Wilcox & -3.1 & -.083 & TBWE (1960) \\
\hline 167 & Nueces & Leona $\mathrm{R}$ & $\begin{array}{l}1.7 \text { mi southeast of Uvalde to } 7.1 \mathrm{mi} \\
\text { southeast of Batesville }\end{array}$ & $8 / 7-9 / 1946$ & 36.3 & 22 & 21 & Carrizo-Wilcox & .3 & .008 & TBWE (1960) \\
\hline 168 & Nueces & Leona $\mathrm{R}$ & $\begin{array}{l}1.7 \text { mi southeast of Uvalde to } 9.5 \mathrm{mi} \\
\text { southeast of Uvalde }\end{array}$ & $3 / 1 / 1947$ & 9.8 & 5 & 5 & Carrizo-Wilcox & 14.91 & 1.521 & TBWE (1960) \\
\hline 169 & Nueces & Leona $\mathrm{R}$ & $\begin{array}{l}1.7 \text { mi southeast of Uvalde to below } \\
\text { Batesville }\end{array}$ & $6 / 21-22 / 1934$ & 34.6 & 13 & 10 & Carrizo-Wilcox & -3.1 & -.09 & TBWE (1960) \\
\hline 170 & Nueces & Leona $\mathrm{R}$ & $\begin{array}{l}1.7 \text { mi southeast of Uvalde to below } \\
\text { Batesville }\end{array}$ & $10 / 18-20 / 1934$ & 34.6 & 14 & 11 & Carrizo-Wilcox & 2.4 & .069 & TBWE (1960) \\
\hline 171 & Nueces & Leona $\mathrm{R}$ & $\begin{array}{l}1.7 \text { mi southeast of Uvalde to below } \\
\text { Batesville }\end{array}$ & $7 / 5-6 / 1939$ & 23 & 14 & 11 & Carrizo-Wilcox & 4.3 & .187 & TBWE (1960) \\
\hline 172 & Nueces & Leona R & $\begin{array}{l}1.7 \text { mi southeast of Uvalde to near } \\
\text { Batesville }\end{array}$ & $11 / 7 / 1932$ & 17 & 6 & 4 & Carrizo-Wilcox & 21.2 & 1.247 & TBWE (1960) \\
\hline 173 & Nueces & Leona $\mathrm{R}$ & $\begin{array}{l}10 \text { mi below Uvalde to below } \\
\text { Batesville }\end{array}$ & 6/8-10/1939 & 26 & 10 & 8 & Carrizo-Wilcox & -3.8 & -.146 & TBWE (1960) \\
\hline
\end{tabular}


Table 1. Characteristics of flow gain-loss studies in Texas-Continued

\begin{tabular}{|c|c|c|c|c|c|c|c|c|c|c|c|}
\hline $\begin{array}{l}\text { Stream- } \\
\text { flow } \\
\text { study } \\
\text { no. }\end{array}$ & $\begin{array}{l}\text { Major } \\
\text { river } \\
\text { basin }\end{array}$ & $\begin{array}{c}\text { Stream } \\
\text { name }\end{array}$ & $\begin{array}{c}\text { Reach } \\
\text { identification }\end{array}$ & $\begin{array}{l}\text { Date of } \\
\text { study }\end{array}$ & $\begin{array}{c}\text { Reach } \\
\text { length } \\
\text { (river } \\
\text { mi) }\end{array}$ & $\begin{array}{c}\text { Total } \\
\text { no. of } \\
\text { measure- } \\
\text { ment } \\
\text { sites }\end{array}$ & $\begin{array}{l}\text { No. of } \\
\text { measure- } \\
\text { ment sites } \\
\text { on main } \\
\text { channel }\end{array}$ & $\begin{array}{c}\text { Major aquifer } \\
\text { outcrop(s) } \\
\text { intersected } \\
\text { by reach }\end{array}$ & $\begin{array}{c}\text { Total } \\
\text { gain or } \\
\text { loss }(-) \\
\text { in reach } \\
\left(\mathrm{ft}^{3} / \mathrm{s}\right)\end{array}$ & $\begin{array}{c}\text { Gain or } \\
\text { loss per } \\
\text { mile of } \\
\text { reach } \\
\left(\mathrm{ft}^{3} / \mathrm{s}-\mathrm{mi}\right)\end{array}$ & $\begin{array}{l}\text { Reference } \\
\text { for data }\end{array}$ \\
\hline 174 & Nueces & Leona $\mathrm{R}$ & $\begin{array}{l}\text { below Kincaid Dam to } 9.5 \mathrm{mi} \\
\text { southeast of Uvalde }\end{array}$ & $2 / 19 / 1946$ & 5.2 & 7 & 7 & Carrizo-Wilcox & 0.7 & 0.135 & TBWE (1960) \\
\hline 175 & Nueces & Leona $\mathrm{R}$ & $\begin{array}{l}\text { Uvalde-Friotown Hwy to near } \\
\text { Batesville }\end{array}$ & $4 / 25-28 / 1925$ & 33.5 & 14 & 11 & Carrizo-Wilcox & 15.89 & 474 & TBWE (1960) \\
\hline 176 & Nueces & Little Seco Cr & 9 mi above mouth to mouth & $4 / 3 / 1958$ & 9 & 7 & 7 & Edwards, Trinity & 0 & 0 & TBWE (1960) \\
\hline 177 & Nueces & $\begin{array}{l}\text { Middle Verde and } \\
\text { Verde } \mathrm{Cr}\end{array}$ & $\begin{array}{l}15.6 \text { mi north of Hondo to } 4.5 \mathrm{mi} \\
\text { north of Hondo }\end{array}$ & $4 / 24 / 1981$ & 16.5 & 10 & 8 & Edwards, Trinity & -116.0 & -7.03 & $\begin{array}{l}\text { Land and others } \\
\text { (1983) }\end{array}$ \\
\hline 178 & Nueces & $\begin{array}{l}\text { Middle Verde and } \\
\text { Verde } \mathrm{Cr}\end{array}$ & $\begin{array}{l}15.6 \mathrm{mi} \text { north of Hondo to } 4.5 \mathrm{mi} \\
\text { north of Hondo }\end{array}$ & $6 / 17 / 1981$ & 16.5 & 10 & 9 & Edwards, Trinity & -161.0 & -9.758 & $\begin{array}{l}\text { Land and others } \\
\text { (1983) }\end{array}$ \\
\hline 179 & Nueces & $\begin{array}{l}\text { Middle Verde and } \\
\text { Verde } \mathrm{Cr}\end{array}$ & $\begin{array}{l}15.6 \text { mi north of Hondo to } 4.5 \mathrm{mi} \\
\text { north of Hondo }\end{array}$ & $9 / 8 / 1981$ & 16.5 & 9 & 7 & Edwards, Trinity & -86.0 & -5.212 & $\begin{array}{l}\text { Land and others } \\
\text { (1983) }\end{array}$ \\
\hline 180 & Nueces & $\begin{array}{l}\text { Middle Verde and } \\
\text { Verde } \mathrm{Cr}\end{array}$ & $\begin{array}{l}5.5 \mathrm{mi} \text { above East Verde } \mathrm{Cr} \text { to } 4 \mathrm{mi} \\
\text { west of Quihi }\end{array}$ & $1 / 14 / 1958$ & 19.5 & 9 & 6 & Edwards, Trinity & -58.3 & -2.99 & TBWE (1960) \\
\hline 181 & Nueces & $\begin{array}{l}\text { Middle Verde and } \\
\text { Verde } \mathrm{Cr}\end{array}$ & $\begin{array}{l}\text { SH } 173 \text { on Middle Verde Cr to } \\
\text { Vandenburg community }\end{array}$ & 9/8/1980 & 16.5 & 11 & 8 & Edwards, Trinity & -86.0 & -5.212 & USGS (1981) \\
\hline 182 & Nueces & Nueces R & $\begin{array}{l}\text { above Laguna ( } 08190000) \text { to } 4.8 \mathrm{mi} \\
\text { southeast of La Pryor }\end{array}$ & $5 / 2-3 / 1940$ & 46.9 & 14 & 13 & $\begin{array}{l}\text { Carrizo-Wilcox, } \\
\text { Edwards }\end{array}$ & -63.8 & -1.36 & TBWE (1960) \\
\hline 183 & Nueces & Nueces R & $\begin{array}{l}\text { above Laguna ( } 08190000) \text { to } 4.8 \mathrm{mi} \\
\text { southeast of La Pryor }\end{array}$ & 7/9-10/1940 & 46.9 & 14 & 13 & $\begin{array}{l}\text { Carrizo-Wilcox, } \\
\text { Edwards }\end{array}$ & -66.7 & -1.422 & TBWE (1960) \\
\hline 184 & Nueces & Nueces R & $\begin{array}{l}\text { above Laguna ( } 08190000) \text { to } 4.8 \mathrm{mi} \\
\text { southeast of La Pryor }\end{array}$ & $8 / 28-29 / 1940$ & 46.8 & 14 & 13 & $\begin{array}{l}\text { Carrizo-Wilcox, } \\
\text { Edwards }\end{array}$ & -52.3 & -1.118 & TBWE (1960) \\
\hline 185 & Nueces & Nueces R & $\begin{array}{l}\text { above Laguna ( } 08190000) \text { to } 4.8 \mathrm{mi} \\
\text { southeast of La Pryor }\end{array}$ & $9 / 26-27 / 1940$ & 46.9 & 14 & 12 & $\begin{array}{l}\text { Carrizo-Wilcox, } \\
\text { Edwards }\end{array}$ & -27.9 & -.595 & TBWE (1960) \\
\hline 186 & Nueces & Nueces R & $\begin{array}{l}\text { Laguna }(08190000) \text { to Uvalde } \\
\quad(08192000)\end{array}$ & 8/10/1981 & 29.4 & 6 & 6 & Edwards & -24.0 & -.816 & $\begin{array}{l}\text { Land and others } \\
\text { (1983) }\end{array}$ \\
\hline 187 & Nueces & Nueces R & Barksdale to Laguna (08190000) & $12 / 13-15 / 1954$ & 25.2 & 37 & 17 & $\begin{array}{l}\text { Edwards-Trinity } \\
\quad \text { (Plateau) }\end{array}$ & -1.01 & -.04 & TBWE (1960) \\
\hline 188 & Nueces & Nueces R & Barksdale to Laguna (08190000) & 9/19-20/1955 & 25.2 & 35 & 19 & $\begin{array}{l}\text { Edwards-Trinity } \\
\quad \text { (Plateau) }\end{array}$ & 15.78 & .626 & TBWE (1960) \\
\hline 189 & Nueces & Nueces R & Barksdale to Laguna (08190000) & $7 / 8-10 / 1957$ & 25.2 & 29 & 17 & $\begin{array}{l}\text { Edwards-Trinity } \\
\quad \text { (Plateau) }\end{array}$ & 18.2 & .722 & TBWE (1960) \\
\hline 190 & Nueces & Nueces R & $\begin{array}{l}\text { below Mathis }(08211100) \text { to above } \\
\text { Calallen }(08211200)\end{array}$ & 2/9/1967 & 8.4 & 6 & 5 & Gulf Coast & 3.0 & .357 & USGS (1968) \\
\hline 191 & Nueces & Nueces R & $\begin{array}{l}\text { below Odley } \mathrm{Cr} \text { near Vance to } \mathrm{La} \\
\text { Pryor }\end{array}$ & $3 / 17-26 / 1924$ & 74.4 & 21 & 18 & $\begin{array}{l}\text { Carrizo-Wilcox, } \\
\text { Edwards, Edwards- } \\
\text { Trinity (Plateau) }\end{array}$ & 19.8 & .266 & TBWE (1960) \\
\hline 192 & Nueces & Nueces R & $\begin{array}{l}\text { Cotulla }(08194000) \text { to Simmons } \\
\quad(08194600)\end{array}$ & $7 / 28-30 / 1981$ & 108.1 & 13 & 10 & Gulf Coast & 7.6 & .07 & USGS (1982a) \\
\hline 193 & Nueces & Nueces R & $\begin{array}{l}\text { Cotulla }(08194000) \text { to Simmons } \\
\quad(08194600)\end{array}$ & $8 / 11-13 / 1981$ & 108.1 & 13 & 10 & Gulf Coast & 9.29 & .086 & USGS (1982a) \\
\hline
\end{tabular}


Table 1. Characteristics of flow gain-loss studies in Texas-Continued

\begin{tabular}{|c|c|c|c|c|c|c|c|c|c|c|c|}
\hline $\begin{array}{l}\text { Stream- } \\
\text { flow } \\
\text { study } \\
\text { no. }\end{array}$ & $\begin{array}{l}\text { Major } \\
\text { river } \\
\text { basin }\end{array}$ & $\begin{array}{l}\text { Stream } \\
\text { name }\end{array}$ & $\begin{array}{c}\text { Reach } \\
\text { identification }\end{array}$ & $\begin{array}{l}\text { Date of } \\
\text { study }\end{array}$ & $\begin{array}{c}\text { Reach } \\
\text { length } \\
\text { (river } \\
\text { mi) }\end{array}$ & $\begin{array}{c}\text { Total } \\
\text { no. of } \\
\text { measure- } \\
\text { ment } \\
\text { sites }\end{array}$ & $\begin{array}{l}\text { No. of } \\
\text { measure- } \\
\text { ment sites } \\
\text { on main } \\
\text { channel }\end{array}$ & $\begin{array}{c}\text { Major aquifer } \\
\text { outcrop(s) } \\
\text { intersected } \\
\text { by reach }\end{array}$ & $\begin{array}{c}\text { Total } \\
\text { gain or } \\
\text { loss }(-) \\
\text { in reach } \\
\left(\mathrm{ft}^{3} / \mathrm{s}\right)\end{array}$ & $\begin{array}{c}\text { Gain or } \\
\text { loss per } \\
\text { mile of } \\
\text { reach } \\
\left(\mathrm{ft}^{3} / \mathrm{s}-\mathrm{mi}\right)\end{array}$ & $\begin{array}{l}\text { Reference } \\
\text { for data }\end{array}$ \\
\hline 194 & Nueces & Nueces $R$ & $\begin{array}{l}\text { Laguna }(08190000) \text { to } 3.8 \mathrm{mi} \\
\text { southeast of Cinonia }\end{array}$ & $6 / 14-30 / 1939$ & 61.6 & 27 & 25 & $\begin{array}{l}\text { Carrizo-Wilcox, } \\
\text { Edwards }\end{array}$ & -23.7 & -0.385 & TBWE (1960) \\
\hline 195 & Nueces & Nueces R & $\begin{array}{l}\text { Laguna }(08190000) \text { to } 5 \mathrm{mi} \\
\text { northeast of La Pryor }\end{array}$ & $11 / 14-16 / 1931$ & 39.6 & 10 & 10 & $\begin{array}{l}\text { Carrizo-Wilcox, } \\
\text { Edwards }\end{array}$ & -60.2 & -1.52 & TBWE (1960) \\
\hline 196 & Nueces & Nueces R & $\begin{array}{l}\text { Laguna ( } 08190000) \text { to } 5 \mathrm{mi} \\
\text { northeast of La Pryor }\end{array}$ & $1 / 24-25 / 1932$ & 39.6 & 11 & 11 & $\begin{array}{l}\text { Carrizo-Wilcox, } \\
\text { Edwards }\end{array}$ & -59.5 & -1.503 & TBWE (1960) \\
\hline 197 & Nueces & Nueces $\mathrm{R}$ & Laguna (08190000) to Cinonia & $4 / 30-5 / 8 / 1925$ & 54.9 & 14 & 14 & $\begin{array}{l}\text { Carrizo-Wilcox, } \\
\text { Edwards }\end{array}$ & -29.9 & -.545 & TBWE (1960) \\
\hline 198 & Nueces & Nueces R & Laguna (08190000) to Cinonia & $5 / 16-17 / 1931$ & 56.5 & 11 & 11 & $\begin{array}{l}\text { Carrizo-Wilcox, } \\
\text { Edwards }\end{array}$ & -76.0 & -1.345 & TBWE (1960) \\
\hline 199 & Nueces & Nueces R & Laguna (08190000) to Cinonia & $6 / 4-6 / 1931$ & 53 & 10 & 10 & $\begin{array}{l}\text { Carrizo-Wilcox, } \\
\text { Edwards }\end{array}$ & -84.0 & -1.585 & TBWE (1960) \\
\hline 200 & Nueces & Nueces R & Laguna (08190000) to Cinonia & $6 / 15-17 / 1931$ & 56.5 & 12 & 12 & $\begin{array}{l}\text { Carrizo-Wilcox, } \\
\text { Edwards }\end{array}$ & -73.6 & -1.303 & TBWE (1960) \\
\hline 201 & Nueces & Nueces R & Laguna (08190000) to Cinonia & $6 / 22-24 / 1931$ & 56.5 & 12 & 12 & $\begin{array}{l}\text { Carrizo-Wilcox, } \\
\text { Edwards }\end{array}$ & -91.9 & -1.627 & TBWE (1960) \\
\hline 202 & Nueces & Nueces $\mathrm{R}$ & Laguna (08190000) to Cinonia & $7 / 2-4 / 1931$ & 56.5 & 12 & 12 & $\begin{array}{l}\text { Carrizo-Wilcox, } \\
\text { Edwards }\end{array}$ & -82.5 & -1.46 & TBWE (1960) \\
\hline 203 & Nueces & Nueces R & $\begin{array}{l}\text { Laguna }(08190000) \text { to Leona } \mathrm{R} \\
\text { springflow near Uvalde } \\
(08204000)\end{array}$ & $5 / 19 / 1931$ & 22.7 & 4 & 4 & Edwards & -79.0 & -3.48 & TBWE (1960) \\
\hline 204 & Nueces & Nueces R & $\begin{array}{l}\text { Laguna }(08190000) \text { to Leona } \mathrm{R} \\
\text { springflow near Uvalde } \\
(08204000)\end{array}$ & 7/9/1931 & 22.7 & 6 & 6 & Edwards & -77.3 & -3.405 & TBWE (1960) \\
\hline 205 & Nueces & Nueces R & $\begin{array}{l}\text { Laguna (08190000) to Leona R } \\
\text { springflow near Uvalde } \\
\text { (08204000) }\end{array}$ & $7 / 16-17 / 1931$ & 22.7 & 6 & 6 & Edwards & -73.0 & -3.216 & TBWE (1960) \\
\hline 206 & Nueces & Nueces $\mathrm{R}$ & Laguna (08190000) to near Cinonia & $11 / 1-4 / 1932$ & 56.5 & 14 & 14 & $\begin{array}{l}\text { Carrizo-Wilcox, } \\
\text { Edwards }\end{array}$ & 28.0 & .496 & TBWE (1960) \\
\hline 207 & Nueces & Nueces R & Laguna (08190000) to near Cinonia & $7 / 23-25 / 1933$ & 56.5 & 14 & 14 & $\begin{array}{l}\text { Carrizo-Wilcox, } \\
\text { Edwards }\end{array}$ & -8.7 & -.154 & TBWE (1960) \\
\hline 208 & Nueces & Nueces R & $\begin{array}{l}\text { Laguna (08190000) to SAU\&G RR } \\
\text { bridge }\end{array}$ & $8 / 29-30 / 1931$ & 31.6 & 8 & 8 & $\begin{array}{l}\text { Carrizo-Wilcox, } \\
\text { Edwards }\end{array}$ & -80.2 & -2.538 & TBWE (1960) \\
\hline 209 & Nueces & Nueces $\mathrm{R}$ & Mathis (08211000) to Calallen & $4 / 20-22 / 1948$ & 25.5 & 18 & 13 & Gulf Coast & -.7 & -.027 & TBWE (1960) \\
\hline 210 & Nueces & Nueces $\mathrm{R}$ & Uvalde $(08204000)$ to Cinonia & 7/13/1931 & 33.8 & 7 & 7 & Carrizo-Wilcox & 4.0 & .118 & TBWE (1960) \\
\hline 211 & Nueces & Nueces $\mathrm{R}$ & $\begin{array}{l}\text { Miller's Ranch to Nueces R at } \\
\text { Highway } 59\end{array}$ & $11 / 12-13 / 1991$ & 16.45 & 7 & 6 & Gulf Coast & 65.89 & 4.005 & unpublished \\
\hline 212 & Nueces & Nueces R & Mathis (08211000) to Calallen Dam & $8 / 28-30 / 1963$ & 25.8 & 13 & 13 & Gulf Coast & -15.0 & -.581 & USGS (1964) \\
\hline 213 & Nueces & Nueces R & Mathis (08211000) to Calallen Dam & $10 / 9-10 / 1963$ & 14.2 & 7 & 7 & Gulf Coast & -5.0 & -.352 & USGS (1964) \\
\hline 214 & Nueces & Nueces $\mathrm{R}$ & Mathis (08211000) to Calallen Dam & $2 / 21-24 / 1966$ & 20.5 & 16 & 12 & Gulf Coast & -8.31 & -.405 & USGS (1967) \\
\hline 215 & Nueces & Nueces R & Mathis $(08211000)$ to Calallen Dam & $8 / 16-18 / 1966$ & 20.5 & 16 & 12 & Gulf Coast & -4.12 & -.201 & USGS (1967) \\
\hline
\end{tabular}


Table 1. Characteristics of flow gain-loss studies in Texas-Continued

\begin{tabular}{|c|c|c|c|c|c|c|c|c|c|c|c|}
\hline $\begin{array}{l}\text { Stream- } \\
\text { flow } \\
\text { study } \\
\text { no. }\end{array}$ & $\begin{array}{l}\text { Major } \\
\text { river } \\
\text { basin }\end{array}$ & $\begin{array}{l}\text { Stream } \\
\text { name }\end{array}$ & $\begin{array}{c}\text { Reach } \\
\text { identification }\end{array}$ & $\begin{array}{l}\text { Date of } \\
\text { study }\end{array}$ & $\begin{array}{c}\text { Reach } \\
\text { length } \\
\text { (river } \\
\text { mi) }\end{array}$ & $\begin{array}{c}\text { Total } \\
\text { no. of } \\
\text { measure- } \\
\text { ment } \\
\text { sites }\end{array}$ & $\begin{array}{l}\text { No. of } \\
\text { measure- } \\
\text { ment sites } \\
\text { on main } \\
\text { channel }\end{array}$ & $\begin{array}{c}\text { Major aquifer } \\
\text { outcrop(s) } \\
\text { intersected } \\
\text { by reach }\end{array}$ & $\begin{array}{c}\text { Total } \\
\text { gain or } \\
\text { loss }(-) \\
\text { in reach } \\
\left(\mathrm{ft}^{3} / \mathrm{s}\right)\end{array}$ & $\begin{array}{c}\text { Gain or } \\
\text { loss per } \\
\text { mile of } \\
\text { reach } \\
\left(\mathrm{ft}^{3} / \mathrm{s}-\mathrm{mi}\right)\end{array}$ & $\begin{array}{l}\text { Reference } \\
\text { for data }\end{array}$ \\
\hline 216 & Nueces & Nueces R & near Vance to La Pryor Crossing & $8 / 11-18 / 1924$ & 66.1 & 13 & 12 & $\begin{array}{l}\text { Carrizo-Wilcox, } \\
\text { Edwards, Edwards- } \\
\text { Trinity (Plateau) }\end{array}$ & 21.0 & 0.318 & TBWE (1960) \\
\hline 217 & Nueces & Nueces R & $\begin{array}{l}\text { Old Spanish Trail crossing to Tom } \\
\text { Nunn Hill Dam }\end{array}$ & 3/10-11/1924 & 3.6 & 3 & 3 & -- & 26.8 & 7.444 & TBWE (1960) \\
\hline 218 & Nueces & Nueces R & $\begin{array}{l}\text { Real-Uvalde Co line to Laguna } \\
\quad(08190000)\end{array}$ & $2 / 16 / 1955$ & 16.8 & 4 & 4 & $\begin{array}{l}\text { Edwards-Trinity } \\
\text { (Plateau) }\end{array}$ & -1.6 & -.095 & TBWE (1960) \\
\hline 219 & Nueces & Nueces $R$ & US 90 to near Crystal City & $11 / 23-25 / 1964$ & 52.2 & 19 & 10 & Carrizo-Wilcox & 13.4 & .257 & USGS (1966) \\
\hline 220 & Nueces & Sabinal R & $\begin{array}{l}1 \text { mi south of Utopia to } 6 \text { mi north } \\
\text { of Sabinal }\end{array}$ & $5 / 7-8 / 1942$ & 17.5 & 7 & 6 & Edwards, Trinity & -32.7 & -1.869 & TBWE (1960) \\
\hline 221 & Nueces & Sabinal R & $\begin{array}{l}8 \text { mi above Vanderpool to at Sabinal } \\
(08198500)\end{array}$ & $4 / 8-11 / 1958$ & 49 & 30 & 19 & $\begin{array}{l}\text { Edwards, Edwards- } \\
\text { Trinity (Plateau), } \\
\text { Trinity }\end{array}$ & -31.6 & -.645 & TBWE (1960) \\
\hline 222 & Nueces & Sabinal R & $\begin{array}{l}8 \mathrm{mi} \text { above Vanderpool to near } \\
\text { Sabinal }(08198000)\end{array}$ & $12 / 15-16 / 1954$ & 31.4 & 24 & 18 & $\begin{array}{l}\text { Edwards-Trinity } \\
\quad \text { (Plateau), Trinity }\end{array}$ & -1.53 & -.049 & TBWE (1960) \\
\hline 223 & Nueces & Sabinal R & $\begin{array}{l}8 \text { mi above Vanderpool to near } \\
\text { Sabinal }(08198000)\end{array}$ & $9 / 10-11 / 1955$ & 31.4 & 24 & 18 & $\begin{array}{l}\text { Edwards-Trinity } \\
\quad \text { (Plateau), Trinity }\end{array}$ & -1.08 & -.034 & TBWE (1960) \\
\hline 224 & Nueces & Sabinal R & below Utopia & $1 / 10 / 1934$ & 18 & 4 & 4 & Trinity & .92 & .051 & TBWE (1960) \\
\hline 225 & Nueces & Sabinal R & $\begin{array}{l}\text { confluence with Onion Cr to } 6 \mathrm{mi} \\
\text { north of Sabinal }\end{array}$ & $5 / 18 / 1942$ & 9.5 & 4 & 4 & Edwards, Trinity & -32.3 & -3.4 & TBWE (1960) \\
\hline 226 & Nueces & Sabinal R & $\begin{array}{l}\text { confluence with Onion Cr to } 6 \mathrm{mi} \\
\text { north of Sabinal }\end{array}$ & $8 / 5 / 1942$ & 9.5 & 4 & 4 & Edwards, Trinity & -6.55 & -.689 & TBWE (1960) \\
\hline 227 & Nueces & Sabinal R & $\begin{array}{l}\text { near Sabinal }(08198000) \text { to at } \\
\quad \text { Sabinal }(08198500)\end{array}$ & $4 / 2 / 1981$ & 19.1 & 4 & 4 & -- & -82.2 & -4.304 & $\begin{array}{l}\text { Land and others } \\
\text { (1983) }\end{array}$ \\
\hline 228 & Nueces & Sabinal R & $\begin{array}{l}\text { near Sabinal }(08198000) \text { to at } \\
\quad \text { Sabinal }(08198500)\end{array}$ & 8/6/1981 & 19.1 & 4 & 4 & -- & -91.2 & -4.775 & $\begin{array}{l}\text { Land and others } \\
\text { (1983) }\end{array}$ \\
\hline 229 & Nueces & $\mathrm{Seco} \mathrm{Cr}$ & $\begin{array}{l}\text { Miller Ranch (08201500) to } \\
\text { D'Hanis (08202700) }\end{array}$ & $6 / 18 / 1981$ & 22.9 & 8 & 7 & Edwards, Trinity & -167.35 & -7.308 & $\begin{array}{l}\text { Land and others } \\
\quad \text { (1983) }\end{array}$ \\
\hline 230 & Nueces & $\mathrm{Seco} \mathrm{Cr}$ & $\begin{array}{l}\text { Miller Ranch (08201500) to } \\
\text { D'Hanis (08202700) }\end{array}$ & $6 / 19 / 1981$ & 22.9 & 8 & 7 & Edwards, Trinity & -159.2 & -6.952 & $\begin{array}{l}\text { Land and others } \\
\text { (1983) }\end{array}$ \\
\hline 231 & Nueces & Seco $\mathrm{Cr}$ & $\begin{array}{l}\text { Miller Ranch }(08201500) \text { to } \\
\text { D'Hanis }(08202700)\end{array}$ & $6 / 22 / 1981$ & 22.9 & 8 & 7 & Edwards, Trinity & -170.2 & -7.432 & $\begin{array}{l}\text { Land and others } \\
\text { (1983) }\end{array}$ \\
\hline 232 & Nueces & $\mathrm{Seco} \mathrm{Cr}$ & $\begin{array}{l}\text { D'Hanis }(08202500) \text { to } 17 \mathrm{mi} \text { below } \\
\quad \text { US } 90\end{array}$ & 11/7/1958 & 36.3 & 13 & 13 & -- & -97.0 & -2.672 & TBWE (1960) \\
\hline 233 & Nueces & $\begin{array}{l}\text { Short Prong Frio } \\
\text { R-[Frio R] }\end{array}$ & $\begin{array}{l}16 \text { mi above Leakey to Concan } \\
(08195000)\end{array}$ & $1 / 4-7 / 1955$ & 39.5 & 26 & 20 & $\begin{array}{l}\text { Edwards, Edwards- } \\
\text { Trinity (Plateau) }\end{array}$ & 10.06 & .255 & TBWE (1960) \\
\hline 234 & Nueces & $\begin{array}{l}\text { Short Prong Frio } \\
\text { R-[Frio R] }\end{array}$ & $\begin{array}{l}16 \text { mi above Leakey to Concan } \\
(08195000)\end{array}$ & 9/8-10/1955 & 39.5 & 28 & 21 & $\begin{array}{l}\text { Edwards, Edwards- } \\
\text { Trinity (Plateau) }\end{array}$ & 8.54 & .216 & TBWE (1960) \\
\hline 235 & Nueces & $\begin{array}{l}\text { West Frio } \mathrm{R}-[\text { Frio } \\
\mathrm{R}]\end{array}$ & $\begin{array}{l}11.6 \mathrm{mi} \text { above confluence with East } \\
\text { Frio R to Concan }(08195000)\end{array}$ & $7 / 8-12 / 1957$ & 35 & 11 & 9 & $\begin{array}{l}\text { Edwards, Edwards- } \\
\text { Trinity (Plateau) }\end{array}$ & 30.86 & .882 & TBWE (1960) \\
\hline
\end{tabular}


Table 1. Characteristics of flow gain-loss studies in Texas-Continued

\begin{tabular}{|c|c|c|c|c|c|c|c|c|c|c|c|}
\hline $\begin{array}{l}\text { Stream- } \\
\text { flow } \\
\text { study } \\
\text { no. }\end{array}$ & $\begin{array}{l}\text { Major } \\
\text { river } \\
\text { basin }\end{array}$ & $\begin{array}{l}\text { Stream } \\
\text { name }\end{array}$ & $\begin{array}{c}\text { Reach } \\
\text { identification }\end{array}$ & $\begin{array}{l}\text { Date of } \\
\text { study }\end{array}$ & $\begin{array}{l}\text { Reach } \\
\text { length } \\
\text { (river } \\
\text { mi) }\end{array}$ & $\begin{array}{l}\text { Total } \\
\text { no. of } \\
\text { measure- } \\
\text { ment } \\
\text { sites }\end{array}$ & $\begin{array}{l}\text { No. of } \\
\text { measure- } \\
\text { ment sites } \\
\text { on main } \\
\text { channel }\end{array}$ & $\begin{array}{l}\text { Major aquifer } \\
\text { outcrop(s) } \\
\text { intersected } \\
\text { by reach }\end{array}$ & $\begin{array}{c}\text { Total } \\
\text { gain or } \\
\text { loss }(-) \\
\text { in reach } \\
\left(\mathrm{ft}^{3} / \mathrm{s}\right)\end{array}$ & $\begin{array}{l}\text { Gain or } \\
\text { loss per } \\
\text { mile of } \\
\text { reach } \\
\left(\mathrm{ft}^{3} / \mathrm{s}-\mathrm{mi}\right)\end{array}$ & $\begin{array}{l}\text { Reference } \\
\text { for data }\end{array}$ \\
\hline 236 & Nueces & $\begin{array}{l}\text { West Frio R- [Frio } \\
\text { R] }\end{array}$ & $\begin{array}{l}8 \text { mi above Leakey to Concan } \\
(08195000)\end{array}$ & $2 / 14-18 / 1955$ & 31.7 & 7 & 5 & $\begin{array}{l}\text { Edwards, Edwards- } \\
\text { Trinity (Plateau) }\end{array}$ & 11.59 & 0.366 & TBWE (1960) \\
\hline 237 & Nueces & West Nueces R & $\begin{array}{l}\text { above Brackettville (08190500) to } \\
\text { near Uvalde }\end{array}$ & $5 / 13 / 1941$ & 37 & 5 & 4 & Edwards & -7.1 & -.192 & TBWE (1960) \\
\hline 238 & Nueces & West Nueces R & $\begin{array}{l}\text { Blackwater Hole to discontinued } \\
\text { station near Brackettville } \\
(08190500)\end{array}$ & $12 / 13-14 / 1954$ & 47.7 & 18 & 15 & $\begin{array}{l}\text { Edwards, Edwards- } \\
\text { Trinity (Plateau) }\end{array}$ & -4.95 & -.104 & TBWE (1960) \\
\hline 239 & Nueces & West Nueces R & $\begin{array}{l}\text { Blackwater Hole to discontinued } \\
\text { station near Brackettville } \\
(08190500)\end{array}$ & $9 / 11-12 / 1955$ & 47.7 & 18 & 16 & $\begin{array}{l}\text { Edwards, Edwards- } \\
\text { Trinity (Plateau) }\end{array}$ & -6.95 & -.146 & TBWE (1960) \\
\hline 240 & Nueces & West Nueces R & $\begin{array}{l}\text { near Brackettville }(08190500) \text { to } \\
\text { Shaw Ranch crossing }\end{array}$ & $6 / 20 / 1981$ & 38.5 & 3 & 2 & Edwards & -286.0 & -7.429 & $\begin{array}{l}\text { Land and others } \\
\text { (1983) }\end{array}$ \\
\hline 241 & Nueces & $\begin{array}{l}\text { West Seco Cr- } \\
\quad[\text { Seco } \mathrm{Cr}]\end{array}$ & near Utopia (08202000) to US 90 & $4 / 1-4 / 1958$ & 39 & 39 & 21 & Edwards, Trinity & -21.04 & -.539 & TBWE (1960) \\
\hline 242 & Nueces & Williams $\mathrm{Cr}$ & $\begin{array}{l}4.7 \text { mi above mouth to } 1,600 \text { feet } \\
\text { above Hondo Cr }\end{array}$ & $4 / 5 / 1958$ & 4.4 & 6 & 3 & Trinity & 6.36 & 1.445 & TBWE (1960) \\
\hline 243 & Red River & Grays $\mathrm{Cr}$ & $\begin{array}{l}2.6 \text { mi north of Marshall to FM } \\
1997\end{array}$ & $6 / 13 / 1964$ & 3.3 & 9 & 2 & Carrizo-Wilcox & -.03 & -.009 & USGS (1966) \\
\hline 244 & Red River & Little Cypress Cr & SH 155 to FM 134 & 6/10-13/1964 & 49.1 & 35 & 10 & Carrizo-Wilcox & 6.52 & .133 & USGS (1966) \\
\hline 245 & Red River & Little Cypress Cr & $\begin{array}{l}\text { northeast of Gilmer to near } \\
\text { Jefferson }\end{array}$ & $1 / 2-3 / 1964$ & 40.5 & 7 & 7 & Carrizo-Wilcox & 24.09 & .595 & USGS (1966) \\
\hline 246 & Red River & North Fk Wichita R & $\begin{array}{l}\text { Truscott }(07311700) \text { to } 4 \mathrm{mi} \text { above } \\
\text { Lake Kemp }\end{array}$ & $2 / 6-7 / 1974$ & 47.6 & 6 & 6 & -- & -1.3 & -.027 & USGS (1975) \\
\hline 247 & Red River & Prairie Dog Town Fk & $\begin{array}{l}\text { Lake Tanglewood to Wayside } \\
\quad(07297910)\end{array}$ & $2 / 6-9 / 1968$ & 40.2 & 68 & 32 & -- & 1.35 & .034 & USGS (1969) \\
\hline 248 & Red River & South Fk Wichita R & $\begin{array}{l}\text { Benjamin }(07311800) \text { to } 4 \mathrm{mi} \text { above } \\
\text { Lake Kemp }\end{array}$ & 2/6-7/1974 & 41 & 6 & 5 & -- & -.8 & -.02 & USGS (1975) \\
\hline 249 & Red River & Sugar $\mathrm{Cr}$ & FM 1403 to SH 154 & $6 / 10-11 / 1964$ & .8 & 3 & 2 & -- & .15 & .188 & USGS (1966) \\
\hline 250 & Red River & Tierra Blanca Cr & near Umbarger to near Canyon & $8 / 31 / 1941$ & 17.9 & 8 & 8 & Ogallala & -4.53 & -.253 & TBWE (1960) \\
\hline 251 & Red River & Tierra Blanca Cr & near Umbarger to near Canyon & 9/28/1941 & 17.9 & 6 & 6 & Ogallala & -2.6 & -.145 & TBWE (1960) \\
\hline 252 & Rio Grande & Big Aguja Canyon & $11.8 \mathrm{mi}$ to $2.2 \mathrm{mi}$ above Toyahvale & $9 / 1 / 1932$ & 9.6 & 4 & 3 & $\begin{array}{l}\text { Cenozoic Pecos } \\
\text { Alluvium, } \\
\text { Edwards-Trinity } \\
\text { (Plateau) }\end{array}$ & -19.2 & -2.0 & TBWE (1960) \\
\hline 253 & Rio Grande & Big Aguja Canyon & $11.8 \mathrm{mi}$ to $2.2 \mathrm{mi}$ above Toyahvale & $9 / 3 / 1932$ & 9.6 & 4 & 3 & $\begin{array}{l}\text { Cenozoic Pecos } \\
\text { Alluvium, } \\
\text { Edwards-Trinity } \\
\text { (Plateau) }\end{array}$ & -26.6 & -2.771 & TBWE (1960) \\
\hline
\end{tabular}


Table 1. Characteristics of flow gain-loss studies in Texas-Continued

\begin{tabular}{|c|c|c|c|c|c|c|c|c|c|c|c|}
\hline $\begin{array}{l}\text { Stream- } \\
\text { flow } \\
\text { study } \\
\text { no. }\end{array}$ & $\begin{array}{l}\text { Major } \\
\text { river } \\
\text { basin }\end{array}$ & $\begin{array}{c}\text { Stream } \\
\text { name }\end{array}$ & $\begin{array}{c}\text { Reach } \\
\text { identification }\end{array}$ & $\begin{array}{l}\text { Date of } \\
\text { study }\end{array}$ & $\begin{array}{l}\text { Reach } \\
\text { length } \\
\text { (river } \\
\text { mi) }\end{array}$ & $\begin{array}{c}\text { Total } \\
\text { no. of } \\
\text { measure- } \\
\text { ment } \\
\text { sites }\end{array}$ & $\begin{array}{l}\text { No. of } \\
\text { measure- } \\
\text { ment sites } \\
\text { on main } \\
\text { channel }\end{array}$ & $\begin{array}{l}\text { Major aquifer } \\
\text { outcrop(s) } \\
\text { intersected } \\
\text { by reach }\end{array}$ & $\begin{array}{c}\text { Total } \\
\text { gain or } \\
\text { loss }(-) \\
\text { in reach } \\
\left(\mathrm{ft}^{3} / \mathrm{s}\right)\end{array}$ & $\begin{array}{c}\text { Gain or } \\
\text { loss per } \\
\text { mile of } \\
\text { reach } \\
\left(\mathrm{ft}^{3} / \mathrm{s}-\mathrm{mi}\right)\end{array}$ & $\begin{array}{c}\text { Reference } \\
\text { for data }\end{array}$ \\
\hline 254 & Rio Grande & Big Aguja Canyon & $11.8 \mathrm{mi}$ to $2.2 \mathrm{mi}$ above Toyahvale & $9 / 13 / 1932$ & 9.6 & 5 & 3 & $\begin{array}{l}\text { Cenozoic Pecos } \\
\text { Alluvium, } \\
\text { Edwards-Trinity } \\
\text { (Plateau) }\end{array}$ & -13.6 & -1.417 & TBWE (1960) \\
\hline 255 & Rio Grande & Big Aguja Canyon & $11.8 \mathrm{mi}$ to $2.2 \mathrm{mi}$ above Toyahvale & $10 / 6 / 1932$ & 9.6 & 11 & 8 & $\begin{array}{l}\text { Cenozoic Pecos } \\
\text { Alluvium, } \\
\text { Edwards-Trinity } \\
\text { (Plateau) }\end{array}$ & -15.4 & -1.604 & TBWE (1960) \\
\hline 256 & Rio Grande & Cherry Canyon & $\begin{array}{l}1.5 \text { mi above to } 2.5 \text { mi below } \\
\text { Madera Canyon near Toyahvale } \\
(08424500)\end{array}$ & $9 / 15 / 1932$ & 2 & 2 & 2 & $\begin{array}{l}\text { Cenozoic Pecos } \\
\text { Alluvium }\end{array}$ & -13.5 & -6.75 & TBWE (1960) \\
\hline 257 & Rio Grande & Cherry Canyon & $\begin{array}{l}1.5 \text { mi above to } 2.5 \text { mi below } \\
\text { Madera Canyon near Toyahvale } \\
(08424500)\end{array}$ & $9 / 21 / 1932$ & 1.5 & 2 & 2 & $\begin{array}{l}\text { Cenozoic Pecos } \\
\text { Alluvium }\end{array}$ & 3.8 & 2.533 & TBWE (1960) \\
\hline 258 & Rio Grande & Cherry Canyon & $\begin{array}{l}1.5 \mathrm{mi} \text { above to } 2.5 \mathrm{mi} \text { below } \\
\text { Madera Canyon near Toyahvale } \\
(08424500)\end{array}$ & $10 / 7 / 1932$ & 2.5 & 2 & 2 & $\begin{array}{l}\text { Cenozoic Pecos } \\
\text { Alluvium }\end{array}$ & -31.2 & -12.48 & TBWE (1960) \\
\hline 259 & Rio Grande & Devils R & $30 \mathrm{mi}$ above Del Rio to mouth & $1 / 26-28 / 1921$ & 27.2 & 5 & 4 & $\begin{array}{l}\text { Edwards-Trinity } \\
\quad \text { (Plateau) }\end{array}$ & 140.0 & 5.147 & TBWE (1960) \\
\hline 260 & Rio Grande & Devils R & $30 \mathrm{mi}$ above Del Rio to mouth & $10 / 6-7 / 1921$ & 7.8 & 4 & 4 & $\begin{array}{l}\text { Edwards-Trinity } \\
\quad \text { (Plateau) }\end{array}$ & 52.0 & 6.667 & TBWE (1960) \\
\hline 261 & Rio Grande & Devils R & Beaver Lake to Juno (08449000) & $8 / 8-13 / 1925$ & 76 & 21 & 17 & $\begin{array}{l}\text { Edwards-Trinity } \\
\quad \text { (Plateau) }\end{array}$ & 426.1 & 5.607 & TBWE (1960) \\
\hline 262 & Rio Grande & Devils R & Dolans $\mathrm{Cr}$ to near Comstock & $2 / 14-20 / 1928$ & 22.3 & 22 & 13 & $\begin{array}{l}\text { Edwards-Trinity } \\
\quad \text { (Plateau) }\end{array}$ & 91.98 & 4.125 & TBWE (1960) \\
\hline 263 & Rio Grande & Devils R & $\begin{array}{l}\text { near Comstock to Southern Pacific } \\
\text { RR bridge }\end{array}$ & $2 / 7-11 / 1928$ & 16.5 & 30 & 10 & $\begin{array}{l}\text { Edwards-Trinity } \\
\quad \text { (Plateau) }\end{array}$ & 97.91 & 5.934 & TBWE (1960) \\
\hline 264 & Rio Grande & Limpia $\mathrm{Cr}$ & $11.7 \mathrm{mi}$ to $28.7 \mathrm{mi}$ below Fort Davis & $11 / 21 / 1932$ & 17 & 7 & 7 & $\begin{array}{l}\text { Edwards-Trinity } \\
\quad \text { (Plateau) }\end{array}$ & -4.0 & -.235 & TBWE (1960) \\
\hline 265 & Rio Grande & Limpia $\mathrm{Cr}$ & $\begin{array}{l}12.3 \mathrm{mi} \text { above to } 18.2 \mathrm{mi} \text { below Fort } \\
\text { Davis }\end{array}$ & $10 / 18-19 / 1932$ & 30.5 & 24 & 18 & $\begin{array}{l}\text { Edwards-Trinity } \\
\quad \text { (Plateau) }\end{array}$ & 12.5 & .41 & TBWE (1960) \\
\hline 266 & Rio Grande & Limpia $\mathrm{Cr}$ & $14.7 \mathrm{mi}$ to $40.2 \mathrm{mi}$ below Fort Davis & $10 / 8 / 1932$ & 25.5 & 9 & 6 & $\begin{array}{l}\text { Edwards-Trinity } \\
\quad \text { (Plateau) }\end{array}$ & -35.5 & -1.392 & TBWE (1960) \\
\hline 267 & Rio Grande & Limpia $\mathrm{Cr}$ & $15.8 \mathrm{mi}$ to $18.7 \mathrm{mi}$ below Fort Davis & $8 / 3 / 1933$ & 2.9 & 3 & 3 & $\begin{array}{l}\text { Edwards-Trinity } \\
\quad \text { (Plateau) }\end{array}$ & -12.6 & -4.345 & TBWE (1960) \\
\hline 268 & Rio Grande & Limpia $\mathrm{Cr}$ & $18.2 \mathrm{mi}$ to $29.2 \mathrm{mi}$ below Fort Davis & $11 / 1 / 1932$ & 11 & 5 & 5 & $\begin{array}{l}\text { Edwards-Trinity } \\
\quad \text { (Plateau) }\end{array}$ & -8.2 & .745 & TBWE (1960) \\
\hline 269 & Rio Grande & Little Aguja Canyon & $11.2 \mathrm{mi}$ to $3 \mathrm{mi}$ above Toyahvale & $10 / 6 / 1932$ & 8.2 & 5 & 4 & $\begin{array}{l}\text { Cenozoic Pecos } \\
\text { Alluvium, } \\
\text { Edwards-Trinity } \\
\text { (Plateau) }\end{array}$ & -27.0 & -3.293 & TBWE (1960) \\
\hline
\end{tabular}


Table 1. Characteristics of flow gain-loss studies in Texas-Continued

\begin{tabular}{|c|c|c|c|c|c|c|c|c|c|c|c|}
\hline $\begin{array}{l}\text { Stream- } \\
\text { flow } \\
\text { study } \\
\text { no. }\end{array}$ & $\begin{array}{l}\text { Major } \\
\text { river } \\
\text { basin }\end{array}$ & $\begin{array}{c}\text { Stream } \\
\text { name }\end{array}$ & $\begin{array}{c}\text { Reach } \\
\text { identification }\end{array}$ & $\begin{array}{l}\text { Date of } \\
\text { study }\end{array}$ & $\begin{array}{l}\text { Reach } \\
\text { length } \\
\text { (river } \\
\text { mi) }\end{array}$ & $\begin{array}{c}\text { Total } \\
\text { no. of } \\
\text { measure- } \\
\text { ment } \\
\text { sites }\end{array}$ & $\begin{array}{l}\text { No. of } \\
\text { measure- } \\
\text { ment sites } \\
\text { on main } \\
\text { channel }\end{array}$ & $\begin{array}{l}\text { Major aquifer } \\
\text { outcrop(s) } \\
\text { intersected } \\
\text { by reach }\end{array}$ & $\begin{array}{c}\text { Total } \\
\text { gain or } \\
\text { loss }(-) \\
\text { in reach } \\
\left(\mathrm{ft}^{3} / \mathrm{s}\right)\end{array}$ & $\begin{array}{c}\text { Gain or } \\
\text { loss per } \\
\text { mile of } \\
\text { reach } \\
\left(\mathrm{ft}^{3} / \mathrm{s}-\mathrm{mi}\right)\end{array}$ & $\begin{array}{c}\text { Reference } \\
\text { for data }\end{array}$ \\
\hline 270 & Rio Grande & Little Aguja Canyon & $11.3 \mathrm{mi}$ to $3.9 \mathrm{mi}$ above Toyahvale & $9 / 13 / 1932$ & 7.4 & 4 & 4 & $\begin{array}{l}\text { Edwards-Trinity } \\
\text { (Plateau) }\end{array}$ & -22.8 & -3.081 & TBWE (1960) \\
\hline 271 & Rio Grande & Little Aguja Canyon & $11.3 \mathrm{mi}$ to $7.7 \mathrm{mi}$ above Toyahvale & $9 / 20 / 1932$ & 3.6 & 6 & 6 & $\begin{array}{l}\text { Edwards-Trinity } \\
\text { (Plateau) }\end{array}$ & -4.1 & -1.139 & TBWE (1960) \\
\hline 272 & Rio Grande & Little Aguja Canyon & $11.3 \mathrm{mi}$ to $8.2 \mathrm{mi}$ above Toyahvale & $9 / 14 / 1932$ & 3.1 & 2 & 2 & $\begin{array}{l}\text { Edwards-Trinity } \\
\quad \text { (Plateau) }\end{array}$ & -11.2 & -3.613 & TBWE (1960) \\
\hline 273 & Rio Grande & Little Aguja Canyon & $12.5 \mathrm{mi}$ to $2.2 \mathrm{mi}$ above Toyahvale & 9/1/1932 & 10.3 & 3 & 2 & $\begin{array}{l}\text { Cenozoic Pecos } \\
\text { Alluvium, } \\
\text { Edwards-Trinity } \\
\text { (Plateau) }\end{array}$ & -18.5 & -1.796 & TBWE (1960) \\
\hline 274 & Rio Grande & Little Aguja Canyon & $15.5 \mathrm{mi}$ to $2.2 \mathrm{mi}$ above Toyahvale & $8 / 17 / 1932$ & 13.3 & 11 & 10 & $\begin{array}{l}\text { Cenozoic Pecos } \\
\text { Alluvium, } \\
\text { Edwards-Trinity } \\
\text { (Plateau) }\end{array}$ & -1.7 & -.128 & TBWE (1960) \\
\hline 275 & Rio Grande & Madera Canyon & $13.3 \mathrm{mi}$ to $3.5 \mathrm{mi}$ above Toyahvale & 9/2/1932 & 9.8 & 4 & 4 & $\begin{array}{l}\text { Edwards-Trinity } \\
\quad \text { (Plateau) }\end{array}$ & -67.0 & -6.837 & TBWE (1960) \\
\hline 276 & Rio Grande & Madera Canyon & $13.3 \mathrm{mi}$ to $3.5 \mathrm{mi}$ above Toyahvale & $9 / 3 / 1932$ & 9.8 & 4 & 4 & $\begin{array}{l}\text { Edwards-Trinity } \\
\text { (Plateau) }\end{array}$ & -37.3 & -3.806 & TBWE (1960) \\
\hline 277 & Rio Grande & Madera Canyon & $13.3 \mathrm{mi}$ to $3.5 \mathrm{mi}$ above Toyahvale & $8 / 27 / 1933$ & 9.8 & 3 & 3 & $\begin{array}{l}\text { Edwards-Trinity } \\
\text { (Plateau) }\end{array}$ & -17.5 & -1.786 & TBWE (1960) \\
\hline 278 & Rio Grande & Madera Canyon & $13.3 \mathrm{mi}$ to $5.1 \mathrm{mi}$ above Toyahvale & 9/1/1932 & 8.2 & 3 & 3 & $\begin{array}{l}\text { Edwards-Trinity } \\
\quad \text { (Plateau) }\end{array}$ & -23.2 & -2.829 & TBWE (1960) \\
\hline 279 & Rio Grande & Madera Canyon & $13.3 \mathrm{mi}$ to $5.1 \mathrm{mi}$ above Toyahvale & $9 / 12 / 1932$ & 8.2 & 4 & 3 & $\begin{array}{l}\text { Edwards-Trinity } \\
\quad \text { (Plateau) }\end{array}$ & -26.7 & -3.256 & TBWE (1960) \\
\hline 280 & Rio Grande & Madera Canyon & $13.3 \mathrm{mi}$ to $5.1 \mathrm{mi}$ above Toyahvale & $9 / 15 / 1932$ & 8.2 & 3 & 3 & $\begin{array}{l}\text { Edwards-Trinity } \\
\text { (Plateau) }\end{array}$ & -14.3 & -1.744 & TBWE (1960) \\
\hline 281 & Rio Grande & Madera Canyon & $13.3 \mathrm{mi}$ to $5.1 \mathrm{mi}$ above Toyahvale & $9 / 21 / 1932$ & 8.2 & 5 & 5 & $\begin{array}{l}\text { Edwards-Trinity } \\
\quad \text { (Plateau) }\end{array}$ & -4.6 & -.561 & TBWE (1960) \\
\hline 282 & Rio Grande & Madera Canyon & $13.3 \mathrm{mi}$ to $9.3 \mathrm{mi}$ above Toyahvale & $10 / 6 / 1932$ & 4 & 5 & 4 & $\begin{array}{l}\text { Edwards-Trinity } \\
\text { (Plateau) }\end{array}$ & -38.8 & -9.7 & TBWE (1960) \\
\hline 283 & Rio Grande & Madera Canyon & $13.3 \mathrm{mi}$ to $9.8 \mathrm{mi}$ above Toyahvale & $9 / 24 / 1932$ & 3.5 & 5 & 4 & $\begin{array}{l}\text { Edwards-Trinity } \\
\quad \text { (Plateau) }\end{array}$ & -6.8 & -1.943 & TBWE (1960) \\
\hline 284 & Rio Grande & $\begin{array}{l}\text { PCWID \#1 canal } \\
\text { system at Fort } \\
\text { Stockton }\end{array}$ & High line canal & $11 / 24 / 1939$ & 7.4 & 7 & 5 & $\begin{array}{l}\text { Cenozoic Pecos } \\
\text { Alluvium }\end{array}$ & -5.5 & -.743 & TBWE (1960) \\
\hline 285 & Rio Grande & $\begin{array}{l}\text { PCWID \#1 canal } \\
\text { system at Fort } \\
\text { Stockton }\end{array}$ & High line canal & $12 / 14 / 1939$ & 2.5 & 3 & 2 & $\begin{array}{c}\text { Cenozoic Pecos } \\
\text { Alluvium }\end{array}$ & .4 & .16 & TBWE (1960) \\
\hline 286 & Rio Grande & $\begin{array}{l}\text { PCWID \#1 canal } \\
\text { system at Fort } \\
\text { Stockton }\end{array}$ & lateral \#2 & $12 / 14 / 1939$ & 2.4 & 5 & 4 & $\begin{array}{c}\text { Cenozoic Pecos } \\
\text { Alluvium }\end{array}$ & -.4 & -.167 & TBWE (1960) \\
\hline
\end{tabular}


Table 1. Characteristics of flow gain-loss studies in Texas-Continued

\begin{tabular}{|c|c|c|c|c|c|c|c|c|c|c|c|}
\hline $\begin{array}{l}\text { Stream- } \\
\text { flow } \\
\text { study } \\
\text { no. }\end{array}$ & $\begin{array}{l}\text { Major } \\
\text { river } \\
\text { basin }\end{array}$ & $\begin{array}{l}\text { Stream } \\
\text { name }\end{array}$ & $\begin{array}{c}\text { Reach } \\
\text { identification }\end{array}$ & $\begin{array}{l}\text { Date of } \\
\text { study }\end{array}$ & $\begin{array}{c}\text { Reach } \\
\text { length } \\
\text { (river } \\
\text { mi) }\end{array}$ & $\begin{array}{c}\text { Total } \\
\text { no. of } \\
\text { measure- } \\
\text { ment } \\
\text { sites }\end{array}$ & $\begin{array}{l}\text { No. of } \\
\text { measure- } \\
\text { ment sites } \\
\text { on main } \\
\text { channel }\end{array}$ & $\begin{array}{l}\text { Major aquifer } \\
\text { outcrop(s) } \\
\text { intersected } \\
\text { by reach }\end{array}$ & $\begin{array}{c}\text { Total } \\
\text { gain or } \\
\text { loss }(-) \\
\text { in reach } \\
\left(\mathrm{ft}^{3} / \mathrm{s}\right)\end{array}$ & $\begin{array}{c}\text { Gain or } \\
\text { loss per } \\
\text { mile of } \\
\text { reach } \\
\left(\mathrm{ft}^{3} / \mathrm{s}-\mathrm{mi}\right)\end{array}$ & $\begin{array}{l}\text { Reference } \\
\text { for data }\end{array}$ \\
\hline 287 & Rio Grande & $\begin{array}{l}\text { PCWID \#1 canal } \\
\text { system at Fort } \\
\text { Stockton }\end{array}$ & lateral \#3 & $12 / 14 / 1939$ & 1.8 & 2 & 2 & $\begin{array}{l}\text { Cenozoic Pecos } \\
\text { Alluvium }\end{array}$ & 0 & 0 & TBWE (1960) \\
\hline 288 & Rio Grande & $\begin{array}{l}\text { PCWID \#1 canal } \\
\text { system at Fort } \\
\text { Stockton }\end{array}$ & Seven-D canal & $12 / 14 / 1939$ & 1.5 & 4 & 3 & $\begin{array}{l}\text { Cenozoic Pecos } \\
\text { Alluvium }\end{array}$ & -1.0 & -.667 & TBWE (1960) \\
\hline 289 & Rio Grande & Pecos R & Angeles (08409500) to Girvin & $5 / 28-30 / 1918$ & 203 & 26 & 18 & $\begin{array}{l}\text { Cenozoic Pecos } \\
\text { Alluvium }\end{array}$ & -105.5 & -.52 & TBWE (1960) \\
\hline 290 & Rio Grande & Pecos R & $\begin{array}{l}\text { Girvin (08446500) to Comstock } \\
\quad \text { (IBWC 08447700) }\end{array}$ & $2 / 6-9 / 1968$ & 193.6 & 34 & 19 & $\begin{array}{l}\text { Cenozoic Pecos } \\
\text { Alluvium, } \\
\text { Edwards-Trinity } \\
\text { (Plateau) }\end{array}$ & 74.97 & .387 & USGS (1969) \\
\hline 291 & Rio Grande & Pecos R & $\begin{array}{l}\text { Orla }(08410000) \text { to Girvin } \\
\quad(08446500)\end{array}$ & $3 / 3-5 / 1964$ & 188.05 & 21 & 11 & $\begin{array}{l}\text { Cenozoic Pecos } \\
\text { Alluvium }\end{array}$ & -184.8 & -.983 & USGS (1965) \\
\hline 292 & Rio Grande & Pecos R & $\begin{array}{l}\text { Orla }(08410000) \text { to Girvin } \\
\quad(08446500)\end{array}$ & $5 / 10-12 / 1965$ & 188.05 & 25 & 16 & $\begin{array}{l}\text { Cenozoic Pecos } \\
\text { Alluvium }\end{array}$ & 8.92 & .047 & USGS (1966) \\
\hline 293 & Rio Grande & Pecos R & $\begin{array}{l}\text { Orla }(08410000) \text { to Girvin } \\
\quad(08446500)\end{array}$ & $4 / 17-19 / 1967$ & 185.5 & 23 & 14 & $\begin{array}{l}\text { Cenozoic Pecos } \\
\text { Alluvium }\end{array}$ & -189.29 & -1.02 & USGS (1968) \\
\hline 294 & Rio Grande & $\begin{array}{l}\text { RCWID \#1 canal } \\
\text { laterals }\end{array}$ & back lateral & $8 / 29 / 1940$ & 2.2 & 4 & 4 & $\begin{array}{l}\text { Cenozoic Pecos } \\
\text { Alluvium }\end{array}$ & 0 & 0 & TBWE (1960) \\
\hline 295 & Rio Grande & $\begin{array}{l}\text { RCWID \#1 canal } \\
\text { laterals }\end{array}$ & Carpenter takeout & $10 / 27 / 1931$ & 1 & 2 & 2 & $\begin{array}{l}\text { Cenozoic Pecos } \\
\text { Alluvium, } \\
\text { Edwards-Trinity } \\
\text { (Plateau) }\end{array}$ & -.08 & -.08 & TBWE (1960) \\
\hline 296 & Rio Grande & $\begin{array}{l}\text { RCWID \#1 canal } \\
\text { laterals }\end{array}$ & Giffin Spring canal & $11 / 18 / 1931$ & .8 & 2 & 2 & $\begin{array}{l}\text { Cenozoic Pecos } \\
\text { Alluvium }\end{array}$ & .26 & .325 & TBWE (1960) \\
\hline 297 & Rio Grande & $\begin{array}{l}\text { RCWID \#1 canal } \\
\text { laterals }\end{array}$ & Greasy Row lateral & $9 / 6 / 1940$ & 1.5 & 5 & 3 & $\begin{array}{l}\text { Cenozoic Pecos } \\
\text { Alluvium }\end{array}$ & -.02 & -.013 & TBWE (1960) \\
\hline 298 & Rio Grande & $\begin{array}{l}\text { RCWID \#1 canal } \\
\text { laterals }\end{array}$ & Halbert corner lateral & $8 / 29 / 1940$ & 1.6 & 7 & 4 & $\begin{array}{l}\text { Cenozoic Pecos } \\
\text { Alluvium }\end{array}$ & .15 & .094 & TBWE (1960) \\
\hline 299 & Rio Grande & $\begin{array}{l}\text { RCWID \#1 canal } \\
\text { laterals }\end{array}$ & highway ditch & $10 / 27 / 1931$ & 1.3 & 4 & 3 & $\begin{array}{l}\text { Cenozoic Pecos } \\
\text { Alluvium }\end{array}$ & .43 & .331 & TBWE (1960) \\
\hline 300 & Rio Grande & $\begin{array}{l}\text { RCWID \#1 canal } \\
\text { laterals }\end{array}$ & Humphrey-Mayer lateral & $8 / 28 / 1940$ & .7 & 4 & 4 & $\begin{array}{l}\text { Cenozoic Pecos } \\
\text { Alluvium }\end{array}$ & .12 & .171 & TBWE (1960) \\
\hline 301 & Rio Grande & $\begin{array}{l}\text { RCWID \#1 canal } \\
\text { laterals }\end{array}$ & Ikens Estate lateral & $8 / 29 / 1940$ & .2 & 2 & 2 & $\begin{array}{l}\text { Cenozoic Pecos } \\
\text { Alluvium }\end{array}$ & -.96 & -4.8 & TBWE (1960) \\
\hline 302 & Rio Grande & $\begin{array}{l}\text { RCWID \#1 canal } \\
\quad \text { laterals }\end{array}$ & Moore canal & $11 / 16 / 1931$ & .5 & 2 & 2 & $\begin{array}{l}\text { Cenozoic Pecos } \\
\text { Alluvium }\end{array}$ & .06 & .12 & TBWE (1960) \\
\hline 303 & Rio Grande & $\begin{array}{l}\text { RCWID \#1 canal } \\
\text { laterals }\end{array}$ & north lateral & $7 / 2 / 1940$ & 4 & 6 & 5 & $\begin{array}{l}\text { Cenozoic Pecos } \\
\text { Alluvium }\end{array}$ & -.44 & -.11 & TBWE (1960) \\
\hline
\end{tabular}


จ Table 1. Characteristics of flow gain-loss studies in Texas-Continued

\begin{tabular}{|c|c|c|c|c|c|c|c|c|c|c|c|}
\hline $\begin{array}{l}\text { Stream- } \\
\text { flow } \\
\text { study } \\
\text { no. }\end{array}$ & $\begin{array}{c}\text { Major } \\
\text { river } \\
\text { basin }\end{array}$ & $\begin{array}{l}\text { Stream } \\
\text { name }\end{array}$ & $\begin{array}{c}\text { Reach } \\
\text { identification }\end{array}$ & $\begin{array}{l}\text { Date of } \\
\text { study }\end{array}$ & $\begin{array}{l}\text { Reach } \\
\text { length } \\
\text { (river } \\
\text { mi) }\end{array}$ & $\begin{array}{l}\text { Total } \\
\text { no. of } \\
\text { measure- } \\
\text { ment } \\
\text { sites }\end{array}$ & $\begin{array}{l}\text { No. of } \\
\text { measure- } \\
\text { ment sites } \\
\text { on main } \\
\text { channel }\end{array}$ & $\begin{array}{l}\text { Major aquifer } \\
\text { outcrop(s) } \\
\text { intersected } \\
\text { by reach }\end{array}$ & $\begin{array}{c}\text { Total } \\
\text { gain or } \\
\text { loss }(-) \\
\text { in reach } \\
\left(\mathrm{ft}^{3} / \mathrm{s}\right)\end{array}$ & $\begin{array}{l}\text { Gain or } \\
\text { loss per } \\
\text { mile of } \\
\text { reach } \\
\left(\mathrm{ft}^{3} / \mathrm{s}-\mathrm{mi}\right)\end{array}$ & $\begin{array}{l}\text { Reference } \\
\text { for data }\end{array}$ \\
\hline 304 & Rio Grande & $\begin{array}{l}\text { RCWID \#1 canal } \\
\text { laterals }\end{array}$ & north lateral & $8 / 28 / 1940$ & 4 & 9 & 7 & $\begin{array}{c}\text { Cenozoic Pecos } \\
\text { Alluvium }\end{array}$ & -0.26 & -0.065 & TBWE (1960) \\
\hline 305 & Rio Grande & $\begin{array}{l}\text { RCWID \#1 canal } \\
\text { laterals }\end{array}$ & reservoir takeout & $10 / 27 / 1931$ & 1 & 2 & 2 & $\begin{array}{l}\text { Cenozoic Pecos } \\
\text { Alluvium }\end{array}$ & -.5 & -.5 & TBWE (1960) \\
\hline 306 & Rio Grande & $\begin{array}{l}\text { RCWID \#1 canal } \\
\text { laterals }\end{array}$ & Saragosa canal & $11 / 16 / 1931$ & .5 & 2 & 2 & $\begin{array}{l}\text { Cenozoic Pecos } \\
\text { Alluvium }\end{array}$ & -.29 & -.58 & TBWE (1960) \\
\hline 307 & Rio Grande & $\begin{array}{l}\text { RCWID \#1 canal } \\
\quad \text { laterals }\end{array}$ & siphon ditch & 10/28/1931 & 2.3 & 2 & 2 & $\begin{array}{l}\text { Cenozoic Pecos } \\
\text { Alluvium }\end{array}$ & -3.13 & -1.361 & TBWE (1960) \\
\hline 308 & Rio Grande & $\begin{array}{l}\text { RCWID \#1 canal } \\
\text { system }\end{array}$ & $\begin{array}{l}\text { below San Solomon Springs to } \\
\text { above Balmorhea Reservoir }\end{array}$ & 2/6-7/1935 & 3.7 & 7 & 5 & $\begin{array}{l}\text { Cenozoic Pecos } \\
\text { Alluvium }\end{array}$ & 5.9 & 1.595 & TBWE (1960) \\
\hline 309 & Rio Grande & $\begin{array}{l}\text { RCWID \#1 main } \\
\text { canal }\end{array}$ & $\begin{array}{l}\text { above first diversion to sixth } \\
\text { diversion, near Balmorhea }\end{array}$ & $4 / 26-27 / 1923$ & 8.85 & 8 & 4 & $\begin{array}{l}\text { Cenozoic Pecos } \\
\text { Alluvium }\end{array}$ & -12.41 & -1.402 & TBWE (1960) \\
\hline 310 & Rio Grande & $\begin{array}{l}\text { RCWID \#1 main } \\
\text { canal }\end{array}$ & $\begin{array}{l}\text { headgate to Barlow diversion, near } \\
\text { Balmorhea }\end{array}$ & $7 / 27 / 1922$ & 5.5 & 8 & 3 & $\begin{array}{l}\text { Cenozoic Pecos } \\
\text { Alluvium }\end{array}$ & -7.0 & -1.273 & TBWE (1960) \\
\hline 311 & Rio Grande & $\begin{array}{l}\text { RCWID \#1 main } \\
\text { canal }\end{array}$ & $\begin{array}{l}\text { headgate to last diversion, near } \\
\text { Balmorhea }\end{array}$ & $9 / 25-26 / 1923$ & 6.3 & 12 & 6 & $\begin{array}{l}\text { Cenozoic Pecos } \\
\text { Alluvium }\end{array}$ & -2.96 & -.47 & TBWE (1960) \\
\hline 312 & Rio Grande & $\begin{array}{l}\text { RCWID \#1 main } \\
\text { canal }\end{array}$ & source to 2.9 mi below source & $3 / 14 / 1933$ & 2.9 & 4 & 2 & $\begin{array}{l}\text { Cenozoic Pecos } \\
\text { Alluvium }\end{array}$ & -.2 & -.069 & TBWE (1960) \\
\hline 313 & Rio Grande & $\begin{array}{l}\text { RCWID \#1 main } \\
\text { canal }\end{array}$ & source to end & $10 / 27-28 / 1931$ & 11.4 & 19 & 5 & $\begin{array}{l}\text { Cenozoic Pecos } \\
\text { Alluvium }\end{array}$ & -6.38 & -.56 & TBWE (1960) \\
\hline 314 & Rio Grande & $\begin{array}{l}\text { RCWID \#1 main } \\
\text { canal }\end{array}$ & source to end & $1 / 11-12 / 1933$ & 9.3 & 13 & 7 & $\begin{array}{l}\text { Cenozoic Pecos } \\
\text { Alluvium }\end{array}$ & -3.9 & -.419 & TBWE (1960) \\
\hline 315 & Rio Grande & $\begin{array}{l}\text { RCWID \#1 main } \\
\text { canal }\end{array}$ & source to end & $3 / 13 / 1933$ & 9.3 & 11 & 7 & $\begin{array}{l}\text { Cenozoic Pecos } \\
\text { Alluvium }\end{array}$ & 7.1 & .763 & TBWE (1960) \\
\hline 316 & Rio Grande & $\begin{array}{l}\text { RCWID \#1 reservoir } \\
\text { outlet canal }\end{array}$ & reservoir to main canal & $7 / 22 / 1932$ & 3.4 & 3 & 2 & $\begin{array}{l}\text { Cenozoic Pecos } \\
\text { Alluvium, } \\
\text { Edwards-Trinity } \\
\text { (Plateau) }\end{array}$ & -3.8 & -1.118 & TBWE (1960) \\
\hline 317 & Rio Grande & $\begin{array}{l}\text { RCWID \#1 reservoir } \\
\text { outlet canal }\end{array}$ & reservoir to main canal & 7/26/1932 & 3.4 & 6 & 5 & $\begin{array}{l}\text { Cenozoic Pecos } \\
\text { Alluvium, } \\
\text { Edwards-Trinity } \\
\text { (Plateau) }\end{array}$ & -.3 & -.088 & TBWE (1960) \\
\hline 318 & Rio Grande & $\begin{array}{l}\text { RCWID \#1 reservoir } \\
\text { outlet canal }\end{array}$ & reservoir to main canal & $8 / 17 / 1932$ & 2.5 & 3 & 3 & $\begin{array}{l}\text { Cenozoic Pecos } \\
\text { Alluvium }\end{array}$ & -.2 & -.08 & TBWE (1960) \\
\hline 319 & Rio Grande & $\begin{array}{l}\text { RCWID \#1 reservoir } \\
\text { outlet canal }\end{array}$ & reservoir to main canal & 7/20/1933 & 2.9 & 2 & 2 & $\begin{array}{l}\text { Cenozoic Pecos } \\
\text { Alluvium }\end{array}$ & -2.6 & -.897 & TBWE (1960) \\
\hline 320 & Rio Grande & $\begin{array}{l}\text { RCWID \#1 reservoir } \\
\text { outlet canal }\end{array}$ & reservoir to main canal & $7 / 26 / 1933$ & 2.7 & 2 & 2 & $\begin{array}{l}\text { Cenozoic Pecos } \\
\text { Alluvium }\end{array}$ & -1.2 & -.444 & TBWE (1960) \\
\hline 321 & Rio Grande & Rio Grande & Comal to Indio Ranch & $1 / 13-3 / 18 / 1928$ & 34 & 3 & 3 & -- & 90.0 & 2.647 & TBWE (1960) \\
\hline 322 & Rio Grande & Rio Grande & Del Rio to Eagle Pass & 2/9-3/3/1926 & 64 & 10 & 4 & $\begin{array}{l}\text { Edwards-Trinity } \\
\quad \text { (Plateau) }\end{array}$ & 116.0 & 1.813 & TBWE (1960) \\
\hline
\end{tabular}


Table 1. Characteristics of flow gain-loss studies in Texas-Continued

\begin{tabular}{|c|c|c|c|c|c|c|c|c|c|c|c|}
\hline $\begin{array}{l}\text { Stream- } \\
\text { flow } \\
\text { study } \\
\text { no. }\end{array}$ & $\begin{array}{l}\text { Major } \\
\text { river } \\
\text { basin }\end{array}$ & $\begin{array}{l}\text { Stream } \\
\text { name }\end{array}$ & $\begin{array}{c}\text { Reach } \\
\text { identification }\end{array}$ & $\begin{array}{l}\text { Date of } \\
\text { study }\end{array}$ & $\begin{array}{l}\text { Reach } \\
\text { length } \\
\text { (river } \\
\text { mi) }\end{array}$ & $\begin{array}{l}\text { Total } \\
\text { no. of } \\
\text { measure- } \\
\text { ment } \\
\text { sites }\end{array}$ & $\begin{array}{l}\text { No. of } \\
\text { measure- } \\
\text { ment sites } \\
\text { on main } \\
\text { channel }\end{array}$ & $\begin{array}{l}\text { Major aquifer } \\
\text { outcrop(s) } \\
\text { intersected } \\
\text { by reach }\end{array}$ & $\begin{array}{c}\text { Total } \\
\text { gain or } \\
\text { loss }(-) \\
\text { in reach } \\
\left(\mathrm{ft}^{3} / \mathrm{s}\right)\end{array}$ & $\begin{array}{l}\text { Gain or } \\
\text { loss per } \\
\text { mile of } \\
\text { reach } \\
\left(\mathrm{ft}^{3} / \mathrm{s}-\mathrm{mi}\right)\end{array}$ & $\begin{array}{l}\text { Reference } \\
\text { for data }\end{array}$ \\
\hline 323 & Rio Grande & Rio Grande & Eagle Pass to Indio Ranch & $1 / 12-4 / 12 / 1928$ & 18 & 2 & 2 & -- & 55.0 & 3.056 & TBWE (1960) \\
\hline 324 & Rio Grande & Rio Grande & Eagle Pass to Indio Ranch & $2 / 2-3 / 14 / 1928$ & 18 & 3 & 3 & -- & 55.0 & 3.056 & TBWE (1960) \\
\hline 325 & Rio Grande & Rio Grande & Eagle Pass to Laredo & 2/22-4/12/1928 & 128 & 6 & 6 & -- & -10.0 & -.078 & TBWE (1960) \\
\hline 326 & Rio Grande & Rio Grande & Eagle Pass to Laredo & $2 / 22-4 / 22 / 1928$ & 128 & 2 & 2 & -- & -25.0 & -.195 & TBWE (1960) \\
\hline 327 & Rio Grande & Rio Grande & Eagle Pass to Laredo & $4 / 3-22 / 1928$ & 128 & 6 & 6 & -- & -75.0 & -.586 & TBWE (1960) \\
\hline 328 & Rio Grande & Rio Grande & Eagle Pass to San Ygnacio & $2 / 12-22 / 1926$ & 167.5 & 22 & 17 & Carrizo-Wilcox & -336.0 & -2.006 & TBWE (1960) \\
\hline 329 & Rio Grande & Rio Grande & $\begin{array}{l}\text { Elephant Butte-Mesilla Valley } \\
\text { Unit, East Canal near Anthony }\end{array}$ & $4 / 21 / 1971$ & 2.51 & 4 & 3 & Hueco-Mesilla Bolson & -3.1 & -1.235 & USGS (1972) \\
\hline 330 & Rio Grande & Rio Grande & $\begin{array}{l}\text { Elephant Butte—Mesilla Valley } \\
\text { Unit, Franklin Canal below } \\
\text { Ysleta }\end{array}$ & $4 / 22 / 1971$ & 2 & 3 & 3 & Hueco-Mesilla Bolson & -.6 & -.3 & USGS (1972) \\
\hline 331 & Rio Grande & Rio Grande & $\begin{array}{l}\text { Elephant Butte-Mesilla Valley } \\
\text { Unit, Franklin Drain below } \\
\text { Sorocco }\end{array}$ & $4 / 22 / 1971$ & 2.4 & 3 & 3 & Hueco-Mesilla Bolson & 2.3 & .958 & USGS (1972) \\
\hline 332 & Rio Grande & Rio Grande & $\begin{array}{l}\text { Elephant Butte—Mesilla Valley } \\
\text { Unit, Nemexas Drain near } \\
\text { Anthony }\end{array}$ & $4 / 21 / 1971$ & 2 & 3 & 3 & Hueco-Mesilla Bolson & 1.81 & .905 & USGS (1972) \\
\hline 333 & Rio Grande & Rio Grande & $\begin{array}{l}\text { Elephant Butte-Mesilla Valley } \\
\text { Unit, West Canal near Anthony }\end{array}$ & $4 / 21 / 1971$ & 2.7 & 4 & 3 & Hueco-Mesilla Bolson & -6.21 & -2.3 & USGS (1972) \\
\hline 334 & Rio Grande & Rio Grande & $\begin{array}{l}\text { Elephant Butte-Mesilla Valley } \\
\text { Unit, West Drain near Anthony }\end{array}$ & $4 / 21 / 1971$ & 1.8 & 3 & 3 & Hueco-Mesilla Bolson & 5.0 & 2.778 & USGS (1972) \\
\hline 335 & Rio Grande & Rio Grande & Lajitas to Del Rio & 2/7-20/1925 & 293.1 & 11 & 8 & $\begin{array}{l}\text { Edwards-Trinity } \\
\quad \text { (Plateau) }\end{array}$ & 783.0 & 2.671 & TBWE (1960) \\
\hline 336 & Rio Grande & Toyah Cr & $\begin{array}{l}1.2 \mathrm{mi} \text { above to } 8.8 \mathrm{mi} \text { below } \\
\text { Toyahvale }\end{array}$ & $11 / 6 / 1932$ & 7.8 & 6 & 4 & $\begin{array}{l}\text { Cenozoic Pecos } \\
\text { Alluvium }\end{array}$ & 5.2 & .667 & TBWE (1960) \\
\hline 337 & Rio Grande & Toyah Cr & $\begin{array}{l}1.2 \mathrm{mi} \text { above to } 8.8 \mathrm{mi} \text { below } \\
\text { Toyahvale }\end{array}$ & $1 / 23 / 1933$ & 5.8 & 5 & 4 & $\begin{array}{l}\text { Cenozoic Pecos } \\
\text { Alluvium }\end{array}$ & 2.3 & .397 & TBWE (1960) \\
\hline 338 & Rio Grande & Toyah Cr & $\begin{array}{l}1.2 \mathrm{mi} \text { above to } 8.8 \mathrm{mi} \text { below } \\
\text { Toyahvale }\end{array}$ & $3 / 14 / 1933$ & 10 & 8 & 5 & $\begin{array}{l}\text { Cenozoic Pecos } \\
\text { Alluvium }\end{array}$ & 2.3 & .23 & TBWE (1960) \\
\hline 339 & Rio Grande & Toyah Cr & $\begin{array}{l}1.2 \text { mi above to } 8.8 \text { mi below } \\
\text { Toyahvale }\end{array}$ & $5 / 16 / 1933$ & 5.8 & 7 & 4 & $\begin{array}{l}\text { Cenozoic Pecos } \\
\text { Alluvium }\end{array}$ & 4.9 & .845 & TBWE (1960) \\
\hline 340 & Rio Grande & Toyah Cr & $\begin{array}{l}1.2 \mathrm{mi} \text { above to } 8.8 \mathrm{mi} \text { below } \\
\text { Toyahvale }\end{array}$ & $7 / 11 / 1933$ & 5.8 & 7 & 4 & $\begin{array}{l}\text { Cenozoic Pecos } \\
\text { Alluvium }\end{array}$ & 4.9 & .845 & TBWE (1960) \\
\hline 341 & Rio Grande & West Sandia Cr & $\begin{array}{l}\text { above East Sandia Spring at } \\
\text { Balmorhea }(08430000)\end{array}$ & $10 / 17 / 1932$ & .76 & 4 & 3 & $\begin{array}{l}\text { Cenozoic Pecos } \\
\text { Alluvium }\end{array}$ & 1.9 & 2.5 & TBWE (1960) \\
\hline 342 & Sabine & Lake $\mathrm{Fk} \mathrm{Cr}$ & SH 182 to US 80 & $8 / 31-9 / 1 / 1981$ & 1.6 & 3 & 3 & Carrizo-Wilcox & -2.6 & -1.625 & USGS (1982b) \\
\hline 343 & Sabine & Sabine and Old $\mathrm{R}$ & Ruliff (08030500) to IH-10 & $10 / 31-11 / 2 / 1966$ & 5.3 & 4 & 2 & Gulf Coast & 27.0 & 5.094 & USGS (1968) \\
\hline 344 & Sabine & Sabine and Old $\mathrm{R}$ & Ruliff (08030500) to IH-10 & $9 / 12-13 / 1967$ & 5.3 & 4 & 3 & Gulf Coast & -206.0 & -38.868 & USGS (1968) \\
\hline 345 & Sabine & Sabine R & FM 1804 to FM 2517 & $9 / 22-24 / 1981$ & 156.4 & 11 & 10 & Carrizo-Wilcox & 127.8 & .817 & USGS (1982b) \\
\hline
\end{tabular}


Table 1. Characteristics of flow gain-loss studies in Texas-Continued

\begin{tabular}{|c|c|c|c|c|c|c|c|c|c|c|c|}
\hline $\begin{array}{l}\text { Stream- } \\
\text { flow } \\
\text { study } \\
\text { no. }\end{array}$ & $\begin{array}{l}\text { Major } \\
\text { river } \\
\text { basin }\end{array}$ & $\begin{array}{l}\text { Stream } \\
\text { name }\end{array}$ & $\begin{array}{l}\text { Reach } \\
\text { identification }\end{array}$ & $\begin{array}{l}\text { Date of } \\
\text { study }\end{array}$ & $\begin{array}{l}\text { Reach } \\
\text { length } \\
\text { (river } \\
\text { mi) }\end{array}$ & $\begin{array}{c}\text { Total } \\
\text { no. of } \\
\text { measure- } \\
\text { ment } \\
\text { sites }\end{array}$ & $\begin{array}{c}\text { No. of } \\
\text { measure- } \\
\text { ment sites } \\
\text { on main } \\
\text { channel }\end{array}$ & $\begin{array}{c}\text { Major aquifer } \\
\text { outcrop(s) } \\
\text { intersected } \\
\text { by reach }\end{array}$ & $\begin{array}{c}\text { Total } \\
\text { gain or } \\
\text { loss }(-) \\
\text { in reach } \\
\left(\mathrm{ft}^{3} / \mathrm{s}\right)\end{array}$ & $\begin{array}{c}\text { Gain or } \\
\text { loss per } \\
\text { mile of } \\
\text { reach } \\
\left(\mathrm{ft}^{3} / \mathrm{s}-\mathrm{mi}\right)\end{array}$ & $\begin{array}{l}\text { Reference } \\
\text { for data }\end{array}$ \\
\hline 346 & Sabine & Sabine R & $\begin{array}{l}\text { Wills Point }(08017410) \text { to Smith- } \\
\text { Upshur Co line at county road } \\
\text { crossing }\end{array}$ & $8 / 31-9 / 2 / 1981$ & 80.5 & 8 & 6 & Carrizo-Wilcox & 427.42 & 5.31 & USGS (1982b) \\
\hline 347 & Sabine & Sabine R & $\begin{array}{l}\text { northeast of Carthage to Ruliff } \\
\quad(08030500)\end{array}$ & $9 / 4-5 / 1963$ & 268 & 98 & 30 & $\begin{array}{l}\text { Carrizo-Wilcox, Gulf } \\
\text { Coast }\end{array}$ & 208.72 & .779 & USGS (1964) \\
\hline 348 & San Antonio & Cibolo $\mathrm{Cr}$ & $\begin{array}{l}7.5 \text { mi above Boerne to gaging } \\
\text { station at Selma }(08185000)\end{array}$ & $1 / 17-30 / 1958$ & 56 & 27 & 19 & Edwards, Trinity & -89.2 & -1.593 & TBWE (1960) \\
\hline 349 & San Antonio & Cibolo $\mathrm{Cr}$ & near Randolph AFB to mouth & $3 / 5-7 / 1963$ & 79.3 & 18 & 13 & $\begin{array}{l}\text { Carrizo-Wilcox, Gulf } \\
\text { Coast }\end{array}$ & 16.68 & .21 & USGS (1964) \\
\hline 350 & San Antonio & Cibolo $\mathrm{Cr}$ & Selma (08185000) to mouth & $3 / 4-8 / 1968$ & 87.1 & 52 & 27 & $\begin{array}{l}\text { Carrizo-Wilcox, Gulf } \\
\text { Coast }\end{array}$ & 59.53 & .683 & USGS (1969) \\
\hline 351 & San Antonio & Salado Cr & $\begin{array}{l}\text { Wilderness Rd (08178585) to Old } \\
\text { Blanco Rd (08178593) }\end{array}$ & $10 / 26 / 1998$ & 3.15 & 2 & 2 & Edwards, Trinity & -60.4 & -19.175 & unpublished \\
\hline 352 & San Antonio & Salado Cr & $\begin{array}{l}\text { Wilderness Rd (08178585) to Old } \\
\text { Blanco Rd (08178593) }\end{array}$ & $10 / 26 / 1998$ & 3.15 & 2 & 2 & Edwards, Trinity & -66.0 & -20.952 & unpublished \\
\hline 353 & San Antonio & Salado Cr & $\begin{array}{l}\text { Wilderness Rd (08178585) to Old } \\
\text { Blanco Rd (08178593) }\end{array}$ & $10 / 27 / 1998$ & 3.15 & 2 & 2 & Edwards, Trinity & -61.6 & -19.556 & unpublished \\
\hline 354 & San Antonio & Salado Cr & $\begin{array}{l}\text { Wilderness Rd (08178585) to Old } \\
\text { Blanco Rd (08178593) }\end{array}$ & $10 / 28 / 1998$ & 7.21 & 3 & 3 & Edwards, Trinity & -98.8 & -13.703 & unpublished \\
\hline 355 & San Antonio & Salado Cr & $\begin{array}{l}\text { Wilderness Rd (08178585) to Old } \\
\text { Blanco Rd (08178593) }\end{array}$ & $10 / 28 / 1998$ & 7.21 & 3 & 3 & Edwards, Trinity & -83.5 & -11.581 & unpublished \\
\hline 356 & San Antonio & Salado Cr & $\begin{array}{l}\text { Wilderness Rd (08178585) to Old } \\
\text { Blanco Rd (08178593) }\end{array}$ & $10 / 29 / 1998$ & 7.21 & 3 & 3 & Edwards, Trinity & -56.4 & -7.822 & unpublished \\
\hline 357 & San Antonio & Salado Cr & $\begin{array}{l}\text { Wilderness Rd (08178585) to Old } \\
\text { Blanco Rd (08178593) }\end{array}$ & $10 / 29 / 1998$ & 7.21 & 3 & 3 & Edwards, Trinity & -60.7 & -8.419 & unpublished \\
\hline 358 & San Antonio & Salado $\mathrm{Cr}$ & $\begin{array}{l}\text { Wilderness Rd (08178585) to Old } \\
\text { Blanco Rd (08178593) }\end{array}$ & $10 / 30 / 1998$ & 3.15 & 2 & 2 & Edwards, Trinity & -29.4 & -9.333 & unpublished \\
\hline 359 & San Antonio & Salado Cr & $\begin{array}{l}\text { Wilderness Rd (08178585) to Old } \\
\text { Blanco Rd (08178593) }\end{array}$ & $10 / 30 / 1998$ & 7.21 & 3 & 3 & Edwards, Trinity & -45.0 & -6.241 & unpublished \\
\hline 360 & San Antonio & San Antonio R & $\begin{array}{l}\text { Hildebrand Ave to San Pedro Cr } \\
\text { mouth }\end{array}$ & $7 / 17 / 1957$ & 8 & 14 & 10 & -- & 12.13 & 1.516 & TBWE (1960) \\
\hline 361 & San Antonio & San Antonio R & San Pedro Cr to Ashley St Bridge & $10 / 27 / 1999$ & 17.54 & 6 & 5 & Carrizo-Wilcox & 9.4 & .536 & unpublished \\
\hline 362 & San Jacinto & $\begin{array}{l}\text { East Fk San Jacinto } \\
\quad \text { R }\end{array}$ & $\begin{array}{l}\text { Cleveland ( } 08070000) \text { to upstream } \\
\text { from Lake Houston }\end{array}$ & $6 / 24 / 1969$ & 18 & 9 & 5 & Gulf Coast & 4.2 & .233 & USGS (1970) \\
\hline 363 & San Jacinto & $\begin{array}{l}\text { West Fk San Jacinto } \\
\quad \mathrm{R}\end{array}$ & $\begin{array}{l}\text { northwest of Conroe to above Lake } \\
\text { Houston }\end{array}$ & $6 / 24 / 1969$ & 37.3 & 22 & 10 & Gulf Coast & 9.82 & .263 & USGS (1970) \\
\hline 364 & Trinity & Big Elkhart Cr & northwest of Grapeland to mouth & $9 / 15-16 / 1965$ & 25.7 & 9 & 7 & -- & 5.18 & .202 & USGS (1966) \\
\hline 365 & Trinity & Little Elkhart Cr & south of Grapeland to mouth & $9 / 16 / 1965$ & 17.5 & 11 & 5 & -- & -1.59 & -.091 & USGS (1966) \\
\hline 366 & Trinity & Trinity R & Riverside to Liberty & $11 / 4-8 / 1952$ & 133.5 & 21 & 5 & Gulf Coast & 37.16 & .278 & TBWE (1960) \\
\hline
\end{tabular}


Table 2. Inventory of daily mean streamflows for active and discontinued streamflow-gaging stations in Texas

[mi ${ }^{2}$, square miles; R, River; --, not applicable; Cr, Creek; nr, near; Fork, Fk; trib, tributary; FTW, Fort Worth]

\begin{tabular}{|c|c|c|c|c|c|c|c|c|c|c|c|c|}
\hline \multirow[b]{2}{*}{$\begin{array}{c}\text { Station } \\
\text { no. }\end{array}$} & \multirow[b]{2}{*}{ Station name } & \multirow[b]{2}{*}{ Latitude } & \multirow[b]{2}{*}{ Longitude } & \multirow[b]{2}{*}{ County } & \multirow{2}{*}{$\begin{array}{l}\text { Contri- } \\
\text { buting } \\
\text { drainage } \\
\text { area } \\
\left(\mathrm{mi}^{2}\right)\end{array}$} & \multirow[b]{2}{*}{$\begin{array}{l}\text { Urban } \\
\text { basin }\end{array}$} & \multicolumn{3}{|c|}{ Years of record ${ }^{2}$} & \multicolumn{3}{|c|}{ Begin and end year ${ }^{2}$} \\
\hline & & & & & & & Total & $\begin{array}{l}\text { Unregu- } \\
\text { lated }\end{array}$ & $\begin{array}{l}\text { Regu- } \\
\text { lated }^{3}\end{array}$ & $\begin{array}{l}\text { First } \\
\text { unregu- } \\
\text { lated }\end{array}$ & $\begin{array}{l}\text { First } \\
\text { regu- } \\
\text { lated }\end{array}$ & $\begin{array}{c}\text { Last } \\
\text { year }\end{array}$ \\
\hline \multicolumn{13}{|c|}{ Arkansas R Basin (Canadian R) } \\
\hline 07227000 & Canadian R at Logan, NM & $35^{\circ} 21^{\prime} 25^{\prime \prime}$ & $103^{\circ} 25^{\prime} 03^{\prime \prime}$ & Quay & 10,000 & -- & 81 & 19 & 62 & 1904 & 1939 & 2000 \\
\hline 07227100 & Revuelto Cr nr Logan, NM & $35^{\circ} 20^{\prime} 29^{\prime \prime}$ & $103^{\circ} 23^{\prime} 37^{\prime \prime}$ & Quay & 786 & -- & 41 & 41 & 0 & 1960 & -- & 2000 \\
\hline 07227448 & Punta De Agua Cr nr Channing, TX & $35^{\circ} 40^{\prime} 03^{\prime \prime}$ & $102^{\circ} 28^{\prime} 48^{\prime \prime}$ & Hartley & 3,570 & -- & 6 & 0 & 6 & -- & 1968 & 1973 \\
\hline 07227470 & Canadian R at Tascosa, TX & $35^{\circ} 31^{\prime} 08^{\prime \prime}$ & $102^{\circ} 15^{\prime} 35^{\prime \prime}$ & Oldham & 14,710 & -- & 9 & 9 & 0 & 1969 & -- & 1977 \\
\hline 07227500 & Canadian R nr Amarillo, TX & $35^{\circ} 28^{\prime} 13^{\prime \prime}$ & $101^{\circ} 52^{\prime} 45^{\prime \prime}$ & Potter & 15,400 & -- & 62 & 0 & 62 & -- & 1939 & 2000 \\
\hline 07227920 & Dixon Cr nr Borger, TX & $35^{\circ} 39^{\prime} 53^{\prime \prime}$ & $101^{\circ} 21^{\prime} 02^{\prime \prime}$ & Hutchinson & 134 & -- & 15 & 15 & 0 & 1975 & -- & 1989 \\
\hline 07228000 & Canadian R nr Canadian, TX & $35^{\circ} 56^{\prime} 06^{\prime \prime}$ & $100^{\circ} 22^{\prime} 13^{\prime \prime}$ & Hemphill & 18,200 & -- & 62 & 25 & 37 & 1939 & 1964 & 2000 \\
\hline 07233500 & Palo Duro Cr nr Spearman, TX & $36^{\circ} 12^{\prime} 08^{\prime \prime}$ & $101^{\circ} 18^{\prime} 20^{\prime \prime}$ & Hansford & 440 & -- & 35 & 35 & 0 & 1946 & -- & 2000 \\
\hline 07235000 & Wolf Cr at Lipscomb, TX & $36^{\circ} 14^{\prime} 19^{\prime \prime}$ & $100^{\circ} 16^{\prime} 31^{\prime \prime}$ & Lipscomb & 475 & -- & 41 & 2 & 39 & 1941 & 1962 & 2000 \\
\hline \multicolumn{13}{|c|}{ Red R Basin } \\
\hline 07295500 & Tierra Blanca Cr above Buffalo Lake nr Umbarger, TX & $34^{\circ} 50^{\prime} 55^{\prime \prime}$ & $102^{\circ} 10^{\prime} 32^{\prime \prime}$ & Deaf Smith & 538 & -- & 20 & 20 & 0 & 1941 & -- & 1973 \\
\hline 07296100 & Tierra Blanca Cr below Buffalo Lake nr Umbarger, TX & $34^{\circ} 55^{\prime} 27^{\prime \prime}$ & $102^{\circ} 05^{\prime} 57^{\prime \prime}$ & Randall & 2,080 & -- & 6 & 0 & 6 & -- & 1968 & 1973 \\
\hline 07297500 & Prairie Dog Town Fk Red R nr Canyon, TX & $35^{\circ} 00^{\prime} 38^{\prime \prime}$ & $101^{\circ} 53^{\prime} 29^{\prime \prime}$ & Randall & 711 & -- & 13 & 1 & 12 & 1925 & 1926 & 1949 \\
\hline 07297910 & Prairie Dog Town Fk Red R nr Wayside, TX & $34^{\circ} 50^{\prime} 15^{\prime \prime}$ & $101^{\circ} 24^{\prime} 49^{\prime \prime}$ & Armstrong & 930 & -- & 33 & 33 & 0 & 1968 & -- & 2000 \\
\hline 07298000 & North Tule Draw at reservoir nr Tulia, TX & $34^{\circ} 33^{\prime} 34^{\prime \prime}$ & $101^{\circ} 42^{\prime} 33^{\prime \prime}$ & Swisher & 65.0 & -- & 32 & 32 & 0 & 1942 & -- & 1973 \\
\hline 07298200 & Tule Cr nr Silverton, TX & $34^{\circ} 32^{\prime} 36^{\prime \prime}$ & $101^{\circ} 25^{\prime} 46^{\prime \prime}$ & Briscoe & 190 & -- & 22 & 9 & 13 & 1965 & 1974 & 1986 \\
\hline 07298500 & Prairie Dog Town Fk Red R nr Brice, TX & $34^{\circ} 37^{\prime} 40^{\prime \prime}$ & $100^{\circ} 56^{\prime} 25^{\prime \prime}$ & Hall & 1,580 & -- & 7 & 7 & 0 & 1940 & -- & 1962 \\
\hline 07299000 & Mulberry Cr nr Brice, TX & $34^{\circ} 40^{\prime} 30^{\prime \prime}$ & $100^{\circ} 55^{\prime} 00^{\prime \prime}$ & Hall & 534 & -- & 1 & 1 & 0 & 1950 & -- & 1950 \\
\hline 07299200 & Prairie Dog Town Fk Red R nr Lakeview, TX & $34^{\circ} 34^{\prime} 23^{\prime \prime}$ & $100^{\circ} 44^{\prime} 43^{\prime \prime}$ & Hall & 2,020 & -- & 17 & 16 & 1 & 1964 & 1980 & 1980 \\
\hline 07299300 & Little Red R nr Turkey, TX & $34^{\circ} 32^{\prime} 27^{\prime \prime}$ & $100^{\circ} 46^{\prime} 13^{\prime \prime}$ & Hall & 139 & -- & 13 & 13 & 0 & 1969 & -- & 1981 \\
\hline 07299500 & Prairie Dog Town Fk Red R nr Estelline, TX & $34^{\circ} 34^{\prime} 20^{\prime \prime}$ & $100^{\circ} 26^{\prime} 10^{\prime \prime}$ & Hall & 2,520 & -- & 9 & 9 & 0 & 1925 & -- & 1946 \\
\hline 07299512 & Jonah $\mathrm{Cr}$ at weir nr Estelline, $\mathrm{TX}$ & $34^{\circ} 34^{\prime} 20^{\prime \prime}$ & $100^{\circ} 20^{\prime} 00^{\prime \prime}$ & Childress & 65.5 & -- & 8 & 8 & 0 & 1975 & -- & 1982 \\
\hline 07299514 & Jonah $\mathrm{Cr}$ below weir nr Estelline, $\mathrm{TX}$ & $34^{\circ} 33^{\prime} 33^{\prime \prime}$ & $100^{\circ} 20^{\prime} 21^{\prime \prime}$ & Childress & 66.6 & -- & 2 & 2 & 0 & 1975 & -- & 1976 \\
\hline 07299530 & Salt Cr nr Estelline, TX & $34^{\circ} 35^{\prime} 26^{\prime \prime}$ & $100^{\circ} 15^{\prime} 08^{\prime \prime}$ & Childress & 142 & -- & 4 & 4 & 0 & 1975 & -- & 1978 \\
\hline 07299540 & Prairie Dog Town Fk Red R nr Childress, TX & $34^{\circ} 34^{\prime} 09^{\prime \prime}$ & $100^{\circ} 11^{\prime} 37^{\prime \prime}$ & Childress & 2,960 & -- & 35 & 13 & 22 & 1966 & 1979 & 2000 \\
\hline 07299570 & Red R nr Quanah, TX & $34^{\circ} 24^{\prime} 47^{\prime \prime}$ & $99^{\circ} 44^{\prime} 03^{\prime \prime}$ & Hardeman & 3,550 & -- & 22 & 18 & 4 & 1961 & 1979 & 1982 \\
\hline 07299670 & Groesbeck Cr at SH 6 nr Quanah, TX & $34^{\circ} 21^{\prime} 16^{\prime \prime}$ & $99^{\circ} 44^{\prime} 24^{\prime \prime}$ & Hardeman & 303 & -- & 38 & 38 & 0 & 1963 & -- & 2000 \\
\hline 07299850 & Salt Fk Red R nr Clarendon, TX & $35^{\circ} 00^{\prime} 10^{\prime \prime}$ & $100^{\circ} 533^{\prime} 30^{\prime \prime}$ & Donley & 457 & -- & 4 & 4 & 0 & 1961 & -- & 1964 \\
\hline 07299890 & Lelia Lake Cr below Bell Cr nr Hedley, TX & $34^{\circ} 56^{\prime} 08^{\prime \prime}$ & $100^{\circ} 41^{\prime} 46^{\prime \prime}$ & Donley & 74 & -- & 3 & 3 & 0 & 2000 & -- & 2000 \\
\hline 07300000 & Salt Fk Red R nr Wellington, TX & $34^{\circ} 57^{\prime} 27^{\prime \prime}$ & $100^{\circ} 13^{\prime} 14^{\prime \prime}$ & Collingsworth & 1,010 & -- & 48 & 15 & 33 & 1953 & 1968 & 2000 \\
\hline 07300500 & Salt Fk Red R at Mangum, OK & $34^{\circ} 51^{\prime} 30^{\prime \prime}$ & $99^{\circ} 30^{\prime} 30^{\prime \prime}$ & Greer & 1,360 & -- & 63 & 63 & 0 & 1938 & -- & 2000 \\
\hline 07301200 & McClellan Cr nr McLean, TX & $35^{\circ} 19^{\prime} 45^{\prime \prime}$ & $100^{\circ} 36^{\prime} 32^{\prime \prime}$ & Gray & 460 & -- & 13 & 0 & 13 & -- & 1968 & 1980 \\
\hline
\end{tabular}

$\tilde{\omega}$ Footnotes at end of table. 
Table 2. Inventory of daily mean streamflows for active and discontinued streamflow-gaging stations in Texas-Continued

\begin{tabular}{|c|c|c|c|c|c|c|c|c|c|c|c|c|}
\hline \multirow[b]{2}{*}{$\begin{array}{c}\text { Station } \\
\text { no. }\end{array}$} & \multirow[b]{2}{*}{ Station name } & \multirow[b]{2}{*}{ Latitude } & \multirow[b]{2}{*}{ Longitude } & \multirow[b]{2}{*}{ County } & \multirow{2}{*}{$\begin{array}{l}\text { Contri- } \\
\text { buting } \\
\text { drainage } \\
\text { area } \\
\left(\mathrm{mi}^{2}\right)\end{array}$} & \multirow[b]{2}{*}{$\begin{array}{l}\text { Urban } \\
\text { basin }\end{array}$} & \multicolumn{3}{|c|}{ Years of record $^{2}$} & \multicolumn{3}{|c|}{ Begin and end year $^{2}$} \\
\hline & & & & & & & Total & $\begin{array}{c}\text { Unregu- } \\
\text { lated }\end{array}$ & $\begin{array}{l}\text { Regu- } \\
\text { lated }^{3}\end{array}$ & $\begin{array}{c}\text { First } \\
\text { unregu- } \\
\text { lated }\end{array}$ & $\begin{array}{l}\text { First } \\
\text { regu- } \\
\text { lated }\end{array}$ & $\begin{array}{l}\text { Last } \\
\text { year }\end{array}$ \\
\hline \multicolumn{13}{|c|}{ Red R Basin-Continued } \\
\hline 07301300 & North Fk Red R nr Shamrock, TX & $35^{\circ} 15^{\prime} 51^{\prime \prime}$ & $100^{\circ} 14^{\prime} 29^{\prime \prime}$ & Wheeler & 703 & -- & 27 & 27 & 0 & 1965 & -- & 1991 \\
\hline 07301410 & Sweetwater Cr nr Kelton, TX & $35^{\circ} 28^{\prime} 23^{\prime \prime}$ & $100^{\circ} 07^{\prime} 14^{\prime \prime}$ & Wheeler & 267 & -- & 38 & 38 & 0 & 1963 & -- & 2000 \\
\hline 07307500 & Quitaque Cr nr Quitaque, TX & $34^{\circ} 14^{\prime} 24^{\prime \prime}$ & $101^{\circ} 07^{\prime} 03^{\prime \prime}$ & Floyd & 35.0 & -- & 14 & 14 & 0 & 1946 & -- & 1959 \\
\hline 07307600 & North Pease R nr Childress, TX & $34^{\circ} 16^{\prime} 30^{\prime \prime}$ & $100^{\circ} 17^{\prime} 05^{\prime \prime}$ & Cottle & 1,430 & -- & 6 & 6 & 0 & 1974 & -- & 1979 \\
\hline 07307750 & Middle Pease R nr Paducah, TX & $34^{\circ} 12^{\prime} 31^{\prime \prime}$ & $100^{\circ} 18^{\prime} 03^{\prime \prime}$ & Cottle & 1,020 & -- & 6 & 6 & 0 & 1974 & -- & 1979 \\
\hline 07307760 & Middle Pease R nr Paducah, TX & $34^{\circ} 11^{\prime} 28^{\prime \prime}$ & $100^{\circ} 12^{\prime} 38^{\prime \prime}$ & Cottle & 1,060 & -- & 3 & 3 & 0 & 1980 & -- & 1982 \\
\hline 07307800 & Pease R nr Childress, TX & $34^{\circ} 13^{\prime} 39^{\prime \prime}$ & $100^{\circ} 04^{\prime} 24^{\prime \prime}$ & Cottle & 2,200 & -- & 35 & 35 & 0 & 1961 & -- & 2000 \\
\hline 07308000 & Pease R nr Crowell, TX & $34^{\circ} 05^{\prime} 45^{\prime \prime}$ & $99^{\circ} 43^{\prime} 47^{\prime \prime}$ & Foard & 2,480 & -- & 20 & 20 & 0 & 1925 & -- & 1946 \\
\hline 07308200 & Pease R nr Vernon, TX & $34^{\circ} 10^{\prime} 45^{\prime \prime}$ & $99^{\circ} 16^{\prime} 40^{\prime \prime}$ & Wilbarger & 2,930 & -- & 30 & 30 & 0 & 1961 & -- & 2000 \\
\hline 07308500 & Red R nr Burkburnett, TX & $34^{\circ} 06^{\prime} 36^{\prime \prime}$ & $98^{\circ} 31^{\prime} 53^{\prime \prime}$ & Wichita & 14,600 & -- & 40 & 40 & 0 & 1961 & -- & 2000 \\
\hline 07311600 & North Wichita R nr Paducah, TX & $33^{\circ} 57^{\prime} 02^{\prime \prime}$ & $100^{\circ} 03^{\prime} 52^{\prime \prime}$ & Cottle & 540 & -- & 27 & 27 & 0 & 1962 & -- & 2000 \\
\hline 07311622 & North Fk Wichita R nr Crowell, TX & $33^{\circ} 52^{\prime} 12^{\prime \prime}$ & $99^{\circ} 56^{\prime} 48^{\prime \prime}$ & Foard & 591 & -- & 6 & 6 & 0 & 1971 & -- & 1976 \\
\hline 07311630 & Middle Wichita R nr Guthrie, TX & $33^{\circ} 47^{\prime} 45^{\prime \prime}$ & $100^{\circ} 04^{\prime} 29^{\prime \prime}$ & King & 50.3 & -- & 4 & 4 & 0 & 1997 & -- & 2000 \\
\hline 07311648 & Middle Fk Wichita R nr Truscott, TX & $33^{\circ} 51^{\prime} 12^{\prime \prime}$ & $99^{\circ} 57^{\prime} 44^{\prime \prime}$ & Foard & 161 & -- & 6 & 6 & 0 & 1971 & -- & 1976 \\
\hline 07311700 & North Wichita R nr Truscott, TX & $33^{\circ} 49^{\prime} 14^{\prime \prime}$ & $99^{\circ} 47^{\prime} 10^{\prime \prime}$ & Knox & 937 & -- & 40 & 40 & 0 & 1961 & -- & 2000 \\
\hline 07311780 & South Fk Wichita R nr Guthrie, TX & $33^{\circ} 37^{\prime} 29^{\prime \prime}$ & $100^{\circ} 13^{\prime} 04^{\prime \prime}$ & King & 239 & -- & 6 & 6 & 0 & 1971 & -- & 1976 \\
\hline 07311782 & South Wichita R at low flow dam nr Guthrie, TX & $33^{\circ} 37^{\prime} 19^{\prime \prime}$ & $100^{\circ} 12^{\prime} 31^{\prime \prime}$ & King & 223 & -- & 14 & 14 & 0 & 1985 & -- & 2000 \\
\hline 07311783 & South Wichita R below low flow dam nr Guthrie, TX & $33^{\circ} 37^{\prime} 19^{\prime \prime}$ & $100^{\circ} 12^{\prime} 31^{\prime \prime}$ & King & 223 & -- & 15 & 15 & 0 & 1986 & -- & 2000 \\
\hline 07311790 & South Wichita R at Ross Ranch nr Benjamin, TX & $33^{\circ} 39^{\prime} 18^{\prime \prime}$ & $100^{\circ} 00^{\prime} 49^{\prime \prime}$ & King & 499 & -- & 9 & 9 & 0 & 1971 & -- & 1979 \\
\hline 07311800 & South Wichita R nr Benjamin, TX & $33^{\circ} 38^{\prime} 39^{\prime \prime}$ & $99^{\circ} 48^{\prime} 02^{\prime \prime}$ & Knox & 584 & -- & 40 & 40 & 0 & 1961 & -- & 2000 \\
\hline 07311900 & Wichita R nr Seymour, TX & $33^{\circ} 42^{\prime} 01^{\prime \prime}$ & $99^{\circ} 23^{\prime} 18^{\prime \prime}$ & Baylor & 1,870 & -- & 23 & 23 & 0 & 1961 & -- & 2000 \\
\hline 07312100 & Wichita R nr Mabelle, TX & $33^{\circ} 45^{\prime} 36^{\prime \prime}$ & $99^{\circ} 08^{\prime} 33^{\prime \prime}$ & Baylor & 2,090 & -- & 41 & 0 & 41 & -- & 1960 & 2000 \\
\hline 07312130 & Wichita R at SH 25 nr Kamay, TX & $33^{\circ} 52^{\prime} 09^{\prime \prime}$ & $98^{\circ} 50^{\prime} 20^{\prime \prime}$ & Wichita & 2,182 & -- & 4 & 0 & 4 & -- & 1997 & 2000 \\
\hline 07312200 & Beaver Cr nr Electra, TX & $33^{\circ} 54^{\prime} 21^{\prime \prime}$ & $98^{\circ} 54^{\prime} 17^{\prime \prime}$ & Wichita & 652 & -- & 40 & 0 & 40 & -- & 1961 & 2000 \\
\hline 07312500 & Wichita R at Wichita Falls, TX & $33^{\circ} 54^{\prime} 34^{\prime \prime}$ & $98^{\circ} 32^{\prime} 00^{\prime \prime}$ & Wichita & 3,140 & -- & 62 & 0 & 62 & -- & 1939 & 2000 \\
\hline 07312700 & Wichita R nr Charlie, TX & $34^{\circ} 03^{\prime} 11^{\prime \prime}$ & $98^{\circ} 17^{\prime} 47^{\prime \prime}$ & Clay & 3,440 & -- & 33 & 0 & 33 & -- & 1968 & 2000 \\
\hline 07314500 & Little Wichita R nr Archer City, TX & $33^{\circ} 39^{\prime} 45^{\prime \prime}$ & $98^{\circ} 36^{\prime} 46^{\prime \prime}$ & Archer & 481 & -- & 57 & 13 & 44 & 1933 & 1946 & 2000 \\
\hline 07314900 & Little Wichita R above Henrietta, TX & $33^{\circ} 49^{\prime} 36^{\prime \prime}$ & $98^{\circ} 14^{\prime} 23^{\prime \prime}$ & Clay & 1,040 & -- & 47 & 13 & 34 & 1954 & 1967 & 2000 \\
\hline 07315200 & East Fk Little Wichita R nr Henrietta, TX & $33^{\circ} 48^{\prime} 46^{\prime \prime}$ & $98^{\circ} 05^{\prime} 05^{\prime \prime}$ & Clay & 178 & -- & 36 & 29 & 7 & 1965 & 1994 & 2000 \\
\hline 07315400 & Little Wichita R nr Ringgold, TX & $33^{\circ} 53^{\prime} 55^{\prime \prime}$ & $98^{\circ} 04^{\prime} 05^{\prime \prime}$ & Montague & 1,350 & -- & 6 & 0 & 6 & -- & 1960 & 1965 \\
\hline 07315500 & Red R nr Terrall, OK & $33^{\circ} 52^{\prime} 43^{\prime \prime}$ & $97^{\circ} 56^{\prime} 03^{\prime \prime}$ & Jefferson & 22,800 & -- & 62 & 0 & 62 & -- & 1938 & 2000 \\
\hline 07316000 & Red R nr Gainesville, TX & $33^{\circ} 43^{\prime} 40^{\prime \prime}$ & $97^{\circ} 09^{\prime} 35^{\prime \prime}$ & Cooke & 24,800 & -- & 60 & 6 & 54 & 1937 & 1943 & 1996 \\
\hline 07316200 & Mineral Cr nr Sadler, TX & $33^{\circ} 42^{\prime} 08^{\prime \prime}$ & $96^{\circ} 50^{\prime} 51^{\prime \prime}$ & Grayson & 26.0 & -- & 8 & 8 & 0 & 1969 & -- & 1976 \\
\hline
\end{tabular}


Table 2. Inventory of daily mean streamflows for active and discontinued streamflow-gaging stations in Texas-Continued

\begin{tabular}{|c|c|c|c|c|c|c|c|c|c|c|c|c|}
\hline \multirow[b]{2}{*}{$\begin{array}{c}\text { Station } \\
\text { no. }\end{array}$} & \multirow[b]{2}{*}{ Station name } & \multirow[b]{2}{*}{ Latitude } & \multirow[b]{2}{*}{ Longitude } & \multirow[b]{2}{*}{ County } & \multirow{2}{*}{$\begin{array}{l}\text { Contri- } \\
\text { buting } \\
\text { drainage } \\
\text { area } \\
\left(\mathrm{mi}^{2}\right)\end{array}$} & \multirow[b]{2}{*}{$\begin{array}{l}\text { Urban } \\
\text { basin }\end{array}$} & \multicolumn{3}{|c|}{ Years of record $^{2}$} & \multicolumn{3}{|c|}{ Begin and end year ${ }^{2}$} \\
\hline & & & & & & & Total & $\begin{array}{l}\text { Unregu- } \\
\text { lated }\end{array}$ & $\begin{array}{l}\text { Regu- } \\
\text { lated }^{3}\end{array}$ & $\begin{array}{c}\text { First } \\
\text { unregu- } \\
\text { lated }\end{array}$ & $\begin{array}{l}\text { First } \\
\text { regu- } \\
\text { lated }\end{array}$ & $\begin{array}{l}\text { Last } \\
\text { year }\end{array}$ \\
\hline \multicolumn{13}{|c|}{ Red R Basin-Continued } \\
\hline 07331600 & Red R at Denison Dam nr Denison, TX & $33^{\circ} 49^{\prime} 08^{\prime \prime}$ & $96^{\circ} 33^{\prime} 47^{\prime \prime}$ & Grayson & 33,800 & -- & 69 & 19 & 50 & 1924 & 1944 & 2000 \\
\hline 07332600 & Bois d'Arc Cr nr Randolph, TX & $33^{\circ} 28^{\prime} 32^{\prime \prime}$ & $96^{\circ} 12^{\prime} 52^{\prime \prime}$ & Fannin & 72.0 & -- & 22 & 22 & 0 & 1964 & -- & 1985 \\
\hline 07335400 & Sanders Cr nr Chicota, TX & $33^{\circ} 51^{\prime} 09^{\prime \prime}$ & $95^{\circ} 32^{\prime} 40^{\prime \prime}$ & Lamar & 175 & -- & 19 & 0 & 19 & -- & 1968 & 1986 \\
\hline 07335500 & Red R at Arthur City, TX & $33^{\circ} 52^{\prime} 32^{\prime \prime}$ & $95^{\circ} 30^{\prime} 06^{\prime \prime}$ & Lamar & 38,600 & -- & 69 & 13 & 57 & 1906 & 1944 & 2000 \\
\hline 07336750 & Little Pine Cr nr Kanawha, TX & $33^{\circ} 50^{\prime} 26^{\prime \prime}$ & $95^{\circ} 15^{\prime} 55^{\prime \prime}$ & Red R & 75.4 & -- & 11 & 11 & 0 & 1970 & -- & 1980 \\
\hline 07336800 & Pecan Bayou nr Clarksville, TX & $33^{\circ} 41^{\prime} 07^{\prime \prime}$ & $94^{\circ} 59^{\prime} 41^{\prime \prime}$ & Red R & 100 & -- & 15 & 15 & 0 & 1963 & -- & 1977 \\
\hline 07336820 & Red R nr De Kalb, TX & $33^{\circ} 40^{\prime} 59^{\prime \prime}$ & $94^{\circ} 41^{\prime} 39^{\prime \prime}$ & Bowie & 41,400 & -- & 31 & 0 & 31 & -- & 1968 & 1998 \\
\hline 07337000 & Red R at Index, Arkansas & $33^{\circ} 33^{\prime} 07^{\prime \prime}$ & $94^{\circ} 02^{\prime} 28^{\prime \prime}$ & Miller & 42,100 & -- & 64 & 7 & 57 & 1937 & 1944 & 2000 \\
\hline 07342465 & South Sulphur R at Commerce, TX & $33^{\circ} 12^{\prime} 42^{\prime \prime}$ & $95^{\circ} 54^{\prime} 50^{\prime \prime}$ & Hunt & 150 & -- & 9 & 9 & 0 & 1992 & -- & 2000 \\
\hline 07342470 & South Sulphur R nr Commerce, TX & $33^{\circ} 13^{\prime} 11^{\prime \prime}$ & $95^{\circ} 51^{\prime} 45^{\prime \prime}$ & Hunt & 189 & -- & 12 & 12 & 0 & 1980 & -- & 1991 \\
\hline 07342480 & Middle Sulphur R at Commerce, TX & $33^{\circ} 15^{\prime} 59^{\prime \prime}$ & $95^{\circ} 54^{\prime} 55^{\prime \prime}$ & Hunt & 44.1 & -- & 9 & 9 & 0 & 1992 & -- & 2000 \\
\hline 07342500 & South Sulphur R nr Cooper, TX & $33^{\circ} 21^{\prime} 23^{\prime \prime}$ & $95^{\circ} 35^{\prime} 41^{\prime \prime}$ & Delta & 527 & -- & 58 & 49 & 9 & 1943 & 1992 & 2000 \\
\hline 07343000 & North Sulphur R nr Cooper, TX & $33^{\circ} 28^{\prime} 29^{\prime \prime}$ & $95^{\circ} 35^{\prime} 15^{\prime \prime}$ & Lamar & 276 & -- & 51 & 51 & 0 & 1950 & -- & 2000 \\
\hline 07343200 & Sulphur R nr Talco, TX & $33^{\circ} 23^{\prime} 26^{\prime \prime}$ & $95^{\circ} 03^{\prime} 44^{\prime \prime}$ & Titus & 1,370 & -- & 44 & 35 & 9 & 1957 & 1992 & 2000 \\
\hline 07343300 & Cuthand Cr nr Bogata, TX & $33^{\circ} 32^{\prime} 51^{\prime \prime}$ & $95^{\circ} 10^{\prime} 22^{\prime \prime}$ & Red R & 69.0 & -- & 11 & 11 & 0 & 1964 & -- & 1974 \\
\hline 07343500 & White Oak Cr nr Talco, TX & $33^{\circ} 19^{\prime} 20^{\prime \prime}$ & $95^{\circ} 05^{\prime} 33^{\prime \prime}$ & Titus & 494 & -- & 50 & 22 & 28 & 1951 & 1973 & 2000 \\
\hline 07344000 & Sulphur R nr Darden, TX & $33^{\circ} 15^{\prime} 00^{\prime \prime}$ & $94^{\circ} 37^{\prime} 00^{\prime \prime}$ & Bowie & 2,770 & -- & 33 & 33 & 0 & 1924 & -- & 1956 \\
\hline 07344210 & Sulphur R nr Texarkana, TX & $33^{\circ} 18^{\prime} 20^{\prime \prime}$ & $94^{\circ} 09^{\prime} 03^{\prime \prime}$ & Bowie & 3,440 & -- & 6 & 0 & 6 & -- & 1980 & 1985 \\
\hline 07344482 & Big Cypress Cr nr Winnsboro, TX & $33^{\circ} 01^{\prime} 24^{\prime \prime}$ & $95^{\circ} 16^{\prime} 12^{\prime \prime}$ & Franklin & 27.2 & -- & 17 & 0 & 17 & -- & 1975 & 1991 \\
\hline 07344486 & Brushy $\mathrm{Cr}$ at Scroggins, $\mathrm{TX}$ & $32^{\circ} 58^{\prime} 32^{\prime \prime}$ & $95^{\circ} 11^{\prime} 03^{\prime \prime}$ & Franklin & 23.4 & -- & 22 & 22 & 0 & 1979 & -- & 2000 \\
\hline 07344500 & Big Cypress Cr nr Pittsburg, TX & $33^{\circ} 01^{\prime} 15^{\prime \prime}$ & $94^{\circ} 52^{\prime} 55^{\prime \prime}$ & Camp & 366 & -- & 41 & 21 & 20 & 1944 & 1970 & 1989 \\
\hline 07345000 & Boggy Cr nr Daingerfield, TX & $33^{\circ} 02^{\prime} 10^{\prime \prime}$ & $94^{\circ} 47^{\prime} 15^{\prime \prime}$ & Morris & 72.0 & -- & 34 & 34 & 0 & 1944 & -- & 1977 \\
\hline 07346000 & Big Cypress Cr nr Jefferson, TX & $32^{\circ} 44^{\prime} 58^{\prime \prime}$ & $94^{\circ} 29^{\prime} 55^{\prime \prime}$ & Marion & 850 & -- & 56 & 33 & 23 & 1925 & 1958 & 2000 \\
\hline 07346045 & Black Cypress Bayou at Jefferson, TX & $32^{\circ} 46^{\prime} 40^{\prime \prime}$ & $94^{\circ} 21^{\prime} 26^{\prime \prime}$ & Marion & 365 & -- & 32 & 32 & 0 & 1969 & -- & 2000 \\
\hline 07346050 & Little Cypress Cr nr Ore City, TX & $32^{\circ} 40^{\prime} 21^{\prime \prime}$ & $94^{\circ} 45^{\prime} 03^{\prime \prime}$ & Upshur & 383 & -- & 36 & 36 & 0 & 1964 & -- & 1999 \\
\hline 07346070 & Little Cypress Cr nr Jefferson, TX & $32^{\circ} 42^{\prime} 46^{\prime \prime}$ & $94^{\circ} 20^{\prime} 45^{\prime \prime}$ & Marion & 675 & -- & 54 & 54 & 0 & 1947 & -- & 2000 \\
\hline 07346140 & Frazier Cr nr Linden, TX & $33^{\circ} 03^{\prime} 14^{\prime \prime}$ & $94^{\circ} 17^{\prime} 24^{\prime \prime}$ & Cass & 48.0 & -- & 26 & 26 & 0 & 1966 & -- & 1991 \\
\hline \multicolumn{13}{|c|}{ Sabine R Basin } \\
\hline 08017200 & Cowleech Fk Sabine R at Greenville, TX & $33^{\circ} 07^{\prime} 58^{\prime \prime}$ & $96^{\circ} 04^{\prime} 36^{\prime \prime}$ & Hunt & 77.7 & -- & 41 & 41 & 0 & 1960 & -- & 2000 \\
\hline 08017300 & South Fk Sabine R nr Quinlan, TX & $32^{\circ} 53^{\prime} 52^{\prime \prime}$ & $96^{\circ} 15^{\prime} 11^{\prime \prime}$ & Hunt & 78.7 & -- & 41 & 41 & 0 & 1960 & -- & 2000 \\
\hline 08017410 & Sabine R nr Wills Point, TX & $32^{\circ} 48^{\prime} 22^{\prime \prime}$ & $95^{\circ} 55^{\prime} 09^{\prime \prime}$ & Van Zandt & 756 & -- & 30 & 0 & 30 & -- & 1971 & 2000 \\
\hline 08017500 & Sabine R nr Emory, TX & $32^{\circ} 46^{\prime} 23^{\prime \prime}$ & $95^{\circ} 47^{\prime} 56^{\prime \prime}$ & Rains & 888 & -- & 21 & 8 & 13 & 1953 & 1961 & 1973 \\
\hline 08018200 & Grand Saline Cr nr Grand Saline, TX & $32^{\circ} 40^{\prime} 20^{\prime \prime}$ & $95^{\circ} 36^{\prime} 36^{\prime \prime}$ & Van Zandt & 91.4 & -- & 5 & 5 & 0 & 1969 & -- & 1973 \\
\hline
\end{tabular}

ऊ Footnotes at end of table. 
Table 2. Inventory of daily mean streamflows for active and discontinued streamflow-gaging stations in Texas-Continued

\begin{tabular}{|c|c|c|c|c|c|c|c|c|c|c|c|c|}
\hline \multirow[b]{2}{*}{$\begin{array}{c}\text { Station } \\
\text { no. }\end{array}$} & \multirow[b]{2}{*}{ Station name } & \multirow[b]{2}{*}{ Latitude } & \multirow[b]{2}{*}{ Longitude } & \multirow[b]{2}{*}{ County } & \multirow{2}{*}{$\begin{array}{l}\text { Contri- } \\
\text { buting } \\
\text { drainage } \\
\text { area } \\
\left(\mathrm{mi}^{2}\right)\end{array}$} & \multirow[b]{2}{*}{$\begin{array}{l}\text { Urban } \\
\text { basin }\end{array}$} & \multicolumn{3}{|c|}{ Years of record $^{2}$} & \multicolumn{3}{|c|}{ Begin and end year $^{2}$} \\
\hline & & & & & & & Total & $\begin{array}{c}\text { Unregu- } \\
\text { lated }\end{array}$ & $\begin{array}{l}\text { Regu- } \\
\text { lated }^{3}\end{array}$ & $\begin{array}{c}\text { First } \\
\text { unregu- } \\
\text { lated }\end{array}$ & $\begin{array}{l}\text { First } \\
\text { regu- } \\
\text { lated }\end{array}$ & $\begin{array}{l}\text { Last } \\
\text { year }\end{array}$ \\
\hline \multicolumn{13}{|c|}{ Sabine R Basin-Continued } \\
\hline 08018500 & Sabine R nr Mineola, TX & $32^{\circ} 36^{\prime} 49^{\prime \prime}$ & $95^{\circ} 29^{\prime} 08^{\prime \prime}$ & Wood & 1,360 & -- & 53 & 20 & 33 & 1940 & 1968 & 2000 \\
\hline 08018730 & Burke Cr nr Yantis, TX & $32^{\circ} 59^{\prime} 26^{\prime \prime}$ & $95^{\circ} 37^{\prime} 18^{\prime \prime}$ & Hopkins & 33.1 & -- & 11 & 11 & 0 & 1979 & -- & 1989 \\
\hline 08019000 & Lake Fk Cr nr Quitman, TX & $32^{\circ} 45^{\prime} 47^{\prime \prime}$ & $95^{\circ} 27^{\prime} 46^{\prime \prime}$ & Wood & 585 & -- & 62 & 41 & 21 & 1925 & 1980 & 2000 \\
\hline 08019200 & Sabine R nr Hawkins, TX & $32^{\circ} 33^{\prime} 35^{\prime \prime}$ & $95^{\circ} 12^{\prime} 23^{\prime \prime}$ & Wood & 2,259 & -- & 3 & 0 & 3 & -- & 1998 & 2000 \\
\hline 08019500 & Big Sandy Cr nr Big Sandy, TX & $32^{\circ} 36^{\prime} 14^{\prime \prime}$ & $95^{\circ} 05^{\prime} 29^{\prime \prime}$ & Upshur & 231 & -- & 61 & 23 & 38 & 1940 & 1963 & 2000 \\
\hline 08020000 & Sabine R nr Gladewater, TX & $32^{\circ} 31^{\prime} 37^{\prime \prime}$ & $94^{\circ} 57^{\prime} 36^{\prime \prime}$ & Gregg & 2,790 & -- & 68 & 28 & 40 & 1933 & 1961 & 2000 \\
\hline 08020200 & Prairie Cr nr Gladewater, TX & $32^{\circ} 28^{\prime} 45^{\prime \prime}$ & $94^{\circ} 57^{\prime} 14^{\prime \prime}$ & Gregg & 48.9 & -- & 8 & 8 & 0 & 1969 & -- & 1976 \\
\hline 08020500 & Sabine R nr Longview, TX & $32^{\circ} 28^{\prime} 00^{\prime \prime}$ & $94^{\circ} 46^{\prime} 50^{\prime \prime}$ & Gregg & 2,950 & -- & 8 & 8 & 0 & 1925 & -- & 1932 \\
\hline 08020700 & Rabbit $\mathrm{Cr}$ at Kilgore, $\mathrm{TX}$ & $32^{\circ} 23^{\prime} 17^{\prime \prime}$ & $94^{\circ} 54^{\prime} 11^{\prime \prime}$ & Gregg & 75.8 & -- & 13 & 13 & 0 & 1964 & -- & 1976 \\
\hline 08020900 & Sabine R below Longview, TX & $32^{\circ} 25^{\prime} 00^{\prime \prime}$ & $94^{\circ} 42^{\prime} 35^{\prime \prime}$ & Gregg & 3,160 & -- & 5 & 0 & 5 & -- & 1996 & 2000 \\
\hline 08020960 & Mill Cr nr Henderson, TX & $32^{\circ} 14^{\prime} 02^{\prime \prime}$ & $94^{\circ} 46^{\prime} 54^{\prime \prime}$ & Rusk & 20.3 & -- & 3 & 3 & 0 & 1979 & -- & 1981 \\
\hline 08020980 & Mill Cr nr Longview, TX & $32^{\circ} 18^{\prime} 18^{\prime \prime}$ & $94^{\circ} 43^{\prime} 41^{\prime \prime}$ & Rusk & 47.9 & -- & 3 & 3 & 0 & 1979 & -- & 1981 \\
\hline 08020990 & Tiawichi Cr nr Longview, TX & $32^{\circ} 19^{\prime} 14^{\prime \prime}$ & $94^{\circ} 43^{\prime} 57^{\prime \prime}$ & Rusk & 62.7 & -- & 3 & 3 & 0 & 1979 & -- & 1981 \\
\hline 08021000 & Cherokee Bayou nr Elderville, TX & $32^{\circ} 20^{\prime} 00^{\prime \prime}$ & $94^{\circ} 42^{\prime} 00^{\prime \prime}$ & Gregg & 120 & -- & 9 & 9 & 0 & 1940 & -- & 1948 \\
\hline 08022040 & Sabine R nr Beckville, TX & $32^{\circ} 19^{\prime} 38^{\prime \prime}$ & $94^{\circ} 21^{\prime} 12^{\prime \prime}$ & Panola & 3,590 & -- & 62 & 22 & 40 & 1939 & 1961 & 2000 \\
\hline 08022070 & Martin Cr nr Tatum, TX & $32^{\circ} 17^{\prime} 44^{\prime \prime}$ & $94^{\circ} 29^{\prime} 29^{\prime \prime}$ & Panola & 148 & -- & 22 & 0 & 22 & -- & 1975 & 1996 \\
\hline 08022300 & Murvaul Bayou nr Gary, TX & $32^{\circ} 02^{\prime} 54^{\prime \prime}$ & $94^{\circ} 22^{\prime} 31^{\prime \prime}$ & Panola & 134 & -- & 25 & 0 & 25 & -- & 1959 & 1983 \\
\hline 08022400 & Socagee Cr nr Carthage, TX & $32^{\circ} 13 ' 54^{\prime \prime}$ & $94^{\circ} 05^{\prime} 31^{\prime \prime}$ & Panola & 82.6 & -- & 11 & 11 & 0 & 1963 & -- & 1973 \\
\hline 08022500 & Sabine R at Logansport, LA & $31^{\circ} 58^{\prime} 20^{\prime \prime}$ & $94^{\circ} 00^{\prime} 22^{\prime \prime}$ & Shelby & 4,840 & -- & 64 & 57 & 7 & 1904 & 1961 & 1967 \\
\hline 08023200 & Tenaha Cr nr Shelbyville, TX & $31^{\circ} 45^{\prime} 56^{\prime \prime}$ & $94^{\circ} 05^{\prime} 02^{\prime \prime}$ & Shelby & 97.8 & -- & 28 & 28 & 0 & 1953 & -- & 1980 \\
\hline 08024400 & Sabine R nr Milam, TX & $31^{\circ} 28^{\prime} 01^{\prime \prime}$ & $93^{\circ} 44^{\prime} 41^{\prime \prime}$ & Sabine & 6,510 & -- & 29 & 22 & 7 & 1924 & 1960 & 1966 \\
\hline 08024500 & Palo Gaucho Bayou nr Hemphill, TX & $31^{\circ} 23^{\prime} 10^{\prime \prime}$ & $93^{\circ} 50^{\prime} 08^{\prime \prime}$ & Sabine & 123 & -- & 13 & 13 & 0 & 1953 & -- & 1965 \\
\hline 08025307 & Mill Cr nr Burkeville, TX & $31^{\circ} 09^{\prime} 23^{\prime \prime}$ & $93^{\circ} 40^{\prime} 35^{\prime \prime}$ & Newton & 18.0 & -- & 5 & 5 & 0 & 1975 & -- & 1979 \\
\hline 08025360 & Sabine R at Toledo Bend Reservoir nr Burkeville, TX & $31^{\circ} 10^{\prime} 25^{\prime \prime}$ & $93^{\circ} 33^{\prime} 57^{\prime \prime}$ & Newton & 7,180 & -- & 29 & 0 & 29 & -- & 1972 & 2000 \\
\hline 08026000 & Sabine R nr Burkeville, TX & $31^{\circ} 03^{\prime} 50^{\prime \prime}$ & $93^{\circ} 31^{\prime} 10^{\prime \prime}$ & Newton & 7,480 & -- & 45 & 10 & 35 & 1956 & 1966 & 2000 \\
\hline 08028500 & Sabine R nr Bon Wier, TX & $30^{\circ} 44^{\prime} 49^{\prime \prime}$ & $93^{\circ} 36^{\prime} 30^{\prime \prime}$ & Newton & 8,230 & -- & 77 & 42 & 35 & 1924 & 1966 & 2000 \\
\hline 08029500 & Big Cow Cr nr Newton, TX & $30^{\circ} 49^{\prime} 08^{\prime \prime}$ & $93^{\circ} 47^{\prime} 07^{\prime \prime}$ & Newton & 128 & -- & 48 & 48 & 0 & 1953 & -- & 2000 \\
\hline 08030000 & Cypress Cr nr Buna, TX & $30^{\circ} 25^{\prime} 52^{\prime \prime}$ & $93^{\circ} 54^{\prime} 28^{\prime \prime}$ & Jasper & 69.2 & -- & 31 & 31 & 0 & 1953 & -- & 1983 \\
\hline 08030500 & Sabine R nr Ruliff, TX & $30^{\circ} 18^{\prime} 13^{\prime \prime}$ & $93^{\circ} 44^{\prime} 37^{\prime \prime}$ & Newton & 9,330 & -- & 76 & 42 & 34 & 1925 & 1967 & 2000 \\
\hline 08031000 & Cow Bayou nr Mauriceville, TX & $30^{\circ} 11^{\prime} 10^{\prime \prime}$ & $93^{\circ} 54^{\prime} 30^{\prime \prime}$ & Orange & 83.3 & -- & 34 & 34 & 0 & 1953 & -- & 1986 \\
\hline \multicolumn{13}{|c|}{ Neches R Basin } \\
\hline 08031200 & Kickapoo Cr nr Brownsboro, TX & $32^{\circ} 18^{\prime} 34^{\prime \prime}$ & $95^{\circ} 36^{\prime} 19^{\prime \prime}$ & Henderson & 232 & -- & 27 & 27 & 0 & 1963 & -- & 1989 \\
\hline 08031500 & Neches R nr Reese, TX & $32^{\circ} 01^{\prime} 30^{\prime \prime}$ & $95^{\circ} 25^{\prime} 40^{\prime \prime}$ & Cherokee & 851 & -- & 3 & 3 & 0 & 1925 & -- & 1927 \\
\hline
\end{tabular}

Footnotes at end of table. 
Table 2. Inventory of daily mean streamflows for active and discontinued streamflow-gaging stations in Texas-Continued

\begin{tabular}{|c|c|c|c|c|c|c|c|c|c|c|c|c|}
\hline \multirow[b]{2}{*}{$\begin{array}{c}\text { Station } \\
\text { no. }\end{array}$} & \multirow[b]{2}{*}{ Station name } & \multirow[b]{2}{*}{ Latitude } & \multirow[b]{2}{*}{ Longitude } & \multirow[b]{2}{*}{ County } & \multirow{2}{*}{$\begin{array}{l}\text { Contri- } \\
\text { buting } \\
\text { drainage } \\
\text { area } \\
\left(\mathrm{mi}^{2}\right)\end{array}$} & \multirow[b]{2}{*}{$\begin{array}{l}\text { Urban } \\
\text { basin }\end{array}$} & \multicolumn{3}{|c|}{ Years of record $^{2}$} & \multicolumn{3}{|c|}{ Begin and end year ${ }^{2}$} \\
\hline & & & & & & & Total & $\begin{array}{l}\text { Unregu- } \\
\text { lated }\end{array}$ & $\begin{array}{l}\text { Regu- } \\
\text { lated }^{3}\end{array}$ & $\begin{array}{c}\text { First } \\
\text { unregu- } \\
\text { lated }\end{array}$ & $\begin{array}{l}\text { First } \\
\text { regu- } \\
\text { lated }\end{array}$ & $\begin{array}{l}\text { Last } \\
\text { year }\end{array}$ \\
\hline \multicolumn{13}{|c|}{ Neches R Basin-Continued } \\
\hline 08032000 & Neches R nr Neches, TX & $31^{\circ} 53^{\prime} 32^{\prime \prime}$ & $95^{\circ} 25^{\prime} 50^{\prime \prime}$ & Anderson & 1,150 & -- & 61 & 22 & 39 & 1940 & 1962 & 2000 \\
\hline 08032500 & Neches R nr Alto, TX & $31^{\circ} 34^{\prime} 45^{\prime \prime}$ & $95^{\circ} 09^{\prime} 55^{\prime \prime}$ & Cherokee & 1,950 & -- & 34 & 17 & 17 & 1945 & 1962 & 1978 \\
\hline 08033000 & Neches R nr Diboll, TX & $31^{\circ} 07^{\prime} 58^{\prime \prime}$ & $94^{\circ} 48^{\prime} 35^{\prime \prime}$ & Polk & 2,720 & -- & 48 & 24 & 24 & 1924 & 1962 & 1985 \\
\hline 08033300 & Piney Cr nr Groveton, TX & $31^{\circ} 08^{\prime} 25^{\prime \prime}$ & $95^{\circ} 05^{\prime} 11^{\prime \prime}$ & Trinity & 79.0 & -- & 28 & 28 & 0 & 1962 & -- & 1989 \\
\hline 08033500 & Neches R nr Rockland, TX & $31^{\circ} 01^{\prime} 29^{\prime \prime}$ & $94^{\circ} 23^{\prime} 55^{\prime \prime}$ & Tyler & 3,640 & -- & 95 & 56 & 39 & 1904 & 1962 & 2000 \\
\hline 08033700 & Striker Cr nr Summerfield, TX & $32^{\circ} 00^{\prime} 10^{\prime \prime}$ & $94^{\circ} 59^{\prime} 35^{\prime \prime}$ & Cherokee & 146 & -- & 9 & 9 & 0 & 1941 & -- & 1949 \\
\hline 08033900 & East Fk Angelina R nr Cushing, TX & $31^{\circ} 51^{\prime} 36^{\prime \prime}$ & $94^{\circ} 49^{\prime} 23^{\prime \prime}$ & Rusk & 158 & -- & 25 & 25 & 0 & 1965 & -- & 1989 \\
\hline 08034500 & Mud Cr nr Jacksonville, TX & $31^{\circ} 58^{\prime} 35^{\prime \prime}$ & $95^{\circ} 09^{\prime} 38^{\prime \prime}$ & Cherokee & 376 & -- & 40 & 9 & 31 & 1940 & 1949 & 1979 \\
\hline 08035000 & Mud Cr at Ponta, TX & $31^{\circ} 53^{\prime} 21^{\prime \prime}$ & $95^{\circ} 05^{\prime} 19^{\prime \prime}$ & Cherokee & 475 & -- & 3 & 3 & 0 & 1925 & -- & 1927 \\
\hline 08036500 & Angelina R nr Alto, TX & $31^{\circ} 40^{\prime} 10^{\prime \prime}$ & $94^{\circ} 57^{\prime} 24^{\prime \prime}$ & Cherokee & 1,280 & -- & 42 & 1 & 41 & 1943 & 1960 & 2000 \\
\hline 08037000 & Angelina R nr Lufkin, TX & $31^{\circ} 27^{\prime} 26^{\prime \prime}$ & $94^{\circ} 43^{\prime} 34^{\prime \prime}$ & Angelina & 1,600 & -- & 51 & 28 & 23 & 1924 & 1957 & 1979 \\
\hline 08037050 & Bayou LaNana at Nacogdoches, TX & $31^{\circ} 36^{\prime} 58^{\prime \prime}$ & $94^{\circ} 38^{\prime} 28^{\prime \prime}$ & Nacogdoches & 31.3 & -- & 27 & 27 & 0 & 1965 & -- & 1993 \\
\hline 08037500 & Arenoso Cr nr San Augustine, TX & $31^{\circ} 35^{\prime} 48^{\prime \prime}$ & $94^{\circ} 16^{\prime} 06^{\prime \prime}$ & San Augustine & 75.3 & -- & 2 & 2 & 0 & 1939 & -- & 1940 \\
\hline 08038000 & Attoyac Bayou nr Chireno, TX & $31^{\circ} 30^{\prime} 15^{\prime \prime}$ & $94^{\circ} 18^{\prime} 15^{\prime \prime}$ & Nacogdoches & 503 & -- & 46 & 46 & 0 & 1925 & -- & 1985 \\
\hline 08038500 & Angelina R nr Zavalla, TX & $31^{\circ} 12^{\prime} 41^{\prime \prime}$ & $94^{\circ} 17^{\prime} 40^{\prime \prime}$ & Angelina & 2,890 & -- & 13 & 13 & 0 & 1952 & -- & 1964 \\
\hline 08039100 & Ayish Bayou nr San Augustine, TX & $31^{\circ} 23^{\prime} 46^{\prime \prime}$ & $94^{\circ} 09^{\prime} 03^{\prime \prime}$ & San Augustine & 89.0 & -- & 26 & 26 & 0 & 1960 & -- & 1985 \\
\hline 08039500 & Angelina R at Horger, TX & $31^{\circ} 00^{\prime} 54^{\prime \prime}$ & $94^{\circ} 09^{\prime} 07^{\prime \prime}$ & Jasper & 3,490 & -- & 30 & 22 & 8 & 1929 & 1965 & 1973 \\
\hline 08040600 & Neches R nr Town Bluff, TX & $30^{\circ} 47^{\prime} 27^{\prime \prime}$ & $94^{\circ} 09^{\prime} 03^{\prime \prime}$ & Tyler & 7,570 & -- & 49 & 0 & 49 & -- & 1952 & 2000 \\
\hline 08041000 & Neches R at Evadale, TX & $30^{\circ} 21^{\prime} 20^{\prime \prime}$ & $94^{\circ} 05^{\prime} 35^{\prime \prime}$ & Jasper & 7,950 & -- & 81 & 31 & 50 & 1905 & 1951 & 2000 \\
\hline 08041500 & Village Cr nr Kountze, TX & $30^{\circ} 23^{\prime} 52^{\prime \prime}$ & $94^{\circ} 15^{\prime} 48^{\prime \prime}$ & Hardin & 860 & -- & 64 & 64 & 0 & 1925 & -- & 2000 \\
\hline 08041700 & Pine Island Bayou nr Sour Lake, TX & $30^{\circ} 06^{\prime} 21^{\prime \prime}$ & $94^{\circ} 20^{\prime} 04^{\prime \prime}$ & Hardin & 336 & -- & 33 & 33 & 0 & 1968 & -- & 2000 \\
\hline \multicolumn{13}{|c|}{ Trinity R Basin } \\
\hline 08042700 & North Cr nr Jacksboro, TX & $33^{\circ} 16^{\prime} 57^{\prime \prime}$ & $98^{\circ} 17^{\prime} 53^{\prime \prime}$ & Jack & 21.6 & -- & 24 & 15 & 9 & 1957 & 1972 & 1980 \\
\hline 08042800 & West Fk Trinity R nr Jacksboro, TX & $33^{\circ} 17^{\prime} 30^{\prime \prime}$ & $98^{\circ} 04^{\prime} 49^{\prime \prime}$ & Jack & 683 & -- & 44 & 17 & 27 & 1957 & 1974 & 2000 \\
\hline 08043100 & West Fk Trinity R at Bridgeport, TX & $33^{\circ} 12^{\prime} 07^{\prime \prime}$ & $97^{\circ} 48^{\prime} 09^{\prime \prime}$ & Wise & 1,110 & -- & 5 & 0 & 5 & -- & 1985 & 1989 \\
\hline 08043500 & West Fk Trinity R at Bridgeport, TX & $33^{\circ} 12^{\prime} 05^{\prime \prime}$ & $97^{\circ} 45^{\prime} 21^{\prime \prime}$ & Wise & 1,150 & -- & 14 & 14 & 0 & 1916 & -- & 1929 \\
\hline 08043950 & Big Sandy Cr nr Chico, TX & $33^{\circ} 16^{\prime} 27^{\prime \prime}$ & $97^{\circ} 40^{\prime} 42^{\prime \prime}$ & Wise & 312 & -- & 64 & 19 & 45 & 1937 & 1956 & 2000 \\
\hline 08044500 & West Fk Trinity R nr Boyd, TX & $33^{\circ} 05^{\prime} 07^{\prime \prime}$ & $97^{\circ} 33^{\prime} 30^{\prime \prime}$ & Wise & 1,730 & -- & 53 & 0 & 53 & -- & 1948 & 2000 \\
\hline 08044800 & Walnut $\mathrm{Cr}$ at Reno, $\mathrm{TX}$ & $32^{\circ} 56^{\prime} 44^{\prime \prime}$ & $97^{\circ} 34^{\prime} 58^{\prime \prime}$ & Parker & 75.6 & -- & 5 & 5 & 0 & 1996 & -- & 2000 \\
\hline 08045500 & West Fk Trinity R at Lake Worth Dam above FTW, TX & $32^{\circ} 47^{\prime} 27^{\prime \prime}$ & $97^{\circ} 24^{\prime} 54^{\prime \prime}$ & Tarrant & 2,070 & -- & 10 & 9 & 1 & 1925 & 1934 & 1934 \\
\hline 08045850 & Clear Fk Trinity R nr Weatherford, TX & $32^{\circ} 44^{\prime} 25^{\prime \prime}$ & $97^{\circ} 39^{\prime} 06^{\prime \prime}$ & Parker & 121 & -- & 8 & 0 & 8 & -- & 1981 & 2000 \\
\hline 08046000 & Clear Fk Trinity R nr Aledo, TX & $32^{\circ} 38^{\prime} 28^{\prime \prime}$ & $97^{\circ} 33^{\prime} 51^{\prime \prime}$ & Parker & 251 & -- & 28 & 9 & 19 & 1948 & 1957 & 1975 \\
\hline 08047000 & Clear Fk Trinity R nr Benbrook, TX & $32^{\circ} 39^{\prime} 54^{\prime \prime}$ & $97^{\circ} 26^{\prime} 30^{\prime \prime}$ & Tarrant & 431 & -- & 53 & 5 & 48 & 1948 & 1953 & 2000 \\
\hline
\end{tabular}

v Footnotes at end of table 
Table 2. Inventory of daily mean streamflows for active and discontinued streamflow-gaging stations in Texas-Continued

\begin{tabular}{|c|c|c|c|c|c|c|c|c|c|c|c|c|}
\hline \multirow[b]{2}{*}{$\begin{array}{c}\text { Station } \\
\text { no. }\end{array}$} & \multirow[b]{2}{*}{ Station name } & \multirow[b]{2}{*}{ Latitude } & \multirow[b]{2}{*}{ Longitude } & \multirow[b]{2}{*}{ County } & \multirow{2}{*}{$\begin{array}{l}\text { Contri- } \\
\text { buting } \\
\text { drainage } \\
\text { area } \\
\left(\mathbf{m i}^{2}\right)\end{array}$} & \multirow[b]{2}{*}{$\begin{array}{l}\text { Urban } \\
\text { basin }\end{array}$} & \multicolumn{3}{|c|}{ Years of record ${ }^{2}$} & \multicolumn{3}{|c|}{ Begin and end year $^{2}$} \\
\hline & & & & & & & Total & $\begin{array}{c}\text { Unregu- } \\
\text { lated }\end{array}$ & $\begin{array}{l}\text { Regu- } \\
\text { lated }^{3}\end{array}$ & $\begin{array}{c}\text { First } \\
\text { unregu- } \\
\text { lated }\end{array}$ & $\begin{array}{l}\text { First } \\
\text { regu- } \\
\text { lated }\end{array}$ & $\begin{array}{l}\text { Last } \\
\text { year }\end{array}$ \\
\hline \multicolumn{13}{|c|}{ Trinity R Basin-Continued } \\
\hline 08047500 & Clear Fk Trinity R at FTW, TX & $32^{\circ} 43^{\prime} 56^{\prime \prime}$ & $97^{\circ} 21^{\prime} 31^{\prime \prime}$ & Tarrant & 518 & -- & 77 & 28 & 48 & 1925 & 1953 & 2000 \\
\hline 08048000 & West Fk Trinity R at FTW, TX & $32^{\circ} 45^{\prime} 39^{\prime \prime}$ & $97^{\circ} 19^{\prime} 56^{\prime \prime}$ & Tarrant & 2,620 & -- & 80 & 0 & 80 & -- & 1921 & 2000 \\
\hline 08048500 & Marine $\mathrm{Cr}$ at FTW, TX & $32^{\circ} 48^{\prime} 16^{\prime \prime}$ & $97^{\circ} 21^{\prime} 48^{\prime \prime}$ & Tarrant & 16.8 & -- & 8 & 7 & 1 & 1951 & 1958 & 1958 \\
\hline 08048520 & Sycamore Cr at IH-35, FTW, TX & $32^{\circ} 39^{\prime} 55^{\prime \prime}$ & $97^{\circ} 19^{\prime} 16^{\prime \prime}$ & Tarrant & 17.7 & Yes & 7 & 7 & 0 & 1970 & -- & 1976 \\
\hline 08048530 & Sycamore Cr trib above Seminary South Shopping Center, FTW, TX & $32^{\circ} 41^{\prime} 08^{\prime \prime}$ & $97^{\circ} 19^{\prime} 44^{\prime \prime}$ & Tarrant & .97 & Yes & 7 & 7 & 0 & 1970 & -- & 1976 \\
\hline 08048540 & Sycamore Cr trib at IH-35, FTW, TX & $32^{\circ} 41^{\prime} 18^{\prime \prime}$ & $97^{\circ} 19^{\prime} 11^{\prime \prime}$ & Tarrant & 1.35 & Yes & 7 & 7 & 0 & 1970 & -- & 1976 \\
\hline 08048543 & West Fk Trinity R at Beach St, FTW, TX & $32^{\circ} 45^{\prime} 06^{\prime \prime}$ & $97^{\circ} 17^{\prime} 21^{\prime \prime}$ & Tarrant & 2,690 & -- & 24 & 0 & 24 & -- & 1977 & 2000 \\
\hline 08048600 & Dry Branch at Fain St, FTW, TX & $32^{\circ} 46^{\prime} 34^{\prime \prime}$ & $97^{\circ} 17^{\prime} 18^{\prime \prime}$ & Tarrant & 2.15 & Yes & 8 & 8 & 0 & 1969 & -- & 1976 \\
\hline 08048800 & Big Fossil Cr at Haltom City, TX & $32^{\circ} 48^{\prime} 26^{\prime \prime}$ & $97^{\circ} 14^{\prime} 54^{\prime \prime}$ & Tarrant & 52.8 & Yes & 14 & 14 & 0 & 1960 & -- & 1973 \\
\hline 08048850 & Little Fossil Cr at Mesquite St, FTW, TX & $32^{\circ} 48^{\prime} 33^{\prime \prime}$ & $97^{\circ} 17^{\prime} 28^{\prime \prime}$ & Tarrant & 12.3 & Yes & 8 & 8 & 0 & 1969 & -- & 1976 \\
\hline 08048970 & Village $\mathrm{Cr}$ at Everman, TX & $32^{\circ} 36^{\prime} 12^{\prime \prime}$ & $97^{\circ} 15^{\prime} 53^{\prime \prime}$ & Tarrant & 84.5 & -- & 11 & 11 & 0 & 1990 & -- & 2000 \\
\hline 08049000 & Village Cr nr Handley, TX & $32^{\circ} 42^{\prime} 00^{\prime \prime}$ & $97^{\circ} 13^{\prime} 00^{\prime \prime}$ & Tarrant & 126 & -- & 4 & 4 & 0 & 1926 & -- & 1929 \\
\hline 08049240 & Rush Cr at Woodland Park Blvd, Arlington, TX & $32^{\circ} 42^{\prime} 50^{\prime \prime}$ & $97^{\circ} 10^{\prime} 19^{\prime \prime}$ & Tarrant & 26.6 & -- & 1 & 1 & 0 & 1994 & -- & 1994 \\
\hline 08049500 & West Fk Trinity R at Grand Prairie, TX & $32^{\circ} 45^{\prime} 46^{\prime \prime}$ & $96^{\circ} 59^{\prime} 42^{\prime \prime}$ & Dallas & 3,070 & -- & 75 & 6 & 69 & 1926 & 1932 & 2000 \\
\hline 08049550 & Big Bear Cr nr Grapevine, TX & $32^{\circ} 54^{\prime} 48^{\prime \prime}$ & $97^{\circ} 07^{\prime} 44^{\prime \prime}$ & Tarrant & 29.6 & -- & 12 & 12 & 0 & 1968 & -- & 1979 \\
\hline 08049565 & Trigg Branch at Dallas-FTW Airport nr Euless, TX & $32^{\circ} 52^{\prime} 02^{\prime \prime}$ & $97^{\circ} 02^{\prime} 20^{\prime \prime}$ & Brooks & 1.73 & Yes & 5 & 5 & 0 & 1983 & -- & 1987 \\
\hline 08049580 & Mountain Cr nr Venus, TX & $32^{\circ} 29^{\prime} 27^{\prime \prime}$ & $97^{\circ} 07^{\prime} 22^{\prime \prime}$ & Dallas & 25.5 & -- & 2 & 2 & 0 & 1986 & -- & 1987 \\
\hline 08049600 & Mountain Cr nr Cedar Hill, TX & $32^{\circ} 35^{\prime} 03^{\prime \prime}$ & $97^{\circ} 01 ' 23^{\prime \prime}$ & Dallas & 119 & -- & 24 & 0 & 24 & -- & 1961 & 1984 \\
\hline 08049700 & Walnut Cr nr Mansfield, TX & $32^{\circ} 34^{\prime} 51^{\prime \prime}$ & $97^{\circ} 06^{\prime} 06^{\prime \prime}$ & Tarrant & 62.8 & -- & 40 & 40 & 0 & 1961 & -- & 2000 \\
\hline 08050000 & Mountain Cr nr Grand Prairie, TX & $32^{\circ} 42^{\prime} 20^{\prime \prime}$ & $96^{\circ} 58^{\prime} 00^{\prime \prime}$ & Dallas & 273 & -- & 8 & 8 & 0 & 1925 & -- & 1932 \\
\hline 08050100 & Mountain Cr at Grand Prairie, TX & $32^{\circ} 44^{\prime} 51^{\prime \prime}$ & $96^{\circ} 55^{\prime} 32^{\prime \prime}$ & Dallas & 298 & -- & 40 & 0 & 40 & -- & 1961 & 2000 \\
\hline 08050300 & Elm Fk Trinity R nr Muenster, TX & $33^{\circ} 36^{\prime} 36^{\prime \prime}$ & $97^{\circ} 22^{\prime} 57^{\prime \prime}$ & Cooke & 46.0 & -- & 17 & 0 & 17 & -- & 1957 & 1973 \\
\hline 08050400 & Elm Fk Trinity R at Gainesville, TX & $33^{\circ} 37^{\prime} 27^{\prime \prime}$ & $97^{\circ} 09^{\prime} 22^{\prime \prime}$ & Cooke & 174 & -- & 15 & 15 & 0 & 1986 & -- & 2000 \\
\hline 08050500 & Elm Fk Trinity R nr Sanger, TX & $33^{\circ} 23^{\prime} 11^{\prime \prime}$ & $97^{\circ} 05^{\prime} 05^{\prime \prime}$ & Denton & 381 & -- & 35 & 4 & 31 & 1950 & 1954 & 1984 \\
\hline 08050800 & Timber Cr nr Collinsville, TX & $33^{\circ} 33^{\prime} 16^{\prime \prime}$ & $96^{\circ} 56^{\prime} 49^{\prime \prime}$ & Cooke & 38.8 & -- & 15 & 15 & 0 & 1986 & -- & 2000 \\
\hline 08050840 & Range $\mathrm{Cr}$ nr Collinsville, TX & $33^{\circ} 31^{\prime} 34^{\prime \prime}$ & $96^{\circ} 48^{\prime} 25^{\prime \prime}$ & Denton & 29.2 & -- & 8 & 8 & 0 & 1993 & -- & 2000 \\
\hline 08051000 & Isle Du Bois Cr nr Pilot Point, TX & $33^{\circ} 24^{\prime} 23^{\prime \prime}$ & $97^{\circ} 00^{\prime} 45^{\prime \prime}$ & Denton & 266 & -- & 35 & 35 & 0 & 1950 & -- & 1984 \\
\hline 08051130 & Elm Fk Trinity R nr Pilot Point, TX & $33^{\circ} 21^{\prime} 01^{\prime \prime}$ & $97^{\circ} 02^{\prime} 49^{\prime \prime}$ & Denton & 692 & -- & 7 & 0 & 7 & -- & 1986 & 1992 \\
\hline 08051500 & Clear Cr nr Sanger, TX & $33^{\circ} 20^{\prime} 10^{\prime \prime}$ & $97^{\circ} 10^{\prime} 45^{\prime \prime}$ & Denton & 295 & -- & 51 & 31 & 20 & 1950 & 1981 & 2000 \\
\hline 08052000 & Elm Fk Trinity R nr Denton, TX & $33^{\circ} 15^{\prime} 02^{\prime \prime}$ & $97^{\circ} 02^{\prime} 42^{\prime \prime}$ & Denton & 1,080 & -- & 2 & 2 & 0 & 1925 & -- & 1926 \\
\hline 08052650 & Little Elm Cr nr Celina, TX & $33^{\circ} 21^{\prime} 55^{\prime \prime}$ & $96^{\circ} 49^{\prime} 25^{\prime \prime}$ & Collin & 46.7 & -- & 10 & 0 & 10 & -- & 1967 & 1976 \\
\hline 08052700 & Little Elm Cr nr Aubrey, TX & $33^{\circ} 17^{\prime} 00^{\prime \prime}$ & $96^{\circ} 53^{\prime} 33^{\prime \prime}$ & Denton & 75.5 & -- & 41 & 9 & 32 & 1957 & 1966 & 2000 \\
\hline 08052780 & Hickory $\mathrm{Cr}$ at Denton, $\mathrm{TX}$ & $33^{\circ} 09^{\prime} 06^{\prime \prime}$ & $97^{\circ} 08^{\prime} 30^{\prime \prime}$ & Denton & 129 & -- & 2 & 0 & 2 & -- & 1986 & 1987 \\
\hline 08053000 & Elm Fk Trinity R nr Lewisville, TX & $33^{\circ} 02^{\prime} 44^{\prime \prime}$ & $96^{\circ} 57^{\prime} 39^{\prime \prime}$ & Denton & 1,670 & -- & 51 & 0 & 51 & -- & 1950 & 2000 \\
\hline
\end{tabular}


Table 2. Inventory of daily mean streamflows for active and discontinued streamflow-gaging stations in Texas-Continued

\begin{tabular}{|c|c|c|c|c|c|c|c|c|c|c|c|c|}
\hline \multirow[b]{2}{*}{$\begin{array}{l}\text { Station } \\
\text { no. }\end{array}$} & \multirow[b]{2}{*}{ Station name } & \multirow[b]{2}{*}{ Latitude } & \multirow[b]{2}{*}{ Longitude } & \multirow[b]{2}{*}{ County } & \multirow{2}{*}{$\begin{array}{l}\text { Contri- } \\
\text { buting } \\
\text { drainage } \\
\text { area } \\
\left(\mathrm{mi}^{2}\right)\end{array}$} & \multirow[b]{2}{*}{$\begin{array}{l}\text { Urban } \\
\text { basin }\end{array}$} & \multicolumn{3}{|c|}{ Years of record $^{2}$} & \multicolumn{3}{|c|}{ Begin and end year ${ }^{2}$} \\
\hline & & & & & & & Total & $\begin{array}{l}\text { Unregu- } \\
\text { lated }\end{array}$ & $\begin{array}{l}\text { Regu- } \\
\text { lated }^{3}\end{array}$ & $\begin{array}{l}\text { First } \\
\text { unregu- } \\
\text { lated }\end{array}$ & $\begin{array}{l}\text { First } \\
\text { regu- } \\
\text { lated }\end{array}$ & $\begin{array}{c}\text { Last } \\
\text { year }\end{array}$ \\
\hline \multicolumn{13}{|c|}{ Trinity R Basin-Continued } \\
\hline 08053010 & Indian $\mathrm{Cr}$ at Hebron Pkwy at Carrollton, TX & $33^{\circ} 01^{\prime} 06^{\prime \prime}$ & $96^{\circ} 55^{\prime} 27^{\prime \prime}$ & Denton & 14.7 & -- & 3 & 3 & 0 & 1986 & -- & 1989 \\
\hline 08053500 & Denton Cr nr Justin, TX & $33^{\circ} 07^{\prime} 08^{\prime \prime}$ & $97^{\circ} 17^{\prime} 25^{\prime \prime}$ & Denton & 400 & -- & 51 & 15 & 36 & 1950 & 1965 & 2000 \\
\hline 08054000 & Denton Cr nr Roanoke, TX & $33^{\circ} 02^{\prime} 24^{\prime \prime}$ & $97^{\circ} 12^{\prime} 17^{\prime \prime}$ & Denton & 621 & -- & 20 & 20 & 0 & 1924 & -- & 1955 \\
\hline 08055000 & Denton Cr nr Grapevine, TX & $32^{\circ} 59^{\prime} 13^{\prime \prime}$ & $97^{\circ} 00^{\prime} 45^{\prime \prime}$ & Tarrant & 705 & -- & 43 & 5 & 38 & 1948 & 1953 & 1990 \\
\hline 08055500 & Elm Fk Trinity R nr Carrollton, TX & $32^{\circ} 57^{\prime} 57^{\prime \prime}$ & $96^{\circ} 56^{\prime} 39^{\prime \prime}$ & Dallas & 2,460 & -- & 93 & 44 & 49 & 1908 & 1952 & 2000 \\
\hline 08055700 & Bachman Branch at Dallas, TX & $32^{\circ} 51^{\prime} 37^{\prime \prime}$ & $96^{\circ} 51^{\prime} 13^{\prime \prime}$ & Dallas & 10.0 & Yes & 16 & 16 & 0 & 1964 & -- & 1979 \\
\hline 08056500 & Turtle Cr at Dallas, TX & $32^{\circ} 48^{\prime} 26^{\prime \prime}$ & $96^{\circ} 48^{\prime} 08^{\prime \prime}$ & Dallas & 7.98 & Yes & 36 & 18 & 18 & 1952 & 1970 & 1991 \\
\hline 08057000 & Trinity $\mathrm{R}$ at Dallas, $\mathrm{TX}$ & $32^{\circ} 46^{\prime} 29^{\prime \prime}$ & $96^{\circ} 49^{\prime} 18^{\prime \prime}$ & Dallas & 6,110 & -- & 98 & 28 & 70 & 1903 & 1931 & 2000 \\
\hline 08057100 & White Rock Cr at Keller Springs Rd, Dallas, TX & $32^{\circ} 58^{\prime} 13^{\prime \prime}$ & $96^{\circ} 48^{\prime} 19^{\prime \prime}$ & Dallas & 29.4 & Yes & 18 & 18 & 0 & 1962 & -- & 1979 \\
\hline 08057200 & White Rock Cr at Greenville Ave, Dallas, TX & $32^{\circ} 533^{\prime} 21^{\prime \prime}$ & $96^{\circ} 45^{\prime} 23^{\prime \prime}$ & Dallas & 66.4 & Yes & 35 & 35 & 0 & 1962 & -- & 2000 \\
\hline 08057300 & White Rock Cr at White Rock Lake, Dallas, TX & $32^{\circ} 48^{\prime} 31^{\prime \prime}$ & $96^{\circ} 43^{\prime} 32^{\prime \prime}$ & Dallas & 100 & Yes & 17 & 0 & 17 & -- & 1963 & 1979 \\
\hline 08057400 & White Rock Cr at Scyene Rd, Dallas, TX & $32^{\circ} 45^{\prime} 57^{\prime \prime}$ & $96^{\circ} 43^{\prime} 49^{\prime \prime}$ & Dallas & 122 & Yes & 17 & 0 & 17 & -- & 1963 & 1979 \\
\hline 08057410 & Trinity R below Dallas, TX & $32^{\circ} 42^{\prime} 26^{\prime \prime}$ & $96^{\circ} 44^{\prime} 08^{\prime \prime}$ & Dallas & 6,280 & -- & 43 & 0 & 43 & -- & 1958 & 2000 \\
\hline 08057445 & Prairie $\mathrm{Cr}$ at US 175, Dallas, TX & $32^{\circ} 42^{\prime} 17^{\prime \prime}$ & $96^{\circ} 40^{\prime} 11^{\prime \prime}$ & Dallas & 9.03 & Yes & 21 & 21 & 0 & 1976 & -- & 2000 \\
\hline 08057450 & Tenmile Cr at SH 342 at Lancaster, TX & $32^{\circ} 34^{\prime} 42^{\prime \prime}$ & $96^{\circ} 45^{\prime} 21^{\prime \prime}$ & Dallas & 52.8 & Yes & 10 & 10 & 0 & 1970 & -- & 1979 \\
\hline 08058500 & Honey Cr nr McKinney, TX & $33^{\circ} 16^{\prime} 42^{\prime \prime}$ & $96^{\circ} 39^{\prime} 27^{\prime \prime}$ & Collin & 39.0 & -- & 22 & 0 & 22 & -- & 1952 & 1973 \\
\hline 08058900 & East Fk Trinity R at McKinney, TX & $33^{\circ} 14^{\prime} 38^{\prime \prime}$ & $96^{\circ} 36^{\prime} 31^{\prime \prime}$ & Collin & 164 & -- & 25 & 0 & 25 & -- & 1976 & 2000 \\
\hline 08059000 & East Fk Trinity R nr McKinney, TX & $33^{\circ} 12^{\prime} 13^{\prime \prime}$ & $96^{\circ} 35^{\prime} 44^{\prime \prime}$ & Collin & 190 & -- & 26 & 5 & 21 & 1950 & 1955 & 1975 \\
\hline 08059400 & Sister Grove Cr nr Blue Ridge, TX & $33^{\circ} 17^{\prime} 40^{\prime \prime}$ & $96^{\circ} 28^{\prime} 58^{\prime \prime}$ & Collin & 83.1 & -- & 25 & 0 & 25 & -- & 1976 & 2000 \\
\hline 08059500 & Sister Grove Cr nr Princeton, TX & $33^{\circ} 11^{\prime} 35^{\prime \prime}$ & $96^{\circ} 28^{\prime} 32^{\prime \prime}$ & Collin & 113 & -- & 25 & 3 & 22 & 1950 & 1953 & 1974 \\
\hline 08060000 & East Fk Trinity R above Pilot Grove, TX & $33^{\circ} 01 ' 23^{\prime \prime}$ & $96^{\circ} 28^{\prime} 32^{\prime \prime}$ & Collin & 324 & -- & 4 & 4 & 0 & 1950 & -- & 1953 \\
\hline 08061000 & East Fk Trinity R nr Lavon, TX & $33^{\circ} 01 ' 25^{\prime \prime}$ & $96^{\circ} 28^{\prime} 31^{\prime \prime}$ & Collin & 773 & -- & 36 & 0 & 36 & -- & 1954 & 1989 \\
\hline 08061500 & East Fk Trinity R nr Rockwall, TX & $32^{\circ} 55^{\prime} 25^{\prime \prime}$ & $96^{\circ} 30^{\prime} 20^{\prime \prime}$ & Rockwall & 840 & -- & 30 & 29 & 1 & 1925 & 1954 & 1954 \\
\hline 08061540 & Rowlett Cr nr Sachse, TX & $32^{\circ} 57^{\prime} 35^{\prime \prime}$ & $96^{\circ} 36^{\prime} 51^{\prime \prime}$ & Dallas & 120 & -- & 32 & 32 & 0 & 1969 & -- & 2000 \\
\hline 08061700 & Duck Cr nr Garland, TX & $32^{\circ} 49^{\prime} 58^{\prime \prime}$ & $96^{\circ} 35^{\prime} 43^{\prime \prime}$ & Dallas & 31.6 & Yes & 34 & 34 & 0 & 1959 & -- & 1992 \\
\hline 08061750 & East Fk Trinity R nr Forney, TX & $32^{\circ} 46^{\prime} 27^{\prime \prime}$ & $96^{\circ} 30^{\prime} 12^{\prime \prime}$ & Kaufman & 1,120 & -- & 27 & 0 & 27 & -- & 1974 & 2000 \\
\hline 08061950 & South Mesquite $\mathrm{Cr}$ at Mercury Rd nr Mesquite, TX & $32^{\circ} 43^{\prime} 32^{\prime \prime}$ & $96^{\circ} 34^{\prime} 12^{\prime \prime}$ & Dallas & 23.0 & Yes & 11 & 11 & 0 & 1969 & -- & 1979 \\
\hline 08062000 & East Fk Trinity R nr Crandall, TX & $32^{\circ} 38^{\prime} 19^{\prime \prime}$ & $96^{\circ} 29^{\prime} 06^{\prime \prime}$ & Kaufman & 1,260 & -- & 51 & 4 & 47 & 1950 & 1954 & 2000 \\
\hline 08062500 & Trinity R nr Rosser, TX & $32^{\circ} 25^{\prime} 35^{\prime \prime}$ & $96^{\circ} 27^{\prime} 46^{\prime \prime}$ & Kaufman & 8,150 & -- & 62 & 0 & 62 & -- & 1925 & 2000 \\
\hline 08062650 & Cedar Cr Reservoir spillway outflow nr Trinidad, TX & $32^{\circ} 14^{\prime} 16^{\prime \prime}$ & $96^{\circ} 08^{\prime} 36^{\prime \prime}$ & Henderson & 1,010 & -- & 17 & 0 & 17 & -- & 1966 & 1982 \\
\hline 08062700 & Trinity $\mathrm{R}$ at Trinidad, $\mathrm{TX}$ & $32^{\circ} 08^{\prime} 05^{\prime \prime}$ & $96^{\circ} 06^{\prime} 20^{\prime \prime}$ & Henderson & 8,540 & -- & 36 & 0 & 36 & -- & 1965 & 2000 \\
\hline 08062800 & Cedar Cr nr Kemp, TX & $32^{\circ} 30^{\prime} 18^{\prime \prime}$ & $96^{\circ} 06^{\prime} 57^{\prime \prime}$ & Kaufman & 189 & -- & 24 & 6 & 18 & 1964 & 1970 & 1987 \\
\hline 08062900 & Kings Cr nr Kaufman, TX & $32^{\circ} 30^{\prime} 48^{\prime \prime}$ & $96^{\circ} 19^{\prime} 44^{\prime \prime}$ & Kaufman & 233 & -- & 24 & 8 & 16 & 1964 & 1972 & 1987 \\
\hline
\end{tabular}

* Footnotes at end of table. 
Table 2. Inventory of daily mean streamflows for active and discontinued streamflow-gaging stations in Texas-Continued

\begin{tabular}{|c|c|c|c|c|c|c|c|c|c|c|c|c|}
\hline \multirow[b]{2}{*}{$\begin{array}{c}\text { Station } \\
\text { no. }\end{array}$} & \multirow[b]{2}{*}{ Station name } & \multirow[b]{2}{*}{ Latitude } & \multirow[b]{2}{*}{ Longitude } & \multirow[b]{2}{*}{ County } & \multirow{2}{*}{$\begin{array}{l}\text { Contri- } \\
\text { buting } \\
\text { drainage } \\
\text { area } \\
\left(\mathrm{mi}^{2}\right)\end{array}$} & \multirow[b]{2}{*}{$\begin{array}{l}\text { Urban } \\
\text { basin }\end{array}$} & \multicolumn{3}{|c|}{ Years of record $^{2}$} & \multicolumn{3}{|c|}{ Begin and end year $^{2}$} \\
\hline & & & & & & & Total & $\begin{array}{c}\text { Unregu- } \\
\text { lated }\end{array}$ & $\begin{array}{l}\text { Regu- } \\
\text { lated }^{3}\end{array}$ & $\begin{array}{c}\text { First } \\
\text { unregu- } \\
\text { lated }\end{array}$ & $\begin{array}{l}\text { First } \\
\text { regu- } \\
\text { lated }\end{array}$ & $\begin{array}{l}\text { Last } \\
\text { year }\end{array}$ \\
\hline \multicolumn{13}{|c|}{ Trinity R Basin-Continued } \\
\hline 08062980 & Lacy Fk nr Mabank, TX & $32^{\circ} 25^{\prime} 27^{\prime \prime}$ & $96^{\circ} 06^{\prime} 33^{\prime \prime}$ & Kaufman & 118 & -- & 1 & 1 & 0 & 1983 & -- & 1983 \\
\hline 08063000 & Cedar Cr nr Mabank, TX & $32^{\circ} 19^{\prime} 45^{\prime \prime}$ & $96^{\circ} 10^{\prime} 05^{\prime \prime}$ & Kaufman & 733 & -- & 27 & 27 & 0 & 1939 & -- & 1965 \\
\hline 08063003 & South Twin Cr nr Eustace, TX & $32^{\circ} 19^{\prime} 18^{\prime \prime}$ & $96^{\circ} 01^{\prime} 43^{\prime \prime}$ & Henderson & 27.4 & -- & 1 & 1 & 0 & 1983 & -- & 1983 \\
\hline 08063020 & Cedar $\mathrm{Cr}$ at Trinidad, TX & $32^{\circ} 09^{\prime} 24^{\prime \prime}$ & $96^{\circ} 03^{\prime} 45^{\prime \prime}$ & Henderson & 1,010 & -- & 6 & 0 & 6 & -- & 1966 & 1971 \\
\hline 08063100 & Richland Cr nr Dawson, TX & $31^{\circ} 56^{\prime} 18^{\prime \prime}$ & $96^{\circ} 40^{\prime} 52^{\prime \prime}$ & Navarro & 333 & -- & 40 & 2 & 38 & 1961 & 1963 & 2000 \\
\hline 08063200 & Pin Oak Cr nr Hubbard, TX & $31^{\circ} 48^{\prime} 01^{\prime \prime}$ & $96^{\circ} 43^{\prime} 02^{\prime \prime}$ & Limestone & 17.6 & -- & 16 & 5 & 11 & 1957 & 1962 & 1972 \\
\hline 08063500 & Richland Cr nr Richland, TX & $31^{\circ} 57^{\prime} 02^{\prime \prime}$ & $96^{\circ} 25^{\prime} 16^{\prime \prime}$ & Navarro & 734 & -- & 49 & 23 & 26 & 1940 & 1963 & 1988 \\
\hline 08063800 & Waxahachie Cr nr Bardwell, TX & $32^{\circ} 14^{\prime} 36^{\prime \prime}$ & $96^{\circ} 38^{\prime} 24^{\prime \prime}$ & Ellis & 178 & -- & 37 & 0 & 37 & -- & 1964 & 2000 \\
\hline 08064100 & Chambers Cr nr Rice, TX & $32^{\circ} 11^{\prime} 54^{\prime \prime}$ & $96^{\circ} 31^{\prime} 12^{\prime \prime}$ & Navarro & 807 & -- & 17 & 0 & 17 & -- & 1984 & 2000 \\
\hline 08064500 & Chambers $\mathrm{Cr}$ nr Corsicana, TX & $32^{\circ} 06^{\prime} 29^{\prime \prime}$ & $96^{\circ} 22^{\prime} 14^{\prime \prime}$ & Navarro & 963 & -- & 45 & 21 & 24 & 1940 & 1961 & 1984 \\
\hline 08064600 & Richland Cr nr Fairfield, TX & $31^{\circ} 57^{\prime} 08^{\prime \prime}$ & $96^{\circ} 05^{\prime} 50^{\prime \prime}$ & Freestone & 1,960 & -- & 10 & 0 & 10 & -- & 1973 & 1982 \\
\hline 08064700 & Tehuacana Cr nr Streetman, TX & $31^{\circ} 50^{\prime} 46^{\prime \prime}$ & $96^{\circ} 17^{\prime} 37^{\prime \prime}$ & Freestone & 142 & -- & 32 & 32 & 0 & 1969 & -- & 2000 \\
\hline 08064800 & Catfish Cr nr Tennessee Colony, TX & $31^{\circ} 52^{\prime} 51^{\prime \prime}$ & $95^{\circ} 52^{\prime} 07^{\prime \prime}$ & Anderson & 207 & -- & 27 & 27 & 0 & 1963 & -- & 1989 \\
\hline 08065000 & Trinity R nr Oakwood, TX & $31^{\circ} 38^{\prime} 54^{\prime \prime}$ & $95^{\circ} 47^{\prime} 21^{\prime \prime}$ & Anderson & 12,800 & -- & 77 & 0 & 77 & -- & 1924 & 2000 \\
\hline 08065200 & Upper Keechi Cr nr Oakwood, TX & $31^{\circ} 34^{\prime} 11^{\prime \prime}$ & $95^{\circ} 53^{\prime} 17^{\prime \prime}$ & Leon & 150 & -- & 38 & 38 & 0 & 1963 & -- & 2000 \\
\hline 08065350 & Trinity R nr Crockett, TX & $31^{\circ} 20^{\prime} 18^{\prime \prime}$ & $95^{\circ} 39^{\prime} 22^{\prime \prime}$ & Houston & 13,900 & -- & 37 & 0 & 37 & -- & 1964 & 2000 \\
\hline 08065500 & Trinity R nr Midway, TX & $31^{\circ} 04^{\prime} 28^{\prime \prime}$ & $95^{\circ} 41^{\prime} 57^{\prime \prime}$ & Madison & 14,500 & -- & 31 & 14 & 17 & 1940 & 1954 & 1970 \\
\hline 08065700 & Caney Cr nr Madisonville, TX & $30^{\circ} 56^{\prime} 12^{\prime \prime}$ & $95^{\circ} 56^{\prime} 07^{\prime \prime}$ & Madison & 112 & -- & 13 & 13 & 0 & 1964 & -- & 1976 \\
\hline 08065800 & Bedias Cr nr Madisonville, TX & $30^{\circ} 53^{\prime} 03^{\prime \prime}$ & $95^{\circ} 46^{\prime} 39^{\prime \prime}$ & Walker & 321 & -- & 33 & 33 & 0 & 1968 & -- & 2000 \\
\hline 08066000 & Trinity R at Riverside, $\mathrm{TX}$ & $30^{\circ} 51^{\prime} 33^{\prime \prime}$ & $95^{\circ} 23^{\prime} 55^{\prime \prime}$ & Walker & 15,600 & -- & 45 & 30 & 15 & 1924 & 1954 & 1968 \\
\hline 08066100 & White Rock Cr nr Trinity, TX & $31^{\circ} 03^{\prime} 06^{\prime \prime}$ & $95^{\circ} 22^{\prime} 40^{\prime \prime}$ & Trinity & 222 & -- & 16 & 16 & 0 & 1967 & -- & 1985 \\
\hline 08066170 & Kickapoo Cr nr Onalaska, TX & $30^{\circ} 54^{\prime} 25^{\prime \prime}$ & $95^{\circ} 05^{\prime} 18^{\prime \prime}$ & Polk & 57.0 & -- & 34 & 34 & 0 & 1967 & -- & 2000 \\
\hline 08066200 & Long King $\mathrm{Cr}$ at Livingston, $\mathrm{TX}$ & $30^{\circ} 42^{\prime} 58^{\prime \prime}$ & $94^{\circ} 57^{\prime} 31^{\prime \prime}$ & Polk & 141 & -- & 37 & 37 & 0 & 1964 & -- & 2000 \\
\hline 08066250 & Trinity R nr Goodrich, TX & $30^{\circ} 34^{\prime} 19^{\prime \prime}$ & $94^{\circ} 56^{\prime} 55^{\prime \prime}$ & Polk & 16,800 & -- & 34 & 0 & 34 & -- & 1967 & 2000 \\
\hline 08066300 & Menard Cr nr Rye, TX & $30^{\circ} 28^{\prime} 52^{\prime \prime}$ & $94^{\circ} 46^{\prime} 46^{\prime \prime}$ & Liberty & 152 & -- & 34 & 34 & 0 & 1967 & -- & 2000 \\
\hline 08066400 & Big Cr nr Shepherd, TX & $30^{\circ} 30^{\prime} 59^{\prime \prime}$ & $94^{\circ} 59^{\prime} 06^{\prime \prime}$ & San Jacinto & 38.8 & -- & 23 & 23 & 0 & 1967 & -- & 1989 \\
\hline 08066500 & Trinity R at Romayer, TX & $30^{\circ} 25^{\prime} 30^{\prime \prime}$ & $94^{\circ} 51^{\prime} 02^{\prime \prime}$ & Liberty & 17,200 & -- & 76 & 29 & 47 & 1925 & 1954 & 2000 \\
\hline \multicolumn{13}{|c|}{ Cedar Bayou Basin (Coastal Basin) } \\
\hline 08067500 & Cedar Bayou nr Crosby, TX & $29^{\circ} 58^{\prime} 21^{\prime \prime}$ & $94^{\circ} 59^{\prime} 08^{\prime \prime}$ & Liberty & 64.9 & -- & 20 & 20 & 0 & 1972 & -- & 1991 \\
\hline \multicolumn{13}{|c|}{ San Jacinto R Basin } \\
\hline 08067650 & West Fk San Jacinto R below Lake Conroe nr Conroe, TX & $30^{\circ} 20^{\prime} 31^{\prime \prime}$ & $95^{\circ} 32^{\prime} 34^{\prime \prime}$ & Montgomery & 451 & -- & 21 & 0 & 21 & -- & 1973 & 2000 \\
\hline 08067700 & Caney Cr nr Dobbin, TX & $30^{\circ} 21^{\prime} 13^{\prime \prime}$ & $95^{\circ} 48^{\prime} 35^{\prime \prime}$ & Montgomery & 40.4 & -- & 2 & 2 & 0 & 1964 & -- & 1965 \\
\hline 08068000 & West Fk San Jacinto R nr Conroe, TX & $30^{\circ} 14^{\prime} 40^{\prime \prime}$ & $95^{\circ} 27^{\prime} 25^{\prime \prime}$ & Montgomery & 828 & -- & 64 & 36 & 28 & 1925 & 1973 & 2000 \\
\hline
\end{tabular}


Table 2. Inventory of daily mean streamflows for active and discontinued streamflow-gaging stations in Texas-Continued

\begin{tabular}{|c|c|c|c|c|c|c|c|c|c|c|c|c|}
\hline \multirow[b]{2}{*}{$\begin{array}{l}\text { Station } \\
\text { no. }\end{array}$} & \multirow[b]{2}{*}{ Station name } & \multirow[b]{2}{*}{ Latitude } & \multirow[b]{2}{*}{ Longitude } & \multirow[b]{2}{*}{ County } & \multirow{2}{*}{$\begin{array}{l}\text { Contri- } \\
\text { buting } \\
\text { drainage } \\
\text { area } \\
\left(\mathrm{mi}^{2}\right)\end{array}$} & \multirow[b]{2}{*}{$\begin{array}{l}\text { Urban } \\
\text { basin }\end{array}$} & \multicolumn{3}{|c|}{ Years of record $^{2}$} & \multicolumn{3}{|c|}{ Begin and end year ${ }^{2}$} \\
\hline & & & & & & & Total & $\begin{array}{l}\text { Unregu- } \\
\text { lated }\end{array}$ & $\begin{array}{l}\text { Regu- } \\
\text { lated }^{3}\end{array}$ & $\begin{array}{l}\text { First } \\
\text { unregu- } \\
\text { lated }\end{array}$ & $\begin{array}{l}\text { First } \\
\text { regu- } \\
\text { lated }\end{array}$ & $\begin{array}{l}\text { Last } \\
\text { year }\end{array}$ \\
\hline \multicolumn{13}{|c|}{ San Jacinto R Basin-Continued } \\
\hline 08068090 & West Fk San Jacinto R above Lake Houston nr Porter, TX & $30^{\circ} 05^{\prime} 09^{\prime \prime}$ & $95^{\circ} 17^{\prime} 59^{\prime \prime}$ & Montgomery & 962 & -- & 16 & 0 & 16 & -- & 1985 & 2000 \\
\hline 08068275 & Spring Cr nr Tomball, TX & $30^{\circ} 07^{\prime} 11^{\prime \prime}$ & $95^{\circ} 38^{\prime} 45^{\prime \prime}$ & Harris & 186 & -- & 1 & 1 & 0 & 2000 & -- & 2000 \\
\hline 08068390 & Bear Branch at Research Forest Blvd, The Woodlands, TX & $30^{\circ} 11^{\prime} 25^{\prime \prime}$ & $95^{\circ} 29^{\prime} 27^{\prime \prime}$ & Montgomery & 15.4 & -- & 1 & 1 & 0 & 2000 & -- & 2000 \\
\hline 08068450 & Panther Branch nr Spring, TX & $30^{\circ} 07^{\prime} 51^{\prime \prime}$ & $95^{\circ} 28^{\prime} 52^{\prime \prime}$ & Harris & 34.5 & -- & 4 & 4 & 0 & 1973 & -- & 1976 \\
\hline 08068500 & Spring Cr nr Spring, TX & $30^{\circ} 06^{\prime} 37^{\prime \prime}$ & $95^{\circ} 26^{\prime} 10^{\prime \prime}$ & Harris & 409 & -- & 61 & 61 & 0 & 1940 & -- & 2000 \\
\hline 08068720 & Cypress Cr at Katy-Hockley Rd nr Hockley, TX & $29^{\circ} 57^{\prime} 00^{\prime \prime}$ & $95^{\circ} 48^{\prime} 29^{\prime \prime}$ & Harris & 110 & -- & 23 & 23 & 0 & 1976 & -- & 2000 \\
\hline 08068740 & Cypress Cr at House-Hahl Rd nr Cypress, TX & $29^{\circ} 57^{\prime} 32^{\prime \prime}$ & $95^{\circ} 43^{\prime} 03^{\prime \prime}$ & Harris & 131 & -- & 25 & 25 & 0 & 1976 & -- & 2000 \\
\hline 08068780 & Little Cypress Cr nr Cypress, TX & $30^{\circ} 00^{\prime} 57^{\prime \prime}$ & $95^{\circ} 41^{\prime} 50^{\prime \prime}$ & Harris & 41.0 & -- & 10 & 10 & 0 & 1983 & -- & 1992 \\
\hline 08068800 & Cypress Cr at Grant Rd nr Cypress, TX & $29^{\circ} 58^{\prime} 24^{\prime \prime}$ & $95^{\circ} 35^{\prime} 54^{\prime \prime}$ & Harris & 214 & -- & 10 & 10 & 0 & 1983 & -- & 1992 \\
\hline 08068900 & Cypress Cr at Stuebner Airline Rd nr Westfield, TX & $30^{\circ} 00^{\prime} 23^{\prime \prime}$ & $95^{\circ} 30^{\prime} 42^{\prime \prime}$ & Harris & 248 & Yes & 2 & 2 & 0 & 1988 & -- & 1989 \\
\hline 08069000 & Cypress Cr nr Westfield, TX & $30^{\circ} 02^{\prime} 08^{\prime \prime}$ & $95^{\circ} 25^{\prime} 43^{\prime \prime}$ & Harris & 285 & Yes & 56 & 56 & 0 & 1945 & -- & 2000 \\
\hline 08069500 & West Fk San Jacinto R nr Humble, TX & $30^{\circ} 01^{\prime} 37^{\prime \prime}$ & $95^{\circ} 15^{\prime} 28^{\prime \prime}$ & Harris & 1,740 & -- & 26 & 26 & 0 & 1929 & -- & 1954 \\
\hline 08070000 & East Fk San Jacinto R nr Cleveland, TX & $30^{\circ} 20^{\prime} 11^{\prime \prime}$ & $95^{\circ} 06^{\prime} 14^{\prime \prime}$ & Liberty & 325 & -- & 61 & 61 & 0 & 1940 & -- & 2000 \\
\hline 08070200 & East Fk San Jacinto R nr New Caney, TX & $30^{\circ} 08^{\prime} 43^{\prime \prime}$ & $95^{\circ} 07^{\prime} 27^{\prime \prime}$ & Montgomery & 388 & -- & 16 & 16 & 0 & 1985 & -- & 2000 \\
\hline 08070500 & Caney $\mathrm{Cr}$ nr Splendora, TX & $30^{\circ} 15^{\prime} 34^{\prime \prime}$ & $95^{\circ} 18^{\prime} 08^{\prime \prime}$ & Montgomery & 105 & -- & 56 & 56 & 0 & 1945 & -- & 2000 \\
\hline 08071000 & Peach $\mathrm{Cr}$ at Splendora, TX & $30^{\circ} 13^{\prime} 57^{\prime \prime}$ & $95^{\circ} 10^{\prime} 05^{\prime \prime}$ & Montgomery & 117 & -- & 35 & 35 & 0 & 1944 & -- & 2000 \\
\hline 08071280 & Luce Bayou above Lake Houston nr Huffman, TX & $30^{\circ} 06^{\prime} 34^{\prime \prime}$ & $95^{\circ} 03^{\prime} 35^{\prime \prime}$ & Liberty & 218 & -- & 16 & 16 & 0 & 1985 & -- & 2000 \\
\hline 08071500 & San Jacinto R nr Huffman, TX & $29^{\circ} 59^{\prime} 40^{\prime \prime}$ & $95^{\circ} 08^{\prime} 00^{\prime \prime}$ & Harris & 2,800 & -- & 17 & 17 & 0 & 1937 & -- & 1953 \\
\hline 08072300 & Buffalo Bayou nr Katy, TX & $29^{\circ} 44^{\prime} 35^{\prime \prime}$ & $95^{\circ} 48^{\prime} 24^{\prime \prime}$ & Fort Bend & 63.3 & -- & 23 & 23 & 0 & 1978 & -- & 2000 \\
\hline 08072730 & Bear Cr nr Barker, TX & $29^{\circ} 49^{\prime} 50^{\prime \prime}$ & $95^{\circ} 41^{\prime} 12^{\prime \prime}$ & Harris & 21.5 & -- & 23 & 23 & 0 & 1978 & -- & 2000 \\
\hline 08073500 & Buffalo Bayou nr Addicks, TX & $29^{\circ} 45^{\prime} 42^{\prime \prime}$ & $95^{\circ} 36^{\prime} 20^{\prime \prime}$ & Harris & 293 & Yes & 55 & 0 & 55 & -- & 1946 & 2000 \\
\hline 08073600 & Buffalo Bayou at West Belt Dr, Houston, TX & $29^{\circ} 45^{\prime} 43^{\prime \prime}$ & $95^{\circ} 33^{\prime} 27^{\prime \prime}$ & Harris & 307 & Yes & 29 & 7 & 22 & 1972 & 1979 & 2000 \\
\hline 08073700 & Buffalo Bayou at Piney Point, TX & $29^{\circ} 44^{\prime} 48^{\prime \prime}$ & $95^{\circ} 31^{\prime} 24^{\prime \prime}$ & Harris & 317 & -- & 29 & 0 & 29 & -- & 1964 & 2000 \\
\hline 08074000 & Buffalo Bayou at Houston, TX & $29^{\circ} 45^{\prime} 36^{\prime \prime}$ & $95^{\circ} 24^{\prime} 30^{\prime \prime}$ & Harris & 358 & Yes & 34 & 9 & 25 & 1937 & 1945 & 1975 \\
\hline 08074150 & Cole Cr at Deihl Rd, Houston, TX & $29^{\circ} 51^{\prime} 04^{\prime \prime}$ & $95^{\circ} 29^{\prime} 16^{\prime \prime}$ & Harris & 7.50 & Yes & 22 & 22 & 0 & 1965 & -- & 1986 \\
\hline 08074250 & Brickhouse Gulley at Costa Rica St, Houston, TX & $29^{\circ} 49^{\prime} 40^{\prime \prime}$ & $95^{\circ} 28^{\prime} 09^{\prime \prime}$ & Harris & 11.4 & Yes & 17 & 17 & 0 & 1965 & -- & 1981 \\
\hline 08074500 & Whiteoak Bayou at Houston, TX & $29^{\circ} 46^{\prime} 30^{\prime \prime}$ & $95^{\circ} 23^{\prime} 49^{\prime \prime}$ & Harris & 86.3 & Yes & 64 & 64 & 0 & 1937 & -- & 2000 \\
\hline 08074800 & Keegans Bayou at Roark Rd nr Houston, TX & $29^{\circ} 39^{\prime} 23^{\prime \prime}$ & $95^{\circ} 33^{\prime} 43^{\prime \prime}$ & Harris & 12.7 & Yes & 20 & 20 & 0 & 1965 & -- & 1984 \\
\hline 08075000 & Brays Bayou at Houston, TX & $29^{\circ} 41^{\prime} 49^{\prime \prime}$ & $95^{\circ} 24^{\prime} 43^{\prime \prime}$ & Harris & 94.9 & Yes & 64 & 64 & 0 & 1937 & -- & 2000 \\
\hline 08075400 & Sims Bayou at Hiram Clarke St, Houston, TX & $29^{\circ} 37^{\prime} 07^{\prime \prime}$ & $95^{\circ} 26^{\prime} 45^{\prime \prime}$ & Harris & 20.2 & Yes & 28 & 28 & 0 & 1965 & -- & 2000 \\
\hline 08075500 & Sims Bayou at Houston, TX & $29^{\circ} 40^{\prime} 27^{\prime \prime}$ & $95^{\circ} 17^{\prime} 21^{\prime \prime}$ & Harris & 63.0 & Yes & 43 & 43 & 0 & 1953 & -- & 1995 \\
\hline 08075650 & Berry Bayou at Forest Oaks St, Houston, TX & $29^{\circ} 40^{\prime} 35^{\prime \prime}$ & $95^{\circ} 14^{\prime} 37^{\prime \prime}$ & Harris & 10.1 & Yes & 2 & 2 & 0 & 1965 & -- & 1966 \\
\hline 08075730 & Vince Bayou at Pasadena, TX & $29^{\circ} 41^{\prime} 40^{\prime \prime}$ & $95^{\circ} 12^{\prime} 58^{\prime \prime}$ & Harris & 7.32 & Yes & 29 & 29 & 0 & 1972 & -- & 2000 \\
\hline 08075770 & Hunting Bayou at IH-610, Houston, TX & $29^{\circ} 47^{\prime} 35^{\prime \prime}$ & $95^{\circ} 16^{\prime} 04^{\prime \prime}$ & Harris & 16.1 & Yes & 36 & 36 & 0 & 1965 & -- & 2000 \\
\hline
\end{tabular}

$\underline{\omega} \quad$ Footnotes at end of table. 
Table 2. Inventory of daily mean streamflows for active and discontinued streamflow-gaging stations in Texas-Continued

\begin{tabular}{|c|c|c|c|c|c|c|c|c|c|c|c|c|}
\hline \multirow[b]{2}{*}{$\begin{array}{c}\text { Station } \\
\text { no. }\end{array}$} & \multirow[b]{2}{*}{ Station name } & \multirow[b]{2}{*}{ Latitude } & \multirow[b]{2}{*}{ Longitude } & \multirow[b]{2}{*}{ County } & \multirow{2}{*}{$\begin{array}{l}\text { Contri- } \\
\text { buting } \\
\text { drainage } \\
\text { area } \\
\left(\mathrm{mi}^{2}\right)\end{array}$} & \multirow[b]{2}{*}{$\begin{array}{l}\text { Urban } \\
\text { basin }\end{array}$} & \multicolumn{3}{|c|}{ Years of record $^{2}$} & \multicolumn{3}{|c|}{ Begin and end year $^{2}$} \\
\hline & & & & & & & Total & $\begin{array}{c}\text { Unregu- } \\
\text { lated }\end{array}$ & $\begin{array}{l}\text { Regu- } \\
\text { lated }^{3}\end{array}$ & $\begin{array}{c}\text { First } \\
\text { unregu- } \\
\text { lated }\end{array}$ & $\begin{array}{l}\text { First } \\
\text { regu- } \\
\text { lated }\end{array}$ & $\begin{array}{l}\text { Last } \\
\text { year }\end{array}$ \\
\hline \multicolumn{13}{|c|}{ San Jacinto R Basin-Continued } \\
\hline 08075900 & Greens Bayou nr US 75 nr Houston, TX & $29^{\circ} 57^{\prime} 24^{\prime \prime}$ & $95^{\circ} 25^{\prime} 04^{\prime \prime}$ & Harris & 36.6 & Yes & 26 & 26 & 0 & 1966 & -- & 1992 \\
\hline 08076000 & Greens Bayou nr Houston, TX & $29^{\circ} 55^{\prime} 05^{\prime \prime}$ & $95^{\circ} 18^{\prime} 24^{\prime \prime}$ & Harris & 68.7 & Yes & 48 & 48 & 0 & 1953 & -- & 2000 \\
\hline 08076180 & Garners Bayou nr Humble, TX & $29^{\circ} 56^{\prime} 03^{\prime \prime}$ & $95^{\circ} 14^{\prime} 02^{\prime \prime}$ & Harris & 31.0 & -- & 7 & 7 & 0 & 1987 & -- & 1993 \\
\hline 08076500 & Halls Bayou at Houston, TX & $29^{\circ} 51^{\prime} 42^{\prime \prime}$ & $95^{\circ} 20^{\prime} 05^{\prime \prime}$ & Harris & 28.7 & Yes & 41 & 41 & 0 & 1953 & -- & 1993 \\
\hline \multicolumn{13}{|c|}{ Clear Cr Basin (Coastal Basin) } \\
\hline 08077000 & Clear Cr nr Pearland, TX & $29^{\circ} 35^{\prime} 50^{\prime \prime}$ & $95^{\circ} 17^{\prime} 11^{\prime \prime}$ & Brazoria & 38.8 & Yes & 41 & 41 & 0 & 1948 & -- & 1992 \\
\hline \multicolumn{13}{|c|}{ Chocolate Bayou Basin (Coastal Basin) } \\
\hline 08078000 & Chocolate Bayou nr Alvin, TX & $29^{\circ} 22^{\prime} 09^{\prime \prime}$ & $95^{\circ} 19^{\prime} 14^{\prime \prime}$ & Brazoria & 87.7 & -- & 41 & 41 & 0 & 1960 & -- & 2000 \\
\hline \multicolumn{13}{|c|}{ Oyster Cr Basin (Coastal Basin) } \\
\hline 08079000 & Oyster Cr nr Angleton, TX & $29^{\circ} 09^{\prime} 30^{\prime \prime}$ & $95^{\circ} 28^{\prime} 32^{\prime \prime}$ & Brazoria & 171 & Yes & 36 & 29 & 7 & 1945 & 1974 & 1980 \\
\hline \multicolumn{13}{|c|}{ Brazos R Basin } \\
\hline 08079500 & North Fk Double Mountain Fk Brazos R at Lubbock, TX & $33^{\circ} 35^{\prime} 08^{\prime \prime}$ & $101^{\circ} 49^{\prime} 40^{\prime \prime}$ & Lubbock & 200 & -- & 10 & 10 & 0 & 1940 & -- & 1949 \\
\hline 08079575 & North Fk Double Mountain Fk Brazos R nr Post, TX & $33^{\circ} 14^{\prime} 55^{\prime \prime}$ & $101^{\circ} 20^{\prime} 17^{\prime \prime}$ & Garza & 438 & -- & 10 & 10 & 0 & 1984 & -- & 1993 \\
\hline 08079600 & Double Mountain Fk Brazos R at Justiceburg, TX & $33^{\circ} 02^{\prime} 18^{\prime \prime}$ & $101^{\circ} 11^{\prime} 50^{\prime \prime}$ & Garza & 244 & -- & 38 & 38 & 0 & 1963 & -- & 2000 \\
\hline 08080000 & Double Mountain Fk Brazos R nr Rotan, TX & $32^{\circ} 55^{\prime} 49^{\prime \prime}$ & $100^{\circ} 29^{\prime} 16^{\prime \prime}$ & Fisher & 1,600 & -- & 1 & 1 & 0 & 1951 & -- & 1951 \\
\hline 08080500 & Double Mountain Fk Brazos R nr Aspermont, TX & $33^{\circ} 00^{\prime} 29^{\prime \prime}$ & $100^{\circ} 10^{\prime} 49^{\prime \prime}$ & Stonewall & 1,860 & -- & 71 & 71 & 0 & 1925 & -- & 2000 \\
\hline 08080540 & McDonald Cr nr Post, TX & $33^{\circ} 21^{\prime} 03^{\prime \prime}$ & $101^{\circ} 13^{\prime} 36^{\prime \prime}$ & Garza & 79.2 & -- & 13 & 13 & 0 & 1966 & -- & 1978 \\
\hline 08080700 & Running Water Draw at Plainview, TX & $34^{\circ} 10^{\prime} 44^{\prime \prime}$ & $101^{\circ} 42^{\prime} 08^{\prime \prime}$ & Hale & 382 & -- & 34 & 34 & 0 & 1940 & -- & 1978 \\
\hline 08080950 & Duck Cr nr Girard, TX & $33^{\circ} 21^{\prime 22 "}$ & $100^{\circ} 42^{\prime} 17^{\prime \prime}$ & Kent & 279 & -- & 25 & 2 & 23 & 1965 & 1967 & 1989 \\
\hline 08081000 & Salt Fk Brazos R nr Peacock, TX & $33^{\circ} 12^{\prime} 43^{\prime \prime}$ & $100^{\circ} 25^{\prime} 53^{\prime \prime}$ & Stonewall & 1,990 & -- & 23 & 0 & 23 & -- & 1951 & 1986 \\
\hline 08081200 & Croton Cr nr Jayton, TX & $33^{\circ} 17^{\prime} 18^{\prime \prime}$ & $100^{\circ} 25^{\prime} 52^{\prime \prime}$ & Stonewall & 290 & -- & 27 & 27 & 0 & 1960 & -- & 1986 \\
\hline 08081500 & Salt Croton Cr nr Aspermont, TX & $33^{\circ} 24^{\prime} 03^{\prime \prime}$ & $100^{\circ} 24^{\prime} 29^{\prime \prime}$ & King & 64.3 & -- & 21 & 21 & 0 & 1957 & -- & 1977 \\
\hline 08082000 & Salt Fk Brazos R nr Aspermont, TX & $33^{\circ} 20^{\prime} 02^{\prime \prime}$ & $100^{\circ} 14^{\prime} 16^{\prime \prime}$ & Stonewall & 2,500 & -- & 61 & 24 & 37 & 1940 & 1964 & 2000 \\
\hline 08082100 & Stinking Cr nr Aspermont, TX & $33^{\circ} 14^{\prime} 00^{\prime \prime}$ & $100^{\circ} 12^{\prime} 47^{\prime \prime}$ & Stonewall & 88.8 & -- & 18 & 18 & 0 & 1966 & -- & 1983 \\
\hline 08082180 & North Croton Cr nr Knox City, TX & $33^{\circ} 22^{\prime} 59^{\prime \prime}$ & $100^{\circ} 04^{\prime} 51^{\prime \prime}$ & Stonewall & 251 & -- & 21 & 21 & 0 & 1966 & -- & 1986 \\
\hline 08082500 & Brazos R at Seymour, TX & $33^{\circ} 34^{\prime} 51^{\prime \prime}$ & $99^{\circ} 16^{\prime} 02^{\prime \prime}$ & Baylor & 5,970 & -- & 76 & 49 & 27 & 1925 & 1974 & 2000 \\
\hline 08082700 & Millers Cr nr Munday, TX & $33^{\circ} 19^{\prime} 45^{\prime \prime}$ & $99^{\circ} 27^{\prime} 53^{\prime \prime}$ & Throckmorton & 104 & -- & 37 & 37 & 0 & 1964 & -- & 2000 \\
\hline 08083000 & Brazos R nr Graham, TX & $33^{\circ} 04^{\prime} 55^{\prime \prime}$ & $98^{\circ} 43^{\prime} 36^{\prime \prime}$ & Young & 7,260 & -- & 2 & 2 & 0 & 1918 & -- & 1919 \\
\hline 08083100 & Clear Fk Brazos R nr Roby, TX & $32^{\circ} 47^{\prime} 15^{\prime \prime}$ & $100^{\circ} 23^{\prime} 18^{\prime \prime}$ & Fisher & 228 & -- & 38 & 38 & 0 & 1963 & -- & 2000 \\
\hline 08083240 & Clear Fk Brazos R at Hawley, TX & $32^{\circ} 35^{\prime} 53^{\prime \prime}$ & $99^{\circ} 48^{\prime} 53^{\prime \prime}$ & Jones & 1,420 & -- & 22 & 22 & 0 & 1968 & -- & 1989 \\
\hline 08083245 & Mulberry Cr nr Hawley, TX & $32^{\circ} 34^{\prime} 04^{\prime \prime}$ & $99^{\circ} 47^{\prime} 32^{\prime \prime}$ & Jones & 205 & -- & 21 & 21 & 0 & 1969 & -- & 1989 \\
\hline 08083300 & Elm Cr nr Abilene, TX & $32^{\circ} 21^{\prime} 08^{\prime \prime}$ & $99^{\circ} 48^{\prime} 27^{\prime \prime}$ & Taylor & 133 & -- & 16 & 0 & 16 & -- & 1964 & 1979 \\
\hline 08083400 & Little Elm Cr nr Abilene, TX & $32^{\circ} 23^{\prime} 29^{\prime \prime}$ & $99^{\circ} 51^{\prime} 08^{\prime \prime}$ & Taylor & 39.1 & -- & 16 & 16 & 0 & 1964 & -- & 1979 \\
\hline 08083420 & Cat Claw Cr at Abilene, TX & $32^{\circ} 28^{\prime} 31^{\prime \prime}$ & $99^{\circ} 44^{\prime} 56^{\prime \prime}$ & Taylor & 13.0 & -- & 9 & 9 & 0 & 1971 & -- & 1979 \\
\hline
\end{tabular}

Footnotes at end of table. 
Table 2. Inventory of daily mean streamflows for active and discontinued streamflow-gaging stations in Texas-Continued

\begin{tabular}{|c|c|c|c|c|c|c|c|c|c|c|c|c|}
\hline \multirow[b]{2}{*}{$\begin{array}{l}\text { Station } \\
\text { no. }\end{array}$} & \multirow[b]{2}{*}{ Station name } & \multirow[b]{2}{*}{ Latitude } & \multirow[b]{2}{*}{ Longitude } & \multirow[b]{2}{*}{ County } & \multirow{2}{*}{$\begin{array}{l}\text { Contri- } \\
\text { buting } \\
\text { drainage } \\
\text { area } \\
\left(\mathrm{mi}^{2}\right)\end{array}$} & \multirow[b]{2}{*}{$\begin{array}{l}\text { Urban } \\
\text { basin }\end{array}$} & \multicolumn{3}{|c|}{ Years of record $^{2}$} & \multicolumn{3}{|c|}{ Begin and end year ${ }^{2}$} \\
\hline & & & & & & & Total & $\begin{array}{l}\text { Unregu- } \\
\text { lated }\end{array}$ & $\begin{array}{l}\text { Regu- } \\
\text { lated }^{3}\end{array}$ & $\begin{array}{l}\text { First } \\
\text { unregu- } \\
\text { lated }\end{array}$ & $\begin{array}{l}\text { First } \\
\text { regu- } \\
\text { lated }\end{array}$ & $\begin{array}{c}\text { Last } \\
\text { year }\end{array}$ \\
\hline \multicolumn{13}{|c|}{ Brazos R Basin-Continued } \\
\hline 08083430 & Elm $\mathrm{Cr}$ at Abilene, TX & $32^{\circ} 30^{\prime} 29^{\prime \prime}$ & $99^{\circ} 44^{\prime} 27^{\prime \prime}$ & Taylor & 422 & -- & 4 & 0 & 4 & -- & 1980 & 1983 \\
\hline 08083470 & Cedar Cr at Abilene, TX & $32^{\circ} 26^{\prime} 56^{\prime \prime}$ & $99^{\circ} 43^{\prime} 13^{\prime \prime}$ & Taylor & 119 & -- & 14 & 0 & 14 & -- & 1971 & 1984 \\
\hline 08084000 & Clear Fk Brazos R at Nugent, TX & $32^{\circ} 41^{\prime} 24^{\prime \prime}$ & $99^{\circ} 40^{\prime} 09^{\prime \prime}$ & Jones & 2,200 & -- & 76 & 15 & 61 & 1925 & 1940 & 2000 \\
\hline 08084800 & California Cr nr Stamford, TX & $32^{\circ} 55^{\prime} 51^{\prime \prime}$ & $99^{\circ} 38^{\prime} 32^{\prime \prime}$ & Jones & 478 & -- & 38 & 38 & 0 & 1963 & -- & 2000 \\
\hline 08085000 & Paint Cr nr Haskell, TX & $33^{\circ} 04^{\prime} 39^{\prime \prime}$ & $99^{\circ} 32^{\prime} 36^{\prime \prime}$ & Haskell & 914 & -- & 1 & 1 & 0 & 1951 & -- & 1951 \\
\hline 08085500 & Clear Fk Brazos R at Fort Griffin, TX & $32^{\circ} 56^{\prime} 04^{\prime \prime}$ & $99^{\circ} 13^{\prime} 27^{\prime \prime}$ & Shackelford & 3,990 & -- & 76 & 15 & 61 & 1925 & 1940 & 2000 \\
\hline 08086000 & Clear Fk Brazos R at Crystal Falls, TX & $32^{\circ} 54^{\prime} 00^{\prime \prime}$ & $98^{\circ} 50^{\prime} 00^{\prime \prime}$ & Stephens & 4,320 & -- & 4 & 4 & 0 & 1924 & -- & 1928 \\
\hline 08086015 & Hubbard Cr nr Sedwick, TX & $32^{\circ} 36^{\prime} 06^{\prime \prime}$ & $99^{\circ} 14^{\prime} 20^{\prime \prime}$ & Shackelford & 128 & -- & 3 & 3 & 0 & 1964 & -- & 1966 \\
\hline 08086050 & Deep Cr at Moran, TX & $32^{\circ} 33^{\prime} 33^{\prime \prime}$ & $99^{\circ} 10^{\prime} 11^{\prime \prime}$ & Shackelford & 228 & -- & 13 & 13 & 0 & 1963 & -- & 1975 \\
\hline 08086100 & Hubbard Cr nr Albany, TX & $32^{\circ} 41^{\prime} 21^{\prime \prime}$ & $99^{\circ} 09^{\prime} 52^{\prime \prime}$ & Shackelford & 454 & -- & 13 & 13 & 0 & 1963 & -- & 1975 \\
\hline 08086120 & Salt Prong Hubbard Cr at US $380 \mathrm{nr}$ Albany, TX & $32^{\circ} 41^{\prime} 01^{\prime \prime}$ & $99^{\circ} 16^{\prime} 05^{\prime \prime}$ & Shackelford & 65.2 & -- & 5 & 0 & 5 & -- & 1964 & 1968 \\
\hline 08086150 & North Fk Hubbard Cr nr Albany, TX & $32^{\circ} 42^{\prime} 27^{\prime \prime}$ & $99^{\circ} 16^{\prime} 29^{\prime \prime}$ & Shackelford & 39.3 & -- & 27 & 27 & 0 & 1964 & -- & 1990 \\
\hline 08086200 & Salt Prong Hubbard Cr nr Albany, TX & $32^{\circ} 42^{\prime} 02^{\prime \prime}$ & $99^{\circ} 12^{\prime} 42^{\prime \prime}$ & Shackelford & 115 & -- & 1 & 1 & 0 & 1963 & -- & 1963 \\
\hline 08086210 & Snailum Cr nr Albany, TX & $32^{\circ} 43^{\prime} 15^{\prime \prime}$ & $99^{\circ} 10^{\prime} 30^{\prime \prime}$ & Shackelford & 22.9 & -- & 3 & 3 & 0 & 1964 & -- & 1966 \\
\hline 08086212 & Hubbard Cr below Albany, TX & $32^{\circ} 43^{\prime} 58^{\prime \prime}$ & $99^{\circ} 08^{\prime} 25^{\prime \prime}$ & Shackelford & 613 & -- & 34 & 34 & 0 & 1967 & -- & 2000 \\
\hline 08086235 & Battle Cr nr Moran, TX & $32^{\circ} 33^{\prime} 10^{\prime \prime}$ & $99^{\circ} 06^{\prime} 32^{\prime \prime}$ & Shackelford & 108 & -- & 2 & 2 & 0 & 1967 & -- & 1968 \\
\hline 08086260 & Pecan Cr nr Eolian, TX & $32^{\circ} 35^{\prime} 01^{\prime \prime}$ & $99^{\circ} 01 ' 57^{\prime \prime}$ & Stephens & 26.4 & -- & 9 & 9 & 0 & 1967 & -- & 1975 \\
\hline 08086290 & Big Sandy Cr above Breckenridge, TX & $32^{\circ} 38^{\prime} 54^{\prime \prime}$ & $99^{\circ} 00^{\prime} 15^{\prime \prime}$ & Stephens & 280 & -- & 38 & 38 & 0 & 1963 & -- & 2000 \\
\hline 08086500 & Hubbard Cr nr Breckenridge, TX & $32^{\circ} 50^{\prime} 13^{\prime \prime}$ & $98^{\circ} 56^{\prime} 52^{\prime \prime}$ & Stephens & 1,090 & -- & 31 & 7 & 24 & 1956 & 1963 & 1986 \\
\hline 08087300 & Clear Fk Brazos R at Eliasville, TX & $32^{\circ} 57^{\prime} 36^{\prime \prime}$ & $98^{\circ} 45^{\prime} 59^{\prime \prime}$ & Young & 5,700 & -- & 47 & 26 & 21 & 1917 & 1962 & 1982 \\
\hline 08088000 & Brazos R nr South Bend, TX & $33^{\circ} 01^{\prime} 27^{\prime \prime}$ & $98^{\circ} 38^{\prime} 37^{\prime \prime}$ & Young & 13,100 & -- & 62 & 23 & 39 & 1939 & 1962 & 2000 \\
\hline 08088100 & Salt $\mathrm{Cr}$ at Olney, TX & $33^{\circ} 22^{\prime} 13^{\prime \prime}$ & $98^{\circ} 44^{\prime} 40^{\prime \prime}$ & Young & 11.8 & -- & 19 & 19 & 0 & 1959 & -- & 1977 \\
\hline 08088200 & Salt Cr nr Newcastle, TX & $33^{\circ} 13^{\prime} 00^{\prime \prime}$ & $98^{\circ} 38^{\prime} 55^{\prime \prime}$ & Young & 120 & -- & 2 & 2 & 0 & 1959 & -- & 1960 \\
\hline 08088300 & Briar Cr nr Graham, TX & $33^{\circ} 12^{\prime} 43^{\prime \prime}$ & $98^{\circ} 37^{\prime} 06^{\prime \prime}$ & Young & 24.2 & -- & 31 & 31 & 0 & 1959 & -- & 1989 \\
\hline 08088450 & Big Cedar Cr nr Ivan, TX & $32^{\circ} 49^{\prime} 39^{\prime \prime}$ & $98^{\circ} 43^{\prime} 25^{\prime \prime}$ & Stephens & 97.0 & -- & 24 & 24 & 0 & 1966 & -- & 1989 \\
\hline 08088610 & Brazos R nr Graford, TX & $32^{\circ} 51^{\prime} 29^{\prime \prime}$ & $98^{\circ} 24^{\prime} 41^{\prime \prime}$ & Palo Pinto & 14,000 & -- & 11 & 0 & 11 & -- & 1990 & 2000 \\
\hline 08089000 & Brazos R nr Palo Pinto, TX & $32^{\circ} 51^{\prime} 45^{\prime \prime}$ & $98^{\circ} 18^{\prime} 08^{\prime \prime}$ & Palo Pinto & 14,200 & -- & 76 & 16 & 60 & 1925 & 1941 & 2000 \\
\hline 08090500 & Palo Pinto Cr nr Santo, TX & $32^{\circ} 37^{\prime} 51^{\prime \prime}$ & $98^{\circ} 10^{\prime} 50^{\prime \prime}$ & Palo Pinto & 573 & -- & 26 & 13 & 13 & 1925 & 1964 & 1976 \\
\hline 08090800 & Brazos R nr Dennis, TX & $32^{\circ} 36^{\prime} 56^{\prime \prime}$ & $97^{\circ} 55^{\prime} 32^{\prime \prime}$ & Parker & 15,700 & -- & 32 & 0 & 32 & -- & 1969 & 2000 \\
\hline 08091000 & Brazos R nr Glen Rose, TX & $32^{\circ} 16^{\prime} 18^{\prime \prime}$ & $97^{\circ} 39^{\prime} 48^{\prime \prime}$ & Somervell & 16,300 & -- & 77 & 17 & 60 & 1924 & 1941 & 2000 \\
\hline 08091500 & Paluxy R at Glen Rose, TX & $32^{\circ} 13^{\prime} 53^{\prime \prime}$ & $97^{\circ} 46^{\prime} 37^{\prime \prime}$ & Somervell & 410 & -- & 54 & 35 & 19 & 1925 & 1982 & 2000 \\
\hline 08091750 & Squaw Cr nr Glen Rose, TX & $32^{\circ} 16^{\prime} 12^{\prime \prime}$ & $97^{\circ} 43^{\prime} 56^{\prime \prime}$ & Somervell & 70.3 & -- & 27 & 5 & 22 & 1974 & 1978 & 2000 \\
\hline 08092000 & Nolan R at Blum, TX & $32^{\circ} 09^{\prime} 02^{\prime \prime}$ & $97^{\circ} 24^{\prime} 09^{\prime \prime}$ & Hill & 282 & -- & 39 & 17 & 22 & 1925 & 1965 & 1986 \\
\hline
\end{tabular}

$\stackrel{\omega}{\omega} \quad$ Footnotes at end of table. 
Table 2. Inventory of daily mean streamflows for active and discontinued streamflow-gaging stations in Texas-Continued

\begin{tabular}{|c|c|c|c|c|c|c|c|c|c|c|c|c|}
\hline \multirow[b]{2}{*}{$\begin{array}{c}\text { Station } \\
\text { no. }\end{array}$} & \multirow[b]{2}{*}{ Station name } & \multirow[b]{2}{*}{ Latitude } & \multirow[b]{2}{*}{ Longitude } & \multirow[b]{2}{*}{ County } & \multirow{2}{*}{$\begin{array}{l}\text { Contri- } \\
\text { buting } \\
\text { drainage } \\
\text { area } \\
\left(\mathrm{mi}^{2}\right)\end{array}$} & \multirow[b]{2}{*}{$\begin{array}{l}\text { Urban } \\
\text { basin }\end{array}$} & \multicolumn{3}{|c|}{ Years of record $^{2}$} & \multicolumn{3}{|c|}{ Begin and end year ${ }^{2}$} \\
\hline & & & & & & & Total & $\begin{array}{l}\text { Unregu- } \\
\text { lated }\end{array}$ & $\begin{array}{l}\text { Regu- } \\
\text { lated }^{3}\end{array}$ & $\begin{array}{c}\text { First } \\
\text { unregu- } \\
\text { lated }\end{array}$ & $\begin{array}{l}\text { First } \\
\text { regu- } \\
\text { lated }\end{array}$ & $\begin{array}{c}\text { Last } \\
\text { year }\end{array}$ \\
\hline \multicolumn{13}{|c|}{ Brazos R Basin-Continued } \\
\hline 08093100 & Brazos R nr Aquilla, TX & $31^{\circ} 48^{\prime} 44^{\prime \prime}$ & $97^{\circ} 17^{\prime} 51^{\prime \prime}$ & Bosque & 17,700 & -- & 62 & 2 & 60 & 1939 & 1941 & 2000 \\
\hline 08093250 & Hackberry $\mathrm{Cr}$ at Hillsboro, TX & $32^{\circ} 00^{\prime} 20^{\prime \prime}$ & $97^{\circ} 08^{\prime} 59^{\prime \prime}$ & Hill & 57.9 & -- & 13 & 13 & 0 & 1980 & -- & 1992 \\
\hline 08093360 & Aquilla $\mathrm{Cr}$ above Aquilla, TX & $31^{\circ} 53^{\prime} 42^{\prime \prime}$ & $97^{\circ} 12^{\prime} 21 "$ & Hill & 255 & -- & 2 & 0 & 2 & -- & 1980 & 1981 \\
\hline 08093400 & Cobb Cr nr Abbott, TX & $31^{\circ} 55^{\prime} 11^{\prime \prime}$ & $97^{\circ} 05^{\prime} 57^{\prime \prime}$ & Hill & 12.4 & Yes & 12 & 12 & 0 & 1968 & -- & 1979 \\
\hline 08093500 & Aquilla $\mathrm{Cr}$ nr Aquilla, TX & $31^{\circ} 50^{\prime} 40^{\prime \prime}$ & $97^{\circ} 12^{\prime} 04^{\prime \prime}$ & Hill & 308 & -- & 61 & 43 & 18 & 1940 & 1983 & 2000 \\
\hline 08093700 & North Bosque R at Stephenville, TX & $32^{\circ} 12^{\prime} 56^{\prime \prime}$ & $98^{\circ} 11^{\prime} 55^{\prime \prime}$ & Erath & 95.9 & -- & 21 & 7 & 14 & 1959 & 1966 & 1979 \\
\hline 08094500 & Green $\mathrm{Cr}$ nr Alexander, TX & $32^{\circ} 04^{\prime} 26^{\prime \prime}$ & $98^{\circ} 13^{\prime} 46^{\prime \prime}$ & Erath & 45.4 & -- & 15 & 0 & 15 & -- & 1959 & 1973 \\
\hline 08094800 & North Bosque R at Hico, TX & $31^{\circ} 58^{\prime} 41^{\prime \prime}$ & $98^{\circ} 02^{\prime} 04^{\prime \prime}$ & Hamilton & 359 & -- & 38 & 0 & 38 & -- & 1963 & 2000 \\
\hline 08095000 & North Bosque R nr Clifton, TX & $31^{\circ} 47^{\prime} 09^{\prime \prime}$ & $97^{\circ} 34^{\prime} 04^{\prime \prime}$ & Bosque & 968 & -- & 77 & 44 & 33 & 1924 & 1968 & 2000 \\
\hline 08095200 & North Bosque R at Valley Mills, TX & $31^{\circ} 40^{\prime} 10^{\prime \prime}$ & $97^{\circ} 28^{\prime} 09^{\prime \prime}$ & Bosque & 1,150 & -- & 41 & 8 & 33 & 1960 & 1968 & 2000 \\
\hline 08095300 & Middle Bosque R nr McGregor, TX & $31^{\circ} 30^{\prime} 33^{\prime \prime}$ & $97^{\circ} 21^{\prime} 56^{\prime \prime}$ & McLennan & 182 & -- & 26 & 26 & 0 & 1960 & -- & 1985 \\
\hline 08095400 & Hog Cr nr Crawford, TX & $31^{\circ} 33^{\prime} 20^{\prime \prime}$ & $97^{\circ} 21^{\prime} 22^{\prime \prime}$ & McLennan & 78.2 & -- & 26 & 20 & 6 & 1960 & 1980 & 1985 \\
\hline 08095500 & South Bosque R nr Speegleville, TX & $31^{\circ} 31^{\prime} 00^{\prime \prime}$ & $97^{\circ} 15^{\prime} 00^{\prime \prime}$ & McLennan & 386 & -- & 5 & 5 & 0 & 1925 & -- & 1929 \\
\hline 08095600 & Bosque R nr Waco, TX & $31^{\circ} 36^{\prime} 04^{\prime \prime}$ & $97^{\circ} 11^{\prime} 36^{\prime \prime}$ & McLennan & 1,660 & -- & 22 & 6 & 16 & 1960 & 1966 & 1981 \\
\hline 08096500 & Brazos R at Waco, TX & $31^{\circ} 32^{\prime} 09^{\prime \prime}$ & $97^{\circ} 04^{\prime} 23^{\prime \prime}$ & McLennan & 20,000 & -- & 102 & 42 & 60 & 1899 & 1941 & 2000 \\
\hline 08097000 & Cow Bayou at Mooreville, TX & $31^{\circ} 18^{\prime} 45^{\prime \prime}$ & $97^{\circ} 08^{\prime} 16^{\prime \prime}$ & Falls & 83.5 & -- & 17 & 0 & 17 & -- & 1959 & 1975 \\
\hline 08097500 & Brazos R nr Marlin, TX & $31^{\circ} 17^{\prime} 18^{\prime \prime}$ & $96^{\circ} 58^{\prime} 10^{\prime \prime}$ & Falls & 20,600 & -- & 13 & 2 & 11 & 1939 & 1941 & 1951 \\
\hline 08098000 & Deer Cr at Chilton, TX & $31^{\circ} 15^{\prime} 58^{\prime \prime}$ & $97^{\circ} 03^{\prime} 30^{\prime \prime}$ & Falls & 84.5 & -- & 2 & 2 & 0 & 1935 & -- & 1936 \\
\hline 08098290 & Brazos R nr Highbank, TX & $31^{\circ} 08^{\prime} 02^{\prime \prime}$ & $96^{\circ} 49^{\prime} 29^{\prime \prime}$ & Falls & 20,900 & -- & 35 & 0 & 35 & -- & 1966 & 2000 \\
\hline 08098300 & Little Pond Cr nr Burlington, TX & $31^{\circ} 01^{\prime} 35^{\prime \prime}$ & $96^{\circ} 59^{\prime} 17^{\prime \prime}$ & Milam & 22.2 & -- & 20 & 20 & 0 & 1963 & -- & 1982 \\
\hline 08099100 & Leon R nr De Leon, TX & $32^{\circ} 10^{\prime} 25^{\prime \prime}$ & $98^{\circ} 31^{\prime} 58^{\prime \prime}$ & Comanche & 479 & -- & 26 & 0 & 26 & -- & 1961 & 1986 \\
\hline 08099300 & Sabana R nr De Leon, TX & $32^{\circ} 06^{\prime} 50^{\prime \prime}$ & $98^{\circ} 36^{\prime} 19^{\prime \prime}$ & Comanche & 264 & -- & 26 & 19 & 7 & 1961 & 1980 & 1986 \\
\hline 08099500 & Leon R nr Hasse, TX & $31^{\circ} 57^{\prime} 28^{\prime \prime}$ & $98^{\circ} 27^{\prime} 32^{\prime \prime}$ & Comanche & 1,260 & -- & 52 & 14 & 38 & 1940 & 1954 & 1991 \\
\hline 08100000 & Leon R nr Hamilton, TX & $31^{\circ} 47^{\prime} 19^{\prime \prime}$ & $98^{\circ} 07^{\prime} 16^{\prime \prime}$ & Hamilton & 1,890 & -- & 46 & 7 & 39 & 1926 & 1962 & 2000 \\
\hline 08100500 & Leon $\mathrm{R}$ at Gatesville, TX & $31^{\circ} 25^{\prime} 58^{\prime \prime}$ & $97^{\circ} 45^{\prime} 42^{\prime \prime}$ & Coryell & 2,340 & -- & 50 & 13 & 37 & 1951 & 1964 & 2000 \\
\hline 08101000 & Cowhouse $\mathrm{Cr}$ at Pidcoke, TX & $31^{\circ} 17^{\prime} 05^{\prime \prime}$ & $97^{\circ} 53^{\prime} 05^{\prime \prime}$ & Coryell & 455 & -- & 50 & 50 & 0 & 1951 & -- & 2000 \\
\hline 08101500 & Cowhouse Cr nr Killeen, TX & $31^{\circ} 12^{\prime} 21^{\prime \prime}$ & $97^{\circ} 42^{\prime} 55^{\prime \prime}$ & Coryell & 667 & -- & 2 & 2 & 0 & 1940 & -- & 1941 \\
\hline 08102500 & Leon R nr Belton, TX & $31^{\circ} 04^{\prime} 12^{\prime \prime}$ & $97^{\circ} 26^{\prime} 28^{\prime \prime}$ & Bell & 3,540 & -- & 77 & 31 & 46 & 1924 & 1955 & 2000 \\
\hline 08102600 & Nolan $\mathrm{Cr}$ at Belton, $\mathrm{TX}$ & $31^{\circ} 03^{\prime} 06^{\prime \prime}$ & $97^{\circ} 27^{\prime} 25^{\prime \prime}$ & Bell & 112 & -- & 8 & 0 & 8 & -- & 1975 & 1982 \\
\hline 08103800 & Lampasas R nr Kempner, TX & $31^{\circ} 04^{\prime} 54^{\prime \prime}$ & $98^{\circ} 00^{\prime} 59^{\prime \prime}$ & Lampasas & 818 & -- & 38 & 11 & 27 & 1963 & 1974 & 2000 \\
\hline 08103900 & South Fk Rocky Cr nr Briggs, TX & $30^{\circ} 54^{\prime} 41^{\prime \prime}$ & $98^{\circ} 02^{\prime} 12^{\prime \prime}$ & Burnet & 33.3 & -- & 37 & 37 & 0 & 1964 & -- & 2000 \\
\hline 08104000 & Lampasas R at Youngsport, TX & $30^{\circ} 57^{\prime} 26^{\prime \prime}$ & $97^{\circ} 42^{\prime} 30^{\prime \prime}$ & Bell & 1,240 & -- & 56 & 49 & 7 & 1925 & 1974 & 1980 \\
\hline 08104100 & Lampasas R nr Belton, TX & $31^{\circ} 00^{\prime} 06^{\prime \prime}$ & $97^{\circ} 29^{\prime} 32^{\prime \prime}$ & Bell & 1,320 & -- & 26 & 3 & 23 & 1964 & 1967 & 1989 \\
\hline 08104500 & Little R nr Little River, TX & $30^{\circ} 57^{\prime} 59^{\prime \prime}$ & $97^{\circ} 20^{\prime} 45^{\prime \prime}$ & Bell & 5,230 & -- & 43 & 5 & 38 & 1924 & 1963 & 2000 \\
\hline
\end{tabular}


Table 2. Inventory of daily mean streamflows for active and discontinued streamflow-gaging stations in Texas-Continued

\begin{tabular}{|c|c|c|c|c|c|c|c|c|c|c|c|c|}
\hline \multirow[b]{2}{*}{$\begin{array}{l}\text { Station } \\
\text { no. }\end{array}$} & \multirow[b]{2}{*}{ Station name } & \multirow[b]{2}{*}{ Latitude } & \multirow[b]{2}{*}{ Longitude } & \multirow[b]{2}{*}{ County } & \multirow{2}{*}{$\begin{array}{l}\text { Contri- } \\
\text { buting } \\
\text { drainage } \\
\text { area } \\
\left(\mathrm{mi}^{2}\right)\end{array}$} & \multirow[b]{2}{*}{$\begin{array}{l}\text { Urban } \\
\text { basin }\end{array}$} & \multicolumn{3}{|c|}{ Years of record $^{2}$} & \multicolumn{3}{|c|}{ Begin and end year ${ }^{2}$} \\
\hline & & & & & & & Total & $\begin{array}{l}\text { Unregu- } \\
\text { lated }\end{array}$ & $\begin{array}{l}\text { Regu- } \\
\text { lated }^{3}\end{array}$ & $\begin{array}{l}\text { First } \\
\text { unregu- } \\
\text { lated }\end{array}$ & $\begin{array}{l}\text { First } \\
\text { regu- } \\
\text { lated }\end{array}$ & $\begin{array}{c}\text { Last } \\
\text { year }\end{array}$ \\
\hline \multicolumn{13}{|c|}{ Brazos R Basin-Continued } \\
\hline 08104700 & North Fk San Gabriel R nr Georgetown, TX & $30^{\circ} 39^{\prime} 42^{\prime \prime}$ & $97^{\circ} 42^{\prime} 40^{\prime \prime}$ & Williamson & 248 & -- & 32 & 11 & 21 & 1969 & 1980 & 2000 \\
\hline 08104900 & South Fk San Gabriel R at Georgetown, TX & $30^{\circ} 37^{\prime} 32^{\prime \prime}$ & $97^{\circ} 41^{\prime} 27^{\prime \prime}$ & Williamson & 133 & -- & 32 & 32 & 0 & 1969 & -- & 2000 \\
\hline 08105000 & San Gabriel R at Georgetown, TX & $30^{\circ} 39^{\prime} 14^{\prime \prime}$ & $97^{\circ} 39^{\prime} 18^{\prime \prime}$ & Williamson & 405 & -- & 39 & 39 & 0 & 1935 & -- & 1973 \\
\hline 08105100 & Berry Cr nr Georgetown, TX & $30^{\circ} 41^{\prime 2} 28^{\prime \prime}$ & $97^{\circ} 39^{\prime} 21^{\prime \prime}$ & Williamson & 83.1 & -- & 33 & 33 & 0 & 1968 & -- & 2000 \\
\hline 08105300 & San Gabriel R nr Weir, TX & $30^{\circ} 38^{\prime} 45^{\prime \prime}$ & $97^{\circ} 35^{\prime} 06^{\prime \prime}$ & Williamson & 563 & -- & 13 & 2 & 11 & 1978 & 1980 & 1990 \\
\hline 08105400 & San Gabriel R nr Circleville, TX & $30^{\circ} 37^{\prime} 43^{\prime \prime}$ & $97^{\circ} 28^{\prime} 23^{\prime \prime}$ & Williamson & 599 & -- & 19 & 19 & 0 & 1925 & -- & 1976 \\
\hline 08105700 & San Gabriel R at Laneport, TX & $30^{\circ} 41^{\prime} 39^{\prime \prime}$ & $97^{\circ} 16^{\prime} 43^{\prime \prime}$ & Williamson & 738 & -- & 34 & 14 & 21 & 1966 & 1980 & 2000 \\
\hline 08106300 & Brushy Cr nr Rockdale, TX & $30^{\circ} 41^{\prime} 38^{\prime \prime}$ & $97^{\circ} 04^{\prime} 42^{\prime \prime}$ & Milam & 505 & -- & 13 & 0 & 13 & -- & 1968 & 1980 \\
\hline 08106310 & San Gabriel R nr Rockdale, TX & $30^{\circ} 43^{\prime} 39^{\prime \prime}$ & $97^{\circ} 02^{\prime} 19^{\prime \prime}$ & Milam & 1,360 & -- & 12 & 0 & 12 & -- & 1981 & 1992 \\
\hline 08106500 & Little $\mathrm{R}$ at Cameron, $\mathrm{TX}$ & $30^{\circ} 50^{\prime} 06^{\prime \prime}$ & $96^{\circ} 56^{\prime} 47^{\prime \prime}$ & Milam & 7,070 & -- & 83 & 36 & 47 & 1918 & 1954 & 2000 \\
\hline 08107000 & Big Elm Cr nr Temple, TX & $31^{\circ} 02^{\prime} 58^{\prime \prime}$ & $97^{\circ} 14^{\prime} 08^{\prime \prime}$ & Bell & 74.7 & -- & 1 & 1 & 0 & 1935 & -- & 1935 \\
\hline 08107500 & Big Elm Cr nr Buckholts, TX & $30^{\circ} 56^{\prime} 50^{\prime \prime}$ & $97^{\circ} 06^{\prime} 14^{\prime \prime}$ & Milam & 171 & -- & 2 & 2 & 0 & 1935 & -- & 1936 \\
\hline 08108000 & North Elm Cr nr Ben Arnold, TX & $30^{\circ} 57^{\prime} 00^{\prime \prime}$ & $97^{\circ} 03^{\prime} 00^{\prime \prime}$ & Milam & 32.2 & -- & 2 & 2 & 0 & 1935 & -- & 1936 \\
\hline 08108200 & North Elm Cr nr Cameron, TX & $30^{\circ} 55^{\prime} 52^{\prime \prime}$ & $97^{\circ} 01^{\prime} 13^{\prime \prime}$ & Milam & 44.8 & -- & 11 & 11 & 0 & 1963 & -- & 1973 \\
\hline 08108700 & Brazos R at SH $21 \mathrm{nr}$ Bryan, TX & $30^{\circ} 37^{\prime} 36^{\prime \prime}$ & $96^{\circ} 32^{\prime} 38^{\prime \prime}$ & Brazos & 29,500 & -- & 7 & 0 & 7 & -- & 1994 & 2000 \\
\hline 08109000 & Brazos R nr Bryan, TX & $30^{\circ} 36^{\prime} 50^{\prime \prime}$ & $96^{\circ} 29^{\prime} 11^{\prime \prime}$ & Brazos & 29,900 & -- & 76 & 25 & 53 & 1900 & 1941 & 1992 \\
\hline 08109700 & Middle Yegua Cr nr Dime Box, TX & $30^{\circ} 20^{\prime} 21^{\prime \prime}$ & $96^{\circ} 54^{\prime} 16^{\prime \prime}$ & Lee & 236 & -- & 38 & 38 & 0 & 1963 & -- & 2000 \\
\hline 08109800 & East Yegua Cr nr Dime Box, TX & $30^{\circ} 24^{\prime} 26^{\prime \prime}$ & $96^{\circ} 49^{\prime} 02^{\prime \prime}$ & Burleson & 244 & -- & 38 & 38 & 0 & 1963 & -- & 2000 \\
\hline 08110000 & Yegua Cr nr Somerville, TX & $30^{\circ} 19^{\prime} 18^{\prime \prime}$ & $96^{\circ} 30^{\prime} 26^{\prime \prime}$ & Burleson & 1,010 & -- & 67 & 42 & 25 & 1925 & 1967 & 1991 \\
\hline 08110100 & Davidson Cr nr Lyons, TX & $30^{\circ} 25^{\prime} 10^{\prime \prime}$ & $96^{\circ} 32^{\prime} 24^{\prime \prime}$ & Burleson & 195 & -- & 38 & 38 & 0 & 1963 & -- & 2000 \\
\hline 08110200 & Brazos R at Washington, TX & $30^{\circ} 21^{\prime} 40^{\prime \prime}$ & $96^{\circ} 09^{\prime} 18^{\prime \prime}$ & Washington & 31,600 & -- & 28 & 0 & 28 & -- & 1967 & 1994 \\
\hline 08110325 & Navasota R above Groesbeck, TX & $31^{\circ} 34^{\prime} 27^{\prime \prime}$ & $96^{\circ} 31^{\prime} 14^{\prime \prime}$ & Limestone & 239 & -- & 22 & 0 & 22 & -- & 1979 & 2000 \\
\hline 08110400 & Navasota R nr Groesbeck, TX & $31^{\circ} 30^{\prime} 44^{\prime \prime}$ & $96^{\circ} 27^{\prime} 01^{\prime \prime}$ & Limestone & 311 & -- & 13 & 0 & 13 & -- & 1966 & 1978 \\
\hline 08110430 & Big Cr nr Freestone, TX & $31^{\circ} 30^{\prime} 24^{\prime \prime}$ & $96^{\circ} 19^{\prime} 28^{\prime \prime}$ & Limestone & 97.2 & -- & 22 & 22 & 0 & 1979 & -- & 2000 \\
\hline 08110500 & Navasota R nr Easterly, TX & $31^{\circ} 10^{\prime} 12^{\prime \prime}$ & $96^{\circ} 17^{\prime} 51^{\prime \prime}$ & Robertson & 968 & -- & 76 & 37 & 39 & 1925 & 1962 & 2000 \\
\hline 08110800 & Navasota $\mathrm{R}$ at Old Spanish Rd nr Bryan, TX & $30^{\circ} 58^{\prime} 25^{\prime \prime}$ & $96^{\circ} 14^{\prime} 29^{\prime \prime}$ & Robertson & 1,287 & -- & 3 & 0 & 3 & -- & 1998 & 2000 \\
\hline 08111000 & Navasota R nr Bryan, TX & $30^{\circ} 52^{\prime} 10^{\prime \prime}$ & $96^{\circ} 11^{\prime} 32^{\prime \prime}$ & Brazos & 1,450 & -- & 46 & 10 & 36 & 1952 & 1962 & 1997 \\
\hline 08111010 & Navasota R nr College Station, TX & $30^{\circ} 36^{\prime} 26^{\prime \prime}$ & $96^{\circ} 10^{\prime} 53^{\prime \prime}$ & Grimes & 1,810 & -- & 8 & 0 & 8 & -- & 1978 & 1985 \\
\hline 08111025 & Burton Cr at Villa Maria Rd, Bryan, TX & $30^{\circ} 38^{\prime} 47^{\prime \prime}$ & $96^{\circ} 20^{\prime} 58^{\prime \prime}$ & Brazos & 1.33 & -- & 2 & 2 & 0 & 1969 & -- & 1970 \\
\hline 08111050 & Hudson Cr nr Bryan, TX & $30^{\circ} 39^{\prime} 37^{\prime \prime}$ & $96^{\circ} 17^{\prime} 58^{\prime \prime}$ & Brazos & 1.94 & -- & 2 & 2 & 0 & 1969 & -- & 1970 \\
\hline 08111500 & Brazos R nr Hempstead, TX & $30^{\circ} 07^{\prime} 44^{\prime \prime}$ & $96^{\circ} 11^{\prime} 15^{\prime \prime}$ & Washington & 34,300 & -- & 62 & 2 & 60 & 1939 & 1941 & 2000 \\
\hline 08111700 & Mill Cr nr Bellville, TX & $29^{\circ} 52^{\prime} 51^{\prime \prime}$ & $96^{\circ} 12^{\prime} 18^{\prime \prime}$ & Austin & 376 & -- & 30 & 30 & 0 & 1964 & -- & 1993 \\
\hline 08114000 & Brazos R at Richmond, TX & $29^{\circ} 34^{\prime} 56^{\prime \prime}$ & $95^{\circ} 45^{\prime} 27^{\prime \prime}$ & Fort Bend & 35,400 & -- & 80 & 20 & 60 & 1904 & 1941 & 2000 \\
\hline 08114500 & Brazos R nr Juliff, TX & $29^{\circ} 27^{\prime} 19^{\prime \prime}$ & $95^{\circ} 31^{\prime} 58^{\prime \prime}$ & Fort Bend & 35,500 & -- & 20 & 0 & 20 & -- & 1950 & 1969 \\
\hline
\end{tabular}


Table 2. Inventory of daily mean streamflows for active and discontinued streamflow-gaging stations in Texas-Continued

\begin{tabular}{|c|c|c|c|c|c|c|c|c|c|c|c|c|}
\hline \multirow[b]{2}{*}{$\begin{array}{l}\text { Station } \\
\text { no. }\end{array}$} & \multirow[b]{2}{*}{ Station name } & \multirow[b]{2}{*}{ Latitude } & \multirow[b]{2}{*}{ Longitude } & \multirow[b]{2}{*}{ County } & \multirow{2}{*}{$\begin{array}{l}\text { Contri- } \\
\text { buting } \\
\text { drainage } \\
\text { area } \\
\left(\mathbf{m i}^{2}\right)\end{array}$} & \multirow[b]{2}{*}{$\begin{array}{l}\text { Urban } \\
\text { basin }\end{array}$} & \multicolumn{3}{|c|}{ Years of record ${ }^{2}$} & \multicolumn{3}{|c|}{ Begin and end year $^{2}$} \\
\hline & & & & & & & Total & $\begin{array}{c}\text { Unregu- } \\
\text { lated }\end{array}$ & $\begin{array}{l}\text { Regu- } \\
\text { lated }^{3}\end{array}$ & $\begin{array}{c}\text { First } \\
\text { unregu- } \\
\text { lated }\end{array}$ & $\begin{array}{l}\text { First } \\
\text { regu- } \\
\text { lated }\end{array}$ & $\begin{array}{l}\text { Last } \\
\text { year }\end{array}$ \\
\hline \multicolumn{13}{|c|}{ Brazos R Basin-Continued } \\
\hline 08115000 & Big Cr nr Needville, TX & $29^{\circ} 28^{\prime} 35^{\prime \prime}$ & $95^{\circ} 48^{\prime} 45^{\prime \prime}$ & Fort Bend & 42.8 & -- & 50 & 50 & 0 & 1948 & -- & 2000 \\
\hline 08115500 & Fairchild Cr nr Needville, TX & $29^{\circ} 26^{\prime} 45^{\prime \prime}$ & $95^{\circ} 45^{\prime} 41^{\prime \prime}$ & Fort Bend & 26.2 & -- & 7 & 7 & 0 & 1948 & -- & 1954 \\
\hline 08116000 & Big Cr nr Guy, TX & $29^{\circ} 24^{\prime} 45^{\prime \prime}$ & $95^{\circ} 42^{\prime} 36^{\prime \prime}$ & Fort Bend & 116 & -- & 2 & 2 & 0 & 1948 & -- & 1949 \\
\hline 08116400 & Dry Cr nr Rosenburg, TX & $29^{\prime \circ} 30^{\prime} 42^{\prime \prime}$ & $95^{\circ} 44^{\prime} 48^{\prime \prime}$ & Fort Bend & 8.65 & -- & 21 & 21 & 0 & 1959 & -- & 1979 \\
\hline 08116500 & Dry Cr nr Richmond, TX & $29^{\circ} 30^{\prime} 19^{\prime \prime}$ & $95^{\circ} 42^{\prime} 41^{\prime \prime}$ & Fort Bend & 12.2 & -- & 3 & 3 & 0 & 1948 & -- & 1957 \\
\hline 08116650 & Brazos R nr Rosharon, TX & $29^{\circ} 20^{\prime} 58^{\prime \prime}$ & $95^{\circ} 34^{\prime} 56^{\prime \prime}$ & Fort Bend & 35,800 & -- & 29 & 0 & 29 & -- & 1968 & 2000 \\
\hline \multicolumn{13}{|c|}{ San Bernard R Basin (Coastal Basin) } \\
\hline 08117500 & San Bernard R nr Boling, TX & $29^{\circ} 18^{\prime} 48^{\prime \prime}$ & $95^{\circ} 53^{\prime} 37^{\prime \prime}$ & Fort Bend & 727 & -- & 46 & 46 & 0 & 1955 & -- & 2000 \\
\hline \multicolumn{13}{|c|}{ Big Boggy Cr Basin (Coastal Basin) } \\
\hline 08117900 & Big Boggy Cr nr Wadsworth, TX & $28^{\circ} 48^{\prime} 26^{\prime \prime}$ & $95^{\circ} 57^{\prime} 02^{\prime \prime}$ & Matagorda & 10.3 & -- & 7 & 7 & 0 & 1971 & -- & 1977 \\
\hline \multicolumn{13}{|c|}{ Colorado R Basin } \\
\hline 08117995 & Colorado R nr Gail, TX & $32^{\circ} 37^{\prime} 43^{\prime \prime}$ & $101^{\circ} 17^{\prime} 06^{\prime \prime}$ & Borden & 498 & -- & 12 & 12 & 0 & 1989 & -- & 2000 \\
\hline 08118500 & Bull Cr nr Ira, TX & $32^{\circ} 36^{\prime} 00^{\prime \prime}$ & $101^{\circ} 05^{\prime} 38^{\prime \prime}$ & Scurry & 26.3 & -- & 10 & 6 & 4 & 1948 & 1959 & 1962 \\
\hline 08119000 & Bluff Cr nr Ira, TX & $32^{\circ} 35^{\prime} 29^{\prime \prime}$ & $101^{\circ} 03^{\prime} 02^{\prime \prime}$ & Scurry & 42.6 & -- & 18 & 18 & 0 & 1948 & -- & 1965 \\
\hline 08119500 & Colorado R nr Ira, TX & $32^{\circ} 32^{\prime} 18^{\prime \prime}$ & $101^{\circ} 03^{\prime} 12^{\prime \prime}$ & Scurry & 1,110 & -- & 36 & 5 & 31 & 1948 & 1959 & 1989 \\
\hline 08120500 & Deep Cr nr Dunn, TX & $32^{\circ} 34^{\prime} 25^{\prime \prime}$ & $100^{\circ} 54^{\prime} 27^{\prime \prime}$ & Scurry & 188 & -- & 33 & 33 & 0 & 1954 & -- & 1986 \\
\hline 08120700 & Colorado R nr Cuthbert, TX & $32^{\circ} 28^{\prime} 38^{\prime \prime}$ & $100^{\circ} 56^{\prime} 58^{\prime \prime}$ & Mitchell & 1,530 & -- & 35 & 0 & 35 & -- & 1966 & 2000 \\
\hline 08121000 & Colorado R at Colorado City, TX & $32^{\circ} 23^{\prime} 33^{\prime \prime}$ & $100^{\circ} 52^{\prime} 42^{\prime \prime}$ & Mitchell & 1,590 & -- & 54 & 5 & 49 & 1924 & 1952 & 2000 \\
\hline 08121500 & Morgan Cr nr Westbrook, TX & $32^{\circ} 23^{\prime} 42^{\prime \prime}$ & $101^{\circ} 01^{\prime} 32^{\prime \prime}$ & Mitchell & 230 & -- & 9 & 9 & 0 & 1955 & -- & 1963 \\
\hline 08122000 & Graze Cr nr Westbrook, TX & $32^{\circ} 35^{\prime} 03^{\prime \prime}$ & $101^{\circ} 01^{\prime} 10^{\prime \prime}$ & Mitchell & 21.7 & -- & 5 & 5 & 0 & 1955 & -- & 1959 \\
\hline 08122500 & Morgan Cr nr Colorado City, TX & $32^{\circ} 23^{\prime} 17^{\prime \prime}$ & $100^{\circ} 56^{\prime} 59^{\prime \prime}$ & Mitchell & 270 & -- & 1 & 1 & 0 & 1948 & -- & 1948 \\
\hline 08123500 & Champion Cr nr Colorado City, TX & $32^{\circ} 19^{\prime} 01^{\prime \prime}$ & $100^{\circ} 49^{\prime} 28^{\prime \prime}$ & Mitchell & 177 & -- & 12 & 12 & 0 & 1948 & -- & 1959 \\
\hline 08123650 & Beals $\mathrm{Cr}$ above Big Spring, TX & $32^{\circ} 15^{\prime} 01^{\prime \prime}$ & $101^{\circ} 29^{\prime} 26^{\prime \prime}$ & Howard & 1,510 & -- & 20 & 0 & 20 & -- & 1960 & 1979 \\
\hline 08123700 & Beals $\mathrm{Cr}$ at Big Spring, TX & $32^{\circ} 15^{\prime} 45^{\prime \prime}$ & $101^{\circ} 26^{\prime} 30^{\prime \prime}$ & Howard & 1,530 & -- & 1 & 0 & 1 & -- & 1958 & 1958 \\
\hline 08123720 & Beals Cr nr Coahoma, TX & $32^{\circ} 14^{\prime} 56^{\prime \prime}$ & $101^{\circ} 21^{\prime} 42^{\prime \prime}$ & Howard & 1,570 & -- & 4 & 0 & 4 & -- & 1984 & 1987 \\
\hline 08123800 & Beals Cr nr Westbrook, TX & $32^{\circ} 11^{\prime} 57^{\prime \prime}$ & $101^{\circ} 00^{\prime} 49^{\prime \prime}$ & Mitchell & 1,990 & -- & 42 & 0 & 42 & 1959 & -- & 2000 \\
\hline 08123850 & Colorado R above Silver, TX & $32^{\circ} 03^{\prime} 13^{\prime \prime}$ & $100^{\circ} 45^{\prime} 42^{\prime \prime}$ & Coke & 4,650 & -- & 33 & 0 & 33 & -- & 1968 & 2000 \\
\hline 08123900 & Colorado R nr Silver, TX & $32^{\circ} 01^{\prime} 10^{\prime \prime}$ & $100^{\circ} 44^{\prime} 08^{\prime \prime}$ & Coke & 4,740 & -- & 14 & 0 & 14 & -- & 1957 & 1970 \\
\hline 08124000 & Colorado R at Robert Lee, TX & $31^{\circ} 53^{\prime} 07^{\prime \prime}$ & $100^{\circ} 28^{\prime} 49^{\prime \prime}$ & Coke & 5,050 & -- & 51 & 17 & 34 & 1925 & 1953 & 2000 \\
\hline 08126380 & Colorado R nr Ballinger, TX & $31^{\circ} 42^{\prime} 55^{\prime \prime}$ & $100^{\circ} 01^{\prime} 34^{\prime \prime}$ & Runnels & 6,110 & -- & 93 & 61 & 32 & 1908 & 1969 & 2000 \\
\hline 08127000 & Elm Cr at Ballinger, TX & $31^{\circ} 44^{\prime} 57^{\prime \prime}$ & $99^{\circ} 56 ' 51^{\prime \prime}$ & Runnels & 450 & -- & 68 & 49 & 19 & 1933 & 1982 & 2000 \\
\hline 08128000 & South Concho R at Christoval, TX & $31^{\circ} 11^{\prime} 13^{\prime \prime}$ & $100^{\circ} 30^{\prime} 06^{\prime \prime}$ & Tom Green & 354 & -- & 65 & 65 & 0 & 1931 & -- & 1995 \\
\hline 08128400 & Middle Concho R above Tankersley, TX & $31^{\circ} 25^{\prime} 38^{\prime \prime}$ & $100^{\circ} 42^{\prime} 39^{\prime \prime}$ & Irion & 1,610 & -- & 34 & 34 & 0 & 1962 & -- & 1995 \\
\hline
\end{tabular}


Table 2. Inventory of daily mean streamflows for active and discontinued streamflow-gaging stations in Texas-Continued

\begin{tabular}{|c|c|c|c|c|c|c|c|c|c|c|c|c|}
\hline \multirow[b]{2}{*}{$\begin{array}{l}\text { Station } \\
\text { no. }\end{array}$} & \multirow[b]{2}{*}{ Station name } & \multirow[b]{2}{*}{ Latitude } & \multirow[b]{2}{*}{ Longitude } & \multirow[b]{2}{*}{ County } & \multirow{2}{*}{$\begin{array}{l}\text { Contri- } \\
\text { buting } \\
\text { drainage } \\
\text { area } \\
\left(\mathrm{mi}^{2}\right)\end{array}$} & \multirow[b]{2}{*}{$\begin{array}{l}\text { Urban } \\
\text { basin }\end{array}$} & \multicolumn{3}{|c|}{ Years of record $^{2}$} & \multicolumn{3}{|c|}{ Begin and end year ${ }^{2}$} \\
\hline & & & & & & & Total & $\begin{array}{l}\text { Unregu- } \\
\text { lated }\end{array}$ & $\begin{array}{l}\text { Regu- } \\
\text { lated }^{3}\end{array}$ & $\begin{array}{l}\text { First } \\
\text { unregu- } \\
\text { lated }\end{array}$ & $\begin{array}{l}\text { First } \\
\text { regu- } \\
\text { lated }\end{array}$ & $\begin{array}{l}\text { Last } \\
\text { year }\end{array}$ \\
\hline \multicolumn{13}{|c|}{ Colorado R Basin-Continued } \\
\hline 08128500 & Middle Concho R nr Tankersley, TX & $31^{\circ} 22^{\prime} 35^{\prime \prime}$ & $100^{\circ} 36^{\prime} 50^{\prime \prime}$ & Tom Green & 1,690 & -- & 30 & 30 & 0 & 1931 & -- & 1960 \\
\hline 08129300 & Spring Cr above Tankersley, TX & $31^{\circ} 19^{\prime} 48^{\prime \prime}$ & $100^{\circ} 38^{\prime} 24^{\prime \prime}$ & Tom Green & 405 & -- & 35 & 35 & 0 & 1961 & -- & 1995 \\
\hline 08129500 & Dove Cr Spring nr Knickerbocker, TX & $31^{\circ} 11^{\prime} 06^{\prime \prime}$ & $100^{\circ} 43^{\prime} 51^{\prime \prime}$ & Irion & -- & & 15 & 15 & 0 & 1958 & & \\
\hline 08130500 & Dove $\mathrm{Cr}$ at Knickerbocker, TX & $31^{\circ} 16^{\prime} 26^{\prime \prime}$ & $100^{\circ} 37^{\prime} 50^{\prime \prime}$ & Tom Green & 198 & -- & 35 & 35 & 0 & 1961 & -- & 1995 \\
\hline 08131000 & Spring Cr nr Tankersley, TX & $31^{\circ} 21^{\prime} 30^{\prime \prime}$ & $100^{\circ} 32^{\prime} 05^{\prime \prime}$ & Tom Green & 671 & -- & 30 & 30 & 0 & 1931 & -- & 1960 \\
\hline 08131400 & Pecan Cr nr San Angelo, TX & $31^{\circ} 18^{\prime} 32^{\prime \prime}$ & $100^{\circ} 26^{\prime} 44^{\prime \prime}$ & Tom Green & 81.1 & -- & 25 & 25 & 0 & 1962 & -- & 1986 \\
\hline 08132500 & South Concho R at San Angelo, TX & $31^{\circ} 26^{\prime} 45^{\prime \prime}$ & $100^{\circ} 25^{\prime} 30^{\prime \prime}$ & Tom Green & 2,690 & -- & 22 & 0 & 22 & -- & 1932 & 1953 \\
\hline 08133500 & North Concho R at Sterling City, TX & $31^{\circ} 49^{\prime} 48^{\prime \prime}$ & $100^{\circ} 59^{\prime} 36^{\prime \prime}$ & Sterling & 568 & -- & 46 & 46 & 0 & 1940 & -- & 1985 \\
\hline 08134000 & North Concho R nr Carlsbad, TX & $31^{\circ} 35^{\prime} 33^{\prime \prime}$ & $100^{\circ} 38^{\prime} 12^{\prime \prime}$ & Tom Green & 1,190 & -- & 76 & 76 & 0 & 1925 & -- & 2000 \\
\hline 08135000 & North Concho R at San Angelo, TX & $31^{\circ} 27^{\prime} 57^{\prime \prime}$ & $100^{\circ} 26^{\prime} 51^{\prime \prime}$ & Tom Green & 1,450 & -- & 55 & 16 & 39 & 1917 & 1952 & 1990 \\
\hline 08136000 & Concho R at San Angelo, TX & $31^{\circ} 27^{\prime} 16^{\prime \prime}$ & $100^{\circ} 24^{\prime} 37^{\prime \prime}$ & Tom Green & 4,410 & -- & 85 & 15 & 70 & 1916 & 1931 & 2000 \\
\hline 08136500 & Concho R at Painted Rock, TX & $31^{\circ} 30^{\prime} 57^{\prime \prime}$ & $99^{\circ} 55^{\prime} 09^{\prime \prime}$ & Concho & 5,440 & -- & 85 & 15 & 70 & 1916 & 1931 & 2000 \\
\hline 08136700 & Colorado R nr Stacy, TX & $31^{\circ} 29^{\prime} 37^{\prime \prime}$ & $99^{\circ} 34^{\prime} 25^{\prime \prime}$ & McCulloch & 12,800 & -- & 32 & 0 & 32 & -- & 1969 & 2000 \\
\hline 08137500 & Mukewater $\mathrm{Cr}$ at Trickham, TX & $31^{\circ} 35^{\prime} 24^{\prime \prime}$ & $99^{\circ} 13^{\prime} 36^{\prime \prime}$ & Coleman & 70.0 & -- & 22 & 9 & 13 & 1952 & 1961 & 1973 \\
\hline 08138000 & Colorado R at Winchell, TX & $31^{\circ} 28^{\prime} 04^{\prime \prime}$ & $99^{\circ} 09^{\prime} 43^{\prime \prime}$ & Brown & 13,800 & -- & 67 & 22 & 45 & 1925 & 1952 & 2000 \\
\hline 08139500 & Deep Cr nr Mercury, TX & $31^{\circ} 24^{\prime} 08^{\prime \prime}$ & $99^{\circ} 07^{\prime} 17^{\prime \prime}$ & McCulloch & 43.9 & -- & 20 & 0 & 20 & -- & 1954 & 1973 \\
\hline 08140500 & Dry Prong Deep Cr nr Mercury, TX & $31^{\circ} 24^{\prime} 09^{\prime \prime}$ & $99^{\circ} 08^{\prime} 13^{\prime \prime}$ & McCulloch & 8.31 & -- & 20 & 0 & 20 & -- & 1952 & 1971 \\
\hline 08140700 & Pecan Bayou nr Cross Cut, TX & $31^{\circ} 58^{\prime} 21^{\prime \prime}$ & $99^{\circ} 07^{\prime} 48^{\prime \prime}$ & Brown & 532 & -- & 10 & 0 & 10 & -- & 1969 & 1978 \\
\hline 08140800 & Jim Ned Cr nr Coleman, TX & $31^{\circ} 58^{\prime} 59^{\prime \prime}$ & $99^{\circ} 24^{\prime} 52^{\prime \prime}$ & Coleman & 333 & -- & 15 & 0 & 15 & -- & 1966 & 1980 \\
\hline 08141500 & Hords Cr nr Valera, TX & $31^{\circ} 50^{\prime} 03^{\prime \prime}$ & $99^{\circ} 32^{\prime} 04^{\prime \prime}$ & Coleman & 54.2 & -- & 43 & 0 & 43 & -- & 1948 & 1990 \\
\hline 08142000 & Hords Cr nr Coleman, TX & $31^{\circ} 50^{\prime} 50^{\prime \prime}$ & $99^{\circ} 25^{\prime} 25^{\prime \prime}$ & Coleman & 107 & -- & 30 & 7 & 23 & 1941 & 1948 & 1970 \\
\hline 08143500 & Pecan Bayou at Brownwood, TX & $31^{\circ} 43^{\prime} 54^{\prime \prime}$ & $98^{\circ} 58^{\prime} 25^{\prime \prime}$ & Brown & 1,660 & -- & 58 & 6 & 52 & 1925 & 1932 & 1983 \\
\hline 08143600 & Pecan Bayou nr Mullin, TX & $31^{\circ} 31^{\prime} 02^{\prime \prime}$ & $98^{\circ} 44^{\prime} 25^{\prime \prime}$ & Mills & 2,070 & -- & 33 & 0 & 33 & -- & 1968 & 2000 \\
\hline 08144500 & San Saba R at Menard, TX & $30^{\circ} 55^{\prime} 08^{\prime \prime}$ & $99^{\circ} 47^{\prime} 07^{\prime \prime}$ & Menard & 1,130 & -- & 81 & 81 & 0 & 1916 & -- & 2000 \\
\hline 08144600 & San Saba R nr Brady, TX & $31^{\circ} 00^{\prime} 14^{\prime \prime}$ & $99^{\circ} 16^{\prime} 07^{\prime \prime}$ & McCulloch & 1,630 & -- & 17 & 17 & 0 & 1980 & -- & 2000 \\
\hline 08144800 & Brady Cr nr Eden, TX & $31^{\circ} 11^{\prime} 03^{\prime \prime}$ & $99^{\circ} 50^{\prime} 27^{\prime \prime}$ & Concho & 101 & -- & 23 & 0 & 23 & -- & 1963 & 1985 \\
\hline 08145000 & Brady Cr at Brady, TX & $31^{\circ} 08^{\prime} 17^{\prime \prime}$ & $99^{\circ} 20^{\prime} 05^{\prime \prime}$ & McCulloch & 588 & -- & 47 & 15 & 32 & 1940 & 1955 & 1986 \\
\hline 08146000 & San Saba R at San Saba, TX & $31^{\circ} 12^{\prime} 47^{\prime \prime}$ & $98^{\circ} 43^{\prime} 09^{\prime \prime}$ & San Saba & 3,040 & -- & 81 & 47 & 34 & 1916 & 1963 & 2000 \\
\hline 08147000 & Colorado R nr San Saba, TX & $31^{\circ} 13^{\prime} 04^{\prime \prime}$ & $98^{\circ} 33^{\prime} 51^{\prime \prime}$ & San Saba & 19,800 & -- & 82 & 12 & 70 & 1917 & 1931 & 2000 \\
\hline 08148500 & North Llano R nr Junction, TX & $30^{\circ} 31^{\prime} 06^{\prime \prime}$ & $99^{\circ} 48^{\prime} 39^{\prime \prime}$ & Kimble & 914 & -- & 62 & 62 & 0 & 1916 & -- & 1977 \\
\hline 08150000 & Llano R nr Junction, TX & $30^{\circ} 30^{\prime} 15^{\prime \prime}$ & $99^{\circ} 44^{\prime} 03^{\prime \prime}$ & Kimble & 1,850 & -- & 80 & 80 & 0 & 1916 & -- & 2000 \\
\hline 08150700 & Llano R nr Mason, TX & $30^{\circ} 39^{\prime} 38^{\prime \prime}$ & $99^{\circ} 06^{\prime} 32^{\prime \prime}$ & Mason & 3,240 & -- & 27 & 27 & 0 & 1969 & -- & 2000 \\
\hline 08150800 & Beaver Cr nr Mason, TX & $30^{\circ} 38^{\prime} 36^{\prime \prime}$ & $99^{\circ} 05^{\prime} 44^{\prime \prime}$ & Mason & 215 & -- & 37 & 37 & 0 & 1964 & -- & 2000 \\
\hline 08151000 & Llano R nr Castell, TX & $30^{\circ} 43^{\prime} 00^{\prime \prime}$ & $98^{\circ} 53^{\prime} 00^{\prime \prime}$ & Llano & 3,740 & -- & 15 & 15 & 0 & 1925 & -- & 1939 \\
\hline
\end{tabular}

$\stackrel{\omega}{\omega}$ Footnotes at end of table. 
Table 2. Inventory of daily mean streamflows for active and discontinued streamflow-gaging stations in Texas-Continued

\begin{tabular}{|c|c|c|c|c|c|c|c|c|c|c|c|c|}
\hline \multirow[b]{2}{*}{$\begin{array}{c}\text { Station } \\
\text { no. }\end{array}$} & \multirow[b]{2}{*}{ Station name } & \multirow[b]{2}{*}{ Latitude } & \multirow[b]{2}{*}{ Longitude } & \multirow[b]{2}{*}{ County } & \multirow{2}{*}{$\begin{array}{l}\text { Contri- } \\
\text { buting } \\
\text { drainage } \\
\text { area } \\
\left(\mathbf{m i}^{2}\right)\end{array}$} & \multirow[b]{2}{*}{$\begin{array}{l}\text { Urban } \\
\text { basin }\end{array}$} & \multicolumn{3}{|c|}{ Years of record $^{2}$} & \multicolumn{3}{|c|}{ Begin and end year ${ }^{2}$} \\
\hline & & & & & & & Total & $\begin{array}{c}\text { Unregu- } \\
\text { lated }\end{array}$ & $\begin{array}{l}\text { Regu- } \\
\text { lated }^{3}\end{array}$ & $\begin{array}{c}\text { First } \\
\text { unregu- } \\
\text { lated }\end{array}$ & $\begin{array}{l}\text { First } \\
\text { regu- } \\
\text { lated }\end{array}$ & $\begin{array}{l}\text { Last } \\
\text { year }\end{array}$ \\
\hline \multicolumn{13}{|c|}{ Colorado R Basin—Continued } \\
\hline 08151500 & Llano R at Llano, TX & $30^{\circ} 45^{\prime} 04^{\prime \prime}$ & $98^{\circ} 40^{\prime} 10^{\prime \prime}$ & Llano & 4,190 & -- & 61 & 61 & 0 & 1940 & -- & 2000 \\
\hline 08152000 & Sandy Cr nr Kingsland, TX & $30^{\circ} 33^{\prime} 27^{\prime \prime}$ & $98^{\circ} 28^{\prime} 19^{\prime \prime}$ & Llano & 346 & -- & 29 & 29 & 0 & 1967 & -- & 2000 \\
\hline 08152900 & Pedernales R nr Fredericksburg, TX & $30^{\circ} 13^{\prime} 13^{\prime \prime}$ & $98^{\circ} 52^{\prime} 10^{\prime \prime}$ & Gillespie & 369 & -- & 16 & 16 & 0 & 1980 & -- & 2000 \\
\hline 08153000 & Pedernales R at Stonewall, TX & $30^{\circ} 15^{\prime} 00^{\prime \prime}$ & $98^{\circ} 40^{\prime} 00^{\prime \prime}$ & Gillespie & 647 & -- & 10 & 10 & 0 & 1925 & -- & 1934 \\
\hline 08153500 & Pedernales R nr Johnson City, TX & $30^{\circ} 17^{\prime} 30^{\prime \prime}$ & $98^{\circ} 23^{\prime} 57^{\prime \prime}$ & Blanco & 901 & -- & 61 & 61 & 0 & 1940 & -- & 2000 \\
\hline 08154000 & Pedernales R nr Spicewood, TX & $30^{\circ} 25^{\prime} 15^{\prime \prime}$ & $98^{\circ} 04^{\prime} 50^{\prime \prime}$ & Travis & 1,290 & -- & 14 & 14 & 0 & 1925 & -- & 1938 \\
\hline 08154510 & Colorado R below Mansfield Dam, Austin, TX & $30^{\circ} 23^{\prime} 30^{\prime \prime}$ & $97^{\circ} 54^{\prime} 28^{\prime \prime}$ & Travis & 27,400 & -- & 16 & 0 & 16 & -- & 1975 & 1990 \\
\hline 08154700 & Bull Cr at Loop $360 \mathrm{nr}$ Austin, TX & $30^{\circ} 22^{\prime} 19^{\prime \prime}$ & $97^{\circ} 47^{\prime} 04^{\prime \prime}$ & Travis & 22.3 & -- & 22 & 22 & 0 & 1979 & -- & 2000 \\
\hline 08155200 & Barton Cr at SH $71 \mathrm{nr}$ Oak Hill, TX & $30^{\circ} 17^{\prime} 46^{\prime \prime}$ & $97^{\circ} 55^{\prime} 31^{\prime \prime}$ & Travis & 89.7 & -- & 15 & 15 & 0 & 1979 & -- & 2000 \\
\hline 08155240 & Barton $\mathrm{Cr}$ at Lost Cr Blvd nr Austin, TX & $30^{\circ} 16^{\prime} 26^{\prime \prime}$ & $97^{\circ} 50^{\prime} 40^{\prime \prime}$ & Travis & 107 & -- & 11 & 11 & 0 & 1990 & -- & 2000 \\
\hline 08155260 & Barton Cr nr Camp Craft Rd nr Austin, TX & $30^{\circ} 16^{\prime} 12^{\prime \prime}$ & $97^{\circ} 49^{\prime} 43^{\prime \prime}$ & Travis & 109 & -- & 6 & 6 & 0 & 1983 & -- & 1988 \\
\hline 08155300 & Barton Cr at Loop 360, Austin, TX & $30^{\circ} 14^{\prime} 40^{\prime \prime}$ & $97^{\circ} 48^{\prime} 07^{\prime \prime}$ & Travis & 116 & -- & 23 & 23 & 0 & 1978 & -- & 2000 \\
\hline 08155500 & Barton Springs at Austin, TX & $30^{\circ} 15^{\prime} 48^{\prime \prime}$ & $97^{\circ} 46^{\prime} 16^{\prime \prime}$ & Travis & -- & Yes & 23 & 23 & 0 & 1979 & -- & 2000 \\
\hline 08156700 & Shoal Cr at Northwest Park at Austin, TX & $30^{\circ} 20^{\prime} 50^{\prime \prime}$ & $97^{\circ} 44^{\prime} 41^{\prime \prime}$ & Travis & 6.52 & Yes & 9 & 9 & 0 & 1976 & -- & 1984 \\
\hline 08156800 & Shoal Cr at West 12th St, Austin, TX & $30^{\circ} 16^{\prime} 35^{\prime \prime}$ & $97^{\circ} 45^{\prime} 00^{\prime \prime}$ & Travis & 12.3 & Yes & 16 & 16 & 0 & 1985 & -- & 2000 \\
\hline 08157000 & Waller Cr at 38th St, Austin, TX & $30^{\circ} 17^{\prime} 49^{\prime \prime}$ & $97^{\circ} 43^{\prime} 36^{\prime \prime}$ & Travis & 2.31 & Yes & 25 & 25 & 0 & 1956 & -- & 1980 \\
\hline 08157500 & Waller Cr at $23 \mathrm{~d} \mathrm{St}$, Austin, TX & $30^{\circ} 17^{\prime} 08^{\prime \prime}$ & $97^{\circ} 44^{\prime} 01^{\prime \prime}$ & Travis & 4.13 & Yes & 25 & 25 & 0 & 1956 & -- & 1980 \\
\hline 08158000 & Colorado R at Austin, TX & $30^{\circ} 14^{\prime} 40^{\prime \prime}$ & $97^{\circ} 41^{\prime} 39^{\prime \prime}$ & Travis & 27,600 & -- & 102 & 38 & 64 & 1899 & 1937 & 2000 \\
\hline 08158050 & Boggy Cr at US 183, Austin, TX & $30^{\circ} 15^{\prime} 47^{\prime \prime}$ & $97^{\circ} 40^{\prime} 20^{\prime \prime}$ & Travis & 13.1 & Yes & 15 & 15 & 0 & 1978 & -- & 2000 \\
\hline 08158600 & Walnut $\mathrm{Cr}$ at Webberville Rd, Austin, TX & $30^{\circ} 16^{\prime} 59^{\prime \prime}$ & $97^{\circ} 39^{\prime} 17^{\prime \prime}$ & Travis & 51.3 & Yes & 34 & 34 & 0 & 1967 & -- & 2000 \\
\hline 08158700 & Onion Cr nr Driftwood, TX & $30^{\circ} 04^{\prime} 58^{\prime \prime}$ & $98^{\circ} 00^{\prime} 27^{\prime \prime}$ & Hays & 124 & -- & 21 & 21 & 0 & 1980 & -- & 2000 \\
\hline 08158800 & Onion $\mathrm{Cr}$ at Buda, $\mathrm{TX}$ & $30^{\circ} 05^{\prime} 09^{\prime \prime}$ & $97^{\circ} 50^{\prime} 52^{\prime \prime}$ & Hays & 166 & -- & 7 & 7 & 0 & 1980 & -- & 1995 \\
\hline 08158810 & Bear Cr below FM 1826 nr Driftwood, TX & $30^{\circ} 09^{\prime} 19^{\prime \prime}$ & $97^{\circ} 56^{\prime} 23^{\prime \prime}$ & Hays & 12.2 & -- & 21 & 21 & 0 & 1980 & -- & 2000 \\
\hline 08158840 & Slaughter Cr at FM $1826 \mathrm{nr}$ Austin, TX & $30^{\circ} 12^{\prime} 32^{\prime \prime}$ & $97^{\circ} 54^{\prime} 11^{\prime \prime}$ & Travis & 8.24 & -- & 22 & 22 & 0 & 1979 & -- & 2000 \\
\hline 08158920 & Williamson Cr at Oak Hill, TX & $30^{\circ} 14^{\prime} 06^{\prime \prime}$ & $97^{\circ} 51^{\prime} 36^{\prime \prime}$ & Travis & 6.30 & Yes & 14 & 14 & 0 & 1979 & -- & 1992 \\
\hline 08158922 & Williamson Cr at Brushy Country Blvd, Oak Hill, TX & $30^{\circ} 13 ' 34^{\prime \prime}$ & $97^{\circ} 50^{\prime} 28^{\prime \prime}$ & Travis & 6.79 & -- & 7 & 7 & 0 & 1994 & -- & 2000 \\
\hline 08158970 & Williamson Cr at Jimmy Clay Rd, Austin, TX & $30^{\circ} 11^{\prime} 21^{\prime \prime}$ & $97^{\circ} 43^{\prime} 56^{\prime \prime}$ & Travis & 27.6 & Yes & 11 & 11 & 0 & 1976 & -- & 1986 \\
\hline 08159000 & Onion $\mathrm{Cr}$ at US 183, Austin, TX & $30^{\circ} 10^{\prime} 40^{\prime \prime}$ & $97^{\circ} 41^{\prime} 18^{\prime \prime}$ & Travis & 321 & Yes & 29 & 29 & 0 & 1925 & -- & 2000 \\
\hline 08159150 & Wilbarger Cr nr Pflugerville, TX & $30^{\circ} 27^{\prime} 16^{\prime \prime}$ & $97^{\circ} 36^{\prime} 02^{\prime \prime}$ & Travis & 4.61 & -- & 17 & 17 & 0 & 1964 & -- & 1980 \\
\hline 08159165 & Big Sandy Cr nr McDade, TX & $30^{\circ} 18^{\prime} 18^{\prime \prime}$ & $97^{\circ} 17^{\prime} 48^{\prime \prime}$ & Bastrop & 38.7 & -- & 6 & 6 & 0 & 1980 & -- & 1985 \\
\hline 08159170 & Big Sandy Cr nr Elgin, TX & $30^{\circ} 15^{\prime} 54^{\prime \prime}$ & $97^{\circ} 19^{\prime} 39^{\prime \prime}$ & Bastrop & 63.8 & -- & 6 & 6 & 0 & 1980 & -- & 1985 \\
\hline 08159200 & Colorado R at Bastrop, TX & $30^{\circ} 06^{\prime} 16^{\prime \prime}$ & $97^{\circ} 19^{\prime} 09^{\prime \prime}$ & Bastrop & 28,600 & -- & 40 & 0 & 40 & -- & 1961 & 2000 \\
\hline 08159500 & Colorado R at Smithville, TX & $30^{\circ} 00^{\prime} 45^{\prime \prime}$ & $97^{\circ} 09^{\prime} 42^{\prime \prime}$ & Bastrop & 29,100 & -- & 48 & 6 & 42 & 1931 & 1937 & 2000 \\
\hline 08160000 & Dry $\mathrm{Cr}$ at Buescher Lake nr Smithville, TX & $30^{\circ} 02^{\prime} 32^{\prime \prime}$ & $97^{\circ} 09^{\prime} 34^{\prime \prime}$ & Bastrop & 1.48 & -- & 27 & 27 & 0 & 1940 & -- & 1966 \\
\hline
\end{tabular}


Table 2. Inventory of daily mean streamflows for active and discontinued streamflow-gaging stations in Texas-Continued

\begin{tabular}{|c|c|c|c|c|c|c|c|c|c|c|c|c|}
\hline \multirow[b]{2}{*}{$\begin{array}{l}\text { Station } \\
\text { no. }\end{array}$} & \multirow[b]{2}{*}{ Station name } & \multirow[b]{2}{*}{ Latitude } & \multirow[b]{2}{*}{ Longitude } & \multirow[b]{2}{*}{ County } & \multirow{2}{*}{$\begin{array}{l}\text { Contri- } \\
\text { buting } \\
\text { drainage } \\
\text { area } \\
\left(\mathbf{m i}^{2}\right)\end{array}$} & \multirow[b]{2}{*}{$\begin{array}{l}\text { Urban } \\
\text { basin }\end{array}$} & \multicolumn{3}{|c|}{ Years of record $^{2}$} & \multicolumn{3}{|c|}{ Begin and end year ${ }^{2}$} \\
\hline & & & & & & & Total & $\begin{array}{c}\text { Unregu- } \\
\text { lated }\end{array}$ & $\begin{array}{l}\text { Regu- } \\
\text { lated }^{3}\end{array}$ & $\begin{array}{l}\text { First } \\
\text { unregu- } \\
\text { lated }\end{array}$ & $\begin{array}{l}\text { First } \\
\text { regu- } \\
\text { lated }\end{array}$ & $\begin{array}{c}\text { Last } \\
\text { year }\end{array}$ \\
\hline \multicolumn{13}{|c|}{ Colorado R Basin—Continued } \\
\hline 08160400 & Colorado R above La Grange, TX & $29^{\circ} 54^{\prime} 44^{\prime \prime}$ & $96^{\circ} 54^{\prime} 13^{\prime \prime}$ & Fayette & 29,500 & -- & 12 & 0 & 12 & -- & 1989 & 2000 \\
\hline 08160500 & Colorado R at La Grange, TX & $29^{\circ} 53^{\prime} 45^{\prime \prime}$ & $96^{\circ} 52^{\prime} 15^{\prime \prime}$ & Fayette & 29,700 & -- & 16 & 0 & 16 & -- & 1940 & 1955 \\
\hline 08160700 & Colorado R above Columbus, TX & $29^{\circ} 43^{\prime} 09^{\prime \prime}$ & $96^{\circ} 34^{\prime} 16^{\prime \prime}$ & Colorado & 29,900 & -- & 2 & 0 & 2 & -- & 1984 & 1985 \\
\hline 08160800 & Redgate $\mathrm{Cr}$ nr Columbus, TX & $29^{\circ} 47^{\prime} 56^{\prime \prime}$ & $96^{\circ} 31^{\prime} 55^{\prime \prime}$ & Colorado & 17.3 & -- & 38 & 38 & 0 & 1963 & -- & 2000 \\
\hline 08161000 & Colorado R at Columbus, TX & $29^{\circ} 42^{\prime} 22^{\prime \prime}$ & $96^{\circ} 32^{\prime} 12^{\prime \prime}$ & Colorado & 30,200 & -- & 84 & 20 & 64 & 1917 & 1937 & 2000 \\
\hline 08162000 & Colorado R at Wharton, TX & $29^{\circ} 18^{\prime} 32^{\prime \prime}$ & $96^{\circ} 06^{\prime} 13^{\prime \prime}$ & Wharton & 30,600 & -- & 62 & 0 & 62 & -- & 1939 & 2000 \\
\hline 08162500 & Colorado R nr Bay City, TX & $28^{\circ} 58^{\prime} 26^{\prime \prime}$ & $96^{\circ} 00^{\prime} 44^{\prime \prime}$ & Matagorda & 30,800 & -- & 52 & 0 & 52 & -- & -- & 2000 \\
\hline \multicolumn{13}{|c|}{ Tres Palacios R Basin (Coastal Basin) } \\
\hline 08162600 & Tres Palacios R nr Midfield, TX & $28^{\circ} 55^{\prime} 40^{\prime \prime}$ & $96^{\circ} 10^{\prime} 15^{\prime \prime}$ & Matagorda & 145 & -- & 30 & 30 & 0 & 1971 & -- & 2000 \\
\hline \multicolumn{13}{|c|}{ Lavaca R Basin } \\
\hline 08163500 & Lavaca R at Hallettsville, TX & $29^{\circ} 26^{\prime} 35^{\prime \prime}$ & $96^{\circ} 56^{\prime} 41^{\prime \prime}$ & Lavaca & 108 & -- & 53 & 53 & 0 & 1940 & -- & 1992 \\
\hline 08164000 & Lavaca R nr Edna, TX & $28^{\circ} 57^{\prime} 35^{\prime \prime}$ & $96^{\circ} 41^{\prime} 10^{\prime \prime}$ & Jackson & 817 & -- & 62 & 62 & 0 & 1939 & -- & 2000 \\
\hline 08164300 & Navidad R nr Hallettsville, TX & $29^{\circ} 28^{\prime} 00^{\prime \prime}$ & $96^{\circ} 48^{\prime} 45^{\prime \prime}$ & Lavaca & 332 & -- & 39 & 39 & 0 & 1962 & -- & 2000 \\
\hline 08164350 & Navidad R nr Speaks, TX & $29^{\circ} 19^{\prime} 18^{\prime \prime}$ & $96^{\circ} 42^{\prime} 32^{\prime \prime}$ & Lavaca & 437 & -- & 12 & 12 & 0 & 1982 & -- & 2000 \\
\hline 08164370 & Navidad R at Morales, TX & $29^{\circ} 08^{\prime} 07^{\prime \prime}$ & $96^{\circ} 44^{\prime} 39^{\prime \prime}$ & Jackson & 549 & -- & 4 & 4 & 0 & 1997 & -- & 2000 \\
\hline 08164390 & Navidad R at Strane Park nr Edna, TX & $29^{\circ} 03^{\prime} 55^{\prime \prime}$ & $96^{\circ} 40^{\prime} 26^{\prime \prime}$ & Jackson & 549 & -- & 4 & 4 & 0 & 1997 & -- & 2000 \\
\hline 08164450 & Sandy Cr nr Ganado, TX & $29^{\circ} 09^{\prime} 36^{\prime \prime}$ & $96^{\circ} 32^{\prime} 46^{\prime \prime}$ & Jackson & 289 & -- & 23 & 23 & 0 & 1978 & -- & 2000 \\
\hline 08164500 & Navidad R nr Ganado, TX & $29^{\circ} 01^{\prime} 32^{\prime \prime}$ & $96^{\circ} 33^{\prime} 08^{\prime \prime}$ & Jackson & 826 & -- & 40 & 40 & 0 & 1940 & -- & 1979 \\
\hline 08164503 & West Mustang Cr nr Ganado, TX & $29^{\circ} 04^{\prime} 17^{\prime \prime}$ & $96^{\circ} 28^{\prime} 01^{\prime \prime}$ & Jackson & 178 & -- & 22 & 22 & 0 & 1978 & -- & 2000 \\
\hline 08164504 & East Mustang Cr nr Louise, TX & $29^{\circ} 04^{\prime} 14^{\prime \prime}$ & $96^{\circ} 25^{\prime} 01^{\prime \prime}$ & Wharton & 90.8 & -- & 4 & 4 & 0 & 1997 & -- & 2000 \\
\hline \multicolumn{13}{|c|}{ Garcitas Cr Basin (Coastal Basin) } \\
\hline 08164600 & Garcitas Cr nr Inez, TX & $28^{\circ} 53^{\prime} 28^{\prime \prime}$ & $96^{\circ} 49^{\prime} 08^{\prime \prime}$ & Victoria & 91.7 & -- & 30 & 30 & 0 & 1971 & -- & 2000 \\
\hline \multicolumn{13}{|c|}{ Placedo Cr Basin (Coastal Basin) } \\
\hline 08164800 & Placedo Cr nr Placedo, TX & $28^{\circ} 43^{\prime} 30^{\prime \prime}$ & $96^{\circ} 46^{\prime} 07^{\prime \prime}$ & Victoria & 68.3 & -- & 30 & 30 & 0 & 1971 & -- & 2000 \\
\hline \multicolumn{13}{|c|}{ Guadalupe R Basin } \\
\hline 08165300 & North Fk Guadalupe R nr Hunt, TX & $30^{\circ} 03^{\prime} 50^{\prime \prime}$ & $99^{\circ} 23^{\prime} 12^{\prime \prime}$ & Kerr & 168 & -- & 33 & 33 & 0 & 1968 & -- & 2000 \\
\hline 08165500 & Guadalupe R at Hunt, TX & $30^{\circ} 04^{\prime} 11^{\prime \prime}$ & $99^{\circ} 19^{\prime} 17^{\prime \prime}$ & Kerr & 288 & -- & 35 & 35 & 0 & 1966 & -- & 2000 \\
\hline 08166000 & Johnson Cr nr Ingram, TX & $30^{\circ} 06^{\prime} 00^{\prime \prime}$ & $99^{\circ} 16^{\prime} 58^{\prime \prime}$ & Kerr & 114 & -- & 50 & 50 & 0 & 1942 & -- & 1993 \\
\hline 08166140 & Guadalupe $\mathrm{R}$ above Bear $\mathrm{Cr}$ at Kerrville, $\mathrm{TX}$ & $30^{\circ} 04^{\prime} 10^{\prime \prime}$ & $99^{\circ} 11^{\prime} 42^{\prime \prime}$ & Kerr & 494 & -- & 7 & 7 & 0 & 1979 & -- & 1985 \\
\hline 08166200 & Guadalupe R at Kerrville, TX & $30^{\circ} 03^{\prime} 11^{\prime \prime}$ & $99^{\circ} 09^{\prime} 47^{\prime \prime}$ & Kerr & 510 & -- & 14 & 14 & 0 & 1987 & -- & 2000 \\
\hline 08166500 & Guadalupe R nr Comfort, TX & $29^{\circ} 56^{\prime} 57^{\prime \prime}$ & $98^{\circ} 53^{\prime} 32^{\prime \prime}$ & Kerr & 762 & -- & 13 & 13 & 0 & 1920 & -- & 1932 \\
\hline 08167000 & Guadalupe R at Comfort, TX & $29^{\circ} 58^{\prime} 10^{\prime \prime}$ & $98^{\circ} 53^{\prime} 33^{\prime \prime}$ & Kendall & 839 & -- & 61 & 61 & 0 & 1940 & -- & 2000 \\
\hline 08167500 & Guadalupe R nr Spring Branch, TX & $29^{\circ} 51^{\prime} 37^{\prime \prime}$ & $98^{\circ} 23^{\prime} 00^{\prime \prime}$ & Comal & 1,320 & -- & 78 & 78 & 0 & 1923 & -- & 2000 \\
\hline
\end{tabular}

$\boldsymbol{\Theta} \quad$ Footnotes at end of table. 
Table 2. Inventory of daily mean streamflows for active and discontinued streamflow-gaging stations in Texas-Continued

\begin{tabular}{|c|c|c|c|c|c|c|c|c|c|c|c|c|}
\hline \multirow[b]{2}{*}{$\begin{array}{c}\text { Station } \\
\text { no. }\end{array}$} & \multirow[b]{2}{*}{ Station name } & \multirow[b]{2}{*}{ Latitude } & \multirow[b]{2}{*}{ Longitude } & \multirow[b]{2}{*}{ County } & \multirow{2}{*}{$\begin{array}{l}\text { Contri- } \\
\text { buting } \\
\text { drainage } \\
\text { area } \\
\left(\mathbf{m i}^{2}\right)\end{array}$} & \multirow[b]{2}{*}{$\begin{array}{l}\text { Urban } \\
\text { basin }\end{array}$} & \multicolumn{3}{|c|}{ Years of record ${ }^{2}$} & \multicolumn{3}{|c|}{ Begin and end year $^{2}$} \\
\hline & & & & & & & Total & $\begin{array}{l}\text { Unregu- } \\
\text { lated }\end{array}$ & $\begin{array}{l}\text { Regu- } \\
\text { lated }^{3}\end{array}$ & $\begin{array}{c}\text { First } \\
\text { unregu- } \\
\text { lated }\end{array}$ & $\begin{array}{l}\text { First } \\
\text { regu- } \\
\text { lated }\end{array}$ & $\begin{array}{l}\text { Last } \\
\text { year }\end{array}$ \\
\hline \multicolumn{13}{|c|}{ Guadalupe R Basin-Continued } \\
\hline 08167600 & Rebecca Cr nr Spring Branch, TX & $29^{\circ} 55^{\prime} 06^{\prime \prime}$ & $98^{\circ} 22^{\prime} 10^{\prime \prime}$ & Comal & 10.9 & -- & 18 & 13 & 5 & 1961 & 1974 & 1978 \\
\hline 08167800 & Guadalupe R at Sattler, TX & $29^{\circ} 51^{\prime} 32^{\prime \prime}$ & $98^{\circ} 10^{\prime} 47^{\prime \prime}$ & Comal & 1,440 & -- & 40 & 2 & 38 & 1961 & 1963 & 2000 \\
\hline 08168500 & Guadalupe R above Comal R at New Braunfels, TX & $29^{\circ} 42^{\prime} 53^{\prime \prime}$ & $98^{\circ} 06^{\prime} 35^{\prime \prime}$ & Comal & 1,520 & -- & 72 & 34 & 38 & 1929 & 1963 & 2000 \\
\hline 08168710 & Comal Springs at New Braunfels, TX & $29^{\circ} 42^{\prime} 21^{\prime \prime}$ & $98^{\circ} 07^{\prime} 20^{\prime \prime}$ & Comal & -- & -- & 72 & 72 & 0 & 1929 & -- & 2000 \\
\hline 08169000 & Comal R at New Braunfels, TX & $29^{\circ} 42^{\prime} 21^{\prime \prime}$ & $98^{\circ} 07^{\prime} 20^{\prime \prime}$ & Comal & 130 & Yes & 72 & 45 & 27 & 1929 & 1974 & 2000 \\
\hline 08169500 & Guadalupe R at New Braunfels, TX & $29^{\circ} 41^{\prime} 52^{\prime \prime}$ & $98^{\circ} 06^{\prime 2} 23^{\prime \prime}$ & Comal & 1,650 & -- & 13 & 13 & 0 & 1916 & -- & 2000 \\
\hline 08170000 & San Marcos R springflow at San Marcos, TX & $29^{\circ} 53^{\prime} 20^{\prime \prime}$ & $97^{\circ} 56^{\prime} 02^{\prime \prime}$ & Hays & -- & Yes & 44 & 44 & 0 & 1957 & -- & 2000 \\
\hline 08170500 & San Marcos R at San Marcos, TX & $29^{\circ} 53^{\prime} 20^{\prime \prime}$ & $97^{\circ} 56^{\prime} 02^{\prime \prime}$ & Hays & 47 & Yes & 6 & 6 & 0 & 1995 & -- & 2000 \\
\hline 08171000 & Blanco R at Wimberley, TX & $29^{\circ} 59^{\prime} 39^{\prime \prime}$ & $98^{\circ} 05^{\prime} 19^{\prime \prime}$ & Hays & 355 & -- & 73 & 73 & 0 & 1925 & -- & 2000 \\
\hline 08171300 & Blanco R nr Kyle, TX & $29^{\circ} 58^{\prime} 45^{\prime \prime}$ & $97^{\circ} 54^{\prime} 35^{\prime \prime}$ & Hays & 412 & -- & 44 & 44 & 0 & 1957 & -- & 2000 \\
\hline 08172000 & San Marcos R at Luling, TX & $29^{\circ} 39^{\prime} 58^{\prime \prime}$ & $97^{\circ} 39^{\prime} 02^{\prime \prime}$ & Caldwell & 838 & -- & 61 & 44 & 17 & 1940 & 1984 & 2000 \\
\hline 08172400 & Plum Cr at Lockhart, TX & $29^{\circ} 55^{\prime} 22^{\prime \prime}$ & $97^{\circ} 40^{\prime} 44^{\prime \prime}$ & Caldwell & 112 & -- & 41 & 4 & 37 & 1960 & 1964 & 2000 \\
\hline 08172500 & Plum Cr nr Lockhart, TX & $29^{\circ} 49^{\prime} 17^{\prime \prime}$ & $97^{\circ} 35^{\prime} 02^{\prime \prime}$ & Caldwell & 184 & -- & 4 & 4 & 0 & 1926 & -- & 1929 \\
\hline 08173000 & Plum Cr nr Luling, TX & $29^{\circ} 41^{\prime} 58^{\prime \prime}$ & $97^{\circ} 36^{\prime} 12^{\prime \prime}$ & Caldwell & 309 & -- & 63 & 33 & 30 & 1931 & 1964 & 1993 \\
\hline 08173500 & San Marcos R at Ottine, TX & $29^{\circ} 35^{\prime} 36^{\prime \prime}$ & $97^{\circ} 35^{\prime} 22^{\prime \prime}$ & Gonzales & 1,250 & -- & 27 & 27 & 0 & 1916 & -- & 1942 \\
\hline 08173900 & Guadalupe R at Gonzales, TX & $29^{\circ} 29^{\prime} 03^{\prime \prime}$ & $97^{\circ} 27^{\prime} 00^{\prime \prime}$ & Gonzales & 3,490 & -- & 4 & 4 & 0 & 1997 & -- & 2000 \\
\hline 08174600 & Peach Cr below Dilworth, TX & $29^{\circ} 28^{\prime} 26^{\prime \prime}$ & $97^{\circ} 18^{\prime} 59^{\prime \prime}$ & Gonzales & 460 & -- & 20 & 20 & 0 & 1960 & -- & 1979 \\
\hline 08175000 & Sandies Cr nr Westhoff, TX & $29^{\circ} 12^{\prime} 54^{\prime \prime}$ & $97^{\circ} 26^{\prime} 57^{\prime \prime}$ & De Witt & 549 & -- & 45 & 45 & 0 & 1931 & -- & 2000 \\
\hline 08175800 & Guadalupe R at Cuero, TX & $29^{\circ} 03^{\prime} 57^{\prime \prime}$ & $97^{\circ} 19^{\prime} 16^{\prime \prime}$ & De Witt & 4,930 & -- & 36 & 0 & 36 & -- & 1965 & 2000 \\
\hline 08176000 & Guadalupe R below Cuero, TX & $29^{\circ} 03^{\prime} 05^{\prime \prime}$ & $97^{\circ} 15^{\prime} 52^{\prime \prime}$ & De Witt & 4,920 & -- & 18 & 18 & 0 & 1906 & -- & 1935 \\
\hline 08176500 & Guadalupe R at Victoria, TX & $28^{\circ} 47^{\prime} 34^{\prime \prime}$ & $97^{\circ} 00^{\prime} 46^{\prime \prime}$ & Victoria & 5,200 & -- & 65 & 26 & 39 & 1936 & 1962 & 2000 \\
\hline 08176550 & Fifteenmile Cr nr Weser, TX & $28^{\circ} 53^{\prime} 51^{\prime \prime}$ & $97^{\circ} 21^{\prime} 17^{\prime \prime}$ & De Witt & 167 & -- & 5 & 5 & 0 & 1985 & -- & 1989 \\
\hline 08176900 & Coleto $\mathrm{Cr}$ at Arnold Rd nr Schroeder, TX & $28^{\circ} 51^{\prime} 41^{\prime \prime}$ & $97^{\circ} 13^{\prime} 34^{\prime \prime}$ & Goliad & 357 & -- & 18 & 18 & 0 & 1979 & -- & 1996 \\
\hline 08176990 & Coleto Cr Reservoir inflow nr Schroeder, TX & $28^{\circ} 50^{\prime} 21^{\prime \prime}$ & $97^{\circ} 11^{\prime} 20^{\prime \prime}$ & Victoria & 354 & -- & 13 & 0 & 13 & -- & 1981 & 1993 \\
\hline 08177000 & Coleto Cr nr Schroeder, TX & $28^{\circ} 49^{\prime} 53^{\prime \prime}$ & $97^{\circ} 11^{\prime} 10^{\prime \prime}$ & Victoria & 369 & -- & 30 & 30 & 0 & 1931 & -- & 1979 \\
\hline 08177300 & Perdido $\mathrm{Cr}$ at FM $622 \mathrm{nr}$ Fannin, TX & $28^{\circ} 45^{\prime} 05^{\prime \prime}$ & $97^{\circ} 19^{\prime} 01^{\prime \prime}$ & Goliad & 28.0 & -- & 13 & 13 & 0 & 1979 & -- & 1991 \\
\hline 08177500 & Coleto Cr nr Victoria, TX & $28^{\circ} 43^{\prime} 51^{\prime \prime}$ & $97^{\circ} 08^{\prime} 18^{\prime \prime}$ & Victoria & 514 & -- & 37 & 17 & 20 & 1940 & 1981 & 2000 \\
\hline 08177520 & Guadalupe R nr Bloomington, TX & $28^{\circ} 39^{\prime} 42^{\prime \prime}$ & $96^{\circ} 57^{\prime} 54^{\prime \prime}$ & Victoria & 5,816 & -- & 1 & 0 & 1 & -- & 2000 & 2000 \\
\hline \multicolumn{13}{|c|}{ San Antonio R Basin } \\
\hline 08177700 & Olmos Cr at Desden Dr, San Antonio, TX & $29^{\circ} 29^{\prime} 56^{\prime \prime}$ & $98^{\circ} 30^{\prime} 36^{\prime \prime}$ & Bexar & 21.2 & Yes & 13 & 13 & 0 & 1969 & -- & 1981 \\
\hline 08178000 & San Antonio R at San Antonio, TX & $29^{\circ} 24^{\prime} 34^{\prime \prime}$ & $98^{\circ} 29^{\prime} 41^{\prime \prime}$ & Bexar & 41.8 & Yes & 71 & 9 & 62 & 1916 & 1926 & 2000 \\
\hline 08178050 & San Antonio R at Mitchell St, San Antonio, & $29^{\circ} 23^{\prime} 34^{\prime \prime}$ & $98^{\circ} 29^{\prime} 40^{\prime \prime}$ & Bexar & 42.4 & Yes & 8 & 0 & 8 & -- & 1993 & 2000 \\
\hline 08178500 & San Pedro Cr at Furnish St, San Antonio, TX & $29^{\circ} 24^{\prime} 22^{\prime \prime}$ & $98^{\circ} 30^{\prime} 38^{\prime \prime}$ & Bexar & 2.64 & -- & 12 & 12 & 0 & 1917 & -- & 1929 \\
\hline 08178565 & San Antonio R at Loop 410 at San Antonio, TX & $29^{\circ} 19^{\prime} 19^{\prime \prime}$ & $98^{\circ} 27^{\prime} 00^{\prime \prime}$ & Bexar & -- & Yes & 14 & 0 & 14 & -- & 1987 & 2000 \\
\hline
\end{tabular}


Table 2. Inventory of daily mean streamflows for active and discontinued streamflow-gaging stations in Texas-Continued

\begin{tabular}{|c|c|c|c|c|c|c|c|c|c|c|c|c|}
\hline \multirow[b]{2}{*}{$\begin{array}{c}\text { Station } \\
\text { no. }\end{array}$} & \multirow[b]{2}{*}{ Station name } & \multirow[b]{2}{*}{ Latitude } & \multirow[b]{2}{*}{ Longitude } & \multirow[b]{2}{*}{ County } & \multirow{2}{*}{$\begin{array}{l}\text { Contri- } \\
\text { buting } \\
\text { drainage } \\
\text { area } \\
\left(\mathrm{mi}^{2}\right)\end{array}$} & \multirow[b]{2}{*}{$\begin{array}{l}\text { Urban } \\
\text { basin }\end{array}$} & \multicolumn{3}{|c|}{ Years of record $^{2}$} & \multicolumn{3}{|c|}{ Begin and end year ${ }^{2}$} \\
\hline & & & & & & & Total & $\begin{array}{l}\text { Unregu- } \\
\text { lated }\end{array}$ & $\begin{array}{l}\text { Regu- } \\
\text { lated }^{3}\end{array}$ & $\begin{array}{c}\text { First } \\
\text { unregu- } \\
\text { lated }\end{array}$ & $\begin{array}{l}\text { First } \\
\text { regu- } \\
\text { lated }\end{array}$ & $\begin{array}{l}\text { Last } \\
\text { year }\end{array}$ \\
\hline \multicolumn{13}{|c|}{ San Antonio R Basin-Continued } \\
\hline 08178585 & Salado Cr at Wilderness Rd at San Antonio, TX & $29^{\circ} 37^{\prime} 50^{\prime \prime}$ & $98^{\circ} 33^{\prime} 55^{\prime \prime}$ & Bexar & 23 & -- & 3 & 0 & 3 & -- & 1998 & 2000 \\
\hline 08178700 & Salado Cr (Upper Station) at San Antonio, TX & $29^{\circ} 30^{\prime} 57^{\prime \prime}$ & $98^{\circ} 25^{\prime} 51^{\prime \prime}$ & Bexar & 137 & Yes & 40 & 12 & 28 & 1961 & 1973 & 2000 \\
\hline 08178800 & Salado Cr (Lower Station) at San Antonio, TX & $29^{\circ} 21^{\prime} 25^{\prime \prime}$ & $98^{\circ} 24^{\prime} 45^{\prime \prime}$ & Bexar & 189 & Yes & 40 & 12 & 28 & 1961 & 1973 & 2000 \\
\hline 08178880 & Medina $\mathrm{R}$ at Bandera, TX & $29^{\circ} 43^{\prime} 25^{\prime \prime}$ & $99^{\circ} 04^{\prime} 11^{\prime \prime}$ & Bandera & 427 & -- & 18 & 18 & 0 & 1983 & -- & 2000 \\
\hline 08179000 & Medina R nr Pipe Cr, TX & $29^{\circ} 40^{\prime} 31^{\prime \prime}$ & $98^{\circ} 58^{\prime} 33^{\prime \prime}$ & Bandera & 474 & -- & 41 & 41 & 0 & 1924 & -- & 1982 \\
\hline 08179100 & Red Bluff Cr nr Pipe Cr, TX & $29^{\circ} 40^{\prime} 51^{\prime \prime}$ & $98^{\circ} 57^{\prime} 19^{\prime \prime}$ & Bandera & 56.3 & -- & 25 & 25 & 0 & 1957 & -- & 1981 \\
\hline 08180500 & Medina R nr Riomedina, TX & $29^{\circ} 29^{\prime} 53^{\prime \prime}$ & $98^{\circ} 54^{\prime} 16^{\prime \prime}$ & Medina & 650 & -- & 32 & 0 & 32 & -- & 1923 & 1973 \\
\hline 08180640 & Medina $\mathrm{R}$ at La Coste, TX & $29^{\circ} 19^{\prime} 26^{\prime \prime}$ & $98^{\circ} 48^{\prime} 46^{\prime \prime}$ & Medina & 805 & -- & 13 & 0 & 13 & -- & 1988 & 2000 \\
\hline 08180700 & Medina R nr MacDona, TX & $29^{\circ} 20^{\prime} 05^{\prime \prime}$ & $98^{\circ} 41^{\prime 2} 22^{\prime \prime}$ & Bexar & 885 & -- & 17 & 0 & 17 & -- & 1982 & 2000 \\
\hline 08180750 & Medio $\mathrm{Cr}$ at Pearsall Rd at San Antonio, TX & $29^{\circ} 19^{\prime} 40^{\prime \prime}$ & $98^{\circ} 38^{\prime} 19^{\prime \prime}$ & Bexar & 47.9 & -- & 8 & 8 & 0 & 1988 & -- & 1995 \\
\hline 08180800 & Medina R nr Somerset, TX & $29^{\circ} 15^{\prime} 43^{\prime \prime}$ & $98^{\circ} 34^{\prime} 52^{\prime \prime}$ & Bexar & 967 & -- & 28 & 0 & 28 & -- & 1971 & 2000 \\
\hline 08181400 & Helotes $\mathrm{Cr}$ at Helotes, $\mathrm{TX}$ & $29^{\circ} 34^{\prime} 42^{\prime \prime}$ & $98^{\circ} 41^{\prime 2} 29^{\prime \prime}$ & Bexar & 15.0 & -- & 32 & 32 & 0 & 1969 & -- & 2000 \\
\hline 08181410 & Ranch Cr nr Helotes, TX & $29^{\circ} 36^{\prime} 06^{\prime \prime}$ & $98^{\circ} 43^{\prime} 26^{\prime \prime}$ & Bexar & .39 & -- & 1 & 1 & 0 & 1978 & -- & 1978 \\
\hline 08181450 & Leon Cr trib at Kelly Air Force Base, TX & $29^{\circ} 23^{\prime} 12^{\prime \prime}$ & $98^{\circ} 36^{\prime} 00^{\prime \prime}$ & Bexar & 1.19 & Yes & 10 & 10 & 0 & 1970 & -- & 1979 \\
\hline 08181480 & Leon $\mathrm{Cr}$ at IH-35 at San Antonio, TX & $29^{\circ} 19^{\prime} 47^{\prime \prime}$ & $98^{\circ} 35^{\prime} 02^{\prime \prime}$ & Bexar & 219 & Yes & 16 & 16 & 0 & 1985 & -- & 2000 \\
\hline 08181500 & Medina R at San Antonio, TX & $29^{\circ} 15^{\prime} 14^{\prime \prime}$ & $98^{\circ} 28^{\prime} 20^{\prime \prime}$ & Bexar & 1,320 & -- & 61 & 0 & 61 & -- & 1940 & 2000 \\
\hline 08181800 & San Antonio R nr Elmendorf, TX & $29^{\circ} 13^{\prime} 19^{\prime \prime}$ & $98^{\circ} 21^{\prime} 20^{\prime \prime}$ & Bexar & 1,740 & -- & 38 & 0 & 38 & -- & 1963 & 2000 \\
\hline 08182500 & Calaveras Cr nr Elemendorf, TX & $29^{\circ} 15^{\prime} 38^{\prime \prime}$ & $98^{\circ} 17^{\prime} 34^{\prime \prime}$ & Bexar & 77.2 & -- & 17 & 0 & 17 & -- & 1955 & 1971 \\
\hline 08183000 & San Antonio R at Calaveras, TX & $29^{\circ} 12^{\prime} 54^{\prime \prime}$ & $98^{\circ} 15^{\prime} 39^{\prime \prime}$ & Wilson & 1,790 & -- & 6 & 0 & 6 & -- & 1919 & 1924 \\
\hline 08183500 & San Antonio R nr Falls City, TX & $28^{\circ} 57^{\prime} 05^{\prime \prime}$ & $98^{\circ} 03^{\prime} 50^{\prime \prime}$ & Karnes & 2,110 & -- & 75 & 0 & 75 & -- & 1926 & 2000 \\
\hline 08183850 & Cibolo $\mathrm{Cr}$ at $\mathrm{IH}-10$ above Boerne, TX & $29^{\circ} 48^{\prime} 52^{\prime \prime}$ & $98^{\circ} 45^{\prime} 12^{\prime \prime}$ & Kendall & 29 & -- & 5 & 0 & 5 & -- & 1996 & 2000 \\
\hline 08183900 & Cibolo Cr nr Boerne, TX & $29^{\circ} 46^{\prime} 26^{\prime \prime}$ & $98^{\circ} 41^{\prime} 50^{\prime \prime}$ & Kendall & 68.4 & -- & 33 & 15 & 18 & 1963 & 1978 & 1995 \\
\hline 08184000 & Cibolo Cr nr Bulverde, TX & $29^{\circ} 43^{\prime} 33^{\prime \prime}$ & $98^{\circ} 25^{\prime} 37^{\prime \prime}$ & Comal & 198 & -- & 19 & 19 & 0 & 1947 & -- & 1965 \\
\hline 08185000 & Cibolo Cr at Selma, TX & $29^{\circ} 35^{\prime} 38^{\prime \prime}$ & $98^{\circ} 18^{\prime} 39^{\prime \prime}$ & Bexar & 274 & -- & 54 & 33 & 21 & 1947 & 1980 & 2000 \\
\hline 08185500 & Cibolo Cr at Sutherland Springs, TX & $29^{\circ} 16^{\prime} 47^{\prime \prime}$ & $98^{\circ} 03^{\prime} 10^{\prime \prime}$ & Wilson & 665 & -- & 4 & 4 & 0 & 1925 & -- & 1928 \\
\hline 08186000 & Cibolo Cr nr Falls City, TX & $29^{\circ} 00^{\prime} 50^{\prime \prime}$ & $97^{\circ} 55^{\prime} 48^{\prime \prime}$ & Karnes & 827 & -- & 70 & 70 & 0 & 1931 & -- & 2000 \\
\hline 08186500 & Ecleto Cr nr Runge, TX & $28^{\circ} 55^{\prime} 12^{\prime \prime}$ & $97^{\circ} 46^{\prime} 19^{\prime \prime}$ & Karnes & 239 & -- & 27 & 27 & 0 & 1963 & -- & 1989 \\
\hline 08187500 & Escondido $\mathrm{Cr}$ at Kenedy, TX & $28^{\circ} 49^{\prime} 11^{\prime \prime}$ & $97^{\circ} 51^{\prime} 32^{\prime \prime}$ & Karnes & 72.4 & -- & 19 & 0 & 19 & -- & 1955 & 1973 \\
\hline 08188000 & Dry Escondido Cr nr Kenedy, TX & $28^{\circ} 51^{\prime} 41^{\prime \prime}$ & $97^{\circ} 50^{\prime} 14^{\prime \prime}$ & Karnes & 9.43 & -- & 5 & 0 & 5 & -- & 1955 & 1959 \\
\hline 08188500 & San Antonio R at Goliad, TX & $28^{\circ} 38^{\prime} 58^{\prime \prime}$ & $97^{\circ} 23^{\prime} 04^{\prime \prime}$ & Goliad & 3,920 & -- & 65 & 0 & 65 & -- & 1925 & 2000 \\
\hline \multicolumn{13}{|c|}{ Copano Cr Basin (Coastal Basin) } \\
\hline 08189200 & Copano Cr nr Refugio, TX & $28^{\circ} 18^{\prime} 12^{\prime \prime}$ & $97^{\circ} 06^{\prime} 44^{\prime \prime}$ & Refugio & 87.8 & -- & 30 & 30 & 0 & 1971 & -- & 2000 \\
\hline
\end{tabular}

$\pm \quad$ Footnotes at end of table. 
Table 2. Inventory of daily mean streamflows for active and discontinued streamflow-gaging stations in Texas-Continued

\begin{tabular}{|c|c|c|c|c|c|c|c|c|c|c|c|c|}
\hline \multirow[b]{2}{*}{$\begin{array}{c}\text { Station } \\
\text { no. }\end{array}$} & \multirow[b]{2}{*}{ Station name } & \multirow[b]{2}{*}{ Latitude } & \multirow[b]{2}{*}{ Longitude } & \multirow[b]{2}{*}{ County } & \multirow{2}{*}{$\begin{array}{l}\text { Contri- } \\
\text { buting } \\
\text { drainage } \\
\text { area } \\
\left(\mathrm{mi}^{2}\right)\end{array}$} & \multirow[b]{2}{*}{$\begin{array}{l}\text { Urban } \\
\text { basin }\end{array}$} & \multicolumn{3}{|c|}{ Years of record $^{2}$} & \multicolumn{3}{|c|}{ Begin and end year $^{2}$} \\
\hline & & & & & & & Total & $\begin{array}{c}\text { Unregu- } \\
\text { lated }\end{array}$ & $\begin{array}{l}\text { Regu- } \\
\text { lated }^{3}\end{array}$ & $\begin{array}{c}\text { First } \\
\text { unregu- } \\
\text { lated }\end{array}$ & $\begin{array}{l}\text { First } \\
\text { regu- } \\
\text { lated }\end{array}$ & $\begin{array}{l}\text { Last } \\
\text { year }\end{array}$ \\
\hline \multicolumn{13}{|c|}{ Mission R Basin } \\
\hline 08189300 & Medio Cr nr Beeville, TX & $28^{\circ} 28^{\prime} 58^{\prime \prime}$ & $97^{\circ} 39^{\prime} 23^{\prime \prime}$ & Bee & 204 & -- & 15 & 15 & 0 & 1963 & -- & 1977 \\
\hline 08189500 & Misson $\mathrm{R}$ at Refugio, $\mathrm{TX}$ & $28^{\circ} 17^{\prime} 30^{\prime \prime}$ & $97^{\circ} 16^{\prime} 44^{\prime \prime}$ & Refugio & 690 & -- & 61 & 61 & 0 & 1940 & -- & 2000 \\
\hline \multicolumn{13}{|c|}{ Aransas R Basin } \\
\hline 08189700 & Aransas R nr Skidmore, TX & $28^{\circ} 28^{\prime} 58^{\prime \prime}$ & $97^{\circ} 39^{\prime} 23^{\prime \prime}$ & Bee & 247 & -- & 36 & 36 & 0 & 1965 & -- & 2000 \\
\hline 08189800 & Chiltipin $\mathrm{Cr}$ at Sinton, TX & $28^{\circ} 17^{\prime} 30^{\prime \prime}$ & $97^{\circ} 16^{\prime} 44^{\prime \prime}$ & San Patricio & 128 & -- & 21 & 21 & 0 & 1971 & -- & 1991 \\
\hline \multicolumn{13}{|c|}{ Nueces R Basin } \\
\hline 08190000 & Nueces $R$ at Laguna, TX & $29^{\circ} 25^{\prime} 42^{\prime \prime}$ & $99^{\circ} 59^{\prime} 49^{\prime \prime}$ & Uvalde & 737 & -- & 77 & 77 & 0 & 1924 & -- & 2000 \\
\hline 08190500 & West Nueces R nr Brackettville, TX & $29^{\circ} 28^{\prime} 21^{\prime \prime}$ & $100^{\circ} 14^{\prime} 10^{\prime \prime}$ & Kinney & 694 & -- & 55 & 55 & 0 & 1940 & -- & 2000 \\
\hline 08192000 & Nueces R below Uvalde, TX & $29^{\circ} 07^{\prime} 25^{\prime \prime}$ & $99^{\circ} 53^{\prime} 40^{\prime \prime}$ & Uvalde & 1,860 & -- & 73 & 73 & 0 & 1928 & -- & 2000 \\
\hline 08192500 & Nueces R nr Cinonia, TX & $28^{\circ} 47^{\prime} 00^{\prime \prime}$ & $99^{\circ} 50^{\prime} 00^{\prime \prime}$ & Zavala & 2,150 & -- & 7 & 7 & 0 & 1916 & -- & 1924 \\
\hline 08193000 & Nueces R nr Asherton, TX & $28^{\circ} 30^{\prime} 00^{\prime \prime}$ & $99^{\circ} 40^{\prime} 54^{\prime \prime}$ & Dimmit & 4,080 & -- & 61 & 9 & 52 & 1940 & 1949 & 2000 \\
\hline 08194000 & Nueces R at Cotulla, TX & $28^{\circ} 25^{\prime} 34^{\prime \prime}$ & $99^{\circ} 14^{\prime} 23^{\prime \prime}$ & La Salle & 5,170 & -- & 74 & 22 & 52 & 1927 & 1949 & 2000 \\
\hline 08194200 & San Casimiro Cr nr Freer, TX & $27^{\circ} 57^{\prime} 53^{\prime \prime}$ & $98^{\circ} 58^{\prime} 00^{\prime \prime}$ & Webb & 469 & -- & 38 & 38 & 0 & 1963 & -- & 2000 \\
\hline 08194500 & Nueces R nr Tilden, TX & $28^{\circ} 18^{\prime} 31^{\prime \prime}$ & $98^{\circ} 33^{\prime} 25^{\prime \prime}$ & McMullen & 8,090 & -- & 57 & 5 & 52 & 1944 & 1949 & 2000 \\
\hline 08194600 & Nueces R at Simmons, TX & $28^{\circ} 25^{\prime} 16^{\prime \prime}$ & $98^{\circ} 17^{\prime} 03^{\prime \prime}$ & Live Oak & 8,560 & -- & 12 & 12 & 0 & 1966 & -- & 1977 \\
\hline 08195000 & Frio $\mathrm{R}$ at Concan, $\mathrm{TX}$ & $29^{\circ} 29^{\prime} 18^{\prime \prime}$ & $99^{\circ} 42^{\prime} 16^{\prime \prime}$ & Uvalde & 389 & -- & 75 & 75 & 0 & 1925 & -- & 2000 \\
\hline 08196000 & Dry Frio R nr Reagan Wells, TX & $29^{\circ} 30^{\prime} 16^{\prime \prime}$ & $99^{\circ} 466^{\prime \prime}$ & Uvalde & 126 & -- & 48 & 48 & 0 & 1953 & -- & 2000 \\
\hline 08197500 & Frio R below Dry Frio R nr Uvalde, TX & $29^{\circ} 14^{\prime} 44^{\prime \prime}$ & $99^{\circ} 40^{\prime} 27^{\prime \prime}$ & Uvalde & 631 & -- & 48 & 48 & 0 & 1953 & -- & 2000 \\
\hline 08198000 & Sabinal R nr Sabinal, TX & $29^{\circ} 29^{\prime} 27^{\prime \prime}$ & $99^{\circ} 29^{\prime} 33^{\prime \prime}$ & Uvalde & 206 & -- & 58 & 58 & 0 & 1943 & -- & 2000 \\
\hline 08198500 & Sabinal R at Sabinal, TX & $29^{\circ} 18^{\prime} 05^{\prime \prime}$ & $99^{\circ} 28^{\prime} 46^{\prime \prime}$ & Uvalde & 241 & -- & 48 & 48 & 0 & 1953 & -- & 200 \\
\hline 08200000 & Hondo Cr nr Tarpley, TX & $29^{\circ} 34^{\prime} 10^{\prime \prime}$ & $99^{\circ} 14^{\prime} 47^{\prime \prime}$ & Medina & 95.6 & -- & 48 & 48 & 0 & 1953 & -- & 2000 \\
\hline 08200500 & Hondo $\mathrm{Cr}$ nr Hondo, TX & $29^{\circ} 27^{\prime} 05^{\prime \prime}$ & $99^{\circ} 11^{\prime} 07^{\prime \prime}$ & Medina & 132 & -- & 12 & 12 & 0 & 1953 & -- & 1964 \\
\hline 08200700 & Hondo Cr at King Waterhole nr Hondo, TX & $29^{\circ} 23^{\prime} 26^{\prime \prime}$ & $99^{\circ} 09^{\prime} 04^{\prime \prime}$ & Medina & 149 & -- & 40 & 40 & 0 & 1961 & -- & 2000 \\
\hline 08201500 & Seco Cr at Miller Ranch nr Utopia, TX & $29^{\circ} 34^{\prime} 23^{\prime \prime}$ & $99^{\circ} 24^{\prime} 10^{\prime \prime}$ & Medina & 45.0 & -- & 39 & 39 & 0 & 1962 & -- & 2000 \\
\hline 08202000 & Seco Cr nr Utopia, TX & $29^{\circ} 33^{\prime} 01^{\prime \prime}$ & $99^{\circ} 24^{\prime} 22^{\prime \prime}$ & Medina & 53.2 & -- & 9 & 9 & 0 & 1953 & -- & 1961 \\
\hline 08202450 & Seco Cr Reservoir inflow nr Utopia, TX & $29^{\circ} 31^{\prime} 34^{\prime \prime}$ & $99^{\circ} 23^{\prime} 42^{\prime \prime}$ & Medina & 59.5 & -- & 7 & 7 & 0 & 1992 & -- & 1998 \\
\hline 08202490 & Seco Cr Reservoir outflow nr Utopia, TX & $29^{\circ} 30^{\prime} 58^{\prime \prime}$ & $99^{\circ} 23^{\prime} 51^{\prime \prime}$ & Medina & 60.5 & -- & 5 & 5 & 0 & 1992 & -- & 1996 \\
\hline 08202500 & Seco Cr nr DíHanis, TX & $29^{\circ} 29^{\prime} 20^{\prime \prime}$ & $99^{\circ} 23^{\prime} 16^{\prime \prime}$ & Medina & 87.4 & -- & 12 & 12 & 0 & 1953 & -- & 1964 \\
\hline 08202700 & Seco Cr at Rowe Ranch nr DíHanis, TX & $29^{\circ} 21^{\prime} 43^{\prime \prime}$ & $99^{\circ} 17^{\prime} 05^{\prime \prime}$ & Medina & 168 & -- & 39 & 39 & 0 & 1962 & -- & 2000 \\
\hline 08202790 & Parkers Cr Reservoir inflow nr DíHanis, TX & $29^{\circ} 27^{\prime} 27^{\prime \prime}$ & $99^{\circ} 15^{\prime} 16^{\prime \prime}$ & Medina & 9.34 & -- & 5 & 5 & 0 & 1992 & -- & 1996 \\
\hline 08202810 & Parkers Cr Reservoir outflow nr DíHanis, TX & $29^{\circ} 26^{\prime} 38^{\prime \prime}$ & $99^{\circ} 15^{\prime} 09^{\prime \prime}$ & Medina & 10.0 & -- & 5 & 0 & 5 & -- & 1992 & 1996 \\
\hline 08202900 & Seco Cr nr Yancey, TX & $29^{\circ} 08^{\prime} 03^{\prime \prime}$ & $99^{\circ} 12^{\prime} 19^{\prime \prime}$ & Medina & 253 & -- & 5 & 5 & 0 & 1992 & -- & 1996 \\
\hline 08204000 & Leona R springflow nr Uvalde, TX & $29^{\circ} 09^{\prime} 15^{\prime \prime}$ & $99^{\circ} 44^{\prime} 35^{\prime \prime}$ & Uvalde & -- & -- & 26 & 26 & 0 & 1940 & -- & 1965 \\
\hline 08204500 & Leona R nr Divot, TX & $28^{\circ} 47^{\prime} 34^{\prime \prime}$ & $99^{\circ} 14^{\prime} 27^{\prime \prime}$ & Frio & 565 & -- & 3 & 3 & 0 & 1925 & -- & 1928 \\
\hline
\end{tabular}


Table 2. Inventory of daily mean streamflows for active and discontinued streamflow-gaging stations in Texas-Continued

\begin{tabular}{|c|c|c|c|c|c|c|c|c|c|c|c|c|}
\hline \multirow[b]{2}{*}{$\begin{array}{l}\text { Station } \\
\text { no. }\end{array}$} & \multirow[b]{2}{*}{ Station name } & \multirow[b]{2}{*}{ Latitude } & \multirow[b]{2}{*}{ Longitude } & \multirow[b]{2}{*}{ County } & \multirow{2}{*}{$\begin{array}{l}\text { Contri- } \\
\text { buting } \\
\text { drainage } \\
\text { area } \\
\left(\mathrm{mi}^{2}\right)\end{array}$} & \multirow[b]{2}{*}{$\begin{array}{l}\text { Urban } \\
\text { basin }\end{array}$} & \multicolumn{3}{|c|}{ Years of record $^{2}$} & \multicolumn{3}{|c|}{ Begin and end year ${ }^{2}$} \\
\hline & & & & & & & Total & $\begin{array}{l}\text { Unregu- } \\
\text { lated }\end{array}$ & $\begin{array}{l}\text { Regu- } \\
\text { lated }^{3}\end{array}$ & $\begin{array}{c}\text { First } \\
\text { unregu- } \\
\text { lated }\end{array}$ & $\begin{array}{l}\text { First } \\
\text { regu- } \\
\text { lated }\end{array}$ & $\begin{array}{c}\text { Last } \\
\text { year }\end{array}$ \\
\hline \multicolumn{13}{|c|}{ Nueces R Basin-Continued } \\
\hline 08205500 & Frio R nr Derby, TX & $28^{\circ} 44^{\prime} 11^{\prime \prime}$ & $99^{\circ} 08^{\prime} 40^{\prime \prime}$ & Frio & 3,430 & -- & 85 & 85 & 0 & 1916 & -- & 2000 \\
\hline 08206600 & Frio $\mathrm{R}$ at Tilden, TX & $28^{\circ} 28^{\prime} 02^{\prime \prime}$ & $98^{\circ} 32^{\prime} 50^{\prime \prime}$ & McMullen & 4,490 & -- & 22 & 22 & 0 & 1979 & -- & 2000 \\
\hline 08206700 & San Miguel Cr nr Tilden, TX & $28^{\circ} 35^{\prime} 14^{\prime \prime}$ & $98^{\circ} 32^{\prime} 44^{\prime \prime}$ & McMullen & 783 & -- & 36 & 36 & 0 & 1965 & -- & 2000 \\
\hline 08207000 & Frio $\mathrm{R}$ at Calliham, TX & $28^{\circ} 29^{\prime} 31^{\prime \prime}$ & $98^{\circ} 20^{\prime} 47^{\prime \prime}$ & McMullen & 5,490 & -- & 49 & 49 & 0 & 1925 & -- & 1980 \\
\hline 08208000 & Atascosa R at Whitsett, TX & $28^{\circ} 37^{\prime} 19^{\prime \prime}$ & $98^{\circ} 16^{\prime} 52^{\prime \prime}$ & Live Oak & 1,170 & -- & 68 & 68 & 0 & 1933 & -- & 2000 \\
\hline 08210000 & Nueces R nr Three Rivers, TX & $28^{\circ} 25^{\prime} 38^{\prime \prime}$ & $98^{\circ} 10^{\prime} 40^{\prime \prime}$ & Live Oak & 15,400 & -- & 85 & 67 & 18 & 1916 & 1983 & 2000 \\
\hline 08210300 & Ramirena Cr nr George West, TX & $28^{\circ} 08^{\prime} 30^{\prime \prime}$ & $98^{\circ} 06^{\prime} 11^{\prime \prime}$ & Live Oak & 84.4 & -- & 3 & 3 & 0 & 1969 & -- & 1971 \\
\hline 08210400 & Lagarto Cr nr George West, TX & $28^{\circ} 03^{\prime} 34^{\prime \prime}$ & $98^{\circ} 05^{\prime} 48^{\prime \prime}$ & Live Oak & 155 & -- & 17 & 17 & 0 & 1973 & -- & 1989 \\
\hline 08211000 & Nueces R nr Mathis, TX & $28^{\circ} 02^{\prime} 17^{\prime \prime}$ & $97^{\circ} 51^{\prime} 36^{\prime \prime}$ & San Patricio & 16,700 & -- & 61 & 0 & 61 & -- & 1940 & 2000 \\
\hline \multicolumn{13}{|c|}{ Oso Cr Basin (Coastal Basin) } \\
\hline 08211520 & Oso $\mathrm{Cr}$ at Corpus Christi, TX & $27^{\circ} 42^{\prime} 40^{\prime \prime}$ & $97^{\circ} 30^{\prime} 06^{\prime \prime}$ & Nueces & 90.3 & -- & 28 & 28 & 0 & 1973 & -- & 2000 \\
\hline \multicolumn{13}{|c|}{ San Fernando $\mathrm{Cr}$ Basin } \\
\hline 08211800 & San Diego Cr at Alice, TX & $27^{\circ} 45^{\prime} 59^{\prime \prime}$ & $98^{\circ} 04^{\prime} 31^{\prime \prime}$ & Jim Wells & 319 & -- & 26 & 0 & 26 & -- & 1964 & 1989 \\
\hline 08211900 & San Fernando Cr at Alice, TX & $27^{\circ} 46^{\prime} 20^{\prime \prime}$ & $98^{\circ} 02^{\prime} 00^{\prime \prime}$ & Jim Wells & 507 & -- & 23 & 0 & 23 & -- & 1966 & 2000 \\
\hline 08212000 & San Fernando Cr nr Alice, TX & $27^{\circ} 43^{\prime} 30^{\prime \prime}$ & $97^{\circ} 59^{\prime} 15^{\prime \prime}$ & Jim Wells & 518 & -- & 1 & 1 & 0 & 1963 & -- & 1963 \\
\hline 08212400 & Los Olmos Cr nr Falfurrias, TX & $27^{\circ} 15^{\prime} 51^{\prime \prime}$ & $98^{\circ} 08^{\prime} 08^{\prime \prime}$ & Brooks & 476 & -- & 18 & 18 & 0 & 1968 & -- & 2000 \\
\hline 08212500 & Upper Madero Canal nr Kingsville, TX & $27^{\circ} 33^{\prime} 28^{\prime \prime}$ & $97^{\circ} 43^{\prime} 25^{\prime \prime}$ & Kleberg & 40 & -- & 4 & 4 & 0 & 1996 & 0 & 1999 \\
\hline 08212600 & Chiltipin Canal nr Kingsville, TX & $27^{\circ} 33^{\prime} 33^{\prime \prime}$ & $97^{\circ} 38^{\prime} 26^{\prime \prime}$ & Kleberg & 35 & -- & 4 & 4 & 0 & 1996 & 0 & 1999 \\
\hline 08212700 & Pinto Cr Canal nr Kingsville, TX & $27^{\circ} 28^{\prime} 57^{\prime \prime}$ & $97^{\circ} 43^{\prime} 29^{\prime \prime}$ & Kleberg & 44 & -- & 4 & 4 & 0 & 1996 & 0 & 1999 \\
\hline 08212800 & Lower Madero Canal nr Kingsville, TX & $27^{\circ} 27^{\prime} 55^{\prime \prime}$ & $97^{\circ} 40^{\prime} 02^{\prime \prime}$ & Kleberg & 38 & -- & 4 & 4 & 0 & 1996 & 0 & 1999 \\
\hline 08212900 & Tunas Cr nr Kingsville, TX & $27^{\circ} 28^{\prime} 23^{\prime \prime}$ & $97^{\circ} 34^{\prime} 42^{\prime \prime}$ & Kleberg & 10 & -- & 4 & 4 & 0 & 1996 & 0 & 1999 \\
\hline \multicolumn{13}{|c|}{ Rio Grande Basin } \\
\hline 08363840 & Rio Grande at Vinton Bridge nr Anthony, TX & $31^{\circ} 57^{\prime} 32^{\prime \prime}$ & $106^{\circ} 36^{\prime} 17^{\prime \prime}$ & El Paso & 28,700 & -- & 4 & 0 & 4 & -- & 1971 & 1974 \\
\hline 08365600 & McKelligon Canyon at El Paso, TX & $31^{\circ} 49^{\prime} 20^{\prime \prime}$ & $106^{\circ} 28^{\prime} 09^{\prime \prime}$ & El Paso & 2.30 & -- & 20 & 0 & 20 & -- & 1958 & 1977 \\
\hline 08365800 & Government Ditch at El Paso, TX & $31^{\circ} 47^{\prime} 02^{\prime \prime}$ & $106^{\circ} 26^{\prime} 41^{\prime \prime}$ & El Paso & 6.40 & Yes & 19 & 19 & 0 & 1959 & -- & 1977 \\
\hline 08373200 & Cibolo Cr nr Presidio, TX & $29^{\circ} 34^{\prime} 50^{\prime \prime}$ & $104^{\circ} 21^{\prime} 55^{\prime \prime}$ & Presidio & 276 & -- & 6 & 6 & 0 & 1972 & -- & 1977 \\
\hline 08376300 & Sanderson Canyon at Sanderson, TX & $30^{\circ} 07^{\prime} 46^{\prime \prime}$ & $102^{\circ} 23^{\prime} 06^{\prime \prime}$ & Terrell & 195 & -- & 12 & 12 & 0 & 1969 & -- & 1980 \\
\hline 08407500 & Pecos R at Red Bluff, NM & $32^{\circ} 04^{\prime} 30^{\prime \prime}$ & $104^{\circ} 02^{\prime} 21^{\prime \prime}$ & Eddy & 19,500 & -- & 63 & 0 & 63 & -- & 1938 & 2000 \\
\hline 08408500 & Delaware R nr Red Bluff, NM & $32^{\circ} 01 ' 23^{\prime \prime}$ & $104^{\circ} 03^{\prime} 15^{\prime \prime}$ & Eddy & 689 & -- & 63 & 63 & 0 & 1938 & -- & 2000 \\
\hline 08409500 & Pecos R nr Angeles, TX & $32^{\circ} 02^{\prime} 00^{\prime \prime}$ & $104^{\circ} 00^{\prime} 00^{\prime \prime}$ & Reeves & 20,500 & -- & 23 & 23 & 0 & -- & 1915 & 1937 \\
\hline 08411500 & Salt Screwbean Draw nr Orla, TX & $31^{\circ} 52^{\prime} 40^{\prime \prime}$ & $103^{\circ} 56^{\prime} 50^{\prime \prime}$ & Reeves & 464 & -- & 15 & 15 & 0 & 1940 & -- & 1957 \\
\hline 08412500 & Pecos R nr Orla, TX & $31^{\circ} 52^{\prime} 21^{\prime \prime}$ & $103^{\circ} 49^{\prime} 52^{\prime \prime}$ & Reeves & 21,200 & -- & 59 & 0 & 59 & -- & 1938 & 1996 \\
\hline 08414000 & Pecos R nr Mentone, TX & $31^{\circ} 40^{\prime} 07^{\prime \prime}$ & $103^{\circ} 37^{\prime} 34^{\prime \prime}$ & Reeves & 21,700 & -- & 6 & 3 & 3 & 1923 & 1970 & 1972 \\
\hline
\end{tabular}

ఉ Footnotes at end of table. 
Table 2. Inventory of daily mean streamflows for active and discontinued streamflow-gaging stations in Texas-Continued

\begin{tabular}{|c|c|c|c|c|c|c|c|c|c|c|c|c|}
\hline \multirow[b]{2}{*}{$\begin{array}{c}\text { Station } \\
\text { no. }\end{array}$} & \multirow[b]{2}{*}{ Station name } & \multirow[b]{2}{*}{ Latitude } & \multirow[b]{2}{*}{ Longitude } & \multirow[b]{2}{*}{ County } & \multirow{2}{*}{$\begin{array}{l}\text { Contri- } \\
\text { buting } \\
\text { drainage } \\
\text { area } \\
\left(\mathrm{mi}^{2}\right)\end{array}$} & \multirow[b]{2}{*}{$\begin{array}{l}\text { Urban } \\
\text { basin }\end{array}$} & \multicolumn{3}{|c|}{ Years of record $^{2}$} & \multicolumn{3}{|c|}{ Begin and end year ${ }^{2}$} \\
\hline & & & & & & & Total & $\begin{array}{c}\text { Unregu- } \\
\text { lated }\end{array}$ & $\begin{array}{l}\text { Regu- } \\
\text { lated }^{3}\end{array}$ & $\begin{array}{c}\text { First } \\
\text { unregu- } \\
\text { lated }\end{array}$ & $\begin{array}{l}\text { First } \\
\text { regu- } \\
\text { lated }\end{array}$ & $\begin{array}{l}\text { Last } \\
\text { year }\end{array}$ \\
\hline \multicolumn{13}{|c|}{ Rio Grande Basin—Continued } \\
\hline 08416500 & Pecos R above Barstow, TX & $31^{\circ} 35^{\prime} 00^{\prime \prime}$ & $103^{\circ} 30^{\prime} 00^{\prime \prime}$ & Reeves & 21,600 & -- & 4 & 4 & 0 & 1917 & -- & 1920 \\
\hline 08420500 & Pecos R at Pecos, TX & $31^{\circ} 26^{\prime} 11^{\prime \prime}$ & $103^{\circ} 28^{\prime} 01^{\prime \prime}$ & Reeves & 22,100 & -- & 21 & 0 & 21 & -- & 1900 & 1954 \\
\hline 08424500 & Madera Canyon nr Toyahvale, TX & $30^{\circ} 52^{\prime} 04^{\prime \prime}$ & $103^{\circ} 58^{\prime} 09^{\prime \prime}$ & Jeff Davis & 53.8 & -- & 17 & 17 & 0 & 1933 & -- & 1949 \\
\hline 08425500 & Phantom Lake Springs nr Toyahvale, TX & $30^{\circ} 56^{\prime} 01^{\prime \prime}$ & $103^{\circ} 50^{\prime} 43^{\prime \prime}$ & Jeff Davis & -- & -- & 25 & 25 & 0 & 1933 & -- & 1966 \\
\hline 08427000 & Giffin Springs at Toyahvale, TX & $30^{\circ} 56^{\prime} 51^{\prime \prime}$ & $103^{\circ} 47^{\prime} 19^{\prime \prime}$ & Reeves & -- & -- & 2 & 2 & 0 & 1932 & -- & 1933 \\
\hline 08427500 & San Solomon Springs at Toyahvale, TX & $30^{\circ} 56^{\prime} 34^{\prime \prime}$ & $103^{\circ} 47^{\prime} 16^{\prime \prime}$ & Reeves & -- & -- & 26 & 26 & 0 & 1932 & -- & 1965 \\
\hline 08430000 & East Sandia Spring at Balmorhea, TX & $30^{\circ} 59^{\prime} 00^{\prime \prime}$ & $103^{\circ} 43^{\prime} 30^{\prime \prime}$ & Reeves & -- & -- & 1 & 1 & 0 & 1933 & -- & 1933 \\
\hline 08431000 & Toyah Cr nr Pecos, TX & $31^{\circ} 16^{\prime} 50^{\prime \prime}$ & $103^{\circ} 27^{\prime} 31^{\prime \prime}$ & Reeves & 1,020 & -- & 3 & 0 & 3 & -- & 1940 & 1945 \\
\hline 08431500 & Salt Draw nr Pecos, TX & $31^{\circ} 18 ' 54^{\prime \prime}$ & $103^{\circ} 29^{\prime} 07^{\prime \prime}$ & Reeves & 1,880 & -- & 3 & 0 & 3 & -- & 1940 & 1945 \\
\hline 08431700 & Limpia $\mathrm{Cr}$ above Fort Davis, TX & $30^{\circ} 36^{\prime} 48^{\prime \prime}$ & $104^{\circ} 00^{\prime} 04^{\prime \prime}$ & Jeff Davis & 52.4 & -- & 20 & 20 & 0 & 1967 & -- & 1986 \\
\hline 08431800 & Limpia Cr below Fort Davis, TX & $30^{\circ} 40^{\prime} 52^{\prime \prime}$ & $103^{\circ} 47^{\prime} 30^{\prime \prime}$ & Jeff Davis & 227 & -- & 15 & 15 & 0 & 1963 & -- & 1977 \\
\hline 08432000 & Limpia Cr nr Fort Davis, TX & $30^{\circ} 47^{\prime} 00^{\prime \prime}$ & $103^{\circ} 45^{\prime} 00^{\prime \prime}$ & Jeff Davis & 303 & -- & 5 & 5 & 0 & 1927 & -- & 1931 \\
\hline 08433000 & Barrilla Draw nr Saragosa, TX & $30^{\circ} 57^{\prime} 28^{\prime \prime}$ & $103^{\circ} 27^{\prime} 33^{\prime \prime}$ & Reeves & 612 & -- & 8 & 8 & 0 & 1976 & -- & 1983 \\
\hline 08434000 & Toyah Cr below Toyah Lake nr Pecos, TX & $31^{\circ} 21^{\prime} 00^{\prime \prime}$ & $103^{\circ} 24^{\prime} 00^{\prime \prime}$ & Reeves & 3,710 & -- & 12 & 0 & 12 & -- & 1940 & 1951 \\
\hline 08435500 & Pecos R below Barstow, TX & $31^{\circ} 25^{\prime} 00^{\prime \prime}$ & $103^{\circ} 15^{\prime} 00^{\prime \prime}$ & Ward & 26,000 & -- & 1 & 0 & 1 & -- & 1940 & 1940 \\
\hline 08435600 & Toronto Cr nr Alpine, TX & $30^{\circ} 21^{\prime} 30^{\prime \prime}$ & $103^{\circ} 42^{\prime} 48^{\prime \prime}$ & Brewster & 27.9 & -- & 5 & 5 & 0 & 1972 & -- & 1976 \\
\hline 08435620 & Alpine $\mathrm{Cr}$ at Alpine, $\mathrm{TX}$ & $30^{\circ} 21^{\prime} 06^{\prime \prime}$ & $103^{\circ} 40^{\prime} 00^{\prime \prime}$ & Brewster & 18.1 & -- & 5 & 5 & 0 & 1972 & -- & 1976 \\
\hline 08435660 & Moss Cr nr Alpine, TX & $30^{\circ} 20^{\prime} 10^{\prime \prime}$ & $103^{\circ} 38^{\prime} 24^{\prime \prime}$ & Brewster & 11.3 & -- & 5 & 5 & 0 & 1972 & -- & 1976 \\
\hline 08435700 & Sunny Glen Canyon nr Alpine, TX & $30^{\circ} 22^{\prime} 52^{\prime \prime}$ & $103^{\circ} 44^{\prime} 08^{\prime \prime}$ & Brewster & 29.7 & -- & 9 & 9 & 0 & 1969 & -- & 1977 \\
\hline 08435800 & Coyanosa Draw nr Fort Stockton, TX & $31^{\circ} 02^{\prime} 27^{\prime \prime}$ & $103^{\circ} 08^{\prime} 15^{\prime \prime}$ & Pecos & 1,180 & -- & 13 & 13 & 0 & 1965 & -- & 1977 \\
\hline 08438100 & Pecos R nr Grandfalls, TX & $31^{\circ} 19^{\prime} 18^{\prime \prime}$ & $102^{\circ} 53^{\prime} 33^{\prime \prime}$ & Ward & 27,800 & -- & 6 & 0 & 6 & -- & 1917 & 1925 \\
\hline 08441500 & Pecos R below Grandfalls, TX & $31^{\circ} 17^{\prime} 00^{\prime \prime}$ & $102^{\circ} 44^{\prime} 32^{\prime \prime}$ & Crane & 27,800 & -- & 20 & 0 & 20 & -- & 1923 & 1956 \\
\hline 08444500 & Comanche Springs at Fort Stockton, TX & $30^{\circ} 53 \prime 20^{\prime \prime}$ & $102^{\circ} 51^{\prime} 59^{\prime \prime}$ & Pecos & -- & -- & 23 & 23 & 0 & 1942 & -- & 1964 \\
\hline 08446500 & Pecos R nr Girvin, TX & $31^{\circ} 06^{\prime} 47^{\prime \prime}$ & $102^{\circ} 25^{\prime} 02^{\prime \prime}$ & Pecos & 29,600 & -- & 61 & 0 & 61 & -- & 1940 & 2000 \\
\hline 08447000 & Pecos R nr Sheffield, TX & $30^{\circ} 39^{\prime} 34^{\prime \prime}$ & $101^{\circ} 46^{\prime} 11^{\prime \prime}$ & Pecos & 31,600 & -- & 13 & 0 & 13 & -- & 1922 & 1949 \\
\hline 08447020 & Independence Cr nr Sheffield, TX & $30^{\circ} 27^{\prime} 07^{\prime \prime}$ & $101^{\circ} 43^{\prime} 58^{\prime \prime}$ & Terrell & 763 & -- & 11 & 11 & 0 & 1975 & -- & 1985 \\
\hline 08449000 & Devils R nr Juno, TX & $29^{\circ} 57^{\prime} 48^{\prime \prime}$ & $101^{\circ} 08^{\prime} 42^{\prime \prime}$ & Val Verde & 2,730 & -- & 34 & 34 & 0 & 1926 & -- & 1973 \\
\hline
\end{tabular}

${ }^{1}$ An urban basin has at least 10-percent impervious cover.

${ }^{2}$ Record might not be continuous from begin year to end year. Partial-record data exist for some stations outside period of record indicated.

${ }^{3} \mathrm{~A}$ regulated basin has at least 10 percent of its contributing drainage area controlled by at least one reservoir.

${ }^{4}$ Inventory includes data through 2000 water year. Most stations with end year of 2000 are still in operation. 
Table 3. Median streamflow and associated hydraulic characteristics for streamflow-gaging stations in Texas

$\left[\mathrm{mi}^{2}\right.$, square miles; $\mathrm{ft}^{3} / \mathrm{s}$, cubic feet per second; ft, feet; $\mathrm{ft}^{2}$, square feet; $\mathrm{ft} / \mathrm{s}$, feet per second; --, not available; FTW, Fort Worth]

\begin{tabular}{|c|c|c|c|c|c|c|c|c|c|c|c|}
\hline \multirow[b]{2}{*}{$\begin{array}{c}\text { Station } \\
\text { no. }\end{array}$} & \multirow[b]{2}{*}{ Station name } & \multirow[b]{2}{*}{ Latitude } & \multirow[b]{2}{*}{ Longitude } & \multirow{2}{*}{$\begin{array}{l}\text { Contri- } \\
\text { buting } \\
\text { drainage } \\
\text { area } \\
\left(\mathrm{mi}^{2}\right)\end{array}$} & \multicolumn{6}{|c|}{ Characteristics for median streamflow } & \multirow[b]{2}{*}{$\begin{array}{l}\text { Elevation } \\
\text { of datum } \\
\text { of gage }\end{array}$} \\
\hline & & & & & $\begin{array}{l}\text { Stream- } \\
\text { flow } \\
\left(\mathrm{ft}^{3} / \mathrm{s}\right)\end{array}$ & $\begin{array}{c}\text { Gage } \\
\text { height } \\
\text { (ft) }\end{array}$ & $\begin{array}{l}\text { Stream } \\
\text { width } \\
\text { (ft) }\end{array}$ & $\begin{array}{c}\text { Cross } \\
\text { section } \\
\text { area } \\
\left(\mathrm{ft}^{2}\right)\end{array}$ & $\begin{array}{l}\text { Mean } \\
\text { velocity }\end{array}$ & $\begin{array}{l}\text { Mean } \\
\text { depth }\end{array}$ & \\
\hline 07227500 & Canadian R nr Amarillo, TX & $35^{\circ} 28^{\prime} 13^{\prime \prime}$ & $101^{\circ} 52^{\prime} 45^{\prime \prime}$ & 15,376 & 27 & 1.99 & 82.50 & 41.80 & 0.67 & 0.51 & $2,989.16$ \\
\hline 07228000 & Canadian R nr Canadian, TX & $35^{\circ} 56^{\prime} 06^{\prime \prime}$ & $100^{\circ} 22^{\prime} 13^{\prime \prime}$ & 18,178 & 40 & 2.67 & 78 & 34 & 1.17 & .44 & $2,301.5$ \\
\hline 07235000 & Wolf $\mathrm{Cr}$ at Lipscomb, TX & $36^{\circ} 14^{\prime} 19^{\prime \prime}$ & $100^{\circ} 16^{\prime} 31^{\prime \prime}$ & 475 & 2.6 & 3.24 & 22.5 & 8.11 & .31 & .36 & $2,371.29$ \\
\hline 07297910 & Prairie Dog Town Fk Red R nr Wayside, TX & $34^{\circ} 50^{\prime} 15^{\prime \prime}$ & $101^{\circ} 24 ' 49^{\prime \prime}$ & 930 & 2.2 & 7.24 & 15.5 & 2.92 & .74 & .19 & $2,463.74$ \\
\hline 07299540 & Prairie Dog Town Fk Red R nr Childress, TX & $34^{\circ} 34^{\prime} 09^{\prime \prime}$ & $100^{\circ} 11^{\prime} 37^{\prime \prime}$ & 2,958 & 4.7 & 8.12 & 43.7 & 7.01 & .71 & .16 & $1,628.4$ \\
\hline 07299670 & Groesbeck Cr at SH $6 \mathrm{nr}$ Quanah, TX & $34^{\circ} 21^{\prime} 16^{\prime \prime}$ & $99^{\circ} 44^{\prime} 24 "$ & 303 & 7.1 & 6.31 & 14 & 9.96 & .76 & .71 & $1,425.69$ \\
\hline 07299890 & Lelia Lake Cr below Bell Cr nr Hedley, TX & $34^{\circ} 56^{\prime} 08^{\prime \prime}$ & $100^{\circ} 41^{\prime} 46^{\prime \prime}$ & 74 & 3.3 & 2.83 & 17.5 & 4.77 & .71 & .27 & 2,417 \\
\hline 07300000 & Salt Fk Red R nr Wellington, TX & $34^{\circ} 57^{\prime} 27^{\prime \prime}$ & $100^{\circ} 13^{\prime} 14^{\prime \prime}$ & 1,013 & 17 & 3.09 & 66.6 & 18.1 & .95 & .27 & $1,941.41$ \\
\hline 07301200 & McClellan Cr nr McLean, TX & $35^{\circ} 19^{\prime} 45^{\prime \prime}$ & $100^{\circ} 36^{\prime} 32^{\prime \prime}$ & 460 & 6.4 & 4.21 & 10.5 & 6.54 & .94 & .62 & $2,545.99$ \\
\hline 07301300 & North Fk Red R nr Shamrock, TX & $35^{\circ} 15^{\prime} 51^{\prime \prime}$ & $100^{\circ} 14^{\prime} 29^{\prime \prime}$ & 703 & 57 & 2.59 & 65 & 36.2 & 1.53 & .56 & $2,165.55$ \\
\hline 07301410 & Sweetwater Cr nr Kelton, TX & $35^{\circ} 28^{\prime} 23^{\prime \prime}$ & $100^{\circ} 07^{\prime} 14^{\prime \prime}$ & 267 & 10 & 6.06 & 19.5 & 20 & .48 & 1.03 & 2,230 \\
\hline 07307800 & Pease R nr Childress, TX & $34^{\circ} 13^{\prime} 39^{\prime \prime}$ & $100^{\circ} 04^{\prime} 24^{\prime \prime}$ & 2,195 & 9.2 & 7.12 & 29 & 13.8 & .69 & .48 & $1,492.98$ \\
\hline 07308200 & Pease R nr Vernon, TX & $34^{\circ} 10^{\prime} 45^{\prime \prime}$ & $99^{\circ} 16^{\prime} 40^{\prime \prime}$ & 2,929 & 16 & 7.58 & 41 & 19.2 & .88 & .47 & $1,166.03$ \\
\hline 07308500 & Red R nr Burkburnett, TX & $34^{\circ} 06^{\prime} 36^{\prime \prime}$ & $98^{\circ} 31^{\prime} 53^{\prime \prime}$ & 14,634 & 313 & 3.25 & 203 & 237 & 1.3 & 1.17 & 952.57 \\
\hline 07311600 & North Wichita R nr Paducah, TX & $33^{\circ} 57^{\prime} 02^{\prime \prime}$ & $100^{\circ} 03^{\prime} 52^{\prime \prime}$ & 540 & 11 & 2.76 & 50 & 13.6 & .81 & .27 & 1,530 \\
\hline 07311630 & Middle Wichita R nr Guthrie, TX & $33^{\circ} 47^{\prime} 45^{\prime \prime}$ & $100^{\circ} 04^{\prime} 29^{\prime \prime}$ & 50.3 & 6.1 & 7.91 & 8.7 & 9.04 & .70 & 1.04 & $1,583.9$ \\
\hline 07311700 & North Wichita R nr Truscott, TX & $33^{\circ} 49^{\prime} 14^{\prime \prime}$ & $99^{\circ} 47^{\prime} 10^{\prime \prime}$ & 937 & 20 & 4.56 & 25.5 & 18.2 & 1.08 & .71 & $1,351.78$ \\
\hline 07311783 & South Wichita R below low flow dam nr Guthrie, TX & $33^{\circ} 37^{\prime} 19^{\prime \prime}$ & $100^{\circ} 12^{\prime} 31^{\prime \prime}$ & 223 & .06 & 2.96 & 1.2 & .22 & .28 & .18 & 1,590 \\
\hline 07311800 & South Wichita R nr Benjamin, TX & $33^{\circ} 38^{\prime} 39^{\prime \prime}$ & $99^{\circ} 48^{\prime} 02^{\prime \prime}$ & 584 & 6.9 & 5.25 & 11.9 & 9.33 & .75 & .78 & $1,334.23$ \\
\hline 07311900 & Wichita R nr Seymour, TX & $33^{\circ} 42^{\prime} 01^{\prime \prime}$ & $99^{\circ} 23^{\prime} 18^{\prime \prime}$ & 1,874 & 31 & 6.49 & 68 & 47.8 & .71 & .70 & $1,152.7$ \\
\hline 07312100 & Wichita R nr Mabelle, TX & $33^{\circ} 45^{\prime} 36^{\prime \prime}$ & $99^{\circ} 08^{\prime} 33^{\prime \prime}$ & 2,086 & 13 & 2.77 & 27 & 11.6 & 1.12 & .43 & $1,062.72$ \\
\hline 07312110 & South Side Canal nr Dundee, TX & $33^{\circ} 48^{\prime} 50^{\prime \prime}$ & $98^{\circ} 55^{\prime} 57^{\prime \prime}$ & 2,194 & 54 & 4.51 & 36 & 25.6 & 2.06 & .71 & $1,039.7$ \\
\hline 07312130 & Wichita R at SH 25 nr Kamay, TX & $33^{\circ} 52^{\prime} 09^{\prime \prime}$ & $98^{\circ} 50^{\prime} 20^{\prime \prime}$ & 2,246 & 9.0 & 8.27 & 14 & 7.99 & 1.08 & .57 & 923 \\
\hline 07312200 & Beaver Cr nr Electra, TX & $33^{\circ} 54^{\prime} 21^{\prime \prime}$ & $98^{\circ} 54^{\prime} 17^{\prime \prime}$ & 652 & 5.6 & 8.71 & 10.7 & 6.06 & 1.0 & .57 & 991.3 \\
\hline 07312500 & Wichita R at Wichita Falls, TX & $33^{\circ} 54^{\prime} 34^{\prime \prime}$ & $98^{\circ} 32^{\prime} 00^{\prime \prime}$ & 1,054 & 83 & 2.8 & 68.3 & 66.6 & 1.27 & .98 & 924.26 \\
\hline 07312700 & Wichita R nr Charlie, TX & $34^{\circ} 03^{\prime} 11^{\prime \prime}$ & $98^{\circ} 17^{\prime} 47^{\prime \prime}$ & 3,439 & 140 & 5.36 & 62.5 & 83.3 & 1.67 & 1.33 & 872.71 \\
\hline 07314500 & Little Wichita R nr Archer City, TX & $33^{\circ} 39^{\prime} 45^{\prime \prime}$ & $98^{\circ} 36^{\prime} 46^{\prime \prime}$ & 481 & .31 & 4.31 & 2.65 & .37 & .84 & .14 & 934.72 \\
\hline 07314900 & Little Wichita R above Henrietta, TX & $33^{\circ} 49^{\prime} 36^{\prime \prime}$ & $98^{\circ} 14^{\prime} 23^{\prime \prime}$ & 1,037 & 0 & -- & -- & -- & -- & -- & 831.57 \\
\hline 07315200 & East Fk Little Wichita R nr Henrietta, TX & $33^{\circ} 48^{\prime} 46^{\prime \prime}$ & $98^{\circ} 05^{\prime} 05^{\prime \prime}$ & 178 & .14 & 5.89 & 3.0 & .45 & .30 & .15 & 825.32 \\
\hline 07331600 & Red River at Denison Dam nr Denison, TX & $33^{\circ} 49^{\prime} 08^{\prime \prime}$ & $96^{\circ} 33^{\prime} 47^{\prime \prime}$ & 39,720 & 2,790 & 6.96 & 333 & 1,589 & 1.48 & 4.77 & 495 \\
\hline 07342465 & South Sulphur R at Commerce, TX & $33^{\circ} 12^{\prime} 42^{\prime \prime}$ & $95^{\circ} 54^{\prime} 50^{\prime \prime}$ & 150 & 2.9 & 4.06 & 9.25 & 3.85 & .80 & .42 & 460.74 \\
\hline 07342480 & Middle Sulphur R at Commerce, TX & $33^{\circ} 15^{\prime} 59^{\prime \prime}$ & $95^{\circ} 54^{\prime} 55^{\prime \prime}$ & 44.1 & .52 & 3.1 & 3.8 & .74 & .69 & .19 & 470 \\
\hline 07342500 & South Sulphur R nr Cooper, TX & $33^{\circ} 21^{\prime} 23^{\prime \prime}$ & $95^{\circ} 35^{\prime} 41^{\prime \prime}$ & 527 & 19 & 2.11 & 20 & 17.4 & 1.07 & .87 & 379.91 \\
\hline 07343000 & North Sulphur R nr Cooper, TX & $33^{\circ} 28^{\prime} 29^{\prime \prime}$ & $95^{\circ} 35^{\prime} 15^{\prime \prime}$ & 276 & 11 & 4.74 & 36 & 28.7 & .38 & .80 & 372.42 \\
\hline
\end{tabular}


Table 3. Median streamflow and associated hydraulic characteristics for streamflow-gaging stations in Texas-Continued

\begin{tabular}{|c|c|c|c|c|c|c|c|c|c|c|c|}
\hline \multirow[b]{2}{*}{$\begin{array}{c}\text { Station } \\
\text { no. }\end{array}$} & \multirow[b]{2}{*}{ Station name } & \multirow[b]{2}{*}{ Latitude } & \multirow[b]{2}{*}{ Longitude } & \multirow{2}{*}{$\begin{array}{l}\text { Contri- } \\
\text { buting } \\
\text { drainage } \\
\text { area } \\
\left(\mathrm{mi}^{2}\right)\end{array}$} & \multicolumn{6}{|c|}{ Characteristics for median streamflow } & \multirow[b]{2}{*}{$\begin{array}{l}\text { Elevation } \\
\text { of datum } \\
\text { of gage }\end{array}$} \\
\hline & & & & & $\begin{array}{l}\text { Stream- } \\
\text { flow } \\
\left(\mathrm{ft}^{3} / \mathrm{s}\right)\end{array}$ & $\begin{array}{c}\text { Gage } \\
\text { height } \\
\text { (ft) }\end{array}$ & $\begin{array}{c}\text { Stream } \\
\text { width } \\
\text { (ft) }\end{array}$ & $\begin{array}{c}\text { Cross } \\
\text { section } \\
\text { area } \\
\left(\mathrm{ft}^{2}\right)\end{array}$ & $\begin{array}{l}\text { Mean } \\
\text { velocity }\end{array}$ & $\begin{array}{l}\text { Mean } \\
\text { depth }\end{array}$ & \\
\hline 07343210 & Sulphur R below Talco, TX & $33^{\circ} 23^{\prime} 26^{\prime \prime}$ & $95^{\circ} 03^{\prime} 44^{\prime \prime}$ & 1,405 & 276 & 9.5 & 76 & 250 & 1.11 & 3.29 & 275.48 \\
\hline 07343500 & White Oak Cr nr Talco, TX & $33^{\circ} 19^{\prime} 20^{\prime \prime}$ & $95^{\circ} 05^{\prime} 33^{\prime \prime}$ & 494 & 57 & 3.17 & 78 & 176 & .33 & 2.26 & 286.45 \\
\hline 07344486 & Brushy Cr at Scroggins, TX & $32^{\circ} 58^{\prime} 32^{\prime \prime}$ & $95^{\circ} 11^{\prime} 03^{\prime \prime}$ & 23.4 & 7.2 & 8.22 & 16 & 14 & .52 & .88 & 343.9 \\
\hline 07344500 & Big Cypress Cr nr Pittsburg, TX & $33^{\circ} 01^{\prime} 15^{\prime \prime}$ & $94^{\circ} 52^{\prime} 55^{\prime \prime}$ & 366 & 33 & 6.03 & 34.3 & 67.7 & .50 & 1.97 & 247.49 \\
\hline 07346000 & Big Cypress Cr nr Jefferson, TX & $32^{\circ} 44^{\prime} 58^{\prime \prime}$ & $94^{\circ} 29^{\prime} 55^{\prime \prime}$ & 850 & 182 & 7.4 & 112 & 658.5 & .29 & 5.91 & 180 \\
\hline 07346045 & Black Cypress Bayou at Jefferson, TX & $32^{\circ} 46^{\prime} 40^{\prime \prime}$ & $94^{\circ} 21^{\prime} 26^{\prime \prime}$ & 365 & 168 & 7.4 & 66.3 & 337 & .51 & 5.09 & 171.47 \\
\hline 07346050 & Little Cypress Creek nr Ore City, TX & $32^{\circ} 40^{\prime} 21^{\prime \prime}$ & $94^{\circ} 45^{\prime} 03^{\prime \prime}$ & 383 & 73 & 4.63 & 70 & 204 & .37 & 2.91 & 232.67 \\
\hline 07346070 & Little Cypress Creek nr Jefferson, TX & $32^{\circ} 42^{\prime} 46^{\prime \prime}$ & $94^{\circ} 20^{\prime} 45^{\prime \prime}$ & 675 & 182 & 5.34 & 81.5 & 403.5 & .42 & 4.95 & 174.6 \\
\hline 08017200 & Cowleech Fk Sabine R at Greenville, TX & $33^{\circ} 07^{\prime} 58^{\prime \prime}$ & $96^{\circ} 04^{\prime} 36^{\prime \prime}$ & 77.7 & 1.4 & 2.78 & 9.0 & 6.21 & .22 & .69 & 485.07 \\
\hline 08017300 & South Fk Sabine R nr Quinlan, TX & $32^{\circ} 53^{\prime} 52^{\prime \prime}$ & $96^{\circ} 15^{\prime} 11^{\prime \prime}$ & 78.7 & .46 & 3.89 & 5.15 & 1.11 & .45 & .21 & 461.4 \\
\hline 08017410 & Sabine R nr Wills Point, TX & $32^{\circ} 48^{\prime} 22^{\prime \prime}$ & $95^{\circ} 55^{\prime} 09^{\prime \prime}$ & 756 & 22 & 1.95 & 28 & 18.7 & 1.29 & 67 & 370 \\
\hline 08018500 & Sabine R nr Mineola, TX & $32^{\circ} 36^{\prime} 49^{\prime \prime}$ & $95^{\circ} 29^{\prime} 08^{\prime \prime}$ & 1,357 & 142 & 4.43 & 49 & 214 & .64 & 4.37 & 304.16 \\
\hline 08019000 & Lake Fk Cr nr Quitman, TX & $32^{\circ} 45^{\prime} 47^{\prime \prime}$ & $95^{\circ} 27^{\prime} 46^{\prime \prime}$ & 585 & 43 & 4.21 & 26 & 66.6 & .71 & 2.56 & 317.42 \\
\hline 08019200 & Sabine R nr Hawkins, TX & $32^{\circ} 33^{\prime} 35^{\prime \prime}$ & $95^{\circ} 12^{\prime} 23^{\prime \prime}$ & 2,259 & 468 & 7.44 & 85 & 628 & .82 & 7.39 & 279 \\
\hline 08019500 & Big Sandy Cr nr Big Sandy, TX & $32^{\circ} 36^{\prime} 14^{\prime \prime}$ & $95^{\circ} 05^{\prime} 29^{\prime \prime}$ & 231 & 78 & 5.74 & 39.2 & 111 & .67 & 2.83 & 278.38 \\
\hline 08020000 & Sabine R nr Gladewater, TX & $32^{\circ} 31^{\prime} 37^{\prime \prime}$ & $94^{\circ} 57^{\prime} 36^{\prime \prime}$ & 2,791 & 552 & 7.62 & 100 & 389 & 1.37 & 3.89 & 243.85 \\
\hline 08020450 & Sabine R above Longview, TX & $32^{\circ} 28^{\prime} 47^{\prime \prime}$ & $94^{\circ} 48^{\prime} 15^{\prime \prime}$ & 2,943 & 161 & 4.63 & 58 & 111 & 1.42 & 1.91 & 230 \\
\hline 08020900 & Sabine R below Longview, TX & $32^{\circ} 25^{\prime} 00^{\prime \prime}$ & $94^{\circ} 42^{\prime} 35^{\prime \prime}$ & 3,155 & 548 & 6.16 & 72 & 387 & 1.46 & 5.38 & 230 \\
\hline 08022040 & Sabine R nr Beckville, TX & $32^{\circ} 19^{\prime} 38^{\prime \prime}$ & $94^{\circ} 21^{\prime} 12^{\prime \prime}$ & 3,589 & 896 & 824 & 150 & 840 & 1.02 & 5.6 & 190 \\
\hline 08025360 & Sabine R at Toledo Bend Reservoir nr Burkeville, TX & $31^{\circ} 10^{\prime} 25^{\prime \prime}$ & $93^{\circ} 33^{\prime} 57^{\prime \prime}$ & 7,178 & 3,940 & 1.68 & 228 & 1,360 & 2.26 & 5.96 & 0 \\
\hline 08026000 & Sabine R nr Burkeville, TX & $31^{\circ} 03^{\prime} 50^{\prime \prime}$ & $93^{\circ} 31^{\prime} 10^{\prime \prime}$ & 7,482 & 2,720 & 17.54 & 297 & 1,460 & 1.84 & 4.92 & 60.59 \\
\hline 08028500 & Sabine R nr Bon Wier, TX & $30^{\circ} 44^{\prime} 49^{\prime \prime}$ & $93^{\circ} 36^{\prime} 30^{\prime \prime}$ & 8,229 & 3,590 & 17.21 & 220 & 2,280 & 1.56 & 10.36 & 33.42 \\
\hline 08029500 & Big Cow Cr nr Newton, TX & $30^{\circ} 49^{\prime} 08^{\prime \prime}$ & $93^{\circ} 47^{\prime} 07^{\prime \prime}$ & 128 & 64 & 6.7 & 43 & 78.3 & .81 & 1.82 & 134.69 \\
\hline 08030500 & Sabine R nr Ruliff, TX & $30^{\circ} 18^{\prime} 13^{\prime \prime}$ & $93^{\circ} 44^{\prime} 37^{\prime \prime}$ & 9,329 & 4,710 & 20.44 & 365 & 5,430 & .86 & 14.88 & -5.92 \\
\hline 08032000 & Neches R nr Neches, TX & $31^{\circ} 53^{\prime} 32^{\prime \prime}$ & $95^{\circ} 25^{\prime} 50^{\prime \prime}$ & 1,145 & 263 & 6.19 & 740 & 364 & .74 & .49 & 264.06 \\
\hline 08033000 & Neches R nr Diboll, TX & $31^{\circ} 07^{\prime} 58^{\prime \prime}$ & $94^{\circ} 48^{\prime} 35^{\prime \prime}$ & 2,724 & 625 & 5.33 & 104 & 1,020 & .62 & 9.81 & 136.46 \\
\hline 08033500 & Neches R nr Rockland, TX & $31^{\circ} 01^{\prime} 29^{\prime \prime}$ & $94^{\circ} 23^{\prime} 55^{\prime \prime}$ & 3,636 & 935 & 5.3 & 147 & 686 & 1.38 & 4.67 & 88.41 \\
\hline 08036500 & Angelina R nr Alto, TX & $31^{\circ} 40^{\prime} 10^{\prime \prime}$ & $94^{\circ} 57^{\prime} 24^{\prime \prime}$ & 1,726 & 345 & 4.43 & 93.5 & 318 & 1.09 & 3.4 & 204.3 \\
\hline 08038000 & Attoyac Bayou nr Chireno, TX & $31^{\circ} 30^{\prime} 15^{\prime \prime}$ & $94^{\circ} 18^{\prime} 15^{\prime \prime}$ & 503 & 151 & 5.33 & 61 & 311 & .48 & 5.1 & 169.58 \\
\hline 08039100 & Ayish Bayou nr San Augustine, TX & $31^{\circ} 23^{\prime} 46^{\prime \prime}$ & $94^{\circ} 09^{\prime} 03^{\prime \prime}$ & 89 & 18 & 2.34 & 21.5 & 24.4 & .76 & 1.13 & 190.22 \\
\hline 08040600 & Neches R nr Town Bluff, TX & $30^{\circ} 47^{\prime} 27^{\prime \prime}$ & $94^{\circ} 09^{\prime} 03^{\prime \prime}$ & 7,574 & 2,980 & 51.05 & 177 & 2,415 & 1.24 & 13.61 & 0 \\
\hline 08041500 & Village Cr nr Kountze, TX & $30^{\circ} 23^{\prime} 52^{\prime \prime}$ & $94^{\circ} 15^{\prime} 48^{\prime \prime}$ & 860 & 336 & 4.58 & 85 & 288 & 1.17 & 3.39 & 25.12 \\
\hline 08041700 & Pine Island Bayou nr Sour Lake, TX & $30^{\circ} 06^{\prime} 21^{\prime \prime}$ & $94^{\circ} 20^{\prime} 04^{\prime \prime}$ & 336 & 84 & 14.36 & 61 & 132 & .64 & 2.16 & 0 \\
\hline 08042800 & West Fk Trinity R nr Jacksboro, TX & $33^{\circ} 17^{\prime} 30^{\prime \prime}$ & $98^{\circ} 04^{\prime} 49^{\prime \prime}$ & 683 & .99 & 4.7 & 14.5 & 4.68 & .21 & .32 & 869.28 \\
\hline 08043950 & Big Sandy Cr nr Chico, TX & $33^{\circ} 16^{\prime} 27^{\prime \prime}$ & $97^{\circ} 40^{\prime} 42^{\prime \prime}$ & 312 & 6.6 & 2.6 & 13 & 5.8 & 1.09 & .45 & 754 \\
\hline
\end{tabular}


Table 3. Median streamflow and associated hydraulic characteristics for streamflow-gaging stations in Texas-Continued

\begin{tabular}{|c|c|c|c|c|c|c|c|c|c|c|c|}
\hline \multirow[b]{2}{*}{$\begin{array}{c}\text { Station } \\
\text { no. }\end{array}$} & \multirow[b]{2}{*}{ Station name } & \multirow[b]{2}{*}{ Latitude } & \multirow[b]{2}{*}{ Longitude } & \multirow{2}{*}{$\begin{array}{l}\text { Contri- } \\
\text { buting } \\
\text { drainage } \\
\text { area } \\
\left(\mathrm{mi}^{2}\right)\end{array}$} & \multicolumn{6}{|c|}{ Characteristics for median streamflow } & \multirow[b]{2}{*}{$\begin{array}{c}\text { Elevation } \\
\text { of datum } \\
\text { of gage }\end{array}$} \\
\hline & & & & & $\begin{array}{l}\text { Stream- } \\
\text { flow } \\
\left(\mathrm{ft}^{3} / \mathrm{s}\right)\end{array}$ & $\begin{array}{c}\text { Gage } \\
\text { height } \\
\text { (ft) }\end{array}$ & $\begin{array}{c}\text { Stream } \\
\text { width } \\
\text { (ft) }\end{array}$ & $\begin{array}{c}\text { Cross } \\
\text { section } \\
\text { area } \\
\left(\mathrm{ft}^{2}\right)\end{array}$ & $\begin{array}{l}\text { Mean } \\
\text { velocity }\end{array}$ & $\begin{array}{l}\text { Mean } \\
\text { depth }\end{array}$ & \\
\hline 08044500 & West Fk Trinity R nr Boyd, TX & $33^{\circ} 05^{\prime} 07^{\prime \prime}$ & $97^{\circ} 33^{\prime} 30^{\prime \prime}$ & 1,725 & 68 & 6.24 & 52 & 119 & 0.54 & 2.29 & 660.57 \\
\hline 08044800 & Walnut $\mathrm{Cr}$ at Reno, $\mathrm{TX}$ & $32^{\circ} 56^{\prime} 44^{\prime \prime}$ & $97^{\circ} 34^{\prime} 58^{\prime \prime}$ & 75.6 & 3.7 & 3.45 & 14 & 6.49 & .51 & .46 & 681.11 \\
\hline 08045850 & Clear Fk Trinity R nr Weatherford, TX & $32^{\circ} 44^{\prime} 25^{\prime \prime}$ & $97^{\circ} 39^{\prime} 06^{\prime \prime}$ & 121 & 3.2 & 8.33 & 12 & 11.2 & .29 & .93 & 810 \\
\hline 08047000 & Clear Fk Trinity R nr Benbrook, TX & $32^{\circ} 39^{\prime} 54^{\prime \prime}$ & $97^{\circ} 26^{\prime} 30^{\prime \prime}$ & 431 & 6.7 & 2.9 & 22 & 12.4 & .50 & .56 & 604.22 \\
\hline 08047050 & Marys $\mathrm{Cr}$ at Benbrook, $\mathrm{TX}$ & $32^{\circ} 41^{\prime} 42^{\prime \prime}$ & $97^{\circ} 26^{\prime} 49^{\prime \prime}$ & 54 & 2.3 & 1.2 & 18.3 & 2.8 & .74 & .15 & 635 \\
\hline 08047500 & Clear Fk Trinity R at FTW, TX & $32^{\circ} 43^{\prime} 56^{\prime \prime}$ & $97^{\circ} 21^{\prime} 31^{\prime \prime}$ & 518 & 16 & 8.41 & 20 & 6.93 & 2.26 & .35 & 532.91 \\
\hline 08048000 & West Fk Trinity R at FTW, TX & $32^{\circ} 45^{\prime} 39^{\prime \prime}$ & $97^{\circ} 19^{\prime} 56^{\prime \prime}$ & 2,615 & 40 & .79 & 29 & 15.7 & 2.54 & .54 & 519.24 \\
\hline 08048543 & West Fk Trinity R at Beach St, FTW, TX & $32^{\circ} 45^{\prime} 06^{\prime \prime}$ & $97^{\circ} 17^{\prime} 21^{\prime \prime}$ & 2,685 & 52 & 3.45 & 38 & 38.3 & 1.36 & 1.01 & 478.7 \\
\hline 08048970 & Village $\mathrm{Cr}$ at Everman, $\mathrm{TX}$ & $32^{\circ} 36^{\prime} 12^{\prime \prime}$ & $97^{\circ} 15^{\prime} 53^{\prime \prime}$ & 84.5 & 3.7 & 3.11 & 17 & 3.51 & 1.15 & .21 & 589.93 \\
\hline 08049500 & West Fk Trinty R at Grand Prairie, TX & $32^{\circ} 45^{\prime} 46^{\prime \prime}$ & $96^{\circ} 59^{\prime} 42^{\prime \prime}$ & 3,065 & 178 & 3.05 & 89 & 171 & 1.04 & 1.92 & 405.42 \\
\hline 08049580 & Mountain Cr nr Venus, TX & $32^{\circ} 29^{\prime} 27^{\prime \prime}$ & $97^{\circ} 07^{\prime} 22^{\prime \prime}$ & 25.5 & .01 & 4.26 & 1.0 & .12 & .08 & .12 & 445 \\
\hline 08049700 & Walnut Cr nr Mansfield, TX & $32^{\circ} 34^{\prime} 51^{\prime \prime}$ & $97^{\circ} 06^{\prime} 06^{\prime \prime}$ & 62.8 & .26 & 5.07 & 4.2 & .39 & .69 & .09 & 531.08 \\
\hline 08050100 & Mountain Cr at Grand Prairie, TX & $32^{\circ} 44^{\prime} 51^{\prime \prime}$ & $96^{\circ} 55^{\prime} 32^{\prime \prime}$ & 298 & 1.3 & 3.24 & 7.8 & 2.44 & .49 & .31 & 404.31 \\
\hline 08050400 & Elm Fk Trinity R at Gainesville, TX & $33^{\circ} 37^{\prime} 27^{\prime \prime}$ & $97^{\circ} 09^{\prime} 22^{\prime \prime}$ & 174 & 12 & 1.64 & 30.8 & 23.6 & .52 & .77 & 700 \\
\hline 08050800 & Timber Cr nr Collinsville, TX & $33^{\circ} 33^{\prime} 16^{\prime \prime}$ & $96^{\circ} 56^{\prime} 49^{\prime \prime}$ & 38.8 & 1.6 & 6.54 & 4.4 & 1.8 & .90 & .41 & 640 \\
\hline 08050840 & Range Cr nr Collinsville, TX & $33^{\circ} 31^{\prime} 34^{\prime \prime}$ & $96^{\circ} 48^{\prime} 25^{\prime \prime}$ & 29.2 & .04 & 3.4 & 2.1 & .36 & .13 & .17 & 621.08 \\
\hline 08051500 & Clear Cr nr Sanger, TX & $33^{\circ} 20^{\prime} 10^{\prime \prime}$ & $97^{\circ} 10^{\prime} 45^{\prime \prime}$ & 295 & 25 & 6.02 & 36.3 & 61.4 & .42 & 1.69 & 582.23 \\
\hline 08052700 & Little Elm Cr nr Aubrey, TX & $33^{\circ} 17^{\prime} 00^{\prime \prime}$ & $96^{\circ} 53^{\prime} 33^{\prime \prime}$ & 75.5 & .95 & 4.8 & 5.8 & 5.83 & .15 & 1.01 & 534.76 \\
\hline 08053000 & Elm Fk nr Trinity R nr Lewisville, TX & $33^{\circ} 02^{\prime} 44^{\prime \prime}$ & $96^{\circ} 57^{\prime} 39^{\prime \prime}$ & 1,673 & 220 & 4.68 & 48 & 218 & .99 & 4.54 & 432.39 \\
\hline 08053500 & Denton Cr nr Justin, TX & $33^{\circ} 07^{\prime} 08^{\prime \prime}$ & $97^{\circ} 17^{\prime} 25^{\prime \prime}$ & 400 & 19 & 3.36 & 46.5 & 106 & .18 & 2.28 & 606.66 \\
\hline 08055500 & Elm Fk Trinity R nr Carrollton, TX & $32^{\circ} 57^{\prime} 57^{\prime \prime}$ & $96^{\circ} 56^{\prime} 39^{\prime \prime}$ & 2,459 & 148 & 3.41 & 62.7 & 72.2 & 1.99 & 1.15 & 431.4 \\
\hline 08057000 & Trinity $\mathrm{R}$ at Dallas, $\mathrm{TX}$ & $32^{\circ} 466^{\prime \prime \prime}$ & $96^{\circ} 49^{\prime} 18^{\prime \prime}$ & 6,106 & 418 & 13.2 & 84 & 351 & 1.19 & 4.18 & 368.02 \\
\hline 08057200 & White Rock Cr at Greenville Ave, Dallas, TX & $32^{\circ} 53 \prime 21^{\prime \prime}$ & $96^{\circ} 45^{\prime} 23^{\prime \prime}$ & 66.4 & 13 & 71.27 & 27 & 13.6 & .95 & .50 & 0 \\
\hline 08057445 & Prairie $\mathrm{Cr}$ at US 175 , Dallas, TX & $32^{\circ} 42^{\prime} 17^{\prime \prime}$ & $96^{\circ} 40^{\prime} 11^{\prime \prime}$ & 9.03 & .94 & 10.55 & 13 & 6.21 & .16 & .48 & 390 \\
\hline 08058900 & East Fk Trinity R at McKinney, TX & $33^{\circ} 14^{\prime} 38^{\prime \prime}$ & $96^{\circ} 36^{\prime} 31^{\prime \prime}$ & 164 & 14 & 3.78 & 17 & 18.1 & .72 & 1.06 & 528.74 \\
\hline 08059400 & Sister Grove Cr nr Blue Ridge, TX & $33^{\prime \circ} 17^{\prime} 40^{\prime \prime}$ & $96^{\circ} 28^{\prime} 58^{\prime \prime}$ & 83.1 & 14 & 11.62 & 24 & 38.7 & .36 & 1.61 & 526.29 \\
\hline 08061540 & Rowlett Cr nr Sachse, TX & $32^{\circ} 57^{\prime} 35^{\prime \prime}$ & $96^{\circ} 36^{\prime} 51^{\prime \prime}$ & 120 & 46 & 6.31 & 44 & 34.4 & 1.3 & .78 & 450 \\
\hline 08061750 & East Fk Trinity R nr Forney, TX & $32^{\circ} 46^{\prime} 27^{\prime \prime}$ & $96^{\circ} 30^{\prime} 12^{\prime \prime}$ & 1,118 & 60 & 3.66 & 64 & 144 & .41 & 2.25 & 374.86 \\
\hline 08062000 & East Fk Trinity R nr Crandall, TX & $32^{\circ} 38^{\prime} 19^{\prime \prime}$ & $96^{\circ} 29^{\prime} 06^{\prime \prime}$ & 1,256 & 94 & 2.69 & 46 & 170 & .55 & 3.7 & 338.69 \\
\hline 08062500 & Trinity R nr Rosser, TX & $32^{\circ} 25^{\prime} 35^{\prime \prime}$ & $96^{\circ} 27^{\prime} 46^{\prime \prime}$ & 8,147 & 915 & 2.89 & 94 & 518 & 1.79 & 5.51 & 297.65 \\
\hline 08062700 & Trinity R at Trinidad, TX & $32^{\circ} 08^{\prime} 05^{\prime \prime}$ & $96^{\circ} 06^{\prime} 20^{\prime \prime}$ & 8,538 & 1,290 & 4.63 & 81 & 476 & 2.5 & 5.88 & 239.21 \\
\hline 08063100 & Richland Cr nr Dawson, TX & $31^{\circ} 56^{\prime} 18^{\prime \prime}$ & $96^{\circ} 40^{\prime} 52^{\prime \prime}$ & 333 & 2.0 & 2.52 & 13.5 & 10.6 & .17 & .79 & 367.52 \\
\hline 08063800 & Waxahachie Cr nr Bardwell, TX & $32^{\circ} 14^{\prime} 36^{\prime \prime}$ & $96^{\circ} 38^{\prime} 24^{\prime \prime}$ & 178 & 1.2 & .56 & 1.5 & .44 & 2.77 & .29 & 370.18 \\
\hline 08064100 & Chambers Cr nr Rice, TX & $32^{\circ} 11^{\prime} 54^{\prime \prime}$ & $96^{\circ} 31^{\prime} 12^{\prime \prime}$ & 807 & 50 & 6.38 & 40 & 88 & .52 & 2.2 & 340 \\
\hline 08064700 & Tehuacana Cr nr Streetman, TX & $31^{\circ} 50^{\prime} 46^{\prime \prime}$ & $96^{\circ} 17^{\prime} 37^{\prime \prime}$ & 142 & 1.8 & 3.29 & 8.4 & 3.06 & .64 & .36 & 280.13 \\
\hline
\end{tabular}


Table 3. Median streamflow and associated hydraulic characteristics for streamflow-gaging stations in Texas-Continued

\begin{tabular}{|c|c|c|c|c|c|c|c|c|c|c|c|}
\hline \multirow[b]{2}{*}{$\begin{array}{c}\text { Station } \\
\text { no. }\end{array}$} & \multirow[b]{2}{*}{ Station name } & \multirow[b]{2}{*}{ Latitude } & \multirow[b]{2}{*}{ Longitude } & \multirow{2}{*}{$\begin{array}{l}\text { Contri- } \\
\text { buting } \\
\text { drainage } \\
\text { area } \\
\left(\mathrm{mi}^{2}\right)\end{array}$} & \multicolumn{6}{|c|}{ Characteristics for median streamflow } & \multirow[b]{2}{*}{$\begin{array}{l}\text { Elevation } \\
\text { of datum } \\
\text { of gage }\end{array}$} \\
\hline & & & & & $\begin{array}{l}\text { Stream- } \\
\text { flow } \\
\left(\mathrm{ft}^{3} / \mathrm{s}\right)\end{array}$ & $\begin{array}{c}\text { Gage } \\
\text { height } \\
\text { (ft) }\end{array}$ & $\begin{array}{c}\text { Stream } \\
\text { width } \\
\text { (ft) }\end{array}$ & $\begin{array}{c}\text { Cross } \\
\text { section } \\
\text { area } \\
\left(\mathrm{ft}^{2}\right)\end{array}$ & $\begin{array}{l}\text { Mean } \\
\text { velocity }\end{array}$ & $\begin{array}{l}\text { Mean } \\
\text { depth }\end{array}$ & \\
\hline 08065000 & Trinity R nr Oakwood, TX & $31^{\circ} 38^{\prime} 54^{\prime \prime}$ & $95^{\circ} 47^{\prime} 21^{\prime \prime}$ & 12,833 & 1,490 & 8.36 & 100 & 958 & 1.45 & 9.58 & 175.06 \\
\hline 08065200 & Upper Keechi Cr nr Oakwood, TX & $31^{\circ} 34^{\prime} 11^{\prime \prime}$ & $95^{\circ} 53^{\prime} 17^{\prime \prime}$ & 150 & 11 & 6.36 & 19.5 & 14.8 & .79 & .76 & 240.11 \\
\hline 08065350 & Trinity R nr Crockett, TX & $31^{\circ} 20^{\prime} 18^{\prime \prime}$ & $95^{\circ} 39^{\prime} 22^{\prime \prime}$ & 13,911 & 2,400 & 11 & 182 & 1,270 & 1.86 & 6.98 & 141.15 \\
\hline 08065800 & Bedias Cr nr Madisonville, TX & $30^{\circ} 53^{\prime} 03^{\prime \prime}$ & $95^{\circ} 46^{\prime} 39^{\prime \prime}$ & 321 & 9.0 & 4.66 & 12 & 5.65 & 1.6 & .47 & 150 \\
\hline 08066170 & Kickapoo Cr nr Onalaska, TX & $30^{\circ} 54^{\prime} 25^{\prime \prime}$ & $95^{\circ} 05^{\prime} 18^{\prime \prime}$ & 57 & 3.6 & 3.61 & 22 & 11.3 & .32 & .51 & 139.85 \\
\hline 08066200 & Long King $\mathrm{Cr}$ at Livingston, $\mathrm{TX}$ & $30^{\circ} 42^{\prime} 58^{\prime \prime}$ & $94^{\circ} 57^{\prime} 31^{\prime \prime}$ & 141 & 13 & 3.75 & 21 & 9.97 & 1.33 & .47 & 100.12 \\
\hline 08066250 & Trinity R nr Goodrich, TX & $30^{\circ} 34^{\prime} 19^{\prime \prime}$ & $94^{\circ} 56^{\prime} 55^{\prime \prime}$ & 16,844 & 2,770 & 8.6 & 265 & 2,380 & 1.16 & 8.98 & 40 \\
\hline 08066300 & Menard Cr nr Rye, TX & $30^{\circ} 28^{\prime} 52^{\prime \prime}$ & $94^{\circ} 46^{\prime} 46^{\prime \prime}$ & 152 & 50 & 9.26 & 34 & 63.4 & .79 & 1.86 & 62.32 \\
\hline 08066500 & Trinity R at Romayor, TX & $30^{\circ} 25^{\prime} 30^{\prime \prime}$ & $94^{\circ} 51^{\prime} 02^{\prime \prime}$ & 17,186 & 2,710 & 13.38 & 260 & 6,170 & .45 & 23.73 & 25.92 \\
\hline 08067000 & Trinity R at Liberty, TX & $30^{\circ} 03^{\prime} 27^{\prime \prime}$ & $94^{\circ} 49^{\prime} 05^{\prime \prime}$ & 17,468 & 20,400 & 25.51 & 455 & 10,200 & 1.98 & 22.42 & -2.22 \\
\hline 08067500 & Cedar Bayou nr Crosby, TX & $29^{\circ} 58^{\prime} 20^{\prime \prime}$ & $94^{\circ} 59^{\prime} 10^{\prime \prime}$ & 64.9 & 14 & 7.88 & 25.2 & 14 & .99 & .56 & 31.31 \\
\hline 08067650 & West Fk San Jacinto R below Lake Conroe nr Conroe, TX & $30^{\circ} 20^{\prime} 31 "$ & $95^{\circ} 23^{\prime} 34^{\prime \prime}$ & 451 & 8.8 & 14.34 & 24.2 & 13.8 & .64 & .57 & 116.06 \\
\hline 08068000 & West Fk San Jacinto R nr Conroe, TX & $30^{\circ} 14^{\prime} 40^{\prime \prime}$ & $95^{\circ} 27^{\prime} 25^{\prime \prime}$ & 828 & 95 & 3.66 & 62 & 71.05 & 1.35 & 1.15 & 95.03 \\
\hline 08068090 & West Fk San Jacinto R above Lake Houston nr Porter, TX & $30^{\circ} 05^{\prime} 09^{\prime \prime}$ & $95^{\circ} 17^{\prime} 59^{\prime \prime}$ & 962 & 115 & 11.03 & 88 & 111 & 1.04 & 1.26 & 33 \\
\hline 08068450 & Panther Branch nr Spring, TX & $30^{\circ} 077^{\prime} 51 "$ & $95^{\circ} 28^{\prime} 52^{\prime \prime}$ & 34.5 & 1.4 & 4.68 & 7.1 & 1.64 & .83 & .23 & 0 \\
\hline 08068500 & Spring Cr nr Spring, TX & $30^{\circ} 06^{\prime} 37^{\prime \prime}$ & $95^{\circ} 26^{\prime} 10^{\prime \prime}$ & 409 & 43 & 3.11 & 36 & 31.8 & 1.31 & .88 & 73.1 \\
\hline 08068720 & Cypress Cr at Katy-Hockley Rd nr Hockley, TX & $29^{\circ} 57^{\prime} 00^{\prime \prime}$ & $95^{\circ} 48^{\prime} 29^{\prime \prime}$ & 110 & 3.0 & 47.36 & 9.4 & 5.33 & .60 & .57 & 100 \\
\hline 08068740 & Cypress Cr at House-Hahl Rd nr Cypress, TX & $29^{\circ} 57^{\prime} 32^{\prime \prime}$ & $95^{\circ} 43^{\prime} 03^{\prime \prime}$ & 131 & 5.9 & 29.88 & 11 & 4.62 & 1.28 & .42 & 100 \\
\hline 08068780 & Little Cypress Cr nr Cypress, TX & $30^{\circ} 00^{\prime} 57^{\prime \prime}$ & $95^{\circ} 41^{\prime} 50^{\prime \prime}$ & 41 & .73 & 64.87 & 3.0 & .87 & .92 & .29 & 80 \\
\hline 08068800 & Cypress Cr at Grant Rd nr Cypress, TX & $29^{\circ} 58^{\prime} 24^{\prime \prime}$ & $95^{\circ} 35^{\prime} 54^{\prime \prime}$ & 214 & 9.9 & 25.02 & 24.7 & 13.9 & .69 & .56 & 80 \\
\hline 08068900 & Cypress $\mathrm{Cr}$ at Stuebner-Airline Rd nr Westfield, TX & $30^{\circ} 00^{\prime} 23^{\prime \prime}$ & $95^{\circ} 30^{\prime} 42^{\prime \prime}$ & 248 & 43 & 14.78 & 29.5 & 28.9 & 1.47 & .98 & 70 \\
\hline 08069000 & Cypress Cr nr Westfield, TX & $30^{\circ} 02^{\prime} 08^{\prime \prime}$ & $95^{\circ} 25^{\prime} 43^{\prime \prime}$ & 285 & 27 & 3.21 & 26.5 & 27.6 & 1.0 & 1.04 & 63.89 \\
\hline 08070000 & East Fk San Jacinto R nr Cleveland, TX & $30^{\circ} 20^{\prime} 11^{\prime \prime}$ & $95^{\circ} 06^{\prime} 14^{\prime \prime}$ & 325 & 50 & 4.92 & 50 & 54.4 & .88 & 1.09 & 107.98 \\
\hline 08070200 & East Fk San Jacinto R nr New Caney, TX & $30^{\circ} 08^{\prime} 43^{\prime \prime}$ & $95^{\circ} 07^{\prime} 27^{\prime \prime}$ & 388 & 87 & 4.96 & 40.5 & 75.6 & 1.18 & 1.87 & 43.98 \\
\hline 08070500 & Caney Cr nr Splendora, TX & $30^{\circ} 15^{\prime} 34^{\prime \prime}$ & $95^{\circ} 18^{\prime} 08^{\prime \prime}$ & 105 & 28 & 3.22 & 33 & 28.7 & 1.01 & .87 & 118.44 \\
\hline 08071000 & Peach Cr at Splendora, TX & $30^{\circ} 13^{\prime} 57^{\prime \prime}$ & $95^{\circ} 10^{\prime} 05^{\prime \prime}$ & 117 & 25 & 6.86 & 32 & 34.9 & .72 & 1.09 & 81.61 \\
\hline 08071280 & Luce Bayou above Lake Houston nr Huffman, TX & $30^{\circ} 06^{\prime} 34^{\prime \prime}$ & $95^{\circ} 03^{\prime} 35^{\prime \prime}$ & 218 & 9.6 & 8.96 & 17 & 13.5 & .72 & .79 & 39.91 \\
\hline 08072300 & Buffalo Bayou nr Katy, TX & $29^{\circ} 44^{\prime} 35^{\prime \prime}$ & $95^{\circ} 48^{\prime} 24^{\prime \prime}$ & 63.3 & 8.0 & 22.72 & 19.8 & 23.3 & .35 & 1.18 & 75.02 \\
\hline 08072730 & Bear Cr nr Barker, TX & $29^{\circ} 49^{\prime} 50^{\prime \prime}$ & $95^{\circ} 41^{\prime} 12^{\prime \prime}$ & 21.5 & 1.9 & 2.75 & 7.7 & 2.14 & .91 & .28 & 100 \\
\hline 08072760 & Langham Cr at West Little York Rd nr Addicks, TX ( 2 yrs. data) & $29^{\circ} 52^{\prime} 01^{\prime \prime}$ & $95^{\circ} 38^{\prime} 47^{\prime \prime}$ & 24.6 & 10 & 14.96 & 16.4 & 11.7 & .81 & .71 & 90 \\
\hline 08073500 & Buffalo Bayou nr Addicks, TX & $29^{\circ} 45^{\prime} 42^{\prime \prime}$ & $95^{\circ} 36^{\prime} 20^{\prime \prime}$ & 293 & 49 & 50.19 & 40 & 43 & 1.15 & 1.08 & -1.4 \\
\hline 08073600 & Buffalo Bayou at West Belt Dr at Houston, TX & $29^{\circ} 45^{\prime} 43^{\prime \prime}$ & $95^{\circ} 33^{\prime} 27^{\prime \prime}$ & 307 & 110 & 39.98 & 36.6 & 56.5 & 1.96 & 1.54 & -.67 \\
\hline 08073700 & Buffalo Bayou at Piney Point, TX & $29^{\circ} 44^{\prime} 48^{\prime \prime}$ & $95^{\circ} 31^{\prime} 24^{\prime \prime}$ & 317 & 108 & 33.24 & 39 & 91.8 & 1.15 & 2.35 & -1.35 \\
\hline 08074150 & Cole Cr at Deihl Rd, Houston, TX & $29^{\circ} 51^{\prime} 04^{\prime \prime}$ & $95^{\circ} 29^{\prime} 16^{\prime \prime}$ & 7.5 & 1.6 & 67.6 & 14 & 4.4 & .36 & .31 & 0 \\
\hline 08074250 & Brickhouse Gully at Costa Rica St, Houston, TX & $29^{\circ} 49^{\prime} 40^{\prime \prime}$ & $95^{\circ} 28^{\prime} 09^{\prime \prime}$ & 11.4 & 3.5 & 53.38 & 19.1 & 8.2 & .42 & .43 & 0 \\
\hline
\end{tabular}


Table 3. Median streamflow and associated hydraulic characteristics for streamflow-gaging stations in Texas-Continued

\begin{tabular}{|c|c|c|c|c|c|c|c|c|c|c|c|}
\hline \multirow[b]{2}{*}{$\begin{array}{c}\text { Station } \\
\text { no. }\end{array}$} & \multirow[b]{2}{*}{ Station name } & \multirow[b]{2}{*}{ Latitude } & \multirow[b]{2}{*}{ Longitude } & \multirow{2}{*}{$\begin{array}{l}\text { Contri- } \\
\text { buting } \\
\text { drainage } \\
\text { area } \\
\left(\mathrm{mi}^{2}\right)\end{array}$} & \multicolumn{6}{|c|}{ Characteristics for median streamflow } & \multirow[b]{2}{*}{$\begin{array}{l}\text { Elevation } \\
\text { of datum } \\
\text { of gage }\end{array}$} \\
\hline & & & & & $\begin{array}{c}\text { Stream- } \\
\text { flow } \\
\left(\mathrm{ft}^{3} / \mathrm{s}\right)\end{array}$ & $\begin{array}{c}\text { Gage } \\
\text { height } \\
\text { (ft) }\end{array}$ & $\begin{array}{c}\text { Stream } \\
\text { width } \\
\text { (ft) }\end{array}$ & $\begin{array}{c}\text { Cross } \\
\text { section } \\
\text { area } \\
\left(\mathrm{ft}^{2}\right)\end{array}$ & $\begin{array}{l}\text { Mean } \\
\text { velocity }\end{array}$ & $\begin{array}{l}\text { Mean } \\
\text { depth }\end{array}$ & \\
\hline 08074500 & Whiteoak Bayou at Houston, TX & $29^{\circ} 46^{\prime} 30^{\prime \prime}$ & $95^{\circ} 23^{\prime} 49^{\prime \prime}$ & 86.3 & 29 & 16.7 & 12.4 & 14.8 & 2.03 & 1.19 & -7.35 \\
\hline 08074800 & Keegans Bayou at Roark Rd nr Houston, TX & $29^{\circ} 39^{\prime} 23 "$ & $95^{\circ} 33^{\prime} 43^{\prime \prime}$ & 12.7 & 4.1 & 61.88 & 9.5 & 3.55 & 1.18 & .37 & 0 \\
\hline 08074810 & Brays Bayou at Gessner Dr, Houston, TX & $29^{\circ} 40^{\prime} 21^{\prime \prime}$ & $95^{\circ} 31^{\prime} 41^{\prime \prime}$ & 52.5 & 72 & 43.17 & 51.4 & 44.3 & 1.63 & .86 & 0 \\
\hline 08075000 & Brays Bayou at Houston, TX & $29^{\circ} 41^{\prime} 49^{\prime \prime}$ & $95^{\circ} 24^{\prime} 43^{\prime \prime}$ & 94.9 & 67 & 25.4 & 63 & 73.4 & .98 & 1.17 & -7.16 \\
\hline 08075400 & Sims Bayou at Hiram Clarke St, Houston, TX & $29^{\circ} 37^{\prime} 07^{\prime \prime}$ & $95^{\circ} 26^{\prime} 45^{\prime \prime}$ & 20.16 & 12 & 35.41 & 19.6 & 15 & .84 & .77 & 0 \\
\hline 08075500 & Sims Bayou at Houston, TX & $29^{\circ} 40^{\prime} 27^{\prime \prime}$ & $95^{\circ} 17^{\prime} 21 "$ & 63 & 40 & 2.9 & 51 & 33.6 & 1.17 & .66 & -3.09 \\
\hline 08075730 & Vince Bayou at Pasadena, TX & $29^{\circ} 41^{\prime} 40^{\prime \prime}$ & $95^{\circ} 12^{\prime} 58^{\prime \prime}$ & 8.26 & 2.2 & 8.27 & 26.4 & 2.98 & .72 & .11 & -2.54 \\
\hline 08075770 & Hunting Bayou at IH-610, Houston, TX & $29^{\circ} 47^{\prime} 35^{\prime \prime}$ & $95^{\circ} 16^{\prime} 04^{\prime \prime}$ & 16.1 & 7.0 & 21.16 & 16.5 & 6.84 & 1.0 & .41 & 0 \\
\hline 08075900 & Greens Bayou nr US 75 nr Houston, TX & $29^{\circ} 57^{\prime} 24^{\prime \prime}$ & $95^{\circ} 25^{\prime} 04^{\prime \prime}$ & 36.6 & 15 & 70.66 & 25 & 11.8 & 1.25 & .47 & 0 \\
\hline 08076000 & Greens Bayou nr Houston, TX & $29^{\circ} 55^{\prime} 05^{\prime \prime}$ & $95^{\circ} 18^{\prime} 24^{\prime \prime}$ & 68.7 & 24 & 44.6 & 29.5 & 23.2 & 1.03 & .79 & -.66 \\
\hline 08076180 & Garners Bayou nr Humble, TX & $29^{\circ} 56^{\prime} 03^{\prime \prime}$ & $95^{\circ} 14^{\prime} 02^{\prime \prime}$ & 31 & 12 & 36.38 & 15 & 13 & .95 & .87 & 0 \\
\hline 08076500 & Halls Bayou at Houston, TX & $29^{\circ} 51^{\prime} 42^{\prime \prime}$ & $95^{\circ} 20^{\prime} 05^{\prime \prime}$ & 28.7 & 9.0 & 45.9 & 31 & 22.8 & .39 & .74 & -.66 \\
\hline 08076700 & Greens Bayou at Ley Rd, Houston, TX & $29^{\circ} 50^{\prime} 13^{\prime \prime}$ & $95^{\circ} 13^{\prime} 59^{\prime \prime}$ & 182 & 322 & 4.96 & 57 & 237 & 1.31 & 4.16 & -2.13 \\
\hline 08078000 & Chocolate Bayou nr Alvin, TX & $29^{\circ} 22^{\prime} 09^{\prime \prime}$ & $95^{\circ} 19^{\prime} 14^{\prime \prime}$ & 87.7 & 32 & 11.2 & 21.6 & 25.2 & 1.3 & 1.17 & .31 \\
\hline 08079600 & Double Mountain Fk Brazos R at Justiceburg, TX & $33^{\circ} 02^{\prime} 18^{\prime \prime}$ & $101^{\circ} 11^{\prime} 50^{\prime \prime}$ & 244 & .02 & 5.5 & .89 & .03 & .70 & .03 & $2,222.47$ \\
\hline 08080500 & Double Mountain Fk Brazos R nr Aspermont, TX & $33^{\circ} 00^{\prime} 29^{\prime \prime}$ & $100^{\circ} 10^{\prime} 49^{\prime \prime}$ & 1,864 & 1.5 & 1.36 & 8.5 & 2.54 & .55 & .3 & $1,624.79$ \\
\hline 08082000 & Salt Fk Brazos R nr Aspermont, TX & $33^{\circ} 20^{\prime} 02^{\prime \prime}$ & $100^{\circ} 14^{\prime} 16^{\prime \prime}$ & 2,496 & 7.1 & 2.65 & 27.5 & 9.32 & .77 & .34 & $1,588.7$ \\
\hline 08082500 & Brazos R at Seymour, TX & $33^{\circ} 34^{\prime} 51^{\prime \prime}$ & $99^{\circ} 16^{\prime} 02^{\prime \prime}$ & 5,972 & 50 & 1.97 & 101 & 46 & 1.12 & .46 & $1,238.97$ \\
\hline 08082700 & Millers Cr nr Munday, TX & $33^{\circ} 19^{\prime} 45^{\prime \prime}$ & $99^{\circ} 27^{\prime} 53^{\prime \prime}$ & 104 & 0 & -- & -- & -- & -- & -- & 1,350 \\
\hline 08083100 & Clear Fk Brazos R nr Roby, TX & $32^{\circ} 47^{\prime} 15^{\prime \prime}$ & $100^{\circ} 23^{\prime} 18^{\prime \prime}$ & 228 & 1.6 & 2.45 & 3.0 & 1.42 & 1.1 & .47 & $1,885.09$ \\
\hline 08083420 & Cat Claw $\mathrm{Cr}$ at Abilene, $\mathrm{TX}$ & $32^{\circ} 28^{\prime} 31^{\prime \prime}$ & $99^{\circ} 44^{\prime} 56^{\prime \prime}$ & 13 & 0 & -- & -- & -- & -- & -- & $1,682.32$ \\
\hline 08084000 & Clear Fk Brazos R at Nugent, TX & $32^{\circ} 41^{\prime} 24^{\prime \prime}$ & $99^{\circ} 40^{\prime} 09^{\prime \prime}$ & 2,199 & 12 & 1.95 & 48.5 & 18.8 & .65 & .39 & $1,531.91$ \\
\hline 08084800 & California Cr nr Stamford, TX & $32^{\circ} 55^{\prime} 51^{\prime \prime}$ & $99^{\circ} 38^{\prime} 32^{\prime \prime}$ & 478 & 2.6 & 6.49 & 8.2 & 2.12 & 1.18 & .26 & 1,470 \\
\hline 08085500 & Clear Fk Brazos R at Fort Griffin, TX & $32^{\circ} 56^{\prime} 04^{\prime \prime}$ & $99^{\circ} 13^{\prime} 27^{\prime \prime}$ & 3,988 & 25 & 2.45 & 28 & 13 & 2.0 & .46 & $1,174.09$ \\
\hline 08086212 & Hubbard Cr below Albany, TX & $32^{\circ} 43^{\prime} 58^{\prime \prime}$ & $99^{\circ} 08^{\prime} 15^{\prime \prime}$ & 613 & 1.3 & 3.37 & 12 & 2.67 & .49 & .22 & $1,184.99$ \\
\hline 08086290 & Big Sandy Cr above Breckenridge, TX & $32^{\circ} 38^{\prime} 54^{\prime \prime}$ & $99^{\circ} 00^{\prime} 15^{\prime \prime}$ & 280 & .08 & .82 & 1.2 & .07 & 1.25 & .06 & $1,185.83$ \\
\hline 08088000 & Brazos R nr South Bend, TX & $33^{\circ} 01^{\prime} 27^{\prime \prime}$ & $98^{\circ} 38^{\prime} 37^{\prime \prime}$ & 22,673 & 131 & 4.06 & 112 & 106 & 1.16 & .94 & $1,002.98$ \\
\hline 08088610 & Brazos R nr Graford, TX & $32^{\circ} 51^{\prime} 29^{\prime \prime}$ & $98^{\circ} 14^{\prime} 41^{\prime \prime}$ & 14,030 & 264 & 66.92 & 100 & 243 & 1.19 & 2.43 & 800 \\
\hline 08089000 & Brazos R nr Palo Pinto, TX & $32^{\circ} 51^{\prime} 45^{\prime \prime}$ & $98^{\circ} 18^{\prime} 08^{\prime \prime}$ & 14,245 & 216 & 2.68 & 108 & 110 & 2.03 & 1.02 & 834.23 \\
\hline 08090800 & Brazos R nr Dennis, TX & $32^{\circ} 36^{\prime} 56^{\prime \prime}$ & $97^{\circ} 55^{\prime} 32^{\prime \prime}$ & 15,671 & 281 & 3.08 & 240 & 982 & .28 & 4.09 & 697.67 \\
\hline 08091000 & Brazos R nr Glen Rose, TX & $32^{\circ} 16^{\prime} 18^{\prime \prime}$ & $97^{\circ} 39^{\prime} 48^{\prime \prime}$ & 16,252 & 353 & 5.71 & 218 & 281 & 1.17 & 1.29 & 567.82 \\
\hline 08091500 & Paluxy R at Glen Rose, TX & $32^{\circ} 13^{\prime} 53^{\prime \prime}$ & $97^{\circ} 46^{\prime} 37^{\prime \prime}$ & 410 & 21 & 2.13 & 77 & 37.7 & .60 & .49 & 609.66 \\
\hline 08091750 & Squaw Cr nr Glen Rose, TX & $32^{\circ} 16^{\prime} 12^{\prime \prime}$ & $97^{\circ} 43^{\prime} 56^{\prime \prime}$ & 70.3 & 4.0 & 2.2 & 20 & 6.2 & .63 & .31 & 599 \\
\hline 08092000 & Nolan R at Blum, TX & $32^{\circ} 09^{\prime} 02^{\prime \prime}$ & $97^{\circ} 24^{\prime} 09^{\prime \prime}$ & 282 & 12 & 1.91 & 67 & 24.7 & .43 & .37 & 551.48 \\
\hline 08093100 & Brazos R nr Aquilla, TX & $31^{\circ} 48^{\prime} 44^{\prime \prime}$ & $97^{\circ} 17^{\prime} 51^{\prime \prime}$ & 17,678 & 633 & 7.69 & 241 & 634 & 1.05 & 2.63 & 404.29 \\
\hline
\end{tabular}


Table 3. Median streamflow and associated hydraulic characteristics for streamflow-gaging stations in Texas-Continued

\begin{tabular}{|c|c|c|c|c|c|c|c|c|c|c|c|}
\hline \multirow[b]{2}{*}{$\begin{array}{c}\text { Station } \\
\text { no. }\end{array}$} & \multirow[b]{2}{*}{ Station name } & \multirow[b]{2}{*}{ Latitude } & \multirow[b]{2}{*}{ Longitude } & \multirow{2}{*}{$\begin{array}{l}\text { Contri- } \\
\text { buting } \\
\text { drainage } \\
\text { area } \\
\left(\mathrm{mi}^{2}\right)\end{array}$} & \multicolumn{6}{|c|}{ Characteristics for median streamflow } & \multirow[b]{2}{*}{$\begin{array}{l}\text { Elevation } \\
\text { of datum } \\
\text { of gage }\end{array}$} \\
\hline & & & & & $\begin{array}{l}\text { Stream- } \\
\text { flow } \\
\left(\mathrm{ft}^{3} / \mathrm{s}\right)\end{array}$ & $\begin{array}{c}\text { Gage } \\
\text { height } \\
\text { (ft) }\end{array}$ & $\begin{array}{c}\text { Stream } \\
\text { width } \\
\text { (ft) }\end{array}$ & $\begin{array}{l}\text { Cross } \\
\text { section } \\
\text { area } \\
\left(\mathrm{ft}^{2}\right)\end{array}$ & $\begin{array}{c}\text { Mean } \\
\text { velocity }\end{array}$ & $\begin{array}{l}\text { Mean } \\
\text { depth }\end{array}$ & \\
\hline 08093500 & Aquilla $\mathrm{Cr}$ nr Aquilla, TX & $31^{\circ} 50^{\prime} 40^{\prime \prime}$ & $97^{\circ} 12^{\prime} 04^{\prime \prime}$ & 308 & 6.0 & 4.38 & 7.8 & 3.7 & 1.66 & 0.47 & 451.48 \\
\hline 08094800 & North Bosque R at Hico, TX & $31^{\circ} 58^{\prime} 41^{\prime \prime}$ & $98^{\circ} 02^{\prime} 04^{\prime \prime}$ & 359 & 5.5 & .64 & 32.5 & 13.6 & .39 & .42 & 982.46 \\
\hline 08095000 & North Bosque R nr Clifton, TX & $31^{\circ} 47^{\prime} 09^{\prime \prime}$ & $97^{\circ} 34^{\prime} 04^{\prime \prime}$ & 968 & 29 & 1.93 & 53 & 32.8 & .86 & .62 & 605.43 \\
\hline 08095200 & North Bosque R at Valley Mills, TX & $31^{\circ} 40^{\prime} 10^{\prime \prime}$ & $97^{\circ} 28^{\prime} 09^{\prime \prime}$ & 1,146 & 43 & 3.66 & 57.5 & 40.1 & 1.07 & .70 & 524.55 \\
\hline 08095300 & Middle Bosque R nr McGregor, TX & $31^{\circ} 30^{\prime} 33^{\prime \prime}$ & $97^{\circ} 21^{\prime} 56^{\prime \prime}$ & 182 & 20 & 2.8 & 31.8 & 15.8 & 1.27 & .50 & 530.51 \\
\hline 08095400 & Hog Cr nr Crawford, TX & $31^{\circ} 33^{\prime} 20^{\prime \prime}$ & $97^{\circ} 21^{\prime} 22^{\prime \prime}$ & 78.2 & 4.3 & 2.14 & 30 & 15 & .30 & .50 & 560.54 \\
\hline 08096500 & Brazos R at Waco, TX & $31^{\circ} 32^{\prime} 09^{\prime \prime}$ & $97^{\circ} 04^{\prime} 23^{\prime \prime}$ & 20,007 & 844 & 4.99 & 308 & 670 & 1.25 & 2.18 & 349.34 \\
\hline 08098290 & Brazos R nr Highbank, TX & $31^{\circ} 08^{\prime} 02^{\prime \prime}$ & $96^{\circ} 49^{\prime} 29^{\prime \prime}$ & 20,870 & 1,040 & 3.84 & 345 & 564 & 1.88 & 1.63 & 249.29 \\
\hline 08099100 & Leon R nr De Leon, TX & $32^{\circ} 10^{\prime} 25^{\prime \prime}$ & $98^{\circ} 31^{\prime} 58^{\prime \prime}$ & 479 & 1.0 & 1.5 & 12 & 2.81 & .32 & .23 & $1,209.93$ \\
\hline 08099300 & Sabana R nr De Leon, TX & $32^{\circ} 06 ' 50^{\prime \prime}$ & $98^{\circ} 36^{\prime} 19^{\prime \prime}$ & 264 & 1.3 & 2.82 & 17.5 & 4.4 & .29 & .25 & $1,209.59$ \\
\hline 08100000 & Leon R nr Hamilton, TX & $31^{\circ} 47^{\prime} 19^{\prime \prime}$ & $98^{\circ} 07^{\prime} 16^{\prime \prime}$ & 1,891 & 18 & 5.83 & 24 & 18.3 & 1.03 & .76 & 955.38 \\
\hline 08100500 & Leon R at Gatesville, TX & $31^{\circ} 25^{\prime} 58^{\prime \prime}$ & $97^{\circ} 45^{\prime} 42^{\prime \prime}$ & 2,342 & 48 & 2.64 & 38 & 40 & 1.24 & 1.05 & 723.85 \\
\hline 08101000 & Cowhouse $\mathrm{Cr}$ at Pidcoke, TX & $31^{\circ} 17^{\prime} 05^{\prime \prime}$ & $97^{\circ} 53^{\prime} 05^{\prime \prime}$ & 455 & 6.5 & 3.48 & 18.9 & 8.74 & .70 & .46 & 736.71 \\
\hline 08102500 & Leon R nr Belton, TX & $31^{\circ} 04^{\prime} 12^{\prime \prime}$ & $97^{\circ} 26^{\prime} 28^{\prime \prime}$ & 3,542 & 44 & 3.82 & 60 & 35.8 & 1.14 & .60 & 476.68 \\
\hline 08103800 & Lampasas R nr Kempner, TX & $31^{\circ} 04^{\prime} 54^{\prime \prime}$ & $98^{\circ} 00^{\prime} 59^{\prime \prime}$ & 818 & 32 & 3.53 & 59 & 25.8 & 1.25 & .44 & 828.38 \\
\hline 08103900 & South Fk Rocky Cr nr Briggs, TX & $30^{\circ} 54^{\prime} 41^{\prime \prime}$ & $98^{\circ} 02^{\prime} 12^{\prime \prime}$ & 33.3 & .71 & 1.18 & 5.0 & 1.01 & .66 & .20 & 955.8 \\
\hline 08104100 & Lampasas R nr Belton, TX & $31^{\circ} 00^{\prime} 06^{\prime \prime}$ & $97^{\circ} 29^{\prime} 32^{\prime \prime}$ & 1,321 & 13 & 5.17 & 28 & 11.3 & 1.18 & .40 & 476.58 \\
\hline 08104500 & Little R nr Little River, TX & $30^{\circ} 57^{\prime} 59^{\prime \prime}$ & $97^{\circ} 20^{\prime} 45^{\prime \prime}$ & 5,228 & 272 & 3.31 & 85 & 167 & 1.61 & 1.96 & 400.11 \\
\hline 08104700 & North Fk San Gabriel R nr Georgetown, TX & $30^{\circ} 39^{\prime} 42^{\prime \prime}$ & $97^{\circ} 42^{\prime} 40^{\prime \prime}$ & 248 & 6.8 & 4.2 & 11.2 & 4.6 & 1.46 & .41 & 689.06 \\
\hline 08104900 & South Fk San Gabriel R at Georgetown, TX & $30^{\circ} 37^{\prime} 32^{\prime \prime}$ & $97^{\circ} 41^{\prime} 27^{\prime \prime}$ & 133 & 13 & 2.12 & 21 & 11.6 & 1.06 & .55 & 687.72 \\
\hline 08105100 & Berry Cr nr Georgetown, TX & $30^{\circ} 41^{\prime 2} 28^{\prime \prime}$ & $97^{\circ} 39^{\prime} 21^{\prime \prime}$ & 83.1 & 4.8 & 2.95 & 20 & 3.89 & 1.21 & .19 & 659.97 \\
\hline 08105700 & San Gabriel R at Laneport, TX & $30^{\circ} 41^{\prime} 39^{\prime \prime}$ & $97^{\circ} 16^{\prime} 43^{\prime \prime}$ & 738 & 36 & 5.34 & 40 & 25.8 & 1.48 & .65 & 412.6 \\
\hline 08106350 & Little R nr Rockdale, TX & $30^{\circ} 45^{\prime} 38^{\prime \prime}$ & $97^{\circ} 00^{\prime} 49^{\prime \prime}$ & 6,959 & 185 & 4.86 & 60.5 & 87.1 & 2.05 & 1.44 & 299.12 \\
\hline 08106500 & Little $\mathrm{R}$ at Cameron, $\mathrm{TX}$ & $30^{\circ} 50^{\prime} 06^{\prime \prime}$ & $96^{\circ} 56^{\prime} 47^{\prime \prime}$ & 7,065 & 498 & 3.84 & 99 & 505 & .97 & 5.1 & 281.89 \\
\hline 08108700 & Brazos R at SH $21 \mathrm{nr}$ Bryan, TX & $30^{\circ} 37^{\prime} 36^{\prime \prime}$ & $96^{\circ} 32^{\prime} 38^{\prime \prime}$ & 29,483 & 1,540 & 10.33 & 215 & 1,400 & 1.04 & 6.51 & 188.65 \\
\hline 08109700 & Middle Yegua Cr nr Dime Box, TX & $30^{\circ} 20^{\prime} 21^{\prime \prime}$ & $96^{\circ} 54^{\prime} 16^{\prime \prime}$ & 236 & 6.1 & 2.69 & 8.7 & 7.27 & .87 & .84 & 295.4 \\
\hline 08109800 & East Yegua Cr nr Dime Box, TX & $30^{\circ} 24^{\prime} 26^{\prime \prime}$ & $96^{\circ} 49^{\prime} 02^{\prime \prime}$ & 244 & 11 & 1.91 & 18.3 & 23.5 & .51 & 1.28 & 284 \\
\hline 08110100 & Davidson Cr nr Lyons, TX & $30^{\circ} 25^{\prime} 10^{\prime \prime}$ & $96^{\circ} 32^{\prime} 24^{\prime \prime}$ & 195 & 2.6 & 2.97 & 11.6 & 4.1 & .60 & .35 & 220.26 \\
\hline 08110325 & Navasota $R$ above Groesbeck, TX & $31^{\circ} 34^{\prime} 27^{\prime \prime}$ & $96^{\circ} 31^{\prime} 14^{\prime \prime}$ & 239 & 1.1 & 1.63 & 8.0 & 1.7 & .61 & .21 & 396.65 \\
\hline 08110430 & Big Cr nr Freestone, TX & $31^{\circ} 30^{\prime} 24^{\prime \prime}$ & $96^{\circ} 19^{\prime} 28^{\prime \prime}$ & 97.2 & 3.3 & 3.65 & 10.8 & 5.4 & .62 & .50 & 362.94 \\
\hline 08110500 & Navasota R nr Easterly, TX & $31^{\circ} 10^{\prime} 12^{\prime \prime}$ & $96^{\circ} 17^{\prime} 51^{\prime \prime}$ & 968 & 29 & 3.97 & 27 & 25.2 & 1.1 & .93 & 271.46 \\
\hline 08110800 & Navasota $R$ at Old Spanish Rd nr Bryan, TX & $30^{\circ} 58^{\prime} 25^{\prime \prime}$ & $96^{\circ} 14^{\prime} 29^{\prime \prime}$ & 1,287 & 84 & 3.41 & 44.5 & 91.7 & .93 & 2.06 & 245 \\
\hline 08111500 & Brazos R nr Hempstead, TX & $30^{\circ} 07^{\prime} 44^{\prime \prime}$ & $96^{\circ} 11^{\prime} 15^{\prime \prime}$ & 34,314 & 2,530 & 14.74 & 280 & 2,940 & .87 & 10.5 & 107.9 \\
\hline 08111700 & Mill Cr nr Bellville, TX & $29^{\circ} 52^{\prime} 51^{\prime \prime}$ & $96^{\circ} 12^{\prime} 18^{\prime \prime}$ & 376 & 36 & 4.83 & 25.5 & 24.8 & 1.42 & .97 & 122.82 \\
\hline 08114000 & Brazos R at Richmond, TX & $29^{\circ} 34^{\prime} 56^{\prime \prime}$ & $95^{\circ} 45^{\prime} 27^{\prime \prime}$ & 35,441 & 2,950 & 12.96 & 242 & 2,580 & 1.15 & 10.66 & 27.94 \\
\hline
\end{tabular}


Table 3. Median streamflow and associated hydraulic characteristics for streamflow-gaging stations in Texas-Continued

\begin{tabular}{|c|c|c|c|c|c|c|c|c|c|c|c|}
\hline \multirow[b]{2}{*}{$\begin{array}{c}\text { Station } \\
\text { no. }\end{array}$} & \multirow[b]{2}{*}{ Station name } & \multirow[b]{2}{*}{ Latitude } & \multirow[b]{2}{*}{ Longitude } & \multirow{2}{*}{$\begin{array}{l}\text { Contri- } \\
\text { buting } \\
\text { drainage } \\
\text { area } \\
\left(\mathrm{mi}^{2}\right)\end{array}$} & \multicolumn{6}{|c|}{ Characteristics for median streamflow } & \multirow[b]{2}{*}{$\begin{array}{l}\text { Elevation } \\
\text { of datum } \\
\text { of gage }\end{array}$} \\
\hline & & & & & $\begin{array}{c}\text { Stream- } \\
\text { flow } \\
\left(\mathrm{ft}^{3} / \mathrm{s}\right)\end{array}$ & $\begin{array}{c}\text { Gage } \\
\text { height } \\
\text { (ft) }\end{array}$ & $\begin{array}{c}\text { Stream } \\
\text { width } \\
\text { (ft) }\end{array}$ & $\begin{array}{c}\text { Cross } \\
\text { section } \\
\text { area } \\
\left(\mathrm{ft}^{2}\right)\end{array}$ & $\begin{array}{l}\text { Mean } \\
\text { velocity }\end{array}$ & $\begin{array}{l}\text { Mean } \\
\text { depth }\end{array}$ & \\
\hline 08115000 & Big Cr nr Needville, TX & $29^{\circ} 28^{\prime} 35^{\prime \prime}$ & $95^{\circ} 48^{\prime} 45^{\prime \prime}$ & 42.8 & 1.7 & 6.44 & 9.43 & 3.53 & 0.48 & 0.37 & 59.39 \\
\hline 08116650 & Brazos R nr Rosharon, TX & $29^{\circ} 20^{\prime} 58^{\prime \prime}$ & $95^{\circ} 34^{\prime} 56^{\prime \prime}$ & 35,773 & 3,430 & 11.78 & 170 & 1,580 & 2.18 & 9.29 & 0 \\
\hline 08117500 & San Bernard R nr Boling, TX & $29^{\circ} 18^{\prime} 48^{\prime \prime}$ & $95^{\circ} 53^{\prime} 37^{\prime \prime}$ & 727 & 126 & 4.4 & 57.5 & 117 & 1.04 & 2.03 & 30.81 \\
\hline 08117995 & Colorado R nr Gail, TX & $32^{\circ} 37^{\prime} 43^{\prime \prime}$ & $101^{\circ} 17^{\prime} 06^{\prime \prime}$ & 498 & 0 & -- & -- & -- & -- & -- & 2,240 \\
\hline 08120700 & Colorado R nr Cuthbert, TX & $32^{\circ} 28^{\prime} 38^{\prime \prime}$ & $100^{\circ} 56^{\prime} 58^{\prime \prime}$ & 1,531 & 4.0 & 2.13 & 11 & 4.58 & .82 & .42 & $2,073.49$ \\
\hline 08121000 & Colorado R at Colorado City, TX & $32^{\circ} 23^{\prime} 33^{\prime \prime}$ & $100^{\circ} 52^{\prime} 42^{\prime \prime}$ & 1,585 & .52 & 2.56 & 3.0 & .60 & .82 & .20 & $2,030.16$ \\
\hline 08123800 & Beals Cr nr Westbrook, TX & $32^{\circ} 11^{\prime} 57^{\prime \prime}$ & $101^{\circ} 00^{\prime} 49^{\prime \prime}$ & 1,988 & 2.1 & 1.94 & 10.4 & 3.82 & .58 & .37 & $2,048.74$ \\
\hline 08123850 & Colorado R above Silver, TX & $32^{\circ} 03^{\prime} 13^{\prime \prime}$ & $100^{\circ} 45^{\prime} 42^{\prime \prime}$ & 4,650 & 8.7 & 1.92 & 33 & 20.4 & .42 & .62 & $1,907.66$ \\
\hline 08124000 & Colorado R at Robert Lee, TX & $31^{\circ} 53^{\prime} 07^{\prime \prime}$ & $100^{\circ} 28^{\prime} 49^{\prime \prime}$ & 5,047 & .69 & 1.14 & 5.3 & 1.26 & .58 & .24 & $1,771.7$ \\
\hline 08126380 & Colorado R nr Ballinger, TX & $31^{\circ} 42^{\prime} 55^{\prime \prime}$ & $100^{\circ} 01^{\prime} 34^{\prime \prime}$ & 6,098 & 14 & 4.29 & 34 & 14.1 & 1.0 & .41 & $1,606.51$ \\
\hline 08127000 & Elm Cr at Ballinger, TX & $31^{\circ} 44^{\prime} 57^{\prime \prime}$ & $99^{\circ} 56^{\prime} 51^{\prime \prime}$ & 450 & 2.7 & 3.84 & 21 & 3.73 & .66 & .18 & $1,617.72$ \\
\hline 08128000 & South Concho R at Christoval, TX & $31^{\circ} 11^{\prime} 13^{\prime \prime}$ & $100^{\circ} 30^{\prime} 06^{\prime \prime}$ & 354 & 14 & 1.97 & 16.5 & 14.2 & .98 & .86 & $2,010.22$ \\
\hline 08128400 & Middle Concho R above Tankersley, TX & $31^{\circ} 25^{\prime} 38^{\prime \prime}$ & $100^{\circ} 42^{\prime} 39^{\prime \prime}$ & 1,116 & 1.5 & 6.44 & 5.7 & 1.5 & .99 & .26 & $1,986.47$ \\
\hline 08129300 & Spring Cr above Tankersley, TX & $31^{\circ} 19^{\prime} 48^{\prime \prime}$ & $100^{\circ} 38^{\prime} 14^{\prime \prime}$ & 405 & 5.8 & 4.04 & 15.5 & 6.14 & .94 & .40 & $1,964.72$ \\
\hline 08130500 & Dove $\mathrm{Cr}$ at Knickerbocker, $\mathrm{TX}$ & $31^{\circ} 16^{\prime} 26^{\prime \prime}$ & $100^{\circ} 37^{\prime} 50^{\prime \prime}$ & 218 & 10 & 3.13 & 17 & 14.4 & .69 & .85 & $2,001.45$ \\
\hline 08133500 & North Concho R at Sterling City, TX & $31^{\circ} 49^{\prime} 48^{\prime \prime}$ & $100^{\circ} 59^{\prime} 36^{\prime \prime}$ & 568 & 0 & -- & -- & -- & -- & -- & $2,242.36$ \\
\hline 08134000 & North Concho R nr Carlsbad, TX & $31^{\circ} 35^{\prime} 33^{\prime \prime}$ & $100^{\circ} 38^{\prime} 12^{\prime \prime}$ & 1,191 & 1.5 & 4.88 & 12 & 2.36 & .69 & .20 & $1,968.02$ \\
\hline 08136000 & Concho R at San Angelo, TX & $31^{\circ} 2716^{\prime \prime}$ & $100^{\prime \circ} 24^{\prime} 37^{\prime \prime}$ & 4,411 & 7.3 & 1.6 & 7.0 & 3.9 & 1.91 & .56 & $1,776.79$ \\
\hline 08136500 & Concho R at Paint Rock, TX & $31^{\circ} 30^{\prime} 57^{\prime \prime}$ & $99^{\circ} 55^{\prime} 09^{\prime \prime}$ & 6,574 & 26 & 12.84 & 32 & 23.6 & 1.15 & .74 & $1,574.36$ \\
\hline 08136700 & Colorado R nr Stacy, TX & $31^{\circ} 29^{\prime} 37^{\prime \prime}$ & $99^{\circ} 34^{\prime} 25^{\prime \prime}$ & 12,802 & 46 & 4.47 & 51 & 28.2 & 1.73 & .55 & $1,394.66$ \\
\hline 08138000 & Colorado R at Winchell, TX & $31^{\circ} 28^{\prime} 04^{\prime \prime}$ & $99^{\circ} 09^{\prime} 43^{\prime \prime}$ & 25,179 & 60 & 2.91 & 131 & 81.3 & .74 & .62 & $1,264.86$ \\
\hline 08143600 & Pecan Bayou nr Mullin, TX & $31^{\circ} 31^{\prime} 02^{\prime \prime}$ & $98^{\circ} 44^{\prime} 25^{\prime \prime}$ & 2,073 & 15 & .92 & 29.2 & 20.7 & .73 & .71 & $1,202.93$ \\
\hline 08144500 & San Saba R at Menard, TX & $30^{\circ} 55^{\prime} 08^{\prime \prime}$ & $99^{\circ} 47^{\prime} 07^{\prime \prime}$ & 1,128 & 23 & 3.45 & 56 & 33.4 & .72 & 60 & $1,863.05$ \\
\hline 08144600 & San Saba R nr Brady, TX & $31^{\circ} 00^{\prime} 14^{\prime \prime}$ & $99^{\circ} 16^{\prime} 07^{\prime \prime}$ & 1,626 & 40 & 2.32 & 71 & 38.8 & 1.02 & .55 & $1,530.98$ \\
\hline 08146000 & San Saba R at San Saba, TX & $31^{\circ} 12^{\prime} 47^{\prime \prime}$ & $98^{\circ} 43^{\prime} 09^{\prime \prime}$ & 3,039 & 88 & 3.27 & 58 & 91.6 & .97 & 1.58 & $1,162.16$ \\
\hline 08147000 & Colorado R nr San Saba, TX & $31^{\circ} 13^{\prime} 04^{\prime \prime}$ & $98^{\circ} 33^{\prime} 51^{\prime \prime}$ & 19,819 & 225 & 2.65 & 136 & 88.1 & 2.46 & .65 & $1,096.22$ \\
\hline 08150000 & Llano R nr Junction, TX & $30^{\circ} 30^{\prime} 15^{\prime \prime}$ & $99^{\circ} 44^{\prime} 03^{\prime \prime}$ & 1,849 & 99 & .48 & 73 & 54.2 & 1.82 & .74 & $1,636.32$ \\
\hline 08150700 & Llano R nr Mason, TX & $30^{\circ} 39^{\prime} 38^{\prime \prime}$ & $99^{\circ} 06^{\prime} 32^{\prime \prime}$ & 3,242 & 179 & .82 & 65 & 158 & 1.13 & 2.43 & $1,230.36$ \\
\hline 08150800 & Beaver Cr nr Mason, TX & $30^{\circ} 38^{\prime} 36^{\prime \prime}$ & $99^{\circ} 05^{\prime} 44^{\prime \prime}$ & 215 & 3.2 & 1.24 & 19 & 7.35 & .45 & .39 & $1,253.24$ \\
\hline 08151500 & Llano R at Llano, TX & $30^{\circ} 45^{\prime} 04^{\prime \prime}$ & $98^{\circ} 40^{\prime} 10^{\prime \prime}$ & 4,192 & 157 & 1.42 & 174 & 195 & .81 & 1.12 & 970.01 \\
\hline 08152000 & Sandy Cr nr Kingsland, TX & $30^{\circ} 33^{\prime} 27^{\prime \prime}$ & $98^{\circ} 18^{\prime} 19^{\prime \prime}$ & 346 & 12 & 5.16 & 32.8 & 8.74 & 1.4 & .27 & 862.31 \\
\hline 08152900 & Pedernales R nr Fredericksburg, TX & $30^{\circ} 13^{\prime} 13^{\prime \prime}$ & $98^{\circ} 52^{\prime} 10^{\prime \prime}$ & 369 & 22 & 3.34 & 43 & 13 & 1.6 & .30 & $1,564.96$ \\
\hline 08153500 & Pedernales R nr Johnson City, TX & $30^{\circ} 17^{\prime} 30^{\prime \prime}$ & $98^{\circ} 13^{\prime} 57^{\prime \prime}$ & 901 & 51 & 10.47 & 34 & 38.3 & 1.28 & 1.13 & $1,096.7$ \\
\hline 08154700 & Bull Cr at Loop $360 \mathrm{nr}$ Austin, TX & $30^{\circ} 22^{\prime} 19^{\prime \prime}$ & $97^{\circ} 47^{\prime} 04^{\prime \prime}$ & 22.3 & 4.2 & 2.69 & 8.5 & 4.16 & 1.08 & .49 & 534.08 \\
\hline 08155200 & Barton $\mathrm{Cr}$ at SH $71 \mathrm{nr}$ Oak Hill, TX & $30^{\circ} 17^{\prime} 46^{\prime \prime}$ & $97^{\circ} 55^{\prime} 31^{\prime \prime}$ & 89.7 & 4.6 & 2.6 & 20.8 & 7.47 & .65 & .36 & 737.04 \\
\hline
\end{tabular}


Table 3. Median streamflow and associated hydraulic characteristics for streamflow-gaging stations in Texas-Continued

\begin{tabular}{|c|c|c|c|c|c|c|c|c|c|c|c|}
\hline \multirow[b]{2}{*}{$\begin{array}{c}\text { Station } \\
\text { no. }\end{array}$} & \multirow[b]{2}{*}{ Station name } & \multirow[b]{2}{*}{ Latitude } & \multirow[b]{2}{*}{ Longitude } & \multirow{2}{*}{$\begin{array}{l}\text { Contri- } \\
\text { buting } \\
\text { drainage } \\
\text { area } \\
\left(\mathrm{mi}^{2}\right)\end{array}$} & \multicolumn{6}{|c|}{ Characteristics for median streamflow } & \multirow[b]{2}{*}{$\begin{array}{l}\text { Elevation } \\
\text { of datum } \\
\text { of gage }\end{array}$} \\
\hline & & & & & $\begin{array}{l}\text { Stream- } \\
\text { flow } \\
\left(\mathrm{ft}^{3} / \mathrm{s}\right)\end{array}$ & $\begin{array}{c}\text { Gage } \\
\text { height } \\
\text { (ft) }\end{array}$ & $\begin{array}{c}\text { Stream } \\
\text { width } \\
\text { (ft) }\end{array}$ & $\begin{array}{c}\text { Cross } \\
\text { section } \\
\text { area } \\
\left(\mathrm{ft}^{2}\right)\end{array}$ & $\begin{array}{l}\text { Mean } \\
\text { velocity }\end{array}$ & $\begin{array}{l}\text { Mean } \\
\text { depth }\end{array}$ & \\
\hline 08155240 & Barton $\mathrm{Cr}$ at Lost Cr Blvd nr Austin, TX & $30^{\circ} 16^{\prime} 26^{\prime \prime}$ & $97^{\circ} 50^{\prime} 40^{\prime \prime}$ & 107 & 6.5 & 2.14 & 25 & 12.1 & 0.55 & 0.48 & 600 \\
\hline 08155300 & Barton Cr at Loop 360, Austin, TX & $30^{\circ} 14^{\prime} 40^{\prime \prime}$ & $97^{\circ} 48^{\prime} 07^{\prime \prime}$ & 116 & 0 & -- & -- & -- & -- & -- & 510.32 \\
\hline 08156800 & Shoal Cr at West 12th St, Austin, TX & $30^{\circ} 16^{\prime} 35^{\prime \prime}$ & $97^{\circ} 45^{\prime} 00^{\prime \prime}$ & 12.3 & .02 & 1.99 & 2.0 & .08 & .24 & .04 & 455.33 \\
\hline 08157600 & East Bouldin Cr at South 1st St, Austin, TX & $30^{\circ} 15^{\prime} 07^{\prime \prime}$ & $97^{\circ} 45^{\prime} 14^{\prime \prime}$ & 2.4 & .3 & 1.14 & 3.4 & .29 & 1.0 & .09 & -- \\
\hline 08157700 & Blunn Cr nr Little Stacy Park, Austin, TX & $30^{\circ} 14^{\prime} 50^{\prime \prime}$ & $97^{\circ} 44^{\prime} 37^{\prime \prime}$ & 1.2 & .21 & .81 & 2.08 & .29 & .73 & .14 & -- \\
\hline 08158000 & Colorado R at Austin, TX & $30^{\circ} 14^{\prime} 40^{\prime \prime}$ & $97^{\circ} 41^{\prime} 39^{\prime \prime}$ & 27,606 & 1,140 & 3.74 & 305 & 1,150 & .97 & 3.77 & 402.27 \\
\hline 08158050 & Boggy Cr at US 183, Austin, TX & $30^{\circ} 15^{\prime} 47^{\prime \prime}$ & $97^{\circ} 40^{\prime} 20^{\prime \prime}$ & 13.1 & .29 & 5.18 & 2.7 & .45 & .64 & .17 & 411.29 \\
\hline 08158600 & Walnut $\mathrm{Cr}$ at Webberville Rd, Austin, TX & $30^{\circ} 16 ' 59^{\prime \prime}$ & $97^{\circ} 39^{\prime} 17^{\prime \prime}$ & 51.3 & 7.5 & 4.39 & 10.5 & 5.1 & 1.57 & .49 & 425.96 \\
\hline 08158700 & Onion Cr nr Driftwood, TX & $30^{\circ} 04^{\prime} 58^{\prime \prime}$ & $98^{\circ} 00^{\prime} 27^{\prime \prime}$ & 124 & 9.6 & .92 & 31.5 & 8.08 & 1.14 & .26 & 878.13 \\
\hline 08158810 & Bear Cr below FM 1826 nr Driftwood, TX & $30^{\circ} 09^{\prime} 19^{\prime \prime}$ & $97^{\circ} 56^{\prime} 23^{\prime \prime}$ & 12.2 & 1.2 & 0 & 5.4 & 1.93 & .64 & .36 & 860 \\
\hline 08158840 & Slaughter Cr at FM 1826 nr Austin, TX & $30^{\circ} 12^{\prime} 32^{\prime \prime}$ & $97^{\circ} 54^{\prime} 11^{\prime \prime}$ & 8.24 & .39 & 3.9 & 7.8 & 1.1 & .32 & .14 & 876.14 \\
\hline 08158922 & Williamson $\mathrm{Cr}$ at Brush Country Blvd, Oak Hill, TX & $30^{\circ} 13 \prime 34^{\prime \prime}$ & $97^{\circ} 50^{\prime} 28^{\prime \prime}$ & 6.79 & 0 & -- & -- & -- & -- & -- & 740.25 \\
\hline 08158930 & Williamson $\mathrm{Cr}$ at Manchaca Rd, Austin, TX & $30^{\circ} 13^{\prime} 16^{\prime \prime}$ & $97^{\circ} 47^{\prime} 36^{\prime \prime}$ & 19 & 0 & -- & -- & -- & -- & -- & 618.39 \\
\hline 08159000 & Onion Cr at US 183, Austin, TX & $30^{\circ} 10^{\prime} 40^{\prime \prime}$ & $97^{\circ} 41^{\prime} 18^{\prime \prime}$ & 321 & 6.3 & 5.26 & 9.8 & 6.7 & .92 & .68 & 442.85 \\
\hline 08159200 & Colorado R at Bastrop, TX & $30^{\circ} 06^{\prime} 16^{\prime \prime}$ & $97^{\circ} 19^{\prime} 09^{\prime \prime}$ & 28,576 & 1,570 & 4.31 & 270 & 1,340 & 1.17 & 4.96 & 307.38 \\
\hline 08159500 & Colorado R at Smithville, TX & $30^{\circ} 00^{\prime} 45^{\prime \prime}$ & $97^{\circ} 09^{\prime} 42^{\prime \prime}$ & 27,000 & 1,640 & 3.9 & 240 & 1,186 & 1.44 & 4.94 & 270.14 \\
\hline 08160400 & Colorado R above La Grange, TX & $29^{\circ} 54^{\prime} 44^{\prime \prime}$ & $96^{\circ} 54^{\prime} 13^{\prime \prime}$ & 29,471 & 1,520 & 5.78 & 229 & 815 & 1.84 & 3.57 & 210.04 \\
\hline 08160800 & Redgate Cr nr Columbus, TX & $29^{\circ} 47^{\prime} 56^{\prime \prime}$ & $96^{\circ} 31^{\prime} 55^{\prime \prime}$ & 17.3 & .88 & 9.84 & 4.5 & 1.04 & .81 & .23 & 210.82 \\
\hline 08161000 & Colorado R at Columbus, TX & $29^{\circ} 42^{\prime} 22^{\prime \prime}$ & $96^{\circ} 32^{\prime} 12^{\prime \prime}$ & 30,327 & 1,630 & 12.15 & 230 & 1,393 & 1.1 & 6.06 & 155.52 \\
\hline 08162000 & Colorado R at Wharton, TX & $29^{\circ} 18^{\prime} 32^{\prime \prime}$ & $96^{\circ} 06^{\prime} 13^{\prime \prime}$ & 30,600 & 1,330 & 11.16 & 209 & 644 & 2.03 & 3.08 & 52.42 \\
\hline 08162500 & Colorado R nr Bay City, TX & $28^{\circ} 58^{\prime} 26^{\prime \prime}$ & $96^{\circ} 00^{\prime} 44^{\prime \prime}$ & 30,837 & 909 & 5.52 & 150 & 747 & 1.24 & 4.98 & 0 \\
\hline 08162600 & Tres Palacios R nr Midfield, TX & $28^{\circ} 55^{\prime} 40^{\prime \prime}$ & $96^{\circ} 10^{\prime} 15^{\prime \prime}$ & 145 & 23 & 3.67 & 19 & 13.8 & 1.7 & .73 & 5.38 \\
\hline 08164000 & Lavaca R nr Edna, TX & $28^{\circ} 57^{\prime} 35^{\prime \prime}$ & $96^{\circ} 41^{\prime} 10^{\prime \prime}$ & 817 & 54 & 4.51 & 48 & 58.1 & .92 & 1.21 & 14.1 \\
\hline 08164300 & Navidad R nr Halletsville, TX & $29^{\circ} 28^{\prime} 00^{\prime \prime}$ & $96^{\circ} 48^{\prime} 45^{\prime \prime}$ & 332 & 22 & 5.15 & 39 & 22.2 & .98 & .57 & 159.28 \\
\hline 08164350 & Navidad R nr Speaks, TX & $29^{\circ} 19^{\prime} 18^{\prime \prime}$ & $96^{\circ} 42^{\prime} 32^{\prime \prime}$ & 437 & 30 & 3.71 & 29 & 22.6 & 1.35 & .78 & 120 \\
\hline 08164370 & Navidad R at Morales, TX & $29^{\circ} 08^{\prime} 07^{\prime \prime}$ & $96^{\circ} 44^{\prime} 39^{\prime \prime}$ & 549 & 58 & 7.69 & 40 & 94.5 & .61 & 2.36 & 65.44 \\
\hline 08164390 & Navidad R at Strane Park nr Edna, TX & $29^{\circ} 03^{\prime} 55^{\prime \prime}$ & $96^{\circ} 40^{\prime} 26^{\prime \prime}$ & 579 & 66 & 5.49 & 42.5 & 52.6 & 1.24 & 1.24 & 42.53 \\
\hline 08164450 & Sandy Cr nr Ganado, TX & $29^{\circ} 09^{\prime} 36^{\prime \prime}$ & $96^{\circ} 32^{\prime} 46^{\prime \prime}$ & 289 & 21 & 5.89 & 29 & 19 & 1.19 & .66 & 59.72 \\
\hline 08164503 & West Mustang Cr nr Ganado, TX & $29^{\circ} 04^{\prime} 17^{\prime \prime}$ & $96^{\circ} 28^{\prime} 01^{\prime \prime}$ & 178 & 23 & 5.53 & 40 & 48.5 & .44 & 1.21 & 40.12 \\
\hline 08164504 & East Mustang Cr nr Louise, TX & $29^{\circ} 04^{\prime} 14^{\prime \prime}$ & $96^{\circ} 25^{\prime} 01^{\prime \prime}$ & 90.8 & 3.6 & 4.46 & 7.0 & 2.15 & 1.64 & .31 & 43.02 \\
\hline 08164600 & Garcitas Cr nr Inez, TX & $28^{\circ} 53^{\prime} 28^{\prime \prime}$ & $96^{\circ} 49^{\prime} 08^{\prime \prime}$ & 91.7 & 3.2 & 4.94 & 8.4 & 3.43 & .93 & .41 & 29.16 \\
\hline 08164800 & Placedo Cr nr Placedo, TX & $28^{\circ} 43^{\prime} 30^{\prime \prime}$ & $96^{\circ} 46^{\prime} 07^{\prime \prime}$ & 68.3 & 1.6 & 5.18 & 9.4 & 3.0 & .51 & .32 & 5.58 \\
\hline 08165300 & North Fk Guadalupe R nr Hunt, TX & $30^{\circ} 03^{\prime} 50^{\prime \prime}$ & $99^{\circ} 23^{\prime} 12^{\prime \prime}$ & 169 & 24 & 1.75 & 35 & 36.1 & .65 & 1.03 & $1,800.1$ \\
\hline 08165500 & Guadalupe R at Hunt, TX & $30^{\circ} 04^{\prime} 11^{\prime \prime}$ & $99^{\circ} 19^{\prime} 17^{\prime \prime}$ & 288 & 49 & 7.88 & 42 & 29.7 & 1.67 & .71 & $1,722.7$ \\
\hline 08166000 & Johnson Cr nr Ingram, TX & $30^{\circ} 06^{\prime} 00^{\prime \prime}$ & $99^{\circ} 16^{\prime} 58^{\prime \prime}$ & 114 & 14 & .53 & 38 & 32.1 & .45 & .84 & $1,721.3$ \\
\hline
\end{tabular}


Table 3. Median streamflow and associated hydraulic characteristics for streamflow-gaging stations in Texas-Continued

\begin{tabular}{|c|c|c|c|c|c|c|c|c|c|c|c|}
\hline \multirow[b]{2}{*}{$\begin{array}{c}\text { Station } \\
\text { no. }\end{array}$} & \multirow[b]{2}{*}{ Station name } & \multirow[b]{2}{*}{ Latitude } & \multirow[b]{2}{*}{ Longitude } & \multirow{2}{*}{$\begin{array}{l}\text { Contri- } \\
\text { buting } \\
\text { drainage } \\
\text { area } \\
\left(\mathrm{mi}^{2}\right)\end{array}$} & \multicolumn{6}{|c|}{ Characteristics for median streamflow } & \multirow[b]{2}{*}{$\begin{array}{l}\text { Elevation } \\
\text { of datum } \\
\text { of gage }\end{array}$} \\
\hline & & & & & $\begin{array}{l}\text { Stream- } \\
\text { flow } \\
\left(\mathrm{ft}^{3} / \mathrm{s}\right)\end{array}$ & $\begin{array}{c}\text { Gage } \\
\text { height } \\
\text { (ft) }\end{array}$ & $\begin{array}{l}\text { Stream } \\
\text { width } \\
(\mathrm{ft})\end{array}$ & $\begin{array}{c}\text { Cross } \\
\text { section } \\
\text { area } \\
\left(\mathrm{ft}^{2}\right)\end{array}$ & $\begin{array}{l}\text { Mean } \\
\text { velocity }\end{array}$ & $\begin{array}{l}\text { Mean } \\
\text { depth }\end{array}$ & \\
\hline 08166140 & Guadalupe $\mathrm{R}$ above $\mathrm{Bear} \mathrm{Cr}$ at Kerrville, TX & $30^{\circ} 04^{\prime} 10^{\prime \prime}$ & $99^{\circ} 11^{\prime} 42^{\prime \prime}$ & 494 & 81 & 2.99 & 133 & 68.3 & 1.16 & 0.51 & 1,630 \\
\hline 08166200 & Guadalupe R at Kerrville, TX & $30^{\circ} 03^{\prime} 11^{\prime \prime}$ & $99^{\circ} 09^{\prime} 47^{\prime \prime}$ & 510 & 96 & 1.8 & 143 & 63.6 & 1.44 & .44 & 1,601 \\
\hline 08167000 & Guadalupe R at Comfort, TX & $29^{\circ} 58^{\prime} 10^{\prime \prime}$ & $98^{\circ} 53^{\prime} 33^{\prime \prime}$ & 839 & 131 & 2.47 & 35 & 43 & 3.16 & 1.23 & $1,369.83$ \\
\hline 08167500 & Guadalupe R nr Spring Branch, TX & $29^{\circ} 51^{\prime} 37^{\prime \prime}$ & $98^{\circ} 13^{\prime} 00^{\prime \prime}$ & 1,315 & 199 & 2.92 & 73 & 134 & 1.5 & 1.84 & 948.1 \\
\hline 08167800 & Guadalupe R at Sattler, TX & $29^{\circ} 51^{\prime} 32^{\prime \prime}$ & $98^{\circ} 10^{\prime} 47^{\prime \prime}$ & 1,436 & 215 & 4.86 & 83 & 165 & 1.28 & 1.99 & 742.24 \\
\hline 08168500 & Guadalupe R above Comal R at New Braunfels, TX & $29^{\circ} 42^{\prime} 53^{\prime \prime}$ & $98^{\circ} 06^{\prime} 35^{\prime \prime}$ & 1,518 & 287 & 2.34 & 104 & 253 & 1.13 & 2.43 & 586.65 \\
\hline 08169000 & Comal $\mathrm{R}$ at New Braunfels, $\mathrm{TX}$ & $29^{\circ} 42^{\prime} 21^{\prime \prime}$ & $98^{\circ} 07^{\prime} 20^{\prime \prime}$ & 130 & 308 & 4.2 & 93 & 161 & 1.86 & 1.73 & 582.8 \\
\hline 08170500 & San Marcos R at San Marcos, Tx & $29^{\circ} 53^{\prime} 20^{\prime \prime}$ & $97^{\circ} 56^{\prime} 02^{\prime \prime}$ & 93 & 162 & 5.21 & 79 & 73.4 & 2.22 & .93 & 557.67 \\
\hline 08171000 & Blanco R at Wimberley, TX & $29^{\circ} 59^{\prime} 39^{\prime \prime}$ & $98^{\circ} 05^{\prime} 19^{\prime \prime}$ & 355 & 53 & 3.84 & 112 & 89.1 & .59 & .80 & 797.23 \\
\hline 08171300 & Blanco R nr Kyle, TX & $29^{\circ} 58^{\prime} 45^{\prime \prime}$ & $97^{\circ} 54^{\prime} 35^{\prime \prime}$ & 412 & 52 & 4.88 & 40.2 & 22.3 & 2.39 & .55 & 620.12 \\
\hline 08172000 & San Marcos R at Luling, TX & $29^{\circ} 39^{\prime} 58^{\prime \prime}$ & $97^{\circ} 39^{\prime} 02^{\prime \prime}$ & 838 & 234 & 6.88 & 66 & 103 & 2.23 & 1.56 & 322.05 \\
\hline 08172400 & Plum Cr at Lockhart, TX & $29^{\circ} 55^{\prime} 22^{\prime \prime}$ & $97^{\circ} 40^{\prime} 44^{\prime \prime}$ & 112 & .35 & 1.31 & 3.0 & .42 & .81 & .14 & 431.19 \\
\hline 08173900 & Guadalupe $\mathrm{R}$ at Gonzales, TX & $29^{\circ} 29^{\prime} 03^{\prime \prime}$ & $97^{\circ} 27^{\prime} 00^{\prime \prime}$ & 3,490 & 1,070 & 13.45 & 79 & 370 & 2.6 & 4.68 & 250 \\
\hline 08175000 & Sandies Cr nr Westhoff, TX & $29^{\circ} 12^{\prime} 54^{\prime \prime}$ & $97^{\circ} 26^{\prime} 57^{\prime \prime}$ & 549 & 9.1 & 2.58 & 48.5 & 10.4 & .90 & .21 & 178.27 \\
\hline 08175800 & Guadalupe R at Cuero, TX & $29^{\circ} 03^{\prime} 57^{\prime \prime}$ & $97^{\circ} 19^{\prime} 16^{\prime \prime}$ & 4,934 & 1,060 & 5.18 & 125 & 702 & 1.51 & 5.62 & 128.64 \\
\hline 08176500 & Guadalupe R at Victoria, TX & $28^{\circ} 47^{\prime} 34^{\prime \prime}$ & $97^{\circ} 00^{\prime} 46^{\prime \prime}$ & 5,198 & 990 & 6.77 & 136 & 700 & 1.41 & 5.15 & 29.15 \\
\hline 08176900 & Coleto Cr at Arnold Rd nr Schroeder, TX & $28^{\circ} 51^{\prime} 41^{\prime \prime}$ & $97^{\circ} 13^{\prime} 34^{\prime \prime}$ & 357 & 12 & 4.54 & 16.2 & 8.88 & 1.32 & .55 & 100.43 \\
\hline 08177500 & Coleto $\mathrm{Cr}$ nr Victoria, $\mathrm{TX}$ & $28^{\circ} 43^{\prime} 51^{\prime \prime}$ & $97^{\circ} 08^{\prime} 18^{\prime \prime}$ & 514 & 5.0 & 2.79 & 17.7 & 5.92 & .90 & .33 & 44.18 \\
\hline 08178050 & San Antonio R at Mitchell St, San Antonio, TX & $29^{\circ} 23^{\prime} 34^{\prime \prime}$ & $98^{\circ} 19^{\prime} 40^{\prime \prime}$ & 42.4 & 26 & .78 & 39 & 13.5 & 1.97 & .35 & 585.07 \\
\hline 08178565 & San Antonio R at Loop 410 at San Antonio, TX & $29^{\circ} 19^{\prime} 19^{\prime \prime}$ & $98^{\circ} 17^{\prime} 00^{\prime \prime}$ & 125 & 43 & 6.14 & 33 & 21.4 & 2.08 & .65 & 488.11 \\
\hline 08178700 & Salado Cr (Upper Station) at San Antonio, TX & $29^{\circ} 30^{\prime} 57^{\prime \prime}$ & $98^{\circ} 15^{\prime} 51^{\prime \prime}$ & 137 & .75 & 2.65 & 13 & 4.6 & .15 & .35 & 684.6 \\
\hline 08178800 & Salado Cr (Lower Station) at San Antonio, TX & $29^{\circ} 21^{\prime} 25^{\prime \prime}$ & $98^{\circ} 14^{\prime} 45^{\prime \prime}$ & 189 & 22 & 6.89 & 18.5 & 17.9 & 1.28 & .97 & 526.95 \\
\hline 08178880 & Medina $\mathrm{R}$ at Bandera, $\mathrm{TX}$ & $29^{\circ} 43 \prime 25^{\prime \prime}$ & $99^{\circ} 04^{\prime} 11^{\prime \prime}$ & 427 & 63 & 4.44 & 32 & 35.6 & 1.78 & 1.11 & $1,189.46$ \\
\hline 08180640 & Medina $\mathrm{R}$ at La Coste, $\mathrm{TX}$ & $29^{\circ} 19^{\prime} 26^{\prime \prime}$ & $98^{\circ} 48^{\prime} 46^{\prime \prime}$ & 805 & 43 & 5.81 & 36 & 70.1 & .65 & 1.95 & 667.15 \\
\hline 08180700 & Medina R nr MacDona, TX & $29^{\circ} 20^{\prime} 05^{\prime \prime}$ & $98^{\circ} 41^{\prime} 22^{\prime \prime}$ & 885 & 54 & 2.89 & 47 & 23.4 & 2.38 & .50 & 589.86 \\
\hline 08180800 & Medina R nr Somerset, TX & $29^{\circ} 15^{\prime} 43^{\prime \prime}$ & $98^{\circ} 34^{\prime} 52^{\prime \prime}$ & 967 & 77 & 5.08 & 34 & 71.1 & 1.09 & 2.09 & 493.56 \\
\hline 08181400 & Helotes $\mathrm{Cr}$ at Helotes, TX & $29^{\circ} 34^{\prime} 42^{\prime \prime}$ & $98^{\circ} 41^{\prime} 29^{\prime \prime}$ & 15 & 0 & -- & -- & -- & -- & -- & $1,014.82$ \\
\hline 08181480 & Leon Cr at IH-35 at San Antonio, TX & $29^{\circ} 19^{\prime} 47^{\prime \prime}$ & $98^{\circ} 35^{\prime} 02^{\prime \prime}$ & 219 & 7.9 & 3.1 & 27 & 11.5 & .71 & .43 & 573.49 \\
\hline 08181500 & Medina R at San Antonio, TX & $29^{\circ} 15^{\prime} 14^{\prime \prime}$ & $98^{\circ} 18^{\prime} 10^{\prime \prime}$ & 1,317 & 96 & 5.25 & 38 & 67.6 & 1.4 & 1.78 & 439 \\
\hline 08181800 & San Antonio R nr Elmendorf, TX & $29^{\circ} 13^{\prime} 19^{\prime \prime}$ & $98^{\circ} 11^{\prime} 20^{\prime \prime}$ & 1,743 & 316 & 12.23 & 53 & 305 & 1.04 & 5.75 & 380 \\
\hline 08183500 & San Antonio R nr Falls City, TX & $28^{\circ} 57^{\prime} 05^{\prime \prime}$ & $98^{\circ} 03^{\prime} 50^{\prime \prime}$ & 2,113 & 249 & 1.26 & 102 & 121 & 2.07 & 1.19 & 285.49 \\
\hline 08183850 & Cibolo $\mathrm{Cr}$ at $\mathrm{IH}-10$ above Boerne, $\mathrm{TX}$ & $29^{\circ} 48^{\prime} 52^{\prime \prime}$ & $98^{\circ} 45^{\prime} 12^{\prime \prime}$ & 29 & 3.5 & 1.28 & 32 & 9.11 & .39 & .28 & $1,428.5$ \\
\hline 08185000 & Cibolo $\mathrm{Cr}$ at Selma, TX & $29^{\circ} 35^{\prime} 38^{\prime \prime}$ & $98^{\circ} 18^{\prime} 39^{\prime \prime}$ & 274 & 0 & -- & -- & -- & -- & -- & 728.34 \\
\hline 08186000 & Cibolo Cr nr Falls City, TX & $29^{\circ} 00^{\prime} 50^{\prime \prime}$ & $97^{\circ} 55^{\prime} 48^{\prime \prime}$ & 827 & 27 & 1.37 & 40 & 34 & .78 & .85 & 827 \\
\hline 08188500 & San Antonio R at Goliad, TX & $28^{\circ} 38^{\prime} 58^{\prime \prime}$ & $97^{\circ} 23^{\prime} 04^{\prime \prime}$ & 3,921 & 331 & 4.56 & 82 & 216 & 1.54 & 2.63 & 91.08 \\
\hline
\end{tabular}


Table 3. Median streamflow and associated hydraulic characteristics for streamflow-gaging stations in Texas-Continued

\begin{tabular}{|c|c|c|c|c|c|c|c|c|c|c|c|}
\hline \multirow[b]{2}{*}{$\begin{array}{c}\text { Station } \\
\text { no. }\end{array}$} & \multirow[b]{2}{*}{ Station name } & \multirow[b]{2}{*}{ Latitude } & \multirow[b]{2}{*}{ Longitude } & \multirow{2}{*}{$\begin{array}{l}\text { Contri- } \\
\text { buting } \\
\text { drainage } \\
\text { area } \\
\left(\mathrm{mi}^{2}\right)\end{array}$} & \multicolumn{6}{|c|}{ Characteristics for median streamflow } & \multirow[b]{2}{*}{$\begin{array}{l}\text { Elevation } \\
\text { of datum } \\
\text { of gage }\end{array}$} \\
\hline & & & & & $\begin{array}{c}\text { Stream- } \\
\text { flow } \\
\left(\mathrm{ft}^{3} / \mathrm{s}\right)\end{array}$ & $\begin{array}{c}\text { Gage } \\
\text { height } \\
\text { (ft) }\end{array}$ & $\begin{array}{l}\text { Stream } \\
\text { width } \\
\text { (ft) }\end{array}$ & $\begin{array}{c}\text { Cross } \\
\text { section } \\
\text { area } \\
\left(\mathrm{ft}^{2}\right)\end{array}$ & $\begin{array}{l}\text { Mean } \\
\text { velocity }\end{array}$ & $\begin{array}{l}\text { Mean } \\
\text { depth }\end{array}$ & \\
\hline 08189200 & Copano Cr nr Refugio, TX & $28^{\circ} 18^{\prime} 12^{\prime \prime}$ & $97^{\circ} 06^{\prime} 44^{\prime \prime}$ & 87.8 & 0.07 & 2.89 & 1.0 & 0.18 & 0.39 & 0.18 & 17.25 \\
\hline 08189500 & Mission R at Refugio, TX & $28^{\circ} 17^{\prime} 30^{\prime \prime}$ & $97^{\circ} 16^{\prime} 44^{\prime \prime}$ & 690 & 11 & 2.38 & 33.5 & 19.6 & .56 & .59 & 1.0 \\
\hline 08189700 & Aransas R nr Skidmore, TX & $28^{\circ} 16^{\prime} 56^{\prime \prime}$ & $97^{\circ} 37^{\prime} 14^{\prime \prime}$ & 247 & 4.0 & .64 & 7.2 & 3.74 & 1.07 & .52 & 72.37 \\
\hline 08190000 & Nueces R at Laguna, TX & $29^{\circ} 25^{\prime} 42^{\prime \prime}$ & $99^{\circ} 59^{\prime} 49^{\prime \prime}$ & 737 & 77 & 3.72 & 79.3 & 84.1 & .92 & 1.06 & $1,119.72$ \\
\hline 08190500 & West Nueces R nr Bracketville, TX & $29^{\circ} 28^{\prime} 21^{\prime \prime}$ & $100^{\circ} 14^{\prime} 10^{\prime \prime}$ & 694 & .03 & 1.08 & 1.0 & .08 & .45 & .08 & $1,326.79$ \\
\hline 08192000 & Nueces R below Uvalde, TX & $29^{\circ} 07^{\prime} 25^{\prime \prime}$ & $99^{\circ} 53^{\prime} 40^{\prime \prime}$ & 1,861 & 27 & 2.84 & 56.5 & 34.2 & .78 & .61 & 796.12 \\
\hline 08193000 & Nueces R nr Asherton, TX & $28^{\circ} 30^{\prime} 00^{\prime \prime}$ & $99^{\circ} 40^{\prime} 54^{\prime \prime}$ & 4,082 & .26 & 1.52 & 6.0 & 1.12 & .24 & .19 & 470.92 \\
\hline 08194000 & Nueces R at Cotulla, TX & $28^{\circ} 25^{\prime} 34^{\prime \prime}$ & $99^{\circ} 14^{\prime} 23^{\prime \prime}$ & 5,171 & .61 & 7.56 & 13 & 1.13 & .55 & .09 & 368.08 \\
\hline 08194200 & San Casimiro Cr nr Freer, TX & $27^{\circ} 57^{\prime} 53^{\prime \prime}$ & $98^{\circ} 58^{\prime} 00^{\prime \prime}$ & 469 & 0 & -- & -- & -- & -- & -- & 298 \\
\hline 08194500 & Nueces R nr Tilden, TX & $28^{\circ} 18^{\prime} 31^{\prime \prime}$ & $98^{\circ} 33^{\prime} 25^{\prime \prime}$ & 8,093 & 7.3 & 2.53 & 21 & 9.88 & .70 & .47 & 183.5 \\
\hline 08195000 & Frio $\mathrm{R}$ at Concan, $\mathrm{TX}$ & $29^{\circ} 29^{\prime} 18^{\prime \prime}$ & $99^{\circ} 42^{\prime} 16^{\prime \prime}$ & 389 & 69 & 3.72 & 78 & 68.2 & 1.01 & .87 & $1,203.71$ \\
\hline 08196000 & Dry Frio R nr Reagan Wells, TX & $29^{\circ} 30^{\prime} 16^{\prime \prime}$ & $99^{\circ} 466^{\prime \prime}$ & 126 & 15 & 2.1 & 45 & 35.5 & .42 & .79 & $1,335.2$ \\
\hline 08197500 & Frio R below Dry Frio R nr Uvalde, Tx & $29^{\circ} 14^{\prime} 44^{\prime \prime}$ & $99^{\circ} 40^{\prime} 27^{\prime \prime}$ & 631 & 0 & -- & -- & -- & -- & -- & 882.47 \\
\hline 08198000 & Sabinal R nr Sabinal, TX & $29^{\circ} 29^{\prime} 27^{\prime \prime}$ & $99^{\circ} 29^{\prime} 33^{\prime \prime}$ & 206 & 26 & 4.94 & 37.3 & 27.9 & .90 & .75 & $1,131.2$ \\
\hline 08198500 & Sabinal R at Sabinal, TX & $29^{\circ} 18^{\prime} 05^{\prime \prime}$ & $99^{\circ} 28^{\prime} 46^{\prime \prime}$ & 241 & 1.6 & 3.11 & 10.3 & 5.84 & .26 & .57 & 882.17 \\
\hline 08200000 & Hondo Cr nr Tarpley, TX & $29^{\circ} 34^{\prime} 10^{\prime \prime}$ & $99^{\circ} 14^{\prime} 47^{\prime \prime}$ & 95.6 & 12 & .25 & 39 & 22.6 & .50 & .58 & $1,169.1$ \\
\hline 08200700 & Hondo Cr at King Waterhole nr Hondo, TX & $29^{\circ} 23^{\prime} 26^{\prime \prime}$ & $99^{\circ} 09^{\prime} 04^{\prime \prime}$ & 149 & 0 & -- & -- & -- & -- & -- & 897.87 \\
\hline 08201500 & Seco Cr at Miller Ranch nr Utopia, TX & $29^{\circ} 34^{\prime} 23^{\prime \prime}$ & $99^{\circ} 24^{\prime} 10^{\prime \prime}$ & 45 & 5.6 & 1.58 & 36.6 & 12 & .46 & .33 & $1,265.8$ \\
\hline 08202700 & Seco Cr at Rowe Ranch nr D'Hanis, TX & $29^{\circ} 21^{\prime} 43^{\prime \prime}$ & $99^{\circ} 17^{\prime} 05^{\prime \prime}$ & 168 & 0 & -- & -- & -- & -- & -- & 900.88 \\
\hline 08205500 & Frio R nr Derby, TX & $28^{\circ} 44^{\prime} 11^{\prime \prime}$ & $99^{\circ} 08^{\prime} 40^{\prime \prime}$ & 9,429 & 5.4 & .74 & 13.5 & 10.3 & .52 & .76 & 449.11 \\
\hline 08206600 & Frio $\mathrm{R}$ at Tilden, $\mathrm{TX}$ & $28^{\circ} 28^{\prime} 02^{\prime \prime}$ & $98^{\circ} 32^{\prime} 50^{\prime \prime}$ & 4,493 & 28 & 2.72 & 26 & 20.8 & 1.34 & .80 & 216.04 \\
\hline 08206700 & San Miguel Cr nr Tilden, TX & $28^{\circ} 35^{\prime} 14^{\prime \prime}$ & $98^{\circ} 32^{\prime} 44^{\prime \prime}$ & 783 & 2.0 & 2.13 & 9.5 & 2.58 & .71 & .27 & 242.95 \\
\hline 08206910 & Choke Canyon Reservoir OWC nr Three Rivers, TX & $28^{\circ} 29^{\prime} 09^{\prime \prime}$ & $98^{\circ} 14^{\prime} 29^{\prime \prime}$ & 5,490 & 34 & 4.47 & 26 & 18.1 & 1.89 & .70 & 124.6 \\
\hline 08208000 & Atascosa R at Whitsett, TX & $28^{\circ} 37^{\prime} 19^{\prime \prime}$ & $98^{\circ} 16^{\prime} 52^{\prime \prime}$ & 1,171 & 11 & 2.27 & 21 & 14.2 & .77 & .68 & 159.04 \\
\hline 08210000 & Nueces R nr Three Rivers, TX & $28^{\circ} 25^{\prime} 38^{\prime \prime}$ & $97^{\circ} 10^{\prime} 40^{\prime \prime}$ & 15,427 & 92 & 2.11 & 43 & 55.5 & 1.66 & 1.29 & 99.26 \\
\hline 08211000 & Nueces R nr Mathis, TX & $28^{\circ} 02^{\prime} 17^{\prime \prime}$ & $97^{\circ} 51^{\prime} 36^{\prime \prime}$ & 16,660 & 131 & 1.53 & 58 & 115 & 1.14 & 1.98 & 26.53 \\
\hline 08211200 & Nueces R at Bluntzer, TX & $27^{\circ} 56^{\prime} 15^{\prime \prime}$ & $97^{\circ} 46^{\prime} 32^{\prime \prime}$ & 16,772 & 164 & 7.75 & 63 & 255 & .64 & 4.05 & 0 \\
\hline 08211500 & Nueces R at Calallen, TX & $27^{\circ} 52^{\prime} 34^{\prime \prime}$ & $97^{\circ} 37^{\prime} 32^{\prime \prime}$ & 16,920 & 13 & 4.66 & 148.5 & 13.13 & 1.04 & .09 & .84 \\
\hline 08211520 & Oso $\mathrm{Cr}$ at Corpus Christi, TX & $27^{\circ} 42^{\prime} 40^{\prime \prime}$ & $97^{\circ} 30^{\prime} 06^{\prime \prime}$ & 90.3 & 2.3 & 4.83 & 12.5 & 9.49 & .24 & .76 & -1.91 \\
\hline 08211900 & San Fernando Cr at Alice, TX & $27^{\circ} 46^{\prime} 20^{\prime \prime}$ & $98^{\circ} 02^{\prime} 00^{\prime \prime}$ & 507 & 2.0 & 1.01 & 10 & 2.93 & .68 & .29 & 161.68 \\
\hline 08212400 & Los Olmos Cr nr Falfurrias, TX & $27^{\circ} 15^{\prime} 51^{\prime \prime}$ & $98^{\circ} 08^{\prime} 08^{\prime \prime}$ & 476 & 0 & -- & -- & -- & -- & -- & 116.58 \\
\hline 08446500 & Pecos R nr Girvin, TX & $31^{\circ} 06^{\prime} 47^{\prime \prime}$ & $102^{\circ} 25^{\prime} 02^{\prime \prime}$ & 29,560 & 28 & 1.51 & 30 & 32.5 & .85 & 1.08 & $2,269.65$ \\
\hline
\end{tabular}


Table 4. Gains and losses from gain-loss studies in Texas

$\left[\mathrm{ft}^{3} / \mathrm{s}\right.$, cubic feet per second; mi, miles; --, not applicable; SCS, Soil Conservation Service; ft, feet; IBWC, International Boundary and Water Commission]

Note: Eight digit streamflow-gaging stations used to identify some subreaches

\begin{tabular}{|c|c|c|c|c|c|c|c|c|c|}
\hline $\begin{array}{l}\text { Stream- } \\
\text { flow } \\
\text { study } \\
\text { no. }\end{array}$ & $\begin{array}{c}\text { Latitude of } \\
\text { upstream } \\
\text { end of } \\
\text { subreach }\end{array}$ & $\begin{array}{l}\text { Longitude } \\
\text { of upstream } \\
\text { end of } \\
\text { subreach }\end{array}$ & $\begin{array}{c}\text { Major aquifer } \\
\text { outcrop } \\
\text { intersected } \\
\text { by subreach }\end{array}$ & & $\begin{array}{c}\text { Minor aquifer } \\
\text { outcrop } \\
\text { intercepted } \\
\text { by subreach }\end{array}$ & $\begin{array}{c}\text { Gain or } \\
\text { loss }(-) \text { in } \\
\text { subreach } \\
\left(\mathrm{ft}^{3} / \mathrm{s}\right)\end{array}$ & $\begin{array}{l}\text { Length } \\
\text { of subreach } \\
\text { (river mi) }\end{array}$ & $\begin{array}{l}\text { Location of } \\
\text { upstream } \\
\text { end of } \\
\text { subreach } \\
\text { (river mi) }\end{array}$ & $\begin{array}{l}\text { Descriptive location } \\
\text { of upstream end of } \\
\text { selected subreaches }\end{array}$ \\
\hline 1 & $30^{\circ} 52^{\prime} 47^{\prime \prime}$ & $97^{\circ} 55^{\prime} 11^{\prime \prime}$ & -- & -- & & 0 & 4.9 & 30.5 & at US 183 bridge \\
\hline 1 & $30^{\circ} 50^{\prime} 34^{\prime \prime}$ & $97^{\circ} 51^{\prime} 32^{\prime \prime}$ & -- & -- & & 0 & 3.2 & 25.6 & \\
\hline 1 & $30^{\circ} 48^{\prime} 37^{\prime \prime}$ & $97^{\circ} 49^{\prime} 32^{\prime \prime}$ & -- & -- & & .05 & 3.0 & 22.4 & \\
\hline 1 & $30^{\circ} 46^{\prime} 44^{\prime \prime}$ & $97^{\circ} 47^{\prime} 45^{\prime \prime}$ & -- & -- & & -.05 & 2.5 & 19.4 & \\
\hline 1 & $30^{\circ} 45^{\prime} 49^{\prime \prime}$ & $97^{\circ} 45^{\prime} 13^{\prime \prime}$ & Edwards & -- & & -.12 & 2.5 & 16.9 & \\
\hline 1 & $30^{\circ} 44^{\prime} 51^{\prime \prime}$ & $97^{\circ} 43^{\prime} 55^{\prime \prime}$ & Edwards & - & & -.02 & 1.6 & 14.4 & \\
\hline 1 & $30^{\circ} 43^{\prime} 38^{\prime \prime}$ & $97^{\circ} 44^{\prime} 26^{\prime \prime}$ & Edwards & -- & & 0 & 1.3 & 12.8 & \\
\hline 1 & $30^{\circ} 43^{\prime} 05^{\prime \prime}$ & $97^{\circ} 43^{\prime} 43^{\prime \prime}$ & Edwards & -- & & -.83 & 6.6 & 11.5 & \\
\hline 1 & $30^{\circ} 42^{\prime} 11^{\prime \prime}$ & $97^{\circ} 39^{\prime} 58^{\prime \prime}$ & Edwards & -- & & .18 & 1.3 & 4.9 & \\
\hline 1 & $30^{\circ} 41^{\prime 2} 27^{\prime \prime}$ & $97^{\circ} 39^{\prime} 18^{\prime \prime}$ & -- & -- & & 3.04 & 3.2 & 3.6 & \\
\hline 2 & $30^{\circ} 52^{\prime} 47^{\prime \prime}$ & $97^{\circ} 55^{\prime} 11^{\prime \prime}$ & -- & -- & & 0 & 4.9 & 30.5 & at US 183 bridge \\
\hline 2 & $30^{\circ} 50^{\prime} 34^{\prime \prime}$ & $97^{\circ} 51^{\prime} 32^{\prime \prime}$ & -- & -- & & 0 & 3.2 & 25.6 & \\
\hline 2 & $30^{\circ} 48^{\prime} 37^{\prime \prime}$ & $97^{\circ} 49^{\prime} 32^{\prime \prime}$ & -- & -- & & 0 & 3.0 & 22.4 & \\
\hline 2 & $30^{\circ} 46^{\prime} 44^{\prime \prime}$ & $97^{\circ} 47^{\prime} 45^{\prime \prime}$ & -- & -- & & 0 & 2.5 & 19.4 & \\
\hline 2 & $30^{\circ} 45^{\prime} 49^{\prime \prime}$ & $97^{\circ} 45^{\prime} 13^{\prime \prime}$ & Edwards & -- & & 0 & 2.5 & 16.9 & \\
\hline 2 & $30^{\circ} 44^{\prime} 51^{\prime \prime}$ & $97^{\circ} 43^{\prime} 55^{\prime \prime}$ & Edwards & -- & & 0 & 1.6 & 14.4 & \\
\hline 2 & $30^{\circ} 43^{\prime} 38^{\prime \prime}$ & $97^{\circ} 44^{\prime} 26^{\prime \prime}$ & Edwards & -- & & 0 & 1.3 & 12.8 & \\
\hline 2 & $30^{\circ} 43^{\prime} 05^{\prime \prime}$ & $97^{\circ} 43^{\prime} 43^{\prime \prime}$ & Edwards & -- & & -.21 & 6.6 & 11.5 & \\
\hline 2 & $30^{\circ} 42^{\prime} 11^{\prime \prime}$ & $97^{\circ} 39^{\prime} 58^{\prime \prime}$ & Edwards & -- & & 0 & 1.3 & 4.9 & \\
\hline 2 & $30^{\circ} 41^{\prime} 27^{\prime \prime}$ & $97^{\circ} 39^{\prime} 18^{\prime \prime}$ & -- & -- & & 0 & 3.2 & 3.6 & \\
\hline 3 & $30^{\circ} 52^{\prime} 47^{\prime \prime}$ & $97^{\circ} 55^{\prime} 11^{\prime \prime}$ & -- & -- & & 4.19 & 4.9 & 30.5 & at US 183 bridge \\
\hline 3 & $30^{\circ} 50^{\prime} 34^{\prime \prime}$ & $97^{\circ} 51^{\prime} 32^{\prime \prime}$ & -- & -- & & 5.34 & 3.2 & 25.6 & \\
\hline 3 & $30^{\circ} 48^{\prime} 37^{\prime \prime}$ & $97^{\circ} 49^{\prime} 32^{\prime \prime}$ & -- & -- & & 7.79 & 3.0 & 22.4 & \\
\hline 3 & $30^{\circ} 46^{\prime} 44^{\prime \prime}$ & $97^{\circ} 47^{\prime} 45^{\prime \prime}$ & -- & -- & & .8 & 2.5 & 19.4 & \\
\hline 3 & $30^{\circ} 45^{\prime} 49^{\prime \prime}$ & $97^{\circ} 45^{\prime} 13^{\prime \prime}$ & Edwards & -- & & .83 & 2.5 & 16.9 & \\
\hline 3 & $30^{\circ} 44^{\prime} 51^{\prime \prime}$ & $97^{\circ} 43^{\prime} 55^{\prime \prime}$ & Edwards & -- & & -9.26 & 1.6 & 14.4 & \\
\hline 3 & $30^{\circ} 43^{\prime} 38^{\prime \prime}$ & $97^{\circ} 44^{\prime 2} 26^{\prime \prime}$ & Edwards & -- & & -18.6 & 1.3 & 12.8 & \\
\hline 3 & $30^{\circ} 43^{\prime} 05^{\prime \prime}$ & $97^{\circ} 43^{\prime} 43^{\prime \prime}$ & Edwards & -- & & 20.5 & 6.6 & 11.5 & \\
\hline 3 & $30^{\circ} 42^{\prime} 11^{\prime \prime}$ & $97^{\circ} 39^{\prime} 58^{\prime \prime}$ & Edwards & -- & & 6.2 & 1.3 & 4.9 & \\
\hline 3 & $30^{\circ} 41^{\prime} 27^{\prime \prime}$ & $97^{\circ} 39^{\prime} 18^{\prime \prime}$ & -- & -- & & 13.5 & 3.2 & 3.6 & \\
\hline 4 & $30^{\circ} 52^{\prime} 47^{\prime \prime}$ & $97^{\circ} 55^{\prime} 11^{\prime \prime}$ & -- & -- & & .37 & 4.9 & 30.5 & at US 183 bridge \\
\hline 4 & $30^{\circ} 50^{\prime} 34^{\prime \prime}$ & $97^{\circ} 51^{\prime} 32^{\prime \prime}$ & -- & -- & & .77 & 3.2 & 25.6 & \\
\hline 4 & $30^{\circ} 48^{\prime} 37^{\prime \prime}$ & $97^{\circ} 49^{\prime} 32^{\prime \prime}$ & -- & -- & & .83 & 3.0 & 22.4 & \\
\hline 4 & $30^{\circ} 46^{\prime} 44^{\prime \prime}$ & $97^{\circ} 47^{\prime} 45^{\prime \prime}$ & -- & -- & & .56 & 2.5 & 19.4 & \\
\hline
\end{tabular}


Table 4. Gains and losses from gain-loss studies in Texas-Continued

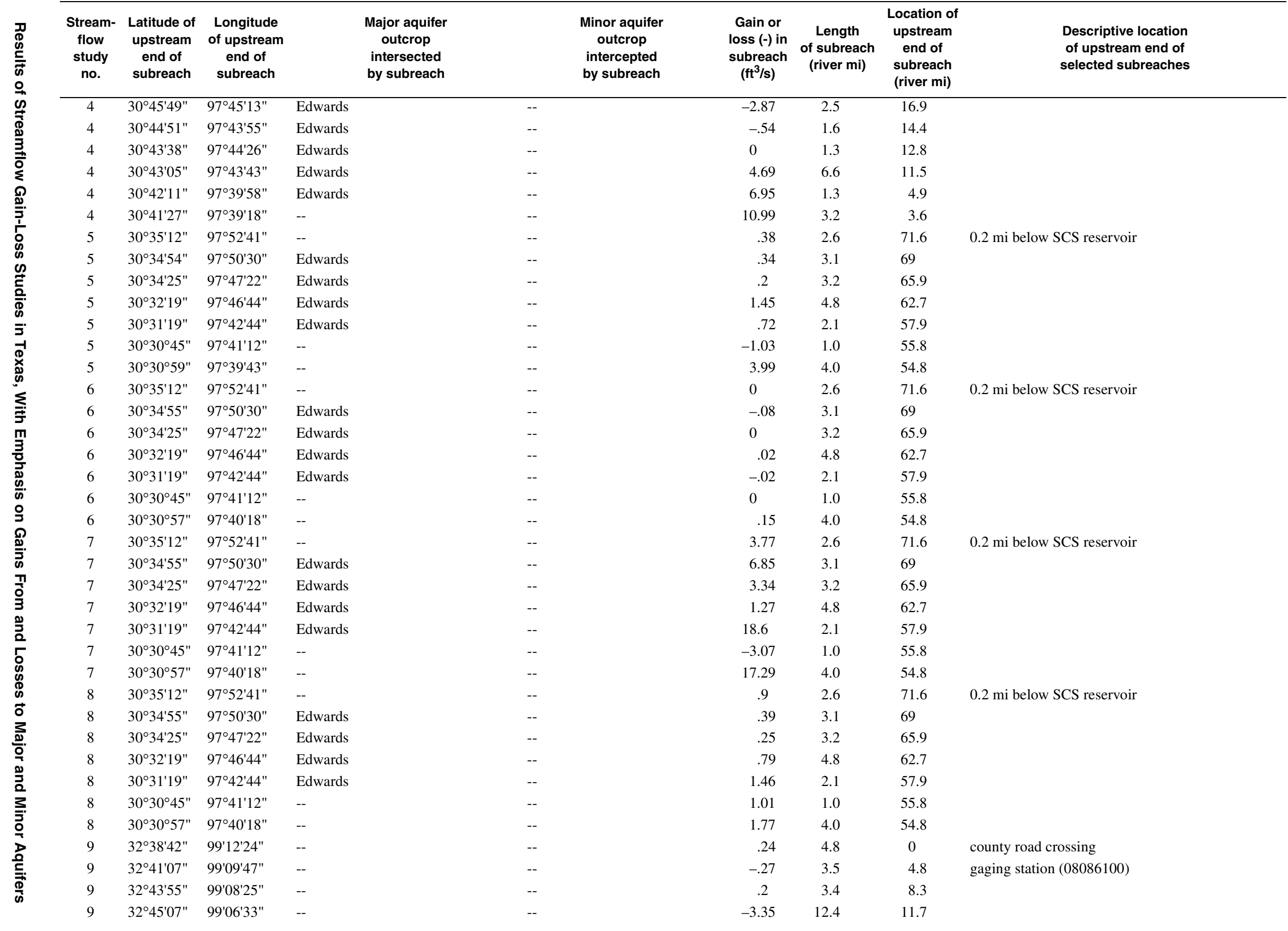


Table 4. Gains and losses from gain-loss studies in Texas-Continued

\begin{tabular}{|c|c|c|c|c|c|c|c|c|c|c|}
\hline $\begin{array}{l}\text { Stream- } \\
\text { flow } \\
\text { study } \\
\text { no. }\end{array}$ & $\begin{array}{l}\text { Latitude of } \\
\text { upstream } \\
\text { end of } \\
\text { subreach }\end{array}$ & $\begin{array}{l}\text { Longitude } \\
\text { of upstream } \\
\text { end of } \\
\text { subreach }\end{array}$ & & $\begin{array}{c}\text { Major aquifer } \\
\text { outcrop } \\
\text { intersected } \\
\text { by subreach }\end{array}$ & & $\begin{array}{c}\text { Minor aquifer } \\
\text { outcrop } \\
\text { intercepted } \\
\text { by subreach }\end{array}$ & $\begin{array}{c}\text { Gain or } \\
\text { loss }(-) \text { in } \\
\text { subreach } \\
\left(\mathrm{ft}^{3} / \mathrm{s}\right)\end{array}$ & $\begin{array}{l}\text { Length } \\
\text { of subreach } \\
\text { (river mi) }\end{array}$ & $\begin{array}{l}\text { Location of } \\
\text { upstream } \\
\text { end of } \\
\text { subreach } \\
\text { (river mi) }\end{array}$ & $\begin{array}{l}\text { Descriptive location } \\
\text { of upstream end of } \\
\text { selected subreaches }\end{array}$ \\
\hline 10 & $31^{\circ} 05^{\prime} 49^{\prime \prime}$ & $98^{\circ} 02^{\prime} 22^{\prime \prime}$ & Trinity & & -- & & -0.75 & 2.3 & 80.1 & $1 \mathrm{mi}$ above mouth of Sulphur Creek \\
\hline 10 & $31^{\circ} 04^{\prime} 37^{\prime \prime}$ & $98^{\circ} 00^{\prime} 45^{\prime \prime}$ & Trinity & & -- & & -1.38 & 12.9 & 77.8 & \\
\hline 10 & $30^{\circ} 59^{\prime} 40^{\prime \prime}$ & $97^{\circ} 55^{\prime} 31^{\prime \prime}$ & Trinity & & -- & & .46 & 12.8 & 64.9 & \\
\hline 10 & $30^{\circ} 58^{\prime} 39^{\prime \prime}$ & $97^{\circ} 47^{\prime} 51^{\prime \prime}$ & Trinity & & -- & & -.77 & 2.5 & 52.1 & \\
\hline 10 & $30^{\circ} 58^{\prime} 19^{\prime \prime}$ & $97^{\circ} 46^{\prime} 37^{\prime \prime}$ & Trinity & & -- & & .34 & 8.0 & 49.6 & \\
\hline 10 & $30^{\circ} 57^{\prime} 09^{\prime \prime}$ & $97^{\circ} 41^{\prime} 47^{\prime \prime}$ & Trinity & & -- & & -1.85 & 7.8 & 41.6 & \\
\hline 10 & $30^{\circ} 59^{\prime} 08^{\prime \prime}$ & $97^{\circ} 39^{\prime} 06^{\prime \prime}$ & -- & & -- & & .76 & 11.8 & 33.8 & \\
\hline 10 & $31^{\circ} 01^{\prime} 40^{\prime \prime}$ & $97^{\circ} 34^{\prime} 59^{\prime \prime}$ & -- & & -- & & -.46 & 2.8 & 22 & \\
\hline 10 & $31^{\circ} 00^{\prime} 37^{\prime \prime}$ & $97^{\circ} 33^{\prime} 52^{\prime \prime}$ & Edwards & & -- & & .95 & 4.4 & 19.2 & \\
\hline 10 & $31^{\circ} 01^{\prime} 14^{\prime \prime}$ & $97^{\circ} 30^{\prime} 43^{\prime \prime}$ & Edwards & & -- & & -1.99 & 2.2 & 14.8 & \\
\hline 10 & $31^{\circ} 00^{\prime} 06^{\prime \prime}$ & $97^{\circ} 29^{\prime} 31^{\prime \prime}$ & Edwards & & -- & & -.81 & 12.3 & 12.6 & \\
\hline 11 & $31^{\circ} 02^{\prime} 27^{\prime \prime}$ & $97^{\circ} 32^{\prime} 08^{\prime \prime}$ & Edwards & & -- & & 8.2 & 2.6 & 15.8 & 1,500 ft below Stillhouse Hollow Dam \\
\hline 11 & $31^{\circ} 00^{\prime} 07^{\prime \prime}$ & $97^{\circ} 29^{\prime} 31^{\prime \prime}$ & Edwards & & -- & & 5.2 & 8.4 & 13.2 & gaging station (08001041) \\
\hline 11 & $30^{\circ} 59^{\prime} 23^{\prime \prime}$ & $97^{\circ} 26^{\prime} 40^{\prime \prime}$ & Edwards & & -- & & 2.48 & 4.7 & 4.8 & \\
\hline 12 & $31^{\circ} 06^{\prime} 19^{\prime \prime}$ & $97^{\circ} 28^{\prime} 21^{\prime \prime}$ & -- & & -- & & 3.36 & 4.4 & 16.7 & $300 \mathrm{ft}$ below Belton Dam \\
\hline 12 & $31^{\circ} 04^{\prime} 11^{\prime \prime}$ & $97^{\circ} 26^{\prime} 28^{\prime \prime}$ & -- & & -- & & -52.94 & 4.4 & 12.3 & \\
\hline 12 & $31^{\circ} 02^{\prime} 02^{\prime \prime}$ & $97^{\circ} 26^{\prime} 11^{\prime \prime}$ & -- & & -- & & 52.06 & .2 & 7.9 & \\
\hline 13 & $32^{\circ} 22^{\prime} 17^{\prime \prime}$ & $98^{\circ} 46^{\prime} 04^{\prime \prime}$ & -- & & -- & & -.26 & 5.2 & 0 & $300 \mathrm{ft}$ below Olden Lake Dam \\
\hline 13 & $32^{\circ} 21^{\prime} 52^{\prime \prime}$ & $98^{\circ} 42^{\prime} 56^{\prime \prime}$ & -- & & -- & & .01 & 1.4 & 5.2 & \\
\hline 13 & $32^{\circ} 20^{\prime} 39^{\prime \prime}$ & $98^{\circ} 41^{\prime} 54^{\prime \prime}$ & -- & & -- & & -.22 & 5.4 & 6.6 & \\
\hline 13 & $32^{\circ} 20^{\prime} 13^{\prime \prime}$ & $98^{\circ} 39^{\prime} 08^{\prime \prime}$ & -- & & -- & & 0 & 3.4 & 12 & \\
\hline 13 & $32^{\circ} 17^{\prime} 53^{\prime \prime}$ & $98^{\circ} 38^{\prime} 31^{\prime \prime}$ & -- & & -- & & -.02 & 4.4 & 15.4 & \\
\hline 13 & $32^{\circ} 15^{\prime} 17^{\prime \prime}$ & $98^{\circ} 36^{\prime} 11^{\prime \prime}$ & -- & & -- & & .32 & 3.8 & 19.8 & \\
\hline 13 & $32^{\circ} 13^{\prime} 14^{\prime \prime}$ & $98^{\circ} 34^{\prime} 37^{\prime \prime}$ & Trinity & & -- & & -.11 & 9.0 & 23.6 & \\
\hline 13 & $32^{\circ} 06^{\prime} 47^{\prime \prime}$ & $98^{\circ} 29^{\prime} 48^{\prime \prime}$ & Trinity & & -- & & .31 & 2.6 & 32.6 & \\
\hline 13 & $32^{\circ} 04^{\prime} 34^{\prime \prime}$ & $98^{\circ} 29^{\prime} 30^{\prime \prime}$ & Trinity & & -- & & .29 & 5.8 & 35.2 & \\
\hline 13 & $31^{\circ} 59^{\prime} 44^{\prime \prime}$ & $98^{\circ} 29^{\prime} 24^{\prime \prime}$ & Trinity & & -- & & -.06 & 4.5 & 41 & \\
\hline 14 & $30^{\circ} 44^{\prime} 07^{\prime \prime}$ & $97^{\circ} 54^{\prime} 55^{\prime \prime}$ & Trinity & & -- & & .73 & 4.0 & 82.5 & $50 \mathrm{ft}$ below county road crossing \\
\hline 14 & $30^{\circ} 41^{\prime} 59^{\prime \prime}$ & $97^{\circ} 52^{\prime} 27^{\prime \prime}$ & Trinity & & -- & & .23 & 1.0 & 78.5 & \\
\hline 14 & $30^{\circ} 41^{\prime} 56^{\prime \prime}$ & $97^{\circ} 51^{\prime} 32^{\prime \prime}$ & Trinity & & -- & & -.22 & .9 & 77.5 & \\
\hline 14 & $30^{\circ} 41^{\prime} 51^{\prime \prime}$ & $97^{\circ} 50^{\prime} 43^{\prime \prime}$ & Trinity & & -- & & .34 & 2.5 & 76.6 & \\
\hline 14 & $30^{\circ} 41^{\prime} 26^{\prime \prime}$ & $97^{\circ} 48^{\prime} 45^{\prime \prime}$ & Edwards & & -- & & 1.81 & 4.6 & 74.1 & \\
\hline 14 & $30^{\circ} 40^{\prime} 19^{\prime \prime}$ & $97^{\circ} 45^{\prime} 01^{\prime \prime}$ & Edwards & & -- & & .6 & 2.6 & 69.5 & \\
\hline 14 & $30^{\circ} 39^{\prime} 44^{\prime \prime}$ & $97^{\circ} 43^{\prime} 02^{\prime \prime}$ & Edwards & & -- & & .16 & 1.4 & 66.9 & \\
\hline 15 & $30^{\circ} 44^{\prime} 07^{\prime \prime}$ & $97^{\circ} 54^{\prime} 55^{\prime \prime}$ & Trinity & & -- & & 0 & 4.0 & 82.5 & $50 \mathrm{ft}$ below county road crossing \\
\hline 15 & $30^{\circ} 42^{\prime} 10^{\prime \prime}$ & $97^{\circ} 52^{\prime} 36^{\prime \prime}$ & Trinity & & -- & & 0 & 1.0 & 78.5 & \\
\hline 15 & $30^{\circ} 41^{\prime} 56^{\prime \prime}$ & $97^{\circ} 51^{\prime} 32^{\prime \prime}$ & Trinity & & -- & & 0 & .9 & 77.5 & \\
\hline 15 & $30^{\circ} 41^{\prime} 51^{\prime \prime}$ & $97^{\circ} 50^{\prime} 43^{\prime \prime}$ & Trinity & & -- & & .03 & 2.5 & 76.6 & \\
\hline
\end{tabular}


Table 4. Gains and losses from gain-loss studies in Texas-Continued

\begin{tabular}{|c|c|c|c|c|c|c|c|c|c|c|}
\hline $\begin{array}{l}\text { Stream- } \\
\text { flow } \\
\text { study } \\
\text { no. }\end{array}$ & $\begin{array}{l}\text { Latitude of } \\
\text { upstream } \\
\text { end of } \\
\text { subreach }\end{array}$ & $\begin{array}{l}\text { Longitude } \\
\text { of upstream } \\
\text { end of } \\
\text { subreach }\end{array}$ & & $\begin{array}{l}\text { Major aquifer } \\
\text { outcrop } \\
\text { intersected } \\
\text { by subreach }\end{array}$ & & $\begin{array}{l}\text { Minor aquifer } \\
\text { outcrop } \\
\text { intercepted } \\
\text { by subreach }\end{array}$ & $\begin{array}{l}\text { Gain or } \\
\text { loss }(-) \text { in } \\
\text { subreach } \\
\left(\mathrm{ft}^{3} / \mathrm{s}\right)\end{array}$ & $\begin{array}{l}\text { Length } \\
\text { of subreach } \\
\text { (river mi) }\end{array}$ & $\begin{array}{l}\text { Location of } \\
\text { upstream } \\
\text { end of } \\
\text { subreach } \\
\text { (river mi) }\end{array}$ & $\begin{array}{l}\text { Descriptive location } \\
\text { of upstream end of } \\
\text { selected subreaches }\end{array}$ \\
\hline 15 & $30^{\circ} 41^{\prime} 26^{\prime \prime}$ & $97^{\circ} 48^{\prime} 45^{\prime \prime}$ & Edwards & & -- & & 0.3 & 4.6 & 74.1 & \multirow{7}{*}{$50 \mathrm{ft}$ below county road crossing } \\
\hline 15 & $30^{\circ} 40^{\prime} 19^{\prime \prime}$ & $97^{\circ} 45^{\prime} 01^{\prime \prime}$ & Edwards & & -- & & -.12 & 2.6 & 69.5 & \\
\hline 15 & $30^{\circ} 39^{\prime} 44^{\prime \prime}$ & $97^{\circ} 43^{\prime} 02^{\prime \prime}$ & Edwards & & -- & & -.14 & 1.4 & 66.9 & \\
\hline 16 & $30^{\circ} 44^{\prime} 07^{\prime \prime}$ & $97^{\circ} 54^{\prime} 55^{\prime \prime}$ & Trinity & & -- & & .4 & 4.0 & 82.5 & \\
\hline 16 & $30^{\circ} 42^{\prime} 10^{\prime \prime}$ & $97^{\circ} 52^{\prime} 36^{\prime \prime}$ & Trinity & & -- & & 4.4 & 1.0 & 78.5 & \\
\hline 16 & $30^{\circ} 41^{\prime} 56^{\prime \prime}$ & $97^{\circ} 51^{\prime} 32^{\prime \prime}$ & Trinity & & -- & & 7.0 & .9 & 77.5 & \\
\hline 16 & $30^{\circ} 41^{\prime} 51^{\prime \prime}$ & $97^{\circ} 50^{\prime} 43^{\prime \prime}$ & Trinity & & -- & & 6.46 & 2.5 & 76.6 & \\
\hline 16 & $30^{\circ} 41^{\prime} 26^{\prime \prime}$ & $97^{\circ} 48^{\prime} 45^{\prime \prime}$ & Edwards & & -- & & .42 & 4.6 & 74.1 & \multirow{7}{*}{$50 \mathrm{ft}$ below county road crossing } \\
\hline 16 & $30^{\circ} 40^{\prime} 19^{\prime \prime}$ & $97^{\circ} 45^{\prime} 01^{\prime \prime}$ & Edwards & & -- & & 18.0 & 2.6 & 69.5 & \\
\hline 16 & $30^{\circ} 39^{\prime} 44^{\prime \prime}$ & $97^{\circ} 43^{\prime} 02^{\prime \prime}$ & Edwards & & -- & & -2.94 & 1.4 & 66.9 & \\
\hline 17 & $30^{\circ} 44^{\prime} 07^{\prime \prime}$ & $97^{\circ} 54^{\prime} 55^{\prime \prime}$ & Trinity & & -- & & 1.8 & 4.0 & 82.5 & \\
\hline 17 & $30^{\circ} 42^{\prime} 10^{\prime \prime}$ & $97^{\circ} 52^{\prime} 36^{\prime \prime}$ & Trinity & & -- & & 1.3 & 1.0 & 78.5 & \\
\hline 17 & $30^{\circ} 41^{\prime} 56^{\prime \prime}$ & $97^{\circ} 51^{\prime} 32^{\prime \prime}$ & Trinity & & -- & & -3.6 & .9 & 77.5 & \\
\hline 17 & $30^{\circ} 41^{\prime} 51^{\prime \prime}$ & $97^{\circ} 50^{\prime} 43^{\prime \prime}$ & Trinity & & -- & & 5.85 & 2.5 & 76.6 & \\
\hline 17 & $30^{\circ} 41^{\prime} 26^{\prime \prime}$ & $97^{\circ} 48^{\prime} 45^{\prime \prime}$ & Edwards & & -- & & 1.42 & 4.6 & 74.1 & \multirow{7}{*}{ at US 183 bridge } \\
\hline 17 & $30^{\circ} 40^{\prime} 19^{\prime \prime}$ & $97^{\circ} 45^{\prime} 01^{\prime \prime}$ & Edwards & & -- & & 3.0 & 2.6 & 69.5 & \\
\hline 17 & $30^{\circ} 39^{\prime} 44^{\prime \prime}$ & $97^{\circ} 43^{\prime} 02^{\prime \prime}$ & Edwards & & -- & & -.37 & 1.4 & 66.9 & \\
\hline 18 & $30^{\circ} 42^{\prime} 11^{\prime \prime}$ & $97^{\circ} 52^{\prime} 37^{\prime \prime}$ & Trinity & & -- & & -.83 & 1.0 & 69 & \\
\hline 18 & $30^{\circ} 41^{\prime} 56^{\prime \prime}$ & $97^{\circ} 51^{\prime} 32^{\prime \prime}$ & Trinity & & -- & & -.23 & .8 & 68 & \\
\hline 18 & $30^{\circ} 41^{\prime} 51^{\prime \prime}$ & $97^{\circ} 50^{\prime} 43^{\prime \prime}$ & Trinity & & -- & & .71 & 2.8 & 67.2 & \\
\hline 18 & $30^{\circ} 41^{\prime} 25^{\prime \prime}$ & $97^{\circ} 48^{\prime} 44^{\prime \prime}$ & Edwards & & -- & & 1.29 & 4.6 & 64.4 & \\
\hline 18 & $30^{\circ} 40^{\prime} 43^{\prime \prime}$ & $97^{\circ} 46^{\prime} 29^{\prime \prime}$ & Edwards & & -- & & 1.04 & 5.2 & 59.8 & \multirow{3}{*}{ at $\mathrm{SH} 195$} \\
\hline 19 & $30^{\circ} 49^{\prime} 59^{\prime \prime}$ & $97^{\circ} 47^{\prime 2} 22^{\prime \prime}$ & Edwards & & -- & & 0 & 8.9 & 36 & \\
\hline 19 & $30^{\circ} 49^{\prime} 36^{\prime \prime}$ & $97^{\circ} 41^{\prime} 38^{\prime \prime}$ & Edwards & & -- & & -.08 & 3.6 & 27.1 & \\
\hline 19 & $30^{\circ} 49^{\prime} 35^{\prime \prime}$ & $97^{\circ} 38^{\prime} 36^{\prime \prime}$ & Edwards & & -- & & .47 & 3.0 & 23.5 & \multirow{14}{*}{$\begin{array}{l}\text { at SH } 195 \\
\text { at County Road } 232 \\
\text { at County Road } 309\end{array}$} \\
\hline 19 & $30^{\circ} 51^{\prime} 54^{\prime \prime}$ & $97^{\circ} 38^{\prime} 03^{\prime \prime}$ & Edwards & & -- & & 1.2 & 3.7 & 20.5 & \\
\hline 19 & $30^{\circ} 53^{\prime} 49^{\prime \prime}$ & $97^{\circ} 36^{\prime} 52^{\prime \prime}$ & Edwards & & -- & & 2.53 & 6.6 & 16.8 & \\
\hline 19 & $30^{\circ} 56^{\prime} 48^{\prime \prime}$ & $97^{\circ} 33^{\prime} 15^{\prime \prime}$ & Edwards & & -- & & 11.17 & .4 & 10.2 & \\
\hline 20 & $30^{\circ} 51^{\prime} 05^{\prime \prime}$ & $97^{\circ} 48^{\prime} 18^{\prime \prime}$ & Edwards & & -- & & 0 & 8.9 & 36 & \\
\hline 20 & $30^{\circ} 49^{\prime} 36^{\prime \prime}$ & $97^{\circ} 41^{\prime} 38^{\prime \prime}$ & Edwards & & -- & & 0 & 3.6 & 27.1 & \\
\hline 20 & $30^{\circ} 49^{\prime} 35^{\prime \prime}$ & $97^{\circ} 38^{\prime} 36^{\prime \prime}$ & Edwards & & -- & & .11 & 3.0 & 23.5 & \\
\hline 20 & $30^{\circ} 51^{\prime} 54^{\prime \prime}$ & $97^{\circ} 38^{\prime} 03^{\prime \prime}$ & Edwards & & -- & & .3 & 3.7 & 20.5 & \\
\hline 20 & $30^{\circ} 53^{\prime} 49^{\prime \prime}$ & 97³6'52" & Edwards & & -- & & -.69 & 6.6 & 16.8 & \\
\hline 20 & $30^{\circ} 56^{\prime} 48^{\prime \prime}$ & 97³3'15" & Edwards & & -- & & 9.24 & .4 & 10.2 & \\
\hline 21 & $30^{\circ} 51^{\prime} 05^{\prime \prime}$ & $97^{\circ} 48^{\prime} 18^{\prime \prime}$ & Edwards & & -- & & 5.93 & 8.9 & 36 & \\
\hline 21 & $30^{\circ} 49^{\prime} 36^{\prime \prime}$ & $97^{\circ} 41^{\prime} 38^{\prime \prime}$ & Edwards & & -- & & -7.61 & 3.6 & 27.1 & \\
\hline 21 & $30^{\circ} 49^{\prime} 35^{\prime \prime}$ & $97^{\circ} 38^{\prime} 36^{\prime \prime}$ & Edwards & & -- & & 2.24 & 3.0 & 23.5 & \\
\hline 21 & $30^{\circ} 51^{\prime} 54^{\prime \prime}$ & $97^{\circ} 38^{\prime} 03^{\prime \prime}$ & Edwards & & -- & & 13.64 & 3.7 & 20.5 & \\
\hline
\end{tabular}


Table 4. Gains and losses from gain-loss studies in Texas-Continued

\begin{tabular}{|c|c|c|c|c|c|c|c|c|c|}
\hline $\begin{array}{l}\text { Stream- } \\
\text { flow } \\
\text { study } \\
\text { no. }\end{array}$ & $\begin{array}{l}\text { Latitude of } \\
\text { upstream } \\
\text { end of } \\
\text { subreach }\end{array}$ & $\begin{array}{l}\text { Longitude } \\
\text { of upstream } \\
\text { end of } \\
\text { subreach }\end{array}$ & $\begin{array}{c}\text { Major aquifer } \\
\text { outcrop } \\
\text { intersected } \\
\text { by subreach }\end{array}$ & & $\begin{array}{c}\text { Minor aquifer } \\
\text { outcrop } \\
\text { intercepted } \\
\text { by subreach }\end{array}$ & $\begin{array}{c}\text { Gain or } \\
\text { loss }(-) \text { in } \\
\text { subreach } \\
\left(\mathrm{ft}^{3} / \mathrm{s}\right)\end{array}$ & $\begin{array}{l}\text { Length } \\
\text { of subreach } \\
\text { (river mi) }\end{array}$ & $\begin{array}{l}\text { Location of } \\
\text { upstream } \\
\text { end of } \\
\text { subreach } \\
\text { (river mi) }\end{array}$ & $\begin{array}{l}\text { Descriptive location } \\
\text { of upstream end of } \\
\text { selected subreaches }\end{array}$ \\
\hline 21 & $30^{\circ} 53^{\prime} 49^{\prime \prime}$ & $97^{\circ} 36^{\prime} 52^{\prime \prime}$ & Edwards & -- & & 23.8 & 6.6 & 16.8 & \\
\hline 21 & $30^{\circ} 56^{\prime} 48^{\prime \prime}$ & $97^{\circ} 33^{\prime} 15^{\prime \prime}$ & Edwards & -- & & 25.0 & .4 & 10.2 & \\
\hline 22 & $30^{\circ} 51^{\prime} 05^{\prime \prime}$ & $97^{\circ} 48^{\prime} 18^{\prime \prime}$ & Edwards & -- & & .41 & 8.9 & 36 & at SH 195 \\
\hline 22 & $30^{\circ} 49^{\prime} 36^{\prime \prime}$ & $97^{\circ} 41^{\prime} 38^{\prime \prime}$ & Edwards & -- & & -1.6 & 3.6 & 27.1 & at County Road 232 \\
\hline 22 & $30^{\circ} 49^{\prime} 35^{\prime \prime}$ & $97^{\circ} 38^{\prime} 36^{\prime \prime}$ & Edwards & -- & & 1.53 & 3.0 & 23.5 & at County Road 309 \\
\hline 22 & $30^{\circ} 51^{\prime} 54^{\prime \prime}$ & $97^{\circ} 38^{\prime} 03^{\prime \prime}$ & Edwards & -- & & 5.54 & 3.7 & 20.5 & \\
\hline 22 & $30^{\circ} 53^{\prime} 49^{\prime \prime}$ & $97^{\circ} 36^{\prime} 52^{\prime \prime}$ & Edwards & -- & & 7.11 & 6.6 & 16.8 & \\
\hline 22 & $30^{\circ} 56^{\prime} 48^{\prime \prime}$ & $97^{\circ} 33^{\prime} 15^{\prime \prime}$ & Edwards & -- & & 36.1 & .4 & 10.2 & \\
\hline 23 & $32^{\circ} 40^{\prime} 39^{\prime \prime}$ & $99^{\circ} 16^{\prime} 49^{\prime \prime}$ & -- & -- & & .88 & 4.1 & 8.3 & $8.3 \mathrm{mi}$ above mouth \\
\hline 23 & $32^{\circ} 41^{\prime} 00^{\prime \prime}$ & $99^{\circ} 16^{\prime} 05^{\prime \prime}$ & -- & -- & & -.04 & 3.7 & 4.2 & gaging station $(08086200)$ \\
\hline 24 & $30^{\circ} 39^{\prime} 18^{\prime \prime}$ & $97^{\circ} 38^{\prime} 06^{\prime \prime}$ & -- & -- & & -2.06 & 9.0 & 51.8 & gaging station $(08105000)$ \\
\hline 24 & $30^{\circ} 38^{\prime} 09^{\prime \prime}$ & $97^{\circ} 33^{\prime} 00^{\prime \prime}$ & -- & -- & & 2.71 & 5.0 & 42.8 & \\
\hline 24 & $30^{\circ} 37^{\prime} 52^{\prime \prime}$ & $97^{\circ} 28^{\prime} 13^{\prime \prime}$ & -- & -- & & 1.7 & 2.2 & 37.8 & \\
\hline 24 & $30^{\circ} 38^{\prime} 38^{\prime \prime}$ & $97^{\circ} 26^{\prime} 19^{\prime \prime}$ & -- & -- & & 6.28 & 9.6 & 35.6 & SH 95 \\
\hline 24 & $30^{\circ} 41^{\prime} 10^{\prime \prime}$ & $97^{\circ} 22^{\prime} 06^{\prime \prime}$ & -- & -- & & 1.75 & 4.0 & 26 & \\
\hline 24 & $30^{\circ} 41^{\prime} 39^{\prime \prime}$ & $97^{\circ} 16^{\prime} 42^{\prime \prime}$ & -- & -- & & 2.7 & 6.0 & 22 & \\
\hline 24 & $30^{\circ} 41^{\prime} 14^{\prime \prime}$ & $97^{\circ} 11^{\prime} 34^{\prime \prime}$ & -- & -- & & -3.4 & 4.0 & 16 & FM 486 \\
\hline 24 & $30^{\circ} 41^{\prime} 30^{\prime \prime}$ & $97^{\circ} 08^{\prime} 15^{\prime \prime}$ & -- & -- & & -.84 & 2.4 & 12 & \\
\hline 24 & $30^{\circ} 42^{\prime} 36^{\prime \prime}$ & $97^{\circ} 06^{\prime} 40^{\prime \prime}$ & -- & -- & & 4.8 & 4.8 & 9.6 & \\
\hline 24 & $30^{\circ} 43^{\prime} 38^{\prime \prime}$ & $97^{\circ} 02^{\prime} 18^{\prime \prime}$ & -- & -- & & -3.1 & 2.0 & 4.8 & FM 487 \\
\hline 25 & $30^{\circ} 37^{\prime} 14^{\prime \prime}$ & $97^{\circ} 51^{\prime} 38^{\prime \prime}$ & Edwards & -- & & .4 & 5.4 & 12.8 & at US 183 \\
\hline 25 & $30^{\circ} 36^{\prime} 56^{\prime \prime}$ & $97^{\circ} 47^{\prime} 01^{\prime \prime}$ & Edwards & -- & & 1.21 & 4.4 & 7.4 & \\
\hline 25 & $30^{\circ} 37^{\prime} 12^{\prime \prime}$ & $97^{\circ} 42^{\prime} 31^{\prime \prime}$ & Edwards & -- & & -.23 & 3.0 & 3 & \\
\hline 26 & $30^{\circ} 42^{\prime} 55^{\prime \prime}$ & $98^{\circ} 03^{\prime} 02^{\prime \prime}$ & Trinity & -- & & .24 & 2.0 & 31.2 & at FM 1174 near Bertram \\
\hline 26 & $30^{\circ} 42^{\prime} 09^{\prime \prime}$ & $98^{\circ} 01^{\prime} 52^{\prime \prime}$ & Trinity & -- & & .11 & 3.1 & 29.2 & \\
\hline 26 & $30^{\circ} 41^{\prime} 17^{\prime \prime}$ & $97^{\circ} 59^{\prime} 00^{\prime \prime}$ & Trinity & -- & & .06 & 2.2 & 26.1 & \\
\hline 26 & $30^{\circ} 40^{\prime} 31^{\prime \prime}$ & $97^{\circ} 57^{\prime} 45^{\prime \prime}$ & Trinity & -- & & -.09 & 2.2 & 23.9 & \\
\hline 26 & $30^{\circ} 39^{\prime} 32^{\prime \prime}$ & $97^{\circ} 56^{\prime} 13^{\prime \prime}$ & Trinity & -- & & .25 & 2.3 & 21.7 & \\
\hline 26 & $30^{\circ} 38^{\prime} 53^{\prime \prime}$ & $97^{\circ} 54^{\prime} 35^{\prime \prime}$ & Trinity & -- & & .83 & 4.9 & 19.4 & \\
\hline 26 & $30^{\circ} 37^{\prime} 14^{\prime \prime}$ & $97^{\circ} 51^{\prime} 38^{\prime \prime}$ & -- & -- & & -.07 & 2.8 & 14.5 & \\
\hline 26 & $30^{\circ} 36^{\prime} 42^{\prime \prime}$ & $97^{\circ} 49^{\prime} 07^{\prime \prime}$ & Edwards & -- & & .25 & 3.0 & 11.7 & \\
\hline 26 & $30^{\circ} 37^{\prime} 10^{\prime \prime}$ & $97^{\circ} 46^{\prime} 37^{\prime \prime}$ & Edwards & -- & & -3.03 & 3.9 & 8.7 & \\
\hline 26 & $30^{\circ} 37^{\prime} 13^{\prime \prime}$ & $97^{\circ} 43^{\prime} 38^{\prime \prime}$ & Edwards & -- & & 1.78 & 1.0 & 4.8 & \\
\hline 26 & $30^{\circ} 37^{\prime} 12^{\prime \prime}$ & $97^{\circ} 42^{\prime} 43^{\prime \prime}$ & Edwards & -- & & 3.66 & 1.5 & 3.8 & \\
\hline 26 & $30^{\circ} 37^{\prime} 34^{\prime \prime}$ & $97^{\circ} 41^{\prime} 35^{\prime \prime}$ & Edwards & -- & & 1.1 & .8 & 2.3 & \\
\hline 27 & $30^{\circ} 42^{\prime} 55^{\prime \prime}$ & $98^{\circ} 03^{\prime} 02^{\prime \prime}$ & Trinity & -- & & 0 & 2.0 & 31.2 & at FM 1174 near Bertram \\
\hline 27 & $30^{\circ} 42^{\prime} 09^{\prime \prime}$ & $98^{\circ} 01^{\prime} 52^{\prime \prime}$ & Trinity & -- & & 0 & 3.1 & 29.2 & \\
\hline 27 & $30^{\circ} 41^{\prime} 17^{\prime \prime}$ & $97^{\circ} 59^{\prime} 00^{\prime \prime}$ & Trinity & -- & & .01 & 2.2 & 26.1 & \\
\hline
\end{tabular}


I Table 4. Gains and losses from gain-loss studies in Texas-Continued

\begin{tabular}{|c|c|c|c|c|c|c|c|c|}
\hline $\begin{array}{l}\text { Stream- } \\
\text { flow } \\
\text { study } \\
\text { no. }\end{array}$ & $\begin{array}{l}\text { Latitude of } \\
\text { upstream } \\
\text { end of } \\
\text { subreach }\end{array}$ & $\begin{array}{l}\text { Longitude } \\
\text { of upstream } \\
\text { end of } \\
\text { subreach }\end{array}$ & $\begin{array}{l}\text { Major aquifer } \\
\text { outcrop } \\
\text { intersected } \\
\text { by subreach }\end{array}$ & $\begin{array}{c}\text { Minor aquifer } \\
\text { outcrop } \\
\text { intercepted } \\
\text { by subreach }\end{array}$ & $\begin{array}{l}\text { Gain or } \\
\text { loss }(-) \text { in } \\
\text { subreach } \\
\left(\mathrm{ft}^{3} / \mathrm{s}\right)\end{array}$ & 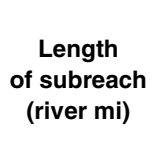 & $\begin{array}{l}\text { Location of } \\
\text { upstream } \\
\text { end of } \\
\text { subreach } \\
\text { (river mi) }\end{array}$ & $\begin{array}{l}\text { Descriptive location } \\
\text { of upstream end of } \\
\text { selected subreaches }\end{array}$ \\
\hline 27 & $30^{\circ} 40^{\prime} 31^{\prime \prime}$ & $97^{\circ} 57^{\prime} 45^{\prime \prime}$ & Trinity & -- & -0.01 & 2.2 & 23.9 & \\
\hline 27 & $30^{\circ} 39^{\prime} 32^{\prime \prime}$ & $97^{\circ} 56^{\prime} 13^{\prime \prime}$ & Trinity & -- & 0 & 2.3 & 21.7 & \\
\hline 27 & $30^{\circ} 38^{\prime} 53^{\prime \prime}$ & $97^{\circ} 54^{\prime} 35^{\prime \prime}$ & Trinity & -- & 20.01 & 4.9 & 19.4 & \\
\hline 27 & $30^{\circ} 37^{\prime} 14^{\prime \prime}$ & $97^{\circ} 51^{\prime} 38^{\prime \prime}$ & -- & -- & -20.01 & 2.8 & 14.5 & \\
\hline 27 & $30^{\circ} 36^{\prime} 42^{\prime \prime}$ & $97^{\circ} 49^{\prime} 07^{\prime \prime}$ & Edwards & -- & 0 & 3.0 & 11.7 & \\
\hline 27 & $30^{\circ} 37^{\prime} 10^{\prime \prime}$ & $97^{\circ} 46^{\prime} 37^{\prime \prime}$ & Edwards & -- & 0 & 3.9 & 8.7 & \\
\hline 27 & $30^{\circ} 37^{\prime} 13^{\prime \prime}$ & $97^{\circ} 43^{\prime} 38^{\prime \prime}$ & Edwards & -- & .02 & 1.0 & 4.8 & \\
\hline 27 & $30^{\circ} 37^{\prime} 12^{\prime \prime}$ & $97^{\circ} 42^{\prime} 43^{\prime \prime}$ & Edwards & -- & 0 & 1.5 & 3.8 & \\
\hline 27 & $30^{\circ} 37^{\prime} 34^{\prime \prime}$ & $97^{\circ} 41^{\prime} 35^{\prime \prime}$ & Edwards & -- & -.01 & .8 & 2.3 & \\
\hline 28 & $30^{\circ} 42^{\prime} 55^{\prime \prime}$ & $98^{\circ} 03^{\prime} 02^{\prime \prime}$ & Trinity & -- & 1.38 & 2.0 & 31.2 & \multirow[t]{12}{*}{ at FM 1174 near Bertram } \\
\hline 28 & $30^{\circ} 42^{\prime} 09^{\prime \prime}$ & $98^{\circ} 01^{\prime} 52^{\prime \prime}$ & Trinity & -- & 1.19 & 3.1 & 29.2 & \\
\hline 28 & $30^{\circ} 41^{\prime} 17^{\prime \prime}$ & $97^{\circ} 59^{\prime} 00^{\prime \prime}$ & Trinity & -- & 1.0 & 2.2 & 26.1 & \\
\hline 28 & $30^{\circ} 40^{\prime} 31^{\prime \prime}$ & $97^{\circ} 57^{\prime} 45^{\prime \prime}$ & Trinity & -- & -.64 & 2.2 & 23.9 & \\
\hline 28 & $30^{\circ} 39^{\prime} 32^{\prime \prime}$ & $97^{\circ} 56^{\prime} 13^{\prime \prime}$ & Trinity & -- & 9.0 & 2.3 & 21.7 & \\
\hline 28 & $30^{\circ} 38^{\prime} 53^{\prime \prime}$ & $97^{\circ} 54^{\prime} 35^{\prime \prime}$ & Trinity & -- & 4.82 & 4.9 & 19.4 & \\
\hline 28 & $30^{\circ} 37^{\prime} 14^{\prime \prime}$ & $97^{\circ} 51^{\prime} 38^{\prime \prime}$ & -- & -- & 6.0 & 2.8 & 14.5 & \\
\hline 28 & $30^{\circ} 36^{\prime} 42^{\prime \prime}$ & $97^{\circ} 49^{\prime} 07^{\prime \prime}$ & Edwards & -- & 1.2 & 3.0 & 11.7 & \\
\hline 28 & $30^{\circ} 37^{\prime} 10^{\prime \prime}$ & $97^{\circ} 46^{\prime} 37^{\prime \prime}$ & Edwards & -- & -46.93 & 3.9 & 8.7 & \\
\hline 28 & $30^{\circ} 37^{\prime} 13^{\prime \prime}$ & $97^{\circ} 43^{\prime} 38^{\prime \prime}$ & Edwards & -- & 55.0 & 1.0 & 4.8 & \\
\hline 28 & $30^{\circ} 37^{\prime} 12^{\prime \prime}$ & $97^{\circ} 42^{\prime} 43^{\prime \prime}$ & Edwards & -- & .1 & 1.5 & 3.8 & \\
\hline 28 & $30^{\circ} 37^{\prime} 34^{\prime \prime}$ & $97^{\circ} 41^{\prime} 35^{\prime \prime}$ & Edwards & -- & 1.1 & .8 & 2.3 & \\
\hline 29 & $30^{\circ} 42^{\prime} 55^{\prime \prime}$ & $98^{\circ} 03^{\prime} 02^{\prime \prime}$ & Trinity & -- & .23 & 2.0 & 31.2 & \multirow[t]{13}{*}{ at FM 1174 near Bertram } \\
\hline 29 & $30^{\circ} 42^{\prime} 09^{\prime \prime}$ & $98^{\circ} 01^{\prime} 52^{\prime \prime}$ & Trinity & -- & .43 & 3.1 & 29.2 & \\
\hline 29 & $30^{\circ} 41^{\prime} 17^{\prime \prime}$ & $97^{\circ} 59^{\prime} 00^{\prime \prime}$ & Trinity & -- & .33 & 2.2 & 26.1 & \\
\hline 29 & $30^{\circ} 40^{\prime} 31^{\prime \prime}$ & $97^{\circ} 57^{\prime} 45^{\prime \prime}$ & Trinity & -- & -2.62 & .9 & 23.9 & \\
\hline 29 & $30^{\circ} 40^{\prime} 44^{\prime \prime}$ & $97^{\circ} 57^{\prime} 00^{\prime \prime}$ & Trinity & -- & 3.71 & 1.3 & 23 & \\
\hline 29 & $30^{\circ} 39^{\prime} 32^{\prime \prime}$ & $97^{\circ} 56^{\prime} 13^{\prime \prime}$ & Trinity & -- & -.54 & 2.3 & 21.7 & \\
\hline 29 & $30^{\circ} 38^{\prime} 53^{\prime \prime}$ & $97^{\circ} 54^{\prime} 35^{\prime \prime}$ & Trinity & -- & 2.27 & 4.9 & 19.4 & \\
\hline 29 & $30^{\circ} 37^{\prime} 14^{\prime \prime}$ & $97^{\circ} 51^{\prime} 38^{\prime \prime}$ & -- & -- & 1.41 & 2.8 & 14.5 & \\
\hline 29 & $30^{\circ} 36^{\prime} 42^{\prime \prime}$ & $97^{\circ} 49^{\prime} 07^{\prime \prime}$ & Edwards & -- & .33 & 3.0 & 11.7 & \\
\hline 29 & $30^{\circ} 37^{\prime} 10^{\prime \prime}$ & $97^{\circ} 46^{\prime} 37^{\prime \prime}$ & Edwards & -- & -8.86 & 3.9 & 8.7 & \\
\hline 29 & $30^{\circ} 37^{\prime} 13^{\prime \prime}$ & $97^{\circ} 43^{\prime} 38^{\prime \prime}$ & Edwards & -- & 11.7 & 1.0 & 4.8 & \\
\hline 29 & $30^{\circ} 37^{\prime} 12^{\prime \prime}$ & $97^{\circ} 42^{\prime} 43^{\prime \prime}$ & Edwards & -- & .6 & 1.5 & 3.8 & \\
\hline 29 & $30^{\circ} 37^{\prime} 34^{\prime \prime}$ & $97^{\circ} 41^{\prime} 35^{\prime \prime}$ & Edwards & -- & -.4 & .8 & 2.3 & \\
\hline 30 & $31^{\circ} 03^{\prime} 00^{\prime \prime}$ & $98^{\circ} 11^{\prime} 05^{\prime \prime}$ & Trinity & Marble Falls & 2.7 & .53 & 0 & \multirow[t]{4}{*}{ just south of Hancock Park } \\
\hline 30 & $31^{\circ} 03^{\prime} 17^{\prime \prime}$ & $98^{\circ} 11^{\prime} 13^{\prime \prime}$ & Trinity & -- & .7 & .24 & .53 & \\
\hline 30 & $31^{\circ} 03^{\prime} 17^{\prime \prime}$ & $98^{\circ} 10^{\prime} 59^{\prime \prime}$ & Trinity & -- & 3.1 & .1 & .77 & \\
\hline 30 & $31^{\circ} 03^{\prime} 18^{\prime \prime}$ & $98^{\circ} 10^{\prime} 56^{\prime \prime}$ & Trinity & -- & 3.3 & .08 & .87 & \\
\hline
\end{tabular}


Table 4. Gains and losses from gain-loss studies in Texas-Continued

\begin{tabular}{|c|c|c|c|c|c|c|c|c|}
\hline $\begin{array}{l}\text { Stream- } \\
\text { flow } \\
\text { study } \\
\text { no. }\end{array}$ & $\begin{array}{l}\text { Latitude of } \\
\text { upstream } \\
\text { end of } \\
\text { subreach }\end{array}$ & $\begin{array}{l}\text { Longitude } \\
\text { of upstream } \\
\text { end of } \\
\text { subreach }\end{array}$ & $\begin{array}{c}\text { Major aquifer } \\
\text { outcrop } \\
\text { intersected } \\
\text { by subreach }\end{array}$ & $\begin{array}{c}\text { Minor aquifer } \\
\text { outcrop } \\
\text { intercepted } \\
\text { by subreach }\end{array}$ & $\begin{array}{l}\text { Gain or } \\
\text { loss }(-) \text { in } \\
\text { subreach } \\
\left(\mathrm{ft}^{3} / \mathrm{s}\right)\end{array}$ & $\begin{array}{l}\text { Length } \\
\text { of subreach } \\
\text { (river mi) }\end{array}$ & $\begin{array}{l}\text { Location of } \\
\text { upstream } \\
\text { end of } \\
\text { subreach } \\
\text { (river mi) }\end{array}$ & $\begin{array}{l}\text { Descriptive location } \\
\text { of upstream end of } \\
\text { selected subreaches }\end{array}$ \\
\hline 30 & $31^{\circ} 03^{\prime} 19^{\prime \prime}$ & $98^{\circ} 10^{\prime} 53^{\prime \prime}$ & Trinity & -- & 5.4 & 2.72 & 0.95 & \\
\hline 31 & $31^{\circ} 03^{\prime} 00^{\prime \prime}$ & $98^{\circ} 11^{\prime} 05^{\prime \prime}$ & Trinity & Marble Falls & 1.8 & .53 & 0 & just south of Hancock Park \\
\hline 31 & $31^{\circ} 03^{\prime} 17^{\prime \prime}$ & $98^{\circ} 11^{\prime} 13^{\prime \prime}$ & Trinity & -- & 3.5 & .24 & .53 & \\
\hline 31 & $31^{\circ} 03^{\prime} 17^{\prime \prime}$ & $98^{\circ} 10^{\prime} 59^{\prime \prime}$ & Trinity & -- & 3.2 & .1 & .77 & \\
\hline 31 & $31^{\circ} 03^{\prime} 18^{\prime \prime}$ & $98^{\circ} 10^{\prime} 56^{\prime \prime}$ & Trinity & -- & 1.9 & .08 & .87 & \\
\hline 31 & $31^{\circ} 03^{\prime} 19^{\prime \prime}$ & $98^{\circ} 10^{\prime} 53^{\prime \prime}$ & Trinity & -- & 1.3 & 2.72 & .95 & \\
\hline 32 & $30^{\circ} 14^{\prime} 40^{\prime \prime}$ & $97^{\circ} 48^{\prime} 07^{\prime \prime}$ & Edwards & -- & -14.04 & 1.1 & 4.6 & at Loop 360, gaging station (08155300) \\
\hline 32 & $30^{\circ} 14^{\prime} 41^{\prime \prime}$ & $97^{\circ} 47^{\prime} 19^{\prime \prime}$ & Edwards & -- & -2.74 & .9 & 3.5 & \\
\hline 32 & $30^{\circ} 15^{\prime} 08^{\prime \prime}$ & $97^{\circ} 47^{\prime} 44^{\prime \prime}$ & Edwards & -- & -.22 & .9 & 2.6 & \\
\hline 32 & $30^{\circ} 15^{\prime} 31^{\prime \prime}$ & $97^{\circ} 47^{\prime} 02^{\prime \prime}$ & Edwards & -- & 0 & 6 & 1.7 & \\
\hline 33 & $30^{\circ} 14^{\prime} 40^{\prime \prime}$ & $97^{\circ} 48^{\prime} 07^{\prime \prime}$ & Edwards & -- & -4.07 & 1.1 & 4.6 & at Loop 360, gaging station (08155300) \\
\hline 33 & $30^{\circ} 14^{\prime} 41^{\prime \prime}$ & $97^{\circ} 47^{\prime} 19^{\prime \prime}$ & Edwards & -- & -1.41 & .9 & 3.5 & \\
\hline 33 & $30^{\circ} 15^{\prime} 08^{\prime \prime}$ & $97^{\circ} 47^{\prime} 44^{\prime \prime}$ & Edwards & -- & .14 & .9 & 2.6 & \\
\hline 33 & $30^{\circ} 15^{\prime} 31^{\prime \prime}$ & $97^{\circ} 47^{\prime} 02^{\prime \prime}$ & Edwards & -- & -.09 & .6 & 1.7 & \\
\hline 34 & $30^{\circ} 13^{\prime} 32^{\prime \prime}$ & $98^{\circ} 09^{\prime} 51^{\prime \prime}$ & Trinity & -- & .08 & .6 & 50 & 2 mi northeast of Henly at county road crossing \\
\hline 34 & $30^{\circ} 13^{\prime} 36^{\prime \prime}$ & $98^{\circ} 09^{\prime} 12^{\prime \prime}$ & Trinity & -- & .11 & .4 & 49.4 & \\
\hline 34 & $30^{\circ} 13^{\prime} 52^{\prime \prime}$ & $98^{\circ} 09^{\prime} 03^{\prime \prime}$ & Trinity & -- & .09 & 1.8 & 49 & \\
\hline 34 & $30^{\circ} 14^{\prime} 36^{\prime \prime}$ & $98^{\circ} 08^{\prime} 08^{\prime \prime}$ & Trinity & -- & .78 & 4.1 & 47.2 & \\
\hline 34 & $30^{\circ} 14^{\prime} 39^{\prime \prime}$ & $98^{\circ} 07^{\prime} 18^{\prime \prime}$ & Trinity & -- & 1.79 & 3.7 & 43.1 & \\
\hline 34 & $30^{\circ} 14^{\prime} 15^{\prime \prime}$ & $98^{\circ} 03^{\prime} 58^{\prime \prime}$ & Trinity & -- & 1.89 & 5.3 & 39.4 & \\
\hline 34 & $30^{\circ} 14^{\prime} 31^{\prime \prime}$ & $98^{\circ} 00^{\prime} 38^{\prime \prime}$ & Trinity & -- & 3.09 & 13.1 & 34.1 & \\
\hline 34 & $30^{\circ} 17^{\prime} 45^{\prime \prime}$ & $97^{\circ} 55^{\prime} 33^{\prime \prime}$ & Trinity & -- & -9.64 & 4.1 & 21 & \\
\hline 34 & $30^{\circ} 17^{\prime} 32^{\prime \prime}$ & $97^{\circ} 53^{\prime} 13^{\prime \prime}$ & Trinity & -- & 0 & 3.3 & 16.9 & \\
\hline 34 & $30^{\circ} 18^{\prime} 13^{\prime \prime}$ & $97^{\circ} 51^{\prime} 52^{\prime \prime}$ & Trinity & -- & 0 & 2.2 & 13.6 & \\
\hline 34 & $30^{\circ} 17^{\prime} 26^{\prime \prime}$ & $97^{\circ} 50^{\prime} 48^{\prime \prime}$ & Trinity & -- & 0 & .8 & 11.4 & \\
\hline 34 & $30^{\circ} 17^{\prime} 17^{\prime \prime}$ & $97^{\circ} 50^{\prime} 46^{\prime \prime}$ & Trinity & -- & 10.78 & 1.5 & 10.6 & \\
\hline 34 & $30^{\circ} 16^{\prime} 30^{\prime \prime}$ & $97^{\circ} 50^{\prime} 42^{\prime \prime}$ & Trinity & -- & -10.9 & .3 & 9.1 & \\
\hline 34 & $30^{\circ} 16^{\prime} 25^{\prime \prime}$ & $97^{\circ} 50^{\prime} 29^{\prime \prime}$ & Trinity & -- & 0 & .4 & 8.8 & \\
\hline 34 & $30^{\circ} 16^{\prime} 16^{\prime \prime}$ & $97^{\circ} 49^{\prime} 53^{\prime \prime}$ & Trinity & -- & 9.1 & .4 & 8.4 & \\
\hline 34 & $30^{\circ} 16^{\prime} 09^{\prime \prime}$ & $97^{\circ} 49^{\prime} 28^{\prime \prime}$ & Edwards, Trinity & -- & -3.36 & 1.2 & 8 & \\
\hline 34 & $30^{\circ} 15^{\prime} 14^{\prime \prime}$ & $97^{\circ} 49^{\prime} 03^{\prime \prime}$ & Edwards & -- & -5.74 & 1.0 & 6.8 & \\
\hline 34 & $30^{\circ} 14^{\prime} 24^{\prime \prime}$ & $97^{\circ} 48^{\prime} 37^{\prime \prime}$ & Edwards & -- & 0 & 1.3 & 5.8 & \\
\hline 34 & $30^{\circ} 14^{\prime} 40^{\prime \prime}$ & $97^{\circ} 48^{\prime} 07^{\prime \prime}$ & Edwards & -- & 0 & 2.2 & 4.5 & \\
\hline 34 & $30^{\circ} 15^{\prime} 17^{\prime \prime}$ & $97^{\circ} 47^{\prime} 26^{\prime \prime}$ & Edwards & -- & .04 & .3 & 2.3 & \\
\hline 34 & $30^{\circ} 15^{\prime} 24^{\prime \prime}$ & $97^{\circ} 47^{\prime} 11^{\prime \prime}$ & Edwards & -- & -.04 & 1.0 & 2 & \\
\hline 34 & $30^{\circ} 15^{\prime} 48^{\prime \prime}$ & $97^{\circ} 46^{\prime} 26^{\prime \prime}$ & Edwards & -- & 1.49 & .2 & 1 & \\
\hline 34 & $30^{\circ} 15^{\prime} 48^{\prime \prime}$ & $97^{\circ} 46^{\prime} 20^{\prime \prime}$ & -- & -- & 99.51 & .3 & .8 & \\
\hline 35 & $30^{\circ} 13^{\prime} 32^{\prime \prime}$ & $98^{\circ} 09^{\prime} 51^{\prime \prime}$ & Trinity & -- & 0 & 6 & 50 & 2 mi northeast of Henly at county road crossing \\
\hline
\end{tabular}


Table 4. Gains and losses from gain-loss studies in Texas-Continued

\begin{tabular}{|c|c|c|c|c|c|c|c|c|c|}
\hline $\begin{array}{l}\text { Stream- } \\
\text { flow } \\
\text { study } \\
\text { no. }\end{array}$ & $\begin{array}{l}\text { Latitude of } \\
\text { upstream } \\
\text { end of } \\
\text { subreach }\end{array}$ & $\begin{array}{l}\text { Longitude } \\
\text { of upstream } \\
\text { end of } \\
\text { subreach }\end{array}$ & $\begin{array}{l}\text { Major aquifer } \\
\text { outcrop } \\
\text { intersected } \\
\text { by subreach }\end{array}$ & & $\begin{array}{l}\text { Minor aquifer } \\
\text { outcrop } \\
\text { intercepted } \\
\text { by subreach }\end{array}$ & $\begin{array}{l}\text { Gain or } \\
\text { loss }(-) \text { in } \\
\text { subreach } \\
\left(\mathrm{ft}^{3} / \mathrm{s}\right)\end{array}$ & $\begin{array}{l}\text { Length } \\
\text { of subreach } \\
\text { (river mi) }\end{array}$ & $\begin{array}{l}\text { Location of } \\
\text { upstream } \\
\text { end of } \\
\text { subreach } \\
\text { (river mi) }\end{array}$ & $\begin{array}{l}\text { Descriptive location } \\
\text { of upstream end of } \\
\text { selected subreaches }\end{array}$ \\
\hline 35 & $30^{\circ} 13^{\prime} 36^{\prime \prime}$ & $98^{\circ} 09^{\prime} 12^{\prime \prime}$ & Trinity & -- & & 0 & 0.4 & 49.4 & \\
\hline 35 & $30^{\circ} 13^{\prime} 51^{\prime \prime}$ & $98^{\circ} 09^{\prime} 05^{\prime \prime}$ & Trinity & -- & & .01 & 1.8 & 49 & \\
\hline 35 & $30^{\circ} 14^{\prime} 36^{\prime \prime}$ & $98^{\circ} 08^{\prime} 08^{\prime \prime}$ & Trinity & -- & & .15 & 4.1 & 47.2 & \\
\hline 35 & $30^{\circ} 14^{\prime} 39^{\prime \prime}$ & $98^{\circ} 07^{\prime} 18^{\prime \prime}$ & Trinity & -- & & .15 & 3.7 & 43.1 & \\
\hline 35 & $30^{\circ} 14^{\prime} 15^{\prime \prime}$ & $98^{\circ} 03^{\prime} 58^{\prime \prime}$ & Trinity & -- & & .28 & 5.3 & 39.4 & \\
\hline 35 & $30^{\circ} 14^{\prime} 31^{\prime \prime}$ & $98^{\circ} 00^{\prime} 38^{\prime \prime}$ & Trinity & -- & & .64 & 13.1 & 34.1 & \\
\hline 35 & $30^{\circ} 17^{\prime} 45^{\prime \prime}$ & $97^{\circ} 55^{\prime} 33^{\prime \prime}$ & Trinity & -- & & -1.29 & 4.1 & 21 & \\
\hline 35 & $30^{\circ} 17^{\prime} 32^{\prime \prime}$ & $97^{\circ} 53^{\prime} 13^{\prime \prime}$ & Trinity & -- & & .11 & 3.3 & 16.9 & \\
\hline 35 & $30^{\circ} 18^{\prime} 13^{\prime \prime}$ & $97^{\circ} 51^{\prime} 52^{\prime \prime}$ & Trinity & -- & & -.11 & 2.2 & 13.6 & \\
\hline 35 & $30^{\circ} 17^{\prime} 26^{\prime \prime}$ & $97^{\circ} 50^{\prime} 48^{\prime \prime}$ & Trinity & -- & & .02 & .8 & 11.4 & \\
\hline 35 & $30^{\circ} 17^{\prime} 17^{\prime \prime}$ & $97^{\circ} 50^{\prime} 46^{\prime \prime}$ & Trinity & -- & & -.02 & 1.5 & 10.6 & \\
\hline 35 & $30^{\circ} 16^{\prime} 30^{\prime \prime}$ & $97^{\circ} 50^{\prime} 42^{\prime \prime}$ & Trinity & -- & & .01 & .3 & 9.1 & \\
\hline 35 & $30^{\circ} 16^{\prime} 31^{\prime \prime}$ & $97^{\circ} 50^{\prime} 16^{\prime \prime}$ & Trinity & -- & & -.01 & .4 & 8.8 & \\
\hline 35 & $30^{\circ} 16^{\prime} 16^{\prime \prime}$ & $97^{\circ} 49^{\prime} 53^{\prime \prime}$ & Trinity & -- & & 0 & .4 & 8.4 & \\
\hline 35 & $30^{\circ} 16^{\prime} 09^{\prime \prime}$ & $97^{\circ} 49^{\prime} 28^{\prime \prime}$ & Edwards, Trinity & -- & & 0 & 1.2 & 8 & \\
\hline 35 & $30^{\circ} 15^{\prime} 14^{\prime \prime}$ & $97^{\circ} 49^{\prime} 03^{\prime \prime}$ & Edwards & -- & & 0 & 1.0 & 6.8 & \\
\hline 35 & $30^{\circ} 14^{\prime} 24^{\prime \prime}$ & $97^{\circ} 48^{\prime} 37^{\prime \prime}$ & Edwards & -- & & 0 & 1.3 & 5.8 & \\
\hline 35 & $30^{\circ} 14^{\prime} 40^{\prime \prime}$ & $97^{\circ} 48^{\prime} 07^{\prime \prime}$ & Edwards & -- & & 0 & 2.2 & 4.5 & \\
\hline 35 & $30^{\circ} 15^{\prime} 17^{\prime \prime}$ & $97^{\circ} 47^{\prime} 26^{\prime \prime}$ & Edwards & -- & & 0 & .3 & 2.3 & \\
\hline 35 & $30^{\circ} 15^{\prime} 24^{\prime \prime}$ & $97^{\circ} 47^{\prime} 11^{\prime \prime}$ & Edwards & -- & & 0 & 1.0 & 2 & \\
\hline 35 & $30^{\circ} 15^{\prime} 48^{\prime \prime}$ & $97^{\circ} 46^{\prime} 26^{\prime \prime}$ & Edwards & -- & & .36 & .2 & 1 & \\
\hline 35 & $30^{\circ} 15^{\prime} 48^{\prime \prime}$ & $97^{\circ} 46^{\prime} 20^{\prime \prime}$ & -- & -- & & 82.14 & .3 & .8 & includes flow from spring \\
\hline 36 & $30^{\circ} 17^{\prime} 45^{\prime \prime}$ & $97^{\circ} 55^{\prime} 31^{\prime \prime}$ & Trinity & -- & & 1.0 & 3.1 & 21 & at SH 71, gaging station (08155200) \\
\hline 36 & $30^{\circ} 18^{\prime} 01^{\prime \prime}$ & $97^{\circ} 54^{\prime} 01^{\prime \prime}$ & Trinity & -- & & 6.0 & 3.6 & 17.9 & \\
\hline 36 & $30^{\circ} 17^{\prime} 53^{\prime \prime}$ & $97^{\circ} 52^{\prime} 40^{\prime \prime}$ & Trinity & -- & & 5.0 & 3.4 & 14.3 & \\
\hline 36 & $30^{\circ} 17^{\prime} 46^{\prime \prime}$ & $97^{\circ} 51^{\prime} 05^{\prime \prime}$ & Trinity & -- & & -.1 & .9 & 10.9 & \\
\hline 36 & $30^{\circ} 16^{\prime} 57^{\prime \prime}$ & $97^{\circ} 51^{\prime} 07^{\prime \prime}$ & Trinity & -- & & .72 & .9 & 10 & \\
\hline 36 & $30^{\circ} 16^{\prime} 26^{\prime \prime}$ & $97^{\circ} 50^{\prime} 38^{\prime \prime}$ & Trinity & -- & & -.7 & 1.3 & 9.1 & \\
\hline 36 & $30^{\circ} 16^{\prime} 10^{\prime \prime}$ & $97^{\circ} 49^{\prime} 39^{\prime \prime}$ & Edwards, Trinity & -- & & -2.3 & .5 & 7.8 & \\
\hline 36 & $30^{\circ} 15^{\prime} 54^{\prime \prime}$ & $97^{\circ} 49^{\prime} 22^{\prime \prime}$ & Edwards & -- & & -1.2 & .5 & 7.3 & \\
\hline 36 & $30^{\circ} 15^{\prime} 30^{\prime \prime}$ & $97^{\circ} 49^{\prime} 21^{\prime \prime}$ & Edwards & -- & & -7.1 & .7 & 6.8 & \\
\hline 36 & $30^{\circ} 15^{\prime} 09^{\prime \prime}$ & $97^{\circ} 48^{\prime} 54^{\prime \prime}$ & Edwards & -- & & -14.3 & 1.5 & 6.1 & \\
\hline 36 & $30^{\circ} 14^{\prime} 40^{\prime \prime}$ & $97^{\circ} 48^{\prime} 07^{\prime \prime}$ & Edwards & -- & & -8.1 & 1.1 & 4.6 & gaging station (08155300) \\
\hline 36 & $30^{\circ} 14^{\prime} 41^{\prime \prime}$ & $97^{\circ} 47^{\prime} 19^{\prime \prime}$ & Edwards & -- & & -2.1 & .9 & 3.5 & \\
\hline 36 & $30^{\circ} 15^{\prime} 08^{\prime \prime}$ & $97^{\circ} 47^{\prime} 44^{\prime \prime}$ & Edwards & -- & & 2.4 & .9 & 2.6 & \\
\hline 36 & $30^{\circ} 15^{\prime} 31^{\prime \prime}$ & $97^{\circ} 47^{\prime} 02^{\prime \prime}$ & Edwards & -- & & 2.0 & .6 & 1.7 & \\
\hline 36 & $30^{\circ} 15^{\prime} 45^{\prime \prime}$ & $97^{\circ} 46^{\prime} 45^{\prime \prime}$ & Edwards & -- & & 29.8 & 1.1 & 1.1 & \\
\hline 37 & $32^{\circ} 15^{\prime} 14^{\prime \prime}$ & $101^{\circ} 28^{\prime} 48^{\prime \prime}$ & Ogallala & -- & & -.04 & 4.2 & 59.2 & \\
\hline
\end{tabular}


Table 4. Gains and losses from gain-loss studies in Texas-Continued

\begin{tabular}{|c|c|c|c|c|c|c|c|c|}
\hline $\begin{array}{l}\text { Stream- } \\
\text { flow } \\
\text { study } \\
\text { no. }\end{array}$ & $\begin{array}{l}\text { Latitude of } \\
\text { upstream } \\
\text { end of } \\
\text { subreach }\end{array}$ & $\begin{array}{l}\text { Longitude } \\
\text { of upstream } \\
\text { end of } \\
\text { subreach }\end{array}$ & $\begin{array}{c}\text { Major aquifer } \\
\text { outcrop } \\
\text { intersected } \\
\text { by subreach }\end{array}$ & $\begin{array}{c}\text { Minor aquifer } \\
\text { outcrop } \\
\text { intercepted } \\
\text { by subreach }\end{array}$ & $\begin{array}{l}\text { Gain or } \\
\text { loss }(-) \text { in } \\
\text { subreach } \\
\left(\mathrm{ft}^{3} / \mathrm{s}\right)\end{array}$ & $\begin{array}{l}\text { Length } \\
\text { of subreach } \\
\text { (river mi) }\end{array}$ & $\begin{array}{l}\text { Location of } \\
\text { upstream } \\
\text { end of } \\
\text { subreach } \\
\text { (river mi) }\end{array}$ & $\begin{array}{l}\text { Descriptive location } \\
\text { of upstream end of } \\
\text { selected subreaches }\end{array}$ \\
\hline 37 & $32^{\circ} 15^{\prime} 25^{\prime \prime}$ & $101^{\circ} 28^{\prime} 22^{\prime \prime}$ & Ogallala & -- & 5.15 & 6.2 & 55 & discontinued gaging station (08123650) \\
\hline 37 & $32^{\circ} 15^{\prime} 27^{\prime \prime}$ & $101^{\circ} 25^{\prime} 48^{\prime \prime}$ & Ogallala & -- & -5.14 & 2.2 & 48.8 & \\
\hline 37 & $32^{\circ} 15^{\prime} 01^{\prime \prime}$ & $101^{\circ} 23^{\prime} 52^{\prime \prime}$ & Ogallala & Dockum & .07 & 6.7 & 46.6 & discontinued gaging station (08123720) \\
\hline 37 & $32^{\circ} 15^{\prime} 14^{\prime \prime}$ & $101^{\circ} 19^{\prime} 26^{\prime \prime}$ & -- & Dockum & .26 & 7.1 & 39.9 & \\
\hline 37 & $32^{\circ} 13^{\prime} 26^{\prime \prime}$ & $101^{\circ} 15^{\prime} 57^{\prime \prime}$ & -- & Dockum & -.23 & 17.6 & 32.8 & \\
\hline 37 & $32^{\circ} 11^{\prime} 56^{\prime \prime}$ & $101^{\circ} 00^{\prime} 46^{\prime \prime}$ & -- & Dockum & -.11 & 14.7 & 15.2 & gaging station $(08123800)$ \\
\hline 38 & $32^{\circ} 15^{\prime} 14^{\prime \prime}$ & $101^{\circ} 28^{\prime} 48^{\prime \prime}$ & Ogallala & -- & 7.52 & 4.2 & 59.2 & \\
\hline 38 & $32^{\circ} 15^{\prime} 25^{\prime \prime}$ & $101^{\circ} 28^{\prime} 22^{\prime \prime}$ & Ogallala & -- & 5.5 & 6.2 & 55 & discontinued gaging station (08123650) \\
\hline 38 & $32^{\circ} 15^{\prime} 27^{\prime \prime}$ & $101^{\circ} 25^{\prime} 48^{\prime \prime}$ & Ogallala & -- & -16.55 & 2.2 & 48.8 & \\
\hline 38 & $32^{\circ} 15^{\prime} 01^{\prime \prime}$ & $101^{\circ} 23^{\prime} 52^{\prime \prime}$ & Ogallala & Dockum & -.23 & 6.7 & 46.6 & discontinued gaging station (08123720) \\
\hline 38 & $32^{\circ} 15^{\prime} 14^{\prime \prime}$ & $101^{\circ} 19^{\prime} 26^{\prime \prime}$ & -- & Dockum & 1.32 & 7.1 & 39.9 & \\
\hline 38 & $32^{\circ} 13^{\prime} 26^{\prime \prime}$ & $101^{\circ} 15^{\prime} 57^{\prime \prime}$ & -- & Dockum & 3.36 & 17.6 & 32.8 & \\
\hline 38 & $32^{\circ} 11^{\prime} 56^{\prime \prime}$ & $101^{\circ} 00^{\prime} 46^{\prime \prime}$ & -- & Dockum & 1.93 & 14.7 & 15.2 & gaging station (08123800) \\
\hline 39 & $32^{\circ} 15^{\prime} 34^{\prime \prime}$ & $101^{\circ} 28^{\prime} 04^{\prime \prime}$ & Ogallala & -- & 5.46 & 4.2 & 59.2 & discontinued gagingstation (08123650) \\
\hline 39 & $32^{\circ} 15^{\prime} 21 "$ & $101^{\circ} 25^{\prime} 48^{\prime \prime}$ & Ogallala & -- & -5.34 & 6.2 & 55 & \\
\hline 39 & $32^{\circ} 14^{\prime} 49^{\prime \prime}$ & $101^{\circ} 23^{\prime} 45^{\prime \prime}$ & Ogallala & Dockum & 2.15 & 2.2 & 48.8 & discontinued gaging station (08123720) \\
\hline 39 & $32^{\circ} 14^{\prime} 57^{\prime \prime}$ & $101^{\circ} 19^{\prime} 26^{\prime \prime}$ & -- & Dockum & .1 & 6.7 & 46.6 & \\
\hline 39 & $32^{\circ} 13^{\prime} 14^{\prime \prime}$ & $101^{\circ} 15^{\prime} 57^{\prime \prime}$ & -- & Dockum & .17 & 24.7 & 39.9 & \\
\hline 39 & $32^{\circ} 12^{\prime} 05^{\prime \prime}$ & $101^{\circ} 00^{\prime} 46^{\prime \prime}$ & -- & Dockum & 1.34 & 14.7 & 15.2 & gaging station $(08123800)$ \\
\hline 40 & $30^{\circ} 09^{\prime} 21^{\prime \prime}$ & $97^{\circ} 56^{\prime} 32^{\prime \prime}$ & Trinity & -- & 17.66 & 2.0 & 11 & gaging station $(08158810)$ \\
\hline 40 & $30^{\circ} 10^{\prime} 06^{\prime \prime}$ & $97^{\circ} 55^{\prime} 31^{\prime \prime}$ & Edwards, Trinity & -- & 12.1 & 1.4 & 9 & \\
\hline 40 & $30^{\circ} 09^{\prime} 45^{\prime \prime}$ & $97^{\circ} 54^{\prime} 32^{\prime \prime}$ & Edwards & -- & -10.7 & 1.6 & 7.6 & \\
\hline 40 & $30^{\circ} 09^{\prime} 31^{\prime \prime}$ & $97^{\circ} 53^{\prime} 41^{\prime \prime}$ & Edwards & -- & -3.6 & 1.1 & 6 & \\
\hline 40 & $30^{\circ} 09^{\prime} 20^{\prime \prime}$ & $97^{\circ} 52^{\prime} 57^{\prime \prime}$ & Edwards & -- & -9.0 & 1.1 & 4.9 & \\
\hline 40 & $30^{\circ} 08^{\prime} 54^{\prime \prime}$ & $97^{\circ} 51^{\prime} 55^{\prime \prime}$ & Edwards & -- & -3.4 & 1.2 & 3.8 & \\
\hline 40 & $30^{\circ} 08^{\prime} 24^{\prime \prime}$ & $97^{\circ} 50^{\prime} 49^{\prime \prime}$ & -- & -- & -6.86 & 1.7 & 2.6 & gaging station $(08158820)$ \\
\hline 41 & $31^{\circ} 08^{\prime} 16^{\prime \prime}$ & $99^{\circ} 20^{\prime} 06^{\prime \prime}$ & -- & Ellenburger-San Saba & 1.0 & 13.0 & 28 & at Brady \\
\hline 41 & $31^{\circ} 06^{\prime} 00^{\prime \prime}$ & $99^{\circ} 12^{\prime} 21^{\prime \prime}$ & -- & $\begin{array}{l}\text { Ellenburger-San Saba, Marble } \\
\text { Falls }\end{array}$ & .7 & 15.0 & 15 & \\
\hline 42 & $32^{\circ} 35^{\prime} 03^{\prime \prime}$ & $101^{\circ} 07^{\prime} 48^{\prime \prime}$ & -- & Dockum & .01 & 11.7 & 239.1 & \\
\hline 42 & $32^{\circ} 32^{\circ} 20^{\prime \prime}$ & $101^{\circ} 03^{\prime} 14^{\prime \prime}$ & -- & Dockum & -.36 & 9.3 & 227.4 & discontinued gaging station (08119500) \\
\hline 42 & $32^{\circ} 32^{\circ} 39^{\prime \prime}$ & $100^{\circ} 58^{\prime} 04^{\prime \prime}$ & -- & Dockum & .93 & 9.3 & 218.1 & \\
\hline 42 & $32^{\circ} 28^{\prime} 41^{\prime \prime}$ & $100^{\circ} 56^{\prime} 52^{\prime \prime}$ & -- & Dockum & -4.65 & 9.5 & 208.8 & \\
\hline 42 & $32^{\circ} 27^{\prime} 28^{\prime \prime}$ & $100^{\circ} 57^{\prime} 36^{\prime \prime}$ & -- & Dockum & 7.95 & 2.0 & 199.3 & \\
\hline 42 & $32^{\circ} 25^{\prime} 25^{\prime \prime}$ & $100^{\circ} 53^{\prime} 49^{\prime \prime}$ & -- & Dockum & -8.2 & .9 & 197.3 & \\
\hline 42 & $32^{\circ} 24^{\prime} 51^{\prime \prime}$ & $100^{\circ} 54^{\prime} 25^{\prime \prime}$ & -- & Dockum & .28 & 2.6 & 196.4 & \\
\hline 42 & $32^{\circ} 23^{\prime} 33^{\prime \prime}$ & $100^{\circ} 52^{\prime} 40^{\prime \prime}$ & -- & Dockum & .5 & 2.5 & 193.8 & \\
\hline 42 & $32^{\circ} 22^{\prime} 35^{\prime \prime}$ & $100^{\circ} 51^{\prime} 39^{\prime \prime}$ & -- & Dockum & .87 & 7.0 & 191.3 & \\
\hline
\end{tabular}


\$ Table 4. Gains and losses from gain-loss studies in Texas-Continued

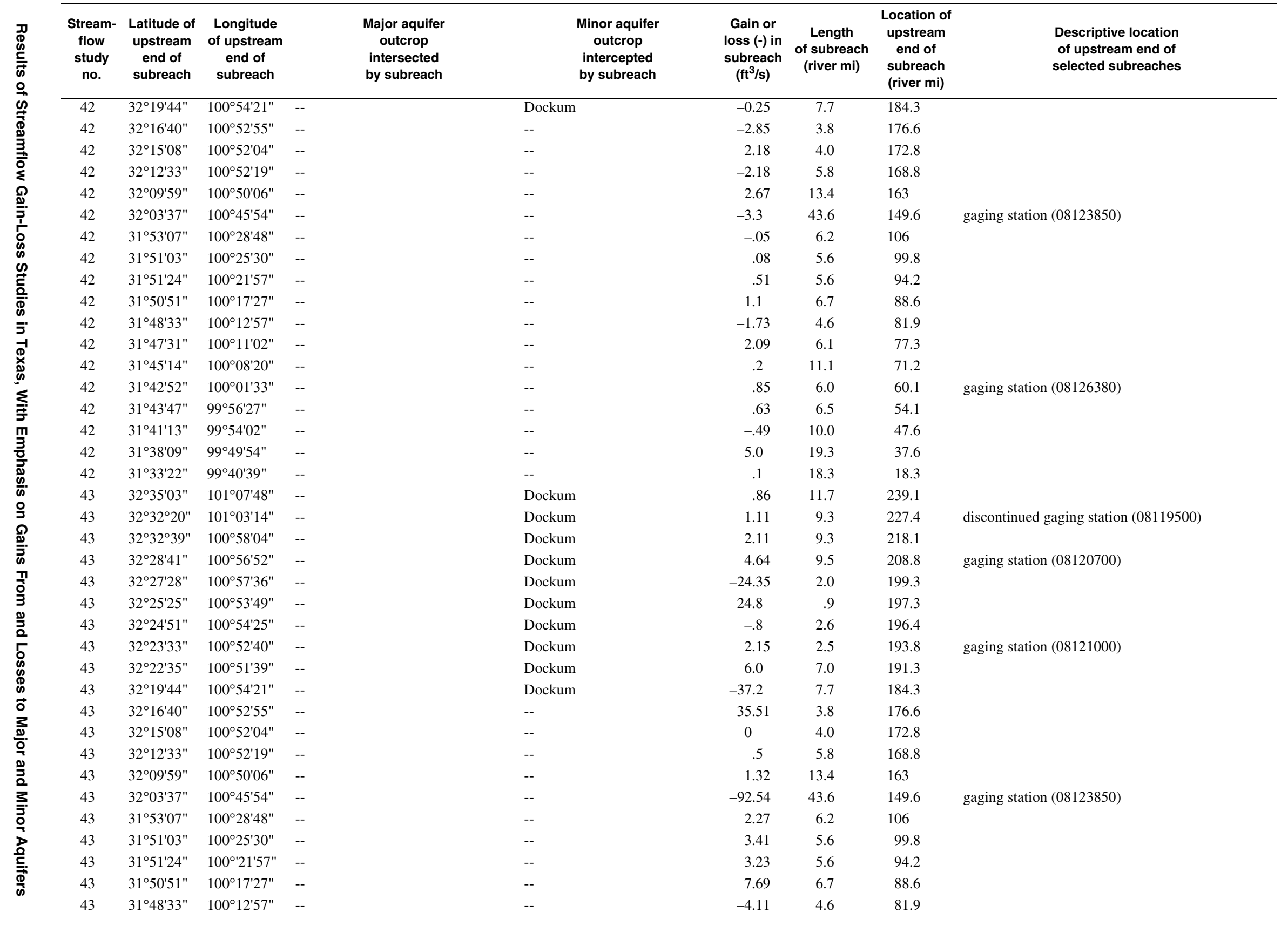


Table 4. Gains and losses from gain-loss studies in Texas-Continued

\begin{tabular}{|c|c|c|c|c|c|c|c|c|}
\hline $\begin{array}{l}\text { Stream- } \\
\text { flow } \\
\text { study } \\
\text { no. }\end{array}$ & $\begin{array}{l}\text { Latitude of } \\
\text { upstream } \\
\text { end of } \\
\text { subreach }\end{array}$ & $\begin{array}{l}\text { Longitude } \\
\text { of upstream } \\
\text { end of } \\
\text { subreach }\end{array}$ & $\begin{array}{l}\text { Major aquifer } \\
\text { outcrop } \\
\text { intersected } \\
\text { by subreach }\end{array}$ & $\begin{array}{c}\text { Minor aquifer } \\
\text { outcrop } \\
\text { intercepted } \\
\text { by subreach }\end{array}$ & $\begin{array}{l}\text { Gain or } \\
\text { loss }(-) \text { in } \\
\text { subreach } \\
\left(\mathrm{ft}^{3} / \mathrm{s}\right)\end{array}$ & $\begin{array}{l}\text { Length } \\
\text { of subreach } \\
\text { (river mi) }\end{array}$ & $\begin{array}{l}\text { Location of } \\
\text { upstream } \\
\text { end of } \\
\text { subreach } \\
\text { (river mi) }\end{array}$ & $\begin{array}{l}\text { Descriptive location } \\
\text { of upstream end of } \\
\text { selected subreaches }\end{array}$ \\
\hline 43 & $31^{\circ} 47^{\prime} 31^{\prime \prime}$ & $100^{\circ} 11^{\prime} 02^{\prime \prime}$ & -- & -- & 7.7 & 6.1 & 77.3 & \\
\hline 43 & $31^{\circ} 45^{\prime} 14^{\prime \prime}$ & $100^{\circ} 08^{\prime} 20^{\prime \prime}$ & -- & -- & -1.75 & 11.1 & 71.2 & \\
\hline 43 & $31^{\circ} 43^{\prime} 04^{\prime \prime}$ & $100^{\circ} 01^{\prime} 55^{\prime \prime}$ & -- & -- & 4.0 & 6.0 & 60.1 & gaging station (08126380) \\
\hline 43 & $31^{\circ} 43^{\prime} 04^{\prime \prime}$ & $100^{\circ} 01^{\prime} 55^{\prime \prime}$ & -- & -- & -150.4 & 6.5 & 54.1 & \\
\hline 43 & $31^{\circ} 41^{\prime} 13^{\prime \prime}$ & $99^{\circ} 54^{\prime} 02^{\prime \prime}$ & -- & -- & 151.0 & 10.0 & 47.6 & \\
\hline 43 & $31^{\circ} 38^{\prime} 09^{\prime \prime}$ & $99^{\circ} 49^{\prime} 54^{\prime \prime}$ & -- & -- & 19.1 & 19.3 & 37.6 & \\
\hline 43 & $31^{\circ} 33^{\prime} 22^{\prime \prime}$ & $99^{\circ} 40^{\prime} 39^{\prime \prime}$ & -- & -- & 10.11 & 18.3 & 18.3 & \\
\hline 44 & $32^{\circ} 35^{\prime} 03^{\prime \prime}$ & $101^{\circ} 07^{\prime} 48^{\prime \prime}$ & -- & Dockum & .78 & 11.7 & 239.1 & \\
\hline 44 & $32^{\circ} 32^{\circ} 20^{\prime \prime}$ & $100^{\circ} 03^{\prime} 14^{\prime \prime}$ & -- & Dockum & .69 & 9.3 & 227.4 & discontinued gaging station (08119500) \\
\hline 44 & $32^{\circ} 32^{\circ} 39^{\prime \prime}$ & $100^{\circ} 58^{\prime} 04^{\prime \prime}$ & -- & Dockum & 4.56 & 9.3 & 218.1 & \\
\hline 44 & $32^{\circ} 28^{\prime} 41^{\prime \prime}$ & $100^{\circ} 562^{\prime \prime}$ & -- & Dockum & -.1 & 9.5 & 208.8 & gaging station (08120700) \\
\hline 44 & $32^{\circ} 27^{\prime} 28^{\prime \prime}$ & $100^{\circ} 57^{\prime} 36^{\prime \prime}$ & -- & Dockum & 8.12 & 2.0 & 199.3 & \\
\hline 44 & $32^{\circ} 25^{\prime} 25^{\prime \prime}$ & $100^{\circ} 53^{\prime} 49^{\prime \prime}$ & -- & Dockum & -22.64 & .9 & 197.3 & \\
\hline 44 & $32^{\circ} 24^{\prime} 51^{\prime \prime}$ & $100^{\circ} 54^{\prime} 25^{\prime \prime}$ & -- & Dockum & 1.75 & 2.6 & 196.4 & \\
\hline 44 & $32^{\circ} 23^{\prime} 33^{\prime \prime}$ & $100^{\circ} 52^{\prime} 40^{\prime \prime}$ & -- & Dockum & .72 & 2.5 & 193.8 & gaging station (08121000) \\
\hline 44 & $32^{\circ} 22^{\prime} 35^{\prime \prime}$ & $100^{\circ} 51^{\prime} 39^{\prime \prime}$ & -- & Dockum & 4.44 & 7.0 & 191.3 & \\
\hline 44 & $32^{\circ} 19^{\prime} 44^{\prime \prime}$ & $100^{\circ} 54^{\prime} 21^{\prime \prime}$ & -- & Dockum & -9.38 & 7.7 & 184.3 & \\
\hline 44 & $32^{\circ} 16^{\prime} 40^{\prime \prime}$ & $100^{\circ} 52^{\prime} 55^{\prime \prime}$ & -- & -- & -.05 & 3.8 & 176.6 & \\
\hline 44 & $32^{\circ} 15^{\prime} 08^{\prime \prime}$ & $100^{\circ} 52^{\prime} 04^{\prime \prime}$ & -- & -- & 24.3 & 4.0 & 172.8 & \\
\hline 44 & $32^{\circ} 12^{\prime} 33^{\prime \prime}$ & $100^{\circ} 52^{\prime} 19^{\prime \prime}$ & -- & -- & -7.76 & 5.8 & 168.8 & \\
\hline 44 & $32^{\circ} 09^{\prime} 59^{\prime \prime}$ & $100^{\circ} 50^{\prime} 06^{\prime \prime}$ & -- & -- & 3.15 & 13.4 & 163 & \\
\hline 44 & $32^{\circ} 03^{\prime} 37^{\prime \prime}$ & $100^{\circ} 45^{\prime} 54^{\prime \prime}$ & -- & -- & -27.66 & 43.6 & 149.6 & gaging station (08123850) \\
\hline 44 & $31^{\circ} 53^{\prime} 07^{\prime \prime}$ & $100^{\circ} 28^{\prime} 48^{\prime \prime}$ & -- & -- & .22 & 6.2 & 106 & gaging station $(08124000)$ \\
\hline 44 & $31^{\circ} 51^{\prime} 03^{\prime \prime}$ & $100^{\circ} 25^{\prime} 30^{\prime \prime}$ & -- & -- & -.52 & 5.6 & 99.8 & \\
\hline 44 & $31^{\circ} 51^{\prime} 24^{\prime \prime}$ & $100^{\circ} 21^{\prime} 57^{\prime \prime}$ & -- & -- & 1.67 & 5.6 & 94.2 & \\
\hline 44 & $31^{\circ} 50^{\prime} 51^{\prime \prime}$ & $100^{\circ} 17^{\prime} 27^{\prime \prime}$ & -- & -- & 3.28 & 6.7 & 88.6 & \\
\hline 44 & $31^{\circ} 48^{\prime} 33^{\prime \prime}$ & $100^{\circ} 12^{\prime} 57^{\prime \prime}$ & -- & -- & -.05 & 4.6 & 81.9 & \\
\hline 44 & $31^{\circ} 47^{\prime} 31^{\prime \prime}$ & $100^{\circ} 11^{\prime} 02^{\prime \prime}$ & -- & -- & 1.08 & 6.1 & 77.3 & \\
\hline 44 & $31^{\circ} 45^{\prime} 14^{\prime \prime}$ & $100^{\circ} 08^{\prime} 20^{\prime \prime}$ & -- & -- & 2.63 & 11.1 & 71.2 & \\
\hline 44 & $31^{\circ} 43^{\prime} 04^{\prime \prime}$ & $100^{\circ} 01^{\prime} 55^{\prime \prime}$ & -- & -- & 6.0 & 6.0 & 60.1 & \\
\hline 44 & $31^{\circ} 43^{\prime} 04^{\prime \prime}$ & $100^{\circ} 01^{\prime} 55^{\prime \prime}$ & -- & -- & .36 & 6.5 & 54.1 & \\
\hline 44 & $31^{\circ} 41^{\prime} 13^{\prime \prime}$ & $99^{\circ} 54^{\prime} 02^{\prime \prime}$ & -- & -- & -2.6 & 10.0 & 47.6 & \\
\hline 44 & $31^{\circ} 38^{\prime} 09^{\prime \prime}$ & $99^{\circ} 49^{\prime} 54^{\prime \prime}$ & -- & -- & .8 & 19.3 & 37.6 & \\
\hline 44 & $31^{\circ} 33^{\prime} 22^{\prime \prime}$ & $99^{\circ} 40^{\prime} 39^{\prime \prime}$ & -- & -- & .8 & 18.3 & 18.3 & \\
\hline 45 & $32^{\circ} 34^{\prime} 58^{\prime \prime}$ & $101^{\circ} 05^{\prime} 42^{\prime \prime}$ & -- & Dockum & .15 & .1 & 831.8 & $50 \mathrm{ft}$ above Bull Creek \\
\hline 45 & $32^{\circ} 34^{\prime} 54^{\prime \prime}$ & $101^{\circ} 05^{\prime} 42^{\prime \prime}$ & -- & Dockum & -.04 & 2.9 & 831.7 & \\
\hline 45 & $32^{\circ} 34^{\prime} 17^{\prime \prime}$ & $101^{\circ} 03^{\prime} 18^{\prime \prime}$ & -- & Dockum & .16 & 2.5 & 828.8 & \\
\hline 45 & $32^{\circ} 32^{\circ} 17^{\prime \prime}$ & $101^{\circ} 03^{\prime} 10^{\prime \prime}$ & -- & Dockum & .18 & 2.3 & 826.3 & \\
\hline
\end{tabular}


Table 4. Gains and losses from gain-loss studies in Texas-Continued

\begin{tabular}{|c|c|c|c|c|c|c|c|c|c|}
\hline $\begin{array}{l}\text { Stream- } \\
\text { flow } \\
\text { study } \\
\text { no. }\end{array}$ & $\begin{array}{l}\text { Latitude of } \\
\text { upstream } \\
\text { end of } \\
\text { subreach }\end{array}$ & $\begin{array}{l}\text { Longitude } \\
\text { of upstream } \\
\text { end of } \\
\text { subreach }\end{array}$ & & $\begin{array}{c}\text { Major aquifer } \\
\text { outcrop } \\
\text { intersected } \\
\text { by subreach }\end{array}$ & $\begin{array}{c}\text { Minor aquifer } \\
\text { outcrop } \\
\text { intercepted } \\
\text { by subreach }\end{array}$ & $\begin{array}{l}\text { Gain or } \\
\text { loss }(-) \text { in } \\
\text { subreach } \\
\left(\mathrm{ft}^{3} / \mathrm{s}\right)\end{array}$ & $\begin{array}{l}\text { Length } \\
\text { of subreach } \\
\text { (river mi) }\end{array}$ & $\begin{array}{l}\text { Location of } \\
\text { upstream } \\
\text { end of } \\
\text { subreach } \\
\text { (river mi) }\end{array}$ & $\begin{array}{l}\text { Descriptive location } \\
\text { of upstream end of } \\
\text { selected subreaches }\end{array}$ \\
\hline 45 & $32^{\circ} 30^{\prime} 42^{\prime \prime}$ & $101^{\circ} 01^{\prime} 40^{\prime \prime}$ & -- & & Dockum & -0.17 & 6.2 & 824 & \multirow{18}{*}{$50 \mathrm{ft}$ above Bull Creek } \\
\hline 45 & $32^{\circ} 32^{\circ} 25^{\prime \prime}$ & $100^{\circ} 56^{\prime} 52^{\prime \prime}$ & -- & & Dockum & .24 & 3.5 & 817.8 & \\
\hline 45 & $32^{\circ} 30^{\prime} 51^{\prime \prime}$ & $100^{\circ} 54^{\prime} 43^{\prime \prime}$ & -- & & Dockum & 3.52 & 3.7 & 814.3 & \\
\hline 45 & $32^{\circ} 28^{\prime} 41^{\prime \prime}$ & $100^{\circ} 56^{\prime} 52^{\prime \prime}$ & -- & & Dockum & -.78 & 6.2 & 810.6 & \\
\hline 45 & $32^{\circ} 26^{\prime} 35^{\prime \prime}$ & $100^{\circ} 56^{\prime} 42^{\prime \prime}$ & -- & & Dockum & .56 & 2.3 & 804.4 & \\
\hline 45 & $32^{\circ} 25^{\prime} 50^{\prime \prime}$ & $100^{\circ} 54^{\prime} 57^{\prime \prime}$ & -- & & Dockum & 2.05 & 3.2 & 802.1 & \\
\hline 45 & $32^{\circ} 24^{\prime} 51^{\prime \prime}$ & $100^{\circ} 54^{\prime} 25^{\prime \prime}$ & -- & & Dockum & 2.11 & 2.6 & 798.9 & \\
\hline 46 & $32^{\circ} 34^{\prime} 58^{\prime \prime}$ & $101^{\circ} 05^{\prime} 42^{\prime \prime}$ & -- & & Dockum & .05 & .1 & 831.8 & \\
\hline 46 & $32^{\circ} 34^{\prime} 54^{\prime \prime}$ & $101^{\circ} 05^{\prime} 42^{\prime \prime}$ & -- & & Dockum & -.01 & 2.9 & 831.7 & \\
\hline 46 & $32^{\circ} 34^{\prime} 17^{\prime \prime}$ & $101^{\circ} 03^{\prime} 18^{\prime \prime}$ & -- & & Dockum & .08 & 2.5 & 828.8 & \\
\hline 46 & $32^{\circ} 32^{\circ} 17^{\prime \prime}$ & $101^{\circ} 03^{\prime} 10^{\prime \prime}$ & -- & & Dockum & .18 & 2.3 & 826.3 & \\
\hline 46 & $32^{\circ} 30^{\prime} 42^{\prime \prime}$ & $101^{\circ} 01^{\prime} 40^{\prime \prime}$ & -- & & Dockum & -.04 & 6.2 & 824 & \\
\hline 46 & $32^{\circ} 32^{\circ} 25^{\prime \prime}$ & $100^{\circ} 56^{\prime} 52^{\prime \prime}$ & -- & & Dockum & .09 & 3.5 & 817.8 & \\
\hline 46 & $32^{\circ} 30^{\prime} 51^{\prime \prime}$ & $100^{\circ} 54^{\prime} 43^{\prime \prime}$ & -- & & Dockum & .82 & 3.7 & 814.3 & \\
\hline 46 & $32^{\circ} 28^{\prime} 41^{\prime \prime}$ & $100^{\circ} 56^{\prime} 52^{\prime \prime}$ & -- & & Dockum & .63 & 6.2 & 810.6 & \\
\hline 46 & $32^{\circ} 26^{\prime} 35^{\prime \prime}$ & $100^{\circ} 56^{\prime} 42^{\prime \prime}$ & -- & & Dockum & .1 & 2.3 & 804.4 & \\
\hline 46 & $32^{\circ} 25^{\prime} 50^{\prime \prime}$ & $100^{\circ} 54^{\prime} 57^{\prime \prime}$ & -- & & Dockum & 2.71 & 3.2 & 802.1 & \\
\hline 46 & $32^{\circ} 24^{\prime} 51^{\prime \prime}$ & $100^{\circ} 54^{\prime} 25^{\prime \prime}$ & -- & & Dockum & .35 & 2.6 & 798.9 & \\
\hline 47 & $32^{\circ} 34^{\prime} 58^{\prime \prime}$ & $101^{\circ} 05^{\prime} 42^{\prime \prime}$ & -- & & Dockum & .12 & .1 & 831.8 & \multirow[t]{11}{*}{$50 \mathrm{ft}$ above Bull Creek } \\
\hline 47 & $32^{\circ} 34^{\prime} 54^{\prime \prime}$ & $101^{\circ} 05^{\prime} 42^{\prime \prime}$ & -- & & Dockum & .06 & 2.9 & 831.7 & \\
\hline 47 & $32^{\circ} 34^{\prime} 17^{\prime \prime}$ & $101^{\circ} 03^{\prime} 18^{\prime \prime}$ & -- & & Dockum & .01 & 2.5 & 828.8 & \\
\hline 47 & $32^{\circ} 32^{\circ} 17^{\prime \prime}$ & $101^{\circ} 03^{\prime} 10^{\prime \prime}$ & -- & & Dockum & .34 & 2.3 & 826.3 & \\
\hline 47 & $32^{\circ} 30^{\prime} 42^{\prime \prime}$ & $100^{\circ} 01^{\prime} 40^{\prime \prime}$ & -- & & Dockum & -.1 & 6.2 & 824 & \\
\hline 47 & $32^{\circ} 32^{\circ} 25^{\prime \prime}$ & $100^{\circ} 56^{\prime} 52^{\prime \prime}$ & -- & & Dockum & -.01 & 3.5 & 817.8 & \\
\hline 47 & $32^{\circ} 30^{\prime} 51^{\prime \prime}$ & $100^{\circ} 54^{\prime} 43^{\prime \prime}$ & -- & & Dockum & 1.02 & 3.7 & 814.3 & \\
\hline 47 & $32^{\circ} 28^{\prime} 41^{\prime \prime}$ & $100^{\circ} 56^{\prime} 52^{\prime \prime}$ & -- & & Dockum & .57 & 6.2 & 810.6 & \\
\hline 47 & $32^{\circ} 26^{\prime} 35^{\prime \prime}$ & $100^{\circ} 56^{\prime} 42^{\prime \prime}$ & -- & & Dockum & .07 & 2.3 & 804.4 & \\
\hline 47 & $32^{\circ} 25^{\prime} 50^{\prime \prime}$ & $100^{\circ} 54^{\prime} 57^{\prime \prime}$ & -- & & Dockum & -4.4 & 3.2 & 802.1 & \\
\hline 47 & $32^{\circ} 24^{\prime} 51^{\prime \prime}$ & $100^{\circ} 54^{\prime} 25^{\prime \prime}$ & -- & & Dockum & .08 & 2.6 & 798.9 & \\
\hline 48 & $32^{\circ} 34^{\prime} 58^{\prime \prime}$ & $101^{\circ} 05^{\prime} 42^{\prime \prime}$ & -- & & Dockum & .1 & .1 & 831.8 & \multirow[t]{9}{*}{$50 \mathrm{ft}$ above Bull Creek } \\
\hline 48 & $32^{\circ} 34^{\prime} 54^{\prime \prime}$ & $100^{\circ} 05^{\prime} 42^{\prime \prime}$ & -- & & Dockum & -.01 & 2.9 & 831.7 & \\
\hline 48 & $32^{\circ} 34^{\prime} 17^{\prime \prime}$ & $101^{\circ} 03^{\prime} 18^{\prime \prime}$ & -- & & Dockum & .08 & 2.5 & 828.8 & \\
\hline 48 & $32^{\circ} 32^{\circ} 17^{\prime \prime}$ & $101^{\circ} 03^{\prime} 10^{\prime \prime}$ & -- & & Dockum & .17 & 2.3 & 826.3 & \\
\hline 48 & $32^{\circ} 30^{\prime} 42^{\prime \prime}$ & $101^{\circ} 01^{\prime} 40^{\prime \prime}$ & -- & & Dockum & -.24 & 6.2 & 824 & \\
\hline 48 & $32^{\circ} 32^{\circ} 25^{\prime \prime}$ & $100^{\circ} 56^{\prime} 52^{\prime \prime}$ & -- & & Dockum & .28 & 3.5 & 817.8 & \\
\hline 48 & $32^{\circ} 30^{\prime} 51^{\prime \prime}$ & $100^{\circ} 54^{\prime} 43^{\prime \prime}$ & -- & & Dockum & 1.63 & 3.7 & 814.3 & \\
\hline 48 & $32^{\circ} 28^{\prime} 41^{\prime \prime}$ & $100^{\circ} 56^{\prime} 52^{\prime \prime}$ & -- & & Dockum & .53 & 6.2 & 810.6 & \\
\hline 48 & $32^{\circ} 26^{\prime} 35^{\prime \prime}$ & $100^{\circ} 56^{\prime} 42^{\prime \prime}$ & -- & & Dockum & -.24 & 2.3 & 804.4 & \\
\hline
\end{tabular}


Table 4. Gains and losses from gain-loss studies in Texas-Continued

\begin{tabular}{|c|c|c|c|c|c|c|c|c|}
\hline $\begin{array}{c}\text { Stream- } \\
\text { flow } \\
\text { study } \\
\text { no. }\end{array}$ & $\begin{array}{l}\text { Latitude of } \\
\text { upstream } \\
\text { end of } \\
\text { subreach }\end{array}$ & $\begin{array}{l}\text { Longitude } \\
\text { of upstream } \\
\text { end of } \\
\text { subreach }\end{array}$ & $\begin{array}{c}\text { Major aquifer } \\
\text { outcrop } \\
\text { intersected } \\
\text { by subreach }\end{array}$ & $\begin{array}{c}\text { Minor aquifer } \\
\text { outcrop } \\
\text { intercepted } \\
\text { by subreach }\end{array}$ & $\begin{array}{l}\text { Gain or } \\
\text { loss }(-) \text { in } \\
\text { subreach } \\
\left(\mathrm{ft}^{3} / \mathrm{s}\right)\end{array}$ & $\begin{array}{l}\text { Length } \\
\text { of subreach } \\
\text { (river mi) }\end{array}$ & $\begin{array}{l}\text { Location of } \\
\text { upstream } \\
\text { end of } \\
\text { subreach } \\
\text { (river mi) }\end{array}$ & $\begin{array}{l}\text { Descriptive location } \\
\text { of upstream end of } \\
\text { selected subreaches }\end{array}$ \\
\hline 48 & $32^{\circ} 25^{\prime} 50^{\prime \prime}$ & $100^{\circ} 54^{\prime} 57^{\prime \prime}$ & -- & Dockum & -4.74 & 3.2 & 802.1 & \\
\hline 48 & $32^{\circ} 24^{\prime} 51^{\prime \prime}$ & $100^{\circ} 54^{\prime} 25^{\prime \prime}$ & -- & Dockum & .19 & 2.6 & 798.9 & \\
\hline 49 & $30^{\circ} 14^{\prime} 42^{\prime \prime}$ & $97^{\circ} 41^{\prime} 27^{\prime \prime}$ & Carrizo-Wilcox & -- & -257.95 & 68.5 & 290.1 & gaging station (08158000) \\
\hline 49 & $30^{\circ} 10^{\prime} 02^{\prime \prime}$ & $97^{\circ} 24^{\prime} 09^{\prime \prime}$ & Carrizo-Wilcox & -- & 70.0 & 15.1 & 221.6 & at FM 969 \\
\hline 49 & $30^{\circ} 07^{\prime} 05^{\prime \prime}$ & $97^{\circ} 19^{\prime} 54^{\prime \prime}$ & Carrizo-Wilcox & Sparta & -91.1 & 26.7 & 236.7 & gaging station (08159200) \\
\hline 49 & $30^{\circ} 00^{\prime} 55^{\prime \prime}$ & $97^{\circ} 08^{\prime} 46^{\prime \prime}$ & -- & Sparta & 0 & 35.5 & 210 & \\
\hline 49 & $29^{\circ} 54^{\prime} 46^{\prime \prime}$ & $96^{\circ} 53^{\prime} 44^{\prime \prime}$ & Gulf Coast & -- & -40.15 & 30.9 & 174.5 & discontinued partial-record station $(08160500)$ \\
\hline 49 & $29^{\circ} 42^{\prime} 55^{\prime \prime}$ & $96^{\circ} 32^{\prime} 31^{\prime \prime}$ & Gulf Coast & -- & 158.23 & 8.5 & 143.6 & gaging station $(08160700)$ \\
\hline 49 & $29^{\circ} 42^{\prime} 17^{\prime \prime}$ & $96^{\circ} 32^{\prime} 12^{\prime \prime}$ & Gulf Coast & -- & -20.0 & 21.7 & 135.1 & gaging station (08161000) \\
\hline 49 & $29^{\circ} 34^{\prime} 47^{\prime \prime}$ & $96^{\circ} 25^{\prime} 02^{\prime \prime}$ & Gulf Coast & -- & -770.0 & 10.5 & 113.4 & \\
\hline 49 & $29^{\circ} 27^{\prime} 11^{\prime \prime}$ & $96^{\circ} 23^{\prime} 46^{\prime \prime}$ & Gulf Coast & -- & -90.0 & 27.0 & 102.9 & \\
\hline 49 & $29^{\circ} 20^{\prime} 09^{\prime \prime}$ & $96^{\circ} 11^{\prime} 52^{\prime \prime}$ & Gulf Coast & -- & -119.0 & 9.3 & 75.9 & \\
\hline 49 & $29^{\circ} 18^{\prime} 20^{\prime \prime}$ & $96^{\circ} 06^{\prime} 34^{\prime \prime}$ & Gulf Coast & -- & -474.27 & 34.1 & 66.6 & gaging station (08162000) \\
\hline 50 & $32^{\circ} 01^{\prime} 09^{\prime \prime}$ & $100^{\circ} 44^{\prime} 06^{\prime \prime}$ & -- & -- & -.45 & 14.4 & 0 & gaging station near Silver (08123900) \\
\hline 50 & $31^{\circ} 58^{\prime} 51^{\prime \prime}$ & $100^{\circ} 36^{\prime} 21^{\prime \prime}$ & -- & -- & -.6 & 1.0 & 14.4 & \\
\hline 50 & $31^{\circ} 58^{\prime} 21^{\prime \prime}$ & $100^{\circ} 35^{\prime} 31^{\prime \prime}$ & -- & -- & -1.92 & 19.3 & 15.4 & \\
\hline 51 & $32^{\circ} 01^{\prime} 09^{\prime \prime}$ & $100^{\circ} 44^{\prime} 06^{\prime \prime}$ & -- & -- & -.84 & 1.2 & 0 & gaging station near Silver (08123900) \\
\hline 51 & $32^{\circ} 00^{\prime} 39^{\prime \prime}$ & $100^{\circ} 43^{\prime} 08^{\prime \prime}$ & -- & -- & .54 & 6.3 & 1.2 & \\
\hline 51 & $31^{\circ} 58^{\prime} 31^{\prime \prime}$ & $100^{\circ} 39^{\prime} 43^{\prime \prime}$ & -- & -- & .03 & 3.0 & 7.5 & \\
\hline 51 & $31^{\circ} 58^{\prime} 24^{\prime \prime}$ & $100^{\circ} 38^{\prime} 27^{\prime \prime}$ & -- & -- & -.83 & 3.9 & 10.5 & \\
\hline 51 & $31^{\circ} 58^{\prime} 51^{\prime \prime}$ & $100^{\circ} 36^{\prime} 21^{\prime \prime}$ & -- & -- & -.19 & 1.0 & 14.4 & \\
\hline 51 & $31^{\circ} 58^{\prime} 21^{\prime \prime}$ & $100^{\circ} 35^{\prime} 31^{\prime \prime}$ & -- & -- & -.1 & 1.7 & 15.4 & \\
\hline 51 & $31^{\circ} 57^{\prime} 16^{\prime \prime}$ & $100^{\circ} 35^{\prime} 13^{\prime \prime}$ & -- & -- & .17 & 3.1 & 17.1 & \\
\hline 51 & $31^{\circ} 56^{\prime} 07^{\prime \prime}$ & $100^{\circ} 34^{\prime} 01^{\prime \prime}$ & -- & -- & .02 & 2.8 & 20.2 & \\
\hline 51 & $31^{\circ} 54^{\prime} 46^{\prime \prime}$ & $100^{\circ} 34^{\prime} 01^{\prime \prime}$ & -- & -- & -1.49 & 11.7 & 23 & \\
\hline 52 & $32^{\circ} 35^{\prime} 03^{\prime \prime}$ & $101^{\circ} 07^{\prime} 48^{\prime \prime}$ & -- & Dockum & .45 & 10.3 & 836.6 & below Lake J.B. Thomas near Vincent (08118000) \\
\hline 52 & $32^{\circ} 32^{\circ} 17^{\prime \prime}$ & $101^{\circ} 03^{\prime} 10^{\prime \prime}$ & -- & Dockum & -.05 & 2.3 & 826.3 & \\
\hline 52 & $32^{\circ} 30^{\prime} 42^{\prime \prime}$ & $101^{\circ} 01^{\prime} 40^{\prime \prime}$ & -- & Dockum & -.2 & 5.0 & 824 & \\
\hline 52 & $32^{\circ} 32^{\circ} 39^{\prime \prime}$ & $100^{\circ} 58^{\prime} 04^{\prime \prime}$ & -- & Dockum & -.02 & 5.6 & 819 & \\
\hline 52 & $32^{\circ} 30^{\prime} 27^{\prime \prime}$ & $100^{\circ} 55^{\prime} 04^{\prime \prime}$ & -- & Dockum & .38 & 2.8 & 813.4 & \\
\hline 52 & $32^{\circ} 28^{\prime} 41^{\prime \prime}$ & $100^{\circ} 56^{\prime} 52^{\prime \prime}$ & -- & Dockum & .71 & 2.8 & 810.6 & \\
\hline 52 & $32^{\circ} 27^{\prime} 21^{\prime \prime}$ & $100^{\circ} 57^{\prime} 07^{\prime \prime}$ & -- & Dockum & -.77 & 3.4 & 807.8 & \\
\hline 52 & $32^{\circ} 26^{\prime} 34^{\prime \prime}$ & $100^{\circ} 56^{\prime} 45^{\prime \prime}$ & -- & Dockum & .33 & 4.3 & 804.4 & \\
\hline 52 & $32^{\circ} 25^{\prime} 36^{\prime \prime}$ & $100^{\circ} 53^{\prime} 49^{\prime \prime}$ & -- & Dockum & .18 & 3.8 & 800.1 & \\
\hline 52 & $32^{\circ} 23^{\prime} 33^{\prime \prime}$ & $100^{\circ} 52^{\prime} 40^{\prime \prime}$ & -- & Dockum & 1.36 & 8.0 & 796.3 & \\
\hline 52 & $32^{\circ} 19^{\prime} 41^{\prime \prime}$ & $100^{\circ} 54^{\prime} 28^{\prime \prime}$ & -- & Dockum & -.07 & 7.5 & 788.3 & \\
\hline 52 & $32^{\circ} 16^{\prime} 41^{\prime \prime}$ & $100^{\circ} 52^{\prime} 19^{\prime \prime}$ & -- & -- & -.31 & 10.4 & 780.8 & \\
\hline 52 & $32^{\circ} 11^{\prime} 03^{\prime \prime}$ & $100^{\circ} 51^{\prime} 00^{\prime \prime}$ & -- & -- & 0 & .9 & 770.4 & \\
\hline
\end{tabular}


\& Table 4. Gains and losses from gain-loss studies in Texas-Continued

\begin{tabular}{|c|c|c|c|c|c|c|c|c|}
\hline $\begin{array}{l}\text { Stream- } \\
\text { flow } \\
\text { study } \\
\text { no. }\end{array}$ & $\begin{array}{l}\text { Latitude of } \\
\text { upstream } \\
\text { end of } \\
\text { subreach }\end{array}$ & $\begin{array}{l}\text { Longitude } \\
\text { of upstream } \\
\text { end of } \\
\text { subreach }\end{array}$ & $\begin{array}{l}\text { Major aquifer } \\
\text { outcrop } \\
\text { intersected } \\
\text { by subreach }\end{array}$ & $\begin{array}{c}\text { Minor aquifer } \\
\text { outcrop } \\
\text { intercepted } \\
\text { by subreach }\end{array}$ & $\begin{array}{c}\text { Gain or } \\
\text { loss }(-) \text { in } \\
\text { subreach } \\
\left(\mathrm{ft}^{3} / \mathrm{s}\right)\end{array}$ & $\begin{array}{l}\text { Length } \\
\text { of subreach } \\
\text { (river mi) }\end{array}$ & $\begin{array}{l}\text { Location of } \\
\text { upstream } \\
\text { end of } \\
\text { subreach } \\
\text { (river mi) }\end{array}$ & $\begin{array}{l}\text { Descriptive location } \\
\text { of upstream end of } \\
\text { selected subreaches }\end{array}$ \\
\hline 52 & $32^{\circ} 10^{\prime} 45^{\prime \prime}$ & $100^{\circ} 51^{\prime} 18^{\prime \prime}$ & -- & -- & -1.74 & 9.2 & 769.5 & \multirow{38}{*}{$\begin{array}{l}\text { at Robert Lee } \\
\text { near Robert Lee gaging station }(08124000)\end{array}$} \\
\hline 52 & $32^{\circ} 05^{\prime} 13^{\prime \prime}$ & $100^{\circ} 47^{\prime} 24^{\prime \prime}$ & -- & -- & -.21 & 3.7 & 760.3 & \\
\hline 52 & $32^{\circ} 03^{\prime} 12^{\prime \prime}$ & $100^{\circ} 45^{\prime} 39^{\prime \prime}$ & -- & -- & .67 & 8.8 & 756.6 & \\
\hline 52 & $32^{\circ} 01^{\prime} 09^{\prime \prime}$ & $100^{\circ} 44^{\prime} 06^{\prime \prime}$ & -- & -- & .63 & 1.2 & 747.8 & \\
\hline 52 & $32^{\circ} 00^{\prime} 39^{\prime \prime}$ & $100^{\circ} 43^{\prime} 08^{\prime \prime}$ & -- & -- & -.01 & 6.3 & 746.6 & \\
\hline 52 & $31^{\circ} 58^{\prime} 31^{\prime \prime}$ & $100^{\circ} 39^{\prime} 43^{\prime \prime}$ & -- & -- & -.72 & 3.0 & 740.3 & \\
\hline 52 & $31^{\circ} 58^{\prime} 24^{\prime \prime}$ & $100^{\circ} 38^{\prime} 27^{\prime \prime}$ & -- & -- & -.79 & 2.8 & 737.3 & \\
\hline 52 & $31^{\circ} 58^{\prime} 01^{\prime \prime}$ & $100^{\circ} 36^{\prime} 36^{\prime \prime}$ & -- & -- & -.1 & 1.1 & 734.5 & \\
\hline 53 & $31^{\circ} 52^{\prime} 51^{\prime \prime}$ & $100^{\circ} 29^{\prime} 16^{\prime \prime}$ & -- & -- & 0 & 9.0 & 0 & \\
\hline 53 & $31^{\circ} 51^{\prime} 25^{\prime \prime}$ & $100^{\circ} 22^{\prime} 58^{\prime \prime}$ & -- & -- & .1 & 7.0 & 9 & \\
\hline 53 & $31^{\circ} 50^{\prime} 34^{\prime \prime}$ & $100^{\circ} 18^{\prime} 57^{\prime \prime}$ & -- & -- & -.1 & 14.0 & 16 & \\
\hline 53 & $31^{\circ} 48^{\prime} 26^{\prime \prime}$ & $100^{\circ} 13^{\prime} 37^{\prime \prime}$ & -- & -- & .2 & 20.0 & 30 & \\
\hline 53 & $31^{\circ} 43^{\prime} 55^{\prime \prime}$ & $99^{\circ} 56^{\prime} 59^{\prime \prime}$ & -- & -- & .3 & 7.0 & 50 & \\
\hline 53 & $31^{\circ} 41^{\prime} 15^{\prime \prime}$ & $99^{\circ} 54^{\prime} 05^{\prime \prime}$ & -- & -- & 0 & 2.0 & 57 & \\
\hline 53 & $31^{\circ} 40^{\prime} 55^{\prime \prime}$ & $99^{\circ} 52^{\prime} 08^{\prime \prime}$ & -- & -- & 1.1 & 4.0 & 59 & \\
\hline 53 & $31^{\circ} 35^{\prime} 52^{\prime \prime}$ & $99^{\circ} 46^{\prime} 42^{\prime \prime}$ & -- & -- & 3.9 & 6.0 & 63 & \\
\hline 53 & $31^{\circ} 34^{\prime} 14^{\prime \prime}$ & $99^{\circ} 43^{\prime} 28^{\prime \prime}$ & -- & -- & 2.5 & 3.0 & 69 & \\
\hline 53 & $31^{\circ} 31^{\circ} 54^{\prime \prime}$ & $99^{\circ} 41^{\prime} 17^{\prime \prime}$ & -- & -- & 2.5 & 3.0 & 72 & \\
\hline 53 & $31^{\circ} 30^{\prime} 10^{\prime \prime}$ & $99^{\circ} 40^{\prime} 34^{\prime \prime}$ & -- & -- & -.4 & 1.0 & 75 & \\
\hline 53 & $31^{\circ} 29^{\prime} 51^{\prime \prime}$ & $99^{\circ} 39^{\prime} 49^{\prime \prime}$ & -- & -- & -.2 & 8.0 & 76 & \\
\hline 53 & $31^{\circ} 25^{\prime} 28^{\prime \prime}$ & $99^{\circ} 30^{\prime} 46^{\prime \prime}$ & -- & -- & 1.2 & 12.0 & 84 & \\
\hline 53 & $31^{\circ} 29^{\prime} 51^{\prime \prime}$ & $99^{\circ} 36^{\prime} 31^{\prime \prime}$ & -- & -- & 1.9 & 8.0 & 96 & \\
\hline 53 & $31^{\circ} 26^{\prime} 41^{\prime \prime}$ & 99²9'39" & -- & -- & -.4 & 14.0 & 104 & \\
\hline 53 & $31^{\circ} 25^{\prime} 52^{\prime \prime}$ & $99^{\circ} 21^{\prime} 27^{\prime \prime}$ & -- & -- & 4.7 & 16.0 & 118 & \\
\hline 53 & $31^{\circ} 27^{\prime} 12^{\prime \prime}$ & $99^{\circ} 06^{\prime} 14^{\prime \prime}$ & -- & -- & -.9 & 10.0 & 134 & \\
\hline 53 & $31^{\circ} 28^{\prime} 48^{\prime \prime}$ & $99^{\circ} 00^{\prime} 07^{\prime \prime}$ & -- & -- & -1.0 & 15.0 & 144 & \\
\hline 53 & $31^{\circ} 25^{\prime} 03^{\prime \prime}$ & $98^{\circ} 51^{\prime} 10^{\prime \prime}$ & -- & -- & 2.4 & 15.0 & 159 & \\
\hline 53 & $31^{\circ} 24^{\prime} 39^{\prime \prime}$ & $98^{\circ} 43^{\prime} 40^{\prime \prime}$ & -- & -- & 4.2 & 11.0 & 174 & \\
\hline 53 & $31^{\circ} 21^{\prime} 10^{\prime \prime}$ & $98^{\circ} 40^{\prime} 17^{\prime \prime}$ & -- & -- & 1.2 & 10.0 & 185 & \\
\hline 53 & $31^{\circ} 18^{\prime} 45^{\prime \prime}$ & $98^{\circ} 36^{\prime} 14^{\prime \prime}$ & -- & -- & -3.5 & 3.0 & 195 & \\
\hline 53 & $31^{\circ} 17^{\prime} 50^{\prime \prime}$ & $98^{\circ} 35^{\prime} 49^{\prime \prime}$ & -- & -- & -.1 & 4.0 & 198 & \\
\hline 53 & $31^{\circ} 13^{\prime} 24^{\prime \prime}$ & $98^{\circ} 33^{\prime} 46^{\prime \prime}$ & -- & -- & 8.43 & 16.0 & 202 & \\
\hline 53 & $31^{\circ} 05^{\prime} 59^{\prime \prime}$ & $98^{\circ} 30^{\prime} 50^{\prime \prime}$ & -- & Ellenburger-San Saba & 20.9 & 25.0 & 218 & \\
\hline 53 & $30^{\circ} 51^{\prime} 12^{\prime \prime}$ & $98^{\circ} 25^{\prime} 09^{\prime \prime}$ & -- & -- & -11.7 & 8.0 & 243 & \\
\hline 53 & $30^{\circ} 50^{\prime} 39^{\prime \prime}$ & $98^{\circ} 22^{\prime} 36^{\prime \prime}$ & -- & Hickory & -29.9 & 11.0 & 251 & \\
\hline 53 & $30^{\circ} 45^{\prime} 22^{\prime \prime}$ & $98^{\circ} 22^{\prime} 31^{\prime \prime}$ & -- & -- & 50.7 & 13.0 & 262 & \\
\hline 53 & $30^{\circ} 38^{\prime} 29^{\prime \prime}$ & $98^{\circ} 24^{\prime} 59^{\prime \prime}$ & -- & -- & -17.0 & 5.0 & 275 & \\
\hline 53 & $30^{\circ} 34^{\prime} 05^{\prime \prime}$ & $98^{\circ} 24^{\prime} 26^{\prime \prime}$ & -- & -- & -16.2 & 5.0 & 280 & \\
\hline
\end{tabular}


Table 4. Gains and losses from gain-loss studies in Texas-Continued

\begin{tabular}{|c|c|c|c|c|c|c|c|c|}
\hline $\begin{array}{l}\text { Stream- } \\
\text { flow } \\
\text { study } \\
\text { no. }\end{array}$ & $\begin{array}{l}\text { Latitude of } \\
\text { upstream } \\
\text { end of } \\
\text { subreach }\end{array}$ & $\begin{array}{l}\text { Longitude } \\
\text { of upstream } \\
\text { end of } \\
\text { subreach }\end{array}$ & $\begin{array}{c}\text { Major aquifer } \\
\text { outcrop } \\
\text { intersected } \\
\text { by subreach }\end{array}$ & $\begin{array}{l}\text { Minor aquifer } \\
\text { outcrop } \\
\text { intercepted } \\
\text { by subreach }\end{array}$ & $\begin{array}{l}\text { Gain or } \\
\text { loss }(-) \text { in } \\
\text { subreach } \\
\left(\mathrm{ft}^{3} / \mathbf{s}\right)\end{array}$ & $\begin{array}{l}\text { Length } \\
\text { of subreach } \\
\text { (river mi) }\end{array}$ & $\begin{array}{l}\text { Location of } \\
\text { upstream } \\
\text { end of } \\
\text { subreach } \\
\text { (river mi) }\end{array}$ & $\begin{array}{l}\text { Descriptive location } \\
\text { of upstream end of } \\
\text { selected subreaches }\end{array}$ \\
\hline 53 & $30^{\circ} 33^{\prime} 13^{\prime \prime}$ & $98^{\circ} 20^{\prime} 34^{\prime \prime}$ & -- & $\begin{array}{l}\text { Ellenburger-San Saba, Marble } \\
\text { Falls }\end{array}$ & 9.3 & 5.0 & 285 & \multirow{37}{*}{$\begin{array}{l}\text { at Robert Lee } \\
\text { gaging station at Ballinger ( } 08126380)\end{array}$} \\
\hline 53 & $30^{\circ} 33^{\prime} 45^{\prime \prime}$ & $98^{\circ} 15^{\prime} 36^{\prime \prime}$ & Trinity & Ellenburger-San Saba & 53.1 & 26.0 & 290 & \\
\hline 53 & $30^{\circ} 25^{\prime} 53^{\prime \prime}$ & $98^{\circ} 03^{\prime} 28^{\prime \prime}$ & Trinity & -- & -13.4 & 6.0 & 316 & \\
\hline 53 & $30^{\circ} 24^{\prime} 49^{\prime \prime}$ & $98^{\circ} 00^{\prime} 47^{\prime \prime}$ & Trinity & -- & 11.8 & 3.0 & 322 & \\
\hline 53 & $30^{\circ} 23^{\prime} 56^{\prime \prime}$ & $97^{\circ} 57^{\prime} 45^{\prime \prime}$ & Trinity & -- & 9.9 & 9.0 & 325 & \\
\hline 53 & $30^{\circ} 27^{\prime} 52^{\prime \prime}$ & $97^{\circ} 54^{\prime} 43^{\prime \prime}$ & Edwards, Trinity & -- & 1.0 & 31.0 & 334 & \\
\hline 54 & $31^{\circ} 52^{\prime} 51^{\prime \prime}$ & $100^{\circ} 29^{\prime} 16^{\prime \prime}$ & -- & -- & 0 & 14.0 & 0 & \\
\hline 54 & $31^{\circ} 50^{\prime} 52^{\prime \prime}$ & $100^{\circ} 17^{\prime} 45^{\prime \prime}$ & -- & -- & 0 & 6.0 & 14 & \\
\hline 54 & $31^{\circ} 48^{\prime} 26^{\prime \prime}$ & $100^{\circ} 13^{\prime} 37^{\prime \prime}$ & -- & -- & 0 & 22.0 & 20 & \\
\hline 54 & $31^{\circ} 43^{\prime} 47^{\prime \prime}$ & $99^{\circ} 56^{\prime} 27^{\prime \prime}$ & -- & -- & 0 & 19.0 & 42 & \\
\hline 54 & $31^{\circ} 34^{\prime} 15^{\prime \prime}$ & $99^{\circ} 43^{\prime} 28^{\prime \prime}$ & -- & -- & 0 & 19.0 & 61 & \\
\hline 54 & $31^{\circ} 29^{\prime} 29^{\prime \prime}$ & $99^{\circ} 34^{\prime} 43^{\prime \prime}$ & -- & -- & 0 & 16.0 & 80 & \\
\hline 54 & $31^{\circ} 28^{\prime} 10^{\prime \prime}$ & $99^{\circ} 26^{\prime} 57^{\prime \prime}$ & -- & -- & 0 & 14.0 & 96 & \\
\hline 54 & $31^{\circ} 25^{\prime} 53^{\prime \prime}$ & $99^{\circ} 17^{\prime} 58^{\prime \prime}$ & -- & -- & 0 & 16.0 & 110 & \\
\hline 54 & $31^{\circ} 27^{\prime} 44^{\prime \prime}$ & $99^{\circ} 07^{\prime} 49^{\prime \prime}$ & -- & -- & 0 & 8.0 & 126 & \\
\hline 54 & $31^{\circ} 28^{\prime} 48^{\prime \prime}$ & $99^{\circ} 00^{\prime} 16^{\prime \prime}$ & -- & -- & 3.8 & 30.0 & 134 & \\
\hline 54 & $31^{\circ} 25^{\prime} 36^{\prime \prime}$ & $98^{\circ} 43^{\prime} 26^{\prime \prime}$ & -- & -- & 7.7 & 26.0 & 164 & \\
\hline 54 & $31^{\circ} 15^{\prime} 14^{\prime \prime}$ & $98^{\circ} 35^{\prime} 37^{\prime \prime}$ & -- & -- & -1.2 & 3.0 & 190 & \\
\hline 54 & $31^{\circ} 15^{\prime} 12^{\prime \prime}$ & $98^{\circ} 34^{\prime} 28^{\prime \prime}$ & -- & -- & -.1 & 15.0 & 193 & \\
\hline 54 & $31^{\circ} 05^{\prime} 19^{\prime \prime}$ & $98^{\circ} 31^{\prime} 26^{\prime \prime}$ & -- & Ellenburger-San Saba & 3.5 & 24.0 & 208 & \\
\hline 54 & $30^{\circ} 55^{\prime} 40^{\prime \prime}$ & $98^{\circ} 24^{\prime} 59^{\prime \prime}$ & -- & Ellenburger-San Saba & 0 & 9.0 & 232 & \\
\hline 54 & $30^{\circ} 48^{\prime} 51^{\prime \prime}$ & $98^{\circ} 28^{\prime} 30^{\prime \prime}$ & -- & -- & -.9 & 7.0 & 241 & \\
\hline 54 & $30^{\circ} 44^{\prime} 53^{\prime \prime}$ & $98^{\circ} 23^{\prime} 50^{\prime \prime}$ & -- & -- & .9 & 15.0 & 248 & \\
\hline 54 & $30^{\circ} 37^{\prime} 47^{\prime \prime}$ & $98^{\circ} 25^{\prime} 12^{\prime \prime}$ & -- & -- & -.1 & 5.0 & 263 & \\
\hline 54 & $30^{\circ} 33^{\prime} 32^{\prime \prime}$ & $98^{\circ} 25^{\prime} 33^{\prime \prime}$ & -- & -- & -.6 & 8.0 & 268 & \\
\hline 54 & $30^{\circ} 33^{\prime} 21^{\prime \prime}$ & $98^{\circ} 20^{\prime} 12^{\prime \prime}$ & Trinity & Ellenburger-San Saba & 6.0 & 28.0 & 276 & \\
\hline 54 & $30^{\circ} 25^{\prime} 59^{\prime \prime}$ & $98^{\circ} 03^{\prime} 32^{\prime \prime}$ & Trinity & -- & .3 & 6.0 & 304 & \\
\hline 54 & $30^{\circ} 25^{\prime} 14^{\prime \prime}$ & $98^{\circ} 02^{\prime} 03^{\prime \prime}$ & Trinity & -- & -1.7 & 4.0 & 310 & \\
\hline 54 & $30^{\circ} 22^{\prime} 37^{\prime \prime}$ & $97^{\circ} 59^{\prime} 43^{\prime \prime}$ & Trinity & -- & -.9 & 9.0 & 314 & \\
\hline 54 & $30^{\circ} 27^{\prime} 15^{\prime \prime}$ & $97^{\circ} 55^{\prime} 25^{\prime \prime}$ & Trinity & -- & 1.4 & 13.0 & 323 & \\
\hline 54 & $30^{\circ} 20^{\prime} 53^{\prime \prime}$ & $97^{\circ} 52^{\prime} 13^{\prime \prime}$ & Edwards, Trinity & -- & 11.5 & 12.0 & 336 & \\
\hline 54 & $30^{\circ} 17^{\prime} 36^{\prime \prime}$ & $97^{\circ} 47^{\prime} 10^{\prime \prime}$ & Edwards & -- & 3.7 & 1.0 & 348 & \\
\hline 54 & $30^{\circ} 16^{\prime} 54^{\prime \prime}$ & $97^{\circ} 466^{\prime} 54^{\prime \prime}$ & Edwards & -- & .4 & 2.0 & 349 & \\
\hline 54 & $30^{\circ} 15^{\prime} 38^{\prime \prime}$ & $97^{\circ} 44^{\prime} 43^{\prime \prime}$ & -- & -- & 27.4 & 16.0 & 351 & \\
\hline 54 & $30^{\circ} 15^{\prime} 43^{\prime \prime}$ & 97³9'09" & -- & -- & -.3 & 2.0 & 367 & \\
\hline 54 & $30^{\circ} 12^{\prime} 33^{\prime \prime}$ & $97^{\circ} 35^{\prime} 43^{\prime \prime}$ & -- & -- & 15.1 & 14.0 & 369 & \\
\hline 54 & $30^{\circ} 12^{\prime} 18^{\prime \prime}$ & $97^{\circ} 28^{\prime} 59^{\prime \prime}$ & Carrizo-Wilcox & -- & 20.2 & 20.0 & 383 & \\
\hline
\end{tabular}


o Table 4. Gains and losses from gain-loss studies in Texas-Continued

\begin{tabular}{|c|c|c|c|c|c|c|c|c|}
\hline $\begin{array}{c}\text { Stream- } \\
\text { flow } \\
\text { study } \\
\text { no. }\end{array}$ & $\begin{array}{l}\text { Latitude of } \\
\text { upstream } \\
\text { end of } \\
\text { subreach }\end{array}$ & $\begin{array}{l}\text { Longitude } \\
\text { of upstream } \\
\text { end of } \\
\text { subreach }\end{array}$ & $\begin{array}{c}\text { Major aquifer } \\
\text { outcrop } \\
\text { intersected } \\
\text { by subreach }\end{array}$ & $\begin{array}{l}\text { Minor aquifer } \\
\text { outcrop } \\
\text { intercepted } \\
\text { by subreach }\end{array}$ & $\begin{array}{c}\text { Gain or } \\
\text { loss }(-) \text { in } \\
\text { subreach } \\
\left(\mathrm{ft}^{3} / \mathbf{s}\right)\end{array}$ & $\begin{array}{l}\text { Length } \\
\text { of subreach } \\
\text { (river mi) }\end{array}$ & $\begin{array}{l}\text { Location of } \\
\text { upstream } \\
\text { end of } \\
\text { subreach } \\
\text { (river mi) }\end{array}$ & $\begin{array}{l}\text { Descriptive location } \\
\text { of upstream end of } \\
\text { selected subreaches }\end{array}$ \\
\hline 54 & $30^{\circ} 05^{\prime} 34^{\prime \prime}$ & $97^{\circ} 20^{\prime} 22^{\prime \prime}$ & Carrizo-Wilcox & -- & 17.3 & 23.0 & 403 & \\
\hline 54 & $30^{\circ} 00^{\prime} 45^{\prime \prime}$ & 9709'39" & -- & -- & 1.0 & 12.0 & 426 & \\
\hline 54 & $29^{\circ} 58^{\prime} 59^{\prime \prime}$ & $97^{\circ} 01^{\prime} 59^{\prime \prime}$ & -- & -- & 21.0 & 15.0 & 438 & \\
\hline 54 & $29^{\circ} 56^{\prime} 27^{\prime \prime}$ & $96^{\circ} 54^{\prime} 31^{\prime \prime}$ & Gulf Coast & -- & 9.0 & 17.0 & 453 & \\
\hline 54 & $29^{\circ} 46^{\prime} 44^{\prime \prime}$ & $96^{\circ} 42^{\prime} 06^{\prime \prime}$ & Gulf Coast & -- & 12.0 & 17.0 & 470 & \\
\hline 54 & $29^{\circ} 42^{\prime} 55^{\prime \prime}$ & $96^{\circ} 32^{\prime} 30^{\prime \prime}$ & Gulf Coast & -- & 27.2 & 24.0 & 487 & \\
\hline 54 & $29^{\circ} 34^{\prime} 24^{\prime \prime}$ & $96^{\circ} 24^{\prime} 22^{\prime \prime}$ & Gulf Coast & -- & 6.2 & 9.0 & 511 & \\
\hline 54 & $29^{\circ} 28^{\prime} 28^{\prime \prime}$ & $96^{\circ} 23^{\prime} 07^{\prime \prime}$ & Gulf Coast & -- & 50.0 & 21.0 & 520 & \\
\hline 54 & $29^{\circ} 20^{\prime} 50^{\prime \prime}$ & $96^{\circ} 13^{\prime} 12^{\prime \prime}$ & Gulf Coast & -- & 14.8 & 6.0 & 541 & \\
\hline 54 & $29^{\circ} 18^{\prime} 44^{\prime \prime}$ & $96^{\circ} 06^{\prime} 59^{\prime \prime}$ & Gulf Coast & -- & 85.2 & 28.0 & 547 & \\
\hline 54 & $28^{\circ} 56^{\prime} 18^{\prime \prime}$ & $96^{\circ} 00^{\prime} 35^{\prime \prime}$ & Gulf Coast & -- & .8 & 18.0 & 575 & \\
\hline 55 & $31^{\circ} 27^{\prime} 32^{\prime \prime}$ & $100^{\circ} 25^{\prime} 15^{\prime \prime}$ & -- & Lipan & 7.25 & 15.5 & 58.2 & gaging station (08136000) \\
\hline 55 & $31^{\circ} 32^{\prime} 01^{\prime \prime}$ & $100^{\circ} 14^{\prime} 38^{\prime \prime}$ & -- & Lipan & -6.42 & 11.5 & 42.7 & \\
\hline 55 & $31^{\circ} 31^{\circ} 12^{\prime \prime}$ & $100^{\circ} 05^{\prime} 34^{\prime \prime}$ & -- & Lipan & 4.09 & 12.9 & 31.2 & \\
\hline 55 & $31^{\circ} 30^{\prime} 56^{\prime \prime}$ & $99^{\circ} 55^{\prime} 09^{\prime \prime}$ & -- & -- & -5.78 & 8.3 & 18.3 & gaging station $(08136500)$ \\
\hline 55 & $31^{\circ} 32^{\prime} 14^{\prime \prime}$ & $99^{\circ} 48^{\prime} 50^{\prime \prime}$ & -- & -- & 8.11 & 6.5 & 10 & \\
\hline 56 & $31^{\circ} 27^{\prime} 11^{\prime \prime}$ & $100^{\circ} 24^{\prime} 36^{\prime \prime}$ & -- & Lipan & 25.8 & 15.5 & 58.2 & gaging station $(08136000)$ \\
\hline 56 & $31^{\circ} 32^{\prime} 01^{\prime \prime}$ & $100^{\circ} 14^{\prime} 38^{\prime \prime}$ & -- & Lipan & 6.6 & 11.5 & 42.7 & \\
\hline 56 & $31^{\circ} 31^{\circ} 12^{\prime \prime}$ & $100^{\circ} 05^{\prime} 34^{\prime \prime}$ & -- & Lipan & 2.96 & 12.9 & 31.2 & \\
\hline 56 & $31^{\circ} 30^{\prime} 56^{\prime \prime}$ & $99^{\circ} 55^{\prime} 09^{\prime \prime}$ & -- & -- & 3.57 & 8.3 & 18.3 & gaging station $(08136500)$ \\
\hline 56 & $31^{\circ} 32^{\prime} 14^{\prime \prime}$ & $99^{\circ} 48^{\prime} 50^{\prime \prime}$ & -- & -- & -2.7 & 6.5 & 10 & \\
\hline 57 & $31^{\circ} 27^{\prime} 11^{\prime \prime}$ & $100^{\circ} 24^{\prime} 36^{\prime \prime}$ & -- & Lipan & 17.84 & 15.5 & 58.2 & gaging station $(08136000)$ \\
\hline 57 & $31^{\circ} 32^{\prime} 01^{\prime \prime}$ & $100^{\circ} 14^{\prime} 38^{\prime \prime}$ & -- & Lipan & 14.5 & 11.5 & 42.7 & \\
\hline 57 & $31^{\circ} 32^{\prime} 01^{\prime \prime}$ & $100^{\circ} 14^{\prime} 38^{\prime \prime}$ & -- & Lipan & -3.44 & 12.9 & 31.2 & \\
\hline 57 & $31^{\circ} 30^{\prime} 56^{\prime \prime}$ & $99^{\circ} 55^{\prime} 09^{\prime \prime}$ & -- & -- & 5.7 & 8.3 & 18.3 & gaging station $(08136500)$ \\
\hline 57 & $31^{\circ} 32^{\prime} 14^{\prime \prime}$ & $99^{\circ} 48^{\prime} 50^{\prime \prime}$ & -- & -- & 3.2 & 6.5 & 10 & \\
\hline 58 & $31^{\circ} 27^{\prime} 15^{\prime \prime}$ & $100^{\circ} 24^{\prime} 57^{\prime \prime}$ & -- & Lipan & 1.3 & .2 & 0 & at confluence of North and South Concho Rivers \\
\hline 58 & $31^{\circ} 27^{\prime} 11^{\prime \prime}$ & $100^{\circ} 24^{\prime} 36^{\prime \prime}$ & -- & Lipan & -.9 & 13.8 & .2 & \\
\hline 58 & $31^{\circ} 31^{\circ} 54^{\prime \prime}$ & $100^{\circ} 15^{\prime} 14^{\prime \prime}$ & -- & Lipan & 2.0 & 3.0 & 14 & \\
\hline 58 & $31^{\circ} 32^{\prime} 08^{\prime \prime}$ & $100^{\circ} 13^{\prime} 04^{\prime \prime}$ & -- & Lipan & 3.7 & 1.0 & 17 & \\
\hline 58 & $31^{\circ} 31^{\circ} 35^{\prime \prime}$ & $100^{\circ} 12^{\prime} 39^{\prime \prime}$ & -- & Lipan & -.8 & 14.0 & 18 & \\
\hline 58 & $31^{\circ} 30^{\prime} 56^{\prime \prime}$ & $99^{\circ} 55^{\prime} 09^{\prime \prime}$ & -- & -- & -.7 & 19.5 & 32 & \\
\hline 59 & $31^{\circ} 27^{\prime} 11^{\prime \prime}$ & $100^{\circ} 24^{\prime} 36^{\prime \prime}$ & -- & Lipan & -.3 & 8.0 & 0 & at gaging station near San Angelo (08136000) \\
\hline 59 & $31^{\circ} 27^{\prime} 00^{\prime \prime}$ & $100^{\circ} 22^{\prime} 55^{\prime \prime}$ & -- & Lipan & -3.1 & 6.0 & 8 & \\
\hline 59 & $31^{\circ} 29^{\prime} 03^{\prime \prime}$ & $100^{\circ} 18^{\prime} 43^{\prime \prime}$ & -- & Lipan & .3 & 2.0 & 14 & \\
\hline 59 & $31^{\circ} 30^{\prime} 09^{\prime \prime}$ & $100^{\circ} 18^{\prime} 18^{\prime \prime}$ & -- & Lipan & 2.9 & 1.0 & 16 & \\
\hline 59 & $31^{\circ} 30^{\prime} 05^{\prime \prime}$ & $100^{\circ} 18^{\prime} 00^{\prime \prime}$ & -- & Lipan & 6.7 & 1.0 & 17 & \\
\hline 59 & $31^{\circ} 30^{\prime} 22^{\prime \prime}$ & $100^{\circ} 17^{\prime} 16^{\prime \prime}$ & -- & Lipan & -1.5 & .5 & 18 & \\
\hline
\end{tabular}


Table 4. Gains and losses from gain-loss studies in Texas-Continued

\begin{tabular}{|c|c|c|c|c|c|c|c|c|c|}
\hline $\begin{array}{l}\text { Stream- } \\
\text { flow } \\
\text { study } \\
\text { no. }\end{array}$ & $\begin{array}{l}\text { Latitude of } \\
\text { upstream } \\
\text { end of } \\
\text { subreach }\end{array}$ & $\begin{array}{l}\text { Longitude } \\
\text { of upstream } \\
\text { end of } \\
\text { subreach }\end{array}$ & $\begin{array}{c}\text { Major aquifer } \\
\text { outcrop } \\
\text { intersected } \\
\text { by subreach }\end{array}$ & & $\begin{array}{c}\text { Minor aquifer } \\
\text { outcrop } \\
\text { intercepted } \\
\text { by subreach }\end{array}$ & $\begin{array}{c}\text { Gain or } \\
\text { loss }(-) \text { in } \\
\text { subreach } \\
\left(\mathrm{ft}^{3} / \mathrm{s}\right)\end{array}$ & $\begin{array}{l}\text { Length } \\
\text { of subreach } \\
\text { (river mi) }\end{array}$ & $\begin{array}{l}\text { Location of } \\
\text { upstream } \\
\text { end of } \\
\text { subreach } \\
\text { (river mi) }\end{array}$ & $\begin{array}{l}\text { Descriptive location } \\
\text { of upstream end of } \\
\text { selected subreaches }\end{array}$ \\
\hline 59 & $31^{\circ} 30^{\prime} 42^{\prime \prime}$ & $100^{\circ} 17^{\prime} 24^{\prime \prime}$ & -- & Lipan & & 0 & 1.9 & 18.5 & \\
\hline 59 & $31^{\circ} 31^{\circ} 01^{\prime \prime}$ & $100^{\circ} 16^{\prime} 51^{\prime \prime}$ & -- & Lipan & & 0 & 5.3 & 20.4 & \\
\hline 59 & $31^{\circ} 31^{\circ} 20^{\prime \prime}$ & $100^{\circ} 15^{\prime} 43^{\prime \prime}$ & -- & Lipan & & .2 & 8.3 & 25.7 & \\
\hline 59 & $31^{\circ} 31^{\circ} 00^{\prime \prime}$ & $99^{\circ} 58^{\prime} 40^{\prime \prime}$ & -- & -- & & .5 & 3.0 & 34 & \\
\hline 59 & $31^{\circ} 30^{\prime} 56^{\prime \prime}$ & $99^{\circ} 55^{\prime} 08^{\prime \prime}$ & -- & -- & & .9 & 17.5 & 37 & \\
\hline 60 & $31^{\circ} 19^{\prime} 49^{\prime \prime}$ & $100^{\circ} 36^{\prime} 00^{\prime \prime}$ & -- & -- & & -.6 & 1.1 & 0 & just below spring source \\
\hline 60 & $31^{\circ} 19^{\prime} 22^{\prime \prime}$ & $100^{\circ} 36^{\prime} 18^{\prime \prime}$ & -- & -- & & 11.0 & 3.3 & 1.1 & \\
\hline 60 & $31^{\circ} 18^{\prime} 41^{\prime \prime}$ & $100^{\circ} 37^{\prime} 15^{\prime \prime}$ & -- & -- & & 5.8 & 4.3 & 4.4 & \\
\hline 60 & $31^{\circ} 15^{\prime} 51^{\prime \prime}$ & $100^{\circ} 38^{\prime} 20^{\prime \prime}$ & Edwards-Trinity Plateau & -- & & -.5 & 3.1 & 8.7 & \\
\hline 61 & $31^{\circ} 58^{\prime} 45^{\prime \prime}$ & $99^{\circ} 51^{\prime} 59^{\prime \prime}$ & -- & -- & & .04 & 3.0 & 21.2 & FM 1770 \\
\hline 61 & $31^{\circ} 56^{\prime} 03^{\prime \prime}$ & $99^{\circ} 52^{\prime} 04^{\prime \prime}$ & -- & -- & & -.04 & 6.0 & 18.2 & FM 53 \\
\hline 61 & $31^{\circ} 51^{\prime} 47^{\prime \prime}$ & $99^{\circ} 53^{\prime} 52^{\prime \prime}$ & -- & -- & & .09 & .7 & 12.2 & FM 2647 \\
\hline 61 & $31^{\circ} 51^{\prime} 27^{\prime \prime}$ & $99^{\circ} 54^{\prime} 12 "$ & -- & -- & & -.88 & 5.1 & 11.5 & \\
\hline 61 & $31^{\circ} 47^{\prime} 49^{\prime \prime}$ & $99^{\circ} 56^{\prime} 00^{\prime \prime}$ & -- & -- & & .79 & 1.5 & 6.4 & US 83 \\
\hline 61 & $31^{\circ} 47^{\prime} 29^{\prime \prime}$ & $99^{\circ} 56^{\prime} 33^{\prime \prime}$ & -- & -- & & -.53 & 2.1 & 4.9 & \\
\hline 62 & $31^{\circ} 58^{\prime} 45^{\prime \prime}$ & $99^{\circ} 51^{\prime} 59^{\prime \prime}$ & -- & -- & & 3.06 & 3.0 & 21.2 & FM 1770 \\
\hline 62 & $31^{\circ} 56^{\prime} 03^{\prime \prime}$ & $99^{\circ} 52^{\prime} 04^{\prime \prime}$ & -- & -- & & 3.81 & 6.0 & 18.2 & FM 53 \\
\hline 62 & $31^{\circ} 51^{\prime} 47^{\prime \prime}$ & $99^{\circ} 53^{\prime} 52^{\prime \prime}$ & -- & -- & & -6.87 & .7 & 12.2 & FM 2647 \\
\hline 62 & $31^{\circ} 51^{\prime} 27^{\prime \prime}$ & $99^{\circ} 54^{\prime} 12^{\prime \prime}$ & -- & -- & & 9.5 & 5.1 & 11.5 & \\
\hline 62 & $31^{\circ} 47^{\prime} 49^{\prime \prime}$ & $99^{\circ} 56^{\prime} 00^{\prime \prime}$ & -- & -- & & -36.2 & 1.5 & 6.4 & US 83 \\
\hline 62 & $31^{\circ} 47^{\prime} 29^{\prime \prime}$ & $99^{\circ} 56^{\prime} 33^{\prime \prime}$ & -- & -- & & 39.4 & 2.1 & 4.9 & \\
\hline 63 & $31^{\circ} 58^{\prime} 45^{\prime \prime}$ & $99^{\circ} 51^{\prime} 59^{\prime \prime}$ & -- & -- & & .01 & 3.0 & 21.2 & FM 1770 \\
\hline 63 & $31^{\circ} 56^{\prime} 03^{\prime \prime}$ & $99^{\circ} 52^{\prime} 04^{\prime \prime}$ & -- & -- & & -.01 & 6.0 & 18.2 & FM 53 \\
\hline 63 & $31^{\circ} 51^{\prime} 47^{\prime \prime}$ & $99^{\circ} 53^{\prime} 52^{\prime \prime}$ & -- & -- & & 0 & .7 & 12.2 & FM 2647 \\
\hline 63 & $31^{\circ} 51^{\prime} 27^{\prime \prime}$ & $99^{\circ} 54^{\prime} 12^{\prime \prime}$ & -- & -- & & -1.58 & 5.1 & 11.5 & \\
\hline 63 & $31^{\circ} 47^{\prime} 49^{\prime \prime}$ & $99^{\circ} 56^{\prime} 00^{\prime \prime}$ & -- & -- & & 2.04 & 1.5 & 6.4 & US 83 \\
\hline 63 & $31^{\circ} 47^{\prime} 29^{\prime \prime}$ & $99^{\circ} 56^{\prime} 33^{\prime \prime}$ & -- & -- & & .22 & 2.1 & 4.9 & \\
\hline 64 & $30^{\circ} 30^{\circ} 23^{\prime \prime}$ & $99^{\circ} 40^{\prime} 47^{\prime \prime}$ & Edwards-Trinity Plateau & -- & & 7.0 & .9 & 0 & at springs $8.3 \mathrm{mi}$ above Segovia \\
\hline 64 & $30^{\circ} 29^{\prime} 42^{\prime \prime}$ & $99^{\circ} 40^{\prime} 19^{\prime \prime}$ & Edwards-Trinity Plateau & -- & & 3.5 & 3.1 & .9 & \\
\hline 64 & $30^{\circ} 27^{\prime} 40^{\prime \prime}$ & $99^{\circ} 40^{\prime} 28^{\prime \prime}$ & Edwards-Trinity Plateau & -- & & .8 & 1.3 & 4 & \\
\hline 64 & $30^{\circ} 27^{\prime} 04^{\prime \prime}$ & $99^{\circ} 40^{\prime} 27^{\prime \prime}$ & Edwards-Trinity Plateau & -- & & .2 & 1.4 & 5.3 & \\
\hline 64 & $30^{\circ} 26^{\prime} 04^{\prime \prime}$ & $99^{\circ} 40^{\prime} 26^{\prime \prime}$ & Edwards-Trinity Plateau & -- & & -1.0 & 3.3 & 6.7 & \\
\hline 64 & $30^{\circ} 23^{\prime} 51^{\prime \prime}$ & $99^{\circ} 39^{\prime} 28^{\prime \prime}$ & Edwards-Trinity Plateau & -- & & -.8 & 2.1 & 10 & \\
\hline 64 & $30^{\circ} 22^{\prime} 10^{\prime \prime}$ & $99^{\circ} 38^{\prime} 30^{\prime \prime}$ & Edwards-Trinity Plateau & -- & & -.8 & 4.7 & 12.1 & \\
\hline 65 & $30^{\circ} 29^{\prime} 21^{\prime \prime}$ & $99^{\circ} 45^{\prime} 32^{\prime \prime}$ & Edwards-Trinity Plateau & -- & & 0 & 3.1 & 0 & $0.2 \mathrm{mi}$ above confluence with North Llano River \\
\hline 65 & $30^{\circ} 30^{\circ} 26^{\prime \prime}$ & $99^{\circ} 44^{\prime} 09^{\prime \prime}$ & Edwards-Trinity Plateau & -- & & -4.0 & .9 & 3.1 & \\
\hline 65 & $30^{\circ} 29^{\prime} 45^{\prime \prime}$ & $99^{\circ} 43^{\prime} 19^{\prime \prime}$ & Edwards-Trinity Plateau & -- & & 6.0 & 2.3 & 4 & gaging station $(08150000)$ \\
\hline 65 & $30^{\circ} 30^{\circ} 52^{\prime \prime}$ & $99^{\circ} 42^{\prime} 00^{\prime \prime}$ & Edwards-Trinity Plateau & -- & & 4.2 & 3.8 & 6.3 & \\
\hline
\end{tabular}


Table 4. Gains and losses from gain-loss studies in Texas-Continued

\begin{tabular}{|c|c|c|c|c|c|c|c|c|}
\hline $\begin{array}{l}\text { Stream- } \\
\text { flow } \\
\text { study } \\
\text { no. }\end{array}$ & $\begin{array}{l}\text { Latitude of } \\
\text { upstream } \\
\text { end of } \\
\text { subreach }\end{array}$ & $\begin{array}{l}\text { Longitude } \\
\text { of upstream } \\
\text { end of } \\
\text { subreach }\end{array}$ & $\begin{array}{c}\text { Major aquifer } \\
\text { outcrop } \\
\text { intersected } \\
\text { by subreach }\end{array}$ & $\begin{array}{c}\text { Minor aquifer } \\
\text { outcrop } \\
\text { intercepted } \\
\text { by subreach }\end{array}$ & $\begin{array}{l}\text { Gain or } \\
\text { loss }(-) \text { in } \\
\text { subreach } \\
\left(\mathrm{ft}^{3} / \mathrm{s}\right)\end{array}$ & $\begin{array}{l}\text { Length } \\
\text { of subreach } \\
\text { (river mi) }\end{array}$ & $\begin{array}{l}\text { Location of } \\
\text { upstream } \\
\text { end of } \\
\text { subreach } \\
\text { (river mi) }\end{array}$ & $\begin{array}{l}\text { Descriptive location } \\
\text { of upstream end of } \\
\text { selected subreaches }\end{array}$ \\
\hline 65 & $30^{\circ} 31^{\prime} 07^{\prime \prime}$ & $99^{\circ} 40^{\prime} 14^{\prime \prime}$ & Edwards-Trinity Plateau & -- & -6.0 & 1.9 & 10.1 & \\
\hline 65 & $30^{\circ} 31^{\prime} 37^{\prime \prime}$ & $99^{\circ} 38^{\prime} 37^{\prime \prime}$ & Edwards-Trinity Plateau & -- & 1.65 & 3.8 & 12 & \\
\hline 65 & $30^{\circ} 33^{\prime} 09^{\prime \prime}$ & $99^{\circ} 36^{\prime} 02^{\prime \prime}$ & Edwards-Trinity Plateau & -- & 4.0 & 2.7 & 15.8 & \\
\hline 65 & $30^{\circ} 34^{\prime} 44^{\prime \prime}$ & $99^{\circ} 35^{\prime} 48^{\prime \prime}$ & Edwards-Trinity Plateau & -- & .98 & 3.5 & 18.5 & \\
\hline 65 & $30^{\circ} 35^{\prime} 08^{\prime \prime}$ & $99^{\circ} 33^{\prime} 35^{\prime \prime}$ & -- & $\begin{array}{l}\text { Ellenburger-San Saba, Marble } \\
\text { Falls }\end{array}$ & 2.95 & 3.1 & 22 & \\
\hline 65 & $30^{\circ} 37^{\prime} 42^{\prime \prime}$ & $99^{\circ} 30^{\prime} 55^{\prime \prime}$ & -- & Ellenburger-San Saba & -7.61 & 8.5 & 25.1 & \\
\hline 65 & $30^{\circ} 41^{\prime} 03^{\prime \prime}$ & $99^{\circ} 23^{\prime} 47^{\prime \prime}$ & -- & Ellenburger-San Saba & 10.0 & 6.4 & 33.6 & \\
\hline 65 & $30^{\circ} 39^{\prime} 28^{\prime \prime}$ & $99^{\circ} 19^{\prime} 26^{\prime \prime}$ & -- & Ellenburger-San Saba & -4.11 & 4.6 & 40 & \\
\hline 65 & $30^{\circ} 38^{\prime} 58^{\prime \prime}$ & $99^{\circ} 14^{\prime} 31^{\prime \prime}$ & -- & Hickory & -8.7 & 5.4 & 44.6 & \\
\hline 65 & $30^{\circ} 38^{\prime} 10^{\prime \prime}$ & $99^{\circ} 10^{\prime} 31^{\prime \prime}$ & -- & Hickory & -1.0 & 5.1 & 50 & \\
\hline 65 & $30^{\circ} 39^{\prime} 42^{\prime \prime}$ & $99^{\circ} 05^{\prime} 24^{\prime \prime}$ & -- & -- & 7.5 & 5.8 & 55.1 & \\
\hline 65 & $30^{\circ} 42^{\prime} 01^{\prime \prime}$ & $99^{\circ} 01^{\prime 2} 27^{\prime \prime}$ & -- & -- & -2.0 & 6.5 & 60.9 & \\
\hline 65 & $30^{\circ} 42^{\prime} 20^{\prime \prime}$ & $98^{\circ} 55^{\prime} 40^{\prime \prime}$ & -- & -- & -3.22 & 6.9 & 67.4 & \\
\hline 65 & $30^{\circ} 43^{\prime} 34^{\prime \prime}$ & $98^{\circ} 48^{\prime} 41^{\prime \prime}$ & -- & -- & 3.7 & 6.8 & 74.3 & \\
\hline 65 & $30^{\circ} 45^{\prime} 09^{\prime \prime}$ & $98^{\circ} 40^{\prime} 24^{\prime \prime}$ & -- & -- & 3.5 & 2.4 & 81.1 & \\
\hline 66 & $30^{\circ} 29^{\prime} 31^{\prime \prime}$ & $99^{\circ} 45^{\prime} 20^{\prime \prime}$ & Edwards-Trinity Plateau & -- & -3.03 & 10.0 & 0 & near Junction gaging station (08150000) \\
\hline 66 & $30^{\circ} 32^{\prime} 13^{\prime \prime}$ & $99^{\circ} 37^{\prime} 34^{\prime \prime}$ & Edwards-Trinity Plateau & -- & -2.2 & 6.0 & 10 & \\
\hline 66 & $30^{\circ} 35^{\prime} 19^{\prime \prime}$ & $99^{\circ} 35^{\prime} 50^{\prime \prime}$ & Edwards-Trinity Plateau & Ellenburger-San Saba & -7.93 & 19.0 & 16 & \\
\hline 66 & $30^{\circ} 38^{\prime} 52^{\prime \prime}$ & $99^{\circ} 18^{\prime} 07^{\prime \prime}$ & -- & Ellenburger-San Saba & -3.1 & 4.0 & 35 & \\
\hline 66 & $30^{\circ} 38^{\prime} 16^{\prime \prime}$ & $99^{\circ} 13^{\prime} 28^{\prime \prime}$ & -- & -- & -1.84 & 8.0 & 39 & \\
\hline 66 & $30^{\circ} 39^{\prime} 38^{\prime \prime}$ & $99^{\circ} 06^{\prime} 33^{\prime \prime}$ & -- & -- & -.72 & 12.0 & 47 & \\
\hline 66 & $30^{\circ} 42^{\prime} 12^{\prime \prime}$ & $98^{\circ} 57^{\prime} 30^{\prime \prime}$ & -- & -- & -.01 & 5.0 & 59 & \\
\hline 66 & $30^{\circ} 42^{\prime} 41^{\prime \prime}$ & $98^{\circ} 53^{\prime} 03^{\prime \prime}$ & -- & -- & 0 & 12.0 & 64 & \\
\hline 66 & $30^{\circ} 45^{\prime} 05^{\prime \prime}$ & $98^{\circ} 42^{\prime} 22^{\prime \prime}$ & -- & -- & 0 & 3.0 & 76 & \\
\hline 67 & $30^{\circ} 29^{\prime} 27^{\prime \prime}$ & $99^{\circ} 45^{\prime} 29^{\prime \prime}$ & Edwards-Trinity Plateau & -- & 11.6 & 3.0 & 0 & just above Llano River \\
\hline 67 & $30^{\circ} 29^{\prime} 45^{\prime \prime}$ & $99^{\circ} 43^{\prime} 19^{\prime \prime}$ & Edwards-Trinity Plateau & -- & -.9 & 4.0 & 3 & \\
\hline 67 & $30^{\circ} 30^{\circ} 30^{\prime \prime}$ & $99^{\circ} 40^{\prime} 55^{\prime \prime}$ & Edwards-Trinity Plateau & -- & -3.6 & 13.0 & 7 & \\
\hline 67 & $30^{\circ} 34^{\prime} 55^{\prime \prime}$ & $99^{\circ} 34^{\prime} 19^{\prime \prime}$ & Edwards-Trinity Plateau & Ellenburger-San Saba & -2.3 & 15.0 & 20 & \\
\hline 67 & $30^{\circ} 40^{\prime} 56^{\prime \prime}$ & $99^{\circ} 23^{\prime} 53^{\prime \prime}$ & -- & Ellenburger-San Saba & 1.6 & 8.0 & 35 & \\
\hline 67 & $30^{\circ} 38^{\prime} 53^{\prime \prime}$ & $99^{\circ} 15^{\prime} 07^{\prime \prime}$ & -- & -- & -4.9 & 11.0 & 43 & \\
\hline 67 & $30^{\circ} 39^{\prime} 41^{\prime \prime}$ & $99^{\circ} 06^{\prime} 18^{\prime \prime}$ & -- & -- & 7.4 & 10.0 & 54 & \\
\hline 67 & $30^{\circ} 42^{\prime} 11^{\prime \prime}$ & $98^{\circ} 57^{\prime} 25^{\prime \prime}$ & -- & -- & 5.5 & 9.5 & 64 & \\
\hline 67 & $30^{\circ} 43^{\prime} 50^{\prime \prime}$ & $98^{\circ} 48^{\prime} 01^{\prime \prime}$ & -- & -- & 5.6 & 8.5 & 73.5 & \\
\hline 67 & $30^{\circ} 45^{\prime} 05^{\prime \prime}$ & $98^{\circ} 40^{\prime} 06^{\prime \prime}$ & -- & -- & 9.3 & 1.0 & 82 & \\
\hline 67 & $30^{\circ} 45^{\prime} 03^{\prime \prime}$ & $98^{\circ} 39^{\prime} 53^{\prime \prime}$ & -- & -- & 0 & 9.0 & 83 & \\
\hline 67 & $30^{\circ} 45^{\prime} 56^{\prime \prime}$ & $98^{\circ} 32^{\prime} 49^{\prime \prime}$ & -- & -- & -7.0 & 14.0 & 92 & \\
\hline 68 & $30^{\circ} 29^{\prime} 31^{\prime \prime}$ & $99^{\circ} 45^{\prime} 20^{\prime \prime}$ & Edwards-Trinity Plateau & -- & 3.3 & .2 & 0 & 6.8 mi below Junction \\
\hline
\end{tabular}


Table 4. Gains and losses from gain-loss studies in Texas-Continued

\begin{tabular}{|c|c|c|c|c|c|c|c|c|}
\hline $\begin{array}{l}\text { Stream- } \\
\text { flow } \\
\text { study } \\
\text { no. }\end{array}$ & $\begin{array}{c}\text { Latitude of } \\
\text { upstream } \\
\text { end of } \\
\text { subreach }\end{array}$ & $\begin{array}{l}\text { Longitude } \\
\text { of upstream } \\
\text { end of } \\
\text { subreach }\end{array}$ & $\begin{array}{c}\text { Major aquifer } \\
\text { outcrop } \\
\text { intersected } \\
\text { by subreach }\end{array}$ & $\begin{array}{c}\text { Minor aquifer } \\
\text { outcrop } \\
\text { intercepted } \\
\text { by subreach }\end{array}$ & $\begin{array}{c}\text { Gain or } \\
\text { loss }(-) \text { in } \\
\text { subreach } \\
\left(\mathrm{ft}^{3} / \mathrm{s}\right)\end{array}$ & $\begin{array}{l}\text { Length } \\
\text { of subreach } \\
\text { (river mi) }\end{array}$ & $\begin{array}{l}\text { Location of } \\
\text { upstream } \\
\text { end of } \\
\text { subreach } \\
\text { (river mi) }\end{array}$ & $\begin{array}{l}\text { Descriptive location } \\
\text { of upstream end of } \\
\text { selected subreaches }\end{array}$ \\
\hline 68 & $30^{\circ} 29^{\prime} 34^{\prime \prime}$ & $99^{\circ} 45^{\prime} 14^{\prime \prime}$ & Edwards-Trinity Plateau & -- & 0 & 2.8 & 0.2 & \\
\hline 68 & $30^{\circ} 29^{\prime} 45^{\prime \prime}$ & $99^{\circ} 43^{\prime} 19^{\prime \prime}$ & Edwards-Trinity Plateau & -- & -2.2 & 3.8 & 3 & \\
\hline 68 & $30^{\circ} 30^{\circ} 28^{\prime \prime}$ & $99^{\circ} 40^{\prime} 55^{\prime \prime}$ & Edwards-Trinity Plateau & -- & -4.0 & 8.2 & 6.8 & \\
\hline 68 & $30^{\circ} 33^{\prime} 10^{\prime \prime}$ & $99^{\circ} 36^{\prime} 02^{\prime \prime}$ & Edwards-Trinity Plateau & Ellenburger-San Saba & 6.3 & 22.0 & 15 & \\
\hline 68 & $30^{\circ} 41^{\prime} 14^{\prime \prime}$ & $99^{\circ} 21^{\prime} 25^{\prime \prime}$ & -- & Ellenburger-San Saba & -3.8 & 7.0 & 37 & \\
\hline 68 & $30^{\circ} 38^{\prime} 57^{\prime \prime}$ & $99^{\circ} 14^{\prime} 48^{\prime \prime}$ & -- & -- & 4.0 & 10.2 & 44 & \\
\hline 68 & $30^{\circ} 39^{\prime} 41^{\prime \prime}$ & $99^{\circ} 06^{\prime} 18^{\prime \prime}$ & -- & -- & -.7 & 10.7 & 54.2 & \\
\hline 68 & $30^{\circ} 42^{\prime} 34^{\prime \prime}$ & $98^{\circ} 58^{\prime} 40^{\prime \prime}$ & -- & -- & 4.0 & 4.8 & 64.9 & \\
\hline 68 & $30^{\circ} 42^{\prime} 20^{\prime \prime}$ & $98^{\circ} 55^{\prime} 40^{\prime \prime}$ & -- & -- & -8.0 & 5.0 & 69.7 & Castell gaging station (08151000) \\
\hline 68 & $30^{\circ} 42^{\prime} 58^{\prime \prime}$ & $98^{\circ} 50^{\prime} 51^{\prime \prime}$ & -- & -- & 5.0 & 7.6 & 74.7 & \\
\hline 68 & $30^{\circ} 45^{\prime} 00^{\prime \prime}$ & $98^{\circ} 42^{\prime} 42^{\prime \prime}$ & -- & -- & -1.0 & .7 & 82.3 & \\
\hline 68 & $30^{\circ} 45^{\prime} 10^{\prime \prime}$ & $98^{\circ} 40^{\prime} 30^{\prime \prime}$ & -- & -- & -3.1 & 10.0 & 83 & \\
\hline 68 & $30^{\circ} 46^{\prime} 07^{\prime \prime}$ & $98^{\circ} 33^{\prime} 05^{\prime \prime}$ & -- & -- & 4.9 & 1.2 & 93 & \\
\hline 68 & $30^{\circ} 44^{\prime} 38^{\prime \prime}$ & $98^{\circ} 32^{\prime} 58^{\prime \prime}$ & -- & -- & -1.0 & 4.5 & 94.2 & \\
\hline 68 & $30^{\circ} 42^{\prime} 06^{\prime \prime}$ & $98^{\circ} 29^{\prime} 39^{\prime \prime}$ & -- & -- & 2.0 & 6.3 & 98.7 & \\
\hline 69 & $31^{\circ} 26^{\prime} 43^{\prime \prime}$ & $101^{\circ} 08^{\prime} 09^{\prime \prime}$ & -- & -- & -.1 & 6.8 & 0 & mouth of Kiowa Creek \\
\hline 69 & $31^{\circ} 24^{\prime} 34^{\prime \prime}$ & $101^{\circ} 03^{\prime} 57^{\prime \prime}$ & -- & -- & 0 & 1.0 & 6.8 & \\
\hline 69 & $31^{\circ} 24^{\prime} 27^{\prime \prime}$ & $101^{\circ} 03^{\prime} 03^{\prime \prime}$ & -- & -- & .3 & .7 & 7.8 & \\
\hline 69 & $31^{\circ} 24^{\prime} 23^{\prime \prime}$ & $100^{\circ} 02^{\prime} 24^{\prime \prime}$ & -- & -- & -.3 & 3.1 & 8.5 & \\
\hline 69 & $31^{\circ} 23^{\prime} 07^{\prime \prime}$ & $101^{\circ} 00^{\prime} 46^{\prime \prime}$ & -- & -- & .9 & 1.5 & 11.6 & \\
\hline 69 & $31^{\circ} 22^{\prime} 21^{\prime \prime}$ & $101^{\circ} 00^{\prime} 00^{\prime \prime}$ & -- & -- & .1 & 10.6 & 13.1 & \\
\hline 69 & $31^{\circ} 25^{\prime} 06^{\prime \prime}$ & $100^{\circ} 47^{\prime} 34^{\prime \prime}$ & -- & -- & -.5 & 6.0 & 23.7 & \\
\hline 69 & $31^{\circ} 25^{\prime} 15^{\prime \prime}$ & $100^{\circ} 43^{\prime} 08^{\prime \prime}$ & -- & -- & -1.1 & 6.8 & 29.7 & \\
\hline 69 & $31^{\circ} 24^{\prime} 40^{\prime \prime}$ & $100^{\circ} 37^{\prime} 44^{\prime \prime}$ & -- & -- & -.1 & 1.7 & 36.5 & \\
\hline 69 & $31^{\circ} 23^{\prime} 50^{\prime \prime}$ & $100^{\circ} 37^{\prime} 01^{\prime \prime}$ & -- & -- & 2.1 & 7.0 & 38.2 & \\
\hline 69 & $31^{\circ} 22^{\prime} 04^{\prime \prime}$ & $100^{\circ} 33^{\prime} 54^{\prime \prime}$ & -- & -- & 5.2 & .5 & 45.2 & \\
\hline 69 & $31^{\circ} 20^{\prime} 42^{\prime \prime}$ & $100^{\circ} 32^{\prime} 49^{\prime \prime}$ & -- & -- & -7.3 & 2.0 & 45.7 & \\
\hline 70 & $31^{\circ} 24^{\prime} 05^{\prime \prime}$ & $100^{\circ} 50^{\prime} 34^{\prime \prime}$ & -- & -- & 3.9 & 22.0 & 0 & at San Angelo - Mertzon Rd crossing \\
\hline 71 & $31^{\circ} 52^{\prime} 14^{\prime \prime}$ & $100^{\circ} 04^{\prime} 12^{\prime \prime}$ & -- & -- & 1.0 & .75 & 0 & at McIntyres Dam - $13 \mathrm{mi}$ above Sterling, estimate \\
\hline 71 & $31^{\circ} 52^{\prime} 04^{\prime \prime}$ & $100^{\circ} 04^{\prime} 12^{\prime \prime}$ & -- & -- & 1.4 & 2.25 & .75 & \\
\hline 71 & $31^{\circ} 51^{\prime} 03^{\prime \prime}$ & $100^{\circ} 03^{\prime} 21^{\prime \prime}$ & -- & -- & -.2 & 1.0 & 3 & \\
\hline 71 & $31^{\circ} 50^{\prime} 27^{\prime \prime}$ & $100^{\circ} 02^{\prime} 56^{\prime \prime}$ & -- & -- & .3 & .5 & 4 & \\
\hline 71 & $31^{\circ} 50^{\prime} 24^{\prime \prime}$ & $100^{\circ} 02^{\prime} 42^{\prime \prime}$ & -- & -- & -.2 & 2.0 & 4.5 & \\
\hline 71 & $31^{\circ} 50^{\prime} 02^{\prime \prime}$ & $100^{\circ} 01^{\prime} 22^{\prime \prime}$ & -- & -- & 1.9 & 1.0 & 6.5 & \\
\hline 71 & $31^{\circ} 50^{\prime} 01^{\prime \prime}$ & $100^{\circ} 00^{\prime} 32^{\prime \prime}$ & -- & -- & .2 & 1.5 & 7.5 & estimate \\
\hline 71 & $31^{\circ} 50^{\prime} 03^{\prime \prime}$ & $100^{\circ} 01^{\prime} 15^{\prime \prime}$ & -- & -- & .6 & .5 & 9 & \\
\hline 71 & $31^{\circ} 50^{\prime} 03^{\prime \prime}$ & $100^{\circ} 01^{\prime} 01^{\prime \prime}$ & -- & -- & 0 & 1.5 & 9.5 & estimate \\
\hline 71 & $31^{\circ} 49^{\prime} 59^{\prime \prime}$ & $100^{\circ} 00^{\prime} 36^{\prime \prime}$ & -- & -- & -.1 & 2.0 & 11 & estimate \\
\hline
\end{tabular}


A Table 4. Gains and losses from gain-loss studies in Texas-Continued

\begin{tabular}{|c|c|c|c|c|c|c|c|c|}
\hline $\begin{array}{l}\text { Stream- } \\
\text { flow } \\
\text { study } \\
\text { no. }\end{array}$ & $\begin{array}{l}\text { Latitude of } \\
\text { upstream } \\
\text { end of } \\
\text { subreach }\end{array}$ & $\begin{array}{l}\text { Longitude } \\
\text { of upstream } \\
\text { end of } \\
\text { subreach }\end{array}$ & $\begin{array}{c}\text { Major aquifer } \\
\text { outcrop } \\
\text { intersected } \\
\text { by subreach }\end{array}$ & $\begin{array}{c}\text { Minor aquifer } \\
\text { outcrop } \\
\text { intercepted } \\
\text { by subreach }\end{array}$ & $\begin{array}{l}\text { Gain or } \\
\text { loss }(-) \text { in } \\
\text { subreach } \\
\left(\mathrm{ft}^{3} / \mathbf{s}\right)\end{array}$ & $\begin{array}{l}\text { Length } \\
\text { of subreach } \\
\text { (river } \mathrm{mi} \text { ) }\end{array}$ & $\begin{array}{l}\text { Location of } \\
\text { upstream } \\
\text { end of } \\
\text { subreach } \\
\text { (river mi) }\end{array}$ & $\begin{array}{l}\text { Descriptive location } \\
\text { of upstream end of } \\
\text { selected subreaches }\end{array}$ \\
\hline 71 & $31^{\circ} 50^{\prime} 06^{\prime \prime}$ & $100^{\circ} 00^{\prime} 14^{\prime \prime}$ & -- & -- & -0.1 & 1.0 & 13 & \\
\hline 71 & $31^{\circ} 49^{\prime} 47^{\prime \prime}$ & $100^{\circ} 59^{\prime} 31^{\prime \prime}$ & -- & -- & 0 & 6.0 & 14 & \\
\hline 71 & $31^{\circ} 47^{\prime} 52^{\prime \prime}$ & $100^{\circ} 56^{\prime} 13^{\prime \prime}$ & -- & -- & 0 & 3.0 & 20 & \\
\hline 71 & $31^{\circ} 46^{\prime} 51^{\prime \prime}$ & $100^{\circ} 54^{\prime} 32^{\prime \prime}$ & -- & -- & .3 & 1.0 & 23 & \\
\hline 71 & $31^{\circ} 46^{\prime} 31^{\prime \prime}$ & $100^{\circ} 53^{\prime} 56^{\prime \prime}$ & -- & -- & -.3 & 8.0 & 24 & \\
\hline 71 & $31^{\circ} 43^{\prime} 59^{\prime \prime}$ & $100^{\circ} 50^{\prime} 06^{\prime \prime}$ & -- & -- & 0 & 7.0 & 32 & \\
\hline 71 & $31^{\circ} 42^{\prime} 13^{\prime \prime}$ & $100^{\circ} 48^{\prime} 18^{\prime \prime}$ & -- & -- & .9 & 5.0 & 39 & \\
\hline 71 & $31^{\circ} 40^{\prime} 01^{\prime \prime}$ & $100^{\circ} 45^{\prime} 10^{\prime \prime}$ & -- & -- & .9 & 1.0 & 44 & \\
\hline 71 & $31^{\circ} 39^{\prime} 37^{\prime \prime}$ & $100^{\circ} 44^{\prime} 13^{\prime \prime}$ & -- & -- & .9 & .5 & 45 & \\
\hline 71 & 31'39'31" & $100^{\circ} 43^{\prime} 51^{\prime \prime}$ & -- & -- & -1.0 & 6.0 & 45.5 & \\
\hline 71 & $31^{\circ} 36^{\prime} 59^{\prime \prime}$ & $100^{\circ} 40^{\prime} 26^{\prime \prime}$ & -- & -- & -.1 & 4.5 & 51.5 & \\
\hline 71 & $31^{\circ} 35^{\prime} 42^{\prime \prime}$ & $100^{\circ} 38^{\prime} 49^{\prime \prime}$ & -- & -- & 0 & .5 & 56 & \\
\hline 71 & $31^{\circ} 35^{\prime} 32^{\prime \prime}$ & $100^{\circ} 38^{\prime} 27^{\prime \prime}$ & -- & -- & -.2 & 4.0 & 56.5 & \\
\hline 71 & $31^{\circ} 34^{\prime} 00^{\prime \prime}$ & $100^{\circ} 36^{\prime} 14^{\prime \prime}$ & -- & -- & -1.3 & 4.5 & 60.5 & \\
\hline 71 & $31^{\circ} 32^{\prime} 30^{\prime \prime}$ & $100^{\circ} 33^{\prime} 14^{\prime \prime}$ & -- & -- & .2 & 1.0 & 65 & \\
\hline 71 & $31^{\circ} 32^{\prime} 17^{\prime \prime}$ & $100^{\circ} 32^{\prime} 42^{\prime \prime}$ & -- & -- & -.1 & 5.0 & 66 & estimate \\
\hline 71 & $31^{\circ} 29^{\prime} 42^{\prime \prime}$ & $100^{\circ} 29^{\prime} 38^{\prime \prime}$ & -- & Lipan & .1 & 6.0 & 71 & estimate \\
\hline 71 & $31^{\circ} 27^{\prime} 56^{\prime \prime}$ & $100^{\circ} 26^{\prime} 49^{\prime \prime}$ & -- & Lipan & 0 & 1.0 & 77 & \\
\hline 72 & $31^{\circ} 53^{\prime} 18^{\prime \prime}$ & $100^{\circ} 04^{\prime} 51^{\prime \prime}$ & -- & -- & .2 & 2.0 & 0 & 2 mi above McIntyres Dam \\
\hline 72 & $31^{\circ} 52^{\prime} 14^{\prime \prime}$ & $100^{\circ} 04^{\prime} 12^{\prime \prime}$ & -- & -- & 1.0 & 1.5 & 2 & estimate \\
\hline 72 & $31^{\circ} 51^{\prime} 31^{\prime \prime}$ & $100^{\circ} 03^{\prime} 14^{\prime \prime}$ & -- & -- & .2 & 8.1 & 3.5 & \\
\hline 72 & $31^{\circ} 49^{\prime} 59^{\prime \prime}$ & $100^{\circ} 59^{\prime} 38^{\prime \prime}$ & -- & -- & 0 & .5 & 11.6 & \\
\hline 72 & $31^{\circ} 49^{\prime} 44^{\prime \prime}$ & $100^{\circ} 59^{\prime} 24^{\prime \prime}$ & -- & -- & -.4 & 11.4 & 12.1 & \\
\hline 72 & $31^{\circ} 46^{\prime} 03^{\prime \prime}$ & $100^{\circ} 52^{\prime} 22^{\prime \prime}$ & -- & -- & .9 & 3.4 & 23.5 & \\
\hline 72 & $31^{\circ} 44^{\prime} 33^{\prime \prime}$ & $100^{\circ} 50^{\prime} 20^{\prime \prime}$ & -- & -- & 1.3 & 4.4 & 26.9 & \\
\hline 72 & $31^{\circ} 39^{\prime} 53^{\prime \prime}$ & $100^{\circ} 45^{\prime} 03^{\prime \prime}$ & -- & -- & .7 & 5.7 & 31.3 & \\
\hline 72 & $31^{\circ} 35^{\prime} 33^{\prime \prime}$ & $100^{\circ} 38^{\prime} 09^{\prime \prime}$ & -- & -- & -.8 & 8.6 & 37 & \\
\hline 72 & $31^{\circ} 32^{\prime} 25^{\prime \prime}$ & $100^{\circ} 32^{\prime} 52^{\prime \prime}$ & -- & Lipan & -.6 & 8.8 & 45.6 & \\
\hline 72 & $31^{\circ} 27^{\prime} 57^{\prime \prime}$ & $100^{\circ} 26^{\prime} 49^{\prime \prime}$ & -- & Lipan & -.2 & .9 & 54.4 & gaging station (08133500) \\
\hline 73 & $31^{\circ} 49^{\prime} 41^{\prime \prime}$ & $100^{\circ} 58^{\prime} 37^{\prime \prime}$ & -- & -- & -.1 & 3.0 & 0 & at Sterling City \\
\hline 73 & $31^{\circ} 48^{\prime} 30^{\prime \prime}$ & $100^{\circ} 57^{\prime} 07^{\prime \prime}$ & -- & -- & -1.0 & 6.0 & 3 & estimate \\
\hline 73 & $31^{\circ} 46^{\prime} 17^{\prime \prime}$ & $100^{\circ} 53^{\prime} 20^{\prime \prime}$ & -- & -- & 1.3 & 11.0 & 9 & \\
\hline 73 & $31^{\circ} 39^{\prime} 37^{\prime \prime}$ & $100^{\circ} 43^{\prime} 04^{\prime \prime}$ & -- & -- & .7 & 7.0 & 20 & \\
\hline 73 & $31^{\circ} 35^{\prime} 57^{\prime \prime}$ & $100^{\circ} 39^{\prime} 03^{\prime \prime}$ & -- & -- & -2.0 & 3.0 & 27 & estimate \\
\hline 73 & $31^{\circ} 34^{\prime} 54^{\prime \prime}$ & $100^{\circ} 37^{\prime} 04^{\prime \prime}$ & -- & Lipan & 0 & 18.0 & 30 & \\
\hline 74 & $30^{\circ} 27^{\prime} 30^{\prime \prime}$ & $100^{\circ} 11^{\prime} 20^{\prime \prime}$ & Edwards-Trinity Plateau & -- & 12.7 & 2.1 & 0 & at road crossing $10.2 \mathrm{mi}$ above Roosevelt \\
\hline 74 & $30^{\circ} 28^{\prime} 33^{\prime \prime}$ & $100^{\circ} 09^{\prime} 50^{\prime \prime}$ & Edwards-Trinity Plateau & -- & 2.8 & 8.1 & 2.1 & \\
\hline 74 & $30^{\circ} 29^{\prime} 29^{\prime \prime}$ & $100^{\circ} 03^{\prime} 03^{\prime \prime}$ & Edwards-Trinity Plateau & -- & -.2 & 1.9 & 10.2 & \\
\hline
\end{tabular}


Table 4. Gains and losses from gain-loss studies in Texas-Continued

\begin{tabular}{|c|c|c|c|c|c|c|c|c|c|}
\hline $\begin{array}{l}\text { Stream- } \\
\text { flow } \\
\text { study } \\
\text { no. }\end{array}$ & $\begin{array}{l}\text { Latitude of } \\
\text { upstream } \\
\text { end of } \\
\text { subreach }\end{array}$ & $\begin{array}{l}\text { Longitude } \\
\text { of upstream } \\
\text { end of } \\
\text { subreach }\end{array}$ & $\begin{array}{c}\text { Major aquifer } \\
\text { outcrop } \\
\text { intersected } \\
\text { by subreach }\end{array}$ & & $\begin{array}{l}\text { Minor aquifer } \\
\text { outcrop } \\
\text { intercepted } \\
\text { by subreach }\end{array}$ & $\begin{array}{l}\text { Gain or } \\
\text { loss }(-) \text { in } \\
\text { subreach } \\
\left(\mathrm{ft}^{3} / \mathrm{s}\right)\end{array}$ & $\begin{array}{l}\text { Length } \\
\text { of subreach } \\
\text { (river mi) }\end{array}$ & $\begin{array}{l}\text { Location of } \\
\text { upstream } \\
\text { end of } \\
\text { subreach } \\
\text { (river mi) }\end{array}$ & $\begin{array}{l}\text { Descriptive location } \\
\text { of upstream end of } \\
\text { selected subreaches }\end{array}$ \\
\hline 74 & $30^{\circ} 29^{\prime} 00^{\prime \prime}$ & $100^{\circ} 01^{\prime} 51^{\prime \prime}$ & Edwards-Trinity Plateau & -- & & 0.2 & 3.9 & 12.1 & \\
\hline 74 & $30^{\circ} 29^{\prime} 23^{\prime \prime}$ & $99^{\circ} 58^{\prime} 39^{\prime \prime}$ & Edwards-Trinity Plateau & -- & & -.3 & .7 & 16 & \\
\hline 74 & $30^{\circ} 29^{\prime} 25^{\prime \prime}$ & $99^{\circ} 57^{\prime} 52^{\prime \prime}$ & Edwards-Trinity Plateau & -- & & 0 & 4.3 & 16.7 & \\
\hline 74 & $30^{\circ} 30^{\circ} 07^{\prime \prime}$ & $99^{\circ} 54^{\prime} 15^{\prime \prime}$ & Edwards-Trinity Plateau & -- & & .9 & 4.0 & 21 & \\
\hline 74 & $30^{\circ} 30^{\circ} 52^{\prime \prime}$ & $99^{\circ} 52^{\prime} 03^{\prime \prime}$ & Edwards-Trinity Plateau & -- & & -.5 & 2.3 & 25 & \\
\hline 74 & $30^{\circ} 31^{\prime} 05^{\prime \prime}$ & $99^{\circ} 48^{\prime} 42^{\prime \prime}$ & Edwards-Trinity Plateau & -- & & -8.7 & 4.5 & 27.3 & \\
\hline 75 & $30^{\circ} 09^{\prime} 38^{\prime \prime}$ & $98^{\circ} 05^{\prime} 27^{\prime \prime}$ & Trinity & -- & & 33.0 & 10.0 & 0 & at FM 12, estimate \\
\hline 75 & $30^{\circ} 07^{\prime} 39^{\prime \prime}$ & $98^{\circ} 00^{\prime} 36^{\prime \prime}$ & Trinity & -- & & 2.2 & 4.0 & 10 & \\
\hline 75 & $30^{\circ} 05^{\prime} 00^{\prime \prime}$ & $98^{\circ} 00^{\prime} 25^{\prime \prime}$ & Edwards, Trinity & -- & & 2.9 & 6.0 & 14 & \\
\hline 75 & $30^{\circ} 03^{\prime} 06^{\prime \prime}$ & $97^{\circ} 57^{\prime} 36^{\prime \prime}$ & Edwards & -- & & -6.7 & 5.6 & 20 & \\
\hline 75 & $30^{\circ} 04^{\prime} 11 "$ & $97^{\circ} 53^{\prime} 10^{\prime \prime}$ & Edwards & -- & & -3.0 & 3.4 & 25.6 & \\
\hline 75 & $30^{\circ} 05^{\prime} 07^{\prime \prime}$ & $97^{\circ} 50^{\prime} 53^{\prime \prime}$ & -- & -- & & 13.6 & 12.1 & 29 & \\
\hline 75 & $30^{\circ} 09^{\prime} 32^{\prime \prime}$ & $97^{\circ} 46^{\prime} 07^{\prime \prime}$ & -- & -- & & 14.5 & 14.0 & 41.1 & \\
\hline 75 & $30^{\circ} 11^{\prime} 19^{\prime \prime}$ & $97^{\circ} 37^{\prime} 09^{\prime \prime}$ & -- & -- & & -81.4 & 4.1 & 55.1 & \\
\hline 76 & $30^{\circ} 04^{\prime} 59^{\prime \prime}$ & $98^{\circ} 00^{\prime} 28^{\prime \prime}$ & Trinity & -- & & 7.6 & 1.8 & 46 & at FM 150 \\
\hline 76 & $30^{\circ} 05^{\prime} 15^{\prime \prime}$ & $97^{\circ} 59^{\prime} 06^{\prime \prime}$ & Edwards, Trinity & -- & & -5.8 & 1.5 & 44.2 & \\
\hline 76 & $30^{\circ} 04^{\prime} 37^{\prime \prime}$ & $97^{\circ} 58^{\prime} 43^{\prime \prime}$ & Edwards & -- & & -2.0 & 1.4 & 42.7 & \\
\hline 76 & $30^{\circ} 03^{\prime} 37^{\prime \prime}$ & 9758'39" & Edwards & -- & & -1.0 & 1.4 & 41.3 & \\
\hline 76 & $30^{\circ} 03^{\prime} 06^{\prime \prime}$ & $97^{\circ} 57^{\prime} 34^{\prime \prime}$ & Edwards & -- & & -34.5 & 1.4 & 39.9 & \\
\hline 76 & $30^{\circ} 03^{\prime} 00^{\prime \prime}$ & $97^{\circ} 56^{\prime} 15^{\prime \prime}$ & Edwards & -- & & -21.3 & 1.1 & 38.5 & \\
\hline 76 & $30^{\circ} 03^{\prime} 41^{\prime \prime}$ & $97^{\circ} 55^{\prime} 34^{\prime \prime}$ & Edwards & -- & & -35.7 & 3.4 & 37.4 & \\
\hline 76 & $30^{\circ} 04^{\prime} 12^{\prime \prime}$ & $97^{\circ} 53^{\prime} 09^{\prime \prime}$ & Edwards & -- & & .06 & 1.3 & 34 & \\
\hline 76 & $30^{\circ} 04^{\prime} 24^{\prime \prime}$ & $97^{\circ} 52^{\prime} 07^{\prime \prime}$ & -- & -- & & .97 & 1.2 & 32.7 & \\
\hline 76 & $30^{\circ} 04^{\prime} 35^{\prime \prime}$ & $97^{\circ} 51^{\prime} 05^{\prime \prime}$ & -- & -- & & .29 & .7 & 31.5 & \\
\hline 76 & $30^{\circ} 05^{\prime} 08^{\prime \prime}$ & $97^{\circ} 50^{\prime} 51^{\prime \prime}$ & -- & -- & & 3.5 & 7.1 & 30.8 & \\
\hline 76 & $30^{\circ} 08^{\prime} 06^{\prime \prime}$ & $97^{\circ} 47^{\prime} 51^{\prime \prime}$ & -- & -- & & 5.9 & 7.8 & 23.7 & \\
\hline 76 & $30^{\circ} 10^{\prime} 40^{\prime \prime}$ & $97^{\circ} 44^{\prime} 40^{\prime \prime}$ & -- & -- & & -.72 & 5.3 & 15.9 & \\
\hline 77 & $30^{\circ} 17^{\prime} 04^{\prime \prime}$ & $99^{\circ} 13^{\prime} 41^{\prime \prime}$ & Edwards-Trinity Plateau & -- & & .3 & .5 & 0 & 2 mi below Harper \\
\hline 77 & $30^{\circ} 16^{\prime} 43^{\prime \prime}$ & $99^{\circ} 13^{\prime} 24^{\prime \prime}$ & Edwards-Trinity Plateau, Trinity & -- & & 0 & 1.8 & .5 & \\
\hline 77 & $30^{\circ} 15^{\prime} 56^{\prime \prime}$ & $99^{\circ} 12^{\prime} 00^{\prime \prime}$ & Trinity & -- & & 0 & 1.7 & 2.3 & \\
\hline 77 & $30^{\circ} 15^{\prime} 54^{\prime \prime}$ & $99^{\circ} 10^{\prime} 28^{\prime \prime}$ & Trinity & -- & & -.05 & 1.7 & 4 & \\
\hline 77 & $30^{\circ} 14^{\prime} 59^{\prime \prime}$ & $99^{\circ} 10^{\prime} 00^{\prime \prime}$ & Trinity & -- & & -.55 & 2.6 & 5.7 & \\
\hline 77 & $30^{\circ} 14^{\prime} 29^{\prime \prime}$ & $99^{\circ} 07^{\prime} 59^{\prime \prime}$ & Trinity & -- & & .2 & .9 & 8.3 & \\
\hline 77 & $30^{\circ} 14^{\prime} 24^{\prime \prime}$ & $99^{\circ} 07^{\prime} 14^{\prime \prime}$ & Trinity & -- & & -.1 & 2.2 & 9.2 & \\
\hline 77 & $30^{\circ} 14^{\prime} 15^{\prime \prime}$ & $99^{\circ} 06^{\prime} 04^{\prime \prime}$ & Trinity & -- & & -.1 & 1.9 & 11.4 & \\
\hline 77 & $30^{\circ} 14^{\prime} 27^{\prime \prime}$ & $99^{\circ} 04^{\prime} 43^{\prime \prime}$ & Trinity & -- & & 0 & 1.4 & 13.3 & \\
\hline 77 & $30^{\circ} 13^{\prime} 54^{\prime \prime}$ & $99^{\circ} 03 ' 37^{\prime \prime}$ & Trinity & -- & & 0 & 1.8 & 14.7 & \\
\hline 77 & $30^{\circ} 13^{\prime} 24^{\prime \prime}$ & $99^{\circ} 02^{\prime} 18^{\prime \prime}$ & Trinity & -- & & .05 & 1.5 & 16.5 & \\
\hline
\end{tabular}


Table 4. Gains and losses from gain-loss studies in Texas-Continued

\begin{tabular}{|c|c|c|c|c|c|c|c|c|}
\hline $\begin{array}{l}\text { Stream- } \\
\text { flow } \\
\text { study } \\
\text { no. }\end{array}$ & $\begin{array}{l}\text { Latitude of } \\
\text { upstream } \\
\text { end of } \\
\text { subreach }\end{array}$ & $\begin{array}{l}\text { Longitude } \\
\text { of upstream } \\
\text { end of } \\
\text { subreach }\end{array}$ & $\begin{array}{l}\text { Major aquifer } \\
\text { outcrop } \\
\text { intersected } \\
\text { by subreach }\end{array}$ & $\begin{array}{c}\text { Minor aquifer } \\
\text { outcrop } \\
\text { intercepted } \\
\text { by subreach }\end{array}$ & $\begin{array}{l}\text { Gain or } \\
\text { loss }(-) \text { in } \\
\text { subreach } \\
\left(\mathrm{ft}^{3} / \mathrm{s}\right)\end{array}$ & $\begin{array}{l}\text { Length } \\
\text { of subreach } \\
\text { (river mi) }\end{array}$ & $\begin{array}{l}\text { Location of } \\
\text { upstream } \\
\text { end of } \\
\text { subreach } \\
\text { (river mi) }\end{array}$ & $\begin{array}{l}\text { Descriptive location } \\
\text { of upstream end of } \\
\text { selected subreaches }\end{array}$ \\
\hline 77 & $30^{\circ} 12^{\prime} 52^{\prime \prime}$ & $99^{\circ} 01^{\prime} 19^{\prime \prime}$ & Trinity & -- & 0.05 & 1.5 & 18 & \\
\hline 77 & $30^{\circ} 12^{\prime} 33^{\prime \prime}$ & $99^{\circ} 00^{\prime} 17^{\prime \prime}$ & Trinity & -- & .3 & 1.1 & 19.5 & \\
\hline 77 & $30^{\circ} 12^{\prime} 32^{\prime \prime}$ & $98^{\circ} 59^{\prime} 26^{\prime \prime}$ & Trinity & -- & .7 & .8 & 20.6 & \\
\hline 77 & $30^{\circ} 12^{\prime} 26^{\prime \prime}$ & $98^{\circ} 58^{\prime} 45^{\prime \prime}$ & Trinity & -- & -.1 & .1 & 21.4 & \\
\hline 77 & $30^{\circ} 12^{\prime} 21^{\prime \prime}$ & $98^{\circ} 58^{\prime} 41^{\prime \prime}$ & Trinity & -- & .2 & 2.0 & 21.5 & \\
\hline 77 & $30^{\circ} 12^{\prime} 29^{\prime \prime}$ & $98^{\circ} 57^{\prime} 00^{\prime \prime}$ & Trinity & -- & .08 & 1.6 & 23.5 & \\
\hline 77 & $30^{\circ} 13^{\prime} 28^{\prime \prime}$ & $98^{\circ} 55^{\prime} 49^{\prime \prime}$ & Trinity & -- & .29 & .9 & 25.1 & \\
\hline 77 & $30^{\circ} 13^{\prime} 14^{\prime \prime}$ & $98^{\circ} 55^{\prime} 09^{\prime \prime}$ & Trinity & -- & -.06 & 1.1 & 26 & \\
\hline 77 & $30^{\circ} 13^{\prime} 21^{\prime \prime}$ & $98^{\circ} 54^{\prime} 20^{\prime \prime}$ & Trinity & -- & .14 & 1.7 & 27.1 & \\
\hline 77 & $30^{\circ} 13^{\prime} 11^{\prime \prime}$ & $98^{\circ} 52^{\prime} 33^{\prime \prime}$ & Trinity & -- & -.24 & 2.2 & 28.8 & \\
\hline 77 & $30^{\circ} 13^{\prime} 23^{\prime \prime}$ & $98^{\circ} 51^{\prime} 18^{\prime \prime}$ & Trinity & -- & -.09 & 1.3 & 31 & \\
\hline 77 & $30^{\circ} 13^{\prime} 07^{\prime \prime}$ & $98^{\circ} 49^{\prime} 45^{\prime \prime}$ & Trinity & -- & -.06 & 2.1 & 32.3 & \\
\hline 77 & $30^{\circ} 13^{\prime} 28^{\prime \prime}$ & $98^{\circ} 49^{\prime} 04^{\prime \prime}$ & Trinity & -- & .41 & 2.2 & 34.4 & \\
\hline 77 & $30^{\circ} 13^{\prime} 28^{\prime \prime}$ & $98^{\circ} 49^{\prime} 04^{\prime \prime}$ & Trinity & -- & .05 & 1.8 & 36.6 & \\
\hline 77 & $30^{\circ} 13^{\prime} 52^{\prime \prime}$ & $98^{\circ} 48^{\prime} 24^{\prime \prime}$ & Trinity & -- & 1.6 & 1.6 & 38.4 & \\
\hline 77 & $30^{\circ} 13^{\prime} 46^{\prime \prime}$ & $98^{\circ} 44^{\prime} 57^{\prime \prime}$ & Trinity & -- & 1.16 & 1.5 & 40 & \\
\hline 77 & $30^{\circ} 13^{\prime} 48^{\prime \prime}$ & $98^{\circ} 43^{\prime} 56^{\prime \prime}$ & Trinity & -- & -.05 & 3.1 & 41.5 & \\
\hline 77 & $30^{\circ} 13^{\prime} 43^{\prime \prime}$ & $98^{\circ} 42^{\prime} 09^{\prime \prime}$ & Trinity & -- & -.27 & 2.1 & 44.6 & \\
\hline 77 & $30^{\circ} 13^{\prime} 55^{\prime \prime}$ & $98^{\circ} 41^{\prime} 16^{\prime \prime}$ & Trinity & -- & 1.13 & 2.1 & 46.7 & \\
\hline 77 & $30^{\circ} 14^{\prime} 31^{\prime \prime}$ & $98^{\circ} 39^{\prime} 17^{\prime \prime}$ & Trinity & -- & -.86 & 1.2 & 48.8 & \\
\hline 77 & $30^{\circ} 14^{\prime} 16^{\prime \prime}$ & $98^{\circ} 37^{\prime} 13^{\prime \prime}$ & Trinity & -- & 1.4 & 2.2 & 50 & \\
\hline 77 & $30^{\circ} 15^{\prime} 43^{\prime \prime}$ & $98^{\circ} 34^{\prime} 32^{\prime \prime}$ & -- & -- & -.12 & 4.3 & 52.2 & \\
\hline 77 & $30^{\circ} 16^{\prime} 19^{\prime \prime}$ & $98^{\circ} 32^{\prime} 58^{\prime \prime}$ & -- & -- & -.45 & 1.7 & 56.5 & \\
\hline 77 & $30^{\circ} 16^{\prime} 06^{\prime \prime}$ & $98^{\circ} 31^{\prime} 34^{\prime \prime}$ & -- & -- & -.32 & 1.6 & 58.2 & \\
\hline 77 & $30^{\circ} 16^{\prime} 10^{\prime \prime}$ & $98^{\circ} 31^{\prime} 13^{\prime \prime}$ & -- & -- & -.03 & 2.2 & 59.8 & \\
\hline 77 & $30^{\circ} 18^{\prime} 11^{\prime \prime}$ & $98^{\circ} 30^{\prime} 24^{\prime \prime}$ & -- & -- & .51 & 1.3 & 62 & \\
\hline 77 & $30^{\circ} 19^{\prime} 10^{\prime \prime}$ & $98^{\circ} 29^{\prime} 20^{\prime \prime}$ & -- & Ellenburger-San Saba & -.36 & 6.7 & 63.3 & \\
\hline 78 & $30^{\circ} 17^{\prime} 04^{\prime \prime}$ & $99^{\circ} 13^{\prime} 41^{\prime \prime}$ & Edwards-Trinity Plateau & -- & .5 & .5 & 0 & 2 mi below Harper \\
\hline 78 & $30^{\circ} 16^{\prime} 43^{\prime \prime}$ & $99^{\circ} 13^{\prime} 23^{\prime \prime}$ & Edwards-Trinity Plateau, Trinity & -- & .62 & 1.9 & .5 & \\
\hline 78 & $30^{\circ} 15^{\prime} 56^{\prime \prime}$ & $99^{\circ} 12^{\prime} 00^{\prime \prime}$ & Trinity & -- & -.28 & 2.8 & 2.4 & \\
\hline 78 & $30^{\circ} 15^{\prime} 27^{\prime \prime}$ & $99^{\circ} 10^{\prime} 06^{\prime \prime}$ & Trinity & -- & .61 & 3.1 & 5.2 & \\
\hline 78 & $30^{\circ} 14^{\prime} 29^{\prime \prime}$ & $99^{\circ} 07^{\prime} 59^{\prime \prime}$ & Trinity & -- & -.22 & 2.1 & 8.3 & \\
\hline 78 & $30^{\circ} 14^{\prime} 48^{\prime \prime}$ & $99^{\circ} 06^{\prime} 25^{\prime \prime}$ & Trinity & -- & -.18 & 3.9 & 10.4 & \\
\hline 78 & $30^{\circ} 14^{\prime} 05^{\prime \prime}$ & $99^{\circ} 03^{\prime} 52^{\prime \prime}$ & Trinity & -- & -.59 & 3.7 & 14.3 & \\
\hline 79 & $30^{\circ} 14^{\prime} 05^{\prime \prime}$ & $99^{\circ} 03^{\prime} 52^{\prime \prime}$ & Trinity & -- & .36 & 3.7 & 14.3 & county road crossing \\
\hline 79 & $30^{\circ} 14^{\prime} 05^{\prime \prime}$ & $99^{\circ} 03^{\prime} 52^{\prime \prime}$ & Trinity & -- & -.37 & 2.6 & 18 & \\
\hline 79 & $30^{\circ} 12^{\prime} 32^{\prime \prime}$ & $98^{\circ} 59^{\prime} 27^{\prime \prime}$ & Trinity & -- & .39 & 2.4 & 20.6 & $1 \mathrm{mi}$ above SH 16 \\
\hline 79 & $30^{\circ} 12^{\prime} 28^{\prime \prime}$ & $98^{\circ} 57^{\prime} 22^{\prime \prime}$ & Trinity & -- & 1.23 & 4.7 & 23 & \\
\hline
\end{tabular}


Table 4. Gains and losses from gain-loss studies in Texas-Continued

\begin{tabular}{|c|c|c|c|c|c|c|c|c|}
\hline $\begin{array}{l}\text { Stream- } \\
\text { flow } \\
\text { study } \\
\text { no. }\end{array}$ & $\begin{array}{l}\text { Latitude of } \\
\text { upstream } \\
\text { end of } \\
\text { subreach }\end{array}$ & $\begin{array}{l}\text { Longitude } \\
\text { of upstream } \\
\text { end of } \\
\text { subreach }\end{array}$ & $\begin{array}{c}\text { Major aquifer } \\
\text { outcrop } \\
\text { intersected } \\
\text { by subreach }\end{array}$ & $\begin{array}{l}\text { Minor aquifer } \\
\text { outcrop } \\
\text { intercepted } \\
\text { by subreach }\end{array}$ & $\begin{array}{c}\text { Gain or } \\
\text { loss }(-) \text { in } \\
\text { subreach } \\
\left(\mathrm{ft}^{3} / \mathrm{s}\right)\end{array}$ & $\begin{array}{l}\text { Length } \\
\text { of subreach } \\
\text { (river mi) }\end{array}$ & $\begin{array}{l}\text { Location of } \\
\text { upstream } \\
\text { end of } \\
\text { subreach } \\
\text { (river mi) }\end{array}$ & $\begin{array}{l}\text { Descriptive location } \\
\text { of upstream end of } \\
\text { selected subreaches }\end{array}$ \\
\hline 79 & $30^{\circ} 13^{\prime} 07^{\prime \prime}$ & $98^{\circ} 53^{\prime} 47^{\prime \prime}$ & Trinity & -- & 0.12 & 3.7 & 27.7 & \\
\hline 79 & $30^{\circ} 13^{\prime} 24^{\prime \prime}$ & $98^{\circ} 50^{\prime} 26^{\prime \prime}$ & Trinity & -- & -.51 & 3.0 & 31.4 & \\
\hline 79 & $30^{\circ} 13^{\prime} 37^{\prime \prime}$ & $98^{\circ} 49^{\prime} 05^{\prime \prime}$ & Trinity & -- & 1.52 & 4.0 & 34.4 & US 290 \\
\hline 79 & $30^{\circ} 14^{\prime} 17^{\prime \prime}$ & $98^{\circ} 47^{\prime} 01^{\prime \prime}$ & Trinity & -- & .72 & 4.7 & 38.4 & \\
\hline 79 & $30^{\circ} 14^{\prime} 04^{\prime \prime}$ & $98^{\circ} 45^{\prime} 21^{\prime \prime}$ & Trinity & -- & 2.68 & 2.6 & 43.1 & \\
\hline 79 & $30^{\circ} 14^{\prime} 03^{\prime \prime}$ & $98^{\circ} 41^{\prime 2} 29^{\prime \prime}$ & Trinity & -- & 2.3 & 3.7 & 45.7 & \\
\hline 79 & $30^{\circ} 14^{\prime} 22^{\prime \prime}$ & $98^{\circ} 39^{\prime} 07^{\prime \prime}$ & Trinity & -- & -.9 & 3.8 & 49.4 & \\
\hline 79 & $30^{\circ} 14^{\prime} 42^{\prime \prime}$ & $98^{\circ} 35^{\prime} 43^{\prime \prime}$ & Trinity & -- & .7 & 3.8 & 53.2 & \\
\hline 79 & $30^{\circ} 16^{\prime} 19^{\prime \prime}$ & $98^{\circ} 32^{\prime} 42^{\prime \prime}$ & -- & -- & -1.17 & 3.0 & 57 & FM 1320 \\
\hline 79 & $30^{\circ} 17^{\prime} 05^{\prime \prime}$ & $98^{\circ} 30^{\prime} 04^{\prime \prime}$ & -- & -- & .42 & 3.1 & 60 & \\
\hline 79 & $30^{\circ} 19^{\prime} 13^{\prime \prime}$ & $98^{\circ} 29^{\prime} 25^{\prime \prime}$ & -- & -- & -1.4 & 2.1 & 63.1 & \\
\hline 79 & $30^{\circ} 19^{\prime} 24^{\prime \prime}$ & $98^{\circ} 27^{\prime} 52^{\prime \prime}$ & -- & Ellenburger-San Saba & .3 & 2.6 & 65.2 & \\
\hline 79 & $30^{\circ} 18^{\prime} 16^{\prime \prime}$ & $98^{\circ} 25^{\prime} 18^{\prime \prime}$ & -- & Ellenburger-San Saba & -1.87 & 2.2 & 67.8 & \\
\hline 79 & $30^{\circ} 17^{\prime} 27^{\prime \prime}$ & $98^{\circ} 24^{\prime} 00^{\prime \prime}$ & Trinity & Ellenburger-San Saba & .3 & 3.7 & 70 & gaging station (08153500) \\
\hline 79 & $30^{\circ} 17^{\prime} 54^{\prime \prime}$ & $98^{\circ} 21^{\prime} 46^{\prime \prime}$ & Trinity & Ellenburger-San Saba & 1.3 & 9.4 & 73.7 & \\
\hline 79 & $30^{\circ} 18^{\prime} 10^{\prime \prime}$ & $98^{\circ} 18^{\prime} 34^{\prime \prime}$ & Trinity & $\begin{array}{l}\text { Ellenburger-San Saba, Marble } \\
\text { Falls }\end{array}$ & 4.15 & 7.6 & 83.1 & \\
\hline 79 & $30^{\circ} 18^{\prime} 42^{\prime \prime}$ & $98^{\circ} 14^{\prime} 13^{\prime \prime}$ & Trinity & -- & 1.2 & 5.8 & 90.7 & \\
\hline 79 & $30^{\circ} 20^{\prime} 12^{\prime \prime}$ & $98^{\circ} 11^{\prime} 45^{\prime \prime}$ & Trinity & -- & .84 & 6.5 & 96.5 & \\
\hline 80 & $30^{\circ} 49^{\prime} 57^{\prime \prime}$ & $100^{\circ} 06^{\prime} 50^{\prime \prime}$ & Edwards-Trinity Plateau & -- & 2.5 & 1.0 & 0 & $0.5 \mathrm{mi}$ above Fort McKavett \\
\hline 80 & $30^{\circ} 50^{\prime} 03^{\prime \prime}$ & $100^{\circ} 05^{\prime} 34^{\prime \prime}$ & Edwards-Trinity Plateau & -- & .5 & 7.0 & 1 & \\
\hline 80 & $30^{\circ} 52^{\prime} 13^{\prime \prime}$ & $100^{\circ} 00^{\prime} 10^{\prime \prime}$ & Edwards-Trinity Plateau & -- & -.3 & 5.5 & 8 & \\
\hline 80 & $30^{\circ} 54^{\prime} 00^{\prime \prime}$ & $99^{\circ} 54^{\prime} 43^{\prime \prime}$ & Edwards-Trinity Plateau & -- & -.7 & 3.5 & 13.5 & \\
\hline 80 & $30^{\circ} 54^{\prime} 27^{\prime \prime}$ & $99^{\circ} 52^{\prime} 03^{\prime \prime}$ & Edwards-Trinity Plateau & -- & -1.6 & 4.0 & 17 & \\
\hline 80 & $30^{\circ} 55^{\prime} 07^{\prime \prime}$ & $99^{\circ} 47^{\prime} 06^{\prime \prime}$ & Edwards-Trinity Plateau & -- & 1.1 & 5.0 & 21 & \\
\hline 80 & $30^{\circ} 55^{\prime} 12^{\prime \prime}$ & $99^{\circ} 43^{\prime} 20^{\prime \prime}$ & Edwards-Trinity Plateau & -- & -4.0 & 5.0 & 26 & \\
\hline 80 & $30^{\circ} 533^{\prime} 24^{\prime \prime}$ & $99^{\circ} 41^{\prime 22 "}$ & Edwards-Trinity Plateau & -- & -2.6 & 4.0 & 31 & \\
\hline 80 & $30^{\circ} 52^{\prime} 35^{\prime \prime}$ & $99^{\circ} 39^{\prime} 11^{\prime \prime}$ & Edwards-Trinity Plateau & -- & -3.1 & 6.0 & 35 & \\
\hline 80 & $30^{\circ} 52^{\prime} 47^{\prime \prime}$ & $99^{\circ} 35^{\prime} 31^{\prime \prime}$ & -- & Ellenburger-San Saba & -.8 & 9.0 & 41 & \\
\hline 80 & $30^{\circ} 55^{\prime} 01^{\prime \prime}$ & $99^{\circ} 27^{\prime} 20^{\prime \prime}$ & -- & Ellenburger-San Saba & .1 & 10.0 & 50 & \\
\hline 80 & $31^{\circ} 00^{\prime} 06^{\prime \prime}$ & $99^{\circ} 15^{\prime} 13^{\prime \prime}$ & -- & -- & .6 & 4.0 & 60 & \\
\hline 80 & $31^{\circ} 00^{\prime} 53^{\prime \prime}$ & $99^{\circ} 11^{\prime} 54^{\prime \prime}$ & -- & Hickory & -.1 & 5.0 & 64 & \\
\hline 80 & $31^{\circ} 02^{\prime} 01^{\prime \prime}$ & $99^{\circ} 08^{\prime} 11^{\prime \prime}$ & -- & Ellenburger-San Saba, Hickory & -.9 & 3.0 & 69 & \\
\hline 80 & $31^{\circ} 03^{\prime} 27^{\prime \prime}$ & $99^{\circ} 05^{\prime} 10^{\prime \prime}$ & -- & Ellenburger-San Saba & -1.3 & 1.0 & 72 & \\
\hline 80 & $31^{\circ} 02^{\prime} 56^{\prime \prime}$ & $99^{\circ} 03^{\prime} 55^{\prime \prime}$ & -- & $\begin{array}{l}\text { Ellenburger-San Saba, Marble } \\
\text { Falls }\end{array}$ & 18.3 & 7.0 & 73 & \\
\hline 80 & $31^{\circ} 07^{\prime} 38^{\prime \prime}$ & $98^{\circ} 59^{\prime} 13^{\prime \prime}$ & -- & Marble Falls & 0 & .1 & 80 & \\
\hline 81 & $30^{\circ} 50^{\prime} 03^{\prime \prime}$ & $100^{\circ} 05^{\prime} 34^{\prime \prime}$ & Edwards-Trinity Plateau & -- & -3.0 & 5.7 & 1 & at first McKavett - Menard crossing \\
\hline
\end{tabular}


Table 4. Gains and losses from gain-loss studies in Texas-Continued

\begin{tabular}{|c|c|c|c|c|c|c|c|c|}
\hline $\begin{array}{l}\text { Stream- } \\
\text { flow } \\
\text { study } \\
\text { no. }\end{array}$ & $\begin{array}{l}\text { Latitude of } \\
\text { upstream } \\
\text { end of } \\
\text { subreach }\end{array}$ & $\begin{array}{l}\text { Longitude } \\
\text { of upstream } \\
\text { end of } \\
\text { subreach }\end{array}$ & $\begin{array}{c}\text { Major aquifer } \\
\text { outcrop } \\
\text { intersected } \\
\text { by subreach }\end{array}$ & $\begin{array}{c}\text { Minor aquifer } \\
\text { outcrop } \\
\text { intercepted } \\
\text { by subreach }\end{array}$ & $\begin{array}{l}\text { Gain or } \\
\text { loss }(-) \text { in } \\
\text { subreach } \\
\left(\mathrm{ft}^{3} / \mathrm{s}\right)\end{array}$ & $\begin{array}{l}\text { Length } \\
\text { of subreach } \\
\text { (river mi) }\end{array}$ & $\begin{array}{l}\text { Location of } \\
\text { upstream } \\
\text { end of } \\
\text { subreach } \\
\text { (river mi) }\end{array}$ & $\begin{array}{l}\text { Descriptive location } \\
\text { of upstream end of } \\
\text { selected subreaches }\end{array}$ \\
\hline 81 & $30^{\circ} 52^{\prime} 02^{\prime \prime}$ & $100^{\circ} 01^{\prime} 15^{\prime \prime}$ & Edwards-Trinity Plateau & -- & 4.6 & 6.0 & 6.7 & \\
\hline 81 & $30^{\circ} 54^{\prime} 04^{\prime \prime}$ & $99^{\circ} 55^{\prime} 02^{\prime \prime}$ & Edwards-Trinity Plateau & -- & -.2 & 2.7 & 12.7 & \\
\hline 81 & $30^{\circ} 54^{\prime} 37^{\prime \prime}$ & $99^{\circ} 51^{\prime} 34^{\prime \prime}$ & Edwards-Trinity Plateau & -- & -1.8 & 1.6 & 15.4 & \\
\hline 81 & $30^{\circ} 55^{\prime} 27^{\prime \prime}$ & $99^{\circ} 50^{\prime} 43^{\prime \prime}$ & Edwards-Trinity Plateau & -- & 4.4 & 4.9 & 17 & \\
\hline 82 & $31^{\circ} 12^{\prime} 06^{\prime \prime}$ & $98^{\circ} 52^{\prime} 52^{\prime \prime}$ & -- & -- & -.9 & 14.0 & 0 & damsite at Dorans Ranch $20 \mathrm{mi}$ above San Saba \\
\hline 83 & $30^{\circ} 50^{\prime} 10^{\prime \prime}$ & $100^{\circ} 04^{\prime} 55^{\prime \prime}$ & Edwards-Trinity Plateau & -- & 5.7 & 13.8 & 0 & 1 mi below Fort McKavett Springs \\
\hline 83 & $30^{\circ} 53^{\prime} 56^{\prime \prime}$ & $99^{\circ} 54^{\prime} 36^{\prime \prime}$ & Edwards-Trinity Plateau & -- & 2.0 & 7.2 & 13.8 & \\
\hline 83 & $30^{\circ} 55^{\prime} 07^{\prime \prime}$ & $99^{\circ} 47^{\prime} 06^{\prime \prime}$ & Edwards-Trinity Plateau & -- & 16.0 & 20.0 & 21 & \\
\hline 83 & $30^{\circ} 52^{\prime} 47^{\prime \prime}$ & $99^{\circ} 35^{\prime} 31^{\prime \prime}$ & -- & Ellenburger-San Saba & -2.9 & 15.0 & 41 & \\
\hline 83 & $30^{\circ} 55^{\prime} 13^{\prime \prime}$ & $99^{\circ} 26^{\prime} 49^{\prime \prime}$ & -- & $\begin{array}{l}\text { Ellenburger-San Saba, Marble } \\
\text { Falls }\end{array}$ & 6.9 & 20.0 & 56 & \\
\hline 83 & $31^{\circ} 07^{\prime} 38^{\prime \prime}$ & $98^{\circ} 59^{\prime} 13^{\prime \prime}$ & -- & Marble Falls & -2.6 & 5.0 & 76 & \\
\hline 83 & $31^{\circ} 09^{\prime} 22^{\prime \prime}$ & $98^{\circ} 55^{\prime} 26^{\prime \prime}$ & -- & Marble Falls & 1.0 & 14.0 & 81 & \\
\hline 83 & $31^{\circ} 12^{\prime} 46^{\prime \prime}$ & $98^{\circ} 43^{\prime} 05^{\prime \prime}$ & -- & -- & 2.0 & 2.6 & 95 & \\
\hline 83 & $31^{\circ} 12^{\prime} 05^{\prime \prime}$ & $98^{\circ} 42^{\prime} 44^{\prime \prime}$ & -- & -- & 0 & 1.2 & 97.6 & \\
\hline 83 & $31^{\circ} 12^{\prime} 10^{\prime \prime}$ & $98^{\circ} 41^{\prime} 44^{\prime \prime}$ & -- & -- & -30.6 & 6.2 & 98.8 & \\
\hline 84 & $30^{\circ} 12^{\prime} 32^{\prime \prime}$ & $97^{\circ} 54^{\prime} 10^{\prime \prime}$ & Edwards, Trinity & -- & -47.3 & 5.2 & 12.9 & at FM 1826 \\
\hline 84 & $30^{\circ} 10^{\prime} 08^{\prime \prime}$ & $97^{\circ} 51^{\prime} 32^{\prime \prime}$ & Edwards & -- & -3.9 & .9 & 7.7 & \\
\hline 84 & $30^{\circ} 10^{\prime} 03^{\prime \prime}$ & $97^{\circ} 50^{\prime} 51^{\prime \prime}$ & -- & -- & -.77 & .3 & 6.8 & \\
\hline 84 & $30^{\circ} 09^{\prime} 48^{\prime \prime}$ & $97^{\circ} 50^{\prime} 40^{\prime \prime}$ & -- & -- & -2.73 & .2 & 6.5 & \\
\hline 84 & $30^{\circ} 09^{\prime} 42^{\prime \prime}$ & 9750'33" & -- & -- & 0 & .8 & 6.3 & \\
\hline 85 & $30^{\circ} 12^{\prime} 32^{\prime \prime}$ & $97^{\circ} 54^{\prime} 10^{\prime \prime}$ & Edwards, Trinity & -- & -1.7 & 1.5 & 12.9 & at FM 1826 \\
\hline 85 & $30^{\circ} 11^{\prime} 53^{\prime \prime}$ & $97^{\circ} 52^{\prime} 53^{\prime \prime}$ & Edwards & -- & -5.56 & 1.3 & 11.4 & \\
\hline 85 & $30^{\circ} 11^{\prime} 34^{\prime \prime}$ & $97^{\circ} 51^{\prime} 55^{\prime \prime}$ & Edwards & -- & -2.18 & .2 & 10.1 & \\
\hline 85 & $30^{\circ} 11^{\prime} 24^{\prime \prime}$ & $97^{\circ} 51^{\prime} 54^{\prime \prime}$ & Edwards & -- & -2.36 & .2 & 9.9 & \\
\hline 85 & $30^{\circ} 11^{\prime} 24^{\prime \prime}$ & $97^{\circ} 51^{\prime} 43^{\prime \prime}$ & Edwards & -- & 0 & 1.2 & 9.7 & \\
\hline 85 & $30^{\circ} 10^{\prime} 32^{\prime \prime}$ & $97^{\circ} 51^{\prime} 29^{\prime \prime}$ & Edwards & -- & 0 & .8 & 8.5 & \\
\hline 85 & $30^{\circ} 10^{\prime} 08^{\prime \prime}$ & $97^{\circ} 51^{\prime} 32^{\prime \prime}$ & Edwards & -- & 0 & .9 & 7.7 & \\
\hline 85 & $30^{\circ} 10^{\prime} 03^{\prime \prime}$ & $97^{\circ} 50^{\prime} 51^{\prime \prime}$ & -- & -- & .02 & 1.3 & 6.8 & \\
\hline 85 & $30^{\circ} 09^{\prime} 42^{\prime \prime}$ & $97^{\circ} 49^{\prime} 54^{\prime \prime}$ & -- & -- & 1.33 & 1.4 & 5.5 & \\
\hline 86 & $31^{\circ} 07^{\prime} 38^{\prime \prime}$ & $100^{\circ} 29^{\prime} 49^{\prime \prime}$ & Edwards-Trinity Plateau & -- & 16.5 & 5.0 & 0 & $\begin{array}{l}\text { at Main Springs - } 5 \mathrm{mi} \text { above Christoval, site now } \\
\text { inundated by Lake Nasworthy }\end{array}$ \\
\hline 86 & $31^{\circ} 11^{\prime} 13^{\prime \prime}$ & $100^{\circ} 29^{\prime} 56^{\prime \prime}$ & Edwards-Trinity Plateau & -- & 4.6 & 9.4 & 5 & site now inundated by Lake Nasworthy \\
\hline 86 & $31^{\circ} 20^{\prime} 21^{\prime \prime}$ & $100^{\circ} 28^{\prime} 37^{\prime \prime}$ & -- & Lipan & -1.3 & 6.4 & 14.4 & site now inundated by Lake Nasworthy \\
\hline 86 & $31^{\circ} 23^{\prime} 59^{\prime \prime}$ & $100^{\circ} 26^{\prime} 34^{\prime \prime}$ & -- & Lipan & -.2 & 4.0 & 20.8 & site now inundated by Lake Nasworthy \\
\hline 87 & $31^{\circ} 20^{\prime} 12^{\prime \prime}$ & $100^{\circ} 28^{\prime} 48^{\prime \prime}$ & -- & -- & 2.2 & 1.0 & 11.8 & $\begin{array}{l}\text { just above Middle Concho River, site now } \\
\text { inundated by Lake Nasworthy }\end{array}$ \\
\hline
\end{tabular}


Table 4. Gains and losses from gain-loss studies in Texas-Continued

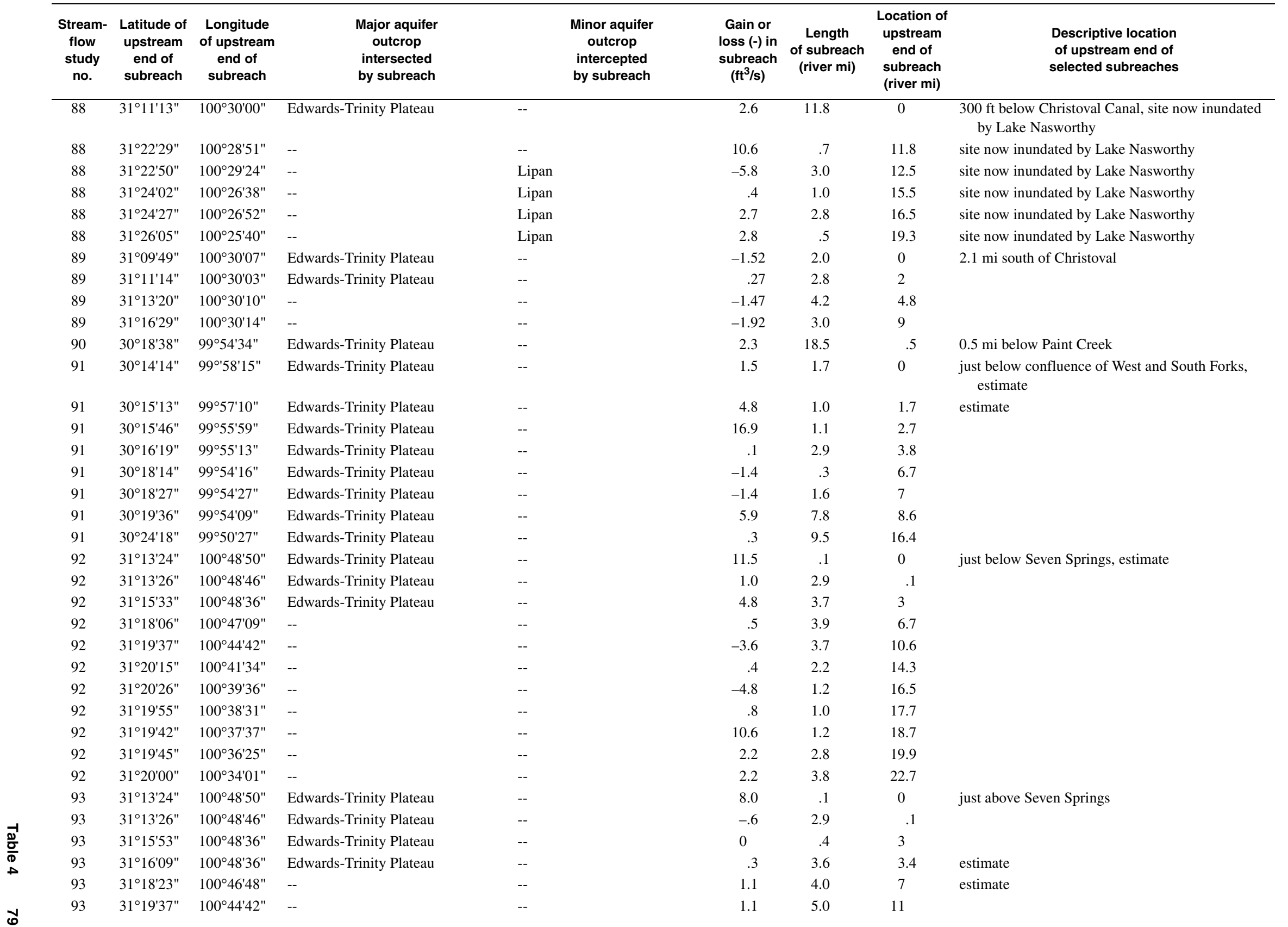


: Table 4. Gains and losses from gain-loss studies in Texas-Continued

\begin{tabular}{|c|c|c|c|c|c|c|c|c|c|}
\hline \multirow[t]{2}{*}{$\begin{array}{c}\text { Stream- } \\
\text { flow } \\
\text { study } \\
\text { no. }\end{array}$} & \multirow{2}{*}{$\begin{array}{c}\begin{array}{c}\text { Latitude of } \\
\text { upstream } \\
\text { end of } \\
\text { subreach }\end{array} \\
31^{\circ} 20^{\prime} 16^{\prime \prime}\end{array}$} & \multirow{2}{*}{$\begin{array}{c}\begin{array}{c}\text { Longitude } \\
\text { of upstream } \\
\text { end of } \\
\text { subreach }\end{array} \\
100^{\circ} 40^{\prime} 37^{\prime \prime}\end{array}$} & \multicolumn{2}{|l|}{$\begin{array}{l}\text { Major aquifer } \\
\text { outcrop } \\
\text { intersected } \\
\text { by subreach }\end{array}$} & \multirow[t]{2}{*}{$\begin{array}{c}\text { Minor aquifer } \\
\text { outcrop } \\
\text { intercepted } \\
\text { by subreach }\end{array}$} & \multirow{2}{*}{$\begin{array}{c}\begin{array}{c}\text { Gain or } \\
\text { loss (-) in } \\
\text { subreach } \\
\left(\mathrm{ft}^{3} / \mathbf{s}\right)\end{array} \\
0.2\end{array}$} & \multirow{2}{*}{$\begin{array}{c}\begin{array}{c}\text { Length } \\
\text { of subreach } \\
\text { (river mi) }\end{array} \\
4.0\end{array}$} & \multirow{2}{*}{$\begin{array}{c}\begin{array}{c}\text { Location of } \\
\text { upstream } \\
\text { end of } \\
\text { subreach } \\
\text { (river mi) }\end{array} \\
16\end{array}$} & \multirow[t]{2}{*}{$\begin{array}{l}\text { Descriptive location } \\
\text { of upstream end of } \\
\text { selected subreaches }\end{array}$} \\
\hline & & & -- & -- & & & & & \\
\hline 93 & $31^{\circ} 19^{\prime} 51^{\prime \prime}$ & $100^{\circ} 36^{\prime} 03^{\prime \prime}$ & -- & -- & & .4 & 5.0 & 20 & \\
\hline 93 & $31^{\circ} 19^{\prime} 52^{\prime \prime}$ & $100^{\circ} 34^{\prime} 30^{\prime \prime}$ & -- & -- & & 0 & 2.0 & 25 & estimate \\
\hline 94 & $30^{\circ} 14^{\prime} 06^{\prime \prime}$ & $97^{\circ} 51^{\prime} 36^{\prime \prime}$ & -- & -- & & -8.2 & 3.7 & 14 & 0.8 mi east of intersection of US 290 and SH 71 \\
\hline 94 & $30^{\circ} 13^{\prime} 10^{\prime \prime}$ & $97^{\circ} 48^{\prime} 47^{\prime \prime}$ & -- & -- & & -3.5 & .5 & 10.3 & \\
\hline 94 & $30^{\circ} 13^{\prime} 17^{\prime \prime}$ & $97^{\circ} 48^{\prime} 18^{\prime \prime}$ & -- & -- & & -.9 & .4 & 9.8 & \\
\hline 94 & $30^{\circ} 13^{\prime} 23^{\prime \prime}$ & $97^{\circ} 47^{\prime} 52^{\prime \prime}$ & -- & -- & & .37 & .8 & 9.4 & \\
\hline 95 & $30^{\circ} 14^{\prime} 12^{\prime \prime}$ & $97^{\circ} 52^{\prime} 25^{\prime \prime}$ & Trinity & -- & & 3.35 & .8 & 14.8 & 1,300 ft above Old Oak Hill - Bee Cave Rd \\
\hline 95 & $30^{\circ} 14^{\prime} 06^{\prime \prime}$ & $97^{\circ} 51^{\prime} 36^{\prime \prime}$ & Edwards & -- & & -4.07 & .5 & 14 & \\
\hline 95 & $30^{\circ} 13^{\prime} 45^{\prime \prime}$ & $97^{\circ} 51^{\prime} 11^{\prime \prime}$ & Edwards & -- & & -1.27 & .9 & 13.5 & \\
\hline 95 & $30^{\circ} 13^{\prime} 30^{\prime \prime}$ & $97^{\circ} 50^{\prime} 35^{\prime \prime}$ & Edwards & -- & & -3.6 & .8 & 12.6 & \\
\hline 95 & $30^{\circ} 13^{\prime} 26^{\prime \prime}$ & $97^{\circ} 49^{\prime} 59^{\prime \prime}$ & Edwards & -- & & -.49 & .6 & 11.8 & \\
\hline 95 & $30^{\circ} 13^{\prime} 22^{\prime \prime}$ & $97^{\circ} 49^{\prime} 26^{\prime \prime}$ & Edwards & -- & & -1.87 & 1.0 & 11.2 & \\
\hline 95 & $30^{\circ} 12^{\prime} 55^{\prime \prime}$ & $97^{\circ} 48^{\prime} 58^{\prime \prime}$ & -- & -- & & .83 & .1 & 10.2 & \\
\hline 95 & $30^{\circ} 13^{\prime} 10^{\prime \prime}$ & $97^{\circ} 48^{\prime} 47^{\prime \prime}$ & -- & -- & & -.83 & .5 & 10.3 & \\
\hline 95 & $30^{\circ} 13^{\prime} 17^{\prime \prime}$ & $97^{\circ} 48^{\prime} 18^{\prime \prime}$ & -- & -- & & 6.4 & .4 & 9.8 & \\
\hline 95 & $30^{\circ} 13^{\prime} 23^{\prime \prime}$ & $97^{\circ} 47^{\prime} 52^{\prime \prime}$ & -- & -- & & -5.78 & .8 & 9.4 & \\
\hline 95 & $30^{\circ} 13^{\prime} 15^{\prime \prime}$ & $97^{\circ} 47^{\prime} 35^{\prime \prime}$ & -- & -- & & -.13 & .5 & 8.6 & \\
\hline 95 & $30^{\circ} 12^{\prime} 59^{\prime \prime}$ & $97^{\circ} 47^{\prime} 18^{\prime \prime}$ & -- & -- & & -.15 & .7 & 8.1 & \\
\hline 95 & $30^{\circ} 12^{\prime} 42^{\prime \prime}$ & $97^{\circ} 46^{\prime} 44^{\prime \prime}$ & -- & -- & & 2.73 & 6.1 & 7.4 & \\
\hline 96 & $30^{\circ} 10^{\prime} 26^{\prime \prime}$ & $99^{\circ} 00^{\prime} 41^{\prime \prime}$ & Trinity & -- & & .37 & 2.3 & 12.1 & $12.1 \mathrm{mi}$ above mouth \\
\hline 96 & $30^{\circ} 10^{\prime} 40^{\prime \prime}$ & $99^{\circ} 00^{\prime} 11^{\prime \prime}$ & Trinity & -- & & -.01 & 1.5 & 9.8 & \\
\hline 96 & $30^{\circ} 11^{\prime} 02^{\prime \prime}$ & $98^{\circ} 59^{\prime} 46^{\prime \prime}$ & Trinity & -- & & 1.64 & 4.7 & 8.3 & \\
\hline 96 & $30^{\circ} 11^{\prime} 57^{\prime \prime}$ & $98^{\circ} 59^{\prime} 08^{\prime \prime}$ & Trinity & -- & & -.1 & 3.6 & 3.6 & \\
\hline 97 & $29^{\circ} 59^{\prime} 39^{\prime \prime}$ & $98^{\circ} 11^{\prime} 45^{\prime \prime}$ & Trinity & -- & & -.2 & 11.3 & 16.3 & at Fishers Store road crossing \\
\hline 97 & $29^{\circ} 59^{\prime} 36^{\prime \prime}$ & $98^{\circ} 05^{\prime} 18^{\prime \prime}$ & Edwards, Trinity & -- & & .3 & 11.0 & 27.6 & gaging station at Wimberley (08171000) \\
\hline 97 & $30^{\circ} 00^{\prime} 24^{\prime \prime}$ & $97^{\circ} 57^{\prime} 24^{\prime \prime}$ & Edwards & -- & & -10.1 & 1.8 & 38.6 & \\
\hline 97 & $30^{\circ} 00^{\prime} 09^{\prime \prime}$ & $97^{\circ} 55^{\prime} 57^{\prime \prime}$ & Edwards & -- & & -.3 & .1 & 40.4 & \\
\hline 97 & $30^{\circ} 00^{\prime} 08^{\prime \prime}$ & $97^{\circ} 55^{\prime} 57^{\prime \prime}$ & Edwards & -- & & 0 & 9.1 & 40.5 & \\
\hline 98 & $30^{\circ} 05^{\prime} 56^{\prime \prime}$ & $98^{\circ} 20^{\prime} 29^{\prime \prime}$ & Trinity & -- & & -1.51 & 5.3 & 0 & at east crossing of Chimney Valley Rd \\
\hline 98 & $30^{\circ} 03^{\prime} 53^{\prime \prime}$ & $98^{\circ} 18^{\prime} 36^{\prime \prime}$ & Trinity & -- & & 0 & 1.6 & 5.3 & \\
\hline 98 & $30^{\circ} 03^{\prime} 04^{\prime \prime}$ & $98^{\circ} 18^{\prime} 46^{\prime \prime}$ & Trinity & -- & & 0 & 2.7 & 6.9 & \\
\hline 98 & $30^{\circ} 02^{\prime} 37^{\prime \prime}$ & $98^{\circ} 14^{\prime} 59^{\prime \prime}$ & Trinity & -- & & 0 & 1.1 & 9.6 & \\
\hline 98 & $30^{\circ} 03^{\prime} 03^{\prime \prime}$ & $98^{\circ} 14^{\prime} 03^{\prime \prime}$ & Trinity & -- & & -.94 & .7 & 10.7 & \\
\hline 98 & $30^{\circ} 02^{\prime} 40^{\prime \prime}$ & $98^{\circ} 13^{\prime} 42^{\prime \prime}$ & Trinity & -- & & .08 & .8 & 11.4 & \\
\hline 98 & $30^{\circ} 01^{\prime} 56^{\prime \prime}$ & $98^{\circ} 13^{\prime} 15^{\prime \prime}$ & Trinity & -- & & 4.08 & .2 & 12.2 & \\
\hline 98 & $30^{\circ} 01^{\prime} 49^{\prime \prime}$ & $98^{\circ} 13^{\prime} 07^{\prime \prime}$ & Trinity & -- & & 2.92 & .9 & 12.4 & \\
\hline 98 & $30^{\circ} 01 ' 24^{\prime \prime}$ & $98^{\circ} 13^{\prime} 04^{\prime \prime}$ & Trinity & -- & & -1.46 & .1 & 13.3 & \\
\hline
\end{tabular}


Table 4. Gains and losses from gain-loss studies in Texas-Continued

\begin{tabular}{|c|c|c|c|c|c|c|c|c|c|}
\hline $\begin{array}{l}\text { Stream- } \\
\text { flow } \\
\text { study } \\
\text { no. }\end{array}$ & $\begin{array}{l}\text { Latitude of } \\
\text { upstream } \\
\text { end of } \\
\text { subreach }\end{array}$ & $\begin{array}{l}\text { Longitude } \\
\text { of upstream } \\
\text { end of } \\
\text { subreach }\end{array}$ & $\begin{array}{l}\text { Major aquifer } \\
\text { outcrop } \\
\text { intersected } \\
\text { by subreach }\end{array}$ & & $\begin{array}{l}\text { Minor aquifer } \\
\text { outcrop } \\
\text { intercepted } \\
\text { by subreach }\end{array}$ & $\begin{array}{l}\text { Gain or } \\
\text { loss (-) in } \\
\text { subreach } \\
\left(\mathrm{ft}^{3} / \mathrm{s}\right)\end{array}$ & $\begin{array}{l}\text { Length } \\
\text { of subreach } \\
\text { (river mi) }\end{array}$ & $\begin{array}{l}\text { Location of } \\
\text { upstream } \\
\text { end of } \\
\text { subreach } \\
\text { (river mi) }\end{array}$ & $\begin{array}{l}\text { Descriptive location } \\
\text { of upstream end of } \\
\text { selected subreaches }\end{array}$ \\
\hline 98 & $30^{\circ} 01^{\prime} 16^{\prime \prime}$ & $98^{\circ} 13^{\prime} 10^{\prime \prime}$ & Trinity & -- & & -2.44 & 0.2 & 13.4 & \\
\hline 98 & $30^{\circ} 01^{\prime} 09^{\prime \prime}$ & $98^{\circ} 13^{\prime} 07^{\prime \prime}$ & Trinity & -- & & .47 & .3 & 13.6 & \\
\hline 98 & $30^{\circ} 01^{\prime} 00^{\prime \prime}$ & $98^{\circ} 13^{\prime} 08^{\prime \prime}$ & Trinity & -- & & -.34 & .3 & 13.9 & \\
\hline 98 & $30^{\circ} 00^{\prime} 53^{\prime \prime}$ & $98^{\circ} 12^{\prime} 55^{\prime \prime}$ & Trinity & -- & & .02 & .6 & 14.2 & \\
\hline 98 & $30^{\circ} 00^{\prime} 51^{\prime \prime}$ & $98^{\circ} 12^{\prime} 24^{\prime \prime}$ & Trinity & -- & & 13.94 & .6 & 14.8 & \\
\hline 98 & $30^{\circ} 00^{\prime} 21^{\prime \prime}$ & $98^{\circ} 12^{\prime} 18^{\prime \prime}$ & Trinity & -- & & .49 & 3.1 & 15.4 & \\
\hline 98 & $29^{\circ} 58^{\prime} 28^{\prime \prime}$ & $98^{\circ} 11^{\prime} 58^{\prime \prime}$ & Trinity & -- & & -1.4 & 1.9 & 18.5 & \\
\hline 98 & $29^{\circ} 57^{\prime} 18^{\prime \prime}$ & $98^{\circ} 10^{\prime} 07^{\prime \prime}$ & Trinity & -- & & 3.0 & 2.8 & 20.4 & \\
\hline 98 & $29^{\circ} 58^{\prime} 07^{\prime \prime}$ & $98^{\circ} 08^{\prime} 07^{\prime \prime}$ & Trinity & -- & & 2.7 & 1.6 & 23.2 & \\
\hline 98 & $29^{\circ} 58^{\prime} 34^{\prime \prime}$ & $98^{\circ} 06^{\prime} 51^{\prime \prime}$ & Trinity & -- & & 6.29 & 2.3 & 24.8 & \\
\hline 99 & $30^{\circ} 00^{\prime} 23^{\prime \prime}$ & $97^{\circ} 57^{\prime 2} 23^{\prime \prime}$ & Edwards & -- & & -4.6 & 2.0 & 11.4 & $1 \mathrm{mi}$ above Halifax Creek \\
\hline 99 & $30^{\circ} 00^{\prime} 20^{\prime \prime}$ & $97^{\circ} 56^{\prime} 03^{\prime \prime}$ & Edwards & -- & & -8.5 & 2.2 & 13.4 & 0.75 mi below Halifax Creek \\
\hline 100 & $30^{\circ} 05^{\prime} 56^{\prime \prime}$ & $98^{\circ} 20^{\prime} 29^{\prime \prime}$ & Trinity & -- & & 0 & 4.8 & 0 & at east crossing of Chimney Valley Rd \\
\hline 100 & $30^{\circ} 03^{\prime} 54^{\prime \prime}$ & $98^{\circ} 18^{\prime} 20^{\prime \prime}$ & Trinity & -- & & 0 & 3.3 & 4.8 & \\
\hline 100 & $30^{\circ} 03^{\prime} 05^{\prime \prime}$ & $98^{\circ} 17^{\prime} 32^{\prime \prime}$ & Trinity & -- & & 0 & .9 & 8.1 & \\
\hline 100 & $30^{\circ} 03^{\prime} 18^{\prime \prime}$ & $98^{\circ} 16^{\prime} 49^{\prime \prime}$ & Trinity & -- & & 0 & .5 & 9 & \\
\hline 100 & $30^{\circ} 03^{\prime} 09^{\prime \prime}$ & $98^{\circ} 16^{\prime} 10^{\prime \prime}$ & Trinity & -- & & -.1 & .2 & 9.5 & \\
\hline 100 & $30^{\circ} 02^{\prime} 43^{\prime \prime}$ & $98^{\circ} 14^{\prime} 56^{\prime \prime}$ & Trinity & -- & & 0 & 2.8 & 9.7 & \\
\hline 100 & $30^{\circ} 01^{\prime} 54^{\prime \prime}$ & $98^{\circ} 13^{\prime} 12^{\prime \prime}$ & Trinity & -- & & 0 & 1.1 & 12.5 & \\
\hline 100 & $30^{\circ} 01^{\prime} 37^{\prime \prime}$ & $98^{\circ} 12^{\prime} 46^{\prime \prime}$ & Trinity & -- & & 2.68 & .1 & 13.6 & \\
\hline 100 & $30^{\circ} 01^{\prime} 21^{\prime \prime}$ & $98^{\circ} 13^{\prime} 11^{\prime \prime}$ & Trinity & -- & & -2.32 & .2 & 13.7 & \\
\hline 100 & $30^{\circ} 01^{\prime} 09^{\prime \prime}$ & $98^{\circ} 13^{\prime} 07^{\prime \prime}$ & Trinity & -- & & -.34 & .2 & 13.9 & \\
\hline 100 & $30^{\circ} 00^{\prime} 55^{\prime \prime}$ & $98^{\circ} 13^{\prime} 08^{\prime \prime}$ & Trinity & -- & & .09 & .2 & 14.1 & \\
\hline 100 & $30^{\circ} 00^{\prime} 57^{\prime \prime}$ & $98^{\circ} 12^{\prime} 49^{\prime \prime}$ & Trinity & -- & & -.11 & .6 & 14.3 & \\
\hline 100 & $30^{\circ} 00^{\prime} 45^{\prime \prime}$ & $98^{\circ} 12^{\prime} 19^{\prime \prime}$ & Trinity & -- & & 7.5 & 1.4 & 14.9 & \\
\hline 100 & 29०59'39" & $98^{\circ} 11^{\prime} 45^{\prime \prime}$ & Trinity & -- & & -.25 & 1.9 & 16.3 & \\
\hline 100 & $29^{\circ} 58^{\prime} 31^{\prime \prime}$ & $98^{\circ} 12^{\prime} 05^{\prime \prime}$ & Trinity & -- & & .05 & 2.2 & 18.2 & \\
\hline 100 & $29^{\circ} 57^{\prime} 26^{\prime \prime}$ & $98^{\circ} 10^{\prime} 17^{\prime \prime}$ & Trinity & -- & & -.17 & 2.8 & 20.4 & \\
\hline 100 & $29^{\circ} 58^{\prime} 02^{\prime \prime}$ & $98^{\circ} 08^{\prime} 20^{\prime \prime}$ & Trinity & -- & & .71 & 2.1 & 23.2 & \\
\hline 100 & $29^{\circ} 58^{\prime} 55^{\prime \prime}$ & $98^{\circ} 06^{\prime} 54^{\prime \prime}$ & Trinity & -- & & .11 & 2.3 & 25.3 & \\
\hline 100 & $29^{\circ} 59^{\prime} 36^{\prime \prime}$ & $98^{\circ} 05^{\prime} 18^{\prime \prime}$ & Trinity & -- & & .5 & 2.5 & 27.6 & gaging station at Wimberley (08171000) \\
\hline 100 & $29^{\circ} 59^{\prime} 07^{\prime \prime}$ & $98^{\circ} 03^{\prime} 56^{\prime \prime}$ & Trinity & -- & & .1 & 2.6 & 30.1 & \\
\hline 100 & $29^{\circ} 59^{\prime} 01^{\prime \prime}$ & $98^{\circ} 03^{\prime} 09^{\prime \prime}$ & Trinity & -- & & -.5 & 2.2 & 32.7 & \\
\hline 100 & $29^{\circ} 58^{\prime} 47^{\prime \prime}$ & $98^{\circ} 01^{\prime} 44^{\prime \prime}$ & Edwards, Trinity & -- & & 0 & 3.7 & 34.9 & \\
\hline 100 & $30^{\circ} 00^{\prime} 24^{\prime \prime}$ & $97^{\circ} 57^{\prime} 24^{\prime \prime}$ & Edwards & -- & & -9.24 & 1.3 & 38.6 & \\
\hline 100 & $30^{\circ} 00^{\prime} 33^{\prime \prime}$ & $97^{\circ} 56^{\prime} 09^{\prime \prime}$ & Edwards & -- & & -1.22 & .5 & 39.9 & \\
\hline 100 & $30^{\circ} 00^{\prime} 09^{\prime \prime}$ & $97^{\circ} 55^{\prime} 57^{\prime \prime}$ & Edwards & -- & & -.14 & .5 & 40.4 & \\
\hline 100 & $29^{\circ} 59^{\prime} 39^{\prime \prime}$ & $97^{\circ} 55^{\prime} 42^{\prime \prime}$ & -- & -- & & 0 & 1.0 & 40.9 & \\
\hline
\end{tabular}


Table 4. Gains and losses from gain-loss studies in Texas-Continued

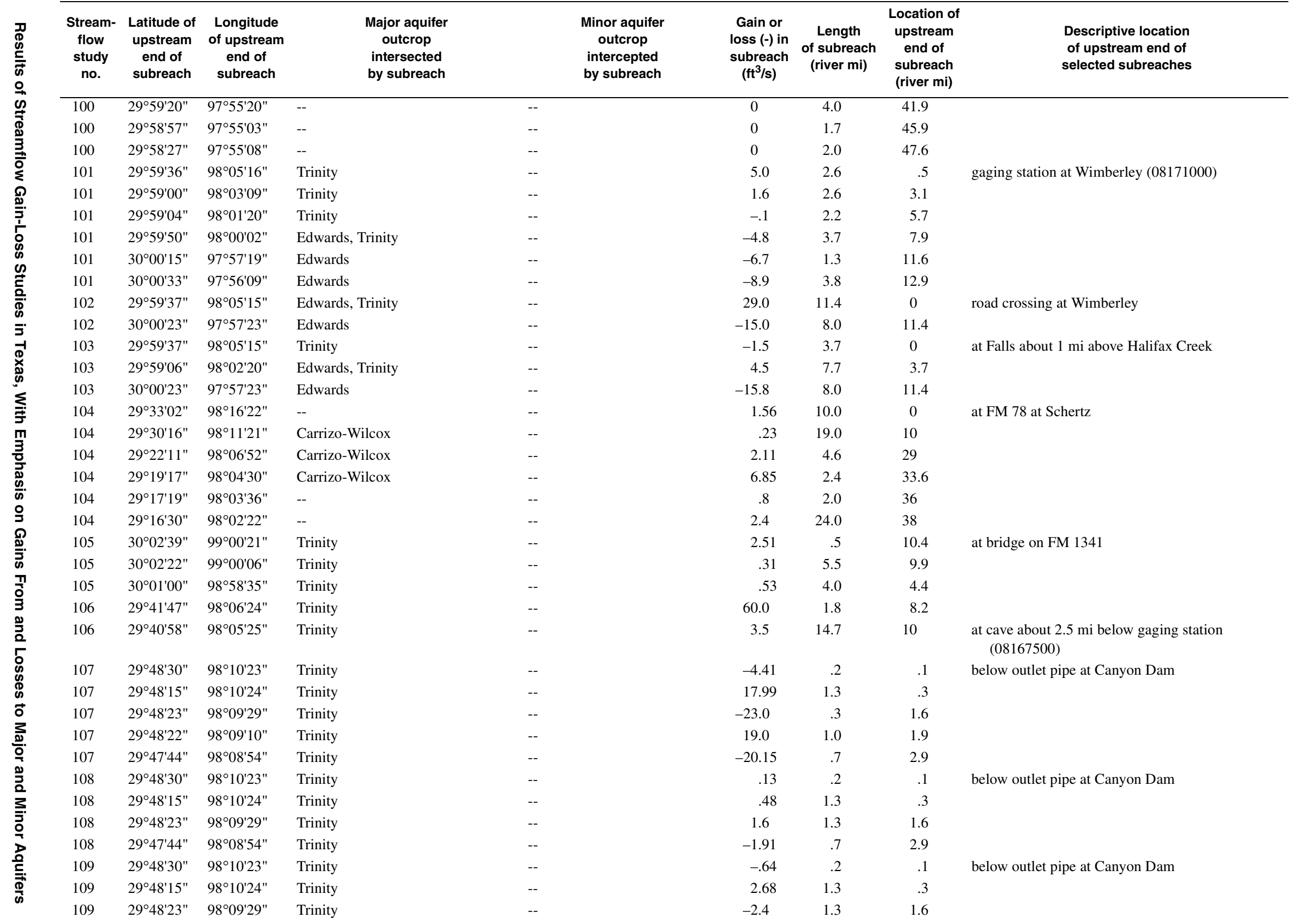


Table 4. Gains and losses from gain-loss studies in Texas-Continued

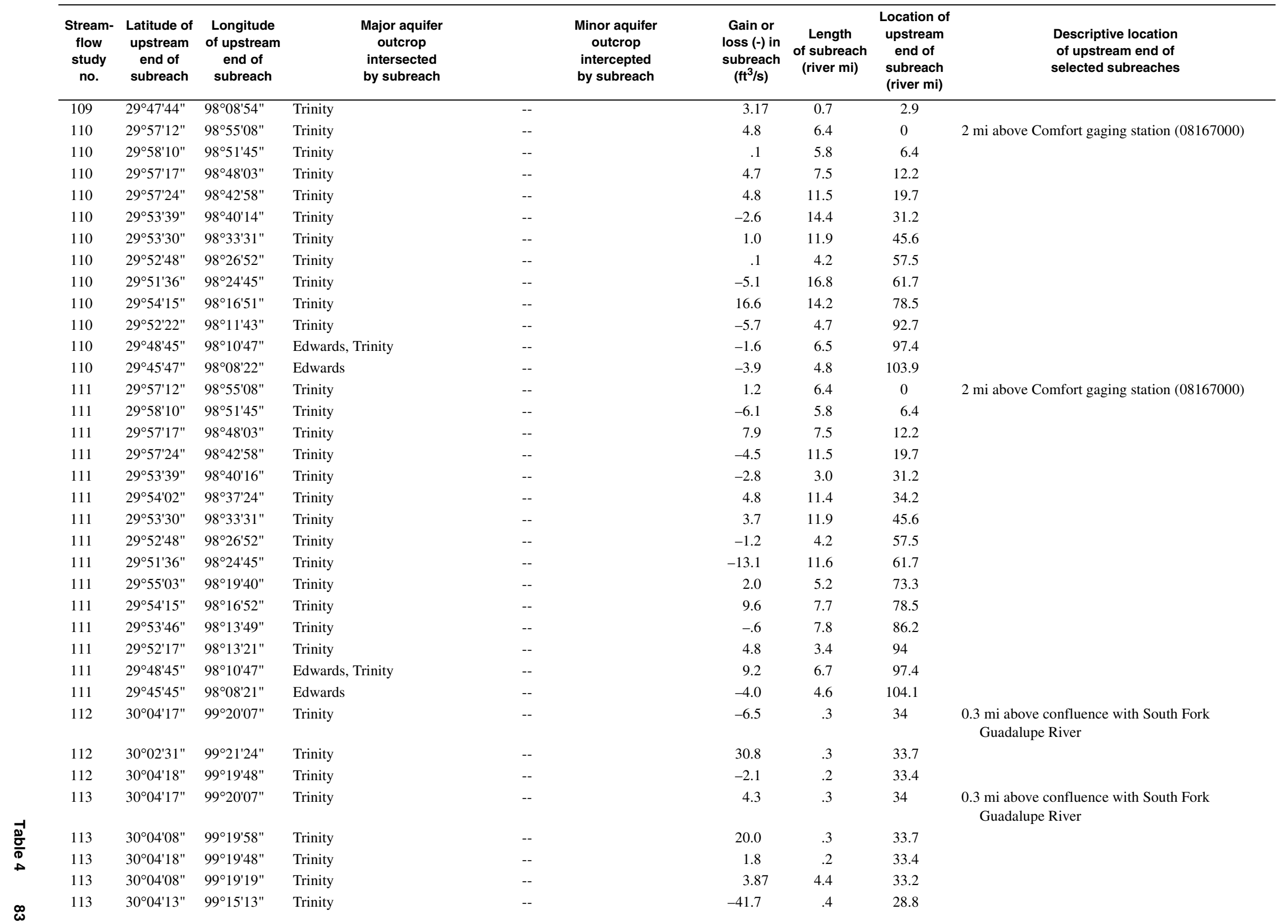




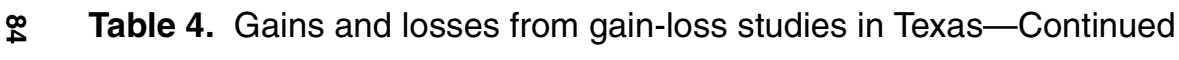

\begin{tabular}{|c|c|c|c|c|c|c|c|c|c|}
\hline $\begin{array}{l}\text { Stream- } \\
\text { flow } \\
\text { study } \\
\text { no. }\end{array}$ & $\begin{array}{l}\text { Latitude of } \\
\text { upstream } \\
\text { end of } \\
\text { subreach }\end{array}$ & $\begin{array}{l}\text { Longitude } \\
\text { of upstream } \\
\text { end of } \\
\text { subreach }\end{array}$ & $\begin{array}{l}\text { Major aquifer } \\
\text { outcrop } \\
\text { intersected } \\
\text { by subreach }\end{array}$ & & $\begin{array}{l}\text { Minor aquifer } \\
\text { outcrop } \\
\text { intercepted } \\
\text { by subreach }\end{array}$ & $\begin{array}{l}\text { Gain or } \\
\text { loss }(-) \text { in } \\
\text { subreach } \\
\left(\mathrm{ft}^{3} / \mathrm{s}\right)\end{array}$ & $\begin{array}{l}\text { Length } \\
\text { of subreach } \\
\text { (river mi) }\end{array}$ & $\begin{array}{l}\text { Location of } \\
\text { upstream } \\
\text { end of } \\
\text { subreach } \\
\text { (river mi) }\end{array}$ & $\begin{array}{l}\text { Descriptive location } \\
\text { of upstream end of } \\
\text { selected subreaches }\end{array}$ \\
\hline 113 & $30^{\circ} 04^{\prime} 34^{\prime \prime}$ & $99^{\circ} 14^{\prime} 50^{\prime \prime}$ & Trinity & -- & & 48.84 & 3.4 & 28.4 & \multirow{38}{*}{ at county road crossing above US 281} \\
\hline 113 & $30^{\circ} 03^{\prime} 59^{\prime \prime}$ & $99^{\circ} 11^{\prime} 27^{\prime \prime}$ & Trinity & -- & & -8.3 & 4.0 & 25 & \\
\hline 113 & $30^{\circ} 01 ' 50^{\prime \prime}$ & $99^{\circ} 08^{\prime} 13^{\prime \prime}$ & Trinity & -- & & 13.79 & 6.95 & 21 & \\
\hline 113 & $29^{\circ} 57^{\prime} 22^{\prime \prime}$ & $99^{\circ} 04^{\prime} 50^{\prime \prime}$ & Trinity & -- & & 8.9 & 3.25 & 14.05 & \\
\hline 113 & $29^{\circ} 56^{\prime} 46^{\prime \prime}$ & $99^{\circ} 02^{\prime} 14^{\prime \prime}$ & Trinity & -- & & -12.05 & 6.6 & 10.8 & \\
\hline 113 & $29^{\circ} 56^{\prime} 22^{\prime \prime}$ & $98^{\circ} 56^{\prime} 52^{\prime \prime}$ & Trinity & -- & & 7.97 & 4.2 & 4.2 & \\
\hline 114 & $29^{\circ} 53^{\prime} 12^{\prime \prime}$ & $98^{\circ} 27^{\prime} 10^{\prime \prime}$ & Trinity & -- & & -2.5 & 2.4 & 0 & \\
\hline 114 & $29^{\circ} 52^{\prime} 05^{\prime \prime}$ & $98^{\circ} 25^{\prime} 54^{\prime \prime}$ & Trinity & -- & & -3.9 & .9 & 2.4 & \\
\hline 114 & $29^{\circ} 51^{\prime} 54^{\prime \prime}$ & $98^{\circ} 24^{\prime} 53^{\prime \prime}$ & Trinity & -- & & -1.6 & 1.4 & 3.3 & \\
\hline 114 & $29^{\circ} 51^{\prime} 22^{\prime \prime}$ & $98^{\circ} 23^{\prime} 49^{\prime \prime}$ & Trinity & -- & & 4.5 & 1.0 & 4.7 & \\
\hline 114 & $29^{\circ} 51^{\prime} 42^{\prime \prime}$ & $98^{\circ} 22^{\prime} 51^{\prime \prime}$ & Trinity & -- & & -4.7 & 1.3 & 5.7 & \\
\hline 114 & $29^{\circ} 51^{\prime} 53^{\prime \prime}$ & $98^{\circ} 22^{\prime} 19^{\prime \prime}$ & Trinity & -- & & -16.3 & 3.0 & 7 & \\
\hline 114 & $29^{\circ} 53^{\prime} 15^{\prime \prime}$ & $98^{\circ} 22^{\prime} 04^{\prime \prime}$ & Trinity & -- & & -6.0 & 2.5 & 10 & \\
\hline 114 & $29^{\circ} 53^{\prime} 13^{\prime \prime}$ & $98^{\circ} 20^{\prime} 40^{\prime \prime}$ & Trinity & -- & & -8.8 & 2.6 & 12.5 & \\
\hline 114 & $29^{\circ} 54^{\prime} 12^{\prime \prime}$ & $98^{\circ} 19^{\prime} 54^{\prime \prime}$ & Trinity & -- & & -1.8 & 1.3 & 15.1 & \\
\hline 114 & $29^{\circ} 54^{\prime} 48^{\prime \prime}$ & $98^{\circ} 20^{\prime} 06^{\prime \prime}$ & Trinity & -- & & -2.2 & 1.6 & 16.4 & \\
\hline 114 & $29^{\circ} 54^{\prime} 50^{\prime \prime}$ & $98^{\circ} 19^{\prime} 05^{\prime \prime}$ & Trinity & -- & & 1.9 & 2.5 & 18 & \\
\hline 114 & $29^{\circ} 54^{\prime} 08^{\prime \prime}$ & $98^{\circ} 18^{\prime} 39^{\prime \prime}$ & Trinity & -- & & -5.9 & 1.8 & 20.5 & \\
\hline 114 & $29^{\circ} 53^{\prime} 32^{\prime \prime}$ & $98^{\circ} 18^{\prime} 18^{\prime \prime}$ & Trinity & -- & & -2.2 & 2.2 & 22.3 & \\
\hline 114 & $29^{\circ} 54^{\prime} 12^{\prime \prime}$ & $98^{\circ} 17^{\prime} 48^{\prime \prime}$ & Trinity & -- & & 0 & .2 & 24.5 & \\
\hline 114 & $29^{\circ} 54^{\prime} 18^{\prime \prime}$ & $98^{\circ} 17^{\prime} 48^{\prime \prime}$ & Trinity & -- & & -1.1 & 2.5 & 24.7 & \\
\hline 114 & $29^{\circ} 53^{\prime} 47^{\prime \prime}$ & $98^{\circ} 16^{\prime} 11^{\prime \prime}$ & Trinity & -- & & 3.9 & 1.8 & 27.2 & \\
\hline 114 & $29^{\circ} 53^{\prime} 10^{\prime \prime}$ & $98^{\circ} 14^{\prime} 58^{\prime \prime}$ & Trinity & -- & & 3.3 & 1.4 & 29 & \\
\hline 114 & $29^{\circ} 53^{\prime} 26^{\prime \prime}$ & $98^{\circ} 13^{\prime} 45^{\prime \prime}$ & Trinity & -- & & 2.8 & 1.9 & 30.4 & \\
\hline 114 & $29^{\circ} 51^{\prime} 49^{\prime \prime}$ & $98^{\circ} 13^{\prime} 04^{\prime \prime}$ & Trinity & -- & & -4.1 & .6 & 32.3 & \\
\hline 114 & $29^{\circ} 51^{\prime} 26^{\prime \prime}$ & $98^{\circ} 12^{\prime} 43^{\prime \prime}$ & Trinity & -- & & 4.0 & 1.4 & 32.9 & \\
\hline 114 & $29^{\circ} 51^{\prime} 40^{\prime \prime}$ & $98^{\circ} 12^{\prime} 00^{\prime \prime}$ & Trinity & -- & & -1.0 & 3.0 & 34.3 & \\
\hline 114 & $29^{\circ} 52^{\prime} 08^{\prime \prime}$ & $98^{\circ} 11^{\prime} 20^{\prime \prime}$ & Trinity & -- & & -2.9 & .8 & 37.3 & \\
\hline 114 & $29^{\circ} 51^{\prime} 31^{\prime \prime}$ & $98^{\circ} 10^{\prime} 45^{\prime \prime}$ & Trinity & -- & & 2.9 & 1.8 & 38.1 & \\
\hline 114 & $29^{\circ} 52^{\prime} 03^{\prime \prime}$ & $98^{\circ} 09^{\prime} 34^{\prime \prime}$ & Trinity & -- & & -2.7 & 1.8 & 39.9 & \\
\hline 114 & $29^{\circ} 50^{\prime} 38^{\prime \prime}$ & $98^{\circ} 09^{\prime} 47^{\prime \prime}$ & Trinity & -- & & -2.7 & 1.1 & 41.7 & \\
\hline 114 & $29^{\circ} 49^{\prime} 57^{\prime \prime}$ & $98^{\circ} 10^{\prime} 19^{\prime \prime}$ & Trinity & -- & & 7.6 & .9 & 42.8 & \\
\hline 114 & $29^{\circ} 49^{\prime} 06^{\prime \prime}$ & $98^{\circ} 10^{\prime} 23^{\prime \prime}$ & Trinity & -- & & -1.4 & 1.8 & 43.7 & \\
\hline 114 & $29^{\circ} 48^{\prime} 06^{\prime \prime}$ & $98^{\circ} 10^{\prime} 21^{\prime \prime}$ & Trinity & -- & & -.9 & 2.5 & 45.5 & \\
\hline 114 & $29^{\circ} 47^{\prime} 40^{\prime \prime}$ & $98^{\circ} 08^{\prime} 58^{\prime \prime}$ & Edwards, Trinity & -- & & -5.0 & 3.5 & 48 & \\
\hline 114 & $29^{\circ} 45^{\prime} 51^{\prime \prime}$ & $98^{\circ} 08^{\prime} 25^{\prime \prime}$ & Edwards & -- & & 3.7 & 1.8 & 51.5 & \\
\hline 114 & $29^{\circ} 45^{\prime} 10^{\prime \prime}$ & $98^{\circ} 07^{\prime} 58^{\prime \prime}$ & Edwards & -- & & -3.5 & 1.8 & 53.3 & \\
\hline 114 & $29^{\circ} 44^{\prime} 44^{\prime \prime}$ & $98^{\circ} 06^{\prime} 38^{\prime \prime}$ & Edwards & -- & & 2.9 & 1.9 & 55.1 & \\
\hline
\end{tabular}


Table 4. Gains and losses from gain-loss studies in Texas-Continued

\begin{tabular}{|c|c|c|c|c|c|c|c|c|c|}
\hline $\begin{array}{l}\text { Stream- } \\
\text { flow } \\
\text { study } \\
\text { no. }\end{array}$ & $\begin{array}{l}\text { Latitude of } \\
\text { upstream } \\
\text { end of } \\
\text { subreach }\end{array}$ & $\begin{array}{l}\text { Longitude } \\
\text { of upstream } \\
\text { end of } \\
\text { subreach }\end{array}$ & $\begin{array}{c}\text { Major aquifer } \\
\text { outcrop } \\
\text { intersected } \\
\text { by subreach }\end{array}$ & & $\begin{array}{c}\text { Minor aquifer } \\
\text { outcrop } \\
\text { intercepted } \\
\text { by subreach }\end{array}$ & $\begin{array}{l}\text { Gain or } \\
\text { loss }(-) \text { in } \\
\text { subreach } \\
\left(\mathrm{ft}^{3} / \mathrm{s}\right)\end{array}$ & $\begin{array}{l}\text { Length } \\
\text { of subreach } \\
\text { (river mi) }\end{array}$ & $\begin{array}{l}\text { Location of } \\
\text { upstream } \\
\text { end of } \\
\text { subreach } \\
\text { (river mi) }\end{array}$ & $\begin{array}{l}\text { Descriptive location } \\
\text { of upstream end of } \\
\text { selected subreaches }\end{array}$ \\
\hline 115 & $29^{\circ} 53^{\prime} 12^{\prime \prime}$ & $98^{\circ} 27^{\prime} 10^{\prime \prime}$ & Trinity & -- & & -1.6 & 5.7 & 0 & at county road crossing above US 281 \\
\hline 115 & $29^{\circ} 51^{\prime} 42^{\prime \prime}$ & $98^{\circ} 22^{\prime} 51^{\prime \prime}$ & Trinity & -- & & -24.54 & 12.3 & 5.7 & \\
\hline 115 & $29^{\circ} 54^{\prime} 02^{\prime \prime}$ & $98^{\circ} 37^{\prime} 24^{\prime \prime}$ & Trinity & -- & & 15.24 & 6.5 & 18 & \\
\hline 115 & $29^{\circ} 54^{\prime} 12^{\prime \prime}$ & $98^{\circ} 17^{\prime} 48^{\prime \prime}$ & Trinity & -- & & 0 & .2 & 24.5 & \\
\hline 115 & $29^{\circ} 54^{\prime} 18^{\prime \prime}$ & $98^{\circ} 17^{\prime} 48^{\prime \prime}$ & Trinity & -- & & 2.9 & 4.3 & 24.7 & \\
\hline 115 & $29^{\circ} 53^{\prime} 10^{\prime \prime}$ & $98^{\circ} 14^{\prime} 58^{\prime \prime}$ & Trinity & -- & & 7.8 & 3.3 & 29 & \\
\hline 115 & $29^{\circ} 51^{\prime} 49^{\prime \prime}$ & $98^{\circ} 13^{\prime} 04^{\prime \prime}$ & Trinity & -- & & -2.2 & 5.0 & 32.3 & \\
\hline 115 & $29^{\circ} 52^{\prime} 02^{\prime \prime}$ & $98^{\circ} 11^{\prime} 00^{\prime \prime}$ & Trinity & -- & & 1.0 & 5.5 & 37.3 & \\
\hline 115 & $29^{\circ} 49^{\prime} 57^{\prime \prime}$ & $98^{\circ} 10^{\prime} 19^{\prime \prime}$ & Trinity & -- & & -1.8 & .9 & 42.8 & \\
\hline 115 & $29^{\circ} 49^{\prime} 06^{\prime \prime}$ & $98^{\circ} 10^{\prime} 23^{\prime \prime}$ & Trinity & -- & & 4.1 & 4.3 & 43.7 & \\
\hline 115 & $29^{\circ} 47^{\prime} 40^{\prime \prime}$ & $98^{\circ} 08^{\prime} 58^{\prime \prime}$ & Edwards, Trinity & -- & & -.8 & 3.5 & 48 & \\
\hline 115 & $29^{\circ} 45^{\prime} 51^{\prime \prime}$ & $98^{\circ} 08^{\prime} 25^{\prime \prime}$ & Edwards & -- & & -1.1 & 5.5 & 51.5 & \\
\hline 116 & $29^{\circ} 53^{\prime} 12^{\prime \prime}$ & $98^{\circ} 27^{\prime} 10^{\prime \prime}$ & Trinity & -- & & -2.7 & 5.7 & 0 & at county road crossing above US 281 \\
\hline 116 & $29^{\circ} 51^{\prime} 42^{\prime \prime}$ & $98^{\circ} 22^{\prime} 51^{\prime \prime}$ & Trinity & -- & & -22.16 & 12.3 & 5.7 & \\
\hline 116 & $29^{\circ} 54^{\prime} 02^{\prime \prime}$ & $98^{\circ} 37^{\prime} 24^{\prime \prime}$ & Trinity & -- & & 23.66 & 6.7 & 18 & \\
\hline 116 & $29^{\circ} 54^{\prime} 18^{\prime \prime}$ & $98^{\circ} 17^{\prime} 48^{\prime \prime}$ & Trinity & -- & & 8.5 & 7.6 & 24.7 & \\
\hline 116 & $29^{\circ} 51^{\prime} 49^{\prime \prime}$ & $98^{\circ} 13^{\prime} 04^{\prime \prime}$ & Trinity & -- & & 3.0 & 15.7 & 32.3 & \\
\hline 116 & $29^{\circ} 47^{\prime} 40^{\prime \prime}$ & $98^{\circ} 08^{\prime} 58^{\prime \prime}$ & Edwards, Trinity & -- & & -3.2 & 3.5 & 48 & \\
\hline 116 & $29^{\circ} 45^{\prime} 51^{\prime \prime}$ & $98^{\circ} 08^{\prime} 25^{\prime \prime}$ & Edwards & -- & & 3.9 & 5.5 & 51.5 & \\
\hline 117 & $29^{\circ} 54^{\prime} 22^{\prime \prime}$ & $98^{\circ} 32^{\prime} 37^{\prime \prime}$ & Trinity & -- & & 2.6 & 10.3 & -10.3 & $300 \mathrm{ft}$ below Bergheim-Kendalia Rd \\
\hline 117 & $29^{\circ} 53^{\prime} 12^{\prime \prime}$ & $98^{\circ} 27^{\prime} 10^{\prime \prime}$ & Trinity & -- & & -3.4 & 5.7 & 0 & at county road crossing above US 281 \\
\hline 117 & $29^{\circ} 51^{\prime} 42^{\prime \prime}$ & $98^{\circ} 22^{\prime} 51^{\prime \prime}$ & Trinity & -- & & -26.6 & 12.3 & 5.7 & \\
\hline 117 & $29^{\circ} 54^{\prime} 02^{\prime \prime}$ & $98^{\circ} 37^{\prime} 24^{\prime \prime}$ & Trinity & -- & & 18.6 & 6.7 & 18 & \\
\hline 117 & $29^{\circ} 54^{\prime} 18^{\prime \prime}$ & $98^{\circ} 17^{\prime} 48^{\prime \prime}$ & Trinity & -- & & 7.4 & 7.6 & 24.7 & \\
\hline 117 & $29^{\circ} 51^{\prime} 49^{\prime \prime}$ & $98^{\circ} 13^{\prime} 04^{\prime \prime}$ & Trinity & -- & & -.1 & 15.7 & 32.3 & \\
\hline 117 & $29^{\circ} 47^{\prime} 40^{\prime \prime}$ & $98^{\circ} 08^{\prime} 58^{\prime \prime}$ & Edwards, Trinity & -- & & -2.1 & 3.5 & 48 & \\
\hline 117 & $29^{\circ} 45^{\prime} 51^{\prime \prime}$ & $98^{\circ} 08^{\prime} 25^{\prime \prime}$ & Edwards & -- & & 2.2 & 5.5 & 51.5 & \\
\hline 118 & $29^{\circ} 53^{\prime} 12^{\prime \prime}$ & $98^{\circ} 27^{\prime} 10^{\prime \prime}$ & Trinity & -- & & -3.5 & 5.7 & 0 & at county road crossing above US 281 \\
\hline 118 & $29^{\circ} 51^{\prime} 42^{\prime \prime}$ & $98^{\circ} 22^{\prime} 51^{\prime \prime}$ & Trinity & -- & & -19.7 & 12.3 & 5.7 & \\
\hline 118 & $29^{\circ} 54^{\prime} 02^{\prime \prime}$ & $98^{\circ} 37^{\prime} 24^{\prime \prime}$ & Trinity & -- & & 15.3 & 6.7 & 18 & \\
\hline 118 & $29^{\circ} 54^{\prime} 18^{\prime \prime}$ & $98^{\circ} 17^{\prime} 48^{\prime \prime}$ & Trinity & -- & & 6.8 & 7.6 & 24.7 & \\
\hline 118 & $29^{\circ} 51^{\prime} 49^{\prime \prime}$ & $98^{\circ} 13^{\prime} 04^{\prime \prime}$ & Trinity & -- & & .3 & 15.7 & 32.3 & \\
\hline 118 & $29^{\circ} 47^{\prime} 40^{\prime \prime}$ & $98^{\circ} 08^{\prime} 58^{\prime \prime}$ & Edwards, Trinity & -- & & -.4 & 3.5 & 48 & \\
\hline 118 & $29^{\circ} 45^{\prime} 51^{\prime \prime}$ & $98^{\circ} 08^{\prime} 25^{\prime \prime}$ & Edwards & -- & & .9 & 5.5 & 51.5 & \\
\hline 119 & $29^{\circ} 53^{\prime} 12^{\prime \prime}$ & $98^{\circ} 27^{\prime} 10^{\prime \prime}$ & Trinity & -- & & -1.9 & 5.7 & 0 & at county road crossing above US 281 \\
\hline 119 & $29^{\circ} 51^{\prime} 42^{\prime \prime}$ & $98^{\circ} 22^{\prime} 51^{\prime \prime}$ & Trinity & -- & & -8.2 & 12.3 & 5.7 & \\
\hline 119 & $29^{\circ} 54^{\prime} 02^{\prime \prime}$ & $98^{\circ} 37^{\prime} 24^{\prime \prime}$ & Trinity & -- & & 6.29 & 6.7 & 18 & \\
\hline 119 & $29^{\circ} 54^{\prime} 18^{\prime \prime}$ & $98^{\circ} 17^{\prime} 48^{\prime \prime}$ & Trinity & -- & & 5.91 & 7.6 & 24.7 & \\
\hline
\end{tabular}


\& Table 4. Gains and losses from gain-loss studies in Texas-Continued

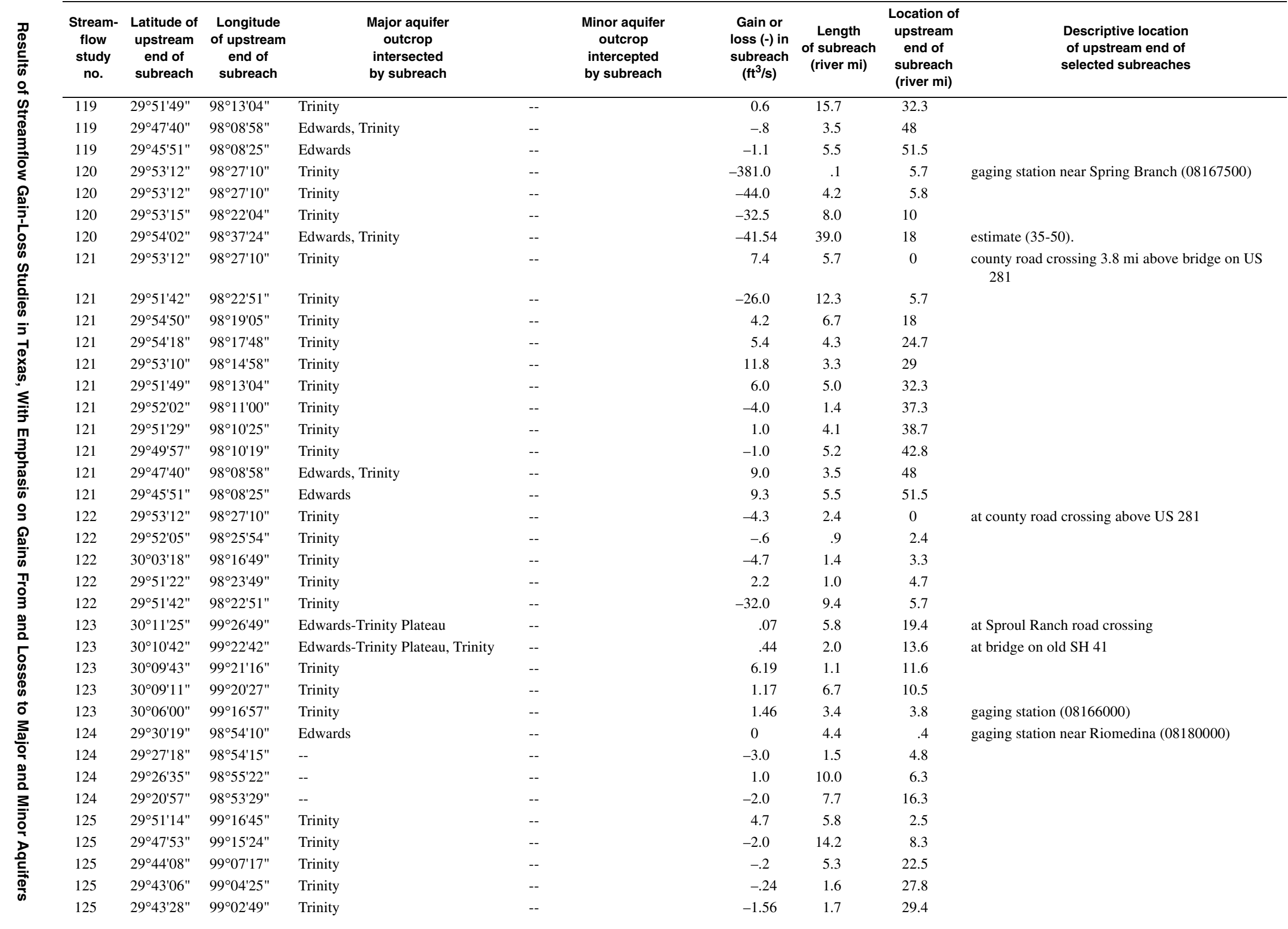


Table 4. Gains and losses from gain-loss studies in Texas-Continued

\begin{tabular}{|c|c|c|c|c|c|c|c|c|c|c|}
\hline $\begin{array}{l}\text { Stream- } \\
\text { flow } \\
\text { study } \\
\text { no. }\end{array}$ & $\begin{array}{l}\text { Latitude of } \\
\text { upstream } \\
\text { end of } \\
\text { subreach }\end{array}$ & $\begin{array}{l}\text { Longitude } \\
\text { of upstream } \\
\text { end of } \\
\text { subreach }\end{array}$ & & $\begin{array}{c}\text { Major aquifer } \\
\text { outcrop } \\
\text { intersected } \\
\text { by subreach }\end{array}$ & & $\begin{array}{c}\text { Minor aquifer } \\
\text { outcrop } \\
\text { intercepted } \\
\text { by subreach }\end{array}$ & $\begin{array}{l}\text { Gain or } \\
\text { loss }(-) \text { in } \\
\text { subreach } \\
\left(\mathrm{ft}^{3} / \mathrm{s}\right)\end{array}$ & $\begin{array}{l}\text { Length } \\
\text { of subreach } \\
\text { (river mi) }\end{array}$ & $\begin{array}{l}\text { Location of } \\
\text { upstream } \\
\text { end of } \\
\text { subreach } \\
\text { (river mi) }\end{array}$ & $\begin{array}{l}\text { Descriptive location } \\
\text { of upstream end of } \\
\text { selected subreaches }\end{array}$ \\
\hline 125 & $29^{\circ} 43^{\prime} 18^{\prime \prime}$ & $99^{\circ} 02^{\prime} 20^{\prime \prime}$ & Trinity & & -- & & -0.6 & 0.1 & 31.1 & \\
\hline 125 & $29^{\circ} 43^{\prime} 08^{\prime \prime}$ & $99^{\circ} 02^{\prime} 17^{\prime \prime}$ & Trinity & & -- & & -.3 & 1.2 & 31.2 & \\
\hline 125 & $29^{\circ} 42^{\prime} 57^{\prime \prime}$ & $99^{\circ} 00^{\prime} 44^{\prime \prime}$ & Trinity & & -- & & -.1 & 1.5 & 32.4 & \\
\hline 125 & $29^{\circ} 43^{\prime} 27^{\prime \prime}$ & $98^{\circ} 59^{\prime} 47^{\prime \prime}$ & Trinity & & -- & & -.01 & 1.8 & 33.9 & \\
\hline 125 & $29^{\circ} 42^{\prime} 35^{\prime \prime}$ & $98^{\circ} 59^{\prime} 08^{\prime \prime}$ & Trinity & & -- & & -.01 & .9 & 35.7 & \\
\hline 125 & $29^{\circ} 42^{\prime} 28^{\prime \prime}$ & $98^{\circ} 58^{\prime} 21^{\prime \prime}$ & Trinity & & -- & & .46 & .7 & 36.6 & \\
\hline 125 & $29^{\circ} 42^{\prime} 00^{\prime \prime}$ & $98^{\circ} 58^{\prime 2} 24^{\prime \prime}$ & Trinity & & -- & & -.59 & 1.7 & 37.3 & \\
\hline 125 & $29^{\circ} 42^{\prime} 00^{\prime \prime}$ & $98^{\circ} 58^{\prime} 24^{\prime \prime}$ & Trinity & & -- & & -1.58 & 4.0 & 39 & \\
\hline 125 & $29^{\circ} 42^{\prime} 00^{\prime \prime}$ & $98^{\circ} 58^{\prime} 24^{\prime \prime}$ & Trinity & & -- & & -2.19 & 3.1 & 43 & \\
\hline 125 & $29^{\circ} 42^{\prime} 00^{\prime \prime}$ & $98^{\circ} 58^{\prime} 24^{\prime \prime}$ & Trinity & & -- & & -.62 & 2.1 & 46.1 & \\
\hline 126 & 2951'36" & $99^{\circ} 24^{\prime} 14^{\prime \prime}$ & Trinity & & -- & & 2.4 & 2.0 & 0 & $5.3 \mathrm{mi}$ northwest of Lima \\
\hline 126 & $29^{\circ} 52^{\prime} 28^{\prime \prime}$ & $99^{\circ} 23^{\prime} 27^{\prime \prime}$ & Trinity & & -- & & 2.0 & 2.5 & 2 & \\
\hline 126 & $29^{\circ} 51^{\prime} 33^{\prime \prime}$ & $99^{\circ} 22^{\prime} 06^{\prime \prime}$ & Trinity & & -- & & -2.0 & 6.6 & 4.5 & \\
\hline 126 & $29^{\circ} 50^{\prime} 41^{\prime \prime}$ & $99^{\circ} 17^{\prime} 45^{\prime \prime}$ & Trinity & & -- & & -1.2 & 1.8 & 11.1 & \\
\hline 126 & $29^{\circ} 51^{\prime} 14^{\prime \prime}$ & $99^{\circ} 16^{\prime} 44^{\prime \prime}$ & Trinity & & -- & & 4.8 & 3.2 & 12.9 & \\
\hline 126 & $29^{\circ} 49^{\prime} 30^{\prime \prime}$ & $99^{\circ} 15^{\prime} 36^{\prime \prime}$ & Trinity & & -- & & .2 & 6.7 & 16.1 & \\
\hline 126 & $29^{\circ} 46^{\prime} 31^{\prime \prime}$ & $99^{\circ} 12^{\prime} 12^{\prime \prime}$ & Trinity & & -- & & 1.7 & 3.1 & 22.8 & \\
\hline 126 & $29^{\circ} 45^{\prime} 33^{\prime \prime}$ & $99^{\circ} 10^{\prime} 47^{\prime \prime}$ & Trinity & & -- & & -1.1 & 8.3 & 25.9 & \\
\hline 126 & $29^{\circ} 43^{\prime} 12^{\prime \prime}$ & $99^{\circ} 05^{\prime} 25^{\prime \prime}$ & Trinity & & -- & & -.5 & 4.4 & 34.2 & \\
\hline 126 & $29^{\circ} 43^{\prime} 10^{\prime \prime}$ & $99^{\circ} 01 ' 23^{\prime \prime}$ & Trinity & & -- & & -2.1 & 3.1 & 38.6 & \\
\hline 126 & $29^{\circ} 40^{\prime} 32^{\prime \prime}$ & 9858'33" & Trinity & & -- & & -2.0 & 2.1 & 41.7 & gaging station (08179000) \\
\hline 127 & $29^{\circ} 51^{\prime} 21^{\prime \prime}$ & $99^{\circ} 18^{\prime} 45^{\prime \prime}$ & Trinity & & -- & & 0 & 2.5 & 0 & $0.5 \mathrm{mi}$ below Rocky Creek \\
\hline 127 & $29^{\circ} 51^{\prime} 14^{\prime \prime}$ & $99^{\circ} 16^{\prime} 44^{\prime \prime}$ & Trinity & & -- & & 1.5 & 5.8 & 2.5 & \\
\hline 127 & $29^{\circ} 47^{\prime} 53^{\prime \prime}$ & $99^{\circ} 15^{\prime} 24^{\prime \prime}$ & Trinity & & -- & & -1.35 & 14.2 & 8.3 & \\
\hline 127 & $29^{\circ} 44^{\prime} 08^{\prime \prime}$ & $99^{\circ} 07^{\prime} 17^{\prime \prime}$ & Trinity & & -- & & -.14 & 5.3 & 22.5 & \\
\hline 127 & $29^{\circ} 43^{\prime} 06^{\prime \prime}$ & $99^{\circ} 04^{\prime} 25^{\prime \prime}$ & Trinity & & -- & & -.01 & 1.7 & 27.8 & \\
\hline 127 & $29^{\circ} 43^{\prime} 34^{\prime \prime}$ & $99^{\circ} 02^{\prime} 44^{\prime \prime}$ & Trinity & & -- & & 0 & 2.9 & 29.5 & \\
\hline 127 & $29^{\circ} 42^{\prime} 57^{\prime \prime}$ & $99^{\circ} 00^{\prime} 44^{\prime \prime}$ & Trinity & & -- & & 0 & 1.5 & 32.4 & \\
\hline 127 & $29^{\circ} 43^{\prime} 27^{\prime \prime}$ & $98^{\circ} 59^{\prime} 47^{\prime \prime}$ & Trinity & & -- & & -.01 & 1.8 & 33.9 & \\
\hline 127 & $29^{\circ} 42^{\prime} 35^{\prime \prime}$ & $98^{\circ} 59^{\prime} 08^{\prime \prime}$ & Trinity & & -- & & 0 & .9 & 35.7 & \\
\hline 127 & $29^{\circ} 42^{\prime} 28^{\prime \prime}$ & $98^{\circ} 58^{\prime} 21^{\prime \prime}$ & Trinity & & -- & & .24 & .7 & 36.6 & \\
\hline 127 & $29^{\circ} 40^{\prime} 23^{\prime \prime}$ & $98^{\circ} 58^{\prime} 23^{\prime \prime}$ & Trinity & & -- & & .1 & 1.7 & 37.3 & \\
\hline 127 & $29^{\circ} 40^{\prime} 51^{\prime \prime}$ & $98^{\circ} 57^{\prime} 18^{\prime \prime}$ & Trinity & & -- & & 0 & .8 & 39 & gaging station (08179000) near Pipe Creek \\
\hline 127 & $29^{\circ} 40^{\prime} 03^{\prime \prime}$ & $98^{\circ} 58^{\prime} 15^{\prime \prime}$ & Trinity & & -- & & -.3 & 3.2 & 39.8 & \\
\hline 127 & $29^{\circ} 38^{\prime} 17^{\prime \prime}$ & $98^{\circ} 58^{\prime} 04^{\prime \prime}$ & Trinity & & -- & & -.7 & .5 & 43 & \\
\hline 127 & $29^{\circ} 37^{\prime} 41^{\prime \prime}$ & $98^{\circ} 58^{\prime} 00^{\prime \prime}$ & Trinity & & -- & & 0 & .6 & 43.5 & \\
\hline 127 & $29^{\circ} 37^{\prime} 39^{\prime \prime}$ & $98^{\circ} 58^{\prime} 34^{\prime \prime}$ & Trinity & & -- & & 0 & .7 & 44.1 & \\
\hline 127 & $29^{\circ} 37^{\prime} 29^{\prime \prime}$ & $98^{\circ} 58^{\prime} 53^{\prime \prime}$ & Trinity & & -- & & 0 & 1.3 & 44.8 & \\
\hline
\end{tabular}


œ Table 4. Gains and losses from gain-loss studies in Texas-Continued

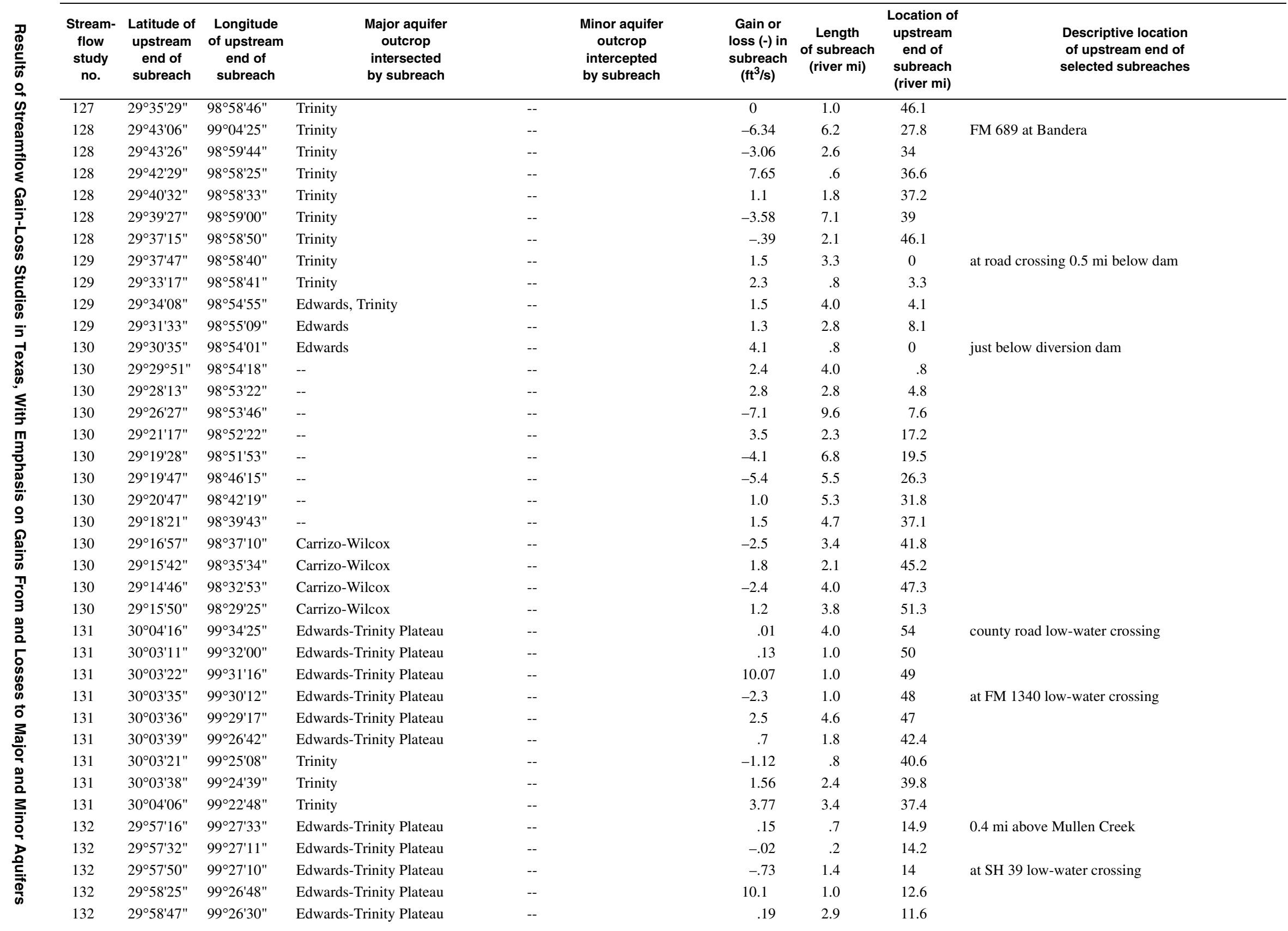


Table 4. Gains and losses from gain-loss studies in Texas-Continued

\begin{tabular}{|c|c|c|c|c|c|c|c|c|c|}
\hline $\begin{array}{l}\text { Stream- } \\
\text { flow } \\
\text { study } \\
\text { no. }\end{array}$ & $\begin{array}{l}\text { Latitude of } \\
\text { upstream } \\
\text { end of } \\
\text { subreach }\end{array}$ & $\begin{array}{l}\text { Longitude } \\
\text { of upstream } \\
\text { end of } \\
\text { subreach }\end{array}$ & $\begin{array}{c}\text { Major aquifer } \\
\text { outcrop } \\
\text { intersected } \\
\text { by subreach }\end{array}$ & & $\begin{array}{c}\text { Minor aquifer } \\
\text { outcrop } \\
\text { intercepted } \\
\text { by subreach }\end{array}$ & $\begin{array}{c}\text { Gain or } \\
\text { loss }(-) \text { in } \\
\text { subreach } \\
\left(\mathrm{ft}^{3} / \mathbf{s}\right)\end{array}$ & $\begin{array}{l}\text { Length } \\
\text { of subreach } \\
\text { (river mi) }\end{array}$ & $\begin{array}{l}\text { Location of } \\
\text { upstream } \\
\text { end of } \\
\text { subreach } \\
\text { (river mi) }\end{array}$ & $\begin{array}{l}\text { Descriptive location } \\
\text { of upstream end of } \\
\text { selected subreaches }\end{array}$ \\
\hline 132 & $30^{\circ} 00^{\prime} 19^{\prime \prime}$ & $99^{\circ} 24^{\prime} 16^{\prime \prime}$ & Edwards-Trinity Plateau & -- & & 0.65 & 1.1 & 8.7 & \\
\hline 132 & $30^{\circ} 00^{\prime} 15^{\prime \prime}$ & $99^{\circ} 23^{\prime} 42^{\prime \prime}$ & Edwards-Trinity Plateau & -- & & .19 & 1.9 & 7.6 & \\
\hline 132 & $30^{\circ} 00^{\prime} 42^{\prime \prime}$ & $99^{\circ} 22^{\prime} 24^{\prime \prime}$ & Edwards-Trinity Plateau, Trinity & -- & & 1.33 & 3.0 & 5.7 & \\
\hline 133 & $29^{\circ} 58^{\prime} 37^{\prime \prime}$ & $99^{\circ} 13^{\prime} 24^{\prime \prime}$ & Trinity & -- & & 9.14 & 3.5 & 11.2 & at bridge on FM 1273 \\
\hline 133 & $29^{\circ} 57^{\prime} 47^{\prime \prime}$ & $99^{\circ} 10^{\prime} 54^{\prime \prime}$ & Trinity & -- & & 2.82 & 5.7 & 7.7 & \\
\hline 134 & $29^{\circ} 53^{\prime} 03^{\prime \prime}$ & $99^{\circ} 08^{\prime} 53^{\prime \prime}$ & Trinity & -- & & 5.63 & 4.2 & 11.2 & at low-water crossing on county road \\
\hline 134 & $29^{\circ} 53^{\prime} 46^{\prime \prime}$ & $99^{\circ} 05^{\prime} 36^{\prime \prime}$ & Trinity & -- & & 1.3 & 1.3 & 7 & \\
\hline 135 & $29^{\circ} 26^{\prime} 29^{\prime \prime}$ & $96^{\circ} 56^{\prime} 40^{\prime \prime}$ & Gulf Coast & -- & & .96 & 5.9 & 77.7 & $200 \mathrm{ft}$ below gaging station ( 08163500$)$ \\
\hline 135 & $29^{\circ} 22^{\prime} 52^{\prime \prime}$ & $96^{\circ} 54^{\prime} 36^{\prime \prime}$ & Gulf Coast & -- & & .87 & 4.1 & 71.8 & \\
\hline 135 & $29^{\circ} 20^{\prime} 56^{\prime \prime}$ & $96^{\circ} 54^{\prime} 55^{\prime \prime}$ & Gulf Coast & -- & & .13 & 1.7 & 67.7 & \\
\hline 135 & $29^{\circ} 20^{\prime} 14^{\prime \prime}$ & $96^{\circ} 54^{\prime} 26^{\prime \prime}$ & Gulf Coast & -- & & 1.2 & 3.7 & 66 & \\
\hline 135 & $29^{\circ} 18^{\prime} 38^{\prime \prime}$ & $96^{\circ} 54^{\prime} 18^{\prime \prime}$ & Gulf Coast & -- & & 2.1 & 4.8 & 62.3 & \\
\hline 135 & $29^{\circ} 18^{\prime} 57^{\prime \prime}$ & $96^{\circ} 51^{\prime} 14^{\prime \prime}$ & Gulf Coast & -- & & -.4 & 8.2 & 57.5 & \\
\hline 135 & $29^{\circ} 14^{\prime} 54^{\prime \prime}$ & $96^{\circ} 51^{\prime} 11^{\prime \prime}$ & Gulf Coast & -- & & 2.9 & 8.7 & 49.3 & \\
\hline 135 & $29^{\circ} 09^{\prime} 28^{\prime \prime}$ & $96^{\circ} 52^{\prime} 27^{\prime \prime}$ & Gulf Coast & -- & & 1.47 & 7.3 & 40.6 & \\
\hline 135 & $29^{\circ} 05^{\prime} 05^{\prime \prime}$ & $96^{\circ} 49^{\prime} 01^{\prime \prime}$ & Gulf Coast & -- & & 3.2 & 4.1 & 33.3 & \\
\hline 135 & $29^{\circ} 03^{\prime} 32^{\prime \prime}$ & $96^{\circ} 46^{\prime} 39^{\prime \prime}$ & Gulf Coast & -- & & 1.2 & 3.9 & 29.2 & \\
\hline 135 & $29^{\circ} 02^{\prime} 29^{\prime \prime}$ & $96^{\circ} 43^{\prime} 47^{\prime \prime}$ & Gulf Coast & -- & & 1.7 & 3.1 & 25.3 & \\
\hline 135 & $29^{\circ} 00^{\prime} 40^{\prime \prime}$ & $96^{\circ} 42^{\prime} 29^{\prime \prime}$ & Gulf Coast & -- & & .5 & 5.4 & 22.2 & \\
\hline 135 & $28^{\circ} 57^{\prime} 38^{\prime \prime}$ & $96^{\circ} 41^{\prime} 12^{\prime \prime}$ & Gulf Coast & -- & & 2.4 & 4.5 & 16.8 & \\
\hline 136 & $28^{\circ} 59^{\prime} 50^{\prime \prime}$ & $96^{\prime} 42^{\prime} 30^{\prime \prime}$ & Gulf Coast & -- & & 2.0 & 3.0 & 0 & \\
\hline 136 & $28^{\circ} 57^{\prime} 32^{\prime \prime}$ & $96^{\circ} 41^{\prime} 10^{\prime \prime}$ & Gulf Coast & -- & & -.2 & 1.1 & 3 & at US 59 gaging station $(08164000)$ \\
\hline 136 & $28^{\circ} 56^{\prime} 34^{\prime \prime}$ & $96^{\circ} 41^{\prime} 18^{\prime \prime}$ & Gulf Coast & -- & & 2.0 & 2.4 & 4.1 & \\
\hline 136 & $28^{\circ} 55^{\prime} 08^{\prime \prime}$ & $96^{\circ} 39^{\prime} 43^{\prime \prime}$ & Gulf Coast & -- & & 1.9 & 3.0 & 6.5 & \\
\hline 137 & $28^{\circ} 59^{\prime} 49^{\prime \prime}$ & $96^{\circ} 42^{\prime} 30^{\prime \prime}$ & Gulf Coast & -- & & 1.48 & 3.0 & 0 & \\
\hline 137 & $28^{\circ} 57^{\prime} 30^{\prime \prime}$ & $96^{\circ} 41^{\prime} 10^{\prime \prime}$ & Gulf Coast & -- & & .4 & 1.1 & 3 & at US 59 gaging station (08164000) \\
\hline 137 & $28^{\circ} 56^{\prime} 34^{\prime \prime}$ & $96^{\circ} 41^{\prime} 17^{\prime \prime}$ & Gulf Coast & -- & & 1.74 & 2.4 & 4.1 & \\
\hline 137 & $28^{\circ} 55^{\prime} 08^{\prime \prime}$ & $96^{\circ} 39^{\prime} 43^{\prime \prime}$ & Gulf Coast & -- & & .86 & 3.0 & 6.5 & \\
\hline 138 & $29^{\circ} 27^{\prime} 57^{\prime \prime}$ & $96^{\circ} 48^{\prime} 38^{\prime \prime}$ & Gulf Coast & -- & & -.26 & 2.8 & 78.2 & $400 \mathrm{ft}$ below gaging station ( 08164300$)$ \\
\hline 138 & $29^{\circ} 25^{\prime} 54^{\prime \prime}$ & $96^{\circ} 48^{\prime} 02^{\prime \prime}$ & Gulf Coast & -- & & 1.7 & 5.4 & 75.4 & \\
\hline 138 & $29^{\circ} 22^{\prime} 55^{\prime \prime}$ & $96^{\circ} 46^{\prime} 44^{\prime \prime}$ & Gulf Coast & -- & & -6.39 & 8.2 & 70 & \\
\hline 138 & $29^{\circ} 19^{\prime} 16^{\prime \prime}$ & $96^{\circ} 42^{\prime 2} 29^{\prime \prime}$ & Gulf Coast & -- & & 7.1 & 4.4 & 61.8 & \\
\hline 138 & $29^{\circ} 16^{\prime} 21^{\prime \prime}$ & $96^{\circ} 42^{\prime} 59^{\prime \prime}$ & Gulf Coast & -- & & 3.33 & 8.7 & 57.4 & \\
\hline 138 & $29^{\circ} 11^{\prime} 50^{\prime \prime}$ & $96^{\circ} 44^{\prime} 24^{\prime \prime}$ & Gulf Coast & -- & & 2.7 & 4.8 & 48.7 & \\
\hline 138 & $29^{\circ} 08^{\prime} 08^{\prime \prime}$ & $96^{\circ} 44^{\prime} 39^{\prime \prime}$ & Gulf Coast & -- & & -1.0 & 4.9 & 43.9 & \\
\hline 138 & $29^{\circ} 05^{\prime} 47^{\prime \prime}$ & $96^{\circ} 43^{\prime} 07^{\prime \prime}$ & Gulf Coast & -- & & 1.9 & 5.9 & 39 & \\
\hline 138 & $29^{\circ} 03^{\prime} 55^{\prime \prime}$ & $96^{\circ} 40^{\prime 2} 24^{\prime \prime}$ & Gulf Coast & -- & & 1.1 & 5.3 & 33.1 & \\
\hline 138 & $29^{\circ} 01^{\prime} 52^{\prime \prime}$ & $96^{\circ} 37^{\prime} 18^{\prime \prime}$ & Gulf Coast & -- & & -.53 & 5.8 & 27.8 & \\
\hline
\end{tabular}


$8 \quad$ Table 4. Gains and losses from gain-loss studies in Texas-Continued

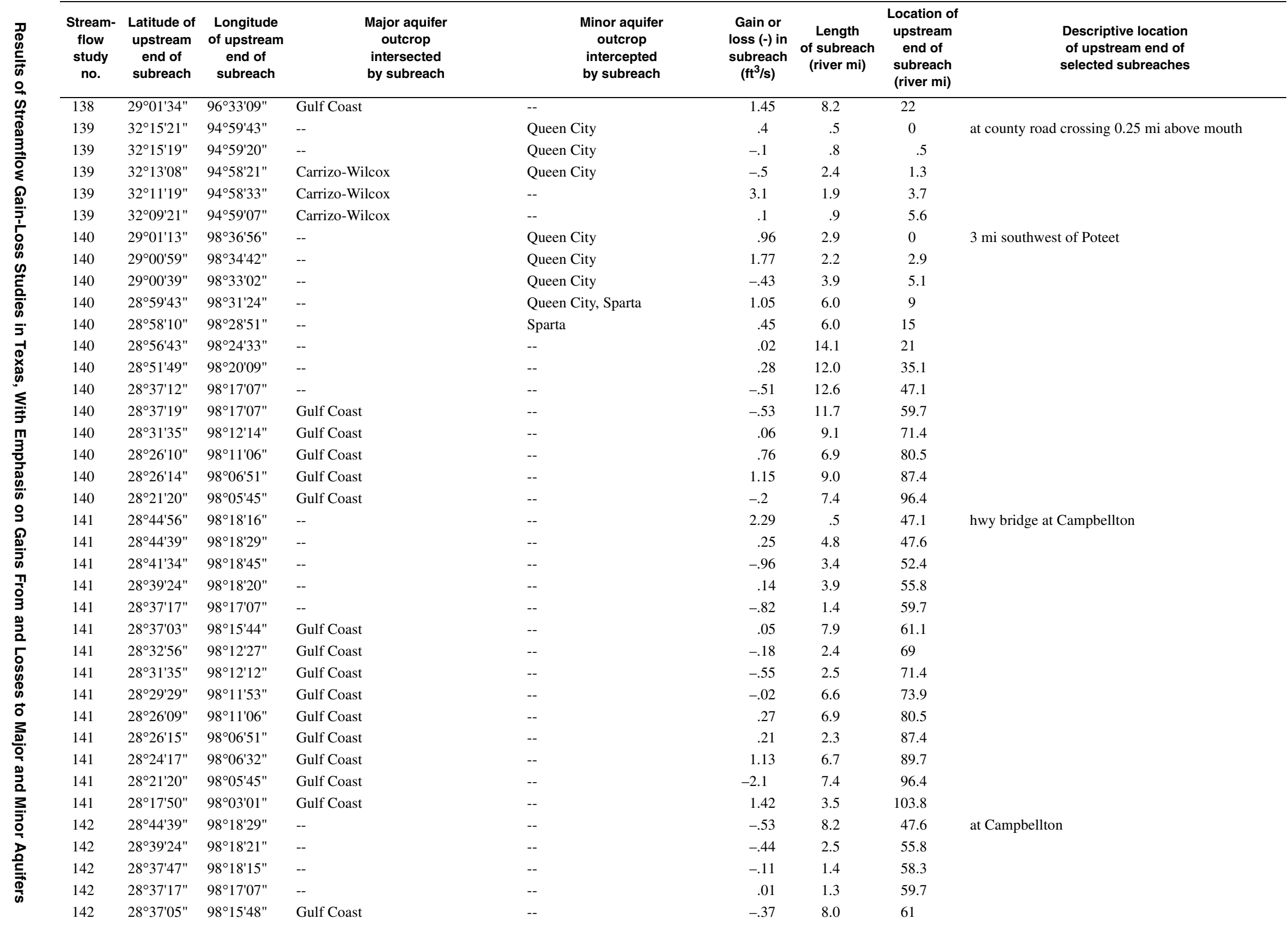


Table 4. Gains and losses from gain-loss studies in Texas-Continued

\begin{tabular}{|c|c|c|c|c|c|c|c|c|}
\hline $\begin{array}{l}\text { Stream- } \\
\text { flow } \\
\text { study } \\
\text { no. }\end{array}$ & $\begin{array}{l}\text { Latitude of } \\
\text { upstream } \\
\text { end of } \\
\text { subreach }\end{array}$ & $\begin{array}{l}\text { Longitude } \\
\text { of upstream } \\
\text { end of } \\
\text { subreach }\end{array}$ & $\begin{array}{c}\text { Major aquifer } \\
\text { outcrop } \\
\text { intersected } \\
\text { by subreach }\end{array}$ & $\begin{array}{c}\text { Minor aquifer } \\
\text { outcrop } \\
\text { intercepted } \\
\text { by subreach }\end{array}$ & $\begin{array}{l}\text { Gain or } \\
\text { loss }(-) \text { in } \\
\text { subreach } \\
\left(\mathrm{ft}^{3} / \mathrm{s}\right)\end{array}$ & $\begin{array}{l}\text { Length } \\
\text { of subreach } \\
\text { (river mi) }\end{array}$ & $\begin{array}{l}\text { Location of } \\
\text { upstream } \\
\text { end of } \\
\text { subreach } \\
\text { (river mi) }\end{array}$ & $\begin{array}{l}\text { Descriptive location } \\
\text { of upstream end of } \\
\text { selected subreaches }\end{array}$ \\
\hline 142 & $28^{\circ} 32^{\prime} 56^{\prime \prime}$ & $98^{\circ} 12^{\prime} 27^{\prime \prime}$ & Gulf Coast & -- & 0.09 & 2.4 & 69 & \\
\hline 142 & $28^{\circ} 31^{\prime} 35^{\prime \prime}$ & $98^{\circ} 12^{\prime} 12^{\prime \prime}$ & Gulf Coast & -- & -.5 & 9.1 & 71.4 & \\
\hline 142 & $28^{\circ} 26^{\prime} 09^{\prime \prime}$ & $98^{\circ} 11^{\prime} 06^{\prime \prime}$ & Gulf Coast & -- & .79 & 6.9 & 80.5 & \\
\hline 142 & $28^{\circ} 26^{\prime} 14^{\prime \prime}$ & $98^{\circ} 06^{\prime} 51^{\prime \prime}$ & Gulf Coast & -- & .55 & 2.3 & 87.4 & \\
\hline 142 & $28^{\circ} 24^{\prime} 17^{\prime \prime}$ & $98^{\circ} 06^{\prime} 32^{\prime \prime}$ & Gulf Coast & -- & .72 & 6.7 & 89.7 & \\
\hline 142 & $28^{\circ} 21^{\prime} 19^{\prime \prime}$ & $98^{\circ} 05^{\prime} 45^{\prime \prime}$ & Gulf Coast & -- & .5 & 7.4 & 96.4 & \\
\hline 142 & $28^{\circ} 17^{\prime} 50^{\prime \prime}$ & $98^{\circ} 03^{\prime} 00^{\prime \prime}$ & Gulf Coast & -- & -.93 & 2.1 & 103.8 & \\
\hline 143 & $29^{\circ} 38^{\prime} 38^{\prime \prime}$ & $99^{\circ} 52^{\prime} 12^{\prime \prime}$ & Edwards-Trinity Plateau & -- & .2 & .7 & 8.2 & \\
\hline 143 & $29^{\circ} 38^{\prime} 17^{\prime \prime}$ & $99^{\circ} 51^{\prime 2} 23^{\prime \prime}$ & Edwards-Trinity Plateau & -- & -.2 & 2.2 & 8.9 & \\
\hline 143 & $29^{\circ} 36^{\prime} 39^{\prime \prime}$ & $99^{\circ} 51^{\prime} 08^{\prime \prime}$ & Edwards-Trinity Plateau & -- & 1.0 & 1.1 & 11.1 & \\
\hline 143 & $29^{\circ} 35^{\prime} 55^{\prime \prime}$ & $99^{\circ} 51^{\prime} 03 "$ & Edwards-Trinity Plateau & -- & -1.0 & .9 & 12.2 & \\
\hline 143 & $29^{\circ} 35^{\prime} 13^{\prime \prime}$ & $99^{\circ} 50^{\prime} 45^{\prime \prime}$ & Edwards-Trinity Plateau & -- & .4 & .2 & 13.1 & \\
\hline 143 & $29^{\circ} 35^{\prime} 02^{\prime \prime}$ & $99^{\circ} 50^{\prime} 44^{\prime \prime}$ & Edwards-Trinity Plateau & -- & -.4 & 6 & 13.3 & \\
\hline 143 & $29^{\circ} 34^{\prime} 27^{\prime \prime}$ & $99^{\circ} 50^{\prime} 24^{\prime \prime}$ & Edwards-Trinity Plateau & -- & .1 & .3 & 13.9 & \\
\hline 143 & $29^{\circ} 34^{\prime} 20^{\prime \prime}$ & $99^{\circ} 50^{\prime} 43^{\prime \prime}$ & Edwards-Trinity Plateau & -- & .9 & 1.1 & 14.2 & \\
\hline 143 & $29^{\circ} 33^{\prime} 40^{\prime \prime}$ & $99^{\circ} 50^{\prime} 54^{\prime \prime}$ & Edwards-Trinity Plateau & -- & .29 & 1.1 & 15.3 & \\
\hline 143 & $29^{\circ} 32^{\prime} 45^{\prime \prime}$ & $99^{\circ} 50^{\prime} 58^{\prime \prime}$ & Edwards-Trinity Plateau & -- & -.05 & 2.3 & 16.4 & \\
\hline 143 & $29^{\circ} 32^{\prime} 27^{\prime \prime}$ & $99^{\circ} 50^{\prime} 51 "$ & Edwards, Edwards-Trinity Plateau & -- & -.42 & 3.9 & 18.7 & \\
\hline 143 & $29^{\circ} 30^{\prime} 49^{\prime \prime}$ & $99^{\circ} 48^{\prime} 40^{\prime \prime}$ & Edwards, Edwards-Trinity Plateau & -- & .66 & 1.5 & 22.6 & \\
\hline 143 & $29^{\circ} 30^{\prime} 01^{\prime \prime}$ & $99^{\circ} 48^{\prime} 39^{\prime \prime}$ & Edwards & -- & -.24 & 2.0 & 24.1 & \\
\hline 143 & $29^{\circ} 30^{\prime} 15^{\prime \prime}$ & $99^{\circ} 46^{\prime} 51^{\prime \prime}$ & Edwards & -- & -1.99 & 2.1 & 26.1 & gaging station (08196000) at river mile 26.2 \\
\hline 144 & $29^{\circ} 42^{\prime} 50^{\prime \prime}$ & $99^{\circ} 53^{\prime} 11^{\prime \prime}$ & Edwards-Trinity Plateau & -- & .56 & .8 & 0 & \\
\hline 144 & $29^{\circ} 42^{\prime} 02^{\prime \prime}$ & $99^{\circ} 53^{\prime 2} 29^{\prime \prime}$ & Edwards-Trinity Plateau & -- & -.32 & 1.7 & .8 & \\
\hline 144 & $29^{\circ} 41^{\prime} 18^{\prime \prime}$ & $99^{\circ} 53^{\prime} 17^{\prime \prime}$ & Edwards-Trinity Plateau & -- & -2.24 & 3.7 & 2.5 & \\
\hline 144 & $29^{\circ} 40^{\prime} 10^{\prime \prime}$ & $99^{\circ} 52^{\prime} 01^{\prime \prime}$ & Edwards-Trinity Plateau & -- & .8 & 1.2 & 6.2 & \\
\hline 144 & $29^{\circ} 39^{\prime} 14^{\prime \prime}$ & $99^{\circ} 52^{\prime} 08^{\prime \prime}$ & Edwards-Trinity Plateau & -- & -.6 & .3 & 7.4 & \\
\hline 144 & $29^{\circ} 39^{\prime} 00^{\prime \prime}$ & $99^{\circ} 52^{\prime} 19^{\prime \prime}$ & Edwards-Trinity Plateau & -- & -.2 & .5 & 7.7 & \\
\hline 144 & $29^{\circ} 38^{\prime} 38^{\prime \prime}$ & $99^{\circ} 52^{\prime} 12^{\prime \prime}$ & Edwards-Trinity Plateau & -- & 1.0 & .7 & 8.2 & \\
\hline 144 & $29^{\circ} 38^{\prime} 17^{\prime \prime}$ & $99^{\circ} 51^{\prime} 24^{\prime \prime}$ & Edwards-Trinity Plateau & -- & -1.0 & 2.2 & 8.9 & \\
\hline 144 & $29^{\circ} 36^{\prime} 39^{\prime \prime}$ & $99^{\circ} 51^{\prime} 09^{\prime \prime}$ & Edwards-Trinity Plateau & -- & 1.0 & 1.1 & 11.1 & \\
\hline 144 & $29^{\circ} 35^{\prime} 55^{\prime \prime}$ & $99^{\circ} 51^{\prime} 03^{\prime \prime}$ & Edwards-Trinity Plateau & -- & -.8 & .8 & 12.2 & \\
\hline 144 & $29^{\circ} 35^{\prime} 26^{\prime \prime}$ & $99^{\circ} 50^{\prime} 45^{\prime \prime}$ & Edwards-Trinity Plateau & -- & 1.3 & .2 & 13 & \\
\hline 144 & $29^{\circ} 35^{\prime} 07^{\prime \prime}$ & $99^{\circ} 50^{\prime} 46^{\prime \prime}$ & Edwards-Trinity Plateau & -- & -1.0 & .3 & 13.2 & \\
\hline 144 & $29^{\circ} 34^{\prime} 48^{\prime \prime}$ & $99^{\circ} 50^{\prime} 38^{\prime \prime}$ & Edwards-Trinity Plateau & -- & -.5 & .2 & 13.5 & \\
\hline 144 & $29^{\circ} 34^{\prime} 38^{\prime \prime}$ & $99^{\circ} 50^{\prime} 27^{\prime \prime}$ & Edwards-Trinity Plateau & -- & 1.0 & .8 & 13.7 & \\
\hline 144 & $29^{\circ} 34^{\prime} 10^{\prime \prime}$ & $99^{\circ} 50^{\prime} 53^{\prime \prime}$ & Edwards-Trinity Plateau & -- & 1.82 & .8 & 14.5 & \\
\hline 144 & $29^{\circ} 33^{\prime} 35^{\prime \prime}$ & $99^{\circ} 50^{\prime} 58^{\prime \prime}$ & Edwards-Trinity Plateau & -- & -1.14 & 3.4 & 15.3 & \\
\hline 144 & $29^{\circ} 32^{\prime} 16^{\prime \prime}$ & 9949'59" & Edwards, Edwards-Trinity Plateau & -- & -.98 & 5.4 & 18.7 & \\
\hline
\end{tabular}


Table 4. Gains and losses from gain-loss studies in Texas-Continued

\begin{tabular}{|c|c|c|c|c|c|c|c|c|c|}
\hline $\begin{array}{l}\text { Stream- } \\
\text { flow } \\
\text { study } \\
\text { no. }\end{array}$ & $\begin{array}{l}\text { Latitude of } \\
\text { upstream } \\
\text { end of } \\
\text { subreach }\end{array}$ & $\begin{array}{l}\text { Longitude } \\
\text { of upstream } \\
\text { end of } \\
\text { subreach }\end{array}$ & $\begin{array}{c}\text { Major aquifer } \\
\text { outcrop } \\
\text { intersected } \\
\text { by subreach }\end{array}$ & & $\begin{array}{c}\text { Minor aquifer } \\
\text { outcrop } \\
\text { intercepted } \\
\text { by subreach }\end{array}$ & $\begin{array}{c}\text { Gain or } \\
\text { loss }(-) \text { in } \\
\text { subreach } \\
\left(\mathrm{ft}^{3} / \mathrm{s}\right)\end{array}$ & $\begin{array}{l}\text { Length } \\
\text { of subreach } \\
\text { (river mi) }\end{array}$ & $\begin{array}{l}\text { Location of } \\
\text { upstream } \\
\text { end of } \\
\text { subreach } \\
\text { (river mi) }\end{array}$ & $\begin{array}{l}\text { Descriptive location } \\
\text { of upstream end of } \\
\text { selected subreaches }\end{array}$ \\
\hline 144 & $29^{\circ} 30^{\prime} 01^{\prime \prime}$ & $99^{\circ} 48^{\prime} 39^{\prime \prime}$ & Edwards & -- & & 0.36 & 2.0 & 24.1 & \multirow{13}{*}{ gaging station (08196000) at river mile 26.2} \\
\hline 144 & $29^{\circ} 30^{\prime} 15^{\prime \prime}$ & $99^{\circ} 46^{\prime} 51^{\prime \prime}$ & Edwards & -- & & -1.24 & 1.9 & 26.1 & \\
\hline 145 & $29^{\circ} 42^{\prime} 50^{\prime \prime}$ & $99^{\circ} 53^{\prime} 11 "$ & Edwards-Trinity Plateau & -- & & .91 & .8 & 0 & \\
\hline 145 & $29^{\circ} 42^{\prime} 02^{\prime \prime}$ & $99^{\circ} 53^{\prime 2} 29^{\prime \prime}$ & Edwards-Trinity Plateau & -- & & -1.01 & 1.7 & .8 & \\
\hline 145 & $29^{\circ} 41^{\prime} 10^{\prime \prime}$ & $99^{\circ} 53^{\prime} 34^{\prime \prime}$ & Edwards-Trinity Plateau & -- & & .15 & 2.3 & 2.5 & \\
\hline 145 & $29^{\circ} 40^{\prime} 53^{\prime \prime}$ & $99^{\circ} 52^{\prime} 27^{\prime \prime}$ & Edwards-Trinity Plateau & -- & & -3.74 & 1.9 & 4.8 & \\
\hline 145 & $29^{\circ} 39^{\prime} 35^{\prime \prime}$ & $99^{\circ} 51^{\prime} 45^{\prime \prime}$ & Edwards-Trinity Plateau & -- & & 4.33 & 1.0 & 6.7 & \\
\hline 145 & $29^{\circ} 39^{\prime} 00^{\prime \prime}$ & $99^{\circ} 52^{\prime} 19^{\prime \prime}$ & Edwards-Trinity Plateau & -- & & 1.1 & 4.3 & 7.7 & \\
\hline 145 & $29^{\circ} 36^{\prime} 12^{\prime \prime}$ & $99^{\circ} 50^{\prime} 45^{\prime \prime}$ & Edwards-Trinity Plateau & -- & & 5.01 & 3.3 & 12 & \\
\hline 145 & $29^{\circ} 33^{\prime} 35^{\prime \prime}$ & $99^{\circ} 50^{\prime} 58^{\prime \prime}$ & Edwards-Trinity Plateau & -- & & 4.08 & 3.4 & 15.3 & \\
\hline 145 & $29^{\circ} 32^{\prime} 16^{\prime \prime}$ & $99^{\circ} 49^{\prime} 59^{\prime \prime}$ & Edwards, Edwards-Trinity Plateau & -- & & .1 & 2.4 & 18.7 & \\
\hline 145 & $29^{\circ} 30^{\prime} 35^{\prime \prime}$ & $99^{\circ} 49^{\prime} 47^{\prime \prime}$ & Edwards & -- & & .4 & 3.0 & 21.1 & \\
\hline 145 & $29^{\circ} 29^{\circ} 58^{\prime \prime}$ & $99^{\circ} 48^{\prime} 38^{\prime \prime}$ & Edwards & -- & & -.32 & 1.9 & 24.1 & \\
\hline 145 & $29^{\circ} 30^{\prime} 15^{\prime \prime}$ & $99^{\circ} 46^{\prime} 51^{\prime \prime}$ & Edwards & -- & & -1.0 & .1 & 26 & \multirow{6}{*}{$\begin{array}{l}\text { gaging station ( } 08196000) \text { at river mile } 26.2 \\
\text { at Clark Ranch house } 6 \mathrm{mi} \text { above Reagan Wells }\end{array}$} \\
\hline 146 & $29^{\circ} 35^{\prime} 32^{\prime \prime}$ & $99^{\circ} 50^{\prime} 46^{\prime \prime}$ & Edwards-Trinity Plateau & -- & & 1.15 & 2.4 & 0 & \\
\hline 146 & $29^{\circ} 34^{\prime} 04^{\prime \prime}$ & $99^{\circ} 50^{\prime} 49^{\prime \prime}$ & Edwards-Trinity Plateau & -- & & 3.01 & 4.1 & 2.4 & \\
\hline 146 & $29^{\circ} 32^{\prime} 06^{\prime \prime}$ & $99^{\circ} 49^{\prime} 41^{\prime \prime}$ & Edwards, Edwards-Trinity Plateau & -- & & 1.58 & 4.0 & 6.5 & \\
\hline 146 & $29^{\circ} 30^{\prime} 28^{\prime \prime}$ & $99^{\circ} 48^{\prime} 31^{\prime \prime}$ & Edwards & -- & & 2.92 & 3.5 & 10.5 & \\
\hline 146 & $29^{\circ} 29^{\circ} 51^{\prime \prime}$ & $99^{\circ} 46^{\prime} 46^{\prime \prime}$ & Edwards & -- & & -9.66 & 1.5 & 14 & \\
\hline 147 & $29^{\circ} 30^{\prime} 15^{\prime \prime}$ & $99^{\circ} 46^{\prime} 51^{\prime \prime}$ & Edwards & -- & & -54.0 & 4.0 & 0 & \multirow[t]{5}{*}{ gaging station (08196000) } \\
\hline 147 & $29^{\circ} 27^{\prime} 25^{\prime \prime}$ & $99^{\circ} 45^{\prime} 30^{\prime \prime}$ & Edwards & -- & & -255.0 & 12.8 & 4 & \\
\hline 147 & $29^{\circ} 22^{\prime} 26^{\prime \prime}$ & $99^{\circ} 42^{\prime} 11^{\prime \prime}$ & -- & -- & & -83.8 & 1.4 & 16.8 & \\
\hline 147 & $29^{\circ} 21^{\prime} 29^{\prime \prime}$ & $99^{\circ} 41^{\prime} 39^{\prime \prime}$ & -- & -- & & -17.4 & 3.1 & 18.2 & \\
\hline 147 & $29^{\circ} 19^{\prime} 48^{\prime \prime}$ & $99^{\circ} 40^{\prime} 44^{\prime \prime}$ & -- & -- & & -15.41 & 3.3 & 21.3 & \\
\hline 148 & $29^{\circ} 48^{\prime} 22^{\prime \prime}$ & $99^{\circ} 39^{\prime} 28^{\prime \prime}$ & Edwards-Trinity Plateau & -- & & -.11 & 4.1 & 11 & \multirow[t]{8}{*}{$11 \mathrm{mi}$ above mouth } \\
\hline 148 & $29^{\circ} 50^{\prime} 31^{\prime \prime}$ & $99^{\circ} 39^{\prime} 52^{\prime \prime}$ & Edwards-Trinity Plateau & -- & & -.61 & .8 & 6.9 & \\
\hline 148 & $29^{\circ} 50^{\prime} 03^{\prime \prime}$ & $99^{\circ} 39^{\prime} 41^{\prime \prime}$ & Edwards-Trinity Plateau & -- & & 1.23 & .9 & 6.1 & \\
\hline 148 & $29^{\circ} 48^{\prime} 31^{\prime \prime}$ & $99^{\circ} 40^{\prime} 43^{\prime \prime}$ & Edwards-Trinity Plateau & -- & & -4.91 & 2.6 & 5.2 & \\
\hline 148 & $29^{\circ} 45^{\prime} 38^{\prime \prime}$ & $99^{\circ} 42^{\prime} 59^{\prime \prime}$ & Edwards-Trinity Plateau & -- & & -2.61 & .6 & 2.6 & \\
\hline 148 & $29^{\circ} 45^{\prime} 35^{\prime \prime}$ & $99^{\circ} 43^{\prime} 28^{\prime \prime}$ & Edwards-Trinity Plateau & -- & & 0 & 1.0 & 2 & \\
\hline 148 & $29^{\circ} 45^{\prime} 00^{\prime \prime}$ & $99^{\circ} 43^{\prime} 44^{\prime \prime}$ & Edwards-Trinity Plateau & -- & & 1.94 & .1 & 1 & \\
\hline 148 & $29^{\circ} 44^{\prime} 57^{\prime \prime}$ & $99^{\circ} 43^{\prime} 53^{\prime \prime}$ & Edwards-Trinity Plateau & -- & & -1.94 & .9 & .9 & \\
\hline 149 & $29^{\circ} 48^{\prime} 22^{\prime \prime}$ & $99^{\circ} 39^{\prime} 28^{\prime \prime}$ & Edwards-Trinity Plateau & -- & & .83 & 2.1 & 11 & \multirow[t]{6}{*}{$11 \mathrm{mi}$ above mouth } \\
\hline 149 & $29^{\circ} 51^{\prime} 36^{\prime \prime}$ & $99^{\circ} 39^{\prime} 27^{\prime \prime}$ & Edwards-Trinity Plateau & -- & & 1.72 & 2.1 & 8.9 & \\
\hline 149 & $29^{\circ} 50^{\prime} 30^{\prime \prime}$ & $99^{\circ} 40^{\prime} 00^{\prime \prime}$ & Edwards-Trinity Plateau & -- & & -.1 & 1.0 & 6.8 & \\
\hline 149 & $29^{\circ} 49^{\prime} 45^{\prime \prime}$ & $99^{\circ} 39^{\prime} 50^{\prime \prime}$ & Edwards-Trinity Plateau & -- & & -.9 & .7 & 5.8 & \\
\hline 149 & $29^{\circ} 48^{\prime} 30^{\prime \prime}$ & $99^{\circ} 40^{\prime} 44^{\prime \prime}$ & Edwards-Trinity Plateau & -- & & -5.3 & 3.1 & 5.1 & \\
\hline 149 & $29^{\circ} 45^{\prime} 35^{\prime \prime}$ & $99^{\circ} 43^{\prime} 28^{\prime \prime}$ & Edwards-Trinity Plateau & -- & & -.36 & 1.0 & 2 & \\
\hline
\end{tabular}


Table 4. Gains and losses from gain-loss studies in Texas-Continued

\begin{tabular}{|c|c|c|c|c|c|c|c|c|c|}
\hline $\begin{array}{l}\text { Stream- } \\
\text { flow } \\
\text { study } \\
\text { no. }\end{array}$ & $\begin{array}{l}\text { Latitude of } \\
\text { upstream } \\
\text { end of } \\
\text { subreach }\end{array}$ & $\begin{array}{l}\text { Longitude } \\
\text { of upstream } \\
\text { end of } \\
\text { subreach }\end{array}$ & $\begin{array}{l}\text { Major aquifer } \\
\text { outcrop } \\
\text { intersected } \\
\text { by subreach }\end{array}$ & & $\begin{array}{l}\text { Minor aquifer } \\
\text { outcrop } \\
\text { intercepted } \\
\text { by subreach }\end{array}$ & $\begin{array}{l}\text { Gain or } \\
\text { loss }(-) \text { in } \\
\text { subreach } \\
\left(\mathrm{ft}^{3} / \mathrm{s}\right)\end{array}$ & $\begin{array}{l}\text { Length } \\
\text { of subreach } \\
\text { (river mi) }\end{array}$ & $\begin{array}{l}\text { Location of } \\
\text { upstream } \\
\text { end of } \\
\text { subreach } \\
\text { (river mi) }\end{array}$ & $\begin{array}{l}\text { Descriptive location } \\
\text { of upstream end of } \\
\text { selected subreaches }\end{array}$ \\
\hline 149 & $29^{\circ} 45^{\prime} 00^{\prime \prime}$ & $99^{\circ} 43^{\prime} 44^{\prime \prime}$ & Edwards-Trinity Plateau & -- & & 0.76 & 0.1 & 1 & \\
\hline 149 & $29^{\circ} 44^{\prime} 57^{\prime \prime}$ & $99^{\circ} 43^{\prime} 53^{\prime \prime}$ & Edwards-Trinity Plateau & -- & & -.76 & .9 & .9 & \\
\hline 150 & $29^{\circ} 48^{\prime} 31^{\prime \prime}$ & $99^{\circ} 40^{\prime} 44^{\prime \prime}$ & Edwards-Trinity Plateau & -- & & -6.95 & 4.2 & 5.2 & road above Cypress Creek \\
\hline 150 & $29^{\circ} 44^{\prime} 58^{\prime \prime}$ & $99^{\circ} 43^{\prime} 51^{\prime \prime}$ & Edwards-Trinity Plateau & -- & & 3.53 & .5 & 1 & \\
\hline 150 & $29^{\circ} 44^{\prime} 56^{\prime \prime}$ & $99^{\circ} 43^{\prime} 54^{\prime \prime}$ & Edwards-Trinity Plateau & -- & & -3.53 & .5 & .5 & \\
\hline 151 & $29^{\circ} 48^{\prime} 32^{\prime \prime}$ & $99^{\circ} 40^{\prime} 44^{\prime \prime}$ & Edwards-Trinity Plateau & -- & & -9.52 & 4.2 & 5.2 & $5.2 \mathrm{mi}$ above Frio River \\
\hline 151 & $29^{\circ} 44^{\prime} 58^{\prime \prime}$ & $99^{\circ} 43^{\prime} 52^{\prime \prime}$ & Edwards-Trinity Plateau & -- & & 3.19 & .5 & 1 & \\
\hline 151 & $29^{\circ} 44^{\prime} 56^{\prime \prime}$ & $99^{\circ} 43^{\prime} 54^{\prime \prime}$ & Edwards-Trinity Plateau & -- & & -2.16 & .5 & .5 & \\
\hline 152 & $29^{\circ} 50^{\prime} 31^{\prime \prime}$ & $99^{\circ} 39^{\prime} 50^{\prime \prime}$ & Edwards-Trinity Plateau & -- & & -1.11 & .1 & 0 & just above Big Spring Creek \\
\hline 152 & $29^{\circ} 50^{\prime} 31^{\prime \prime}$ & $99^{\circ} 39^{\prime} 44^{\prime \prime}$ & Edwards-Trinity Plateau & -- & & 2.64 & 3.2 & .1 & \\
\hline 152 & $29^{\circ} 48^{\prime} 57^{\prime \prime}$ & $99^{\circ} 40^{\prime} 32^{\prime \prime}$ & Edwards-Trinity Plateau & -- & & -2.53 & 5.5 & 3.3 & \\
\hline 152 & $29^{\circ} 45^{\prime} 34^{\prime \prime}$ & $99^{\circ} 43^{\prime} 00^{\prime \prime}$ & Edwards-Trinity Plateau & -- & & -8.91 & 2.0 & 8.8 & \\
\hline 152 & $29^{\circ} 44^{\prime} 03^{\prime \prime}$ & $99^{\circ} 44^{\prime} 57^{\prime \prime}$ & Edwards-Trinity Plateau & -- & & 4.53 & 1.0 & 10.8 & \\
\hline 152 & $29^{\circ} 42^{\prime} 56^{\prime \prime}$ & $99^{\circ} 45^{\prime} 22^{\prime \prime}$ & Edwards-Trinity Plateau & -- & & 6.57 & .5 & 11.8 & \\
\hline 152 & $29^{\circ} 42^{\prime} 18^{\prime \prime}$ & $99^{\circ} 45^{\prime} 11^{\prime \prime}$ & Edwards-Trinity Plateau & -- & & 2.0 & 2.5 & 12.3 & \\
\hline 152 & $29^{\circ} 40^{\prime} 57^{\prime \prime}$ & $99^{\circ} 44^{\prime} 45^{\prime \prime}$ & Edwards-Trinity Plateau & -- & & 5.5 & 8.5 & 14.8 & \\
\hline 152 & $29^{\circ} 36^{\prime} 15^{\prime \prime}$ & $99^{\circ} 44^{\prime} 15^{\prime \prime}$ & Edwards-Trinity Plateau & -- & & 13.1 & 3.5 & 23.3 & \\
\hline 152 & $29^{\circ} 34^{\prime} 36^{\prime \prime}$ & $99^{\circ} 43^{\prime 2} 29^{\prime \prime}$ & Edwards, Edwards-Trinity Plateau & -- & & 1.0 & 4.5 & 26.8 & \\
\hline 152 & $29^{\circ} 29^{\circ} 17^{\prime \prime}$ & $99^{\circ} 42^{\prime} 15^{\prime \prime}$ & Edwards & -- & & -8.0 & 1.0 & 31.3 & \\
\hline 152 & $29^{\circ} 28^{\prime} 41^{\prime \prime}$ & $99^{\circ} 41^{\prime} 50^{\prime \prime}$ & Edwards & -- & & -12.4 & 3.0 & 32.3 & \\
\hline 152 & $29^{\circ} 26^{\prime} 44^{\prime \prime}$ & $99^{\circ} 41^{\prime} 11^{\prime \prime}$ & Edwards & -- & & -13.8 & 2.5 & 35.3 & \\
\hline 152 & $29^{\circ} 26^{\prime} 47^{\prime \prime}$ & $99^{\circ} 39^{\prime} 50^{\prime \prime}$ & Edwards & -- & & -3.8 & .3 & 37.8 & \\
\hline 152 & $29^{\circ} 26^{\prime} 33^{\prime \prime}$ & $99^{\circ} 39^{\prime} 40^{\prime \prime}$ & Edwards & -- & & -2.5 & .2 & 38.1 & \\
\hline 153 & $29^{\circ} 52^{\prime} 03^{\prime \prime}$ & $99^{\circ} 39^{\prime} 47^{\prime \prime}$ & Edwards-Trinity Plateau & -- & & .52 & 4.1 & 0 & on Wolf Ranch $3,300 \mathrm{ft}$ above dam \\
\hline 153 & $29^{\circ} 50^{\prime} 30^{\prime \prime}$ & $99^{\circ} 39^{\prime} 52^{\prime \prime}$ & Edwards-Trinity Plateau & -- & & 13.85 & 16.7 & 4.1 & \\
\hline 153 & $29^{\circ} 37^{\prime} 22^{\prime \prime}$ & $99^{\circ} 45^{\prime} 00^{\prime \prime}$ & Edwards-Trinity Plateau & -- & & -12.8 & 1.7 & 20.8 & \\
\hline 153 & $29^{\circ} 36^{\prime} 14^{\prime \prime}$ & $99^{\circ} 44^{\prime} 15^{\prime \prime}$ & Edwards-Trinity Plateau & -- & & 19.3 & 2.1 & 22.5 & \\
\hline 153 & $29^{\circ} 35^{\prime} 06^{\prime \prime}$ & $99^{\circ} 43^{\prime} 56^{\prime \prime}$ & Edwards-Trinity Plateau & -- & & .2 & 1.9 & 24.6 & \\
\hline 153 & $29^{\circ} 34^{\prime} 25^{\prime \prime}$ & $99^{\circ} 42^{\prime} 57^{\prime \prime}$ & Edwards-Trinity Plateau & -- & & .9 & 3.5 & 26.5 & \\
\hline 153 & $29^{\circ} 32^{\prime} 10^{\prime \prime}$ & $99^{\circ} 42^{\prime} 47^{\prime \prime}$ & Edwards, Edwards-Trinity Plateau & -- & & -2.0 & 4.8 & 30 & \\
\hline 153 & $29^{\circ} 29^{\circ} 17^{\prime \prime}$ & $99^{\circ} 42^{\prime} 15^{\prime \prime}$ & Edwards & -- & & -8.96 & 1.0 & 34.8 & \\
\hline 153 & $29^{\circ} 28^{\prime} 40^{\prime \prime}$ & $99^{\circ} 41^{\prime} 50^{\prime \prime}$ & Edwards & -- & & -5.72 & .7 & 35.8 & \\
\hline 153 & $29^{\circ} 28^{\prime} 11^{\prime \prime}$ & $99^{\circ} 42^{\prime} 01^{\prime \prime}$ & Edwards & -- & & -3.72 & .1 & 36.5 & \\
\hline 153 & $29^{\circ} 28^{\prime} 08^{\prime \prime}$ & $99^{\circ} 42^{\prime} 01^{\prime \prime}$ & Edwards & -- & & 2.71 & .8 & 36.6 & \\
\hline 153 & $29^{\circ} 27^{\prime} 59^{\prime \prime}$ & $99^{\circ} 42^{\prime} 06^{\prime \prime}$ & Edwards & -- & & -1.99 & .7 & 37.4 & \\
\hline 153 & $29^{\circ} 27^{\prime} 44^{\prime \prime}$ & $99^{\circ} 42^{\prime} 12^{\prime \prime}$ & Edwards & -- & & -.72 & .4 & 38.1 & \\
\hline 154 & $28^{\circ} 29^{\prime} 32^{\prime \prime}$ & $98^{\circ} 15^{\prime} 05^{\prime \prime}$ & Gulf Coast & -- & & 1.2 & 1.65 & 0 & at Choke Canyon Reservoir \\
\hline 154 & $28^{\circ} 29^{\prime} 09^{\prime \prime}$ & $98^{\circ} 14^{\prime} 21^{\prime \prime}$ & Gulf Coast & -- & & -1.68 & 5.08 & 1.65 & \\
\hline
\end{tabular}


$\$ \quad$ Table 4. Gains and losses from gain-loss studies in Texas-Continued

\begin{tabular}{|c|c|c|c|c|c|c|c|c|c|}
\hline \multirow[t]{2}{*}{$\begin{array}{l}\text { Stream- } \\
\text { flow } \\
\text { study } \\
\text { no. }\end{array}$} & \multirow{2}{*}{$\begin{array}{c}\text { Latitude of } \\
\text { upstream } \\
\text { end of } \\
\text { subreach }\end{array}$} & \multirow{2}{*}{$\begin{array}{c}\begin{array}{c}\text { Longitude } \\
\text { of upstream } \\
\text { end of } \\
\text { subreach }\end{array} \\
98^{\circ} 11^{\prime} 20^{\prime \prime}\end{array}$} & \multicolumn{2}{|l|}{$\begin{array}{l}\text { Major aquifer } \\
\text { outcrop } \\
\text { intersected } \\
\text { by subreach }\end{array}$} & \multirow[t]{2}{*}{$\begin{array}{c}\text { Minor aquifer } \\
\text { outcrop } \\
\text { intercepted } \\
\text { by subreach }\end{array}$} & \multirow{2}{*}{$\begin{array}{c}\begin{array}{c}\text { Gain or } \\
\text { loss (-) in } \\
\text { subreach } \\
\left(\mathrm{ft}^{3} / \mathbf{s}\right)\end{array} \\
0.6\end{array}$} & \multirow{2}{*}{$\begin{array}{c}\begin{array}{c}\text { Length } \\
\text { of subreach } \\
\text { (river mi) }\end{array} \\
1.86\end{array}$} & \multirow{2}{*}{$\begin{array}{c}\begin{array}{c}\text { Location of } \\
\text { upstream } \\
\text { end of } \\
\text { subreach } \\
\text { (river mi) }\end{array} \\
6.73\end{array}$} & \multirow[t]{2}{*}{$\begin{array}{l}\text { Descriptive location } \\
\text { of upstream end of } \\
\text { selected subreaches }\end{array}$} \\
\hline & & & Gulf Coast & -- & & & & & \\
\hline 155 & $29^{\circ} 29^{\circ} 17^{\prime \prime}$ & $99^{\circ} 42^{\prime} 15^{\prime \prime}$ & Edwards & -- & & -41.0 & 5.6 & 0 & gaging station (08195000) \\
\hline 155 & $29^{\circ} 26^{\prime} 46^{\prime \prime}$ & $99^{\circ} 39^{\prime} 57^{\prime \prime}$ & Edwards & -- & & -66.3 & 6.1 & 5.6 & \\
\hline 155 & $29^{\circ} 23^{\prime} 47^{\prime \prime}$ & $99^{\circ} 38^{\prime} 54^{\prime \prime}$ & -- & -- & & -21.0 & 2.9 & 11.7 & \\
\hline 155 & $29^{\circ} 19^{\prime} 48^{\prime \prime}$ & $99^{\circ} 39^{\prime} 14^{\prime \prime}$ & -- & -- & & -29.9 & 2.2 & 14.6 & \\
\hline 155 & $29^{\circ} 19^{\prime} 48^{\prime \prime}$ & 99³9'14" & -- & -- & & -32.2 & 5.4 & 16.8 & \\
\hline 155 & $29^{\circ} 17^{\prime} 31^{\prime \prime}$ & $99^{\circ} 38^{\prime} 54^{\prime \prime}$ & -- & -- & & -9.74 & 6.2 & 22.2 & \\
\hline 156 & $29^{\circ} 29^{\circ} 17^{\prime \prime}$ & $99^{\circ} 42^{\prime} 15^{\prime \prime}$ & Edwards & -- & & -7.0 & 1.2 & 0 & gaging station at Concan (08195000) \\
\hline 156 & $29^{\circ} 28^{\prime} 36^{\prime \prime}$ & $99^{\circ} 41^{\prime} 55^{\prime \prime}$ & Edwards & -- & & 27.0 & 1.8 & 1.2 & \\
\hline 156 & $29^{\circ} 27^{\prime} 34^{\prime \prime}$ & $99^{\circ} 41^{\prime} 40^{\prime \prime}$ & Edwards & -- & & -33.0 & 2.0 & 3 & \\
\hline 156 & $29^{\circ} 26^{\prime} 44^{\prime \prime}$ & $99^{\circ} 40^{\prime} 06^{\prime \prime}$ & Edwards & -- & & -220.0 & 13.5 & 5 & \\
\hline 157 & $29^{\circ} 29^{\circ} 17^{\prime \prime}$ & $99^{\circ} 42^{\prime} 15^{\prime \prime}$ & Edwards & -- & & -16.0 & 1.2 & 0 & gaging station at Concan (08195000) \\
\hline 157 & $29^{\circ} 28^{\prime} 36^{\prime \prime}$ & $99^{\circ} 41^{\prime} 55^{\prime \prime}$ & Edwards & -- & & 15.0 & 1.8 & 1.2 & \\
\hline 157 & $29^{\circ} 27^{\prime} 34^{\prime \prime}$ & $99^{\circ} 41^{\prime} 40^{\prime \prime}$ & Edwards & -- & & -24.2 & 2.0 & 3 & \\
\hline 157 & $29^{\circ} 26^{\prime} 44^{\prime \prime}$ & $99^{\circ} 40^{\prime} 06^{\prime \prime}$ & Edwards & -- & & -98.8 & 13.5 & 5 & \\
\hline 158 & $29^{\circ} 29^{\circ} 41^{\prime \prime}$ & $99^{\circ} 42^{\prime} 40^{\prime \prime}$ & Edwards & -- & & 2.0 & 1.0 & 0 & at Concan-Leakey road crossing \\
\hline 158 & $29^{\circ} 29^{\circ} 17^{\prime \prime}$ & $99^{\circ} 42^{\prime} 15^{\prime \prime}$ & Edwards & -- & & 2.0 & 1.5 & 1 & \\
\hline 158 & $29^{\circ} 28^{\prime} 25^{\prime \prime}$ & $99^{\circ} 42^{\prime} 01^{\prime \prime}$ & Edwards & -- & & -9.0 & 2.0 & 2.5 & \\
\hline 158 & $29^{\circ} 27^{\prime} 11^{\prime \prime}$ & $99^{\circ} 41^{\prime} 34^{\prime \prime}$ & Edwards & -- & & -20.7 & 1.5 & 4.5 & \\
\hline 159 & $28^{\circ} 28^{\prime} 18^{\prime \prime}$ & $98^{\circ} 48^{\prime} 14^{\prime \prime}$ & -- & -- & & .7 & 9.1 & 62.9 & at SH 97 crossing \\
\hline 159 & $28^{\circ} 25^{\prime} 49^{\prime \prime}$ & $98^{\circ} 41^{\prime} 17^{\prime \prime}$ & -- & -- & & 3.51 & 24.7 & 53.8 & \\
\hline 159 & $28^{\circ} 31^{\prime} 03^{\prime \prime}$ & $98^{\circ} 26^{\prime} 35^{\prime \prime}$ & -- & -- & & 5.46 & 10.1 & 29.1 & \\
\hline 159 & $28^{\circ} 31^{\prime} 24^{\prime \prime}$ & $98^{\circ} 23^{\prime} 22^{\prime \prime}$ & Gulf Coast & -- & & -1.0 & 9.6 & 19 & \\
\hline 159 & $28^{\circ} 29^{\prime} 04^{\prime \prime}$ & $98^{\circ} 14^{\prime} 37^{\prime \prime}$ & Gulf Coast & -- & & 1.96 & 9.4 & 9.4 & \\
\hline 160 & $29^{\circ} 29^{\circ} 17^{\prime \prime}$ & $99^{\circ} 42^{\prime} 15^{\prime \prime}$ & Edwards & -- & & -51.0 & 5.6 & 0 & gaging station (08196000) \\
\hline 160 & $29^{\circ} 26^{\prime} 47^{\prime \prime}$ & $99^{\circ} 39^{\prime} 55^{\prime \prime}$ & Edwards & -- & & -61.0 & 6.1 & 5.6 & \\
\hline 160 & $29^{\circ} 23^{\prime} 22^{\prime \prime}$ & $99^{\circ} 38^{\prime} 57^{\prime \prime}$ & -- & -- & & -41.0 & 2.9 & 11.7 & \\
\hline 160 & $29^{\circ} 21^{\prime} 02^{\prime \prime}$ & $99^{\circ} 39^{\prime} 32^{\prime \prime}$ & -- & -- & & -35.7 & 2.2 & 14.6 & \\
\hline 160 & $29^{\circ} 16^{\prime} 45^{\prime \prime}$ & $99^{\circ} 39^{\prime} 12^{\prime \prime}$ & -- & -- & & -45.0 & 5.4 & 16.8 & \\
\hline 160 & $29^{\circ} 17^{\prime} 30^{\prime \prime}$ & $99^{\circ} 38^{\prime} 43^{\prime \prime}$ & -- & -- & & -25.48 & 6.2 & 22.2 & \\
\hline 161 & $29^{\circ} 29^{\circ} 17^{\prime \prime}$ & $99^{\circ} 42^{\prime} 15^{\prime \prime}$ & Edwards & -- & & 23.0 & 5.6 & 0 & gaging station (08196000) \\
\hline 161 & $29^{\circ} 26^{\prime} 47^{\prime \prime}$ & $99^{\circ} 39^{\prime} 55^{\prime \prime}$ & Edwards & -- & & -48.0 & 6.1 & 5.6 & \\
\hline 161 & $29^{\circ} 23^{\prime} 21^{\prime \prime}$ & $99^{\circ} 38^{\prime} 56^{\prime \prime}$ & -- & -- & & -30.0 & 2.9 & 11.7 & \\
\hline 161 & $29^{\circ} 21^{\prime} 02^{\prime \prime}$ & $99^{\circ} 39^{\prime} 32^{\prime \prime}$ & -- & -- & & -59.0 & 2.2 & 14.6 & \\
\hline 161 & $29^{\circ} 16^{\prime} 45^{\prime \prime}$ & $99^{\circ} 39^{\prime} 12^{\prime \prime}$ & -- & -- & & -75.0 & 5.4 & 16.8 & \\
\hline 161 & $29^{\circ} 17^{\prime} 30^{\prime \prime}$ & $99^{\circ} 38^{\prime} 43^{\prime \prime}$ & -- & -- & & -40.0 & 6.2 & 22.2 & \\
\hline 162 & $29^{\circ} 40^{\prime} 30^{\prime \prime}$ & $99^{\circ} 20^{\prime} 08^{\prime \prime}$ & Trinity & -- & & 2.23 & 3.2 & 0 & \\
\hline 162 & $29^{\circ} 38^{\prime} 45^{\prime \prime}$ & $99^{\circ} 18^{\prime} 17^{\prime \prime}$ & Trinity & -- & & 4.1 & 2.9 & 3.2 & \\
\hline
\end{tabular}


Table 4. Gains and losses from gain-loss studies in Texas-Continued

\begin{tabular}{|c|c|c|c|c|c|c|c|c|c|}
\hline $\begin{array}{c}\text { Stream- } \\
\text { flow } \\
\text { study } \\
\text { no. }\end{array}$ & $\begin{array}{l}\text { Latitude of } \\
\text { upstream } \\
\text { end of } \\
\text { subreach }\end{array}$ & $\begin{array}{l}\text { Longitude } \\
\text { of upstream } \\
\text { end of } \\
\text { subreach }\end{array}$ & $\begin{array}{l}\text { Major aquifer } \\
\text { outcrop } \\
\text { intersected } \\
\text { by subreach }\end{array}$ & & $\begin{array}{c}\text { Minor aquifer } \\
\text { outcrop } \\
\text { intercepted } \\
\text { by subreach }\end{array}$ & $\begin{array}{c}\text { Gain or } \\
\text { loss }(-) \text { in } \\
\text { subreach } \\
\left(\mathrm{ft}^{3} / \mathrm{s}\right)\end{array}$ & $\begin{array}{l}\text { Length } \\
\text { of subreach } \\
\text { (river mi) }\end{array}$ & $\begin{array}{l}\text { Location of } \\
\text { upstream } \\
\text { end of } \\
\text { subreach } \\
\text { (river mi) }\end{array}$ & $\begin{array}{l}\text { Descriptive location } \\
\text { of upstream end of } \\
\text { selected subreaches }\end{array}$ \\
\hline 162 & $29^{\circ} 38^{\prime} 37^{\prime \prime}$ & $99^{\circ} 16^{\prime} 21^{\prime \prime}$ & Trinity & -- & & 0 & 0.1 & 6.1 & \\
\hline 162 & $29^{\circ} 38^{\prime} 35^{\prime \prime}$ & $99^{\circ} 16^{\prime} 14^{\prime \prime}$ & Trinity & -- & & 6.2 & 2.1 & 6.2 & \\
\hline 162 & $29^{\circ} 37^{\prime} 01^{\prime \prime}$ & $99^{\circ} 15^{\prime} 32^{\prime \prime}$ & Trinity & -- & & 4.04 & 1.7 & 8.3 & \\
\hline 162 & $29^{\circ} 35^{\prime} 43^{\prime \prime}$ & $99^{\circ} 15^{\prime} 15^{\prime \prime}$ & Edwards, Trinity & -- & & 3.85 & 2.2 & 10 & \\
\hline 162 & $29^{\circ} 34^{\prime} 09^{\prime \prime}$ & $99^{\circ} 144^{\prime} 47^{\prime \prime}$ & Edwards & -- & & -9.7 & 2.7 & 12.2 & gaging station near Tarpley (08200000) \\
\hline 162 & $29^{\circ} 32^{\prime} 36^{\prime \prime}$ & $99^{\circ} 14^{\prime} 03^{\prime \prime}$ & Edwards & -- & & -5.2 & 3.9 & 14.9 & \\
\hline 162 & $29^{\circ} 30^{\prime} 14^{\prime \prime}$ & $99^{\circ} 12^{\prime} 42^{\prime \prime}$ & Edwards & -- & & -8.3 & 2.6 & 18.8 & \\
\hline 162 & $29^{\circ} 28^{\prime} 22^{\prime \prime}$ & $99^{\circ} 12^{\prime} 21^{\prime \prime}$ & Edwards & -- & & -5.5 & 2.7 & 21.4 & \\
\hline 162 & $29^{\circ} 27^{\prime} 04^{\prime \prime}$ & $99^{\circ} 11^{\prime} 12^{\prime \prime}$ & -- & -- & & -16.5 & 3.5 & 24.1 & \\
\hline 162 & $29^{\circ} 24^{\prime} 21^{\prime \prime}$ & $99^{\circ} 10^{\prime} 22^{\prime \prime}$ & -- & -- & & -2.6 & 3.5 & 27.6 & \\
\hline 162 & $29^{\circ} 22^{\prime} 54^{\prime \prime}$ & $99^{\circ} 08^{\prime 22} "$ & -- & -- & & -1.24 & 3.6 & 31.1 & \\
\hline 162 & $29^{\circ} 22^{\prime} 02^{\prime \prime}$ & $99^{\circ} 05^{\prime} 12^{\prime \prime}$ & -- & -- & & .84 & 2.5 & 34.7 & \\
\hline 163 & $29^{\circ} 34^{\prime} 59^{\prime \prime}$ & $99^{\circ} 15^{\prime} 00^{\prime \prime}$ & Edwards, Trinity & -- & & 7.0 & 1.7 & 10.5 & $50 \mathrm{ft}$ below Bandera Creek \\
\hline 163 & $29^{\circ} 34^{\prime} 00^{\prime \prime}$ & $99^{\circ} 15^{\prime} 00^{\prime \prime}$ & Edwards & -- & & -24.0 & 2.7 & 12.2 & \\
\hline 163 & $29^{\circ} 31^{\prime} 59^{\prime \prime}$ & $99^{\circ} 13^{\prime} 59^{\prime \prime}$ & Edwards & -- & & -25.0 & 3.9 & 14.9 & \\
\hline 163 & $29^{\circ} 30^{\prime} 00^{\prime \prime}$ & $99^{\circ} 12^{\prime} 59^{\prime \prime}$ & Edwards & -- & & -11.0 & 2.6 & 18.8 & \\
\hline 163 & $29^{\circ} 28^{\prime} 00^{\prime \prime}$ & $99^{\circ} 12^{\prime} 59^{\prime \prime}$ & Edwards & -- & & -17.0 & 2.7 & 21.4 & \\
\hline 163 & $29^{\circ} 27^{\prime} 00^{\prime \prime}$ & $99^{\circ} 10^{\prime} 59^{\prime \prime}$ & -- & -- & & -28.0 & 3.5 & 24.1 & \\
\hline 163 & $29^{\circ} 24^{\prime} 00^{\prime \prime}$ & $99^{\circ} 09^{\prime} 59^{\prime \prime}$ & -- & -- & & -3.0 & 2.5 & 27.6 & \\
\hline 163 & $29^{\circ} 23^{\prime} 16^{\prime \prime}$ & $99^{\circ} 08^{\prime} 49^{\prime \prime}$ & -- & -- & & 1.0 & 1.9 & 30.1 & \\
\hline 163 & $29^{\circ} 22^{\prime} 59^{\prime \prime}$ & $99^{\circ} 06^{\prime} 59^{\prime \prime}$ & -- & -- & & 0 & 2.6 & 32 & \\
\hline 163 & $29^{\circ} 22^{\prime} 00^{\prime \prime}$ & $99^{\circ} 04^{\prime} 59^{\prime \prime}$ & -- & -- & & 1.0 & 2.6 & 34.6 & \\
\hline 163 & $29^{\circ} 21^{\prime} 00^{\prime \prime}$ & $99^{\circ} 03^{\prime} 00^{\prime \prime}$ & -- & -- & & 1.0 & 2.8 & 37.2 & \\
\hline 164 & $29^{\circ} 34^{\prime} 09^{\prime \prime}$ & $99^{\circ} 14^{\prime} 47^{\prime \prime}$ & Edwards & -- & & -78.0 & 5.7 & 0 & gaging station (08200000) \\
\hline 164 & $29^{\circ} 31^{\prime} 17^{\prime \prime}$ & $99^{\circ} 13^{\prime} 12^{\prime \prime}$ & Edwards & -- & & -41.7 & 4.0 & 5.7 & \\
\hline 164 & $29^{\circ} 28^{\prime} 23^{\prime \prime}$ & $99^{\circ} 12^{\prime} 15^{\prime \prime}$ & Edwards & -- & & -20.3 & 2.7 & 9.7 & \\
\hline 164 & $29^{\circ} 27^{\prime} 06^{\prime \prime}$ & $99^{\circ} 11^{\prime} 12^{\prime \prime}$ & -- & -- & & -19.4 & 4.4 & 12.4 & \\
\hline 164 & $29^{\circ} 24^{\prime} 16^{\prime \prime}$ & $99^{\circ} 10^{\prime} 22^{\prime \prime}$ & -- & -- & & 1.3 & 2.1 & 16.8 & \\
\hline 165 & $29^{\circ} 11^{\prime} 19^{\prime \prime}$ & $99^{\circ} 46^{\prime} 14^{\prime \prime}$ & -- & -- & & 4.7 & 1.9 & 0 & at old hwy bridge $1.7 \mathrm{mi}$ southeast of Uvalde \\
\hline 165 & $29^{\circ} 10^{\prime} 27^{\prime \prime}$ & $99^{\circ} 45^{\prime} 47^{\prime \prime}$ & -- & -- & & 0 & 1.9 & 1.9 & \\
\hline 165 & $29^{\circ} 09^{\prime} 15^{\prime \prime}$ & $99^{\circ} 44^{\prime} 35^{\prime \prime}$ & Carrizo-Wilcox & -- & & 5.1 & 2.5 & 3.8 & \\
\hline 165 & $29^{\circ} 07^{\prime} 54^{\prime \prime}$ & $99^{\circ} 42^{\prime} 27^{\prime \prime}$ & Carrizo-Wilcox & -- & & -1.8 & 3.0 & 6.3 & \\
\hline 165 & $29^{\circ} 06^{\prime} 49^{\prime \prime}$ & $99^{\circ} 41^{\prime} 07^{\prime \prime}$ & Carrizo-Wilcox & -- & & 5.4 & .5 & 9.3 & \\
\hline 165 & $29^{\circ} 06^{\prime} 35^{\prime \prime}$ & $99^{\circ} 40^{\prime} 50^{\prime \prime}$ & Carrizo-Wilcox & -- & & .2 & .3 & 9.8 & \\
\hline 165 & $29^{\circ} 06^{\prime} 22^{\prime \prime}$ & $99^{\circ} 40^{\prime} 40^{\prime \prime}$ & Carrizo-Wilcox & -- & & 1.0 & .9 & 10.1 & \\
\hline 165 & $29^{\circ} 05^{\prime} 48^{\prime \prime}$ & $99^{\circ} 40^{\prime} 06^{\prime \prime}$ & Carrizo-Wilcox & -- & & -.5 & .3 & 11 & \\
\hline 165 & $29^{\circ} 05^{\prime} 42^{\prime \prime}$ & $99^{\circ} 40^{\prime} 00^{\prime \prime}$ & Carrizo-Wilcox & -- & & -.3 & .3 & 11.3 & \\
\hline 165 & $29^{\circ} 05^{\prime} 31^{\prime \prime}$ & $99^{\circ} 39^{\prime} 45^{\prime \prime}$ & Carrizo-Wilcox & -- & & .2 & .2 & 11.6 & \\
\hline
\end{tabular}


Table 4. Gains and losses from gain-loss studies in Texas-Continued

\begin{tabular}{|c|c|c|c|c|c|c|c|c|c|}
\hline $\begin{array}{l}\text { Stream- } \\
\text { flow } \\
\text { study } \\
\text { no. }\end{array}$ & $\begin{array}{l}\text { Latitude of } \\
\text { upstream } \\
\text { end of } \\
\text { subreach }\end{array}$ & $\begin{array}{l}\text { Longitude } \\
\text { of upstream } \\
\text { end of } \\
\text { subreach }\end{array}$ & $\begin{array}{c}\text { Major aquifer } \\
\text { outcrop } \\
\text { intersected } \\
\text { by subreach }\end{array}$ & & $\begin{array}{l}\text { Minor aquifer } \\
\text { outcrop } \\
\text { intercepted } \\
\text { by subreach }\end{array}$ & $\begin{array}{l}\text { Gain or } \\
\text { loss }(-) \text { in } \\
\text { subreach } \\
\left(\mathrm{ft}^{3} / \mathrm{s}\right)\end{array}$ & 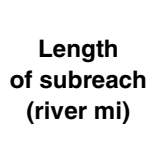 & $\begin{array}{l}\text { Location of } \\
\text { upstream } \\
\text { end of } \\
\text { subreach } \\
\text { (river mi) }\end{array}$ & $\begin{array}{l}\text { Descriptive location } \\
\text { of upstream end of } \\
\text { selected subreaches }\end{array}$ \\
\hline 165 & $29^{\circ} 05^{\prime} 25^{\prime \prime}$ & $99^{\circ} 39^{\prime} 28^{\prime \prime}$ & Carrizo-Wilcox & -- & & 1.5 & 0.5 & 11.8 & \\
\hline 165 & $29^{\circ} 05^{\prime} 23^{\prime \prime}$ & $99^{\circ} 39^{\prime} 24^{\prime \prime}$ & Carrizo-Wilcox & -- & & -3.3 & .6 & 12.3 & \\
\hline 165 & $29^{\circ} 05^{\prime} 10^{\prime \prime}$ & 99³9'19" & Carrizo-Wilcox & -- & & .6 & .6 & 12.9 & \\
\hline 165 & $29^{\circ} 05^{\prime} 06^{\prime \prime}$ & $99^{\circ} 39^{\prime} 29^{\prime \prime}$ & Carrizo-Wilcox & -- & & -.5 & 2.4 & 13.5 & \\
\hline 165 & $29^{\circ} 04^{\prime} 02^{\prime \prime}$ & $99^{\circ} 38^{\prime} 46^{\prime \prime}$ & Carrizo-Wilcox & -- & & .5 & 1.3 & 15.9 & \\
\hline 165 & $29^{\circ} 02^{\prime} 39^{\prime \prime}$ & $99^{\circ} 37^{\prime} 45^{\prime \prime}$ & -- & -- & & -2.0 & 2.1 & 17.2 & \\
\hline 165 & $29^{\circ} 01^{\prime} 40^{\prime \prime}$ & $99^{\circ} 37^{\prime} 26^{\prime \prime}$ & -- & -- & & -.8 & 1.5 & 19.3 & \\
\hline 165 & $29^{\circ} 00^{\prime} 37^{\prime \prime}$ & $99^{\circ} 37^{\prime} 15^{\prime \prime}$ & -- & -- & & .6 & .5 & 20.8 & \\
\hline 165 & $29^{\circ} 00^{\prime} 24^{\prime \prime}$ & 99³7'06" & -- & -- & & -2.8 & 1.9 & 21.3 & \\
\hline 165 & $28^{\circ} 58^{\prime} 57^{\prime \prime}$ & $99^{\circ} 37^{\prime} 06^{\prime \prime}$ & -- & -- & & .3 & 1.3 & 23.2 & \\
\hline 165 & $28^{\circ} 58^{\prime} 03^{\prime \prime}$ & $99^{\circ} 36^{\prime} 55^{\prime \prime}$ & -- & -- & & -.7 & .9 & 24.5 & \\
\hline 165 & $28^{\circ} 57^{\prime} 47^{\prime \prime}$ & $99^{\circ} 36^{\prime} 49^{\prime \prime}$ & -- & -- & & -.2 & .8 & 25.4 & \\
\hline 165 & $28^{\circ} 57^{\prime} 14^{\prime \prime}$ & $99^{\circ} 36^{\prime} 32^{\prime \prime}$ & -- & -- & & -1.0 & 2.2 & 26.2 & \\
\hline 165 & $28^{\circ} 56^{\prime} 13^{\prime \prime}$ & $99^{\circ} 35^{\prime} 41^{\prime \prime}$ & -- & -- & & -.5 & 2.0 & 28.4 & \\
\hline 165 & $28^{\circ} 54^{\prime} 59^{\prime \prime}$ & $99^{\circ} 34^{\prime} 57^{\prime \prime}$ & -- & -- & & -.4 & 1.1 & 30.4 & \\
\hline 165 & $28^{\circ} 54^{\prime} 57^{\prime \prime}$ & $99^{\circ} 34^{\prime} 58^{\prime \prime}$ & -- & -- & & -1.2 & 2.5 & 31.5 & \\
\hline 165 & $28^{\circ} 53^{\prime} 25^{\prime \prime}$ & $99^{\circ} 34^{\prime} 05^{\prime \prime}$ & -- & -- & & .1 & 2.3 & 34 & \\
\hline 165 & $28^{\circ} 52^{\prime} 10^{\prime \prime}$ & 99॰33'34" & -- & -- & & -.4 & 2.0 & 36.3 & \\
\hline 165 & $28^{\circ} 51^{\prime} 49^{\prime \prime}$ & $99^{\circ} 32^{\prime} 14^{\prime \prime}$ & -- & -- & & -.8 & 3.8 & 38.3 & \\
\hline 165 & $28^{\circ} 50^{\prime} 56^{\prime \prime}$ & $99^{\circ} 29^{\prime} 30^{\prime \prime}$ & -- & -- & & -.7 & 4.1 & 42.1 & \\
\hline 165 & $28^{\circ} 50^{\prime} 16^{\prime \prime}$ & $99^{\circ} 27^{\prime} 11^{\prime \prime}$ & -- & -- & & -.3 & 3.2 & 46.2 & \\
\hline 166 & $29^{\circ} 11^{\prime} 19^{\prime \prime}$ & $99^{\circ} 46^{\prime} 15^{\prime \prime}$ & -- & -- & & 4.9 & 2.1 & 0 & at hwy bridge $1.7 \mathrm{mi}$ southeast of Uvalde \\
\hline 166 & $29^{\circ} 10^{\prime} 09^{\prime \prime}$ & $99^{\circ} 45^{\prime} 47^{\prime \prime}$ & -- & -- & & 8.4 & 4.5 & 2.1 & \\
\hline 166 & $29^{\circ} 09^{\prime} 15^{\prime \prime}$ & $99^{\circ} 44^{\prime} 35^{\prime \prime}$ & Carrizo-Wilcox & -- & & -9.2 & 1.5 & 6.6 & \\
\hline 166 & $29^{\circ} 07^{\prime} 03^{\prime \prime}$ & $99^{\circ} 41^{\prime} 25^{\prime \prime}$ & Carrizo-Wilcox & -- & & 11.9 & 2.9 & 8.1 & \\
\hline 166 & $29^{\circ} 05^{\prime} 32^{\prime \prime}$ & $99^{\circ} 39^{\prime} 45^{\prime \prime}$ & Carrizo-Wilcox & -- & & -9.6 & 6.0 & 11 & \\
\hline 166 & $29^{\circ} 00^{\prime} 38^{\prime \prime}$ & $99^{\circ} 37^{\prime} 16^{\prime \prime}$ & -- & -- & & -2.6 & 3.1 & 17 & \\
\hline 166 & $28^{\circ} 58^{\prime} 58^{\prime \prime}$ & $99^{\circ} 37^{\prime} 06^{\prime \prime}$ & -- & -- & & -2.2 & 2.0 & 20.1 & \\
\hline 166 & $28^{\circ} 57^{\prime} 47^{\prime \prime}$ & $99^{\circ} 36^{\prime} 49^{\prime \prime}$ & -- & -- & & -1.1 & 1.2 & 22.1 & \\
\hline 166 & $28^{\circ} 54^{\prime} 19^{\prime \prime}$ & $99^{\circ} 34^{\prime} 37^{\prime \prime}$ & -- & -- & & -1.4 & 3.1 & 23.3 & \\
\hline 166 & $28^{\circ} 53^{\prime} 25^{\prime \prime}$ & $99^{\circ} 34^{\prime} 05^{\prime \prime}$ & -- & -- & & -1.7 & 7.1 & 26.4 & \\
\hline 166 & $28^{\circ} 50^{\prime} 56^{\prime \prime}$ & $99^{\circ} 29^{\prime} 30^{\prime \prime}$ & -- & -- & & -.5 & 4.0 & 33.5 & \\
\hline 167 & $29^{\circ} 11^{\prime} 18^{\prime \prime}$ & $99^{\circ} 46^{\prime} 14^{\prime \prime}$ & -- & -- & & .6 & 1.9 & 0 & at hwy bridge $1.7 \mathrm{mi}$ southeast of Uvalde \\
\hline 167 & $29^{\circ} 10^{\prime} 26^{\prime \prime}$ & $99^{\circ} 45^{\prime} 47^{\prime \prime}$ & -- & -- & & -.1 & 1.9 & 1.9 & \\
\hline 167 & $29^{\circ} 09^{\prime} 14^{\prime \prime}$ & $99^{\circ} 44^{\prime} 35^{\prime \prime}$ & Carrizo-Wilcox & -- & & 2.5 & .8 & 3.8 & \\
\hline 167 & $29^{\circ} 08^{\prime} 47^{\prime \prime}$ & $99^{\circ} 43^{\prime} 46^{\prime \prime}$ & Carrizo-Wilcox & -- & & -.4 & .9 & 4.6 & \\
\hline 167 & $29^{\circ} 08^{\prime} 48^{\prime \prime}$ & $99^{\circ} 43^{\prime} 46^{\prime \prime}$ & Carrizo-Wilcox & -- & & -.5 & .8 & 5.5 & \\
\hline 167 & $29^{\circ} 08^{\prime} 32^{\prime \prime}$ & $99^{\circ} 43^{\prime} 04^{\prime \prime}$ & Carrizo-Wilcox & -- & & -1.4 & 3.0 & 6.3 & \\
\hline
\end{tabular}


Table 4. Gains and losses from gain-loss studies in Texas-Continued

\begin{tabular}{|c|c|c|c|c|c|c|c|c|c|}
\hline $\begin{array}{l}\text { Stream- } \\
\text { flow } \\
\text { study } \\
\text { no. }\end{array}$ & $\begin{array}{l}\text { Latitude of } \\
\text { upstream } \\
\text { end of } \\
\text { subreach }\end{array}$ & $\begin{array}{l}\text { Longitude } \\
\text { of upstream } \\
\text { end of } \\
\text { subreach }\end{array}$ & $\begin{array}{c}\text { Major aquifer } \\
\text { outcrop } \\
\text { intersected } \\
\text { by subreach }\end{array}$ & & $\begin{array}{l}\text { Minor aquifer } \\
\text { outcrop } \\
\text { intercepted } \\
\text { by subreach }\end{array}$ & $\begin{array}{l}\text { Gain or } \\
\text { loss (-) in } \\
\text { subreach } \\
\left(\mathrm{ft}^{3} / \mathrm{s}\right)\end{array}$ & $\begin{array}{l}\text { Length } \\
\text { of subreach } \\
\text { (river } \mathrm{mi} \text { ) }\end{array}$ & $\begin{array}{l}\text { Location of } \\
\text { upstream } \\
\text { end of } \\
\text { subreach } \\
\text { (river mi) }\end{array}$ & $\begin{array}{l}\text { Descriptive location } \\
\text { of upstream end of } \\
\text { selected subreaches }\end{array}$ \\
\hline 167 & $29^{\circ} 06^{\prime} 50^{\prime \prime}$ & $99^{\circ} 41^{\prime} 08^{\prime \prime}$ & Carrizo-Wilcox & -- & & 1.0 & 0.1 & 9.3 & \\
\hline 167 & $29^{\circ} 06^{\prime} 48^{\prime \prime}$ & $99^{\circ} 41^{\prime} 06^{\prime \prime}$ & Carrizo-Wilcox & -- & & 2.7 & .4 & 9.4 & \\
\hline 167 & $29^{\circ} 06^{\prime} 40^{\prime \prime}$ & $99^{\circ} 40^{\prime} 57^{\prime \prime}$ & Carrizo-Wilcox & -- & & .3 & 1.2 & 9.8 & \\
\hline 167 & $29^{\circ} 05^{\prime} 48^{\prime \prime}$ & $99^{\circ} 40^{\prime} 06^{\prime \prime}$ & Carrizo-Wilcox & -- & & -.1 & .6 & 11 & \\
\hline 167 & $29^{\circ} 05^{\prime} 32^{\prime \prime}$ & $99^{\circ} 39^{\prime} 44^{\prime \prime}$ & Carrizo-Wilcox & -- & & -1.0 & 1.3 & 11.6 & \\
\hline 167 & $29^{\circ} 05^{\prime} 09^{\prime \prime}$ & $99^{\circ} 39^{\prime} 18^{\prime \prime}$ & Carrizo-Wilcox & -- & & -1.4 & 3.0 & 12.9 & \\
\hline 167 & $29^{\circ} 04^{\prime} 01^{\prime \prime}$ & $99^{\circ} 38^{\prime} 46^{\prime \prime}$ & Carrizo-Wilcox & -- & & -.5 & 1.3 & 15.9 & \\
\hline 167 & $29^{\circ} 02^{\prime} 39^{\prime \prime}$ & $99^{\circ} 37^{\prime} 46^{\prime \prime}$ & -- & -- & & -1.4 & 2.1 & 17.2 & \\
\hline 167 & $29^{\circ} 01^{\prime} 40^{\prime \prime}$ & $99^{\circ} 37^{\prime} 26^{\prime \prime}$ & -- & -- & & 0 & 1.5 & 19.3 & \\
\hline 167 & $29^{\circ} 00^{\prime} 27^{\prime \prime}$ & $99^{\circ} 36^{\prime} 59^{\prime \prime}$ & -- & -- & & 0 & 2.4 & 20.8 & \\
\hline 167 & $28^{\circ} 58^{\prime} 58^{\prime \prime}$ & $99^{\circ} 37^{\prime} 06^{\prime \prime}$ & -- & -- & & 0 & 2.2 & 23.2 & \\
\hline 167 & $28^{\circ} 57^{\prime} 47^{\prime \prime}$ & $99^{\circ} 36^{\prime} 49^{\prime \prime}$ & -- & -- & & 0 & .8 & 25.4 & \\
\hline 167 & $28^{\circ} 57^{\prime} 14^{\prime \prime}$ & $99^{\circ} 36^{\prime} 32^{\prime \prime}$ & -- & -- & & 0 & 4.2 & 26.2 & \\
\hline 167 & $28^{\circ} 55^{\prime} 38^{\prime \prime}$ & $99^{\circ} 35^{\prime} 10^{\prime \prime}$ & -- & -- & & 0 & 5.9 & 30.4 & \\
\hline 168 & $29^{\circ} 11^{\prime} 19^{\prime \prime}$ & $99^{\circ} 46^{\prime} 15^{\prime \prime}$ & -- & -- & & 6.51 & 3.8 & 0 & at hwy bridge $1.7 \mathrm{mi}$ southeast of Uvalde \\
\hline 168 & $29^{\circ} 09^{\prime} 14^{\prime \prime}$ & $99^{\circ} 44^{\prime} 34^{\prime \prime}$ & Carrizo-Wilcox & -- & & 7.0 & .8 & 3.8 & \\
\hline 168 & $29^{\circ} 07^{\prime} 54^{\prime \prime}$ & $99^{\circ} 42^{\prime} 27^{\prime \prime}$ & Carrizo-Wilcox & -- & & -4.1 & 4.7 & 4.6 & \\
\hline 168 & $29^{\circ} 06^{\prime} 50^{\prime \prime}$ & $99^{\circ} 41^{\prime} 08^{\prime \prime}$ & Carrizo-Wilcox & -- & & 5.5 & .5 & 9.3 & \\
\hline 169 & $29^{\circ} 11^{\prime} 18^{\prime \prime}$ & $99^{\circ} 46^{\prime} 15^{\prime \prime}$ & -- & -- & & 7.6 & 2.1 & 0 & at hwy bridge $1.7 \mathrm{mi}$ southeast of Uvalde \\
\hline 169 & $29^{\circ} 10^{\prime} 09^{\prime \prime}$ & $99^{\circ} 45^{\prime} 47^{\prime \prime}$ & -- & -- & & 4.4 & 4.5 & 2.1 & \\
\hline 169 & $29^{\circ} 09^{\prime} 14^{\prime \prime}$ & $99^{\circ} 44^{\prime} 35^{\prime \prime}$ & Carrizo-Wilcox & -- & & -7.4 & 2.0 & 6.6 & \\
\hline 169 & $29^{\circ} 06^{\prime} 49^{\prime \prime}$ & $99^{\circ} 41^{\prime} 06^{\prime \prime}$ & Carrizo-Wilcox & -- & & 5.2 & 4.4 & 8.6 & \\
\hline 169 & $29^{\circ} 04^{\prime} 02^{\prime \prime}$ & $99^{\circ} 38^{\prime} 46^{\prime \prime}$ & Carrizo-Wilcox & -- & & -4.0 & 4.0 & 13 & \\
\hline 169 & $29^{\circ} 00^{\prime} 37^{\prime \prime}$ & $99^{\circ} 37^{\prime} 15^{\prime \prime}$ & -- & -- & & -3.6 & 3.1 & 17 & \\
\hline 169 & $28^{\circ} 58^{\prime} 58^{\prime \prime}$ & $99^{\circ} 37^{\prime} 06^{\prime \prime}$ & -- & -- & & -2.9 & 3.2 & 20.1 & \\
\hline 169 & $28^{\circ} 54^{\prime} 19^{\prime \prime}$ & $99^{\circ} 34^{\prime} 37^{\prime \prime}$ & -- & -- & & -.8 & 3.1 & 23.3 & \\
\hline 169 & $28^{\circ} 53^{\prime} 25^{\prime \prime}$ & $99^{\circ} 34^{\prime} 05^{\prime \prime}$ & -- & -- & & -1.6 & 8.2 & 26.4 & Ottenhause Ranch \\
\hline 170 & $29^{\circ} 11^{\prime} 19^{\prime \prime}$ & $99^{\circ} 46^{\prime} 14^{\prime \prime}$ & -- & -- & & 5.2 & 2.1 & 0 & at hwy bridge $1.7 \mathrm{mi}$ southeast of Uvalde \\
\hline 170 & $29^{\circ} 10^{\prime} 08^{\prime \prime}$ & $99^{\circ} 45^{\prime} 47^{\prime \prime}$ & -- & -- & & 4.8 & 3.7 & 2.1 & \\
\hline 170 & $29^{\circ} 09^{\prime} 24^{\prime \prime}$ & $99^{\circ} 44^{\prime} 42^{\prime \prime}$ & Carrizo-Wilcox & -- & & -4.3 & 2.3 & 5.8 & \\
\hline 170 & $29^{\circ} 07^{\prime} 03^{\prime \prime}$ & $99^{\circ} 41^{\prime 2} 25^{\prime \prime}$ & Carrizo-Wilcox & -- & & 5.6 & 1.0 & 8.1 & \\
\hline 170 & $29^{\circ} 06^{\prime} 35^{\prime \prime}$ & $99^{\circ} 40^{\prime} 50^{\prime \prime}$ & Carrizo-Wilcox & -- & & -1.2 & 3.9 & 9.1 & \\
\hline 170 & $29^{\circ} 04^{\prime} 02^{\prime \prime}$ & $99^{\circ} 38^{\prime} 46^{\prime \prime}$ & Carrizo-Wilcox & -- & & -2.5 & 4.0 & 13 & \\
\hline 170 & $29^{\circ} 00^{\prime} 37^{\prime \prime}$ & $99^{\circ} 37^{\prime} 15^{\prime \prime}$ & -- & -- & & -1.6 & 3.1 & 17 & \\
\hline 170 & $28^{\circ} 58^{\prime} 58^{\prime \prime}$ & $99^{\circ} 37^{\prime} 06^{\prime \prime}$ & -- & -- & & -1.0 & 3.2 & 20.1 & \\
\hline 170 & $28^{\circ} 54^{\prime} 19^{\prime \prime}$ & $99^{\circ} 34^{\prime} 37^{\prime \prime}$ & -- & -- & & -1.8 & 3.1 & 23.3 & \\
\hline 170 & $28^{\circ} 53^{\prime} 24^{\prime \prime}$ & $99^{\circ} 34^{\prime} 05^{\prime \prime}$ & -- & -- & & -.8 & 8.2 & 26.4 & \\
\hline 171 & $29^{\circ} 11^{\prime} 19^{\prime \prime}$ & $99^{\circ} 46^{\prime} 14^{\prime \prime}$ & -- & -- & & 6.9 & 4.8 & 0 & at hwy bridge $1.7 \mathrm{mi}$ southeast of Uvalde \\
\hline
\end{tabular}


Table 4. Gains and losses from gain-loss studies in Texas-Continued

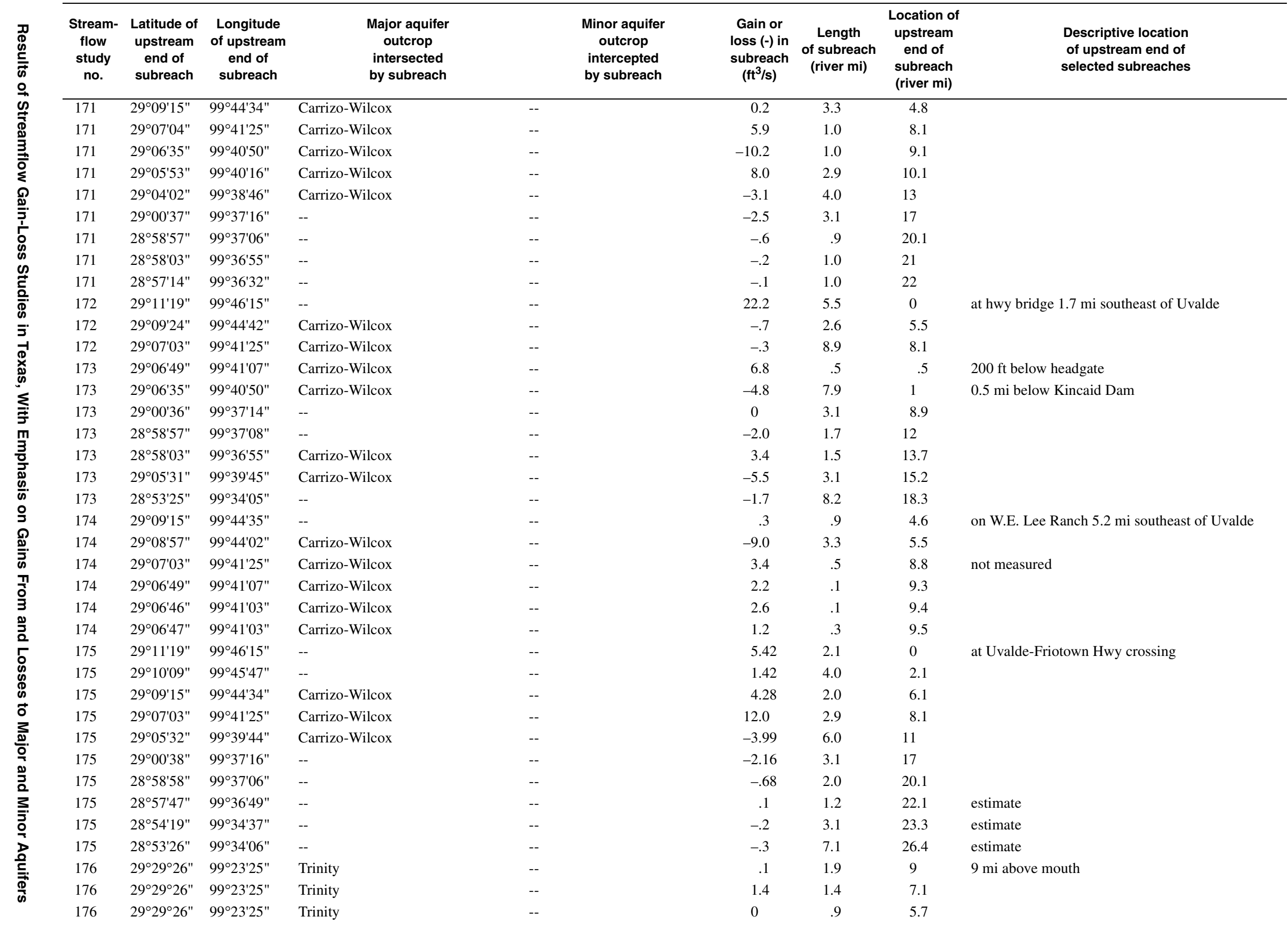


Table 4. Gains and losses from gain-loss studies in Texas-Continued

\begin{tabular}{|c|c|c|c|c|c|c|c|c|c|}
\hline $\begin{array}{l}\text { Stream- } \\
\text { flow } \\
\text { study } \\
\text { no. }\end{array}$ & $\begin{array}{l}\text { Latitude of } \\
\text { upstream } \\
\text { end of } \\
\text { subreach }\end{array}$ & $\begin{array}{l}\text { Longitude } \\
\text { of upstream } \\
\text { end of } \\
\text { subreach }\end{array}$ & $\begin{array}{c}\text { Major aquifer } \\
\text { outcrop } \\
\text { intersected } \\
\text { by subreach }\end{array}$ & & $\begin{array}{c}\text { Minor aquifer } \\
\text { outcrop } \\
\text { intercepted } \\
\text { by subreach }\end{array}$ & $\begin{array}{c}\text { Gain or } \\
\text { loss }(-) \text { in } \\
\text { subreach } \\
\left(\mathrm{ft}^{3} / \mathrm{s}\right)\end{array}$ & $\begin{array}{l}\text { Length } \\
\text { of subreach } \\
\text { (river mi) }\end{array}$ & $\begin{array}{l}\text { Location of } \\
\text { upstream } \\
\text { end of } \\
\text { subreach } \\
\text { (river mi) }\end{array}$ & $\begin{array}{l}\text { Descriptive location } \\
\text { of upstream end of } \\
\text { selected subreaches }\end{array}$ \\
\hline 176 & $29^{\circ} 29^{\circ} 26^{\prime \prime}$ & $99^{\circ} 23^{\prime} 25^{\prime \prime}$ & Trinity & -- & & -1.5 & 0.2 & 4.8 & \\
\hline 176 & $29^{\circ} 29^{\circ} 26^{\prime \prime}$ & $99^{\circ} 23^{\prime} 25^{\prime \prime}$ & Trinity & -- & & 0 & 1.2 & 4.6 & \\
\hline 176 & $29^{\circ} 29^{\circ} 25^{\prime \prime}$ & $99^{\circ} 23^{\prime} 25^{\prime \prime}$ & Edwards, Trinity & -- & & 0 & 3.4 & 3.4 & \\
\hline 177 & $29^{\circ} 31^{\prime} 17^{\prime \prime}$ & $99^{\circ} 05^{\prime} 03^{\prime \prime}$ & Edwards & -- & & -65.0 & 3.7 & 0 & on SH 173 \\
\hline 177 & $29^{\circ} 30^{\prime} 11^{\prime \prime}$ & $99^{\circ} 06^{\prime} 02^{\prime \prime}$ & Edwards, Trinity & -- & & -17.0 & 1.4 & 3.7 & \\
\hline 177 & $29^{\circ} 30^{\prime} 05^{\prime \prime}$ & $99^{\circ} 06^{\prime} 30^{\prime \prime}$ & Edwards & -- & & 111.0 & 2.3 & 5.1 & \\
\hline 177 & $29^{\circ} 34^{\prime} 08^{\prime \prime}$ & $99^{\circ} 05^{\prime} 54^{\prime \prime}$ & Edwards & -- & & -43.0 & .7 & 7.4 & \\
\hline 177 & $29^{\circ} 32^{\prime} 17^{\prime \prime}$ & $99^{\circ} 05^{\prime} 53^{\prime \prime}$ & Edwards & -- & & -80.0 & 1.9 & 8.1 & \\
\hline 177 & $29^{\circ} 29^{\circ} 00^{\prime \prime}$ & $99^{\circ} 07^{\prime} 31^{\prime \prime}$ & Edwards, Trinity & -- & & -14.0 & 2.6 & 10 & \\
\hline 177 & $29^{\circ} 27^{\prime} 00^{\prime \prime}$ & $99^{\circ} 07^{\prime} 30^{\prime \prime}$ & Trinity & -- & & -8.0 & 3.9 & 12.6 & \\
\hline 178 & $29^{\circ} 34^{\prime} 08^{\prime \prime}$ & $99^{\circ} 05^{\prime} 54^{\prime \prime}$ & Edwards & -- & & -3.0 & 3.7 & 0 & on SH 173 \\
\hline 178 & $29^{\circ} 32^{\prime} 17^{\prime \prime}$ & $99^{\circ} 05^{\prime} 03^{\prime \prime}$ & Edwards, Trinity & -- & & -208.0 & .7 & 3.7 & \\
\hline 178 & $29^{\circ} 34^{\prime} 41^{\prime \prime}$ & $99^{\circ} 04^{\prime} 48^{\prime \prime}$ & Edwards, Trinity & -- & & 275.0 & 2.1 & 3 & \\
\hline 178 & $29^{\circ} 31^{\prime} 17^{\prime \prime}$ & $99^{\circ} 05^{\prime} 03^{\prime \prime}$ & Edwards & -- & & -366.0 & 2.3 & 5.1 & \\
\hline 178 & $29^{\circ} 30^{\prime} 11^{\prime \prime}$ & $99^{\circ} 06^{\prime} 02^{\prime \prime}$ & Edwards & -- & & 252.0 & .7 & 7.4 & \\
\hline 178 & $29^{\circ} 30^{\prime} 05^{\prime \prime}$ & 9906'30" & Edwards & -- & & -48.0 & 1.9 & 8.1 & \\
\hline 178 & $29^{\circ} 29^{\circ} 00^{\prime \prime}$ & $99^{\circ} 07^{\prime} 31 "$ & Edwards & -- & & -14.0 & 2.6 & 10 & \\
\hline 178 & $29^{\circ} 27^{\prime} 00^{\prime \prime}$ & $99^{\circ} 07^{\prime} 30^{\prime \prime}$ & Edwards & -- & & -49.0 & 3.9 & 12.6 & \\
\hline 179 & $29^{\circ} 34^{\prime} 08^{\prime \prime}$ & $99^{\circ} 05^{\prime} 54^{\prime \prime}$ & Edwards & -- & & -24.0 & 3.7 & 0 & on SH 173 \\
\hline 179 & $29^{\circ} 32^{\prime} 17^{\prime \prime}$ & $99^{\circ} 05^{\prime} 03^{\prime \prime}$ & Edwards, Trinity & -- & & -33.0 & 1.4 & 3.7 & \\
\hline 179 & $29^{\circ} 31^{\prime} 17^{\prime \prime}$ & $99^{\circ} 05^{\prime} 03^{\prime \prime}$ & Edwards & -- & & -21.0 & 2.3 & 5.1 & \\
\hline 179 & $29^{\circ} 30^{\prime} 11^{\prime \prime}$ & $99^{\circ} 06^{\prime} 02^{\prime \prime}$ & Edwards & -- & & 0 & .7 & 7.4 & \\
\hline 179 & $29^{\circ} 30^{\prime} 05^{\prime \prime}$ & $99^{\circ} 06^{\prime} 30^{\prime \prime}$ & Edwards & -- & & -5.0 & 1.9 & 8.1 & \\
\hline 179 & $29^{\circ} 29^{\circ} 00^{\prime \prime}$ & $99^{\circ} 07^{\prime} 31^{\prime \prime}$ & Edwards & -- & & -3.0 & 6.5 & 10 & \\
\hline 180 & $29^{\circ} 35^{\prime} 15^{\prime \prime}$ & $99^{\circ} 06^{\prime} 32^{\prime \prime}$ & Trinity & -- & & -48.4 & 11.2 & 0 & 1.3 mi below West Verde Creek \\
\hline 180 & $29^{\circ} 28^{\prime} 55^{\prime \prime}$ & $99^{\circ} 07^{\prime} 18^{\prime \prime}$ & Edwards, Trinity & -- & & -5.0 & 5.2 & 11.2 & \\
\hline 180 & $29^{\circ} 24^{\prime} 23^{\prime \prime}$ & $99^{\circ} 07^{\prime} 12^{\prime \prime}$ & Edwards & -- & & -5.0 & 1.3 & 16.4 & \\
\hline 180 & $29^{\circ} 24^{\prime} 25^{\prime \prime}$ & $99^{\circ} 07^{\prime} 24^{\prime \prime}$ & -- & -- & & .1 & .5 & 17.7 & \\
\hline 180 & $29^{\circ} 24^{\prime} 16^{\prime \prime}$ & $99^{\circ} 06^{\prime} 58^{\prime \prime}$ & -- & -- & & 0 & 1.3 & 18.2 & \\
\hline 181 & $29^{\circ} 34^{\prime} 32^{\prime \prime}$ & $99^{\circ} 05^{\prime} 54^{\prime \prime}$ & Edwards & -- & & -24.0 & 3.7 & 24 & $700 \mathrm{ft}$ above bridge on SH 173 \\
\hline 181 & $29^{\circ} 32^{\prime} 34^{\prime \prime}$ & $99^{\circ} 04^{\prime} 46^{\prime \prime}$ & Edwards, Trinity & -- & & -33.0 & 1.4 & 20.3 & \\
\hline 181 & $29^{\circ} 31^{\prime} 30^{\prime \prime}$ & $99^{\circ} 05^{\prime} 03^{\prime \prime}$ & Edwards & -- & & -21.0 & 2.3 & 18.9 & \\
\hline 181 & $29^{\circ} 30^{\prime} 29^{\prime \prime}$ & $99^{\circ} 06^{\prime} 01^{\prime \prime}$ & Edwards & -- & & 0 & .7 & 16.6 & \\
\hline 181 & $29^{\circ} 30^{\prime} 29^{\prime \prime}$ & $99^{\circ} 06 ' 30^{\prime \prime}$ & Edwards & -- & & -5.0 & 1.9 & 15.9 & \\
\hline 181 & $29^{\circ} 29^{\circ} 30^{\prime \prime}$ & $99^{\circ} 07^{\prime} 17^{\prime \prime}$ & Edwards & -- & & -4.0 & 3.0 & 14 & \\
\hline 181 & $29^{\circ} 27^{\prime} 24^{\prime \prime}$ & $99^{\circ} 07^{\prime} 30^{\prime \prime}$ & Edwards & -- & & 1.0 & 3.5 & 11 & \\
\hline 182 & $29^{\circ} 25^{\prime} 41^{\prime \prime}$ & $99^{\circ} 59^{\prime} 48^{\prime \prime}$ & Edwards & -- & & -17.5 & 8.0 & -.4 & $0.4 \mathrm{mi}$ above gaging station at Laguna ( 08190000$)$ \\
\hline 182 & $29^{\circ} 20^{\prime} 50^{\prime \prime}$ & $99^{\circ} 57^{\prime} 24^{\prime \prime}$ & Edwards & -- & & -40.5 & 1.2 & 7.6 & \\
\hline
\end{tabular}


$\overrightarrow{8} \quad$ Table 4. Gains and losses from gain-loss studies in Texas-Continued

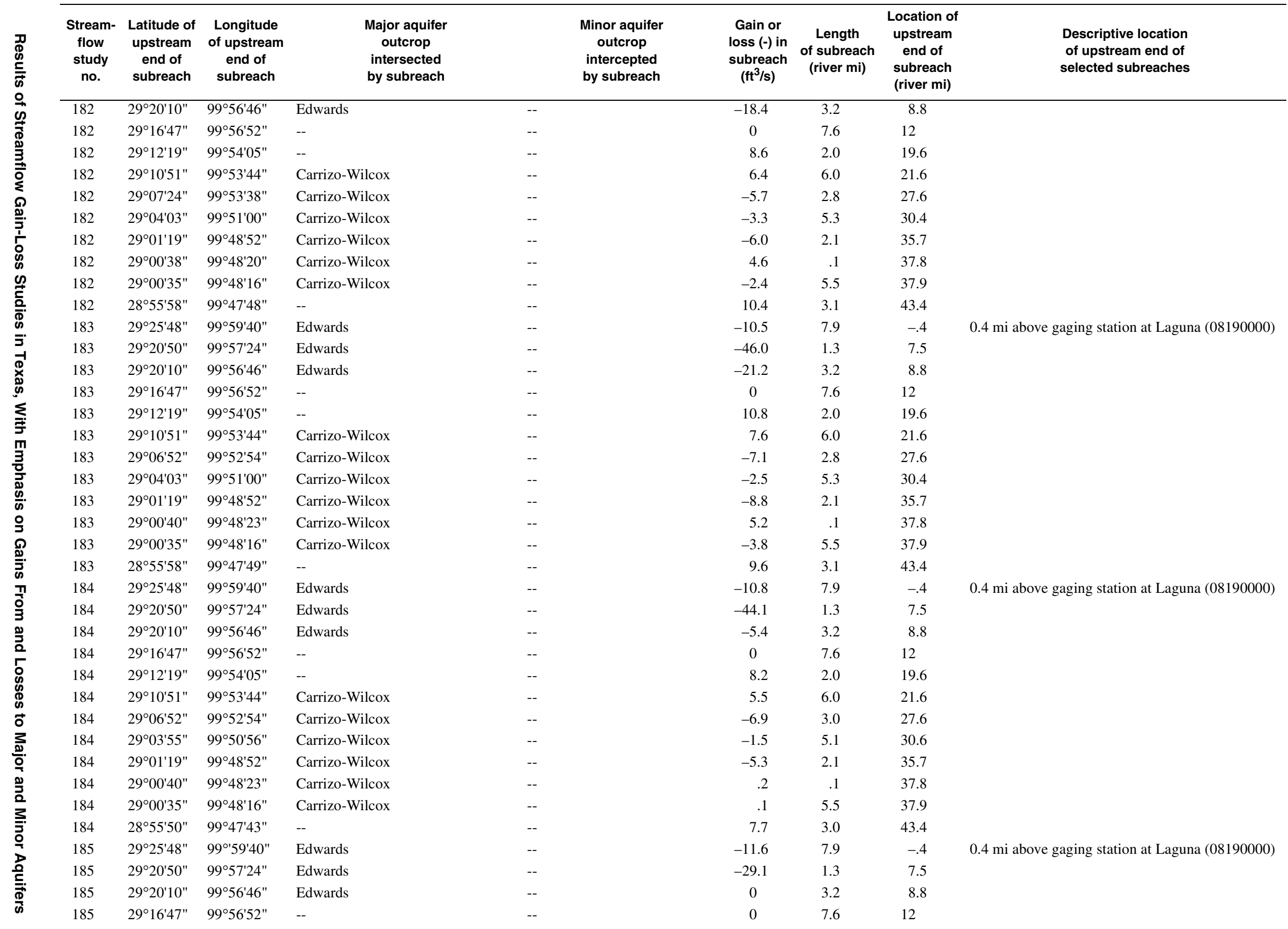


Table 4. Gains and losses from gain-loss studies in Texas-Continued

\begin{tabular}{|c|c|c|c|c|c|c|c|c|c|}
\hline $\begin{array}{l}\text { Stream- } \\
\text { flow } \\
\text { study } \\
\text { no. }\end{array}$ & $\begin{array}{l}\text { Latitude of } \\
\text { upstream } \\
\text { end of } \\
\text { subreach }\end{array}$ & $\begin{array}{l}\text { Longitude } \\
\text { of upstream } \\
\text { end of } \\
\text { subreach }\end{array}$ & $\begin{array}{c}\text { Major aquifer } \\
\text { outcrop } \\
\text { intersected } \\
\text { by subreach }\end{array}$ & & $\begin{array}{c}\text { Minor aquifer } \\
\text { outcrop } \\
\text { intercepted } \\
\text { by subreach }\end{array}$ & $\begin{array}{c}\text { Gain or } \\
\text { loss }(-) \text { in } \\
\text { subreach } \\
\left(\mathrm{ft}^{3} / \mathrm{s}\right)\end{array}$ & $\begin{array}{l}\text { Length } \\
\text { of subreach } \\
\text { (river mi) }\end{array}$ & $\begin{array}{l}\text { Location of } \\
\text { upstream } \\
\text { end of } \\
\text { subreach } \\
\text { (river mi) }\end{array}$ & $\begin{array}{l}\text { Descriptive location } \\
\text { of upstream end of } \\
\text { selected subreaches }\end{array}$ \\
\hline 185 & $29^{\circ} 12^{\prime} 19^{\prime \prime}$ & $99^{\circ} 54^{\prime} 05^{\prime \prime}$ & -- & -- & & 7.0 & 2.0 & 19.6 & \\
\hline 185 & $29^{\circ} 10^{\prime} 51^{\prime \prime}$ & $99^{\circ} 53^{\prime} 44^{\prime \prime}$ & Carrizo-Wilcox & -- & & 5.1 & 6.0 & 21.6 & \\
\hline 185 & $29^{\circ} 06^{\prime} 52^{\prime \prime}$ & $99^{\circ} 52^{\prime} 54^{\prime \prime}$ & Carrizo-Wilcox & -- & & -5.6 & 3.0 & 27.6 & \\
\hline 185 & $29^{\circ} 03^{\prime} 55^{\prime \prime}$ & $99^{\circ} 50^{\prime} 56^{\prime \prime}$ & Carrizo-Wilcox & -- & & -1.4 & 5.1 & 30.6 & \\
\hline 185 & $29^{\circ} 01^{\prime} 19^{\prime \prime}$ & $99^{\circ} 48^{\prime} 52^{\prime \prime}$ & Carrizo-Wilcox & -- & & 0 & 2.2 & 35.7 & \\
\hline 185 & $29^{\circ} 00^{\prime} 35^{\prime \prime}$ & $99^{\circ} 48^{\prime} 16^{\prime \prime}$ & Carrizo-Wilcox & -- & & 0 & 5.3 & 37.9 & \\
\hline 185 & $28^{\circ} 55^{\prime} 58^{\prime \prime}$ & $99^{\circ} 47^{\prime} 49^{\prime \prime}$ & -- & -- & & 7.7 & 3.3 & 43.2 & \\
\hline 186 & $29^{\circ} 25^{\prime} 42^{\prime \prime}$ & $99^{\circ} 59^{\prime} 47^{\prime \prime}$ & Edwards & -- & & -47.0 & 7.8 & 0 & gaging station (08190000) \\
\hline 186 & $29^{\circ} 19^{\prime} 41^{\prime \prime}$ & $99^{\circ} 56^{\prime} 51^{\prime \prime}$ & Edwards & -- & & -4.0 & 9.4 & 7.8 & \\
\hline 186 & $29^{\circ} 14^{\prime} 38^{\prime \prime}$ & $99^{\circ} 54^{\prime} 24 "$ & -- & -- & & -48.0 & 3.3 & 17.2 & \\
\hline 186 & $29^{\circ} 12^{\prime} 19^{\prime \prime}$ & $99^{\circ} 54^{\prime} 06^{\prime \prime}$ & -- & -- & & 11.0 & 2.7 & 20.5 & \\
\hline 186 & $29^{\circ} 10^{\prime} 51^{\prime \prime}$ & $99^{\circ} 53^{\prime} 39^{\prime \prime}$ & -- & -- & & 64.0 & 6.2 & 23.2 & \\
\hline 187 & $29^{\circ} 43^{\prime} 14^{\prime \prime}$ & $100^{\circ} 02^{\prime} 02^{\prime \prime}$ & Edwards-Trinity Plateau & -- & & -3.31 & 1.9 & 0 & Barksdale - $100 \mathrm{ft}$ below hwy \\
\hline 187 & $29^{\circ} 41^{\prime} 54^{\prime \prime}$ & $100^{\circ} 01^{\prime} 48^{\prime \prime}$ & Edwards-Trinity Plateau & -- & & 10.5 & 1.8 & 1.9 & \\
\hline 187 & $29^{\circ} 40^{\prime} 20^{\prime \prime}$ & $100^{\circ} 01^{\prime} 04^{\prime \prime}$ & Edwards-Trinity Plateau & -- & & -1.9 & 2.7 & 3.7 & \\
\hline 187 & $29^{\circ} 38^{\prime} 38^{\prime \prime}$ & $100^{\circ} 00^{\prime} 50^{\prime \prime}$ & Edwards-Trinity Plateau & -- & & 0 & 2.0 & 6.4 & \\
\hline 187 & $29^{\circ} 37^{\prime} 20^{\prime \prime}$ & $100^{\circ} 00^{\prime} 46^{\prime \prime}$ & Edwards-Trinity Plateau & -- & & -4.3 & 3.9 & 8.4 & \\
\hline 187 & $29^{\circ} 34^{\prime} 35^{\prime \prime}$ & $99^{\circ} 59^{\prime} 52^{\prime \prime}$ & Edwards-Trinity Plateau & -- & & -6.4 & 1.5 & 12.3 & \\
\hline 187 & $29^{\circ} 32^{\prime} 53^{\prime \prime}$ & $99^{\circ} 59^{\prime} 38^{\prime \prime}$ & Edwards-Trinity Plateau & -- & & -15.7 & .3 & 13.8 & \\
\hline 187 & $29^{\circ} 32^{\prime} 47^{\prime \prime}$ & $99^{\circ} 59^{\prime} 54^{\prime \prime}$ & Edwards-Trinity Plateau & -- & & 1.5 & .4 & 14.1 & \\
\hline 187 & $29^{\circ} 32^{\prime} 22^{\prime \prime}$ & $99^{\circ} 59^{\prime} 51^{\prime \prime}$ & Edwards-Trinity Plateau & -- & & -.5 & .7 & 14.5 & \\
\hline 187 & $29^{\circ} 32^{\prime} 04^{\prime \prime}$ & $100^{\circ} 00^{\prime} 10^{\prime \prime}$ & Edwards-Trinity Plateau & -- & & 9.0 & .5 & 15.2 & \\
\hline 187 & $29^{\circ} 31^{\prime} 56^{\prime \prime}$ & $100^{\circ} 00^{\prime} 43^{\prime \prime}$ & Edwards-Trinity Plateau & -- & & -10.0 & .5 & 15.7 & \\
\hline 187 & $29^{\circ} 30^{\prime} 57^{\prime \prime}$ & $100^{\circ} 01^{\prime} 26^{\prime \prime}$ & Edwards-Trinity Plateau & -- & & .8 & .9 & 16.2 & \\
\hline 187 & $29^{\circ} 31^{\prime} 12^{\prime \prime}$ & $100^{\circ} 01^{\prime} 08^{\prime \prime}$ & Edwards-Trinity Plateau & -- & & 6.7 & 1.4 & 17.1 & \\
\hline 187 & $29^{\circ} 29^{\circ} 09^{\prime \prime}$ & $100^{\circ} 01^{\prime} 04^{\prime \prime}$ & Edwards-Trinity Plateau & -- & & 3.4 & 2.3 & 18.5 & \\
\hline 187 & $29^{\circ} 28^{\prime} 40^{\prime \prime}$ & $100^{\circ} 01^{\prime} 33^{\prime \prime}$ & Edwards-Trinity Plateau & -- & & 7.4 & 2.5 & 20.8 & \\
\hline 187 & $29^{\circ} 27^{\prime} 37^{\prime \prime}$ & $99^{\circ} 59^{\prime} 52^{\prime \prime}$ & Edwards-Trinity Plateau & -- & & 1.8 & 1.9 & 23.3 & \\
\hline 188 & $29^{\circ} 43^{\prime} 14^{\prime \prime}$ & $100^{\circ} 01^{\prime} 58^{\prime \prime}$ & Edwards-Trinity Plateau & -- & & 3.68 & 1.9 & 0 & Barksdale - $50 \mathrm{ft}$ below hwy \\
\hline 188 & $29^{\circ} 41^{\prime} 54^{\prime \prime}$ & $100^{\circ} 01^{\prime} 48^{\prime \prime}$ & Edwards-Trinity Plateau & -- & & 14.1 & 1.8 & 1.9 & \\
\hline 188 & $29^{\circ} 40^{\prime} 00^{\prime \prime}$ & $100^{\circ} 01^{\prime} 44^{\prime \prime}$ & Edwards-Trinity Plateau & -- & & -.2 & 2.7 & 3.7 & \\
\hline 188 & $29^{\circ} 38^{\prime} 38^{\prime \prime}$ & $100^{\circ} 00^{\prime} 50^{\prime \prime}$ & Edwards-Trinity Plateau & -- & & 2.5 & 2.0 & 6.4 & \\
\hline 188 & $29^{\circ} 37^{\prime} 19^{\prime \prime}$ & $100^{\circ} 00^{\prime} 46^{\prime \prime}$ & Edwards-Trinity Plateau & -- & & -7.0 & 3.9 & 8.4 & \\
\hline 188 & $29^{\circ} 34^{\prime} 35^{\prime \prime}$ & $99^{\circ} 59^{\prime} 52^{\prime \prime}$ & Edwards-Trinity Plateau & -- & & -8.0 & 1.5 & 12.3 & \\
\hline 188 & $29^{\circ} 32^{\prime} 55^{\prime \prime}$ & $99^{\circ} 59^{\prime} 39^{\prime \prime}$ & Edwards-Trinity Plateau & -- & & -19.3 & .3 & 13.8 & \\
\hline 188 & $29^{\circ} 32^{\prime} 47^{\prime \prime}$ & $99^{\circ} 59^{\prime} 54^{\prime \prime}$ & Edwards-Trinity Plateau & -- & & 1.5 & .4 & 14.1 & \\
\hline 188 & $29^{\circ} 32^{\prime} 22^{\prime \prime}$ & 9959'51" & Edwards-Trinity Plateau & -- & & 1.5 & .7 & 14.5 & \\
\hline 188 & $29^{\circ} 32^{\prime} 04^{\prime \prime}$ & $100^{\circ} 00^{\prime} 10^{\prime \prime}$ & Edwards-Trinity Plateau & -- & & 12.0 & .5 & 15.2 & \\
\hline
\end{tabular}


근 Table 4. Gains and losses from gain-loss studies in Texas-Continued

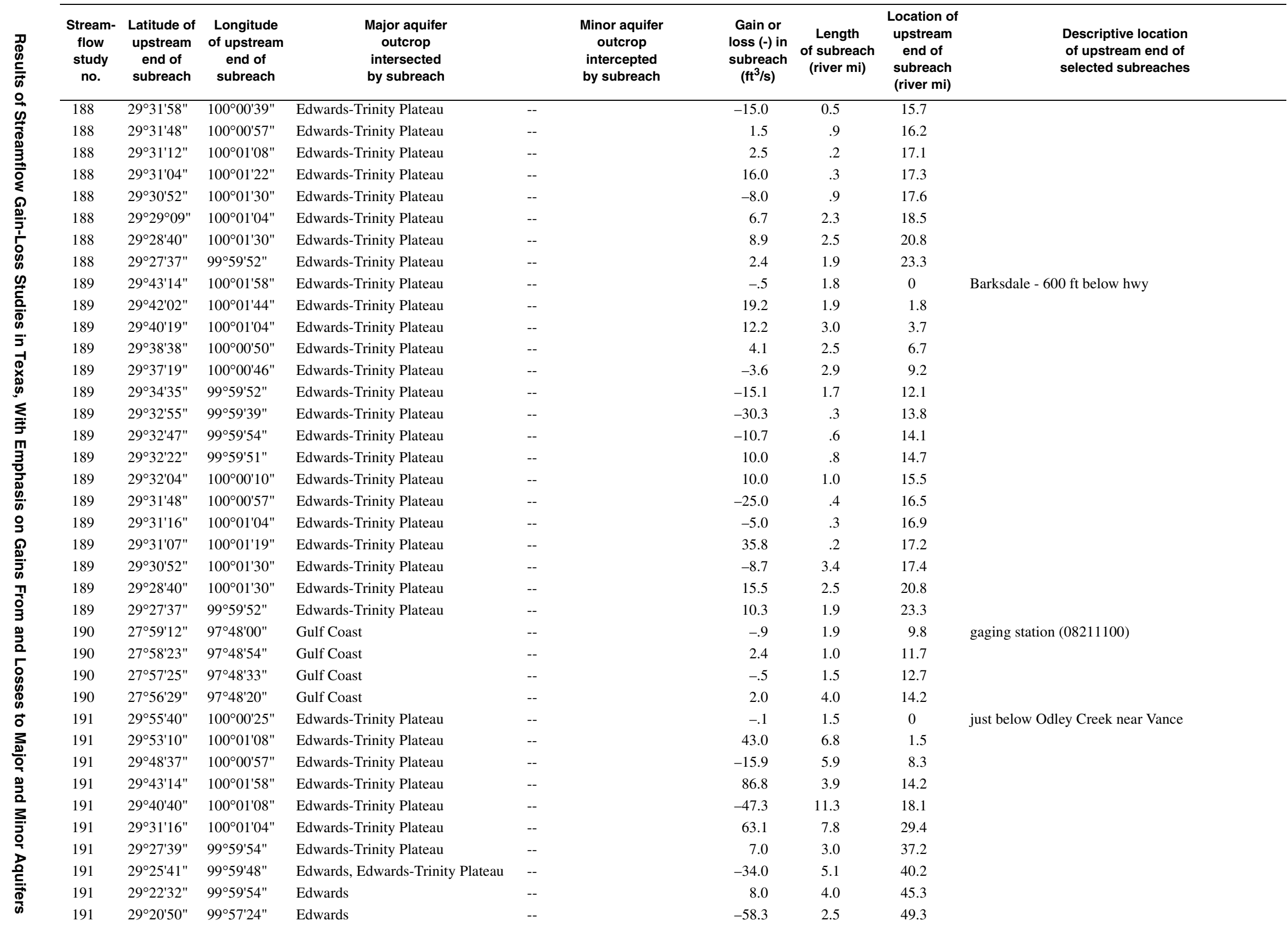


Table 4. Gains and losses from gain-loss studies in Texas-Continued

\begin{tabular}{|c|c|c|c|c|c|c|c|c|c|}
\hline $\begin{array}{l}\text { Stream- } \\
\text { flow } \\
\text { study } \\
\text { no. }\end{array}$ & $\begin{array}{l}\text { Latitude of } \\
\text { upstream } \\
\text { end of } \\
\text { subreach }\end{array}$ & $\begin{array}{l}\text { Longitude } \\
\text { of upstream } \\
\text { end of } \\
\text { subreach }\end{array}$ & $\begin{array}{l}\text { Major aquifer } \\
\text { outcrop } \\
\text { intersected } \\
\text { by subreach }\end{array}$ & & $\begin{array}{l}\text { Minor aquifer } \\
\text { outcrop } \\
\text { intercepted } \\
\text { by subreach }\end{array}$ & $\begin{array}{l}\text { Gain or } \\
\text { loss }(-) \text { in } \\
\text { subreach } \\
\left(\mathrm{ft}^{3} / \mathrm{s}\right)\end{array}$ & $\begin{array}{l}\text { Length } \\
\text { of subreach } \\
\text { (river mi) }\end{array}$ & $\begin{array}{l}\text { Location of } \\
\text { upstream } \\
\text { end of } \\
\text { subreach } \\
\text { (river mi) }\end{array}$ & $\begin{array}{l}\text { Descriptive location } \\
\text { of upstream end of } \\
\text { selected subreaches }\end{array}$ \\
\hline 191 & $29^{\circ} 17^{\prime} 00^{\prime \prime}$ & $99^{\circ} 56^{\prime} 53^{\prime \prime}$ & -- & -- & & -30.1 & 4.5 & 51.8 & \\
\hline 191 & $29^{\circ} 14^{\prime} 37^{\prime \prime}$ & $99^{\circ} 54^{\prime} 24^{\prime \prime}$ & -- & -- & & -33.4 & 2.8 & 56.3 & \\
\hline 191 & $29^{\circ} 11^{\prime} 52^{\prime \prime}$ & $99^{\circ} 54^{\prime} 17^{\prime \prime}$ & -- & -- & & 21.6 & 2.0 & 59.1 & \\
\hline 191 & $29^{\circ} 10^{\prime} 51^{\prime \prime}$ & $99^{\circ} 53^{\prime} 44^{\prime \prime}$ & -- & -- & & .9 & 1.6 & 61.1 & \\
\hline 191 & $29^{\circ} 09^{\prime} 05^{\prime \prime}$ & $99^{\circ} 54^{\prime} 59^{\prime \prime}$ & Carrizo-Wilcox & -- & & 13.9 & 3.9 & 62.7 & \\
\hline 191 & $29^{\circ} 07^{\prime} 09^{\prime \prime}$ & $99^{\circ} 53^{\prime} 15^{\prime \prime}$ & Carrizo-Wilcox & -- & & -8.4 & 3.0 & 66.6 & \\
\hline 191 & $29^{\circ} 04^{\prime} 44^{\prime \prime}$ & 99॰52'17" & Carrizo-Wilcox & -- & & 3.0 & 4.8 & 69.6 & \\
\hline 192 & $28^{\circ} 25^{\prime} 33^{\prime \prime}$ & $99^{\circ} 14^{\prime} 22^{\prime \prime}$ & -- & -- & & 0 & 7.4 & 221.8 & gaging station (08194000) at Cotulla \\
\hline 192 & $28^{\circ} 22^{\prime} 18^{\prime \prime}$ & $99^{\circ} 11^{\prime} 40^{\prime \prime}$ & -- & -- & & -19.7 & 8.8 & 214.4 & \\
\hline 192 & $28^{\circ} 18^{\prime} 02^{\prime \prime}$ & $99^{\circ} 08^{\prime} 20^{\prime \prime}$ & -- & -- & & 7.5 & 11.2 & 205.6 & \\
\hline 192 & $28^{\circ} 14^{\prime} 06^{\prime \prime}$ & $99^{\circ} 04^{\prime} 05^{\prime \prime}$ & -- & -- & & 2.8 & 3.7 & 194.4 & \\
\hline 192 & $28^{\circ} 12^{\prime} 02^{\prime \prime}$ & $99^{\circ} 03^{\prime} 20^{\prime \prime}$ & -- & -- & & 2.4 & 4.3 & 190.7 & \\
\hline 192 & $28^{\circ} 09^{\prime} 36^{\prime \prime}$ & $99^{\circ} 02^{\prime} 04^{\prime \prime}$ & -- & -- & & -1.3 & 33.0 & 186.4 & \\
\hline 192 & $28^{\circ} 11^{\prime} 57^{\prime \prime}$ & $98^{\circ} 41^{\prime} 48^{\prime \prime}$ & -- & -- & & 7.9 & 15.0 & 153.4 & \\
\hline 192 & $28^{\circ} 18^{\prime} 30^{\prime \prime}$ & $98^{\circ} 33^{\prime} 24^{\prime \prime}$ & -- & -- & & 3.0 & 9.3 & 138.4 & \\
\hline 192 & $28^{\circ} 20^{\prime} 12^{\prime \prime}$ & $98^{\circ} 26^{\prime} 24^{\prime \prime}$ & Gulf Coast & -- & & 5.0 & 15.4 & 129.1 & \\
\hline 193 & $28^{\circ} 25^{\prime} 33^{\prime \prime}$ & $99^{\circ} 14^{\prime} 22^{\prime \prime}$ & -- & -- & & -.5 & 7.4 & 221.8 & gaging station (08194000) \\
\hline 193 & $28^{\circ} 22^{\prime} 18^{\prime \prime}$ & $99^{\circ} 11^{\prime} 40^{\prime \prime}$ & -- & -- & & -7.0 & 8.8 & 214.4 & \\
\hline 193 & $28^{\circ} 18^{\prime} 02^{\prime \prime}$ & $99^{\circ} 08^{\prime} 20^{\prime \prime}$ & -- & -- & & 1.2 & 11.2 & 205.6 & \\
\hline 193 & $28^{\circ} 14^{\prime} 06^{\prime \prime}$ & $99^{\circ} 04^{\prime} 05^{\prime \prime}$ & -- & -- & & 4.6 & 8.0 & 194.4 & \\
\hline 193 & $28^{\circ} 09^{\prime} 36^{\prime \prime}$ & $99^{\circ} 02^{\prime} 04^{\prime \prime}$ & -- & -- & & .09 & 17.1 & 186.4 & \\
\hline 193 & $28^{\circ} 05^{\prime} 47^{\prime \prime}$ & $98^{\circ} 51^{\prime} 00^{\prime \prime}$ & -- & -- & & 3.1 & 15.9 & 169.3 & \\
\hline 193 & $28^{\circ} 11^{\prime} 57^{\prime \prime}$ & $98^{\circ} 41^{\prime} 48^{\prime \prime}$ & -- & -- & & 2.3 & 15.0 & 153.4 & \\
\hline 193 & $28^{\circ} 18^{\prime} 30^{\prime \prime}$ & $98^{\circ} 33^{\prime} 24^{\prime \prime}$ & -- & -- & & 6.5 & 9.3 & 138.4 & gaging station (08194500) \\
\hline 193 & $28^{\circ} 20^{\prime} 12^{\prime \prime}$ & $98^{\circ} 26^{\prime} 24^{\prime \prime}$ & Gulf Coast & -- & & -1.0 & 15.4 & 129.1 & \\
\hline 194 & $29^{\circ} 25^{\prime} 48^{\prime \prime}$ & $99^{\circ} 59^{\prime} 40^{\prime \prime}$ & Edwards & -- & & -.9 & .4 & -.2 & $\begin{array}{l}1,200 \mathrm{ft} \text { above gaging station at Laguna } \\
(08190000)\end{array}$ \\
\hline 194 & $29^{\circ} 25^{\prime} 29^{\prime \prime}$ & $99^{\circ} 59^{\prime} 50^{\prime \prime}$ & Edwards & -- & & -4.1 & 1.6 & .2 & \\
\hline 194 & $29^{\circ} 23^{\prime} 52^{\prime \prime}$ & 9959'58" & Edwards & -- & & -3.8 & 1.8 & 1.8 & \\
\hline 194 & $29^{\circ} 23^{\prime} 06^{\prime \prime}$ & $100^{\circ} 00^{\prime} 21^{\prime \prime}$ & Edwards & -- & & -12.2 & 3.0 & 3.6 & \\
\hline 194 & $29^{\circ} 21^{\prime} 34^{\prime \prime}$ & $99^{\circ} 58^{\prime} 13^{\prime \prime}$ & Edwards & -- & & 4.5 & 1.0 & 6.6 & \\
\hline 194 & $29^{\circ} 20^{\prime} 50^{\prime \prime}$ & $99^{\circ} 57^{\prime} 17^{\prime \prime}$ & Edwards & -- & & -10.1 & .6 & 7.6 & \\
\hline 194 & $29^{\circ} 20^{\prime} 10^{\prime \prime}$ & $99^{\circ} 56^{\prime} 46^{\prime \prime}$ & Edwards & -- & & 0 & 3.8 & 8.2 & \\
\hline 194 & $29^{\circ} 17^{\prime} 18^{\prime \prime}$ & $99^{\circ} 56^{\prime} 56^{\prime \prime}$ & -- & -- & & 0 & 4.9 & 12 & \\
\hline 194 & $29^{\circ} 14^{\prime} 38^{\prime \prime}$ & $99^{\circ} 54^{\prime} 25^{\prime \prime}$ & -- & -- & & 0 & 2.7 & 16.9 & \\
\hline 194 & $29^{\circ} 12^{\prime} 19^{\prime \prime}$ & $99^{\circ} 54^{\prime} 05^{\prime \prime}$ & -- & -- & & 0 & 1.0 & 19.6 & \\
\hline 194 & $29^{\circ} 11^{\prime} 38^{\prime \prime}$ & $99^{\circ} 54^{\prime} 19^{\prime \prime}$ & -- & -- & & 8.9 & 1.0 & 20.6 & \\
\hline 194 & $29^{\circ} 10^{\prime} 51^{\prime \prime}$ & $99^{\circ} 53^{\prime} 44^{\prime \prime}$ & -- & -- & & 1.1 & 1.0 & 21.6 & \\
\hline
\end{tabular}


$\vec{\ddagger} \quad$ Table 4. Gains and losses from gain-loss studies in Texas-Continued

\begin{tabular}{|c|c|c|c|c|c|c|c|c|c|}
\hline $\begin{array}{l}\text { Stream- } \\
\text { flow } \\
\text { study } \\
\text { no. }\end{array}$ & $\begin{array}{l}\text { Latitude of } \\
\text { upstream } \\
\text { end of } \\
\text { subreach }\end{array}$ & $\begin{array}{l}\text { Longitude } \\
\text { of upstream } \\
\text { end of } \\
\text { subreach }\end{array}$ & $\begin{array}{c}\text { Major aquifer } \\
\text { outcrop } \\
\text { intersected } \\
\text { by subreach }\end{array}$ & & $\begin{array}{l}\text { Minor aquifer } \\
\text { outcrop } \\
\text { intercepted } \\
\text { by subreach }\end{array}$ & $\begin{array}{l}\text { Gain or } \\
\text { loss }(-) \text { in } \\
\text { subreach } \\
\left(\mathrm{ft}^{3} / \mathrm{s}\right)\end{array}$ & $\begin{array}{l}\text { Length } \\
\text { of subreach } \\
\text { (river mi) }\end{array}$ & $\begin{array}{l}\text { Location of } \\
\text { upstream } \\
\text { end of } \\
\text { subreach } \\
\text { (river mi) }\end{array}$ & $\begin{array}{l}\text { Descriptive location } \\
\text { of upstream end of } \\
\text { selected subreaches }\end{array}$ \\
\hline 194 & $29^{\circ} 09^{\prime} 05^{\prime \prime}$ & $99^{\circ} 54^{\prime} 59^{\prime \prime}$ & -- & -- & & -3.0 & 2.0 & 22.6 & \\
\hline 194 & $29^{\circ} 08^{\prime} 53^{\prime \prime}$ & $99^{\circ} 53^{\prime} 52^{\prime \prime}$ & Carrizo-Wilcox & -- & & 4.3 & 2.0 & 24.6 & \\
\hline 194 & $29^{\circ} 07^{\prime} 24^{\prime \prime}$ & $99^{\circ} 53^{\prime} 38^{\prime \prime}$ & Carrizo-Wilcox & -- & & -1.6 & 1.0 & 26.6 & \\
\hline 194 & $29^{\circ} 06^{\prime} 52^{\prime \prime}$ & $99^{\circ} 52^{\prime} 54^{\prime \prime}$ & Carrizo-Wilcox & -- & & -4.7 & 3.0 & 27.6 & \\
\hline 194 & $29^{\circ} 03^{\prime} 55^{\prime \prime}$ & $99^{\circ} 50^{\prime} 56^{\prime \prime}$ & Carrizo-Wilcox & -- & & -.2 & 5.1 & 30.6 & \\
\hline 194 & $29^{\circ} 01^{\prime} 56^{\prime \prime}$ & $99^{\circ} 49^{\prime} 46^{\prime \prime}$ & Carrizo-Wilcox & -- & & -4.2 & 2.3 & 35.7 & \\
\hline 194 & $29^{\circ} 00^{\prime} 31^{\prime \prime}$ & $99^{\circ} 48^{\prime} 12^{\prime \prime}$ & Carrizo-Wilcox & -- & & -1.1 & 5.2 & 38 & \\
\hline 194 & $28^{\circ} 55^{\prime} 58^{\prime \prime}$ & $99^{\circ} 47^{\prime} 49^{\prime \prime}$ & -- & -- & & 7.0 & 3.3 & 43.2 & \\
\hline 194 & $28^{\circ} 53^{\prime} 40^{\prime \prime}$ & $99^{\circ} 47^{\prime} 15^{\prime \prime}$ & -- & -- & & -2.2 & 3.5 & 46.5 & \\
\hline 194 & $28^{\circ} 51^{\prime} 57^{\prime \prime}$ & $99^{\circ} 46^{\prime} 57^{\prime \prime}$ & -- & -- & & 0 & 3.6 & 50 & \\
\hline 194 & $28^{\circ} 50^{\prime} 35^{\prime \prime}$ & $99^{\circ} 47^{\prime} 34^{\prime \prime}$ & -- & -- & & -.1 & 3.3 & 53.6 & \\
\hline 194 & $28^{\circ} 49^{\prime} 27^{\prime \prime}$ & $99^{\circ} 47^{\prime} 12^{\prime \prime}$ & -- & -- & & -1.3 & 4.5 & 56.9 & \\
\hline 195 & $29^{\circ} 25^{\prime} 41^{\prime \prime}$ & $99^{\circ} 59^{\prime} 48^{\prime \prime}$ & Edwards & -- & & -21.2 & 6.2 & 0 & \multirow[t]{9}{*}{ gaging station at Laguna (08190000) } \\
\hline 195 & $29^{\circ} 21^{\prime} 37^{\prime \prime}$ & $99^{\circ} 58^{\prime} 59^{\prime \prime}$ & Edwards & -- & & -28.1 & 4.2 & 6.2 & \\
\hline 195 & $29^{\circ} 19^{\prime} 17^{\prime \prime}$ & $99^{\circ} 57^{\prime} 03^{\prime \prime}$ & Edwards & -- & & -14.9 & 2.6 & 10.4 & \\
\hline 195 & $29^{\circ} 17^{\prime} 18^{\prime \prime}$ & $99^{\circ} 56^{\prime} 55^{\prime \prime}$ & -- & -- & & 0 & 5.0 & 13 & \\
\hline 195 & $29^{\circ} 14^{\prime} 37^{\prime \prime}$ & $99^{\circ} 54^{\prime} 24^{\prime \prime}$ & -- & -- & & 0 & 2.6 & 18 & \\
\hline 195 & $29^{\circ} 12^{\prime} 19^{\prime \prime}$ & $99^{\circ} 54^{\prime} 05^{\prime \prime}$ & -- & -- & & 9.0 & 2.1 & 20.6 & \\
\hline 195 & $29^{\circ} 07^{\prime} 24^{\prime \prime}$ & $99^{\circ} 53^{\prime} 38^{\prime \prime}$ & -- & -- & & 7.8 & 5.5 & 22.7 & \\
\hline 195 & $29^{\circ} 07^{\prime} 09^{\prime \prime}$ & $99^{\circ} 53^{\prime} 15^{\prime \prime}$ & Carrizo-Wilcox & -- & & -4.1 & 3.4 & 28.2 & \\
\hline 195 & $29^{\circ} 04^{\prime} 42^{\prime \prime}$ & $99^{\circ} 52^{\prime} 18^{\prime \prime}$ & Carrizo-Wilcox & -- & & -8.7 & 8.0 & 31.6 & \\
\hline 196 & $29^{\circ} 25^{\prime} 41^{\prime \prime}$ & $99^{\circ} 59^{\prime} 48^{\prime \prime}$ & Edwards & -- & & -17.8 & 6.2 & 0 & \multirow[t]{10}{*}{ gaging station at Laguna (08190000) } \\
\hline 196 & $29^{\circ} 21^{\prime} 35^{\prime \prime}$ & $99^{\circ} 58^{\prime} 57^{\prime \prime}$ & Edwards & -- & & -23.3 & 4.2 & 6.2 & \\
\hline 196 & $29^{\circ} 19^{\prime} 17^{\prime \prime}$ & $99^{\circ} 57^{\prime} 03^{\prime \prime}$ & Edwards & -- & & -22.9 & 2.6 & 10.4 & \\
\hline 196 & $29^{\circ} 17^{\prime} 18^{\prime \prime}$ & $99^{\circ} 56^{\prime} 56^{\prime \prime}$ & -- & -- & & 0 & 5.0 & 13 & \\
\hline 196 & $29^{\circ} 14^{\prime} 37^{\prime \prime}$ & $99^{\circ} 54^{\prime} 24^{\prime \prime}$ & -- & -- & & 0 & 2.6 & 18 & \\
\hline 196 & $29^{\circ} 12^{\prime} 19^{\prime \prime}$ & $99^{\circ} 54^{\prime} 05^{\prime \prime}$ & -- & -- & & 8.1 & 2.1 & 20.6 & \\
\hline 196 & $29^{\circ} 07^{\prime} 24^{\prime \prime}$ & $99^{\circ} 53^{\prime} 38^{\prime \prime}$ & -- & -- & & 6.3 & 5.5 & 22.7 & \\
\hline 196 & $29^{\circ} 07^{\prime} 09^{\prime \prime}$ & $99^{\circ} 53^{\prime} 15^{\prime \prime}$ & Carrizo-Wilcox & -- & & -1.6 & 3.4 & 28.2 & \\
\hline 196 & $29^{\circ} 04^{\prime} 44^{\prime \prime}$ & $99^{\circ} 52^{\prime} 17^{\prime \prime}$ & Carrizo-Wilcox & -- & & -4.5 & 4.7 & 31.6 & \\
\hline 196 & $29^{\circ} 01 ' 55^{\prime \prime}$ & $99^{\circ} 49^{\prime} 48^{\prime \prime}$ & Carrizo-Wilcox & -- & & -3.8 & 3.3 & 36.3 & \\
\hline 197 & $29^{\circ} 25^{\prime} 42^{\prime \prime}$ & $99^{\circ} 59^{\prime} 47^{\prime \prime}$ & Edwards & -- & & -21.8 & 5.1 & 0 & \multirow[t]{7}{*}{ gaging station at Laguna (08190000) } \\
\hline 197 & $29^{\circ} 22^{\prime} 33^{\prime \prime}$ & $99^{\circ} 59^{\prime} 57^{\prime \prime}$ & Edwards & -- & & -13.5 & 13.8 & 5.1 & \\
\hline 197 & $29^{\circ} 15^{\prime} 08^{\prime \prime}$ & $99^{\circ} 55^{\prime} 55^{\prime \prime}$ & -- & -- & & 4.3 & 2.0 & 18.9 & \\
\hline 197 & $29^{\circ} 12^{\prime} 19^{\prime \prime}$ & $99^{\circ} 54^{\prime} 05^{\prime \prime}$ & -- & -- & & -2.8 & 1.9 & 20.9 & \\
\hline 197 & $29^{\circ} 09^{\prime} 05^{\prime \prime}$ & $99^{\circ} 54^{\prime} 59^{\prime \prime}$ & -- & -- & & 7.5 & 4.0 & 22.8 & \\
\hline 197 & $29^{\circ} 07^{\prime} 09^{\prime \prime}$ & $99^{\circ} 53^{\prime} 15^{\prime \prime}$ & Carrizo-Wilcox & -- & & -6.2 & 3.0 & 26.8 & \\
\hline 197 & $29^{\circ} 04^{\prime} 43^{\prime \prime}$ & $99^{\circ} 52^{\prime} 17^{\prime \prime}$ & Carrizo-Wilcox & -- & & -2.8 & 1.8 & 29.8 & \\
\hline
\end{tabular}


Table 4. Gains and losses from gain-loss studies in Texas-Continued

\begin{tabular}{|c|c|c|c|c|c|c|c|c|c|}
\hline $\begin{array}{c}\text { Stream- } \\
\text { flow } \\
\text { study } \\
\text { no. }\end{array}$ & $\begin{array}{l}\text { Latitude of } \\
\text { upstream } \\
\text { end of } \\
\text { subreach }\end{array}$ & $\begin{array}{l}\text { Longitude } \\
\text { of upstream } \\
\text { end of } \\
\text { subreach }\end{array}$ & $\begin{array}{c}\text { Major aquifer } \\
\text { outcrop } \\
\text { intersected } \\
\text { by subreach }\end{array}$ & & $\begin{array}{c}\text { Minor aquifer } \\
\text { outcrop } \\
\text { intercepted } \\
\text { by subreach }\end{array}$ & $\begin{array}{c}\text { Gain or } \\
\text { loss }(-) \text { in } \\
\text { subreach } \\
\left(\mathrm{ft}^{3} / \mathrm{s}\right)\end{array}$ & $\begin{array}{l}\text { Length } \\
\text { of subreach } \\
\text { (river mi) }\end{array}$ & $\begin{array}{l}\text { Location of } \\
\text { upstream } \\
\text { end of } \\
\text { subreach } \\
\text { (river mi) }\end{array}$ & $\begin{array}{l}\text { Descriptive location } \\
\text { of upstream end of } \\
\text { selected subreaches }\end{array}$ \\
\hline 197 & $29^{\circ} 03^{\prime} 56^{\prime \prime}$ & $99^{\circ} 50^{\prime} 57^{\prime \prime}$ & Carrizo-Wilcox & -- & & 0.5 & 2.9 & 31.6 & \\
\hline 197 & $29^{\circ} 02^{\prime} 43^{\prime \prime}$ & $99^{\circ} 50^{\prime} 03^{\prime \prime}$ & Carrizo-Wilcox & -- & & 0 & .1 & 34.5 & \\
\hline 197 & $29^{\circ} 01^{\prime} 57^{\prime \prime}$ & $99^{\circ} 49^{\prime} 46^{\prime \prime}$ & Carrizo-Wilcox & -- & & -.5 & 6.1 & 34.6 & \\
\hline 197 & $28^{\circ} 57^{\prime} 57^{\prime \prime}$ & $99^{\circ} 47^{\prime} 48^{\prime \prime}$ & -- & -- & & 0 & 2.5 & 40.7 & \\
\hline 197 & $28^{\circ} 54^{\prime} 59^{\prime \prime}$ & $99^{\circ} 47^{\prime} 28^{\prime \prime}$ & -- & -- & & 8.0 & 4.2 & 43.2 & \\
\hline 197 & $28^{\circ} 53^{\prime} 40^{\prime \prime}$ & $99^{\circ} 47^{\prime} 15^{\prime \prime}$ & -- & -- & & -2.6 & 7.5 & 47.4 & \\
\hline 198 & $29^{\circ} 25^{\prime} 41^{\prime \prime}$ & $99^{\circ} 59^{\prime} 47^{\prime \prime}$ & Edwards & -- & & -57.0 & 13.0 & 0 & gaging station at Laguna (08190000) \\
\hline 198 & $29^{\circ} 17^{\prime} 19^{\prime \prime}$ & $99^{\circ} 56^{\prime} 56^{\prime \prime}$ & -- & -- & & -37.0 & 5.0 & 13 & \\
\hline 198 & $29^{\circ} 14^{\prime} 38^{\prime \prime}$ & $99^{\circ} 54^{\prime} 24^{\prime \prime}$ & -- & -- & & -23.0 & 2.6 & 18 & \\
\hline 198 & $29^{\circ} 12^{\prime} 20^{\prime \prime}$ & $99^{\circ} 54^{\prime} 07^{\prime \prime}$ & -- & -- & & 20.0 & 2.1 & 20.6 & \\
\hline 198 & $29^{\circ} 07^{\prime} 24^{\prime \prime}$ & $99^{\circ} 53^{\prime} 37^{\prime \prime}$ & -- & -- & & 10.0 & 5.5 & 22.7 & \\
\hline 198 & $29^{\circ} 07^{\prime} 15^{\prime \prime}$ & $99^{\circ} 53^{\prime} 28^{\prime \prime}$ & Carrizo-Wilcox & -- & & -2.0 & 3.4 & 28.2 & \\
\hline 198 & $29^{\circ} 04^{\prime} 43^{\prime \prime}$ & $99^{\circ} 52^{\prime} 17^{\prime \prime}$ & Carrizo-Wilcox & -- & & -6.0 & 4.7 & 31.6 & \\
\hline 198 & $29^{\circ} 03^{\prime} 55^{\prime \prime}$ & $99^{\circ} 50^{\prime} 57^{\prime \prime}$ & Carrizo-Wilcox & -- & & -2.0 & 3.3 & 36.3 & \\
\hline 198 & $29^{\circ} 02^{\prime} 29^{\prime \prime}$ & $99^{\circ} 50^{\prime} 07^{\prime \prime}$ & Carrizo-Wilcox & -- & & -11.0 & 5.2 & 39.6 & \\
\hline 198 & $28^{\circ} 55^{\prime} 58^{\prime \prime}$ & $99^{\circ} 47^{\prime} 48^{\prime \prime}$ & -- & -- & & 32.0 & 11.7 & 44.8 & \\
\hline 199 & $29^{\circ} 25^{\prime} 38^{\prime \prime}$ & $99^{\circ} 59^{\prime} 43^{\prime \prime}$ & Edwards & -- & & -5.0 & 10.4 & 0 & gaging station at Laguna (08190000) \\
\hline 199 & $29^{\circ} 19^{\prime} 11^{\prime \prime}$ & $99^{\circ} 56^{\prime} 59^{\prime \prime}$ & Edwards & -- & & -30.0 & 2.6 & 10.4 & \\
\hline 199 & $29^{\circ} 17^{\prime} 10^{\prime \prime}$ & $99^{\circ} 56{ }^{\prime} 52^{\prime \prime}$ & -- & -- & & -41.0 & 5.0 & 13 & \\
\hline 199 & $29^{\circ} 14^{\prime} 36^{\prime \prime}$ & $99^{\circ} 54^{\prime} 16^{\prime \prime}$ & -- & -- & & -31.4 & 2.6 & 18 & \\
\hline 199 & $29^{\circ} 12^{\prime} 11^{\prime \prime}$ & $99^{\circ} 54^{\prime} 04^{\prime \prime}$ & -- & -- & & 29.4 & 2.1 & 20.6 & \\
\hline 199 & $29^{\circ} 07^{\prime} 05^{\prime \prime}$ & $99^{\circ} 53^{\prime} 39^{\prime \prime}$ & Carrizo-Wilcox & -- & & 4.0 & 8.9 & 22.7 & \\
\hline 199 & $29^{\circ} 04^{\prime} 50^{\prime \prime}$ & $99^{\circ} 52^{\prime} 21 "$ & Carrizo-Wilcox & -- & & -7.0 & 8.0 & 31.6 & \\
\hline 199 & $29^{\circ} 02^{\prime} 26^{\prime \prime}$ & $99^{\circ} 50^{\prime} 07^{\prime \prime}$ & Carrizo-Wilcox & -- & & -4.0 & 5.2 & 39.6 & \\
\hline 199 & $28^{\circ} 55^{\prime} 54^{\prime \prime}$ & $99^{\circ} 47^{\prime} 46^{\prime \prime}$ & -- & -- & & 1.0 & 8.2 & 44.8 & \\
\hline 200 & $29^{\circ} 25^{\prime} 41^{\prime \prime}$ & $99^{\circ} 59^{\prime} 42^{\prime \prime}$ & Edwards & -- & & -16.0 & 10.4 & 0 & gaging station at Laguna (08190000) \\
\hline 200 & $29^{\circ} 19^{\prime} 09^{\prime \prime}$ & $99^{\circ} 57^{\prime} 08^{\prime \prime}$ & Edwards & -- & & -34.0 & 2.6 & 10.4 & \\
\hline 200 & $29^{\circ} 17^{\prime} 05^{\prime \prime}$ & $99^{\circ} 56^{\prime} 54^{\prime \prime}$ & -- & -- & & -36.3 & 5.0 & 13 & \\
\hline 200 & $29^{\circ} 14^{\prime} 39^{\prime \prime}$ & $99^{\circ} 54^{\prime} 22^{\prime \prime}$ & -- & -- & & -23.1 & 2.6 & 18 & \\
\hline 200 & $29^{\circ} 12^{\prime} 05^{\prime \prime}$ & $99^{\circ} 54^{\prime} 12^{\prime \prime}$ & -- & -- & & 17.1 & 2.1 & 20.6 & \\
\hline 200 & $29^{\circ} 07^{\prime} 07^{\prime \prime}$ & $99^{\circ} 53^{\prime} 35^{\prime \prime}$ & -- & -- & & 17.5 & 5.5 & 22.7 & \\
\hline 200 & $29^{\circ} 07^{\prime} 15^{\prime \prime}$ & $99^{\circ} 53^{\prime} 24 "$ & Carrizo-Wilcox & -- & & -5.5 & 3.4 & 28.2 & \\
\hline 200 & $29^{\circ} 04^{\prime} 43^{\prime \prime}$ & $99^{\circ} 52^{\prime} 17^{\prime \prime}$ & Carrizo-Wilcox & -- & & -10.6 & 8.0 & 31.6 & \\
\hline 200 & $29^{\circ} 02^{\prime} 27^{\prime \prime}$ & $99^{\circ} 50^{\prime} 07^{\prime \prime}$ & Carrizo-Wilcox & -- & & -4.7 & 5.2 & 39.6 & \\
\hline 200 & $28^{\circ} 55^{\prime} 58^{\prime \prime}$ & $99^{\circ} 47^{\prime} 49^{\prime \prime}$ & -- & -- & & 8.6 & 8.2 & 44.8 & \\
\hline 200 & $28^{\circ} 51^{\prime} 56^{\prime \prime}$ & $99^{\circ} 46^{\prime} 56^{\prime \prime}$ & -- & -- & & 13.4 & 3.5 & 53 & \\
\hline 201 & $29^{\circ} 25^{\prime} 40^{\prime \prime}$ & $99^{\circ} 59^{\prime} 48^{\prime \prime}$ & Edwards & -- & & -14.0 & 10.4 & 0 & gaging station at Laguna (08190000) \\
\hline 201 & $29^{\circ} 19^{\prime} 15^{\prime \prime}$ & $99^{\circ} 57^{\prime} 04^{\prime \prime}$ & Edwards & -- & & -39.9 & 2.6 & 10.4 & \\
\hline
\end{tabular}


$\vec{\triangleright} \quad$ Table 4. Gains and losses from gain-loss studies in Texas-Continued

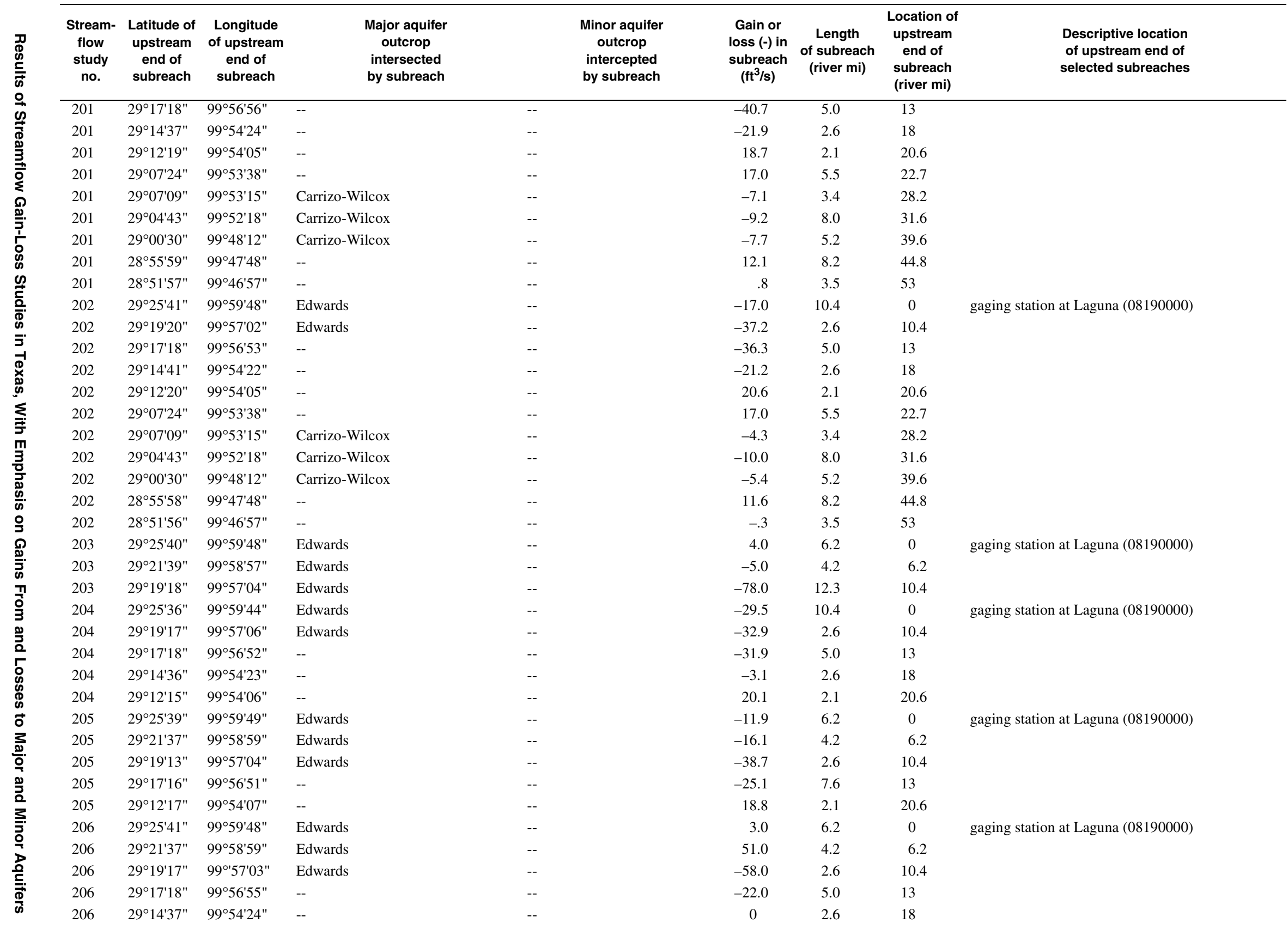


Table 4. Gains and losses from gain-loss studies in Texas-Continued

\begin{tabular}{|c|c|c|c|c|c|c|c|c|c|}
\hline $\begin{array}{l}\text { Stream- } \\
\text { flow } \\
\text { study } \\
\text { no. }\end{array}$ & $\begin{array}{l}\text { Latitude of } \\
\text { upstream } \\
\text { end of } \\
\text { subreach }\end{array}$ & $\begin{array}{l}\text { Longitude } \\
\text { of upstream } \\
\text { end of } \\
\text { subreach }\end{array}$ & $\begin{array}{c}\text { Major aquifer } \\
\text { outcrop } \\
\text { intersected } \\
\text { by subreach }\end{array}$ & & $\begin{array}{c}\text { Minor aquifer } \\
\text { outcrop } \\
\text { intercepted } \\
\text { by subreach }\end{array}$ & $\begin{array}{c}\text { Gain or } \\
\text { loss }(-) \text { in } \\
\text { subreach } \\
\left(\mathrm{ft}^{3} / \mathrm{s}\right)\end{array}$ & $\begin{array}{l}\text { Length } \\
\text { of subreach } \\
\text { (river mi) }\end{array}$ & $\begin{array}{l}\text { Location of } \\
\text { upstream } \\
\text { end of } \\
\text { subreach } \\
\text { (river mi) }\end{array}$ & $\begin{array}{l}\text { Descriptive location } \\
\text { of upstream end of } \\
\text { selected subreaches }\end{array}$ \\
\hline 206 & $29^{\circ} 12^{\prime} 19^{\prime \prime}$ & $99^{\circ} 54^{\prime} 05^{\prime \prime}$ & -- & -- & & 33.0 & 2.1 & 20.6 & \\
\hline 206 & $29^{\circ} 07^{\prime} 24^{\prime \prime}$ & $99^{\circ} 53^{\prime} 38^{\prime \prime}$ & -- & -- & & 11.0 & 5.5 & 22.7 & \\
\hline 206 & $29^{\circ} 07^{\prime} 09^{\prime \prime}$ & $99^{\circ} 53^{\prime} 15^{\prime \prime}$ & Carrizo-Wilcox & -- & & -13.0 & 3.4 & 28.2 & \\
\hline 206 & $29^{\circ} 04^{\prime} 44^{\prime \prime}$ & $99^{\circ} 52^{\prime} 17^{\prime \prime}$ & Carrizo-Wilcox & -- & & 7.0 & 4.7 & 31.6 & \\
\hline 206 & $29^{\circ} 01^{\prime} 57^{\prime \prime}$ & $99^{\circ} 49^{\prime} 46^{\prime \prime}$ & Carrizo-Wilcox & -- & & -10.0 & 3.3 & 36.3 & \\
\hline 206 & $29^{\circ} 00^{\prime} 31^{\prime \prime}$ & $99^{\circ} 48^{\prime} 12^{\prime \prime}$ & Carrizo-Wilcox & -- & & -2.0 & 5.2 & 39.6 & \\
\hline 206 & $28^{\circ} 55^{\prime} 58^{\prime \prime}$ & $99^{\circ} 47^{\prime} 49^{\prime \prime}$ & -- & -- & & 11.0 & 8.2 & 44.8 & \\
\hline 206 & $28^{\circ} 51^{\prime} 57^{\prime \prime}$ & $99^{\circ} 46^{\prime} 57^{\prime \prime}$ & -- & -- & & 17.0 & 3.5 & 53 & \\
\hline 207 & $29^{\circ} 25^{\prime} 41^{\prime \prime}$ & $99^{\circ} 59^{\prime} 48^{\prime \prime}$ & Edwards & -- & & -.2 & 7.8 & 0 & gaging station at Laguna (08190000) \\
\hline 207 & $29^{\circ} 21^{\prime} 29^{\prime \prime}$ & $99^{\circ} 58^{\prime} 03^{\prime \prime}$ & Edwards & -- & & -22.9 & 2.6 & 7.8 & \\
\hline 207 & $29^{\circ} 19^{\prime} 17^{\prime \prime}$ & $99^{\circ} 57^{\prime} 03^{\prime \prime}$ & Edwards & -- & & 0 & 2.6 & 10.4 & \\
\hline 207 & $29^{\circ} 17^{\prime} 18^{\prime \prime}$ & $99^{\circ} 56^{\prime} 55^{\prime \prime}$ & -- & -- & & 0 & 5.0 & 13 & \\
\hline 207 & $29^{\circ} 14^{\prime} 37^{\prime \prime}$ & $99^{\circ} 54^{\prime} 24^{\prime \prime}$ & -- & -- & & 0 & 2.6 & 18 & \\
\hline 207 & $29^{\circ} 12^{\prime} 19^{\prime \prime}$ & $99^{\circ} 54^{\prime} 05^{\prime \prime}$ & -- & -- & & 16.3 & 2.1 & 20.6 & \\
\hline 207 & $29^{\circ} 07^{\prime} 24^{\prime \prime}$ & $99^{\circ} 53^{\prime} 38^{\prime \prime}$ & -- & -- & & 4.3 & 5.5 & 22.7 & \\
\hline 207 & $29^{\circ} 07^{\prime} 09^{\prime \prime}$ & $99^{\circ} 53^{\prime} 15^{\prime \prime}$ & Carrizo-Wilcox & -- & & -4.3 & 3.4 & 28.2 & \\
\hline 207 & $29^{\circ} 04^{\prime} 44^{\prime \prime}$ & $99^{\circ} 52^{\prime} 17^{\prime \prime}$ & Carrizo-Wilcox & -- & & -5.0 & 4.7 & 31.6 & \\
\hline 207 & $29^{\circ} 01^{\prime} 57^{\prime \prime}$ & $99^{\circ} 49^{\prime} 46^{\prime \prime}$ & Carrizo-Wilcox & -- & & -4.1 & 3.3 & 36.3 & \\
\hline 207 & $29^{\circ} 00^{\prime} 31^{\prime \prime}$ & $99^{\circ} 48^{\prime} 12^{\prime \prime}$ & Carrizo-Wilcox & -- & & -3.1 & 5.2 & 39.6 & \\
\hline 207 & $28^{\circ} 55^{\prime} 58^{\prime \prime}$ & $99^{\circ} 47^{\prime} 49^{\prime \prime}$ & -- & -- & & 9.8 & 8.2 & 44.8 & \\
\hline 207 & $28^{\circ} 51^{\prime} 57^{\prime \prime}$ & $99^{\circ} 46 ' 57^{\prime \prime}$ & -- & -- & & .5 & 3.5 & 53 & \\
\hline 208 & $29^{\circ} 25^{\prime} 41^{\prime \prime}$ & $99^{\circ} 59^{\prime} 48^{\prime \prime}$ & Edwards & -- & & -62.2 & 10.4 & 0 & gaging station at Laguna (08190000) \\
\hline 208 & $29^{\circ} 19^{\prime} 17^{\prime \prime}$ & $99^{\circ} 57^{\prime} 03^{\prime \prime}$ & Edwards & -- & & -16.6 & 2.6 & 10.4 & \\
\hline 208 & $29^{\circ} 17^{\prime} 18^{\prime \prime}$ & $99^{\circ} 56^{\prime} 55^{\prime \prime}$ & -- & -- & & -25.0 & 5.0 & 13 & \\
\hline 208 & $29^{\circ} 14^{\prime} 37^{\prime \prime}$ & $99^{\circ} 54^{\prime} 25^{\prime \prime}$ & -- & -- & & -7.4 & 2.6 & 18 & \\
\hline 208 & $29^{\circ} 12^{\prime} 19^{\prime \prime}$ & $99^{\circ} 54^{\prime} 05^{\prime \prime}$ & -- & -- & & 19.1 & 2.1 & 20.6 & \\
\hline 208 & $29^{\circ} 07^{\prime} 24^{\prime \prime}$ & $99^{\circ} 53^{\prime} 38^{\prime \prime}$ & -- & -- & & 9.4 & 5.5 & 22.7 & \\
\hline 208 & $29^{\circ} 07^{\prime} 09^{\prime \prime}$ & $99^{\circ} 53^{\prime} 15^{\prime \prime}$ & Carrizo-Wilcox & -- & & 2.5 & 3.4 & 28.2 & \\
\hline 209 & $28^{\circ} 02^{\prime} 16^{\prime \prime}$ & $97^{\circ} 51^{\prime} 36^{\prime \prime}$ & Gulf Coast & -- & & -4.7 & 1.0 & 0 & gaging station near Mathis (08211000) \\
\hline 209 & $28^{\circ} 02^{\prime} 10^{\prime \prime}$ & $97^{\circ} 50^{\prime} 45^{\prime \prime}$ & Gulf Coast & -- & & 4.4 & 1.6 & 1 & \\
\hline 209 & $28^{\circ} 02^{\prime} 21^{\prime \prime}$ & $97^{\circ} 49^{\prime} 09^{\prime \prime}$ & Gulf Coast & -- & & -1.8 & 2.4 & 2.6 & \\
\hline 209 & $28^{\circ} 02^{\prime} 14^{\prime \prime}$ & $97^{\circ} 48^{\prime} 02^{\prime \prime}$ & Gulf Coast & -- & & -.2 & 2.0 & 5 & \\
\hline 209 & $28^{\circ} 00^{\prime} 52^{\prime \prime}$ & $97^{\circ} 47^{\prime} 22^{\prime \prime}$ & Gulf Coast & -- & & .4 & 1.9 & 7 & \\
\hline 209 & $27^{\circ} 59^{\prime} 43^{\prime \prime}$ & $97^{\circ} 47^{\prime} 53^{\prime \prime}$ & Gulf Coast & -- & & 2.4 & 2.0 & 8.9 & \\
\hline 209 & $27^{\circ} 58^{\prime} 18^{\prime \prime}$ & $97^{\circ} 48^{\prime} 31^{\prime \prime}$ & Gulf Coast & -- & & -1.8 & 2.5 & 10.9 & \\
\hline 209 & $27^{\circ} 56^{\prime} 32^{\prime \prime}$ & $97^{\circ} 48^{\prime} 20^{\prime \prime}$ & Gulf Coast & -- & & 1.9 & 2.3 & 13.4 & \\
\hline 209 & $27^{\circ} 56^{\prime} 25^{\prime \prime}$ & $97^{\circ} 47^{\prime} 44^{\prime \prime}$ & Gulf Coast & -- & & .6 & 2.4 & 15.7 & \\
\hline 209 & $27^{\circ} 56^{\prime} 33^{\prime \prime}$ & $97^{\circ} 47^{\prime} 36^{\prime \prime}$ & Gulf Coast & -- & & 1.5 & 1.8 & 18.1 & \\
\hline
\end{tabular}


$\vec{\Phi} \quad$ Table 4. Gains and losses from gain-loss studies in Texas-Continued

\begin{tabular}{|c|c|c|c|c|c|c|c|c|c|}
\hline $\begin{array}{l}\text { Stream- } \\
\text { flow } \\
\text { study } \\
\text { no. }\end{array}$ & $\begin{array}{l}\text { Latitude of } \\
\text { upstream } \\
\text { end of } \\
\text { subreach }\end{array}$ & $\begin{array}{l}\text { Longitude } \\
\text { of upstream } \\
\text { end of } \\
\text { subreach }\end{array}$ & $\begin{array}{c}\text { Major aquifer } \\
\text { outcrop } \\
\text { intersected } \\
\text { by subreach }\end{array}$ & & $\begin{array}{c}\text { Minor aquifer } \\
\text { outcrop } \\
\text { intercepted } \\
\text { by subreach }\end{array}$ & $\begin{array}{l}\text { Gain or } \\
\text { loss }(-) \text { in } \\
\text { subreach } \\
\left(\mathrm{ft}^{3} / \mathrm{s}\right)\end{array}$ & $\begin{array}{l}\text { Length } \\
\text { of subreach } \\
\text { (river mi) }\end{array}$ & $\begin{array}{l}\text { Location of } \\
\text { upstream } \\
\text { end of } \\
\text { subreach } \\
\text { (river mi) }\end{array}$ & $\begin{array}{l}\text { Descriptive location } \\
\text { of upstream end of } \\
\text { selected subreaches }\end{array}$ \\
\hline 209 & $27^{\circ} 56^{\prime} 14^{\prime \prime}$ & $97^{\circ} 46^{\prime} 19^{\prime \prime}$ & Gulf Coast & -- & & -2.4 & 3.7 & 19.9 & \multirow{8}{*}{ gaging station at Laguna (08190000) } \\
\hline 209 & $27^{\circ} 54^{\prime} 46^{\prime \prime}$ & $97^{\circ} 43^{\prime} 40^{\prime \prime}$ & Gulf Coast & -- & & -1.0 & 1.9 & 23.6 & \\
\hline 210 & $29^{\circ} 07^{\prime} 18^{\prime \prime}$ & $99^{\circ} 53^{\prime} 44^{\prime \prime}$ & Carrizo-Wilcox & -- & & 16.1 & 5.5 & 0 & \\
\hline 210 & $29^{\circ} 07^{\prime} 07^{\prime \prime}$ & $99^{\circ} 53^{\prime} 10^{\prime \prime}$ & Carrizo-Wilcox & -- & & -4.6 & 3.4 & 5.5 & \\
\hline 210 & $29^{\circ} 04^{\prime} 44^{\prime \prime}$ & $99^{\circ} 52^{\prime} 17^{\prime \prime}$ & Carrizo-Wilcox & -- & & -11.1 & 8.0 & 8.9 & \\
\hline 210 & $29^{\circ} 00^{\prime} 31^{\prime \prime}$ & $99^{\circ} 48^{\prime} 11^{\prime \prime}$ & Carrizo-Wilcox & -- & & -6.4 & 5.2 & 16.9 & \\
\hline 210 & $28^{\circ} 55^{\prime} 58^{\prime \prime}$ & $99^{\circ} 47^{\prime} 50^{\prime \prime}$ & -- & -- & & 11.9 & 8.2 & 22.1 & \\
\hline 210 & $28^{\circ} 51^{\prime} 50^{\prime \prime}$ & $99^{\circ} 46^{\prime} 55^{\prime \prime}$ & -- & -- & & -1.9 & 3.5 & 30.3 & \\
\hline 211 & $28^{\circ} 26^{\prime} 13^{\prime \prime}$ & $98^{\circ} 11^{\prime} 19^{\prime \prime}$ & Gulf Coast & -- & & 33.4 & .67 & 9.25 & \multirow[t]{5}{*}{ at Miller's Ranch } \\
\hline 211 & $28^{\circ} 26^{\prime} 09^{\prime \prime}$ & $98^{\circ} 11^{\prime} 06^{\prime \prime}$ & Gulf Coast & -- & & 3.7 & .78 & 9.92 & \\
\hline 211 & $28^{\circ} 25^{\prime} 39^{\prime \prime}$ & $98^{\circ} 10^{\prime} 44^{\prime \prime}$ & Gulf Coast & -- & & -3.4 & 6.1 & 10.7 & \\
\hline 211 & $28^{\circ} 24^{\prime} 53^{\prime \prime}$ & $98^{\circ} 06^{\prime} 12^{\prime \prime}$ & Gulf Coast & -- & & -1.3 & 5.7 & 16.8 & \\
\hline 211 & $28^{\circ} 21^{\prime} 58^{\prime \prime}$ & $98^{\circ} 06^{\prime} 50^{\prime \prime}$ & Gulf Coast & -- & & 33.49 & 3.2 & 22.5 & \\
\hline 212 & $28^{\circ} 02^{\prime} 12^{\prime \prime}$ & $97^{\circ} 51^{\prime} 05^{\prime \prime}$ & Gulf Coast & -- & & -8.0 & 2.8 & 0 & \multirow[t]{12}{*}{ gaging station $(08211000)$} \\
\hline 212 & $28^{\circ} 02^{\prime} 25^{\prime \prime}$ & $97^{\circ} 49^{\prime} 08^{\prime \prime}$ & Gulf Coast & -- & & 1.0 & 1.7 & 2.8 & \\
\hline 212 & $28^{\circ} 01^{\prime} 56^{\prime \prime}$ & $97^{\circ} 47^{\prime} 57^{\prime \prime}$ & Gulf Coast & -- & & 0 & 2.1 & 4.5 & \\
\hline 212 & $28^{\circ} 00^{\prime} 50^{\prime \prime}$ & $97^{\circ} 47^{\prime} 28^{\prime \prime}$ & Gulf Coast & -- & & 2.0 & 2.1 & 6.6 & \\
\hline 212 & $27^{\circ} 59^{\prime} 11^{\prime \prime}$ & $97^{\circ} 48^{\prime} 00^{\prime \prime}$ & Gulf Coast & -- & & 0 & 2.1 & 8.7 & \\
\hline 212 & $27^{\circ} 58^{\prime} 10^{\prime \prime}$ & $97^{\circ} 49^{\prime} 01^{\prime \prime}$ & Gulf Coast & -- & & -7.0 & 2.6 & 10.8 & \\
\hline 212 & $27^{\circ} 56^{\prime} 29^{\prime \prime}$ & $97^{\circ} 48^{\prime} 29^{\prime \prime}$ & Gulf Coast & -- & & -2.0 & 1.0 & 13.4 & \\
\hline 212 & $27^{\circ} 56^{\prime} 07^{\prime \prime}$ & $97^{\circ} 47^{\prime} 55^{\prime \prime}$ & Gulf Coast & -- & & 0 & 2.2 & 14.4 & \\
\hline 212 & $27^{\circ} 56^{\prime} 35^{\prime \prime}$ & $97^{\circ} 46^{\prime} 39^{\prime \prime}$ & Gulf Coast & -- & & 1.0 & 1.6 & 16.6 & \\
\hline 212 & $27^{\circ} 56^{\prime} 02^{\prime \prime}$ & $97^{\circ} 45^{\prime} 44^{\prime \prime}$ & Gulf Coast & -- & & -3.0 & 2.0 & 18.2 & \\
\hline 212 & $27^{\circ} 55^{\prime} 00^{\prime \prime}$ & $97^{\circ} 45^{\prime} 03^{\prime \prime}$ & Gulf Coast & -- & & 2.0 & 2.8 & 20.2 & \\
\hline 212 & $27^{\circ} 54^{\prime} 40^{\prime \prime}$ & $97^{\circ} 44^{\prime} 07^{\prime \prime}$ & Gulf Coast & -- & & -1.0 & 2.8 & 23 & \\
\hline 213 & $28^{\circ} 02^{\prime} 16^{\prime \prime}$ & $97^{\circ} 51^{\prime} 36^{\prime \prime}$ & Gulf Coast & -- & & -6.0 & 1.2 & 0 & \multirow[t]{6}{*}{ gaging station (08211000) } \\
\hline 213 & $28^{\circ} 02^{\prime} 09^{\prime \prime}$ & $97^{\circ} 50^{\prime} 38^{\prime \prime}$ & Gulf Coast & -- & & 5.0 & .8 & 1.2 & \\
\hline 213 & $28^{\circ} 02^{\prime} 10^{\prime \prime}$ & $97^{\circ} 49^{\prime} 44^{\prime \prime}$ & Gulf Coast & -- & & 0 & .8 & 2 & \\
\hline 213 & $28^{\circ} 02^{\prime} 17^{\prime \prime}$ & $97^{\circ} 49^{\prime} 29^{\prime \prime}$ & Gulf Coast & -- & & 2.0 & 7.7 & 2.8 & \\
\hline 213 & $27^{\circ} 58^{\prime} 24^{\prime \prime}$ & $97^{\circ} 48^{\prime} 48^{\prime \prime}$ & Gulf Coast & -- & & -6.0 & 1.2 & 10.5 & \\
\hline 213 & $27^{\circ} 57^{\prime} 32^{\prime \prime}$ & $97^{\circ} 48^{\prime} 37^{\prime \prime}$ & Gulf Coast & -- & & 0 & 2.5 & 11.7 & \\
\hline 214 & $28^{\circ} 02^{\prime} 16^{\prime \prime}$ & $97^{\circ} 51^{\prime} 36^{\prime \prime}$ & Gulf Coast & -- & & -8.0 & 1.7 & 0 & \multirow[t]{5}{*}{ gaging station $(08211000)$} \\
\hline 214 & $28^{\circ} 02^{\prime} 08^{\prime \prime}$ & $97^{\circ} 50^{\prime} 05^{\prime \prime}$ & Gulf Coast & -- & & 3.7 & 2.3 & 1.7 & \\
\hline 214 & $28^{\circ} 02^{\prime} 25^{\prime \prime}$ & $97^{\circ} 48^{\prime} 42^{\prime \prime}$ & Gulf Coast & -- & & -2.01 & 2.6 & 4 & \\
\hline 214 & $28^{\circ} 01^{\prime} 08^{\prime \prime}$ & $97^{\circ} 47^{\prime} 23^{\prime \prime}$ & Gulf Coast & -- & & -3.0 & 2.5 & 6.6 & \\
\hline 214 & $27^{\circ} 59^{\prime} 36^{\prime \prime}$ & $97^{\circ} 47^{\prime} 56^{\prime \prime}$ & Gulf Coast & -- & & 8.0 & .7 & 9.1 & \\
\hline 214 & $27^{\circ} 58^{\prime} 52^{\prime \prime}$ & $97^{\circ} 48^{\prime} 06^{\prime \prime}$ & Gulf Coast & -- & & 0 & 1.0 & 9.8 & \multirow[t]{2}{*}{ gaging station $(08211100)$} \\
\hline 214 & $27^{\circ} 58^{\prime} 21^{\prime \prime}$ & $97^{\circ} 48^{\prime} 35^{\prime \prime}$ & Gulf Coast & -- & & -7.0 & 1.9 & 10.8 & \\
\hline
\end{tabular}


Table 4. Gains and losses from gain-loss studies in Texas-Continued

\begin{tabular}{|c|c|c|c|c|c|c|c|c|}
\hline $\begin{array}{l}\text { Stream- } \\
\text { flow } \\
\text { study } \\
\text { no. }\end{array}$ & $\begin{array}{l}\text { Latitude of } \\
\text { upstream } \\
\text { end of } \\
\text { subreach }\end{array}$ & $\begin{array}{l}\text { Longitude } \\
\text { of upstream } \\
\text { end of } \\
\text { subreach }\end{array}$ & $\begin{array}{l}\text { Major aquifer } \\
\text { outcrop } \\
\text { intersected } \\
\text { by subreach }\end{array}$ & $\begin{array}{l}\text { Minor aquifer } \\
\text { outcrop } \\
\text { intercepted } \\
\text { by subreach }\end{array}$ & $\begin{array}{l}\text { Gain or } \\
\text { loss }(-) \text { in } \\
\text { subreach } \\
\left(\mathrm{ft}^{3} / \mathrm{s}\right)\end{array}$ & $\begin{array}{l}\text { Length } \\
\text { of subreach } \\
\text { (river mi) }\end{array}$ & $\begin{array}{l}\text { Location of } \\
\text { upstream } \\
\text { end of } \\
\text { subreach } \\
\text { (river mi) }\end{array}$ & $\begin{array}{l}\text { Descriptive location } \\
\text { of upstream end of } \\
\text { selected subreaches }\end{array}$ \\
\hline 214 & $27^{\circ} 57^{\prime} 21^{\prime \prime}$ & $97^{\circ} 48^{\prime} 34^{\prime \prime}$ & Gulf Coast & -- & 2.0 & 1.8 & 12.7 & \\
\hline 214 & $27^{\circ} 56^{\prime} 28^{\prime \prime}$ & $97^{\circ} 48^{\prime} 33^{\prime \prime}$ & Gulf Coast & -- & 4.0 & 2.0 & 14.5 & \\
\hline 214 & $27^{\circ} 56^{\prime} 29^{\prime \prime}$ & $97^{\circ} 47^{\prime} 43^{\prime \prime}$ & Gulf Coast & -- & -6.0 & 1.7 & 16.5 & \\
\hline 214 & $27^{\circ} 56^{\prime} 33^{\prime \prime}$ & $97^{\circ} 46^{\prime} 37^{\prime \prime}$ & Gulf Coast & -- & 0 & 2.3 & 18.2 & \\
\hline 215 & $28^{\circ} 02^{\prime} 16^{\prime \prime}$ & $97^{\circ} 51^{\prime} 36^{\prime \prime}$ & Gulf Coast & -- & 1.92 & 1.7 & 0 & gaging station (08211000) \\
\hline 215 & $28^{\circ} 02^{\prime} 08^{\prime \prime}$ & $97^{\circ} 50^{\prime} 04^{\prime \prime}$ & Gulf Coast & -- & 2.0 & 2.3 & 1.7 & \\
\hline 215 & $28^{\circ} 02^{\prime} 16^{\prime \prime}$ & $97^{\circ} 49^{\prime} 30^{\prime \prime}$ & Gulf Coast & -- & -1.04 & 2.6 & 4 & \\
\hline 215 & $28^{\circ} 01^{\prime} 06^{\prime \prime}$ & $97^{\circ} 47^{\prime} 24^{\prime \prime}$ & Gulf Coast & -- & 3.0 & 2.5 & 6.6 & \\
\hline 215 & $27^{\circ} 59^{\prime} 35^{\prime \prime}$ & $97^{\circ} 47^{\prime} 57^{\prime \prime}$ & Gulf Coast & -- & -2.0 & .7 & 9.1 & \\
\hline 215 & $27^{\circ} 58^{\prime} 50^{\prime \prime}$ & $97^{\circ} 48^{\prime} 06^{\prime \prime}$ & Gulf Coast & -- & 0 & 1.0 & 9.8 & gaging station (08211100) \\
\hline 215 & $27^{\circ} 58^{\prime} 20^{\prime \prime}$ & $97^{\circ} 48^{\prime} 34^{\prime \prime}$ & Gulf Coast & -- & -3.0 & 1.9 & 10.8 & \\
\hline 215 & $27^{\circ} 57^{\prime} 21^{\prime \prime}$ & $97^{\circ} 48^{\prime} 34^{\prime \prime}$ & Gulf Coast & -- & 2.0 & 1.8 & 12.7 & \\
\hline 215 & $27^{\circ} 56^{\prime} 29^{\prime \prime}$ & $97^{\circ} 48^{\prime} 33^{\prime \prime}$ & Gulf Coast & -- & 4.0 & 2.0 & 14.5 & \\
\hline 215 & $27^{\circ} 56^{\prime} 29^{\prime \prime}$ & $97^{\circ} 47^{\prime} 44^{\prime \prime}$ & Gulf Coast & -- & -3.0 & 1.7 & 16.5 & \\
\hline 215 & $27^{\circ} 56^{\prime} 32^{\prime \prime}$ & $97^{\circ} 46^{\prime} 36^{\prime \prime}$ & Gulf Coast & -- & -8.0 & 2.3 & 18.2 & \\
\hline 216 & $29^{\circ} 48^{\prime} 37^{\prime \prime}$ & $100^{\circ} 00^{\prime} 57^{\prime \prime}$ & Edwards-Trinity Plateau & -- & 0 & 5.9 & 8.3 & near Vance \\
\hline 216 & $29^{\circ} 43^{\prime} 14^{\prime \prime}$ & $100^{\circ} 02^{\prime} 02^{\prime \prime}$ & Edwards-Trinity Plateau & -- & 20.0 & 3.9 & 14.2 & \\
\hline 216 & $29^{\circ} 40^{\prime} 40^{\prime \prime}$ & $100^{\circ} 01^{\prime} 08^{\prime \prime}$ & Edwards-Trinity Plateau & -- & -20.0 & 11.3 & 18.1 & \\
\hline 216 & $29^{\circ} 31^{\prime} 16^{\prime \prime}$ & $100^{\circ} 01^{\prime} 04^{\prime \prime}$ & Edwards-Trinity Plateau & -- & 24.0 & 7.8 & 29.4 & \\
\hline 216 & $29^{\circ} 27^{\prime} 39^{\prime \prime}$ & $99^{\circ} 59^{\prime} 54^{\prime \prime}$ & Edwards-Trinity Plateau & -- & -6.9 & 3.0 & 37.2 & \\
\hline 216 & $29^{\circ} 25^{\prime} 41^{\prime \prime}$ & $99^{\circ} 59^{\prime} 48^{\prime \prime}$ & Edwards, Edwards-Trinity Plateau & -- & -17.1 & 5.1 & 40.2 & gaging station at Laguna (08190000) \\
\hline 216 & $29^{\circ} 22^{\prime} 32^{\prime \prime}$ & $99^{\circ} 59^{\prime} 54^{\prime \prime}$ & Edwards & -- & 0 & 6.5 & 45.3 & \\
\hline 216 & $29^{\circ} 20^{\prime} 51^{\prime \prime}$ & $99^{\circ} 57^{\prime} 20^{\prime \prime}$ & Edwards & -- & 0 & 4.5 & 51.8 & \\
\hline 216 & $29^{\circ} 14^{\prime} 37^{\prime \prime}$ & $99^{\circ} 54^{\prime} 25^{\prime \prime}$ & -- & -- & 0 & 2.8 & 56.3 & \\
\hline 216 & $29^{\circ} 11^{\prime} 52^{\prime \prime}$ & $99^{\circ} 54^{\prime} 17^{\prime \prime}$ & -- & -- & 27.0 & 2.0 & 59.1 & \\
\hline 216 & $29^{\circ} 10^{\prime} 51^{\prime \prime}$ & $99^{\circ} 53^{\prime} 45^{\prime \prime}$ & Carrizo-Wilcox & -- & -6.0 & 13.3 & 61.1 & \\
\hline 217 & $29^{\circ} 14^{\prime} 37^{\prime \prime}$ & $99^{\circ} 54^{\prime} 25^{\prime \prime}$ & -- & -- & 25.2 & 2.0 & 59.1 & at Old Spanish Trail crossing \\
\hline 217 & $29^{\circ} 10^{\prime} 51^{\prime \prime}$ & $99^{\circ} 53^{\prime} 44^{\prime \prime}$ & -- & -- & 1.6 & 1.6 & 61.1 & \\
\hline 218 & $29^{\circ} 43^{\prime} 14^{\prime \prime}$ & $100^{\circ} 02^{\prime} 02^{\prime \prime}$ & Edwards-Trinity Plateau & -- & -31.1 & 5.7 & 8.4 & $500 \mathrm{ft}$ below county line \\
\hline 218 & $29^{\circ} 32^{\prime} 53^{\prime \prime}$ & 99॰59'39" & Edwards-Trinity Plateau & -- & 17.8 & 6.7 & 14.1 & \\
\hline 218 & $29^{\circ} 28^{\prime} 40^{\prime \prime}$ & $100^{\circ} 01^{\prime} 30^{\prime \prime}$ & Edwards-Trinity Plateau & -- & 11.7 & 4.4 & 20.8 & \\
\hline 219 & $29^{\circ} 12^{\prime} 21^{\prime \prime}$ & $99^{\circ} 54^{\prime} 07^{\prime \prime}$ & -- & -- & 16.6 & 2.8 & 0 & at US 90 \\
\hline 219 & $29^{\circ} 10^{\prime} 51^{\prime \prime}$ & $99^{\circ} 53^{\prime} 44^{\prime \prime}$ & Carrizo-Wilcox & -- & 19.6 & 6.6 & 2.8 & \\
\hline 219 & $29^{\circ} 07^{\prime} 08^{\prime \prime}$ & $99^{\circ} 53^{\prime} 13^{\prime \prime}$ & Carrizo-Wilcox & -- & -4.5 & 3.4 & 9.4 & \\
\hline 219 & $29^{\circ} 04^{\prime} 43^{\prime \prime}$ & $99^{\circ} 52^{\prime} 22^{\prime \prime}$ & Carrizo-Wilcox & -- & .8 & 5.6 & 12.8 & \\
\hline 219 & $29^{\circ} 02^{\prime} 05^{\prime \prime}$ & $99^{\circ} 50^{\prime} 06^{\prime \prime}$ & Carrizo-Wilcox & -- & 1.1 & .6 & 18.4 & \\
\hline 219 & $29^{\circ} 01^{\prime} 43^{\prime \prime}$ & $99^{\circ} 49^{\prime} 26^{\prime \prime}$ & Carrizo-Wilcox & -- & -21.9 & 2.8 & 19 & \\
\hline 219 & $29^{\circ} 00^{\prime} 08^{\prime \prime}$ & $99^{\circ} 47^{\prime} 56^{\prime \prime}$ & Carrizo-Wilcox & -- & -11.7 & 2.9 & 21.8 & \\
\hline
\end{tabular}


Table 4. Gains and losses from gain-loss studies in Texas-Continued

\begin{tabular}{|c|c|c|c|c|c|c|c|c|c|}
\hline $\begin{array}{l}\text { Stream- } \\
\text { flow } \\
\text { study } \\
\text { no. }\end{array}$ & $\begin{array}{l}\text { Latitude of } \\
\text { upstream } \\
\text { end of } \\
\text { subreach }\end{array}$ & $\begin{array}{l}\text { Longitude } \\
\text { of upstream } \\
\text { end of } \\
\text { subreach }\end{array}$ & $\begin{array}{l}\text { Major aquifer } \\
\text { outcrop } \\
\text { intersected } \\
\text { by subreach }\end{array}$ & & $\begin{array}{c}\text { Minor aquifer } \\
\text { outcrop } \\
\text { intercepted } \\
\text { by subreach }\end{array}$ & $\begin{array}{l}\text { Gain or } \\
\text { loss }(-) \text { in } \\
\text { subreach } \\
\left(\mathrm{ft}^{3} / \mathrm{s}\right)\end{array}$ & $\begin{array}{l}\text { Length } \\
\text { of subreach } \\
\text { (river mi) }\end{array}$ & $\begin{array}{l}\text { Location of } \\
\text { upstream } \\
\text { end of } \\
\text { subreach } \\
\text { (river mi) }\end{array}$ & $\begin{array}{l}\text { Descriptive location } \\
\text { of upstream end of } \\
\text { selected subreaches }\end{array}$ \\
\hline 219 & $28^{\circ} 56^{\prime} 55^{\prime \prime}$ & $99^{\circ} 47^{\prime} 20^{\prime \prime}$ & -- & -- & & 7.9 & 5.2 & 24.7 & \\
\hline 219 & $28^{\circ} 54^{\prime} 00^{\prime \prime}$ & $99^{\circ} 47^{\prime} 14^{\prime \prime}$ & -- & -- & & 5.5 & 22.3 & 29.9 & \\
\hline 220 & $29^{\circ} 36^{\prime} 03^{\prime \prime}$ & $99^{\circ} 31^{\prime} 41^{\prime \prime}$ & Trinity & -- & & 4.3 & 3.0 & 0 & $1 \mathrm{mi}$ south of Utopia \\
\hline 220 & $29^{\circ} 34^{\prime} 03^{\prime \prime}$ & $99^{\circ} 31^{\prime} 10^{\prime \prime}$ & Trinity & -- & & 8.5 & 5.0 & 3 & \\
\hline 220 & $29^{\circ} 30^{\prime} 44^{\prime \prime}$ & $99^{\circ} 30^{\prime} 43^{\prime \prime}$ & Trinity & -- & & -.4 & 2.0 & 8 & just below mouth of Onion Creek \\
\hline 220 & $29^{\circ} 29^{\circ} 54^{\prime \prime}$ & $99^{\circ} 29^{\prime} 47^{\prime \prime}$ & Edwards, Trinity & -- & & -10.0 & 2.0 & 10 & \\
\hline 220 & $29^{\circ} 28^{\prime} 16^{\prime \prime}$ & $99^{\circ} 29^{\prime} 34^{\prime \prime}$ & Edwards & -- & & -35.1 & 5.5 & 12 & \\
\hline 221 & $29^{\circ} 49^{\prime} 49^{\prime \prime}$ & $99^{\circ} 34^{\prime} 06^{\prime \prime}$ & Edwards-Trinity Plateau & -- & & .3 & 1.3 & 0 & $8.2 \mathrm{mi}$ above Vanderpool \\
\hline 221 & $29^{\circ} 49^{\prime} 07^{\prime \prime}$ & $99^{\circ} 34^{\prime} 13^{\prime \prime}$ & Edwards-Trinity Plateau, Trinity & -- & & 2.24 & 2.9 & 1.3 & \\
\hline 221 & $29^{\circ} 47^{\prime} 36^{\prime \prime}$ & $99^{\circ} 34^{\prime} 35^{\prime \prime}$ & Trinity & -- & & 2.99 & 4.7 & 4.2 & \\
\hline 221 & $29^{\circ} 43^{\prime} 39^{\prime \prime}$ & $99^{\circ} 32^{\prime} 48^{\prime \prime}$ & Trinity & -- & & 12.17 & .6 & 8.9 & \\
\hline 221 & $29^{\circ} 41^{\prime} 38^{\prime \prime}$ & $99^{\circ} 33^{\prime} 09^{\prime \prime}$ & Trinity & -- & & -.2 & 2.5 & 9.5 & \\
\hline 221 & $29^{\circ} 39^{\prime} 48^{\prime \prime}$ & $99^{\circ} 32^{\prime} 32^{\prime \prime}$ & Trinity & -- & & -44.7 & 2.6 & 12 & \\
\hline 221 & $29^{\circ} 37^{\prime} 55^{\prime \prime}$ & $99^{\circ} 32^{\prime} 10^{\prime \prime}$ & Trinity & -- & & 39.4 & 3.3 & 14.6 & \\
\hline 221 & $29^{\circ} 37^{\prime} 55^{\prime \prime}$ & $99^{\circ} 32^{\prime} 10^{\prime \prime}$ & Trinity & -- & & 0 & .3 & 17.9 & \\
\hline 221 & $29^{\circ} 37^{\prime} 40^{\prime \prime}$ & $99^{\circ} 32^{\prime} 02^{\prime \prime}$ & Trinity & -- & & 3.3 & 3.4 & 18.2 & \\
\hline 221 & $29^{\circ} 35^{\prime} 49^{\prime \prime}$ & $99^{\circ} 31^{\prime} 50^{\prime \prime}$ & Trinity & -- & & 2.9 & 3.0 & 21.6 & \\
\hline 221 & $29^{\circ} 33^{\prime} 56^{\prime \prime}$ & $99^{\circ} 30^{\prime} 17^{\prime \prime}$ & Trinity & -- & & 8.9 & 4.5 & 24.6 & \\
\hline 221 & $29^{\circ} 30^{\prime} 50^{\prime \prime}$ & $99^{\circ} 30^{\prime} 37^{\prime \prime}$ & Trinity & -- & & 2.1 & 2.3 & 29.1 & \\
\hline 221 & $29^{\circ} 29^{\circ} 26^{\prime \prime}$ & $99^{\circ} 29^{\prime} 32^{\prime \prime}$ & Edwards & -- & & -12.7 & 1.8 & 31.4 & near Sabinal gaging station (08198500) \\
\hline 221 & $29^{\circ} 28^{\prime} 16^{\prime \prime}$ & $99^{\circ} 29^{\prime} 34^{\prime \prime}$ & Edwards & -- & & -12.3 & 2.2 & 33.2 & \\
\hline 221 & $29^{\circ} 27^{\prime} 05^{\prime \prime}$ & $99^{\circ} 30^{\prime} 55^{\prime \prime}$ & Edwards & -- & & -29.8 & 3.6 & 35.4 & \\
\hline 221 & $29^{\circ} 24^{\prime} 40^{\prime \prime}$ & $99^{\circ} 29^{\prime} 38^{\prime \prime}$ & -- & -- & & -3.2 & 3.4 & 39 & \\
\hline 221 & $29^{\circ} 22^{\prime} 53^{\prime \prime}$ & 99'29'17" & -- & -- & & -1.6 & 3.4 & 42.4 & \\
\hline 221 & $29^{\circ} 20^{\prime} 25^{\prime \prime}$ & $99^{\circ} 28^{\prime} 48^{\prime \prime}$ & -- & -- & & -1.4 & 3.2 & 45.8 & \\
\hline 222 & $29^{\circ} 49^{\prime} 49^{\prime \prime}$ & $99^{\circ} 34^{\prime} 06^{\prime \prime}$ & Edwards-Trinity Plateau, Trinity & -- & & .2 & 1.2 & 0 & $8.2 \mathrm{mi}$ above Vanderpool \\
\hline 222 & $29^{\circ} 48^{\prime} 54^{\prime \prime}$ & $99^{\circ} 34^{\prime} 18^{\prime \prime}$ & Trinity & -- & & .76 & 3.0 & 1.2 & \\
\hline 222 & $29^{\circ} 47^{\prime} 36^{\prime \prime}$ & $99^{\circ} 34^{\prime} 35^{\prime \prime}$ & Trinity & -- & & -.09 & 4.0 & 4.2 & \\
\hline 222 & $29^{\circ} 44^{\prime} 39^{\prime \prime}$ & $99^{\circ} 33^{\prime} 14^{\prime \prime}$ & Trinity & -- & & -.06 & 1.2 & 8.2 & \\
\hline 222 & $29^{\circ} 43^{\prime} 44^{\prime \prime}$ & $99^{\circ} 32^{\prime} 52^{\prime \prime}$ & Trinity & -- & & -1.84 & .9 & 9.4 & \\
\hline 222 & $29^{\circ} 43^{\prime} 07^{\prime \prime}$ & $99^{\circ} 32^{\prime} 53^{\prime \prime}$ & Trinity & -- & & .5 & 1.3 & 10.3 & \\
\hline 222 & $29^{\circ} 41^{\prime} 59^{\prime \prime}$ & $99^{\circ} 33^{\prime} 02^{\prime \prime}$ & Trinity & -- & & .25 & .9 & 11.6 & \\
\hline 222 & $29^{\circ} 41^{\prime} 20^{\prime \prime}$ & $99^{\circ} 32^{\prime} 59^{\prime \prime}$ & Trinity & -- & & -.75 & .7 & 12.5 & \\
\hline 222 & $29^{\circ} 40^{\prime} 47^{\prime \prime}$ & $99^{\circ} 32^{\prime} 50^{\prime \prime}$ & Trinity & -- & & .5 & 1.2 & 13.2 & \\
\hline 222 & $29^{\circ} 39^{\prime} 48^{\prime \prime}$ & $99^{\circ} 32^{\prime} 32^{\prime \prime}$ & Trinity & -- & & -.5 & .4 & 14.4 & \\
\hline 222 & $29^{\circ} 39^{\prime} 49^{\prime \prime}$ & $99^{\circ} 32^{\prime} 08^{\prime \prime}$ & Trinity & -- & & 0 & .9 & 14.8 & \\
\hline 222 & $29^{\circ} 39^{\prime} 16^{\prime \prime}$ & $99^{\circ} 32^{\prime} 00^{\prime \prime}$ & Trinity & -- & & 0 & 2.2 & 15.7 & \\
\hline 222 & $29^{\circ} 37^{\prime} 47^{\prime \prime}$ & $99^{\circ} 32^{\prime} 03^{\prime \prime}$ & Trinity & -- & & -.5 & 1.1 & 17.9 & \\
\hline
\end{tabular}


Table 4. Gains and losses from gain-loss studies in Texas-Continued

\begin{tabular}{|c|c|c|c|c|c|c|c|c|c|}
\hline $\begin{array}{l}\text { Stream- } \\
\text { flow } \\
\text { study } \\
\text { no. }\end{array}$ & $\begin{array}{l}\text { Latitude of } \\
\text { upstream } \\
\text { end of } \\
\text { subreach }\end{array}$ & $\begin{array}{l}\text { Longitude } \\
\text { of upstream } \\
\text { end of } \\
\text { subreach }\end{array}$ & $\begin{array}{c}\text { Major aquifer } \\
\text { outcrop } \\
\text { intersected } \\
\text { by subreach }\end{array}$ & & $\begin{array}{l}\text { Minor aquifer } \\
\text { outcrop } \\
\text { intercepted } \\
\text { by subreach }\end{array}$ & $\begin{array}{l}\text { Gain or } \\
\text { loss (-) in } \\
\text { subreach } \\
\left(\mathrm{ft}^{3} / \mathrm{s}\right)\end{array}$ & $\begin{array}{l}\text { Length } \\
\text { of subreach } \\
\text { (river mi) }\end{array}$ & $\begin{array}{l}\text { Location of } \\
\text { upstream } \\
\text { end of } \\
\text { subreach } \\
\text { (river mi) }\end{array}$ & $\begin{array}{l}\text { Descriptive location } \\
\text { of upstream end of } \\
\text { selected subreaches }\end{array}$ \\
\hline 222 & $29^{\circ} 36^{\prime} 43^{\prime \prime}$ & $99^{\circ} 31^{\prime} 44^{\prime \prime}$ & Trinity & -- & & 0.5 & 5.5 & 19 & \\
\hline 222 & $29^{\circ} 33^{\prime} 56^{\prime \prime}$ & $99^{\circ} 30^{\prime} 17^{\prime \prime}$ & Trinity & -- & & -.5 & 1.0 & 24.5 & \\
\hline 222 & $29^{\circ} 33^{\prime} 08^{\prime \prime}$ & $99^{\circ} 30^{\prime} 06^{\prime \prime}$ & Trinity & -- & & .5 & 3.3 & 25.5 & \\
\hline 222 & $29^{\circ} 31^{\prime} 04^{\prime \prime}$ & $99^{\circ} 30^{\prime} 25^{\prime \prime}$ & Trinity & -- & & -.5 & 2.6 & 28.8 & \\
\hline 223 & $29^{\circ} 49^{\prime} 49^{\prime \prime}$ & $99^{\circ} 34^{\prime} 06^{\prime \prime}$ & Edwards-Trinity Plateau, Trinity & -- & & 0 & 1.2 & 0 & $8.2 \mathrm{mi}$ above Vanderpool \\
\hline 223 & $29^{\circ} 48^{\prime} 54^{\prime \prime}$ & $99^{\circ} 34^{\prime} 18^{\prime \prime}$ & Trinity & -- & & .26 & 3.0 & 1.2 & \\
\hline 223 & $29^{\circ} 47^{\prime} 36^{\prime \prime}$ & $99^{\circ} 34^{\prime} 35^{\prime \prime}$ & Trinity & -- & & -.08 & 4.0 & 4.2 & \\
\hline 223 & $29^{\circ} 44^{\prime} 39^{\prime \prime}$ & $99^{\circ} 33^{\prime} 14^{\prime \prime}$ & Trinity & -- & & -.05 & 1.2 & 8.2 & \\
\hline 223 & $29^{\circ} 43^{\prime} 44^{\prime \prime}$ & $99^{\circ} 32^{\prime} 52^{\prime \prime}$ & Trinity & -- & & -.4 & .9 & 9.4 & \\
\hline 223 & $29^{\circ} 43^{\prime} 07^{\prime \prime}$ & $99^{\circ} 32^{\prime} 53^{\prime \prime}$ & Trinity & -- & & .1 & 1.3 & 10.3 & \\
\hline 223 & $29^{\circ} 41^{\prime} 59^{\prime \prime}$ & $99^{\circ} 33^{\prime} 02^{\prime \prime}$ & Trinity & -- & & .05 & .9 & 11.6 & \\
\hline 223 & $29^{\circ} 41^{\prime} 20^{\prime \prime}$ & 99॰32'59" & Trinity & -- & & -.15 & .7 & 12.5 & \\
\hline 223 & $29^{\circ} 40^{\prime} 47^{\prime \prime}$ & $99^{\circ} 32^{\prime} 50^{\prime \prime}$ & Trinity & -- & & .3 & 1.2 & 13.2 & \\
\hline 223 & $29^{\circ} 39^{\prime} 48^{\prime \prime}$ & $99^{\circ} 32^{\prime} 32^{\prime \prime}$ & Trinity & -- & & -.2 & .4 & 14.4 & \\
\hline 223 & $29^{\circ} 39^{\prime} 49^{\prime \prime}$ & $99^{\circ} 32^{\prime} 08^{\prime \prime}$ & Trinity & -- & & -.1 & .9 & 14.8 & \\
\hline 223 & $29^{\circ} 39^{\prime} 16^{\prime \prime}$ & $99^{\circ} 32^{\prime} 00^{\prime \prime}$ & Trinity & -- & & 0 & 2.2 & 15.7 & \\
\hline 223 & $29^{\circ} 37^{\prime} 55^{\prime \prime}$ & $99^{\circ} 32^{\prime} 10^{\prime \prime}$ & Trinity & -- & & -.81 & 1.1 & 17.9 & \\
\hline 223 & $29^{\circ} 36^{\prime} 43^{\prime \prime}$ & $99^{\circ} 31^{\prime} 44^{\prime \prime}$ & Trinity & -- & & .3 & 5.5 & 19 & \\
\hline 223 & $29^{\circ} 33^{\prime} 56^{\prime \prime}$ & $99^{\circ} 30^{\prime} 17^{\prime \prime}$ & Trinity & -- & & -.3 & 1.0 & 24.5 & \\
\hline 223 & $29^{\circ} 33^{\prime} 08^{\prime \prime}$ & $99^{\circ} 30^{\prime} 06^{\prime \prime}$ & Trinity & -- & & .1 & 3.3 & 25.5 & \\
\hline 223 & $29^{\circ} 31^{\prime} 04^{\prime \prime}$ & $99^{\circ} 30^{\prime} 25^{\prime \prime}$ & Trinity & -- & & -.1 & 2.6 & 28.8 & \\
\hline 224 & $29^{\circ} 36^{\prime} 12^{\prime \prime}$ & $99^{\circ} 31^{\prime} 33^{\prime \prime}$ & Trinity & -- & & 2.84 & 7.0 & 0 & $0.8 \mathrm{mi}$ below Utopia \\
\hline 224 & $29^{\circ} 30^{\prime} 21^{\prime \prime}$ & $99^{\circ} 30^{\prime} 51^{\prime \prime}$ & Trinity & -- & & -1.36 & 10.5 & 7 & \\
\hline 224 & $29^{\circ} 21^{\prime} 00^{\prime \prime}$ & $99^{\circ} 28^{\prime} 50^{\prime \prime}$ & -- & -- & & -.56 & .5 & 17.5 & \\
\hline 225 & $29^{\circ} 30^{\prime} 46^{\prime \prime}$ & $99^{\circ} 30^{\prime} 43^{\prime \prime}$ & Trinity & -- & & -.5 & 2.0 & 0 & just below Onion Creek \\
\hline 225 & $29^{\circ} 29^{\circ} 38^{\prime \prime}$ & $99^{\circ} 29^{\prime} 47^{\prime \prime}$ & Edwards, Trinity & -- & & -8.8 & 2.0 & 2 & \\
\hline 225 & $29^{\circ} 28^{\prime} 16^{\prime \prime}$ & $99^{\circ} 29^{\prime} 34^{\prime \prime}$ & Edwards & -- & & -23.0 & 5.5 & 4 & \\
\hline 226 & $29^{\circ} 30^{\prime} 45^{\prime \prime}$ & $99^{\circ} 30^{\prime} 43^{\prime \prime}$ & Trinity & -- & & -3.52 & 2.0 & 0 & just below Onion Creek \\
\hline 226 & $29^{\circ} 29^{\circ} 38^{\prime \prime}$ & $99^{\circ} 29^{\prime} 47^{\prime \prime}$ & Edwards, Trinity & -- & & -3.03 & 2.0 & 2 & \\
\hline 226 & $29^{\circ} 28^{\prime} 16^{\prime \prime}$ & $99^{\circ} 29^{\prime} 34^{\prime \prime}$ & Edwards & -- & & 0 & 5.5 & 4 & \\
\hline 227 & $29^{\circ} 29^{\circ} 35^{\prime \prime}$ & $99^{\circ} 29^{\prime} 48^{\prime \prime}$ & -- & -- & & -51.9 & 5.3 & 0 & gaging station $(08190000)$ \\
\hline 227 & $29^{\circ} 25^{\prime} 57^{\prime \prime}$ & $99^{\circ} 31^{\prime} 01^{\prime \prime}$ & -- & -- & & -30.3 & 6.0 & 5.3 & \\
\hline 227 & $29^{\circ} 22^{\prime} 49^{\prime \prime}$ & $99^{\circ} 29^{\prime} 04^{\prime \prime}$ & -- & -- & & 0 & 7.8 & 11.3 & \\
\hline 228 & $29^{\circ} 29^{\circ} 35^{\prime \prime}$ & $99^{\circ} 29^{\prime} 48^{\prime \prime}$ & -- & -- & & -40.1 & 5.3 & 0 & gaging station (90819000) \\
\hline 228 & $29^{\circ} 25^{\prime} 57^{\prime \prime}$ & $99^{\circ} 31^{\prime} 01^{\prime \prime}$ & -- & -- & & -49.4 & 6.0 & 5.3 & \\
\hline 228 & $29^{\circ} 22^{\prime} 49^{\prime \prime}$ & $99^{\circ} 29^{\prime} 04^{\prime \prime}$ & -- & -- & & -1.7 & 7.8 & 11.3 & \\
\hline 229 & $29^{\circ} 34^{\prime} 23^{\prime \prime}$ & $99^{\circ} 24^{\prime} 10^{\prime \prime}$ & Trinity & -- & & -19.0 & 1.3 & 0 & gaging station (08201500) \\
\hline 229 & $29^{\circ} 33^{\prime} 25^{\prime \prime}$ & $99^{\circ} 24^{\prime} 18^{\prime \prime}$ & Trinity & -- & & 42.0 & 2.5 & 1.3 & \\
\hline
\end{tabular}


Table 4. Gains and losses from gain-loss studies in Texas-Continued

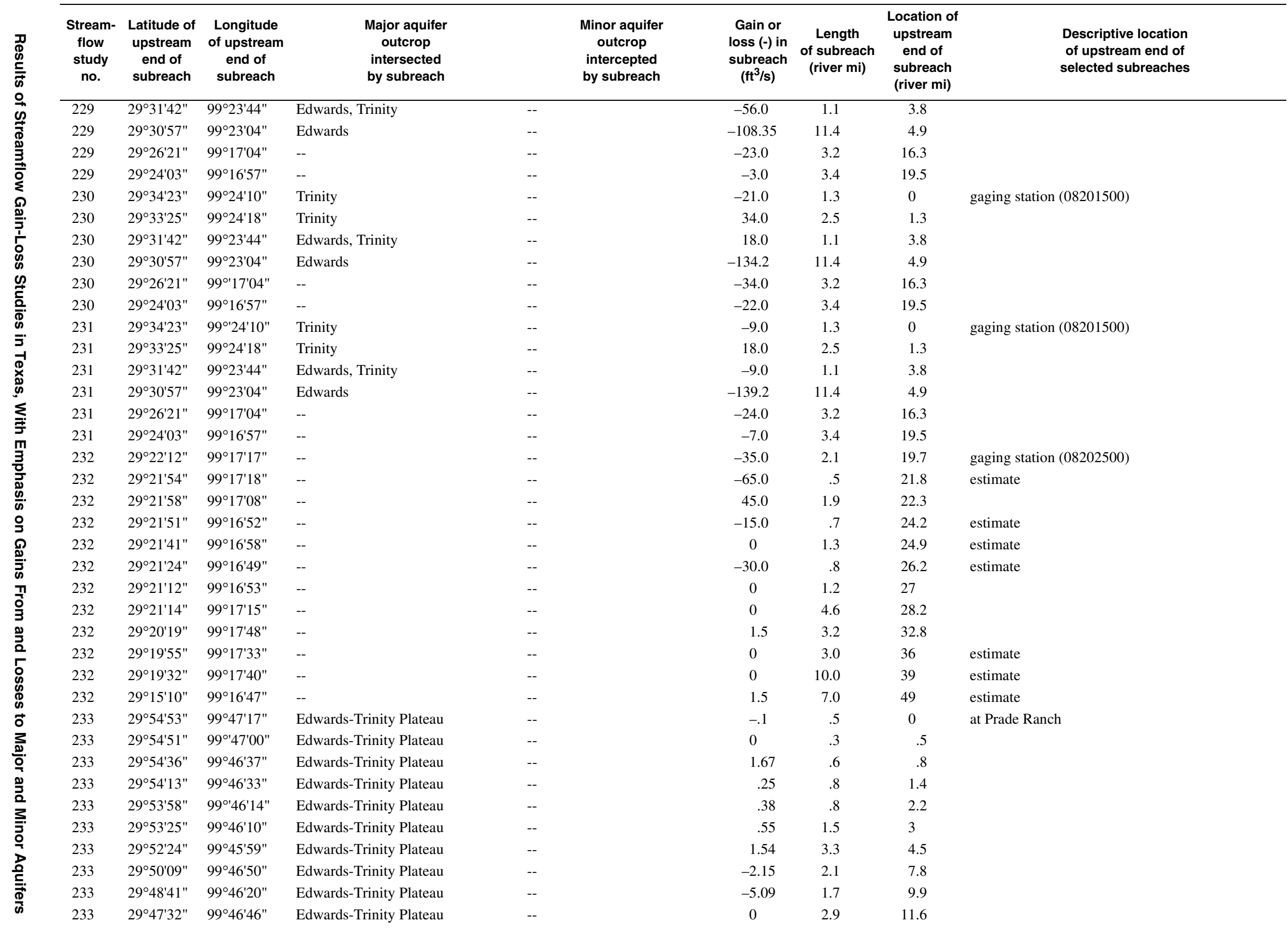


Table 4. Gains and losses from gain-loss studies in Texas-Continued

\begin{tabular}{|c|c|c|c|c|c|c|c|c|c|}
\hline $\begin{array}{l}\text { Stream- } \\
\text { flow } \\
\text { study } \\
\text { no. }\end{array}$ & $\begin{array}{l}\text { Latitude of } \\
\text { upstream } \\
\text { end of } \\
\text { subreach }\end{array}$ & $\begin{array}{l}\text { Longitude } \\
\text { of upstream } \\
\text { end of } \\
\text { subreach }\end{array}$ & $\begin{array}{c}\text { Major aquifer } \\
\text { outcrop } \\
\text { intersected } \\
\text { by subreach }\end{array}$ & & $\begin{array}{c}\text { Minor aquifer } \\
\text { outcrop } \\
\text { intercepted } \\
\text { by subreach }\end{array}$ & $\begin{array}{l}\text { Gain or } \\
\text { loss }(-) \text { in } \\
\text { subreach } \\
\left(\mathrm{ft}^{3} / \mathrm{s}\right)\end{array}$ & $\begin{array}{l}\text { Length } \\
\text { of subreach } \\
\text { (river mi) }\end{array}$ & $\begin{array}{l}\text { Location of } \\
\text { upstream } \\
\text { end of } \\
\text { subreach } \\
\text { (river mi) }\end{array}$ & $\begin{array}{l}\text { Descriptive location } \\
\text { of upstream end of } \\
\text { selected subreaches }\end{array}$ \\
\hline 233 & $29^{\circ} 45^{\prime} 42^{\prime \prime}$ & $99^{\circ} 45^{\prime} 47^{\prime \prime}$ & Edwards-Trinity Plateau & -- & & 0 & 1.6 & 14.5 & \\
\hline 233 & $29^{\circ} 44^{\prime} 27^{\prime \prime}$ & $99^{\circ} 44^{\prime} 58^{\prime \prime}$ & Edwards-Trinity Plateau & -- & & 0 & .9 & 16.1 & \\
\hline 233 & $29^{\circ} 43^{\prime} 50^{\prime \prime}$ & $99^{\circ} 44^{\prime} 49^{\prime \prime}$ & Edwards-Trinity Plateau & -- & & 4.56 & .1 & 17 & \\
\hline 233 & $29^{\circ} 43^{\prime} 46^{\prime \prime}$ & $99^{\circ} 44^{\prime} 44^{\prime \prime}$ & Edwards-Trinity Plateau & -- & & 6.04 & 2.6 & 17.1 & \\
\hline 233 & $29^{\circ} 41^{\prime} 38^{\prime \prime}$ & $99^{\circ} 45^{\prime} 14^{\prime \prime}$ & Edwards-Trinity Plateau & -- & & 1.78 & 2.6 & 19.7 & at Ranch Rd 1120 \\
\hline 233 & $29^{\circ} 39^{\prime} 53^{\prime \prime}$ & $99^{\circ} 44^{\prime} 37^{\prime \prime}$ & Edwards-Trinity Plateau & -- & & -.15 & 4.2 & 22.3 & \\
\hline 233 & $29^{\circ} 36^{\prime} 49^{\prime \prime}$ & $99^{\circ} 44^{\prime} 25^{\prime \prime}$ & Edwards-Trinity Plateau & -- & & 2.26 & 3.0 & 26.5 & \\
\hline 233 & $29^{\circ} 35^{\prime} 06^{\prime \prime}$ & $99^{\circ} 43^{\prime} 56^{\prime \prime}$ & Edwards-Trinity Plateau & -- & & 0 & 6.2 & 29.5 & \\
\hline 233 & $29^{\circ} 31^{\prime} 48^{\prime \prime}$ & $99^{\circ} 42^{\prime} 22^{\prime \prime}$ & Edwards, Edwards-Trinity Plateau & -- & & -1.48 & 3.8 & 35.7 & \\
\hline 234 & $29^{\circ} 57^{\prime} 31^{\prime \prime}$ & $99^{\circ} 44^{\prime} 33^{\prime \prime}$ & Edwards-Trinity Plateau & -- & & -.03 & .5 & 0 & at hydraulic ram on Prade Ranch \\
\hline 234 & $29^{\circ} 57^{\prime} 12^{\prime \prime}$ & $99^{\circ} 44^{\prime} 45^{\prime \prime}$ & Edwards-Trinity Plateau & -- & & 0 & .3 & .5 & \\
\hline 234 & $29^{\circ} 56^{\prime} 59^{\prime \prime}$ & $99^{\circ} 44^{\prime} 53^{\prime \prime}$ & Edwards-Trinity Plateau & -- & & 1.95 & .6 & .8 & \\
\hline 234 & $29^{\circ} 56^{\prime} 43^{\prime \prime}$ & $99^{\circ} 45^{\prime} 05^{\prime \prime}$ & Edwards-Trinity Plateau & -- & & .25 & .8 & 1.4 & \\
\hline 234 & $29^{\circ} 55^{\prime} 55^{\prime \prime}$ & $99^{\circ} 45^{\prime} 26^{\prime \prime}$ & Edwards-Trinity Plateau & -- & & -.79 & .8 & 2.2 & \\
\hline 234 & $29^{\circ} 55^{\prime} 12^{\prime \prime}$ & $99^{\circ} 45^{\prime} 30^{\prime \prime}$ & Edwards-Trinity Plateau & -- & & .39 & 1.5 & 3 & \\
\hline 234 & $29^{\circ} 53^{\prime} 43^{\prime \prime}$ & $99^{\circ} 46^{\prime} 15^{\prime \prime}$ & Edwards-Trinity Plateau & -- & & -.42 & 3.3 & 4.5 & \\
\hline 234 & $29^{\circ} 50^{\prime} 08^{\prime \prime}$ & $99^{\circ} 46^{\prime} 51^{\prime \prime}$ & Edwards-Trinity Plateau & -- & & -1.55 & 2.1 & 7.8 & \\
\hline 234 & $29^{\circ} 48^{\prime} 33^{\prime \prime}$ & $99^{\circ} 46^{\prime} 16^{\prime \prime}$ & Edwards-Trinity Plateau & -- & & -2.11 & 1.7 & 9.9 & \\
\hline 234 & $29^{\circ} 47^{\prime} 34^{\prime \prime}$ & $99^{\circ} 46^{\prime} 48^{\prime \prime}$ & Edwards-Trinity Plateau & -- & & 0 & 2.9 & 11.6 & \\
\hline 234 & $29^{\circ} 45^{\prime} 37^{\prime \prime}$ & $99^{\circ} 45^{\prime} 45^{\prime \prime}$ & Edwards-Trinity Plateau & -- & & 0 & 1.5 & 14.5 & \\
\hline 234 & $29^{\circ} 44^{\prime} 39^{\prime \prime}$ & $99^{\circ} 45^{\prime} 06^{\prime \prime}$ & Edwards-Trinity Plateau & -- & & 0 & .1 & 16 & \\
\hline 234 & $29^{\circ} 44^{\prime} 27^{\prime \prime}$ & $99^{\circ} 44^{\prime} 57^{\prime \prime}$ & Edwards-Trinity Plateau & -- & & 0 & .9 & 16.1 & \\
\hline 234 & $29^{\circ} 43^{\prime} 51^{\prime \prime}$ & $99^{\circ} 44^{\prime} 50^{\prime \prime}$ & Edwards-Trinity Plateau & -- & & 1.86 & .1 & 17 & \\
\hline 234 & $29^{\circ} 43^{\prime} 50^{\prime \prime}$ & $99^{\circ} 44^{\prime} 49^{\prime \prime}$ & Edwards-Trinity Plateau & -- & & 4.54 & 2.6 & 17.1 & \\
\hline 234 & $29^{\circ} 41^{\prime} 38^{\prime \prime}$ & $99^{\circ} 45^{\prime} 13^{\prime \prime}$ & Edwards-Trinity Plateau & -- & & 1.16 & 2.6 & 19.7 & \\
\hline 234 & $29^{\circ} 39^{\prime} 53^{\prime \prime}$ & $99^{\circ} 44^{\prime} 37^{\prime \prime}$ & Edwards-Trinity Plateau & -- & & 1.84 & 4.2 & 22.3 & \\
\hline 234 & $29^{\circ} 36^{\prime} 50^{\prime \prime}$ & $99^{\circ} 44^{\prime} 25^{\prime \prime}$ & Edwards-Trinity Plateau & -- & & 3.56 & 3.0 & 26.5 & \\
\hline 234 & $29^{\circ} 35^{\prime} 11^{\prime \prime}$ & $99^{\circ} 44^{\prime} 07^{\prime \prime}$ & Edwards-Trinity Plateau & -- & & -2.23 & 6.2 & 29.5 & \\
\hline 234 & $29^{\circ} 31^{\prime} 15^{\prime \prime}$ & $99^{\circ} 42^{\prime} 04^{\prime \prime}$ & Edwards, Edwards-Trinity Plateau & -- & & .12 & 3.8 & 35.7 & \\
\hline 235 & $29^{\circ} 56^{\prime} 33^{\prime \prime}$ & $99^{\circ} 45^{\prime} 05^{\prime \prime}$ & Edwards-Trinity Plateau & -- & & .93 & 3.3 & 4.5 & 2.2 mi below West Fork Frio River \\
\hline 235 & $29^{\circ} 50^{\prime} 08^{\prime \prime}$ & $99^{\circ} 46^{\prime} 51^{\prime \prime}$ & Edwards-Trinity Plateau & -- & & -3.95 & 2.1 & 7.8 & \\
\hline 235 & $29^{\circ} 48^{\prime} 34^{\prime \prime}$ & $99^{\circ} 46^{\prime} 17^{\prime \prime}$ & Edwards-Trinity Plateau & -- & & -4.52 & 1.7 & 9.9 & \\
\hline 235 & $29^{\circ} 47^{\prime} 11^{\prime \prime}$ & $99^{\circ} 45^{\prime} 59^{\prime \prime}$ & Edwards-Trinity Plateau & -- & & 0 & 2.9 & 11.6 & \\
\hline 235 & $29^{\circ} 45^{\prime} 20^{\prime \prime}$ & $99^{\circ} 45^{\prime} 36^{\prime \prime}$ & Edwards-Trinity Plateau & -- & & 0 & 1.6 & 14.5 & \\
\hline 235 & $29^{\circ} 44^{\prime} 26^{\prime \prime}$ & $99^{\circ} 44^{\prime} 58^{\prime \prime}$ & Edwards-Trinity Plateau & -- & & 1.59 & .9 & 16.1 & \\
\hline 235 & $29^{\circ} 43^{\prime} 51^{\prime \prime}$ & $99^{\circ} 44^{\prime} 50^{\prime \prime}$ & Edwards-Trinity Plateau & -- & & 9.21 & .1 & 17 & \\
\hline 235 & $29^{\circ} 43^{\prime} 50^{\prime \prime}$ & $99^{\circ} 44^{\prime} 49^{\prime \prime}$ & Edwards, Edwards-Trinity Plateau & -- & & 27.6 & 22.4 & 17.1 & \\
\hline 236 & $29^{\circ} 50^{\prime} 08^{\prime \prime}$ & $99^{\circ} 46^{\prime} 50^{\prime \prime}$ & Edwards-Trinity Plateau & -- & & -5.61 & 8.3 & 7.8 & $500 \mathrm{ft}$ above Kent Creek \\
\hline
\end{tabular}


Table 4. Gains and losses from gain-loss studies in Texas-Continued

\begin{tabular}{|c|c|c|c|c|c|c|c|c|}
\hline $\begin{array}{l}\text { Stream- } \\
\text { flow } \\
\text { study } \\
\text { no. }\end{array}$ & $\begin{array}{l}\text { Latitude of } \\
\text { upstream } \\
\text { end of } \\
\text { subreach }\end{array}$ & $\begin{array}{l}\text { Longitude } \\
\text { of upstream } \\
\text { end of } \\
\text { subreach }\end{array}$ & $\begin{array}{l}\text { Major aquifer } \\
\text { outcrop } \\
\text { intersected } \\
\text { by subreach }\end{array}$ & $\begin{array}{c}\text { Minor aquifer } \\
\text { outcrop } \\
\text { intercepted } \\
\text { by subreach }\end{array}$ & $\begin{array}{c}\text { Gain or } \\
\text { loss }(-) \text { in } \\
\text { subreach } \\
\left(\mathrm{ft}^{3} / \mathrm{s}\right)\end{array}$ & $\begin{array}{l}\text { Length } \\
\text { of subreach } \\
\text { (river mi) }\end{array}$ & $\begin{array}{l}\text { Location of } \\
\text { upstream } \\
\text { end of } \\
\text { subreach } \\
\text { (river mi) }\end{array}$ & $\begin{array}{l}\text { Descriptive location } \\
\text { of upstream end of } \\
\text { selected subreaches }\end{array}$ \\
\hline 236 & $29^{\circ} 44^{\prime 2} 27^{\prime \prime}$ & $99^{\circ} 44^{\prime} 57^{\prime \prime}$ & Edwards-Trinity Plateau & -- & 14.1 & 3.6 & 16.1 & \\
\hline 236 & $29^{\circ} 41^{\prime} 38^{\prime \prime}$ & $99^{\circ} 45^{\prime} 13^{\prime \prime}$ & Edwards-Trinity Plateau & -- & 2.4 & 16.0 & 19.7 & \\
\hline 236 & $29^{\circ} 32^{\prime} 00^{\prime \prime}$ & $99^{\circ} 42^{\prime} 43^{\prime \prime}$ & Edwards, Edwards-Trinity Plateau & -- & .7 & 3.8 & 35.7 & \\
\hline 237 & $29^{\circ} 28^{\prime} 51^{\prime \prime}$ & $100^{\circ} 14^{\prime} 16^{\prime \prime}$ & Edwards & -- & 6.0 & 9.2 & 0 & $\begin{array}{l}0.25 \mathrm{mi} \text { above Brackettville gaging station } \\
(08190500)\end{array}$ \\
\hline 237 & $29^{\circ} 26^{\prime} 27^{\prime \prime}$ & $100^{\circ} 09^{\prime} 54^{\prime \prime}$ & Edwards & -- & 8.0 & 4.2 & 9.2 & \\
\hline 237 & $29^{\circ} 25^{\prime} 39^{\prime \prime}$ & $100^{\circ} 06^{\prime} 14^{\prime \prime}$ & Edwards & -- & -21.1 & 23.6 & 13.4 & \\
\hline 238 & $29^{\circ} 53^{\prime} 10^{\prime \prime}$ & $100^{\circ} 24^{\prime} 46^{\prime \prime}$ & Edwards-Trinity Plateau & -- & 0 & 4.8 & 0 & at Blackwater Hole \\
\hline 238 & $29^{\circ} 49^{\prime} 37^{\prime \prime}$ & $100^{\circ} 23^{\prime} 49^{\prime \prime}$ & Edwards-Trinity Plateau & -- & 0 & 3.0 & 4.8 & \\
\hline 238 & $29^{\circ} 47^{\prime} 35^{\prime \prime}$ & $100^{\circ} 24^{\prime} 39^{\prime \prime}$ & Edwards-Trinity Plateau & -- & .5 & 2.7 & 7.8 & \\
\hline 238 & $29^{\circ} 45^{\prime} 26^{\prime \prime}$ & $100^{\circ} 23^{\prime} 52^{\prime \prime}$ & Edwards-Trinity Plateau & -- & 3.73 & .2 & 10.5 & estimate \\
\hline 238 & $29^{\circ} 45^{\prime} 23^{\prime \prime}$ & $100^{\circ} 23^{\prime} 52^{\prime \prime}$ & Edwards-Trinity Plateau & -- & -6.21 & 2.7 & 10.7 & \\
\hline 238 & $29^{\circ} 43^{\prime} 48^{\prime \prime}$ & $100^{\circ} 24^{\prime} 10^{\prime \prime}$ & Edwards-Trinity Plateau & -- & 0 & 1.1 & 13.4 & \\
\hline 238 & $29^{\circ} 43^{\prime} 19^{\prime \prime}$ & $100^{\circ} 25^{\prime} 04^{\prime \prime}$ & Edwards-Trinity Plateau & -- & 0 & 2.2 & 14.5 & \\
\hline 238 & $29^{\circ} 41^{\prime} 26^{\prime \prime}$ & $100^{\circ} 25^{\prime} 15^{\prime \prime}$ & Edwards-Trinity Plateau & -- & 0 & 7.6 & 16.7 & \\
\hline 238 & $29^{\circ} 36^{\prime} 48^{\prime \prime}$ & $100^{\circ} 22^{\prime} 26^{\prime \prime}$ & Edwards-Trinity Plateau & -- & 0 & 5.0 & 24.3 & \\
\hline 238 & $29^{\circ} 33^{\prime} 49^{\prime \prime}$ & $100^{\circ} 20^{\prime} 56^{\prime \prime}$ & Edwards-Trinity Plateau & -- & 0 & 3.3 & 29.3 & \\
\hline 238 & $29^{\circ} 34^{\prime} 48^{\prime \prime}$ & $100^{\circ} 18^{\prime} 10^{\prime \prime}$ & Edwards-Trinity Plateau & -- & -2.97 & 6.1 & 32.6 & \\
\hline 238 & $29^{\circ} 32^{\prime} 22^{\prime \prime}$ & $100^{\circ} 15^{\prime} 07^{\prime \prime}$ & Edwards-Trinity Plateau & -- & 0 & 4.9 & 38.7 & \\
\hline 238 & $29^{\circ} 30^{\prime} 38^{\prime \prime}$ & $100^{\circ} 16^{\prime} 48^{\prime \prime}$ & Edwards-Trinity Plateau & -- & 0 & 2.5 & 43.6 & \\
\hline 238 & $29^{\circ} 29^{\circ} 30^{\prime \prime}$ & $100^{\circ} 16^{\prime} 40^{\prime \prime}$ & Edwards, Edwards-Trinity Plateau & -- & 0 & 1.6 & 46.1 & \\
\hline 239 & $29^{\circ} 53^{\prime} 10^{\prime \prime}$ & $100^{\circ} 24^{\prime} 46^{\prime \prime}$ & Edwards-Trinity Plateau & -- & 0 & 4.8 & 0 & at Blackwater Hole \\
\hline 239 & $29^{\circ} 49^{\prime} 37^{\prime \prime}$ & $100^{\circ} 23^{\prime} 49^{\prime \prime}$ & Edwards-Trinity Plateau & -- & 0 & 3.0 & 4.8 & \\
\hline 239 & $29^{\circ} 47^{\prime} 35^{\prime \prime}$ & $100^{\circ} 24^{\prime} 39^{\prime \prime}$ & Edwards-Trinity Plateau & -- & 0 & 2.7 & 7.8 & \\
\hline 239 & $29^{\circ} 45^{\prime} 31^{\prime \prime}$ & $100^{\circ} 24^{\prime} 07^{\prime \prime}$ & Edwards-Trinity Plateau & -- & .54 & .3 & 10.5 & \\
\hline 239 & $29^{\circ} 45^{\prime} 23^{\prime \prime}$ & $100^{\circ} 23^{\prime} 52^{\prime \prime}$ & Edwards-Trinity Plateau & -- & -2.35 & 2.6 & 10.8 & \\
\hline 239 & $29^{\circ} 43^{\prime} 48^{\prime \prime}$ & $100^{\circ} 24^{\prime} 10^{\prime \prime}$ & Edwards-Trinity Plateau & -- & 0 & 1.1 & 13.4 & \\
\hline 239 & $29^{\circ} 43^{\prime} 19^{\prime \prime}$ & $100^{\circ} 25^{\prime} 04^{\prime \prime}$ & Edwards-Trinity Plateau & -- & 0 & 2.2 & 14.5 & \\
\hline 239 & $29^{\circ} 41^{\prime} 26^{\prime \prime}$ & $100^{\circ} 25^{\prime} 15^{\prime \prime}$ & Edwards-Trinity Plateau & -- & 0 & 7.6 & 16.7 & \\
\hline 239 & $29^{\circ} 36^{\prime} 48^{\prime \prime}$ & $100^{\circ} 22^{\prime} 26^{\prime \prime}$ & Edwards-Trinity Plateau & -- & 0 & 5.0 & 24.3 & \\
\hline 239 & $29^{\circ} 33^{\prime} 49^{\prime \prime}$ & $100^{\circ} 20^{\prime} 56^{\prime \prime}$ & Edwards-Trinity Plateau & -- & 0 & 3.3 & 29.3 & \\
\hline 239 & $29^{\circ} 34^{\prime} 48^{\prime \prime}$ & $100^{\circ} 18^{\prime} 10^{\prime \prime}$ & Edwards-Trinity Plateau & -- & 0 & 4.9 & 32.6 & \\
\hline 239 & $29^{\circ} 32^{\prime} 45^{\prime \prime}$ & $100^{\circ} 16^{\prime} 26^{\prime \prime}$ & Edwards-Trinity Plateau & -- & -2.41 & 2.6 & 37.5 & \\
\hline 239 & $29^{\circ} 31^{\prime} 12^{\prime \prime}$ & $100^{\circ} 15^{\prime} 03^{\prime \prime}$ & Edwards-Trinity Plateau & -- & -2.73 & 1.5 & 40.1 & \\
\hline 239 & $29^{\circ} 30^{\prime} 56^{\prime \prime}$ & $100^{\circ} 16^{\prime} 33^{\prime \prime}$ & Edwards-Trinity Plateau & -- & 0 & 4.5 & 41.6 & \\
\hline 239 & $29^{\circ} 29^{\circ} 30^{\prime \prime}$ & $100^{\circ} 16^{\prime} 40^{\prime \prime}$ & Edwards, Edwards-Trinity Plateau & -- & 0 & 1.6 & 46.1 & \\
\hline 240 & $29^{\circ} 28^{\prime} 51^{\prime \prime}$ & $100^{\circ} 14^{\prime} 16^{\prime \prime}$ & Edwards & -- & -286.0 & 38.5 & 0 & gaging station $(08190500)$ \\
\hline 241 & $29^{\circ} 41^{\prime} 59^{\prime \prime}$ & $99^{\circ} 26^{\prime} 26^{\prime \prime}$ & Trinity & -- & 1.71 & 2.3 & 0 & FM 470 \\
\hline
\end{tabular}


Table 4. Gains and losses from gain-loss studies in Texas-Continued

\begin{tabular}{|c|c|c|c|c|c|c|c|c|}
\hline $\begin{array}{l}\text { Stream- } \\
\text { flow } \\
\text { study } \\
\text { no. }\end{array}$ & $\begin{array}{l}\text { Latitude of } \\
\text { upstream } \\
\text { end of } \\
\text { subreach }\end{array}$ & $\begin{array}{l}\text { Longitude } \\
\text { of upstream } \\
\text { end of } \\
\text { subreach }\end{array}$ & $\begin{array}{c}\text { Major aquifer } \\
\text { outcrop } \\
\text { intersected } \\
\text { by subreach }\end{array}$ & $\begin{array}{c}\text { Minor aquifer } \\
\text { outcrop } \\
\text { intercepted } \\
\text { by subreach }\end{array}$ & $\begin{array}{l}\text { Gain or } \\
\text { loss }(-) \text { in } \\
\text { subreach } \\
\left(\mathrm{ft}^{3} / \mathrm{s}\right)\end{array}$ & $\begin{array}{l}\text { Length } \\
\text { of subreach } \\
\text { (river mi) }\end{array}$ & $\begin{array}{l}\text { Location of } \\
\text { upstream } \\
\text { end of } \\
\text { subreach } \\
\text { (river mi) }\end{array}$ & $\begin{array}{l}\text { Descriptive location } \\
\text { of upstream end of } \\
\text { selected subreaches }\end{array}$ \\
\hline 241 & $29^{\circ} 40^{\prime} 34^{\prime \prime}$ & $99^{\circ} 25^{\prime} 40^{\prime \prime}$ & Trinity & -- & 4.65 & 1.2 & 2.3 & \\
\hline 241 & $29^{\circ} 39^{\prime} 42^{\prime \prime}$ & $99^{\circ} 25^{\prime} 19^{\prime \prime}$ & Trinity & -- & .5 & 2.0 & 3.5 & \\
\hline 241 & $29^{\circ} 38^{\prime} 21^{\prime \prime}$ & $99^{\circ} 24^{\prime} 51^{\prime \prime}$ & Trinity & -- & 2.5 & 2.1 & 5.5 & \\
\hline 241 & $29^{\circ} 36^{\prime} 51^{\prime \prime}$ & $99^{\circ} 25^{\prime} 02^{\prime \prime}$ & Trinity & -- & .89 & 2.1 & 7.6 & \\
\hline 241 & $29^{\circ} 35^{\prime} 42^{\prime \prime}$ & $99^{\circ} 24^{\prime} 03^{\prime \prime}$ & Trinity & -- & 2.3 & 2.1 & 9.7 & \\
\hline 241 & $29^{\circ} 34^{\prime} 57^{\prime \prime}$ & $99^{\circ} 23^{\prime} 57^{\prime \prime}$ & Trinity & -- & -3.0 & 2.6 & 11.8 & \\
\hline 241 & $29^{\circ} 33^{\prime} 16^{\prime \prime}$ & $99^{\circ} 24^{\prime} 16^{\prime \prime}$ & Trinity & -- & -2.3 & 2.0 & 14.4 & \\
\hline 241 & $29^{\circ} 31^{\prime} 53^{\prime \prime}$ & $99^{\circ} 24^{\prime} 04^{\prime \prime}$ & Edwards, Trinity & -- & -1.0 & 2.1 & 16.4 & \\
\hline 241 & $29^{\circ} 30^{\prime} 35^{\prime \prime}$ & $99^{\circ} 23^{\prime} 29^{\prime \prime}$ & Edwards & -- & -1.9 & 1.2 & 18.5 & \\
\hline 241 & $29^{\circ} 29^{\circ} 16^{\prime \prime}$ & $99^{\circ} 23^{\prime} 11^{\prime \prime}$ & -- & -- & -11.8 & 2.1 & 19.7 & \\
\hline 241 & $29^{\circ} 28^{\prime} 58^{\prime \prime}$ & $99^{\circ} 21^{\prime 2} 20^{\prime \prime}$ & -- & -- & -11.72 & 2.8 & 21.8 & \\
\hline 241 & $29^{\circ} 28^{\prime} 34^{\prime \prime}$ & $99^{\circ} 18^{\prime} 57^{\prime \prime}$ & -- & -- & -.38 & 1.3 & 24.6 & \\
\hline 241 & $29^{\circ} 28^{\prime} 06^{\prime \prime}$ & $99^{\circ} 17^{\prime} 57^{\prime \prime}$ & Edwards & -- & -1.4 & .1 & 25.9 & \\
\hline 241 & $29^{\circ} 28^{\prime} 05^{\prime \prime}$ & $99^{\circ} 17^{\prime} 52^{\prime \prime}$ & Edwards & -- & -.05 & 6 & 26 & \\
\hline 241 & $29^{\circ} 27^{\prime} 50^{\prime \prime}$ & $99^{\circ} 17^{\prime} 39^{\prime \prime}$ & Edwards & -- & -.05 & .2 & 26.6 & \\
\hline 241 & $29^{\circ} 27^{\prime} 41^{\prime \prime}$ & $99^{\circ} 17^{\prime} 34^{\prime \prime}$ & -- & -- & 0 & 1.4 & 26.8 & \\
\hline 241 & $29^{\circ} 26^{\prime} 21^{\prime \prime}$ & $99^{\circ} 17^{\prime} 03^{\prime \prime}$ & -- & -- & 0 & 4.6 & 28.2 & \\
\hline 241 & $29^{\circ} 23^{\prime} 40^{\prime \prime}$ & $99^{\circ} 16^{\prime} 53^{\prime \prime}$ & -- & -- & .2 & 3.2 & 32.8 & \\
\hline 241 & $29^{\circ} 21^{\prime} 13^{\prime \prime}$ & $99^{\circ} 16^{\prime} 53^{\prime \prime}$ & -- & -- & -.19 & 3.0 & 36 & \\
\hline 242 & $29^{\circ} 41^{\prime} 07^{\prime \prime}$ & $99^{\circ} 18^{\prime} 19^{\prime \prime}$ & Trinity & -- & 1.6 & 2.3 & 4.7 & \\
\hline 242 & $29^{\circ} 40^{\prime} 12^{\prime \prime}$ & $99^{\circ} 16^{\prime} 49^{\prime \prime}$ & Trinity & -- & 4.76 & 2.1 & 2.4 & \\
\hline 243 & $32^{\circ} 36^{\prime} 56^{\prime \prime}$ & $94^{\circ} 21^{\prime} 24^{\prime \prime}$ & Carrizo-Wilcox & -- & -.03 & 3.3 & 3.3 & \\
\hline 244 & $32^{\circ} 46^{\prime} 04^{\prime \prime}$ & $94^{\circ} 54^{\prime} 55^{\prime \prime}$ & -- & Queen City & 1.18 & 4.1 & 52.1 & at $\mathrm{SH} 155$ \\
\hline 244 & $32^{\circ} 44^{\prime} 20^{\prime \prime}$ & $94^{\circ} 51^{\prime} 58^{\prime \prime}$ & -- & Queen City & -.04 & 4.2 & 48 & \\
\hline 244 & $32^{\circ} 42^{\prime} 13^{\prime \prime}$ & $94^{\circ} 48^{\prime} 23^{\prime \prime}$ & -- & Queen City & .13 & 4.2 & 43.8 & \\
\hline 244 & $32^{\circ} 40^{\prime} 22^{\prime \prime}$ & $94^{\circ} 45^{\prime} 03^{\prime \prime}$ & -- & Queen City & 1.17 & 7.2 & 39.6 & \\
\hline 244 & $32^{\circ} 38^{\prime} 19^{\prime \prime}$ & $94^{\circ} 41^{\prime} 40^{\prime \prime}$ & -- & Queen City & .02 & 6.4 & 32.4 & \\
\hline 244 & $32^{\circ} 37^{\prime} 24^{\prime \prime}$ & $94^{\circ} 34^{\prime} 36^{\prime \prime}$ & -- & Queen City & -1.18 & 4.0 & 26 & FM 450 \\
\hline 244 & $32^{\circ} 37^{\prime} 36^{\prime \prime}$ & $94^{\circ} 30^{\prime} 55^{\prime \prime}$ & Carrizo-Wilcox & Queen City & 1.74 & 8.9 & 22 & \\
\hline 244 & $32^{\circ} 40^{\prime} 39^{\prime \prime}$ & $94^{\circ} 26^{\prime} 13^{\prime \prime}$ & Carrizo-Wilcox & -- & 2.73 & 5.6 & 13.1 & \\
\hline 244 & $32^{\circ} 42^{\prime} 45^{\prime \prime}$ & $94^{\circ} 20^{\prime} 43^{\prime \prime}$ & Carrizo-Wilcox & -- & .76 & 4.5 & 7.5 & \\
\hline 245 & $32^{\circ} 44^{\prime} 27^{\prime \prime}$ & $94^{\circ} 52^{\prime} 04^{\prime \prime}$ & -- & Queen City & 1.19 & 4.2 & 48 & 0.2 mi above bridge on FM 555 \\
\hline 245 & $32^{\circ} 42^{\prime} 27^{\prime \prime}$ & $94^{\circ} 48^{\prime} 40^{\prime \prime}$ & -- & Queen City & 5.6 & 4.2 & 43.8 & \\
\hline 245 & $32^{\circ} 40^{\prime} 22^{\prime \prime}$ & $94^{\circ} 45^{\prime} 03^{\prime \prime}$ & -- & Queen City & 10.2 & 13.6 & 39.6 & \\
\hline 245 & $32^{\circ} 37^{\prime} 21^{\prime \prime}$ & $94^{\circ} 34^{\prime} 28^{\prime \prime}$ & -- & Queen City & 3.5 & 4.0 & 26 & \\
\hline 245 & $32^{\circ} 37^{\prime} 41^{\prime \prime}$ & $94^{\circ} 30^{\prime} 45^{\prime \prime}$ & -- & -- & .2 & 8.9 & 22 & \\
\hline 245 & $32^{\circ} 40^{\prime} 17^{\prime \prime}$ & $94^{\circ} 26^{\prime} 33^{\prime \prime}$ & Carrizo-Wilcox & -- & 3.4 & 5.6 & 13.1 & \\
\hline 246 & $33^{\circ} 49^{\prime} 14^{\prime \prime}$ & $99^{\circ} 47^{\prime} 09^{\prime \prime}$ & -- & -- & .3 & 13.6 & 188.4 & gaging station (07311700) \\
\hline
\end{tabular}


Table 4. Gains and losses from gain-loss studies in Texas-Continued

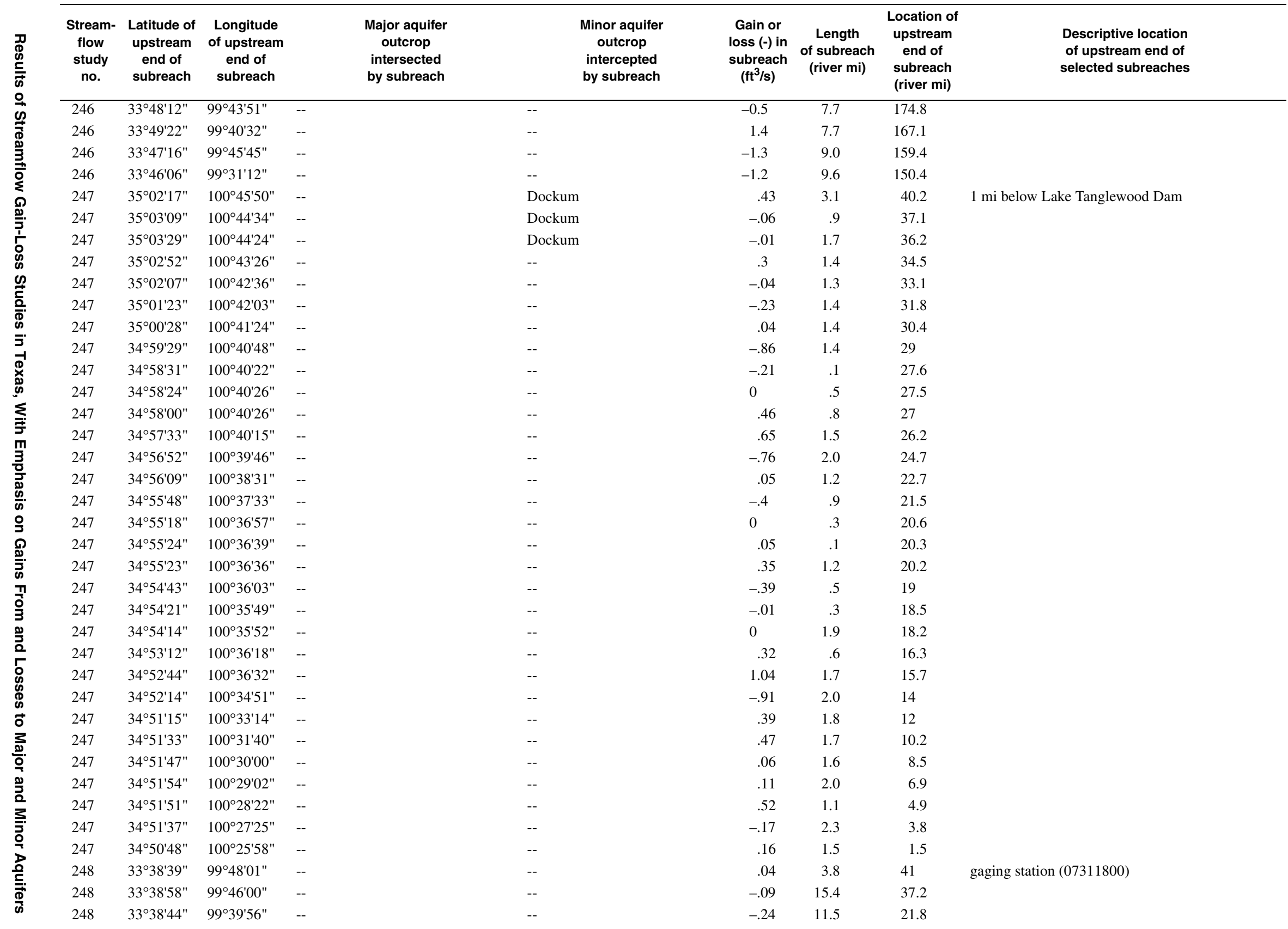


Table 4. Gains and losses from gain-loss studies in Texas-Continued

\begin{tabular}{|c|c|c|c|c|c|c|c|c|}
\hline $\begin{array}{l}\text { Stream- } \\
\text { flow } \\
\text { study } \\
\text { no. }\end{array}$ & $\begin{array}{l}\text { Latitude of } \\
\text { upstream } \\
\text { end of } \\
\text { subreach }\end{array}$ & $\begin{array}{l}\text { Longitude } \\
\text { of upstream } \\
\text { end of } \\
\text { subreach }\end{array}$ & $\begin{array}{l}\text { Major aquifer } \\
\text { outcrop } \\
\text { intersected } \\
\text { by subreach }\end{array}$ & $\begin{array}{c}\text { Minor aquifer } \\
\text { outcrop } \\
\text { intercepted } \\
\text { by subreach }\end{array}$ & $\begin{array}{l}\text { Gain or } \\
\text { loss }(-) \text { in } \\
\text { subreach } \\
\left(\mathrm{ft}^{3} / \mathrm{s}\right)\end{array}$ & $\begin{array}{l}\text { Length } \\
\text { of subreach } \\
\text { (river mi) }\end{array}$ & $\begin{array}{l}\text { Location of } \\
\text { upstream } \\
\text { end of } \\
\text { subreach } \\
\text { (river mi) }\end{array}$ & $\begin{array}{l}\text { Descriptive location } \\
\text { of upstream end of } \\
\text { selected subreaches }\end{array}$ \\
\hline 248 & $33^{\circ} 41^{\prime} 03^{\prime \prime}$ & $99^{\circ} 35^{\prime} 04^{\prime \prime}$ & -- & -- & -0.51 & 10.3 & 10.3 & \\
\hline 249 & $32^{\circ} 41^{\prime} 50^{\prime \prime}$ & $94^{\circ} 56^{\prime} 34^{\prime \prime}$ & -- & Queen City & .15 & .8 & .8 & FM 1403 \\
\hline 250 & $34^{\circ} 55^{\prime} 27^{\prime \prime}$ & $102^{\circ} 05^{\prime} 56^{\prime \prime}$ & Ogallala & -- & 0 & 2.0 & 0 & $600 \mathrm{ft}$ below dam at Buffalo Lake \\
\hline 250 & $34^{\circ} 56^{\prime} 07^{\prime \prime}$ & $102^{\circ} 04^{\prime} 22^{\prime \prime}$ & Ogallala & -- & -.2 & 1.8 & 2 & \\
\hline 250 & $34^{\circ} 56^{\prime} 42^{\prime \prime}$ & $102^{\circ} 02^{\prime} 45^{\prime \prime}$ & Ogallala & -- & -.36 & 1.2 & 3.8 & \\
\hline 250 & $34^{\circ} 57^{\prime} 19^{\prime \prime}$ & $102^{\circ} 01^{\prime} 44^{\prime \prime}$ & Ogallala & -- & -.2 & 1.6 & 5 & \\
\hline 250 & $34^{\circ} 57^{\prime} 33^{\prime \prime}$ & $102^{\circ} 00^{\prime} 43^{\prime \prime}$ & Ogallala & -- & -.03 & 2.6 & 6.6 & \\
\hline 250 & $34^{\circ} 58^{\prime} 01^{\prime \prime}$ & $101^{\circ} 58^{\prime} 58^{\prime \prime}$ & Ogallala & -- & -.83 & 1.7 & 9.2 & \\
\hline 250 & $34^{\circ} 58^{\prime} 19^{\prime \prime}$ & $101^{\circ} 57^{\prime} 32^{\prime \prime}$ & Ogallala & -- & -2.91 & 7.0 & 10.9 & \\
\hline 251 & $34^{\circ} 55^{\prime} 27^{\prime \prime}$ & $102^{\circ} 05^{\prime} 56^{\prime \prime}$ & Ogallala & -- & -.62 & 9.2 & 0 & $600 \mathrm{ft}$ below dam at Buffalo Lake \\
\hline 251 & $34^{\circ} 58^{\prime} 01^{\prime \prime}$ & $101^{\circ} 58^{\prime} 58^{\prime \prime}$ & Ogallala & -- & -.48 & 1.7 & 9.2 & \\
\hline 251 & $34^{\circ} 58^{\prime} 19^{\prime \prime}$ & $101^{\circ} 57^{\prime} 32^{\prime \prime}$ & Ogallala & -- & -.54 & 1.7 & 10.9 & \\
\hline 251 & $34^{\circ} 57^{\prime} 48^{\prime \prime}$ & $101^{\circ} 56^{\prime} 42^{\prime \prime}$ & Ogallala & -- & -.04 & 3.2 & 12.6 & \\
\hline 251 & $34^{\circ} 57^{\prime} 35^{\prime \prime}$ & $101^{\circ} 54^{\prime} 39^{\prime \prime}$ & Ogallala & -- & -.92 & 2.1 & 15.8 & \\
\hline 252 & $30^{\circ} 48^{\prime} 05^{\prime \prime}$ & $103^{\circ} 49^{\prime} 55^{\prime \prime}$ & Edwards-Trinity Plateau & -- & -14.3 & 4.5 & 11.8 & \\
\hline 252 & $30^{\circ} 51^{\prime} 45^{\prime \prime}$ & $103^{\circ} 49^{\prime} 12^{\prime \prime}$ & $\begin{array}{l}\text { Cenozoic Pecos Alluvium, } \\
\text { Edwards-Trinity Plateau }\end{array}$ & -- & -4.9 & 5.1 & 7.3 & \\
\hline 253 & $30^{\circ} 48^{\prime} 05^{\prime \prime}$ & $103^{\circ} 49^{\prime} 55^{\prime \prime}$ & Edwards-Trinity Plateau & -- & -9.7 & 4.5 & 11.8 & \\
\hline 253 & $30^{\circ} 51^{\prime} 45^{\prime \prime}$ & $103^{\circ} 49^{\prime} 12^{\prime \prime}$ & $\begin{array}{l}\text { Cenozoic Pecos Alluvium, } \\
\text { Edwards-Trinity Plateau }\end{array}$ & -- & -16.9 & 5.1 & 7.3 & \\
\hline 254 & $30^{\circ} 48^{\prime} 05^{\prime \prime}$ & $103^{\circ} 49^{\prime} 55^{\prime \prime}$ & Edwards-Trinity Plateau & -- & -3.2 & 4.5 & 11.8 & \\
\hline 254 & $30^{\circ} 51^{\prime} 45^{\prime \prime}$ & $103^{\circ} 49^{\prime} 12^{\prime \prime}$ & $\begin{array}{l}\text { Cenozoic Pecos Alluvium, } \\
\text { Edwards-Trinity Plateau }\end{array}$ & -- & -10.4 & 5.1 & 7.3 & \\
\hline 255 & $30^{\circ} 48^{\prime} 05^{\prime \prime}$ & $103^{\circ} 49^{\prime} 55^{\prime \prime}$ & Edwards-Trinity Plateau & -- & -4.7 & 2.2 & 11.8 & \\
\hline 255 & $30^{\circ} 49^{\prime} 53^{\prime \prime}$ & $103^{\circ} 50^{\prime} 02^{\prime \prime}$ & Edwards-Trinity Plateau & -- & 3.0 & .1 & 9.6 & \\
\hline 255 & $30^{\circ} 50^{\prime} 01^{\prime \prime}$ & $103^{\circ} 50^{\prime} 02^{\prime \prime}$ & Edwards-Trinity Plateau & -- & 6.3 & 2.2 & 9.5 & \\
\hline 255 & $30^{\circ} 51^{\prime} 45^{\prime \prime}$ & $103^{\circ} 49^{\prime} 12^{\prime \prime}$ & $\begin{array}{l}\text { Cenozoic Pecos Alluvium, } \\
\text { Edwards-Trinity Plateau }\end{array}$ & -- & -20.1 & 2.1 & 7.3 & \\
\hline 255 & $30^{\circ} 53^{\prime} 28^{\prime \prime}$ & $103^{\circ} 49^{\prime} 04^{\prime \prime}$ & Cenozoic Pecos Alluvium & -- & 0 & 2.5 & 5.2 & \\
\hline 255 & $30^{\circ} 55^{\prime} 30^{\prime \prime}$ & $103^{\circ} 48^{\prime} 46^{\prime \prime}$ & Cenozoic Pecos Alluvium & -- & .1 & .1 & 2.7 & \\
\hline 255 & $30^{\circ} 55^{\prime} 37^{\prime \prime}$ & $103^{\circ} 48^{\prime} 46^{\prime \prime}$ & Cenozoic Pecos Alluvium & -- & 0 & .4 & 2.6 & \\
\hline 256 & $31^{\circ} 08^{\prime} 25^{\prime \prime}$ & $103^{\circ} 38^{\prime} 09^{\prime \prime}$ & Cenozoic Pecos Alluvium & -- & -13.5 & 2.0 & 0 & $10 \mathrm{mi}$ above US 290 \\
\hline 257 & $31^{\circ} 09^{\prime} 32^{\prime \prime}$ & $103^{\circ} 37^{\prime} 44^{\prime \prime}$ & Cenozoic Pecos Alluvium & -- & 3.8 & 1.5 & -1.5 & \\
\hline 258 & $31^{\circ} 08^{\prime} 25^{\prime \prime}$ & $103^{\circ} 38^{\prime} 09^{\prime \prime}$ & Cenozoic Pecos Alluvium & -- & -31.2 & 2.5 & 0 & at gaging station (08424500) \\
\hline 259 & $29^{\circ} 41^{\prime} 56^{\prime \prime}$ & $101^{\circ} 00^{\prime} 25^{\prime \prime}$ & Edwards-Trinity Plateau & -- & 85.0 & 20.0 & 0 & at Rubbard Ford \\
\hline 259 & $29^{\circ} 34^{\prime} 12^{\prime \prime}$ & $100^{\circ} 58^{\prime} 48^{\prime \prime}$ & Edwards-Trinity Plateau & -- & 24.0 & 5.0 & 20 & \\
\hline 259 & $29^{\circ} 34^{\prime} 01^{\prime \prime}$ & $100^{\circ} 58^{\prime} 48^{\prime \prime}$ & Edwards-Trinity Plateau & -- & 31.0 & 2.2 & 25 & \\
\hline 260 & $29^{\circ} 39^{\prime} 00^{\prime \prime}$ & $100^{\circ} 55^{\prime} 22^{\prime \prime}$ & Edwards-Trinity Plateau & -- & -2.0 & 5.0 & 20 & at Rough Canyon Dam \\
\hline
\end{tabular}


Table 4. Gains and losses from gain-loss studies in Texas-Continued

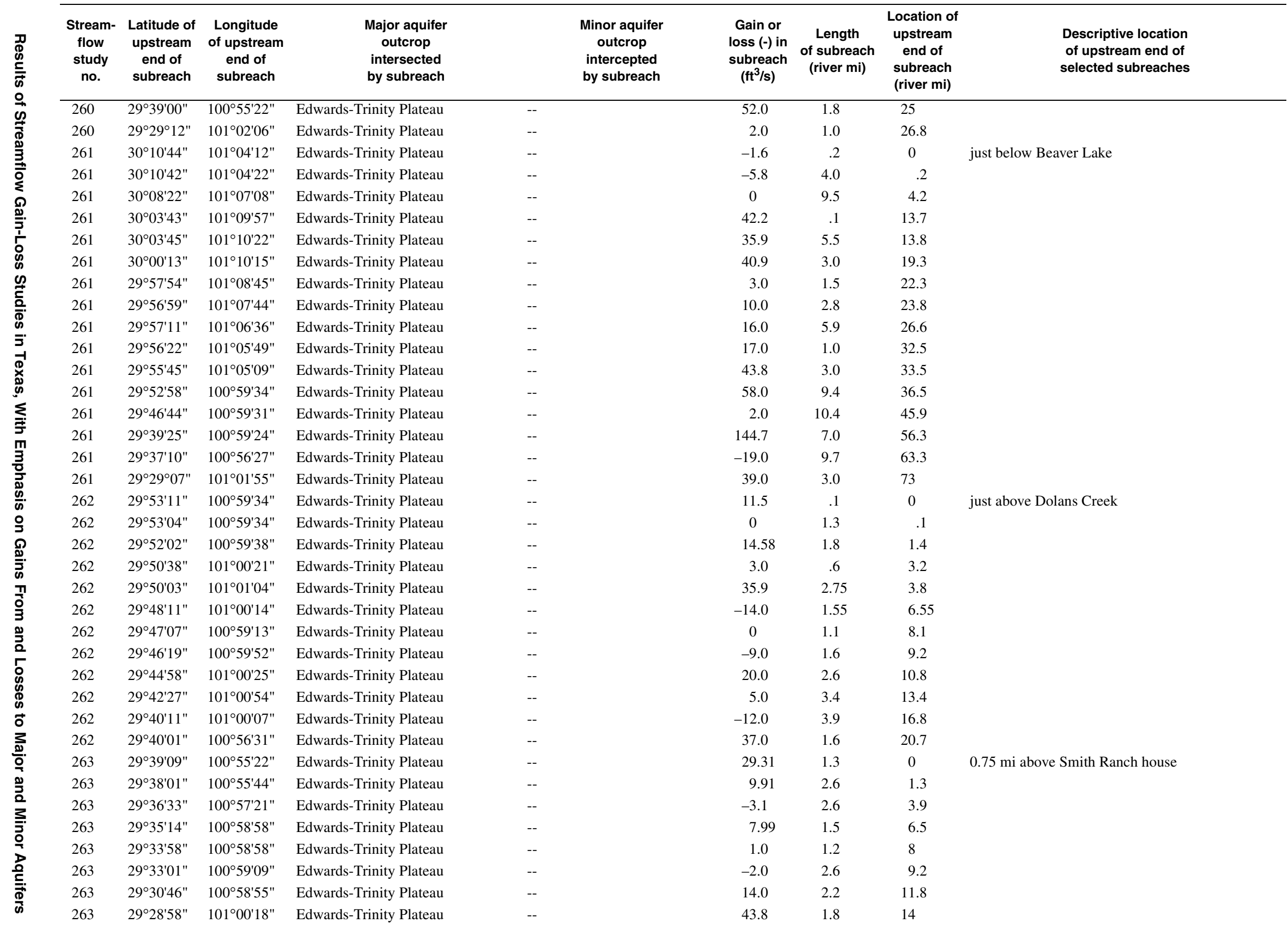


Table 4. Gains and losses from gain-loss studies in Texas-Continued

\begin{tabular}{|c|c|c|c|c|c|c|c|c|}
\hline $\begin{array}{l}\text { Stream- } \\
\text { flow } \\
\text { study } \\
\text { no. }\end{array}$ & $\begin{array}{l}\text { Latitude of } \\
\text { upstream } \\
\text { end of } \\
\text { subreach }\end{array}$ & $\begin{array}{l}\text { Longitude } \\
\text { of upstream } \\
\text { end of } \\
\text { subreach }\end{array}$ & $\begin{array}{l}\text { Major aquifer } \\
\text { outcrop } \\
\text { intersected } \\
\text { by subreach }\end{array}$ & $\begin{array}{c}\text { Minor aquifer } \\
\text { outcrop } \\
\text { intercepted } \\
\text { by subreach }\end{array}$ & $\begin{array}{l}\text { Gain or } \\
\text { loss }(-) \text { in } \\
\text { subreach } \\
\left(\mathrm{ft}^{3} / \mathrm{s}\right)\end{array}$ & $\begin{array}{l}\text { Length } \\
\text { of subreach } \\
\text { (river mi) }\end{array}$ & $\begin{array}{l}\text { Location of } \\
\text { upstream } \\
\text { end of } \\
\text { subreach } \\
\text { (river mi) }\end{array}$ & $\begin{array}{l}\text { Descriptive location } \\
\text { of upstream end of } \\
\text { selected subreaches }\end{array}$ \\
\hline 263 & $29^{\circ} 29^{\circ} 12^{\prime \prime}$ & $101^{\circ} 01^{\prime} 58^{\prime \prime}$ & Edwards-Trinity Plateau & -- & -3.0 & 0.7 & 15.8 & \\
\hline 264 & $30^{\circ} 42^{\prime} 29^{\prime \prime}$ & $103^{\circ} 47^{\prime} 02^{\prime \prime}$ & Edwards-Trinity Plateau & -- & 2.1 & 2.3 & 11.7 & upper end of Wild Rose Canyon \\
\hline 264 & $30^{\circ} 43^{\prime} 00^{\prime \prime}$ & $103^{\circ} 45^{\prime} 57^{\prime \prime}$ & Edwards-Trinity Plateau & -- & -1.4 & 4.2 & 14 & \\
\hline 264 & $30^{\circ} 45^{\prime} 04^{\prime \prime}$ & $103^{\circ} 45^{\prime} 32^{\prime \prime}$ & Edwards-Trinity Plateau & -- & -2.7 & 3.0 & 18.2 & \\
\hline 264 & $30^{\circ} 46^{\prime} 51^{\prime \prime}$ & $103^{\circ} 44^{\prime} 09^{\prime \prime}$ & Edwards-Trinity Plateau & -- & -.9 & 3.0 & 21.2 & \\
\hline 264 & $30^{\circ} 46^{\prime} 23^{\prime \prime}$ & $103^{\circ} 41^{\prime} 49^{\prime \prime}$ & Edwards-Trinity Plateau & -- & 1.3 & 4.0 & 24.2 & \\
\hline 264 & $30^{\circ} 45^{\prime} 17^{\prime \prime}$ & $103^{\circ} 39^{\prime} 03^{\prime \prime}$ & Edwards-Trinity Plateau & -- & -2.4 & .5 & 28.2 & \\
\hline 265 & $30^{\circ} 37^{\prime} 49^{\prime \prime}$ & $104^{\circ} 02^{\prime} 38^{\prime \prime}$ & -- & Igneous & -.1 & .3 & -12.3 & $12.3 \mathrm{mi}$ above old Fort Davis Ln \\
\hline 265 & $30^{\circ} 37^{\prime} 58^{\prime \prime}$ & $104^{\circ} 02^{\prime} 24^{\prime \prime}$ & -- & Igneous & .2 & .7 & -12 & \\
\hline 265 & $30^{\circ} 38^{\prime} 11^{\prime \prime}$ & $104^{\circ} 01^{\prime} 48^{\prime \prime}$ & -- & Igneous & -.2 & .3 & -11.3 & \\
\hline 265 & $30^{\circ} 38^{\prime} 08^{\prime \prime}$ & $104^{\circ} 01^{\prime} 33^{\prime \prime}$ & -- & Igneous & .5 & .7 & -11 & \\
\hline 265 & $30^{\circ} 37^{\prime} 49^{\prime \prime}$ & $104^{\circ} 00^{\prime} 57^{\prime \prime}$ & -- & Igneous & -.5 & .5 & -10.3 & \\
\hline 265 & $30^{\circ} 37^{\prime} 30^{\prime \prime}$ & $104^{\circ} 00^{\prime} 39^{\prime \prime}$ & -- & Igneous & .1 & .1 & -9.8 & \\
\hline 265 & $30^{\circ} 37^{\prime} 25^{\prime \prime}$ & $104^{\circ} 00^{\prime} 39^{\prime \prime}$ & -- & Igneous & .5 & 1.8 & -9.7 & \\
\hline 265 & $30^{\circ} 36^{\prime} 46^{\prime \prime}$ & $103^{\circ} 59^{\prime} 42^{\prime \prime}$ & -- & Igneous & 5.1 & 1.9 & -7.9 & \\
\hline 265 & $30^{\circ} 36^{\prime} 54^{\prime \prime}$ & $103^{\circ} 58^{\prime} 04^{\prime \prime}$ & -- & Igneous & -.5 & 2.3 & -6 & \\
\hline 265 & $30^{\circ} 36^{\prime} 23^{\prime \prime}$ & $103^{\circ} 56^{\prime} 24^{\prime \prime}$ & -- & Igneous & -.4 & 2.4 & -3.7 & \\
\hline 265 & $30^{\circ} 36^{\prime} 08^{\prime \prime}$ & $103^{\circ} 54^{\prime} 50^{\prime \prime}$ & -- & -- & -1.0 & 2.5 & -1.3 & \\
\hline 265 & $30^{\circ} 36^{\prime} 42^{\prime \prime}$ & $103^{\circ} 52^{\prime} 37^{\prime \prime}$ & -- & -- & 3.8 & 3.0 & 1.2 & \\
\hline 265 & $30^{\circ} 38^{\prime} 34^{\prime \prime}$ & $103^{\circ} 50^{\prime} 31^{\prime \prime}$ & -- & -- & .3 & 3.0 & 4.2 & \\
\hline 265 & $30^{\circ} 39^{\prime} 04^{\prime \prime}$ & $103^{\circ} 48^{\prime} 03^{\prime \prime}$ & -- & -- & -.4 & 3.0 & 7.2 & \\
\hline 265 & $30^{\circ} 40^{\prime} 23^{\prime \prime}$ & $103^{\circ} 47^{\prime} 38^{\prime \prime}$ & Edwards-Trinity Plateau & -- & .6 & 1.5 & 10.2 & \\
\hline 265 & $30^{\circ} 42^{\prime} 29^{\prime \prime}$ & $103^{\circ} 47^{\prime} 02^{\prime \prime}$ & Edwards-Trinity Plateau & -- & 3.5 & 2.3 & 11.7 & \\
\hline 265 & $30^{\circ} 43^{\prime} 00^{\prime \prime}$ & $103^{\circ} 45^{\prime} 57^{\prime \prime}$ & Edwards-Trinity Plateau & -- & 1.0 & 4.2 & 14 & \\
\hline 266 & $30^{\circ} 42^{\prime} 41^{\prime \prime}$ & $103^{\circ} 46^{\prime} 19^{\prime \prime}$ & Edwards-Trinity Plateau & -- & 13.2 & 3.5 & 14.7 & 0.8 mi below Wild Rose Canyon \\
\hline 266 & $30^{\circ} 45^{\prime} 04^{\prime \prime}$ & $103^{\circ} 45^{\prime} 32^{\prime \prime}$ & Edwards-Trinity Plateau & -- & -4.2 & 3.0 & 18.2 & \\
\hline 266 & $30^{\circ} 46^{\prime} 52^{\prime \prime}$ & $103^{\circ} 44^{\prime} 06^{\prime \prime}$ & Edwards-Trinity Plateau & -- & 20.6 & 7.0 & 21.2 & \\
\hline 266 & $30^{\circ} 44^{\prime} 22^{\prime \prime}$ & $103^{\circ} 34^{\prime} 58^{\prime \prime}$ & Edwards-Trinity Plateau & -- & -60.9 & 9.0 & 28.2 & \\
\hline 266 & $30^{\circ} 45^{\prime} 37^{\prime \prime}$ & $103^{\circ} 28^{\prime} 19^{\prime \prime}$ & Edwards-Trinity Plateau & -- & -4.2 & 3.0 & 37.2 & \\
\hline 267 & $30^{\circ} 43^{\prime} 20^{\prime \prime}$ & $103^{\circ} 45^{\prime} 50^{\prime \prime}$ & Edwards-Trinity Plateau & -- & -8.6 & 2.4 & 15.8 & $75 \mathrm{ft}$ below mouth of Short Canyon \\
\hline 267 & $30^{\circ} 45^{\prime} 04^{\prime \prime}$ & $103^{\circ} 45^{\prime} 32^{\prime \prime}$ & Edwards-Trinity Plateau & -- & -4.0 & .5 & 18.2 & \\
\hline 268 & $30^{\circ} 45^{\prime} 04^{\prime \prime}$ & $103^{\circ} 45^{\prime} 32^{\prime \prime}$ & Edwards-Trinity Plateau & -- & -2.8 & 3.0 & 18.2 & at Old Limpia post office \\
\hline 268 & $30^{\circ} 46^{\prime} 51^{\prime \prime}$ & $103^{\circ} 44^{\prime} 09^{\prime \prime}$ & Edwards-Trinity Plateau & -- & -.8 & 2.9 & 21.2 & \\
\hline 268 & $30^{\circ} 46^{\prime} 29^{\prime \prime}$ & $103^{\circ} 41^{\prime} 52^{\prime \prime}$ & Edwards-Trinity Plateau & -- & 1.6 & 4.1 & 24.1 & \\
\hline 268 & $30^{\circ} 45^{\prime} 17^{\prime \prime}$ & $103^{\circ} 39^{\prime} 03^{\prime \prime}$ & Edwards-Trinity Plateau & -- & -6.2 & 1.0 & 28.2 & \\
\hline 269 & $30^{\circ} 48^{\prime} 35^{\prime \prime}$ & $103^{\circ} 52^{\prime} 22^{\prime \prime}$ & $\begin{array}{l}\text { Cenozoic Pecos Alluvium, } \\
\text { Edwards-Trinity Plateau }\end{array}$ & -- & -.3 & 5.2 & 11.2 & $200 \mathrm{ft}$ below South Fork \\
\hline
\end{tabular}


Table 4. Gains and losses from gain-loss studies in Texas-Continued

\begin{tabular}{|c|c|c|c|c|c|c|c|c|c|}
\hline $\begin{array}{l}\text { Stream- } \\
\text { flow } \\
\text { study } \\
\text { no. }\end{array}$ & $\begin{array}{l}\text { Latitude of } \\
\text { upstream } \\
\text { end of } \\
\text { subreach }\end{array}$ & $\begin{array}{l}\text { Longitude } \\
\text { of upstream } \\
\text { end of } \\
\text { subreach }\end{array}$ & $\begin{array}{l}\text { Major aquifer } \\
\text { outcrop } \\
\text { intersected } \\
\text { by subreach }\end{array}$ & & $\begin{array}{c}\text { Minor aquifer } \\
\text { outcrop } \\
\text { intercepted } \\
\text { by subreach }\end{array}$ & $\begin{array}{c}\text { Gain or } \\
\text { loss }(-) \text { in } \\
\text { subreach } \\
\left(\mathrm{ft}^{3} / \mathrm{s}\right)\end{array}$ & $\begin{array}{l}\text { Length } \\
\text { of subreach } \\
\text { (river mi) }\end{array}$ & $\begin{array}{l}\text { Location of } \\
\text { upstream } \\
\text { end of } \\
\text { subreach } \\
\text { (river mi) }\end{array}$ & $\begin{array}{l}\text { Descriptive location } \\
\text { of upstream end of } \\
\text { selected subreaches }\end{array}$ \\
\hline 269 & $30^{\circ} 52^{\prime} 15^{\prime \prime}$ & $103^{\circ} 51^{\prime} 28^{\prime \prime}$ & $\begin{array}{l}\text { Cenozoic Pecos Alluvium, } \\
\text { Edwards-Trinity Plateau }\end{array}$ & -- & & -24.5 & 2.1 & 6 & \\
\hline 269 & $30^{\circ} 53^{\prime} 48^{\prime \prime}$ & $103^{\circ} 50^{\prime} 34^{\prime \prime}$ & $\begin{array}{l}\text { Cenozoic Pecos Alluvium, } \\
\text { Edwards-Trinity Plateau }\end{array}$ & -- & & -2.2 & .9 & 3.9 & \\
\hline 270 & $30^{\circ} 48^{\prime} 33^{\prime \prime}$ & $103^{\circ} 52^{\prime} 33^{\prime \prime}$ & Edwards-Trinity Plateau & -- & & -22.8 & 7.0 & 11.3 & $50 \mathrm{ft}$ below South Fork \\
\hline 270 & $30^{\circ} 53^{\prime} 25^{\prime \prime}$ & $103^{\circ} 50^{\prime} 42^{\prime \prime}$ & Edwards-Trinity Plateau & -- & & .1 & .1 & 4.3 & \\
\hline 270 & $30^{\circ} 53^{\prime} 25^{\prime \prime}$ & $103^{\circ} 50^{\prime} 42^{\prime \prime}$ & Edwards-Trinity Plateau & -- & & -.1 & .3 & 4.2 & \\
\hline 271 & $30^{\circ} 48^{\prime} 33^{\prime \prime}$ & $103^{\circ} 52^{\prime} 33^{\prime \prime}$ & Edwards-Trinity Plateau & -- & & -4.1 & 1.8 & 11.3 & $60 \mathrm{ft}$ below South Fork \\
\hline 271 & $30^{\circ} 49^{\prime} 38^{\prime \prime}$ & $103^{\circ} 51^{\prime} 36^{\prime \prime}$ & Edwards-Trinity Plateau & -- & & .6 & .8 & 9.5 & \\
\hline 271 & $30^{\circ} 50^{\prime} 15^{\prime \prime}$ & $103^{\circ} 51^{\prime} 25^{\prime \prime}$ & Edwards-Trinity Plateau & -- & & -.6 & .1 & 8.7 & \\
\hline 271 & $30^{\circ} 50^{\prime} 19^{\prime \prime}$ & $103^{\circ} 51^{\prime} 25^{\prime \prime}$ & Edwards-Trinity Plateau & -- & & .6 & .8 & 8.6 & \\
\hline 271 & $30^{\circ} 50^{\prime} 57^{\prime \prime}$ & $103^{\circ} 51^{\prime} 25^{\prime \prime}$ & Edwards-Trinity Plateau & -- & & -.6 & .1 & 7.8 & \\
\hline 272 & $30^{\circ} 48^{\prime} 33^{\prime \prime}$ & $103^{\circ} 52^{\prime} 33^{\prime \prime}$ & Edwards-Trinity Plateau & -- & & -11.2 & 3.1 & 11.3 & $50 \mathrm{ft}$ below South Fork \\
\hline 273 & $30^{\circ} 48^{\prime} 38^{\prime \prime}$ & $103^{\circ} 53^{\prime} 38^{\prime \prime}$ & $\begin{array}{c}\text { Cenozoic Pecos Alluvium, } \\
\text { Edwards-Trinity Plateau }\end{array}$ & -- & & -18.5 & 10.3 & 12.5 & 3 mi below staff gage \\
\hline 274 & $30^{\circ} 48^{\prime} 45^{\prime \prime}$ & $103^{\circ} 56^{\prime} 24^{\prime \prime}$ & Edwards-Trinity Plateau & -- & & -.3 & 2.8 & 15.5 & \\
\hline 274 & $30^{\circ} 48^{\prime} 37^{\prime \prime}$ & $103^{\circ} 53^{\prime} 52^{\prime \prime}$ & Edwards-Trinity Plateau & -- & & -1.0 & 2.7 & 12.7 & \\
\hline 274 & $30^{\circ} 49^{\prime} 18^{\prime \prime}$ & $103^{\circ} 51^{\prime} 46^{\prime \prime}$ & Edwards-Trinity Plateau & -- & & .2 & 1.5 & 10 & \\
\hline 274 & $30^{\circ} 50^{\prime} 29^{\prime \prime}$ & $103^{\circ} 51^{\prime} 25^{\prime \prime}$ & Edwards-Trinity Plateau & -- & & -.2 & 1.5 & 8.5 & \\
\hline 274 & $30^{\circ} 51^{\prime} 41^{\prime \prime}$ & $103^{\circ} 51^{\prime} 32^{\prime \prime}$ & $\begin{array}{c}\text { Cenozoic Pecos Alluvium, } \\
\text { Edwards-Trinity Plateau }\end{array}$ & -- & & -.2 & 1.1 & 7 & \\
\hline 274 & $30^{\circ} 52^{\prime} 22^{\prime \prime}$ & $103^{\circ} 51^{\prime} 25^{\prime \prime}$ & $\begin{array}{l}\text { Cenozoic Pecos Alluvium, } \\
\text { Edwards-Trinity Plateau }\end{array}$ & -- & & -.1 & 1.6 & 5.9 & \\
\hline 274 & $30^{\circ} 53^{\prime} 25^{\prime \prime}$ & $103^{\circ} 50^{\prime} 42^{\prime \prime}$ & Edwards-Trinity Plateau & -- & & -.1 & .1 & 4.3 & \\
\hline 274 & $30^{\circ} 53^{\prime} 25^{\prime \prime}$ & $103^{\circ} 50^{\prime} 42^{\prime \prime}$ & Edwards-Trinity Plateau & -- & & .2 & .2 & 4.2 & \\
\hline 274 & $30^{\circ} 53^{\prime} 42^{\prime \prime}$ & $103^{\circ} 50^{\prime} 38^{\prime \prime}$ & $\begin{array}{l}\text { Cenozoic Pecos Alluvium, } \\
\text { Edwards-Trinity Plateau }\end{array}$ & -- & & -.2 & 1.8 & 4 & \\
\hline 275 & $30^{\circ} 52^{\prime} 06^{\prime \prime}$ & $103^{\circ} 58^{\prime} 01^{\prime \prime}$ & Edwards-Trinity Plateau & -- & & -25.7 & 6.2 & 13.3 & at gaging station (08424500) \\
\hline 275 & $30^{\circ} 54^{\prime} 26^{\prime \prime}$ & $103^{\circ} 53^{\prime} 20^{\prime \prime}$ & Edwards-Trinity Plateau & -- & & -39.6 & 2.0 & 7.1 & \\
\hline 275 & $30^{\circ} 55^{\prime} 11^{\prime \prime}$ & $103^{\circ} 51^{\prime} 43^{\prime \prime}$ & Edwards-Trinity Plateau & -- & & -1.7 & 1.6 & 5.1 & \\
\hline 276 & $30^{\circ} 52^{\prime} 06^{\prime \prime}$ & $103^{\circ} 58^{\prime} 01^{\prime \prime}$ & Edwards-Trinity Plateau & -- & & -25.7 & 6.2 & 13.3 & at gaging station $(08424500)$ \\
\hline 276 & $30^{\circ} 54^{\prime} 26^{\prime \prime}$ & $103^{\circ} 53^{\prime} 20^{\prime \prime}$ & Edwards-Trinity Plateau & -- & & -7.8 & 2.0 & 7.1 & \\
\hline 276 & $30^{\circ} 55^{\prime} 11^{\prime \prime}$ & $103^{\circ} 51^{\prime} 43^{\prime \prime}$ & Edwards-Trinity Plateau & -- & & -3.8 & 1.6 & 5.1 & \\
\hline 277 & $30^{\circ} 52^{\prime} 06^{\prime \prime}$ & $103^{\circ} 58^{\prime} 01^{\prime \prime}$ & Edwards-Trinity Plateau & -- & & -6.1 & 6.2 & 13.3 & at gaging station $(08424500)$ \\
\hline 277 & $30^{\circ} 54^{\prime} 26^{\prime \prime}$ & $103^{\circ} 53^{\prime} 20^{\prime \prime}$ & Edwards-Trinity Plateau & -- & & -11.4 & 3.6 & 7.1 & \\
\hline 278 & $30^{\circ} 52^{\prime} 06^{\prime \prime}$ & $103^{\circ} 58^{\prime} 01^{\prime \prime}$ & Edwards-Trinity Plateau & -- & & -19.3 & 6.2 & 13.3 & at gaging station (08424500) \\
\hline 278 & $30^{\circ} 54^{\prime} 26^{\prime \prime}$ & $103^{\circ} 53^{\prime} 20^{\prime \prime}$ & Edwards-Trinity Plateau & -- & & -3.9 & 2.0 & 7.1 & \\
\hline 279 & $30^{\circ} 52^{\prime} 06^{\prime \prime}$ & $103^{\circ} 58^{\prime} 01^{\prime \prime}$ & Edwards-Trinity Plateau & -- & & -22.0 & 6.2 & 13.3 & at gaging station $(08424500)$ \\
\hline
\end{tabular}


Table 4. Gains and losses from gain-loss studies in Texas-Continued

\begin{tabular}{|c|c|c|c|c|c|c|c|c|}
\hline $\begin{array}{l}\text { Stream- } \\
\text { flow } \\
\text { study } \\
\text { no. }\end{array}$ & $\begin{array}{l}\text { Latitude of } \\
\text { upstream } \\
\text { end of } \\
\text { subreach }\end{array}$ & $\begin{array}{l}\text { Longitude } \\
\text { of upstream } \\
\text { end of } \\
\text { subreach }\end{array}$ & $\begin{array}{c}\text { Major aquifer } \\
\text { outcrop } \\
\text { intersected } \\
\text { by subreach }\end{array}$ & $\begin{array}{c}\text { Minor aquifer } \\
\text { outcrop } \\
\text { intercepted } \\
\text { by subreach }\end{array}$ & $\begin{array}{c}\text { Gain or } \\
\text { loss }(-) \text { in } \\
\text { subreach } \\
\left(\mathrm{ft}^{3} / \mathrm{s}\right)\end{array}$ & $\begin{array}{l}\text { Length } \\
\text { of subreach } \\
\text { (river mi) }\end{array}$ & $\begin{array}{l}\text { Location of } \\
\text { upstream } \\
\text { end of } \\
\text { subreach } \\
\text { (river mi) }\end{array}$ & $\begin{array}{l}\text { Descriptive location } \\
\text { of upstream end of } \\
\text { selected subreaches }\end{array}$ \\
\hline 279 & $30^{\circ} 54^{\prime} 26^{\prime \prime}$ & $103^{\circ} 53^{\prime} 20^{\prime \prime}$ & Edwards-Trinity Plateau & -- & -4.7 & 2.0 & 7.1 & \\
\hline 280 & $30^{\circ} 52^{\prime} 06^{\prime \prime}$ & $103^{\circ} 58^{\prime} 01^{\prime \prime}$ & Edwards-Trinity Plateau & -- & -14.2 & 6.2 & 13.3 & at gaging station (08424500) \\
\hline 280 & $30^{\circ} 54^{\prime} 26^{\prime \prime}$ & $103^{\circ} 53^{\prime} 20^{\prime \prime}$ & Edwards-Trinity Plateau & -- & -.1 & 2.0 & 7.1 & \\
\hline 281 & $30^{\circ} 52^{\prime} 06^{\prime \prime}$ & $103^{\circ} 58^{\prime} 01^{\prime \prime}$ & Edwards-Trinity Plateau & -- & -5.2 & 5.8 & 13.3 & at gaging station $(08424500)$ \\
\hline 281 & $30^{\circ} 54^{\prime} 24^{\prime \prime}$ & $103^{\circ} 53^{\prime} 45^{\prime \prime}$ & Edwards-Trinity Plateau & -- & .3 & .4 & 7.5 & \\
\hline 281 & $30^{\circ} 54^{\prime} 26^{\prime \prime}$ & $103^{\circ} 53^{\prime} 20^{\prime \prime}$ & Edwards-Trinity Plateau & -- & -.3 & .2 & 7.1 & \\
\hline 281 & $30^{\circ} 54^{\prime} 29^{\prime \prime}$ & $103^{\circ} 53^{\prime} 09^{\prime \prime}$ & Edwards-Trinity Plateau & -- & .6 & 1.8 & 6.9 & \\
\hline 282 & $30^{\circ} 52^{\prime} 06^{\prime \prime}$ & $103^{\circ} 58^{\prime} 01^{\prime \prime}$ & Edwards-Trinity Plateau & -- & -16.2 & 2.6 & 13.3 & at gaging station (08424500) \\
\hline 282 & $30^{\circ} 53^{\prime} 21^{\prime \prime}$ & $103^{\circ} 56^{\prime} 02^{\prime \prime}$ & Edwards-Trinity Plateau & -- & -18.1 & 1.1 & 10.7 & \\
\hline 282 & $30^{\circ} 53^{\prime} 51^{\prime \prime}$ & $103^{\circ} 55^{\prime} 08^{\prime \prime}$ & Edwards-Trinity Plateau & -- & -4.5 & .3 & 9.6 & \\
\hline 283 & $30^{\circ} 52^{\prime} 06^{\prime \prime}$ & $103^{\circ} 58^{\prime} 01^{\prime \prime}$ & Edwards-Trinity Plateau & -- & -.3 & 1.5 & 13.3 & at gaging station (08424500) \\
\hline 283 & $30^{\circ} 52^{\prime} 58^{\prime \prime}$ & $103^{\circ} 57^{\prime} 00^{\prime \prime}$ & Edwards-Trinity Plateau & -- & -5.7 & 1.0 & 11.8 & \\
\hline 283 & $30^{\circ} 53^{\prime} 20^{\prime \prime}$ & $103^{\circ} 56^{\prime} 06^{\prime \prime}$ & Edwards-Trinity Plateau & -- & -.8 & 1.0 & 10.8 & \\
\hline 284 & $31^{\circ} 14^{\prime} 19^{\prime \prime}$ & $102^{\circ} 36^{\prime} 18^{\prime \prime}$ & Cenozoic Pecos Alluvium & -- & -17.2 & 1.0 & -1 & $1.2 \mathrm{mi}$ below headgates \\
\hline 284 & $31^{\circ} 14^{\prime} 02^{\prime \prime}$ & $102^{\circ} 34^{\prime} 55^{\prime \prime}$ & Cenozoic Pecos Alluvium & -- & 12.1 & 1.1 & 0 & \\
\hline 284 & $31^{\circ} 13^{\prime} 55^{\prime \prime}$ & $102^{\circ} 33^{\prime} 54^{\prime \prime}$ & Cenozoic Pecos Alluvium & -- & -.1 & 2.5 & 1.1 & \\
\hline 284 & $31^{\circ} 13^{\prime} 01^{\prime \prime}$ & $102^{\circ} 31^{\prime} 51^{\prime \prime}$ & Cenozoic Pecos Alluvium & -- & -.3 & 2.8 & 3.6 & \\
\hline 285 & $31^{\circ} 14^{\prime} 59^{\prime \prime}$ & $102^{\circ} 37^{\prime} 04^{\prime \prime}$ & Cenozoic Pecos Alluvium & -- & .4 & 2.5 & 0 & at headgates \\
\hline 286 & $31^{\circ} 14^{\prime} 59^{\prime \prime}$ & $102^{\circ} 37^{\prime} 04^{\prime \prime}$ & Cenozoic Pecos Alluvium & -- & 0 & .4 & 0 & at headgates \\
\hline 286 & $31^{\circ} 14^{\prime} 48^{\prime \prime}$ & $102^{\circ} 36^{\prime} 43^{\prime \prime}$ & Cenozoic Pecos Alluvium & -- & 0 & .6 & .4 & \\
\hline 286 & $31^{\circ} 14^{\prime} 29^{\prime \prime}$ & $102^{\circ} 36^{\prime} 18^{\prime \prime}$ & Cenozoic Pecos Alluvium & -- & -.4 & 1.4 & 1 & \\
\hline 287 & $31^{\circ} 14^{\prime} 59^{\prime \prime}$ & $102^{\circ} 37^{\prime} 04^{\prime \prime}$ & Cenozoic Pecos Alluvium & -- & 0 & 1.8 & 0 & at headgates \\
\hline 288 & $31^{\circ} 14^{\prime} 59^{\prime \prime}$ & $102^{\circ} 37^{\prime} 04^{\prime \prime}$ & Cenozoic Pecos Alluvium & -- & -.3 & .1 & 0 & at headgates \\
\hline 288 & $31^{\circ} 14^{\prime} 52^{\prime \prime}$ & $102^{\circ} 36 ' 57^{\prime \prime}$ & Cenozoic Pecos Alluvium & -- & -.7 & 1.4 & .1 & \\
\hline 289 & $31^{\circ} 54^{\prime} 02^{\prime \prime}$ & $103^{\circ} 54^{\prime} 32^{\prime \prime}$ & -- & -- & -7.8 & 22.0 & 0 & near Angeles gaging station (08409500) \\
\hline 289 & $31^{\circ} 49^{\prime} 53^{\prime \prime}$ & $103^{\circ} 48^{\prime} 46^{\prime \prime}$ & Cenozoic Pecos Alluvium & -- & 12.9 & 21.0 & 22 & \\
\hline 289 & $31^{\circ} 44^{\prime} 21^{\prime \prime}$ & $103^{\circ} 44^{\prime} 27^{\prime \prime}$ & Cenozoic Pecos Alluvium & -- & 20.2 & 13.0 & 43 & \\
\hline 289 & $31^{\circ} 40^{\prime} 05^{\prime \prime}$ & $103^{\circ} 37^{\prime} 30^{\prime \prime}$ & Cenozoic Pecos Alluvium & -- & -54.5 & 29.0 & 56 & \\
\hline 289 & $31^{\circ} 26^{\prime} 06^{\prime \prime}$ & $103^{\circ} 27^{\prime} 57^{\prime \prime}$ & Cenozoic Pecos Alluvium & -- & -127.2 & 1.5 & 85 & \\
\hline 289 & $31^{\circ} 25^{\prime} 50^{\prime \prime}$ & $103^{\circ} 25^{\prime} 40^{\prime \prime}$ & Cenozoic Pecos Alluvium & -- & 1.2 & 3.5 & 86.5 & \\
\hline 289 & $31^{\circ} 25^{\prime} 53^{\prime \prime}$ & $103^{\circ} 24^{\prime} 25^{\prime \prime}$ & Cenozoic Pecos Alluvium & -- & 1.2 & .1 & 90 & \\
\hline 289 & $31^{\circ} 25^{\prime} 53^{\prime \prime}$ & $103^{\circ} 24^{\prime} 18^{\prime \prime}$ & Cenozoic Pecos Alluvium & -- & .9 & 11.9 & 90.1 & \\
\hline 289 & $31^{\circ} 25^{\prime} 34^{\prime \prime}$ & $103^{\circ} 23^{\prime} 16^{\prime \prime}$ & Cenozoic Pecos Alluvium & -- & 8.1 & 15.0 & 102 & \\
\hline 289 & $31^{\circ} 25^{\prime} 38^{\prime \prime}$ & $103^{\circ} 15^{\prime} 57^{\prime \prime}$ & -- & -- & -13.1 & 13.0 & 117 & \\
\hline 289 & $31^{\circ} 21^{\prime} 52^{\prime \prime}$ & $103^{\circ} 16^{\prime} 33^{\prime \prime}$ & Cenozoic Pecos Alluvium & -- & 24.5 & 4.0 & 130 & \\
\hline 289 & $31^{\circ} 21^{\prime} 54^{\prime \prime}$ & $103^{\circ} 05^{\prime} 56^{\prime \prime}$ & Cenozoic Pecos Alluvium & -- & -.6 & 3.0 & 134 & \\
\hline 289 & $31^{\circ} 23^{\prime} 02^{\prime \prime}$ & $103^{\circ} 03^{\prime} 18^{\prime \prime}$ & Cenozoic Pecos Alluvium & Capitan Reef Complex & -.8 & 13.0 & 137 & no pumping. \\
\hline 289 & $31^{\circ} 20^{\prime} 17^{\prime \prime}$ & $102^{\circ} 55^{\prime} 37^{\prime \prime}$ & -- & Capitan Reef Complex & 1.2 & 4.0 & 150 & \\
\hline
\end{tabular}


Table 4. Gains and losses from gain-loss studies in Texas-Continued

\begin{tabular}{|c|c|c|c|c|c|c|c|c|}
\hline $\begin{array}{l}\text { Stream- } \\
\text { flow } \\
\text { study } \\
\text { no. }\end{array}$ & $\begin{array}{l}\text { Latitude of } \\
\text { upstream } \\
\text { end of } \\
\text { subreach }\end{array}$ & $\begin{array}{l}\text { Longitude } \\
\text { of upstream } \\
\text { end of } \\
\text { subreach }\end{array}$ & $\begin{array}{l}\text { Major aquifer } \\
\text { outcrop } \\
\text { intersected } \\
\text { by subreach }\end{array}$ & $\begin{array}{c}\text { Minor aquifer } \\
\text { outcrop } \\
\text { intercepted } \\
\text { by subreach }\end{array}$ & $\begin{array}{l}\text { Gain or } \\
\text { loss }(-) \text { in } \\
\text { subreach } \\
\left(\mathrm{ft}^{3} / \mathrm{s}\right)\end{array}$ & $\begin{array}{l}\text { Length } \\
\text { of subreach } \\
\text { (river mi) }\end{array}$ & $\begin{array}{l}\text { Location of } \\
\text { upstream } \\
\text { end of } \\
\text { subreach } \\
\text { (river mi) }\end{array}$ & $\begin{array}{l}\text { Descriptive location } \\
\text { of upstream end of } \\
\text { selected subreaches }\end{array}$ \\
\hline 289 & $31^{\circ} 16^{\prime} 49^{\prime \prime}$ & $102^{\circ} 44^{\prime 2} 20^{\prime \prime}$ & -- & -- & -2.1 & 6.0 & 154 & \\
\hline 289 & $31^{\circ} 18^{\prime} 51^{\prime \prime}$ & $102^{\circ} 39^{\prime} 25^{\prime \prime}$ & Cenozoic Pecos Alluvium & -- & 16.0 & 20.0 & 160 & \\
\hline 289 & $31^{\circ} 12^{\prime} 18^{\prime \prime}$ & $102^{\circ} 26^{\prime} 16^{\prime \prime}$ & Cenozoic Pecos Alluvium & -- & 14.4 & 23.0 & 180 & \\
\hline 290 & $31^{\circ} 06 ' 34^{\prime \prime}$ & $102^{\circ} 24^{\prime} 57^{\prime \prime}$ & Cenozoic Pecos Alluvium & -- & -3.1 & 16.2 & 194.6 & gaging station near Girvin (08446500) \\
\hline 290 & $31^{\circ} 02^{\prime} 04^{\prime \prime}$ & $102^{\circ} 13^{\prime} 12^{\prime \prime}$ & Cenozoic Pecos Alluvium & -- & 1.3 & 8.9 & 178.4 & \\
\hline 290 & $31^{\circ} 00^{\prime} 05^{\prime \prime}$ & $102^{\circ} 07^{\prime} 37^{\prime \prime}$ & $\begin{array}{l}\text { Cenozoic Pecos Alluvium, } \\
\text { Edwards-Trinity Plateau }\end{array}$ & -- & -4.5 & 11.6 & 169.5 & \\
\hline 290 & $30^{\circ} 58^{\prime} 45^{\prime \prime}$ & $101^{\circ} 58^{\prime} 22^{\prime \prime}$ & Edwards-Trinity Plateau & -- & 0 & 9.7 & 157.9 & \\
\hline 290 & $30^{\circ} 54^{\prime} 15^{\prime \prime}$ & $101^{\circ} 52^{\prime} 48^{\prime \prime}$ & Edwards-Trinity Plateau & -- & 7.0 & 10.6 & 148.2 & \\
\hline 290 & $30^{\circ} 47^{\prime} 20^{\prime \prime}$ & $101^{\circ} 50^{\prime} 02^{\prime \prime}$ & Edwards-Trinity Plateau & -- & 5.4 & 11.7 & 137.6 & \\
\hline 290 & $30^{\circ} 39^{\prime} 34^{\prime \prime}$ & $101^{\circ} 46^{\prime} 08^{\prime \prime}$ & Edwards-Trinity Plateau & -- & 4.0 & 9.4 & 125.9 & \\
\hline 290 & $30^{\circ} 37^{\prime} 05^{\prime \prime}$ & $101^{\circ} 39^{\prime} 18^{\prime \prime}$ & Edwards-Trinity Plateau & -- & 2.0 & 5.7 & 116.5 & \\
\hline 290 & $30^{\circ} 32^{\prime} 49^{\prime \prime}$ & $101^{\circ} 39^{\prime} 03^{\prime \prime}$ & Edwards-Trinity Plateau & -- & -3.1 & 2.0 & 110.8 & \\
\hline 290 & $30^{\circ} 31^{\prime} 30^{\prime \prime}$ & $101^{\circ} 40^{\prime} 12^{\prime \prime}$ & Edwards-Trinity Plateau & -- & 17.8 & 9.7 & 108.8 & \\
\hline 290 & $30^{\circ} 26^{\prime} 49^{\prime \prime}$ & $101^{\circ} 43^{\prime} 19^{\prime \prime}$ & Edwards-Trinity Plateau & -- & 18.19 & 7.4 & 99.1 & \\
\hline 290 & $30^{\circ} 22^{\prime} 30^{\prime \prime}$ & $101^{\circ} 41^{\prime} 24^{\prime \prime}$ & Edwards-Trinity Plateau & -- & -8.7 & 10.2 & 91.7 & \\
\hline 290 & $30^{\circ} 18^{\prime} 00^{\prime \prime}$ & $101^{\circ} 44^{\prime} 38^{\prime \prime}$ & Edwards-Trinity Plateau & -- & -7.73 & 20.0 & 81.5 & \\
\hline 290 & $30^{\circ} 07^{\prime} 50^{\prime \prime}$ & $101^{\circ} 34^{\prime} 19^{\prime \prime}$ & Edwards-Trinity Plateau & -- & 33.3 & 4.7 & 61.5 & \\
\hline 290 & $30^{\circ} 07^{\prime} 30^{\prime \prime}$ & $101^{\circ} 30^{\prime} 21^{\prime \prime}$ & Edwards-Trinity Plateau & -- & 17.0 & 13.0 & 56.8 & \\
\hline 290 & $30^{\circ} 00^{\prime} 15^{\prime \prime}$ & $101^{\circ} 30^{\prime} 28^{\prime \prime}$ & Edwards-Trinity Plateau & -- & -10.89 & 17.3 & 43.8 & \\
\hline 290 & $29^{\circ} 53^{\prime} 20^{\prime \prime}$ & $101^{\circ} 27^{\prime} 03^{\prime \prime}$ & Edwards-Trinity Plateau & -- & 3.0 & 11.5 & 26.5 & \\
\hline 290 & $29^{\circ} 48^{\prime} 10^{\prime \prime}$ & $101^{\circ} 26^{\prime} 38^{\prime \prime}$ & Edwards-Trinity Plateau & -- & 4.0 & 14.0 & 15 & \\
\hline 291 & $31^{\circ} 53^{\prime} 57^{\prime \prime}$ & $103^{\circ} 54^{\prime} 18^{\prime \prime}$ & Cenozoic Pecos Alluvium & -- & -143.02 & 43.05 & .35 & gaging station $(08410000)$ \\
\hline 291 & $31^{\circ} 37^{\prime} 57^{\prime \prime}$ & $103^{\circ} 34^{\prime} 19^{\prime \prime}$ & Cenozoic Pecos Alluvium & -- & -8.0 & 9.4 & 43.4 & \\
\hline 291 & $31^{\circ} 35^{\prime} 57^{\prime \prime}$ & $103^{\circ} 30^{\prime} 14^{\prime \prime}$ & Cenozoic Pecos Alluvium & -- & -24.1 & 19.0 & 52.8 & \\
\hline 291 & $31^{\circ} 26^{\prime} 11^{\prime \prime}$ & $103^{\circ} 27^{\prime} 57^{\prime \prime}$ & Cenozoic Pecos Alluvium & -- & 2.52 & 21.8 & 71.8 & \\
\hline 291 & $31^{\circ} 25^{\prime} 01^{\prime \prime}$ & $103^{\circ} 15^{\prime} 25^{\prime \prime}$ & Cenozoic Pecos Alluvium & -- & -4.2 & 12.2 & 93.6 & \\
\hline 291 & $31^{\circ} 21^{\prime} 54^{\prime \prime}$ & $103^{\circ} 06^{\prime} 00^{\prime \prime}$ & Cenozoic Pecos Alluvium & Capitan Reef Complex & -13.6 & 21.6 & 105.8 & \\
\hline 291 & $31^{\circ} 18^{\prime} 20^{\prime \prime}$ & $102^{\circ} 52^{\prime} 30^{\prime \prime}$ & -- & -- & 5.6 & 14.0 & 127.4 & at SH 18 \\
\hline 291 & $31^{\circ} 17^{\prime} 09^{\prime \prime}$ & $102^{\circ} 44^{\prime} 27^{\prime \prime}$ & -- & -- & 2.3 & 8.8 & 141.4 & \\
\hline 291 & $31^{\circ} 18^{\prime} 50^{\prime \prime}$ & $102^{\circ} 39^{\prime} 18^{\prime \prime}$ & Cenozoic Pecos Alluvium & -- & -5.8 & 8.0 & 150.2 & \\
\hline 291 & $31^{\circ} 16^{\prime} 00^{\prime \prime}$ & $102^{\circ} 35^{\prime} 49^{\prime \prime}$ & Cenozoic Pecos Alluvium & -- & 3.5 & 30.2 & 158.2 & \\
\hline 292 & $31^{\circ} 53^{\prime} 57^{\prime \prime}$ & $103^{\circ} 54^{\prime} 18^{\prime \prime}$ & Cenozoic Pecos Alluvium & -- & -2.19 & 13.95 & .35 & gaging station $(08410000)$ \\
\hline 292 & $31^{\circ} 48^{\prime} 14^{\prime \prime}$ & $103^{\circ} 48^{\prime} 25^{\prime \prime}$ & Cenozoic Pecos Alluvium & -- & -.39 & 29.1 & 14.3 & gaging station $(08407500)$ \\
\hline 292 & $31^{\circ} 37^{\prime} 57^{\prime \prime}$ & $103^{\circ} 34^{\prime} 19^{\prime \prime}$ & Cenozoic Pecos Alluvium & -- & 0 & 9.4 & 43.4 & \\
\hline 292 & $31^{\circ} 35^{\prime} 57^{\prime \prime}$ & $103^{\circ} 30^{\prime} 14^{\prime \prime}$ & Cenozoic Pecos Alluvium & -- & 0 & 8.2 & 52.8 & \\
\hline 292 & $31^{\circ} 31^{\circ} 27^{\prime \prime}$ & $103^{\circ} 29^{\prime} 42^{\prime \prime}$ & Cenozoic Pecos Alluvium & -- & 0 & 10.8 & 61 & \\
\hline 292 & $31^{\circ} 26^{\prime} 11^{\prime \prime}$ & $103^{\circ} 27^{\prime} 57^{\prime \prime}$ & Cenozoic Pecos Alluvium & -- & 3.18 & 14.6 & 71.8 & \\
\hline
\end{tabular}


Table 4. Gains and losses from gain-loss studies in Texas-Continued

\begin{tabular}{|c|c|c|c|c|c|c|c|c|}
\hline $\begin{array}{l}\text { Stream- } \\
\text { flow } \\
\text { study } \\
\text { no. }\end{array}$ & $\begin{array}{l}\text { Latitude of } \\
\text { upstream } \\
\text { end of } \\
\text { subreach }\end{array}$ & $\begin{array}{l}\text { Longitude } \\
\text { of upstream } \\
\text { end of } \\
\text { subreach }\end{array}$ & $\begin{array}{c}\text { Major aquifer } \\
\text { outcrop } \\
\text { intersected } \\
\text { by subreach }\end{array}$ & $\begin{array}{l}\text { Minor aquifer } \\
\text { outcrop } \\
\text { intercepted } \\
\text { by subreach }\end{array}$ & $\begin{array}{l}\text { Gain or } \\
\text { loss }(-) \text { in } \\
\text { subreach } \\
\left(\mathrm{ft}^{3} / \mathrm{s}\right)\end{array}$ & $\begin{array}{l}\text { Length } \\
\text { of subreach } \\
\text { (river mi) }\end{array}$ & $\begin{array}{l}\text { Location of } \\
\text { upstream } \\
\text { end of } \\
\text { subreach } \\
\text { (river mi) }\end{array}$ & $\begin{array}{l}\text { Descriptive location } \\
\text { of upstream end of } \\
\text { selected subreaches }\end{array}$ \\
\hline 292 & $31^{\circ} 24^{\prime} 46^{\prime \prime}$ & $103^{\circ} 19^{\prime} 30^{\prime \prime}$ & Cenozoic Pecos Alluvium & -- & -1.19 & 7.2 & 86.4 & \\
\hline 292 & $31^{\circ} 25^{\prime} 01^{\prime \prime}$ & $103^{\circ} 15^{\prime} 25^{\prime \prime}$ & Cenozoic Pecos Alluvium & -- & -1.99 & 12.2 & 93.6 & \\
\hline 292 & $31^{\circ} 21^{\prime} 54^{\prime \prime}$ & $103^{\circ} 06^{\prime} 00^{\prime \prime}$ & Cenozoic Pecos Alluvium & Capitan Reef Complex & .99 & 8.5 & 105.8 & \\
\hline 292 & $31^{\circ} 22^{\prime} 00^{\prime \prime}$ & $103^{\circ} 00^{\prime} 14^{\prime \prime}$ & Cenozoic Pecos Alluvium & Capitan Reef Complex & .6 & 13.1 & 114.3 & \\
\hline 292 & $31^{\circ} 18^{\prime} 20^{\prime \prime}$ & $102^{\circ} 52^{\prime} 30^{\prime \prime}$ & -- & -- & 3.31 & 14.0 & 127.4 & \\
\hline 292 & $31^{\circ} 17^{\prime} 09^{\prime \prime}$ & $102^{\circ} 44^{\prime} 27^{\prime \prime}$ & -- & -- & .54 & 8.8 & 141.4 & \\
\hline 292 & $31^{\circ} 18^{\prime} 50^{\prime \prime}$ & $102^{\circ} 39^{\prime} 18^{\prime \prime}$ & Cenozoic Pecos Alluvium & -- & .31 & 8.0 & 150.2 & \\
\hline 292 & $31^{\circ} 16^{\prime} 00^{\prime \prime}$ & $102^{\circ} 35^{\prime} 49^{\prime \prime}$ & Cenozoic Pecos Alluvium & -- & 5.45 & 15.0 & 158.2 & \\
\hline 292 & $31^{\circ} 12^{\prime} 20^{\prime \prime}$ & $102^{\circ} 27^{\prime} 32^{\prime \prime}$ & Cenozoic Pecos Alluvium & -- & .3 & 15.2 & 173.2 & \\
\hline 293 & $31^{\circ} 52^{\prime} 36^{\prime \prime}$ & $103^{\circ} 52^{\prime} 58^{\prime \prime}$ & Cenozoic Pecos Alluvium & -- & -98.98 & 40.5 & 2.9 & gaging station $(08410000)$ \\
\hline 293 & $31^{\circ} 37^{\prime} 57^{\prime \prime}$ & $103^{\circ} 34^{\prime} 19^{\prime \prime}$ & Cenozoic Pecos Alluvium & -- & -23.5 & 9.4 & 43.4 & \\
\hline 293 & $31^{\circ} 35^{\prime} 57^{\prime \prime}$ & $103^{\circ} 30^{\prime} 14^{\prime \prime}$ & Cenozoic Pecos Alluvium & -- & -34.2 & 8.2 & 52.8 & \\
\hline 293 & $31^{\circ} 31^{\circ} 27^{\prime \prime}$ & $103^{\circ} 29^{\prime} 42^{\prime \prime}$ & Cenozoic Pecos Alluvium & -- & 15.0 & 10.8 & 61 & \\
\hline 293 & $31^{\circ} 26^{\prime} 11^{\prime \prime}$ & $103^{\circ} 27^{\prime} 57^{\prime \prime}$ & Cenozoic Pecos Alluvium & -- & -31.0 & 14.6 & 71.8 & \\
\hline 293 & $31^{\circ} 24^{\prime} 46^{\prime \prime}$ & $103^{\circ} 19^{\prime} 30^{\prime \prime}$ & Cenozoic Pecos Alluvium & -- & -6.01 & 19.4 & 86.4 & \\
\hline 293 & $31^{\circ} 21^{\prime} 54^{\prime \prime}$ & $103^{\circ} 06^{\prime} 00^{\prime \prime}$ & Cenozoic Pecos Alluvium & Capitan Reef Complex & -3.0 & 5.9 & 105.8 & \\
\hline 293 & $31^{\circ} 22^{\prime} 48^{\prime \prime}$ & $103^{\circ} 02^{\prime} 06^{\prime \prime}$ & Cenozoic Pecos Alluvium & Capitan Reef Complex & -15.81 & 15.7 & 111.7 & \\
\hline 293 & $31^{\circ} 18^{\prime} 20^{\prime \prime}$ & $102^{\circ} 52^{\prime} 30^{\prime \prime}$ & -- & -- & 12.21 & 14.0 & 127.4 & \\
\hline 293 & $31^{\circ} 17^{\prime} 09^{\prime \prime}$ & $102^{\circ} 44^{\prime} 27^{\prime \prime}$ & -- & -- & -.1 & 8.8 & 141.4 & \\
\hline 293 & $31^{\circ} 18^{\prime} 50^{\prime \prime}$ & $102^{\circ} 39^{\prime} 18^{\prime \prime}$ & Cenozoic Pecos Alluvium & -- & -3.6 & 8.0 & 150.2 & \\
\hline 293 & $31^{\circ} 16^{\prime} 00^{\prime \prime}$ & $102^{\circ} 35^{\prime} 49^{\prime \prime}$ & Cenozoic Pecos Alluvium & -- & -.3 & 15.0 & 158.2 & \\
\hline 293 & $31^{\circ} 12^{\prime} 20^{\prime \prime}$ & $102^{\circ} 27^{\prime} 32^{\prime \prime}$ & Cenozoic Pecos Alluvium & -- & 0 & 15.2 & 173.2 & \\
\hline 294 & $30^{\circ} 59^{\prime} 39^{\prime \prime}$ & $103^{\circ} 43^{\prime} 19^{\prime \prime}$ & Cenozoic Pecos Alluvium & -- & .23 & 1.1 & 0 & $50 \mathrm{ft}$ below property line of Pacy and Mott tracts \\
\hline 294 & $30^{\circ} 59^{\prime} 21^{\prime \prime}$ & $103^{\circ} 44^{\prime} 13^{\prime \prime}$ & Cenozoic Pecos Alluvium & -- & -.2 & .6 & 1.1 & \\
\hline 294 & $30^{\circ} 59^{\prime} 06^{\prime \prime}$ & $103^{\circ} 44^{\prime} 45^{\prime \prime}$ & Cenozoic Pecos Alluvium & -- & -.03 & .5 & 1.7 & \\
\hline 295 & $30^{\circ} 56^{\prime} 41^{\prime \prime}$ & $103^{\circ} 46^{\prime} 22^{\prime \prime}$ & $\begin{array}{l}\text { Cenozoic Pecos Alluvium, } \\
\text { Edwards-Trinity Plateau }\end{array}$ & -- & -.08 & 1.0 & 0 & at point of diversion \\
\hline 296 & $30^{\circ} 56^{\prime} 42^{\prime \prime}$ & $103^{\circ} 47^{\prime} 24^{\prime \prime}$ & Cenozoic Pecos Alluvium & -- & .26 & .8 & 0 & at weir \\
\hline 297 & $30^{\circ} 58^{\prime} 29^{\prime \prime}$ & $103^{\circ} 45^{\prime} 43^{\prime \prime}$ & Cenozoic Pecos Alluvium & -- & .01 & .5 & 0 & $50 \mathrm{ft}$ below headgate \\
\hline 297 & $30^{\circ} 57^{\prime} 51^{\prime \prime}$ & $103^{\circ} 46^{\prime} 04^{\prime \prime}$ & Cenozoic Pecos Alluvium & -- & -.03 & 1.0 & .5 & \\
\hline 298 & $30^{\circ} 59^{\prime} 31^{\prime \prime}$ & $103^{\circ} 43^{\prime} 55^{\prime \prime}$ & Cenozoic Pecos Alluvium & -- & .07 & .7 & 0 & just below county road crossing \\
\hline 298 & $30^{\circ} 59^{\prime} 15^{\prime \prime}$ & $103^{\circ} 44^{\prime} 20^{\prime \prime}$ & Cenozoic Pecos Alluvium & -- & -.5 & .5 & .7 & \\
\hline 298 & $30^{\circ} 59^{\prime} 08^{\prime \prime}$ & $103^{\circ} 44^{\prime} 38^{\prime \prime}$ & Cenozoic Pecos Alluvium & -- & .58 & .4 & 1.2 & \\
\hline 299 & $30^{\circ} 59^{\prime} 56^{\prime \prime}$ & $103^{\circ} 42^{\prime} 07^{\prime \prime}$ & Cenozoic Pecos Alluvium & -- & .39 & .8 & 0 & at point of diversion \\
\hline 299 & $31^{\circ} 00^{\prime} 05^{\prime \prime}$ & $103^{\circ} 41^{\prime} 52^{\prime \prime}$ & Cenozoic Pecos Alluvium & -- & .04 & .5 & .8 & \\
\hline 300 & $30^{\circ} 59^{\prime} 56^{\prime \prime}$ & $103^{\circ} 42^{\prime} 07^{\prime \prime}$ & Cenozoic Pecos Alluvium & -- & -.41 & .5 & 0 & $50 \mathrm{ft}$ below headgate \\
\hline 300 & $30^{\circ} 59^{\prime} 53^{\prime \prime}$ & $103^{\circ} 42^{\prime} 21^{\prime \prime}$ & Cenozoic Pecos Alluvium & -- & .62 & .1 & .5 & \\
\hline 300 & $30^{\circ} 59^{\prime} 48^{\prime \prime}$ & $103^{\circ} 42^{\prime} 36^{\prime \prime}$ & Cenozoic Pecos Alluvium & -- & -.09 & .1 & .6 & \\
\hline
\end{tabular}


Table 4. Gains and losses from gain-loss studies in Texas-Continued

\begin{tabular}{|c|c|c|c|c|c|c|c|c|}
\hline $\begin{array}{c}\text { Stream- } \\
\text { flow } \\
\text { study } \\
\text { no. }\end{array}$ & $\begin{array}{l}\text { Latitude of } \\
\text { upstream } \\
\text { end of } \\
\text { subreach }\end{array}$ & $\begin{array}{l}\text { Longitude } \\
\text { of upstream } \\
\text { end of } \\
\text { subreach }\end{array}$ & $\begin{array}{l}\text { Major aquifer } \\
\text { outcrop } \\
\text { intersected } \\
\text { by subreach }\end{array}$ & $\begin{array}{c}\text { Minor aquifer } \\
\text { outcrop } \\
\text { intercepted } \\
\text { by subreach }\end{array}$ & $\begin{array}{l}\text { Gain or } \\
\text { loss }(-) \text { in } \\
\text { subreach } \\
\left(\mathrm{ft}^{3} / \mathrm{s}\right)\end{array}$ & $\begin{array}{l}\text { Length } \\
\text { of subreach } \\
\text { (river mi) }\end{array}$ & $\begin{array}{l}\text { Location of } \\
\text { upstream } \\
\text { end of } \\
\text { subreach } \\
\text { (river mi) }\end{array}$ & $\begin{array}{l}\text { Descriptive location } \\
\text { of upstream end of } \\
\text { selected subreaches }\end{array}$ \\
\hline 301 & $30^{\circ} 58^{\prime} 56^{\prime \prime}$ & $103^{\circ} 45^{\prime} 03^{\prime \prime}$ & Cenozoic Pecos Alluvium & -- & -0.96 & 0.2 & 0 & \\
\hline 302 & $30^{\circ} 59^{\prime} 23^{\prime \prime}$ & $103^{\circ} 44^{\prime} 09^{\prime \prime}$ & Cenozoic Pecos Alluvium & -- & .06 & .5 & 0 & $300 \mathrm{ft}$ below dam \\
\hline 303 & $31^{\circ} 00^{\prime} 04^{\prime \prime}$ & $103^{\circ} 42^{\prime} 07^{\prime \prime}$ & Cenozoic Pecos Alluvium & -- & -.18 & .9 & 0 & at US 290 road crossing \\
\hline 303 & $31^{\circ} 00^{\prime} 02^{\prime \prime}$ & $103^{\circ} 41^{\prime} 45^{\prime \prime}$ & Cenozoic Pecos Alluvium & -- & -.02 & 1.3 & .9 & \\
\hline 303 & $31^{\circ} 00^{\prime} 16^{\prime \prime}$ & $103^{\circ} 41^{\prime} 31^{\prime \prime}$ & Cenozoic Pecos Alluvium & -- & -.01 & .7 & 2.2 & \\
\hline 303 & $31^{\circ} 00^{\prime} 23^{\prime \prime}$ & $103^{\circ} 41^{\prime} 13^{\prime \prime}$ & Cenozoic Pecos Alluvium & -- & -.23 & 1.1 & 2.9 & \\
\hline 304 & $31^{\circ} 00^{\prime} 04^{\prime \prime}$ & $103^{\circ} 42^{\prime} 07^{\prime \prime}$ & Cenozoic Pecos Alluvium & -- & -.07 & .4 & 0 & at US 290 road crossing \\
\hline 304 & $31^{\circ} 00^{\prime} 05^{\prime \prime}$ & $103^{\circ} 41^{\prime} 52^{\prime \prime}$ & Cenozoic Pecos Alluvium & -- & -.05 & .5 & .4 & \\
\hline 304 & $31^{\circ} 00^{\prime} 01^{\prime \prime}$ & $103^{\circ} 41^{\prime} 45^{\prime \prime}$ & Cenozoic Pecos Alluvium & -- & -.08 & .8 & .9 & \\
\hline 304 & $31^{\circ} 00^{\prime} 12^{\prime \prime}$ & $103^{\circ} 41^{\prime} 34^{\prime \prime}$ & Cenozoic Pecos Alluvium & -- & 0 & .8 & 1.7 & \\
\hline 304 & $31^{\circ} 00^{\prime} 25^{\prime \prime}$ & $103^{\circ} 41^{\prime} 31^{\prime \prime}$ & Cenozoic Pecos Alluvium & -- & 0 & .4 & 2.5 & \\
\hline 304 & $31^{\circ} 00^{\prime} 23^{\prime \prime}$ & $103^{\circ} 41^{\prime} 13^{\prime \prime}$ & Cenozoic Pecos Alluvium & -- & -.06 & 1.1 & 2.9 & \\
\hline 305 & $30^{\circ} 59^{\prime} 40^{\prime \prime}$ & $103^{\circ} 43^{\prime} 12^{\prime \prime}$ & Cenozoic Pecos Alluvium & -- & -.5 & 1.0 & 0 & at point of diversion \\
\hline 306 & $30^{\circ} 58^{\prime} 47^{\prime \prime}$ & $103^{\circ} 45^{\prime} 25^{\prime \prime}$ & Cenozoic Pecos Alluvium & -- & -.29 & .5 & 0 & $1,000 \mathrm{ft}$ below diversion dam \\
\hline 307 & $30^{\circ} 57^{\prime} 17^{\prime \prime}$ & $103^{\circ} 46^{\prime} 01^{\prime \prime}$ & Cenozoic Pecos Alluvium & -- & -3.13 & 2.3 & 0 & at point of diversion \\
\hline 308 & $31^{\circ} 00^{\prime} 20^{\prime \prime}$ & $103^{\circ} 41^{\prime} 02^{\prime \prime}$ & Cenozoic Pecos Alluvium & -- & 10.9 & 1.1 & .5 & 0.5 mi below San Solomon Springs \\
\hline 308 & $30^{\circ} 59^{\prime} 33^{\prime \prime}$ & $103^{\circ} 43^{\prime} 01^{\prime \prime}$ & Cenozoic Pecos Alluvium & -- & 1.2 & .4 & 1.6 & \\
\hline 308 & $30^{\circ} 59^{\prime} 08^{\prime \prime}$ & $103^{\circ} 42^{\prime} 57^{\prime \prime}$ & Cenozoic Pecos Alluvium & -- & -2.0 & .7 & 2 & \\
\hline 308 & $30^{\circ} 57^{\prime} 14^{\prime \prime}$ & $103^{\circ} 44^{\prime} 49^{\prime \prime}$ & Cenozoic Pecos Alluvium & -- & -4.2 & 1.5 & 2.7 & \\
\hline 309 & $30^{\circ} 59^{\prime} 58^{\prime \prime}$ & $103^{\circ} 42^{\prime} 03^{\prime \prime}$ & Cenozoic Pecos Alluvium & -- & -8.13 & 2.7 & 0 & $400 \mathrm{ft}$ south of Bruce Corner \\
\hline 309 & $30^{\circ} 59^{\prime} 09^{\prime \prime}$ & $103^{\circ} 44^{\prime} 34^{\prime \prime}$ & Cenozoic Pecos Alluvium & -- & -.37 & 2.65 & 2.7 & \\
\hline 309 & $30^{\circ} 57^{\prime} 33^{\prime \prime}$ & $103^{\circ} 46^{\prime} 01^{\prime \prime}$ & Cenozoic Pecos Alluvium & -- & -3.91 & 3.5 & 5.35 & at Scherye Corner \\
\hline 310 & $30^{\circ} 59^{\prime} 58^{\prime \prime}$ & $103^{\circ} 42^{\prime} 00^{\prime \prime}$ & Cenozoic Pecos Alluvium & -- & .47 & 1.9 & 0 & at headgates \\
\hline 310 & $30^{\circ} 59^{\prime} 31^{\prime \prime}$ & $103^{\circ} 43^{\prime} 48^{\prime \prime}$ & Cenozoic Pecos Alluvium & -- & -7.47 & 3.6 & 1.9 & \\
\hline 311 & $30^{\circ} 59^{\prime} 57^{\prime \prime}$ & $103^{\circ} 42^{\prime} 03^{\prime \prime}$ & Cenozoic Pecos Alluvium & -- & -.85 & 1.1 & 0 & San Solomon Springs at outlet \\
\hline 311 & $30^{\circ} 59^{\prime} 41^{\prime \prime}$ & $103^{\circ} 43^{\prime} 04^{\prime \prime}$ & Cenozoic Pecos Alluvium & -- & -1.0 & .4 & 1.1 & \\
\hline 311 & $30^{\circ} 59^{\prime} 37^{\prime \prime}$ & $103^{\circ} 43^{\prime} 30^{\prime \prime}$ & Cenozoic Pecos Alluvium & -- & .34 & 1.6 & 1.5 & \\
\hline 311 & $30^{\circ} 59^{\prime} 02^{\prime \prime}$ & $103^{\circ} 44^{\prime} 52^{\prime \prime}$ & Cenozoic Pecos Alluvium & -- & -.96 & 2.1 & 3.1 & \\
\hline 311 & $30^{\circ} 57^{\prime} 41^{\prime \prime}$ & $103^{\circ} 46^{\prime} 01^{\prime \prime}$ & Cenozoic Pecos Alluvium & -- & -.49 & 1.1 & 5.2 & \\
\hline 312 & $30^{\circ} 59^{\prime} 00^{\prime \prime}$ & $103^{\circ} 44^{\prime} 56^{\prime \prime}$ & Cenozoic Pecos Alluvium & -- & -.2 & 2.9 & 1.8 & $500 \mathrm{ft}$ below Giffin Canal \\
\hline 313 & $30^{\circ} 58^{\prime} 16^{\prime \prime}$ & $103^{\circ} 45^{\prime} 57^{\prime \prime}$ & Cenozoic Pecos Alluvium & -- & -1.61 & 4.0 & 0 & $300 \mathrm{ft}$ below San Solomon Springs \\
\hline 313 & $30^{\circ} 59^{\prime} 43^{\prime \prime}$ & $103^{\circ} 42^{\prime} 57^{\prime \prime}$ & Cenozoic Pecos Alluvium & -- & -3.19 & 3.0 & 4 & \\
\hline 313 & $31^{\circ} 00^{\prime} 04^{\prime \prime}$ & $103^{\circ} 42^{\prime} 07^{\prime \prime}$ & Cenozoic Pecos Alluvium & -- & -1.24 & 1.4 & 7 & \\
\hline 313 & $31^{\circ} 00^{\prime} 21^{\prime \prime}$ & $103^{\circ} 41^{\prime} 06^{\prime \prime}$ & Cenozoic Pecos Alluvium & -- & -.34 & 3.0 & 8.4 & \\
\hline 314 & $30^{\circ} 59^{\prime} 00^{\prime \prime}$ & $103^{\circ} 44^{\prime} 56^{\prime \prime}$ & Cenozoic Pecos Alluvium & -- & -2.5 & 2.9 & 1.8 & $500 \mathrm{ft}$ below Giffin Canal \\
\hline 314 & $30^{\circ} 59^{\prime} 39^{\prime \prime}$ & $103^{\circ} 43^{\prime} 22^{\prime \prime}$ & Cenozoic Pecos Alluvium & -- & .6 & 1.3 & 4.7 & \\
\hline 314 & $30^{\circ} 59^{\prime} 49^{\prime \prime}$ & $103^{\circ} 42^{\prime} 32^{\prime \prime}$ & Cenozoic Pecos Alluvium & -- & -1.2 & 1.1 & 6 & \\
\hline 314 & $30^{\circ} 59^{\prime} 55^{\prime \prime}$ & $103^{\circ} 42^{\prime} 10^{\prime \prime}$ & Cenozoic Pecos Alluvium & -- & 1.0 & 1.0 & 7.1 & \\
\hline
\end{tabular}


Table 4. Gains and losses from gain-loss studies in Texas-Continued

\begin{tabular}{|c|c|c|c|c|c|c|c|c|c|}
\hline $\begin{array}{l}\text { Stream- } \\
\text { flow } \\
\text { study } \\
\text { no. }\end{array}$ & $\begin{array}{l}\text { Latitude of } \\
\text { upstream } \\
\text { end of } \\
\text { subreach }\end{array}$ & $\begin{array}{l}\text { Longitude } \\
\text { of upstream } \\
\text { end of } \\
\text { subreach }\end{array}$ & $\begin{array}{l}\text { Major aquifer } \\
\text { outcrop } \\
\text { intersected } \\
\text { by subreach }\end{array}$ & & $\begin{array}{c}\text { Minor aquifer } \\
\text { outcrop } \\
\text { intercepted } \\
\text { by subreach }\end{array}$ & $\begin{array}{c}\text { Gain or } \\
\text { loss }(-) \text { in } \\
\text { subreach } \\
\left(\mathrm{ft}^{3} / \mathrm{s}\right)\end{array}$ & $\begin{array}{l}\text { Length } \\
\text { of subreach } \\
\text { (river mi) }\end{array}$ & $\begin{array}{l}\text { Location of } \\
\text { upstream } \\
\text { end of } \\
\text { subreach } \\
\text { (river mi) }\end{array}$ & $\begin{array}{l}\text { Descriptive location } \\
\text { of upstream end of } \\
\text { selected subreaches }\end{array}$ \\
\hline 314 & $31^{\circ} 00^{\prime} 03^{\prime \prime}$ & $103^{\circ} 41^{\prime} 49^{\prime \prime}$ & Cenozoic Pecos Alluvium & -- & & -2.1 & 1.6 & 8.1 & \\
\hline 314 & $31^{\circ} 00^{\prime} 21^{\prime \prime}$ & $103^{\circ} 41^{\prime} 09^{\prime \prime}$ & Cenozoic Pecos Alluvium & -- & & .3 & 1.4 & 9.7 & \\
\hline 315 & $30^{\circ} 59^{\prime} 01 "$ & $103^{\circ} 44^{\prime} 56^{\prime \prime}$ & Cenozoic Pecos Alluvium & -- & & 11.2 & 2.9 & 1.8 & $500 \mathrm{ft}$ below Giffin Canal \\
\hline 315 & $30^{\circ} 59^{\prime} 39^{\prime \prime}$ & $103^{\circ} 43^{\prime} 22^{\prime \prime}$ & Cenozoic Pecos Alluvium & -- & & -.4 & 1.6 & 4.7 & \\
\hline 315 & $30^{\circ} 59^{\prime} 51^{\prime \prime}$ & $103^{\circ} 42^{\prime} 28^{\prime \prime}$ & Cenozoic Pecos Alluvium & -- & & 0 & 1.4 & 6.3 & \\
\hline 315 & $31^{\circ} 00^{\prime} 01^{\prime \prime}$ & $103^{\circ} 42^{\prime} 07^{\prime \prime}$ & Cenozoic Pecos Alluvium & -- & & 0 & 1.9 & 7.7 & \\
\hline 315 & $31^{\circ} 00^{\prime} 22^{\prime \prime}$ & $103^{\circ} 41^{\prime} 13^{\prime \prime}$ & Cenozoic Pecos Alluvium & -- & & -4.2 & .5 & 9.6 & \\
\hline 315 & $31^{\circ} 00^{\prime} 15^{\prime \prime}$ & $103^{\circ} 40^{\prime} 37^{\prime \prime}$ & Cenozoic Pecos Alluvium & -- & & .5 & 1.0 & 10.1 & \\
\hline 316 & $30^{\circ} 58^{\prime} 00^{\prime \prime}$ & $103^{\circ} 42^{\prime} 32^{\prime \prime}$ & $\begin{array}{l}\text { Cenozoic Pecos Alluvium, } \\
\text { Edwards-Trinity Plateau }\end{array}$ & -- & & -3.8 & 3.4 & .1 & $0.1 \mathrm{mi}$ below release gate \\
\hline 317 & $30^{\circ} 58^{\prime} 00^{\prime \prime}$ & $103^{\circ} 42^{\prime} 32^{\prime \prime}$ & $\begin{array}{l}\text { Cenozoic Pecos Alluvium, } \\
\text { Edwards-Trinity Plateau }\end{array}$ & -- & & -.9 & .6 & .1 & $0.1 \mathrm{mi}$ below release gate \\
\hline 317 & $30^{\circ} 58^{\prime} 19^{\prime \prime}$ & $103^{\circ} 42^{\prime} 28^{\prime \prime}$ & Cenozoic Pecos Alluvium & -- & & .2 & 1.0 & .7 & \\
\hline 317 & $30^{\circ} 58^{\prime} 50^{\prime \prime}$ & $103^{\circ} 42^{\prime} 36^{\prime \prime}$ & Cenozoic Pecos Alluvium & -- & & -1.3 & .7 & 1.7 & \\
\hline 317 & $30^{\circ} 59^{\prime} 06^{\prime \prime}$ & $103^{\circ} 42^{\prime} 54^{\prime \prime}$ & Cenozoic Pecos Alluvium & -- & & 1.7 & 1.1 & 2.4 & \\
\hline 318 & $30^{\circ} 58^{\prime} 00^{\prime \prime}$ & $103^{\circ} 42^{\prime} 32^{\prime \prime}$ & Cenozoic Pecos Alluvium & -- & & .3 & .3 & .1 & $0.1 \mathrm{mi}$ below release gate \\
\hline 318 & $30^{\circ} 58^{\prime} 22^{\prime \prime}$ & $103^{\circ} 42^{\prime} 39^{\prime \prime}$ & Cenozoic Pecos Alluvium & -- & & -.5 & 2.2 & .4 & \\
\hline 319 & $30^{\circ} 58^{\prime} 07^{\prime \prime}$ & $103^{\circ} 42^{\prime} 36^{\prime \prime}$ & Cenozoic Pecos Alluvium & -- & & -2.6 & 2.9 & .2 & $0.2 \mathrm{mi}$ below release gate \\
\hline 320 & $30^{\circ} 58^{\prime} 08^{\prime \prime}$ & $103^{\circ} 42^{\prime} 36^{\prime \prime}$ & Cenozoic Pecos Alluvium & -- & & -1.2 & 2.7 & .2 & $0.2 \mathrm{mi}$ below release gate \\
\hline 321 & $28^{\circ} 53^{\prime} 40^{\prime \prime}$ & $100^{\circ} 36^{\prime} 03^{\prime \prime}$ & -- & -- & & 30.0 & 16.0 & 481.12 & at Comal, mean discharge for period \\
\hline 321 & $28^{\circ} 42^{\prime} 46^{\prime \prime}$ & $100^{\circ} 30^{\prime} 25^{\prime \prime}$ & -- & -- & & 60.0 & 18.0 & 497.12 & $\begin{array}{l}\text { Eagle Pass IBWC gaging station ( } 08458000) \text {, mean } \\
\text { discharge for period }\end{array}$ \\
\hline 322 & $29^{\circ} 19^{\prime} 41^{\prime \prime}$ & $100^{\circ} 55^{\prime} 48^{\prime \prime}$ & Edwards-Trinity Plateau & -- & & 23.0 & 14.0 & 561.12 & $\begin{array}{l}\text { near Del Rio IBWC gaging station ( } 08451800) \\
\text { mean discharge for period }\end{array}$ \\
\hline 322 & $29^{\circ} 13^{\prime} 41^{\prime \prime}$ & $100^{\circ} 47^{\prime} 09^{\prime \prime}$ & -- & -- & & 113.0 & 29.0 & 575.12 & mean discharge for period \\
\hline 322 & $28^{\circ} 51^{\prime} 22^{\prime \prime}$ & $100^{\circ} 34^{\prime} 48^{\prime \prime}$ & -- & -- & & -20.0 & 21.0 & 604.12 & mean discharge for period \\
\hline 323 & $28^{\circ} 42^{\prime} 46^{\prime \prime}$ & $100^{\circ} 30^{\prime} 25^{\prime \prime}$ & -- & -- & & 55.0 & 18.0 & 497.12 & $\begin{array}{l}\text { Eagle Pass IBWC gaging station ( } 08458000) \text {, mean } \\
\text { discharge for period }\end{array}$ \\
\hline 324 & $28^{\circ} 42^{\prime} 46^{\prime \prime}$ & $100^{\circ} 30^{\prime} 25^{\prime \prime}$ & -- & -- & & 40.0 & 9.0 & 497.12 & $\begin{array}{l}\text { Eagle Pass IBWC gaging station ( } 08458000) \text {, mean } \\
\text { discharge for period }\end{array}$ \\
\hline 324 & $28^{\circ} 36^{\prime} 40^{\prime \prime}$ & $100^{\circ} 26^{\prime} 49^{\prime \prime}$ & -- & -- & & 15.0 & 9.0 & 506.12 & mean discharge for period \\
\hline 325 & $28^{\circ} 42^{\prime} 45^{\prime \prime}$ & $100^{\circ} 30^{\prime} 25^{\prime \prime}$ & -- & -- & & 35.0 & 18.0 & 497.12 & $\begin{array}{l}\text { Eagle Pass IBWC gaging station ( } 08458000) \text {, mean } \\
\text { discharge for period }\end{array}$ \\
\hline 325 & $28^{\circ} 22^{\prime} 44^{\prime \prime}$ & $100^{\circ} 20^{\prime} 06^{\prime \prime}$ & -- & -- & & -10.0 & 69.0 & 515.12 & mean discharge for period \\
\hline 325 & $27^{\circ} 48^{\prime} 08^{\prime \prime}$ & $99^{\circ} 52^{\prime} 36^{\prime \prime}$ & -- & -- & & 60.0 & .25 & 584.12 & mean discharge for period \\
\hline 325 & $27^{\circ} 47^{\prime} 57^{\prime \prime}$ & $99^{\circ} 52^{\prime} 37^{\prime \prime}$ & -- & -- & & -120.0 & 20.75 & 584.37 & mean discharge for period \\
\hline 325 & $27^{\circ} 38^{\prime} 04^{\prime \prime}$ & $99^{\circ} 39^{\prime} 46^{\prime \prime}$ & -- & -- & & 25.0 & 20.0 & 605.12 & mean discharge for period \\
\hline 326 & $28^{\circ} 42^{\prime} 32^{\prime \prime}$ & $100^{\circ} 31^{\prime} 15^{\prime \prime}$ & -- & -- & & -25.0 & 128.0 & 497.12 & $\begin{array}{l}\text { Eagle Pass IBWC gaging station (08458000), mean } \\
\text { discharge for period }\end{array}$ \\
\hline
\end{tabular}


Table 4. Gains and losses from gain-loss studies in Texas-Continued

\begin{tabular}{|c|c|c|c|c|c|c|c|c|}
\hline $\begin{array}{l}\text { Stream- } \\
\text { flow } \\
\text { study } \\
\text { no. }\end{array}$ & $\begin{array}{l}\text { Latitude of } \\
\text { upstream } \\
\text { end of } \\
\text { subreach }\end{array}$ & $\begin{array}{l}\text { Longitude } \\
\text { of upstream } \\
\text { end of } \\
\text { subreach }\end{array}$ & $\begin{array}{c}\text { Major aquifer } \\
\text { outcrop } \\
\text { intersected } \\
\text { by subreach }\end{array}$ & $\begin{array}{c}\text { Minor aquifer } \\
\text { outcrop } \\
\text { intercepted } \\
\text { by subreach }\end{array}$ & $\begin{array}{l}\text { Gain or } \\
\text { loss }(-) \text { in } \\
\text { subreach } \\
\left(\mathrm{ft}^{3} / \mathrm{s}\right)\end{array}$ & $\begin{array}{l}\text { Length } \\
\text { of subreach } \\
\text { (river mi) }\end{array}$ & $\begin{array}{l}\text { Location of } \\
\text { upstream } \\
\text { end of } \\
\text { subreach } \\
\text { (river mi) }\end{array}$ & $\begin{array}{l}\text { Descriptive location } \\
\text { of upstream end of } \\
\text { selected subreaches }\end{array}$ \\
\hline 327 & $28^{\circ} 42^{\prime} 48^{\prime \prime}$ & $100^{\circ} 30^{\prime} 28^{\prime \prime}$ & -- & -- & -25.0 & 87.0 & 497.12 & $\begin{array}{l}\text { Eagle Pass IBWC gaging station ( } 08458000) \text {, mean } \\
\text { discharge for period }\end{array}$ \\
\hline 327 & $27^{\circ} 48^{\prime} 08^{\prime \prime}$ & $99^{\circ} 52^{\prime} 37^{\prime \prime}$ & -- & -- & 60.0 & .25 & 584.12 & mean discharge for period \\
\hline 327 & $27^{\circ} 47^{\prime} 57^{\prime \prime}$ & $99^{\circ} 52^{\prime} 37^{\prime \prime}$ & -- & -- & -50.0 & 12.75 & 584.37 & mean discharge for period \\
\hline 327 & $27^{\circ} 42^{\prime} 23^{\prime \prime}$ & $99^{\circ} 45^{\prime} 03^{\prime \prime}$ & -- & -- & -80.0 & 8.0 & 597.12 & mean discharge for period \\
\hline 327 & $27^{\circ} 38^{\prime} 04^{\prime \prime}$ & $99^{\circ} 39^{\prime} 47^{\prime \prime}$ & -- & -- & 20.0 & 20.0 & 605.12 & mean discharge for period \\
\hline 328 & $28^{\circ} 42^{\prime} 45^{\prime \prime}$ & $100^{\circ} 30^{\prime} 25^{\prime \prime}$ & -- & -- & -161.0 & 11.0 & 497.12 & $\begin{array}{l}\text { Eagle Pass IBWC gaging station (08458000), mean } \\
\text { discharge for period }\end{array}$ \\
\hline 328 & $28^{\circ} 35^{\prime} 26^{\prime \prime}$ & $100^{\circ} 24^{\prime} 43^{\prime \prime}$ & -- & -- & 20.0 & 8.0 & 508.12 & mean discharge for period \\
\hline 328 & $28^{\circ} 22^{\prime} 44^{\prime \prime}$ & $100^{\circ} 20^{\prime} 06^{\prime \prime}$ & -- & -- & 20.0 & 10.0 & 516.12 & mean discharge for period \\
\hline 328 & $28^{\circ} 21^{\prime} 34^{\prime \prime}$ & $100^{\circ} 19^{\prime} 04^{\prime \prime}$ & -- & -- & -30.0 & 12.0 & 526.12 & mean discharge for period \\
\hline 328 & $28^{\circ} 18^{\prime} 45^{\prime \prime}$ & $100^{\circ} 17^{\prime} 06^{\prime \prime}$ & Carrizo-Wilcox & -- & -100.0 & 14.0 & 538.12 & mean discharge for period \\
\hline 328 & $28^{\circ} 09^{\prime} 04^{\prime \prime}$ & $100^{\circ} 06^{\prime} 03^{\prime \prime}$ & Carrizo-Wilcox & -- & 120.0 & 12.0 & 552.12 & mean discharge for period \\
\hline 328 & $27^{\circ} 59^{\prime} 22^{\prime \prime}$ & $99^{\circ} 58^{\prime} 47^{\prime \prime}$ & -- & -- & -110.0 & 10.0 & 564.12 & mean discharge for period \\
\hline 328 & $27^{\circ} 55^{\prime} 27^{\prime \prime}$ & $99^{\circ} 55^{\prime} 24^{\prime \prime}$ & -- & -- & 90.0 & 12.0 & 574.12 & mean discharge for period \\
\hline 328 & $27^{\circ} 48^{\prime} 03^{\prime \prime}$ & $99^{\circ} 52^{\prime} 40^{\prime \prime}$ & -- & -- & -70.0 & 10.0 & 586.12 & mean discharge for period \\
\hline 328 & $27^{\circ} 43^{\prime} 49^{\prime \prime}$ & $99^{\circ} 47^{\prime} 11^{\prime \prime}$ & -- & -- & 15.0 & 12.0 & 596.12 & mean discharge for period \\
\hline 328 & $27^{\circ} 38^{\prime} 04^{\prime \prime}$ & $99^{\circ} 39^{\prime} 45^{\prime \prime}$ & -- & -- & -120.0 & 5.5 & 608.12 & mean discharge for period \\
\hline 328 & $27^{\circ} 37^{\prime} 35^{\prime \prime}$ & $99^{\circ} 35^{\prime} 23^{\prime \prime}$ & -- & -- & -25.0 & 11.0 & 613.62 & mean discharge for period \\
\hline 328 & $27^{\circ} 32^{\prime} 41^{\prime \prime}$ & $99^{\circ} 31^{\prime} 15^{\prime \prime}$ & -- & -- & 5.0 & 12.0 & 624.62 & mean discharge for period \\
\hline 328 & $27^{\circ} 19^{\prime} 24^{\prime \prime}$ & $99^{\circ} 31^{\prime} 32^{\prime \prime}$ & -- & -- & 40.0 & 7.0 & 636.62 & mean discharge for period \\
\hline 328 & $27^{\circ} 12^{\prime} 58^{\prime \prime}$ & $99^{\circ} 26^{\prime} 22^{\prime \prime}$ & -- & -- & -30.0 & 11.0 & 643.62 & mean discharge for period \\
\hline 328 & $27^{\circ} 09^{\prime} 22^{\prime \prime}$ & $99^{\circ} 26^{\prime} 01^{\prime \prime}$ & -- & -- & 0 & 10.0 & 654.62 & mean discharge for period \\
\hline 329 & $32^{\circ} 00^{\prime} 15^{\prime \prime}$ & $106^{\circ} 39^{\prime} 07^{\prime \prime}$ & Hueco-Mesilla Bolson & -- & -2.1 & 1.24 & 0 & at headgate on main canal \\
\hline 329 & $31^{\circ} 59^{\prime} 06^{\prime \prime}$ & $106^{\circ} 38^{\prime} 20^{\prime \prime}$ & Hueco-Mesilla Bolson & -- & -1.0 & 1.27 & 1.24 & \\
\hline 330 & $31^{\circ} 41^{\prime} 17^{\prime \prime}$ & $106^{\circ} 19^{\prime} 08^{\prime \prime}$ & Hueco-Mesilla Bolson & -- & -.4 & 1.0 & 0 & at US 80 in El Paso \\
\hline 330 & $31^{\circ} 40^{\prime} 35^{\prime \prime}$ & $106^{\circ} 18^{\prime} 32^{\prime \prime}$ & Hueco-Mesilla Bolson & -- & -.2 & 1.0 & 1 & \\
\hline 331 & $31^{\circ} 38^{\prime} 12^{\prime \prime}$ & $106^{\circ} 17^{\prime} 06^{\prime \prime}$ & Hueco-Mesilla Bolson & -- & .4 & 1.2 & 0 & $1.2 \mathrm{mi}$ above Passmore $\mathrm{Rd}$ \\
\hline 331 & $31^{\circ} 37^{\prime} 18^{\prime \prime}$ & $106^{\circ} 16^{\prime} 30^{\prime \prime}$ & Hueco-Mesilla Bolson & -- & 1.9 & 1.2 & 1.2 & \\
\hline 332 & $31^{\circ} 58^{\prime} 04^{\prime \prime}$ & $106^{\circ} 38^{\prime} 20^{\prime \prime}$ & Hueco-Mesilla Bolson & -- & 1.24 & .9 & 0 & at bridge on private road $0.9 \mathrm{mi}$ above FM 273 \\
\hline 332 & $31^{\circ} 57^{\prime} 21^{\prime \prime}$ & $106^{\circ} 38^{\prime} 09^{\prime \prime}$ & Hueco-Mesilla Bolson & -- & .57 & 1.1 & .9 & \\
\hline 333 & $32^{\circ} 00^{\prime} 15^{\prime \prime}$ & $106^{\circ} 39^{\prime} 10^{\prime \prime}$ & Hueco-Mesilla Bolson & -- & -5.0 & 1.38 & 0 & at headgate on main canal \\
\hline 333 & $31^{\circ} 59^{\prime} 09^{\prime \prime}$ & $106^{\circ} 38^{\prime} 52^{\prime \prime}$ & Hueco-Mesilla Bolson & -- & -1.21 & 1.32 & 1.38 & \\
\hline 334 & $31^{\circ} 58^{\prime} 14^{\prime \prime}$ & $106^{\circ} 39^{\prime} 36^{\prime \prime}$ & Hueco-Mesilla Bolson & -- & 2.1 & 1.3 & 0 & at private bridge $1.4 \mathrm{mi}$ north of old La Union \\
\hline 334 & $31^{\circ} 57^{\prime} 06^{\prime \prime}$ & $106^{\circ} 39^{\prime} 21^{\prime \prime}$ & Hueco-Mesilla Bolson & -- & 2.9 & .5 & 1.3 & \\
\hline 335 & $29^{\circ} 15^{\prime} 20^{\prime \prime}$ & $103^{\circ} 46^{\prime} 30^{\prime \prime}$ & -- & -- & -20.0 & 17.3 & 279.92 & at Lajitas, mean discharge for period \\
\hline 335 & $29^{\circ} 09^{\prime} 34^{\prime \prime}$ & $103^{\circ} 36^{\prime} 18^{\prime \prime}$ & -- & -- & 0 & 43.2 & 297.22 & mean discharge for period \\
\hline 335 & $28^{\circ} 59^{\prime} 05^{\prime \prime}$ & $103^{\circ} 11^{\prime} 52^{\prime \prime}$ & -- & -- & 50.0 & 19.0 & 340.42 & mean discharge for period \\
\hline
\end{tabular}


Table 4. Gains and losses from gain-loss studies in Texas-Continued

\begin{tabular}{|c|c|c|c|c|c|c|c|c|}
\hline $\begin{array}{l}\text { Stream- } \\
\text { flow } \\
\text { study } \\
\text { no. }\end{array}$ & $\begin{array}{l}\text { Latitude of } \\
\text { upstream } \\
\text { end of } \\
\text { subreach }\end{array}$ & $\begin{array}{l}\text { Longitude } \\
\text { of upstream } \\
\text { end of } \\
\text { subreach }\end{array}$ & $\begin{array}{c}\text { Major aquifer } \\
\text { outcrop } \\
\text { intersected } \\
\text { by subreach }\end{array}$ & $\begin{array}{c}\text { Minor aquifer } \\
\text { outcrop } \\
\text { intercepted } \\
\text { by subreach }\end{array}$ & $\begin{array}{c}\text { Gain or } \\
\text { loss }(-) \text { in } \\
\text { subreach } \\
\left(\mathrm{ft}^{3} / \mathrm{s}\right)\end{array}$ & $\begin{array}{l}\text { Length } \\
\text { of subreach } \\
\text { (river mi) }\end{array}$ & $\begin{array}{l}\text { Location of } \\
\text { upstream } \\
\text { end of } \\
\text { subreach } \\
\text { (river mi) }\end{array}$ & $\begin{array}{l}\text { Descriptive location } \\
\text { of upstream end of } \\
\text { selected subreaches }\end{array}$ \\
\hline 335 & $29^{\circ} 11^{\prime} 03^{\prime \prime}$ & $102^{\circ} 58^{\prime} 55^{\prime \prime}$ & -- & -- & 30.0 & 14.5 & 359.42 & mean discharge for period \\
\hline 335 & $29^{\circ} 16^{\prime} 54^{\prime \prime}$ & $102^{\circ} 53^{\prime} 45^{\prime \prime}$ & -- & -- & 100.0 & 24.9 & 373.92 & mean discharge for period \\
\hline 335 & $29^{\circ} 32^{\prime} 11^{\prime \prime}$ & $102^{\circ} 47^{\prime} 27^{\prime \prime}$ & Edwards-Trinity Plateau & -- & 220.0 & 100.9 & 398.82 & mean discharge for period \\
\hline 335 & $29^{\circ} 47^{\prime} 22^{\prime \prime}$ & $101^{\circ} 34^{\prime} 22^{\prime \prime}$ & Edwards-Trinity Plateau & -- & 403.0 & 73.3 & 499.72 & mean discharge for period \\
\hline 336 & $31^{\circ} 00^{\prime} 44^{\prime \prime}$ & $103^{\circ} 42^{\prime} 46^{\prime \prime}$ & Cenozoic Pecos Alluvium & -- & 5.3 & 3.8 & 1 & at Aloma settlement \\
\hline 336 & $30^{\circ} 59^{\prime} 04^{\prime \prime}$ & $103^{\circ} 45^{\prime} 03^{\prime \prime}$ & Cenozoic Pecos Alluvium & -- & 1.6 & 1.0 & 4.8 & \\
\hline 336 & $30^{\circ} 59^{\prime} 01^{\prime \prime}$ & $103^{\circ} 46^{\prime} 04^{\prime \prime}$ & Cenozoic Pecos Alluvium & -- & -1.7 & 3.0 & 5.8 & \\
\hline 337 & $30^{\circ} 59^{\prime} 59^{\prime \prime}$ & $103^{\circ} 43^{\prime} 48^{\prime \prime}$ & Cenozoic Pecos Alluvium & -- & 4.3 & 1.8 & 3 & $1.8 \mathrm{mi}$ above Balmorhea bridge \\
\hline 337 & $30^{\circ} 59^{\prime} 04^{\prime \prime}$ & $103^{\circ} 45^{\prime} 03^{\prime \prime}$ & Cenozoic Pecos Alluvium & -- & .3 & 1.0 & 4.8 & \\
\hline 337 & $30^{\circ} 59^{\prime} 01^{\prime \prime}$ & $103^{\circ} 46^{\prime} 04^{\prime \prime}$ & Cenozoic Pecos Alluvium & -- & -2.3 & 3.0 & 5.8 & \\
\hline 338 & $31^{\circ} 00^{\prime} 17^{\prime \prime}$ & $103^{\circ} 43^{\prime} 26^{\prime \prime}$ & Cenozoic Pecos Alluvium & -- & -2.5 & 4.2 & -1.2 & at US 290 crossing \\
\hline 338 & $30^{\circ} 59^{\prime} 59^{\prime \prime}$ & $103^{\circ} 43^{\prime} 48^{\prime \prime}$ & Cenozoic Pecos Alluvium & -- & 2.7 & 1.8 & 3 & \\
\hline 338 & $30^{\circ} 59^{\prime} 04^{\prime \prime}$ & $103^{\circ} 45^{\prime} 03^{\prime \prime}$ & Cenozoic Pecos Alluvium & -- & .7 & 1.0 & 4.8 & \\
\hline 338 & $30^{\circ} 59^{\prime} 01 "$ & $103^{\circ} 46^{\prime} 04^{\prime \prime}$ & Cenozoic Pecos Alluvium & -- & 1.4 & 3.0 & 5.8 & \\
\hline 339 & $30^{\circ} 59^{\prime} 59^{\prime \prime}$ & $103^{\circ} 43^{\prime} 48^{\prime \prime}$ & Cenozoic Pecos Alluvium & -- & 2.8 & 1.8 & 3 & $1.8 \mathrm{mi}$ above Balmorhea bridge \\
\hline 339 & $30^{\circ} 59^{\prime} 04^{\prime \prime}$ & $103^{\circ} 45^{\prime} 03^{\prime \prime}$ & Cenozoic Pecos Alluvium & -- & -.3 & 1.0 & 4.8 & \\
\hline 339 & $30^{\circ} 59^{\prime} 01^{\prime \prime}$ & $103^{\circ} 46^{\prime} 01^{\prime \prime}$ & Cenozoic Pecos Alluvium & -- & 2.4 & 3.0 & 5.8 & \\
\hline 340 & $30^{\circ} 59^{\prime} 59^{\prime \prime}$ & $103^{\circ} 43^{\prime} 48^{\prime \prime}$ & Cenozoic Pecos Alluvium & -- & 2.7 & 1.8 & 3 & $1.8 \mathrm{mi}$ below Balmorhea bridge \\
\hline 340 & $30^{\circ} 59^{\prime} 04^{\prime \prime}$ & $103^{\circ} 45^{\prime} 03^{\prime \prime}$ & Cenozoic Pecos Alluvium & -- & .3 & 1.0 & 4.8 & \\
\hline 340 & $30^{\circ} 59^{\prime} 01^{\prime \prime}$ & $103^{\circ} 46^{\prime} 01^{\prime \prime}$ & Cenozoic Pecos Alluvium & -- & 1.9 & 3.0 & 5.8 & \\
\hline 341 & $30^{\circ} 59^{\prime} 24^{\prime \prime}$ & $103^{\circ} 43^{\prime} 44^{\prime \prime}$ & Cenozoic Pecos Alluvium & -- & .4 & .07 & 0 & $300 \mathrm{ft}$ above springs \\
\hline 341 & $30^{\circ} 59^{\prime} 24^{\prime \prime}$ & $103^{\circ} 43^{\prime} 48^{\prime \prime}$ & Cenozoic Pecos Alluvium & -- & 1.5 & .69 & .07 & \\
\hline 342 & $32^{\circ} 48^{\prime} 11^{\prime \prime}$ & $95^{\circ} 32^{\prime} 04^{\prime \prime}$ & Carrizo-Wilcox & Queen City & 1.5 & .8 & 1.6 & SH 182 \\
\hline 342 & $32^{\circ} 42^{\prime} 06^{\prime \prime}$ & $95^{\circ} 24^{\prime} 26^{\prime \prime}$ & -- & Queen City & -4.1 & .8 & .8 & \\
\hline 343 & $30^{\circ} 18^{\prime} 12^{\prime \prime}$ & $93^{\circ} 44^{\prime} 36^{\prime \prime}$ & Gulf Coast & -- & 27.0 & 5.3 & 22.6 & gaging station $(08030500)$ \\
\hline 344 & $30^{\circ} 18^{\prime} 12^{\prime \prime}$ & $93^{\circ} 44^{\prime} 36^{\prime \prime}$ & Gulf Coast & -- & -16.0 & 0 & 22.6 & gaging station (08030500) \\
\hline 344 & $30^{\circ} 18^{\prime} 12^{\prime \prime}$ & $93^{\circ} 44^{\prime} 36^{\prime \prime}$ & Gulf Coast & -- & -190.0 & 5.3 & 22.6 & \\
\hline 345 & $32^{\circ} 36^{\prime} 04^{\prime \prime}$ & $95^{\circ} 23 \prime 30^{\prime \prime}$ & -- & Queen City & 28.2 & 21.2 & 448.6 & discontinued gaging station (08018600) \\
\hline 345 & $32^{\circ} 34^{\prime} 19^{\prime \prime}$ & $95^{\circ} 12^{\prime} 19^{\prime \prime}$ & -- & Queen City & 43.6 & 42.8 & 427.4 & gaging station $(08019200)$ \\
\hline 345 & $32^{\circ} 28^{\prime} 34^{\prime \prime}$ & $94^{\circ} 51^{\prime} 03^{\prime \prime}$ & -- & Queen City & 13.0 & 5.6 & 384.6 & \\
\hline 345 & $32^{\circ} 27^{\prime} 37^{\prime \prime}$ & $94^{\circ} 48^{\prime} 50^{\prime \prime}$ & -- & -- & 19.0 & 6.2 & 379 & \\
\hline 345 & $32^{\circ} 28^{\prime} 02^{\prime \prime}$ & $94^{\circ} 46^{\prime} 51^{\prime \prime}$ & -- & -- & -32.0 & 1.8 & 372.8 & gaging station $(08020500)$ \\
\hline 345 & $32^{\circ} 26^{\prime} 12^{\prime \prime}$ & $94^{\circ} 46^{\prime} 45^{\prime \prime}$ & -- & -- & 12.0 & 8.4 & 371 & \\
\hline 345 & $32^{\circ} 25^{\prime} 00^{\prime \prime}$ & $94^{\circ} 42^{\prime} 33^{\prime \prime}$ & Carrizo-Wilcox & -- & 20.0 & 23.2 & 362.6 & \\
\hline 345 & $32^{\circ} 22^{\prime} 11^{\prime \prime}$ & $94^{\circ} 27^{\prime} 28^{\prime \prime}$ & Carrizo-Wilcox & -- & 22.0 & 36.0 & 339.4 & gaging station $(080220000$ \\
\hline 345 & $32^{\circ} 13^{\prime} 28^{\prime \prime}$ & $94^{\circ} 13 ' 31^{\prime \prime}$ & Carrizo-Wilcox & -- & 2.0 & 11.2 & 303.4 & \\
\hline 346 & $32^{\circ} 46^{\prime} 24^{\prime \prime}$ & $95^{\circ} 47^{\prime} 56^{\prime \prime}$ & Carrizo-Wilcox & -- & -1.1 & 19.5 & 500.7 & discontinued gaging station (08017500) \\
\hline 346 & $32^{\circ} 43^{\prime} 12^{\prime \prime}$ & $95^{\circ} 38^{\prime} 04^{\prime \prime}$ & Carrizo-Wilcox & -- & 2.82 & 9.3 & 481.2 & discontinued gaging station (08018000) \\
\hline
\end{tabular}


$\vec{\infty} \quad$ Table 4. Gains and losses from gain-loss studies in Texas-Continued

\begin{tabular}{|c|c|c|c|c|c|c|c|c|}
\hline $\begin{array}{l}\text { Stream- } \\
\text { flow } \\
\text { study } \\
\text { no. }\end{array}$ & $\begin{array}{l}\text { Latitude of } \\
\text { upstream } \\
\text { end of } \\
\text { subreach }\end{array}$ & $\begin{array}{l}\text { Longitude } \\
\text { of upstream } \\
\text { end of } \\
\text { subreach }\end{array}$ & $\begin{array}{c}\text { Major aquifer } \\
\text { outcrop } \\
\text { intersected } \\
\text { by subreach }\end{array}$ & $\begin{array}{c}\text { Minor aquifer } \\
\text { outcrop } \\
\text { intercepted } \\
\text { by subreach }\end{array}$ & $\begin{array}{c}\text { Gain or } \\
\text { loss }(-) \text { in } \\
\text { subreach } \\
\left(\mathrm{ft}^{3} / \mathrm{s}\right)\end{array}$ & $\begin{array}{l}\text { Length } \\
\text { of subreach } \\
\text { (river mi) }\end{array}$ & $\begin{array}{l}\text { Location of } \\
\text { upstream } \\
\text { end of } \\
\text { subreach } \\
\text { (river mi) }\end{array}$ & $\begin{array}{l}\text { Descriptive location } \\
\text { of upstream end of } \\
\text { selected subreaches }\end{array}$ \\
\hline 346 & $32^{\circ} 40^{\prime} 22^{\prime \prime}$ & $95^{\circ} 34^{\prime} 17^{\prime \prime}$ & -- & Queen City & 37.3 & 23.3 & 471.9 & discontinued gaging station (08018300) \\
\hline 346 & $32^{\circ} 36^{\prime} 04^{\prime \prime}$ & $95^{\circ} 23^{\prime} 30^{\prime \prime}$ & -- & Queen City & -23.3 & 21.2 & 448.6 & discontinued gaging station (08018600) \\
\hline 346 & $32^{\circ} 34^{\prime} 19^{\prime \prime}$ & $95^{\circ} 12^{\prime} 19^{\prime \prime}$ & -- & Queen City & 411.7 & 7.2 & 427.4 & gaging station (08019200) \\
\hline 347 & $32^{\circ} 13^{\prime} 27^{\prime \prime}$ & $94^{\circ} 13 ' 31^{\prime \prime}$ & Carrizo-Wilcox & -- & 1.77 & 12.0 & 308 & at bridge on US 79 \\
\hline 347 & $32^{\circ} 07^{\prime} 29^{\prime \prime}$ & $94^{\circ} 12^{\prime} 04^{\prime \prime}$ & Carrizo-Wilcox & -- & 3.42 & 22.0 & 296 & \\
\hline 347 & $31^{\circ} 59^{\prime} 46^{\prime \prime}$ & $94^{\circ} 04^{\prime} 26^{\prime \prime}$ & Carrizo-Wilcox & -- & .03 & 6.0 & 274 & \\
\hline 347 & $31^{\circ} 58^{\prime} 35^{\prime \prime}$ & $94^{\circ} 00^{\prime} 43^{\prime \prime}$ & Carrizo-Wilcox & -- & 3.9 & 7.0 & 268 & \\
\hline 347 & $31^{\circ} 55^{\prime} 59^{\prime \prime}$ & $93^{\circ} 58^{\prime} 48^{\prime \prime}$ & Carrizo-Wilcox & -- & 1.7 & 5.0 & 261 & \\
\hline 347 & $31^{\circ} 54^{\prime} 05^{\prime \prime}$ & $93^{\circ} 57^{\prime} 33^{\prime \prime}$ & Carrizo-Wilcox & -- & 2.8 & 12.0 & 256 & \\
\hline 347 & $31^{\circ} 50^{\prime} 58^{\prime \prime}$ & $93^{\circ} 55^{\prime} 17^{\prime \prime}$ & Carrizo-Wilcox & -- & .87 & 2.0 & 244 & \\
\hline 347 & $31^{\circ} 50^{\prime} 28^{\prime \prime}$ & $93^{\circ} 55^{\prime} 53^{\prime \prime}$ & Carrizo-Wilcox & -- & -1.4 & 9.0 & 242 & \\
\hline 347 & $31^{\circ} 48^{\prime} 18^{\prime \prime}$ & $93^{\circ} 52^{\prime} 46^{\prime \prime}$ & Carrizo-Wilcox & -- & 6.95 & 7.0 & 233 & \\
\hline 347 & $31^{\circ} 41^{\prime} 10^{\prime \prime}$ & $93^{\circ} 49^{\prime} 29^{\prime \prime}$ & Carrizo-Wilcox & -- & 0 & 9.0 & 226 & \\
\hline 347 & $31^{\circ} 35^{\prime} 42^{\prime \prime}$ & $93^{\circ} 50^{\prime} 23^{\prime \prime}$ & Carrizo-Wilcox & -- & 1.8 & 12.0 & 217 & \\
\hline 347 & $31^{\circ} 34^{\prime} 51^{\prime \prime}$ & $93^{\circ} 49^{\prime} 55^{\prime \prime}$ & Carrizo-Wilcox & -- & 5.34 & 10.0 & 205 & \\
\hline 347 & $31^{\circ} 27^{\prime} 29^{\prime \prime}$ & $93^{\circ} 45^{\prime} 16^{\prime \prime}$ & Carrizo-Wilcox & Sparta & -.7 & 10.0 & 195 & gaging station $(08024400)$ \\
\hline 347 & $31^{\circ} 23^{\prime} 13^{\prime \prime}$ & $93^{\circ} 42^{\prime} 01^{\prime \prime}$ & -- & Sparta & -.27 & 2.0 & 185 & \\
\hline 347 & $31^{\circ} 23^{\prime} 37^{\prime \prime}$ & $93^{\circ} 40^{\prime} 17^{\prime \prime}$ & -- & -- & -.3 & 9.0 & 183 & \\
\hline 347 & $31^{\circ} 21^{\prime} 13^{\prime \prime}$ & $93^{\circ} 39^{\prime} 53^{\prime \prime}$ & -- & -- & 12.0 & 4.0 & 174 & \\
\hline 347 & $31^{\circ} 21^{\prime} 15^{\prime \prime}$ & $93^{\circ} 39^{\prime} 46^{\prime \prime}$ & Gulf Coast & -- & 5.9 & 9.0 & 170 & \\
\hline 347 & $31^{\circ} 09^{\prime} 45^{\prime \prime}$ & $93^{\circ} 36^{\prime} 34^{\prime \prime}$ & Gulf Coast & -- & -1.32 & 7.0 & 161 & \\
\hline 347 & $31^{\circ} 11^{\prime} 20^{\prime \prime}$ & $93^{\circ} 33^{\prime} 05^{\prime \prime}$ & Gulf Coast & -- & 10.38 & 14.0 & 154 & \\
\hline 347 & $31^{\circ} 03^{\prime} 50^{\prime \prime}$ & $93^{\circ} 31^{\prime} 08^{\prime \prime}$ & Gulf Coast & -- & 19.72 & 14.0 & 140 & \\
\hline 347 & $30^{\circ} 59^{\prime} 18^{\prime \prime}$ & $93^{\circ} 33^{\prime} 50^{\prime \prime}$ & Gulf Coast & -- & 5.12 & 11.0 & 126 & \\
\hline 347 & $30^{\circ} 54^{\prime} 58^{\prime \prime}$ & $93^{\circ} 33^{\prime} 12^{\prime \prime}$ & Gulf Coast & -- & .33 & 5.0 & 115 & \\
\hline 347 & $30^{\circ} 51^{\prime} 33^{\prime \prime}$ & $93^{\circ} 33^{\prime} 41^{\prime \prime}$ & Gulf Coast & -- & 3.55 & 7.0 & 110 & \\
\hline 347 & $30^{\circ} 48^{\prime} 04^{\prime \prime}$ & $93^{\circ} 34^{\prime} 30^{\prime \prime}$ & Gulf Coast & -- & 10.23 & 5.0 & 103 & \\
\hline 347 & $30^{\circ} 44^{\prime} 49^{\prime \prime}$ & $93^{\circ} 36^{\prime} 30^{\prime \prime}$ & Gulf Coast & -- & 36.93 & 18.0 & 98 & \\
\hline 347 & $30^{\circ} 38^{\prime} 50^{\prime \prime}$ & $93^{\circ} 40^{\prime} 28^{\prime \prime}$ & Gulf Coast & -- & 2.46 & 4.0 & 80 & \\
\hline 347 & $30^{\circ} 36^{\prime} 59^{\prime \prime}$ & $93^{\circ} 41^{\prime} 08^{\prime \prime}$ & Gulf Coast & -- & -1.1 & 8.0 & 76 & \\
\hline 347 & $30^{\circ} 30^{\circ} 53^{\prime \prime}$ & $93^{\circ} 42^{\prime} 37^{\prime \prime}$ & Gulf Coast & -- & 9.0 & 5.0 & 68 & \\
\hline 347 & $30^{\circ} 27^{\prime} 51^{\prime \prime}$ & $93^{\circ} 42^{\prime} 16^{\prime \prime}$ & Gulf Coast & -- & 69.61 & 23.0 & 63 & \\
\hline 348 & $29^{\circ} 50^{\prime} 06^{\prime \prime}$ & 9850'39" & Trinity & -- & 21.9 & 5.4 & 0 & $75 \mathrm{ft}$ below upper Cibolo Rd \\
\hline 348 & $29^{\circ} 48^{\prime} 53^{\prime \prime}$ & $98^{\circ} 45^{\prime} 18^{\prime \prime}$ & Trinity & -- & 17.8 & 4.2 & 5.4 & \\
\hline 348 & $29^{\circ} 46^{\prime} 25^{\prime \prime}$ & $98^{\circ} 42^{\prime} 32^{\prime \prime}$ & Trinity & -- & -9.7 & 2.9 & 9.6 & \\
\hline 348 & $29^{\circ} 45^{\prime} 57^{\prime \prime}$ & $98^{\circ} 40^{\prime} 19^{\prime \prime}$ & Trinity & -- & -9.0 & 2.1 & 12.5 & \\
\hline 348 & $29^{\circ} 44^{\prime} 46^{\prime \prime}$ & $98^{\circ} 38^{\prime} 40^{\prime \prime}$ & Trinity & -- & -37.8 & 6.9 & 14.6 & \\
\hline 348 & $29^{\circ} 45^{\prime} 00^{\prime \prime}$ & $98^{\circ} 34^{\prime} 26^{\prime \prime}$ & Trinity & -- & -4.3 & 2.1 & 21.5 & \\
\hline
\end{tabular}


Table 4. Gains and losses from gain-loss studies in Texas-Continued

\begin{tabular}{|c|c|c|c|c|c|c|c|c|}
\hline $\begin{array}{l}\text { Stream- } \\
\text { flow } \\
\text { study } \\
\text { no. }\end{array}$ & $\begin{array}{l}\text { Latitude of } \\
\text { upstream } \\
\text { end of } \\
\text { subreach }\end{array}$ & $\begin{array}{l}\text { Longitude } \\
\text { of upstream } \\
\text { end of } \\
\text { subreach }\end{array}$ & $\begin{array}{c}\text { Major aquifer } \\
\text { outcrop } \\
\text { intersected } \\
\text { by subreach }\end{array}$ & $\begin{array}{c}\text { Minor aquifer } \\
\text { outcrop } \\
\text { intercepted } \\
\text { by subreach }\end{array}$ & $\begin{array}{l}\text { Gain or } \\
\text { loss }(-) \text { in } \\
\text { subreach } \\
\left(\mathrm{ft}^{3} / \mathrm{s}\right)\end{array}$ & $\begin{array}{l}\text { Length } \\
\text { of subreach } \\
\text { (river mi) }\end{array}$ & $\begin{array}{l}\text { Location of } \\
\text { upstream } \\
\text { end of } \\
\text { subreach } \\
\text { (river mi) }\end{array}$ & $\begin{array}{l}\text { Descriptive location } \\
\text { of upstream end of } \\
\text { selected subreaches }\end{array}$ \\
\hline 348 & $29^{\circ} 45^{\prime} 36^{\prime \prime}$ & $98^{\circ} 32^{\prime} 58^{\prime \prime}$ & Trinity & -- & -29.2 & 3.4 & 23.6 & \\
\hline 348 & $29^{\circ} 44^{\prime} 35^{\prime \prime}$ & $98^{\circ} 31^{\prime} 40^{\prime \prime}$ & Trinity & -- & -6.7 & 1.7 & 27 & \\
\hline 348 & $29^{\circ} 44^{\prime} 17^{\prime \prime}$ & $98^{\circ} 29^{\prime} 49^{\prime \prime}$ & Trinity & -- & -2.8 & 1.8 & 28.7 & \\
\hline 348 & $29^{\circ} 44^{\prime} 14^{\prime \prime}$ & $98^{\circ} 28^{\prime} 06^{\prime \prime}$ & Trinity & -- & -8.9 & 4.5 & 30.5 & \\
\hline 348 & $29^{\circ} 43^{\prime} 12^{\prime \prime}$ & $98^{\circ} 25^{\prime} 39^{\prime \prime}$ & Trinity & -- & -.4 & 2.0 & 35 & \\
\hline 348 & $29^{\circ} 44^{\prime} 42^{\prime \prime}$ & $98^{\circ} 25^{\prime} 04^{\prime \prime}$ & Trinity & -- & -2.6 & .7 & 37 & \\
\hline 348 & $29^{\circ} 44^{\prime} 54^{\prime \prime}$ & $98^{\circ} 24^{\prime} 18^{\prime \prime}$ & Trinity & -- & -7.5 & 4.9 & 37.7 & \\
\hline 348 & $29^{\circ} 43^{\prime} 57^{\prime \prime}$ & $98^{\circ} 21^{\prime} 22^{\prime \prime}$ & Trinity & -- & 5.2 & 1.4 & 42.6 & \\
\hline 348 & $29^{\circ} 43^{\prime} 22^{\prime \prime}$ & $98^{\circ} 20^{\prime} 26^{\prime \prime}$ & Trinity & -- & 2.3 & 5.5 & 44 & \\
\hline 348 & $29^{\circ} 40^{\prime} 04^{\prime \prime}$ & $98^{\circ} 22^{\prime} 48^{\prime \prime}$ & Edwards & -- & -12.24 & 2.1 & 49.5 & \\
\hline 348 & $29^{\circ} 39^{\prime} 29^{\prime \prime}$ & $98^{\circ} 21^{\prime} 21^{\prime \prime}$ & Edwards & -- & -5.26 & .3 & 51.6 & \\
\hline 348 & $29^{\circ} 39^{\prime} 24^{\prime \prime}$ & $98^{\circ} 21^{\prime} 04^{\prime \prime}$ & Edwards & -- & 0 & 4.1 & 51.9 & \\
\hline 349 & $29^{\circ} 33^{\prime} 02^{\prime \prime}$ & $98^{\circ} 16^{\prime} 23^{\prime \prime}$ & -- & -- & -.21 & .9 & 0 & $0.1 \mathrm{mi}$ above FM 78 at Schertz \\
\hline 349 & $29^{\circ} 32^{\prime} 35^{\prime \prime}$ & $98^{\circ} 15^{\prime} 50^{\prime \prime}$ & -- & -- & 1.77 & .2 & .9 & \\
\hline 349 & $29^{\circ} 32^{\prime} 35^{\prime \prime}$ & $98^{\circ} 15^{\prime} 48^{\prime \prime}$ & -- & -- & -.6 & 11.0 & 1.1 & \\
\hline 349 & $29^{\circ} 30^{\prime} 07^{\prime \prime}$ & $98^{\circ} 11^{\prime} 12^{\prime \prime}$ & Carrizo-Wilcox & -- & 2.93 & 12.9 & 12.1 & \\
\hline 349 & $29^{\circ} 27^{\prime} 08^{\prime \prime}$ & $98^{\circ} 07^{\prime} 24^{\prime \prime}$ & Carrizo-Wilcox & -- & -.48 & 9.1 & 25 & \\
\hline 349 & $29^{\circ} 21^{\prime} 32^{\prime \prime}$ & $98^{\circ} 06^{\prime} 22^{\prime \prime}$ & Carrizo-Wilcox & -- & .8 & 2.6 & 34.1 & \\
\hline 349 & $29^{\circ} 21^{\prime} 03^{\prime \prime}$ & $98^{\circ} 04^{\prime} 26^{\prime \prime}$ & Carrizo-Wilcox & -- & 3.63 & 6.2 & 36.7 & \\
\hline 349 & $29^{\circ} 17^{\prime} 15^{\prime \prime}$ & $98^{\circ} 03^{\prime} 38^{\prime \prime}$ & -- & Queen City & 8.44 & 6.9 & 42.9 & \\
\hline 349 & $29^{\circ} 14^{\prime} 47^{\prime \prime}$ & $98^{\circ} 01^{\prime} 22^{\prime \prime}$ & -- & Queen City & -2.2 & 6.6 & 49.8 & \\
\hline 349 & $29^{\circ} 10^{\prime} 12^{\prime \prime}$ & $97^{\circ} 59^{\prime} 40^{\prime \prime}$ & -- & -- & 1.9 & 7.2 & 56.4 & \\
\hline 349 & $29^{\circ} 05^{\prime} 35^{\prime \prime}$ & $97^{\circ} 58^{\prime} 09^{\prime \prime}$ & -- & -- & -.5 & 8.1 & 63.6 & \\
\hline 349 & $29^{\circ} 00^{\prime} 49^{\prime \prime}$ & $97^{\circ} 55^{\prime} 48^{\prime \prime}$ & Gulf Coast & -- & 1.2 & 7.6 & 71.7 & \\
\hline 350 & $29^{\circ} 35^{\prime} 37^{\prime \prime}$ & $98^{\circ} 18^{\prime} 38^{\prime \prime}$ & -- & -- & 0 & .9 & 89.6 & gaging station at Selma (08185000) \\
\hline 350 & $29^{\circ} 35^{\prime} 04^{\prime \prime}$ & $98^{\circ} 18^{\prime} 14^{\prime \prime}$ & -- & -- & .28 & 1.2 & 88.7 & \\
\hline 350 & $29^{\circ} 34^{\prime} 09^{\prime \prime}$ & $98^{\circ} 18^{\prime} 06^{\prime \prime}$ & -- & -- & -.28 & 2.5 & 87.5 & \\
\hline 350 & $29^{\circ} 33^{\prime} 39^{\prime \prime}$ & $98^{\circ} 17^{\prime} 30^{\prime \prime}$ & -- & -- & -.42 & .8 & 85 & \\
\hline 350 & $29^{\circ} 33^{\prime} 01^{\prime \prime}$ & $98^{\circ} 16^{\prime} 21^{\prime \prime}$ & -- & -- & 1.76 & .7 & 84.2 & \\
\hline 350 & $29^{\circ} 32^{\prime} 35^{\prime \prime}$ & $98^{\circ} 15^{\prime} 53^{\prime \prime}$ & -- & -- & .79 & 1.2 & 83.5 & \\
\hline 350 & $29^{\circ} 32^{\prime} 39^{\prime \prime}$ & $98^{\circ} 15^{\prime} 19^{\prime \prime}$ & -- & -- & 3.58 & 2.6 & 82.3 & \\
\hline 350 & $29^{\circ} 33^{\prime} 01^{\prime \prime}$ & $98^{\circ} 13^{\prime} 49^{\prime \prime}$ & Carrizo-Wilcox & -- & 5.38 & 3.7 & 79.7 & \\
\hline 350 & $29^{\circ} 27^{\prime} 07^{\prime \prime}$ & $98^{\circ} 07^{\prime} 24^{\prime \prime}$ & Carrizo-Wilcox & -- & 4.8 & 3.4 & 76 & \\
\hline 350 & $29^{\circ} 30^{\prime} 07^{\prime \prime}$ & $98^{\circ} 11^{\prime} 10^{\prime \prime}$ & Carrizo-Wilcox & -- & 7.43 & 13.4 & 72.6 & \\
\hline 350 & $29^{\circ} 27^{\prime} 07^{\prime \prime}$ & $98^{\circ} 07^{\prime} 24^{\prime \prime}$ & Carrizo-Wilcox & -- & 5.78 & 9.6 & 59.2 & \\
\hline 350 & $29^{\circ} 21^{\prime} 32^{\prime \prime}$ & $98^{\circ} 06^{\prime} 23^{\prime \prime}$ & Carrizo-Wilcox & -- & 1.7 & 2.8 & 49.6 & \\
\hline 350 & $29^{\circ} 21^{\prime} 03^{\prime \prime}$ & $98^{\circ} 04^{\prime} 26^{\prime \prime}$ & Carrizo-Wilcox & -- & 4.0 & 1.9 & 46.8 & \\
\hline 350 & $29^{\circ} 20^{\prime} 00^{\prime \prime}$ & $98^{\circ} 04^{\prime} 30^{\prime \prime}$ & Carrizo-Wilcox & -- & .6 & 1.9 & 44.9 & \\
\hline
\end{tabular}


Table 4. Gains and losses from gain-loss studies in Texas-Continued

\begin{tabular}{|c|c|c|c|c|c|c|c|c|}
\hline $\begin{array}{l}\text { Stream- } \\
\text { flow } \\
\text { study } \\
\text { no. }\end{array}$ & $\begin{array}{l}\text { Latitude of } \\
\text { upstream } \\
\text { end of } \\
\text { subreach }\end{array}$ & $\begin{array}{l}\text { Longitude } \\
\text { of upstream } \\
\text { end of } \\
\text { subreach }\end{array}$ & $\begin{array}{c}\text { Major aquifer } \\
\text { outcrop } \\
\text { intersected } \\
\text { by subreach }\end{array}$ & $\begin{array}{c}\text { Minor aquifer } \\
\text { outcrop } \\
\text { intercepted } \\
\text { by subreach }\end{array}$ & $\begin{array}{l}\text { Gain or } \\
\text { loss }(-) \text { in } \\
\text { subreach } \\
\left(\mathrm{ft}^{3} / \mathrm{s}\right)\end{array}$ & $\begin{array}{l}\text { Length } \\
\text { of subreach } \\
\text { (river mi) }\end{array}$ & $\begin{array}{l}\text { Location of } \\
\text { upstream } \\
\text { end of } \\
\text { subreach } \\
\text { (river mi) }\end{array}$ & $\begin{array}{l}\text { Descriptive location } \\
\text { of upstream end of } \\
\text { selected subreaches }\end{array}$ \\
\hline 350 & $29^{\circ} 19^{\prime} 09^{\prime \prime}$ & $98^{\circ} 04^{\prime} 30^{\prime \prime}$ & Carrizo-Wilcox & -- & 0 & 1.5 & 43 & \\
\hline 350 & $29^{\circ} 18^{\prime} 19^{\prime \prime}$ & $98^{\circ} 03^{\prime} 42^{\prime \prime}$ & Carrizo-Wilcox & -- & 6.1 & 2.8 & 41.5 & \\
\hline 350 & $29^{\circ} 17^{\prime} 05^{\prime \prime}$ & $98^{\circ} 03^{\prime} 36^{\prime \prime}$ & -- & -- & 1.95 & 1.5 & 38.7 & \\
\hline 350 & $29^{\circ} 16^{\prime} 36^{\prime \prime}$ & $98^{\circ} 03^{\prime} 01^{\prime \prime}$ & -- & Queen City & 2.47 & 4.4 & 37.2 & \\
\hline 350 & $29^{\circ} 14^{\prime} 47^{\prime \prime}$ & $98^{\circ} 01^{\prime} 21^{\prime \prime}$ & -- & Queen City & -.63 & 2.6 & 32.8 & \\
\hline 350 & $29^{\circ} 13^{\prime} 09^{\prime \prime}$ & $98^{\circ} 00^{\prime} 31^{\prime \prime}$ & -- & Queen City & -.1 & 4.2 & 30.2 & \\
\hline 350 & $29^{\circ} 10^{\prime} 12^{\prime \prime}$ & $97^{\circ} 59^{\prime} 40^{\prime \prime}$ & -- & Sparta & 8.5 & 4.5 & 26 & \\
\hline 350 & $29^{\circ} 07^{\prime} 35^{\prime \prime}$ & $97^{\circ} 58^{\prime} 08^{\prime \prime}$ & -- & -- & -3.4 & 2.8 & 21.5 & \\
\hline 350 & $29^{\circ} 05^{\prime} 33^{\prime \prime}$ & $97^{\circ} 58^{\prime} 07^{\prime \prime}$ & -- & -- & 4.9 & 4.6 & 18.7 & \\
\hline 350 & $29^{\circ} 02^{\prime} 42^{\prime \prime}$ & $97^{\circ} 56^{\prime} 52^{\prime \prime}$ & -- & -- & -.96 & 3.7 & 14.1 & \\
\hline 350 & $29^{\circ} 00^{\prime} 49^{\prime \prime}$ & $97^{\circ} 55^{\prime} 48^{\prime \prime}$ & Gulf Coast & -- & .6 & 6.2 & 10.4 & \\
\hline 350 & $28^{\circ} 59^{\prime} 23^{\prime \prime}$ & $97^{\circ} 53^{\prime} 00^{\prime \prime}$ & Gulf Coast & -- & 4.7 & 1.7 & 4.2 & \\
\hline 351 & $29^{\circ} 37^{\prime} 49^{\prime \prime}$ & $98^{\circ} 33^{\prime} 42^{\prime \prime}$ & Edwards, Trinity & -- & -60.4 & 3.15 & 7.21 & gaging station (08178585), a.m., at Wilderness Rd \\
\hline 352 & $29^{\circ} 37^{\prime} 49^{\prime \prime}$ & $98^{\circ} 33^{\prime} 42^{\prime \prime}$ & Edwards, Trinity & -- & -66.0 & 3.15 & 7.21 & gaging station (08178585), p.m., at Wilderness Rd \\
\hline 353 & $29^{\circ} 37^{\prime} 49^{\prime \prime}$ & $98^{\circ} 33^{\prime} 42^{\prime \prime}$ & Edwards, Trinity & -- & -61.6 & 3.15 & 7.21 & gaging station (08178585), a.m., at Wilderness Rd \\
\hline 354 & $29^{\circ} 37^{\prime} 49^{\prime \prime}$ & $98^{\circ} 33^{\prime} 42^{\prime \prime}$ & Edwards, Trinity & -- & -50.1 & 3.15 & 7.21 & gaging station (08178585), a.m., at Wilderness Rd \\
\hline 354 & $29^{\circ} 36^{\prime} 06^{\prime \prime}$ & $98^{\circ} 32^{\prime} 22^{\prime \prime}$ & Edwards & -- & -48.7 & 4.06 & 4.06 & gaging station (08178592), a.m., FM 1604 \\
\hline 355 & $29^{\circ} 37^{\prime} 49^{\prime \prime}$ & $98^{\circ} 33^{\prime} 42^{\prime \prime}$ & Edwards, Trinity & -- & -47.2 & 3.15 & 7.21 & gaging station (08178585), p.m., at Wilderness Rd \\
\hline 355 & $29^{\circ} 36^{\prime} 06^{\prime \prime}$ & $98^{\circ} 32^{\prime} 22^{\prime \prime}$ & Edwards & -- & -36.3 & 4.06 & 4.06 & gaging station (08178592), p.m., FM 1604 \\
\hline 356 & $29^{\circ} 37^{\prime} 49^{\prime \prime}$ & $98^{\circ} 33^{\prime} 42^{\prime \prime}$ & Edwards, Trinity & -- & -32.1 & 3.15 & 7.21 & gaging station (08178585), a.m., at Wilderness Rd \\
\hline 356 & $29^{\circ} 36^{\prime} 06^{\prime \prime}$ & $98^{\circ} 32^{\prime} 22^{\prime \prime}$ & Edwards & -- & -24.3 & 4.06 & 4.06 & gaging station (08178592), a.m., FM 1604 \\
\hline 357 & $29^{\circ} 37^{\prime} 49^{\prime \prime}$ & $98^{\circ} 33^{\prime} 42^{\prime \prime}$ & Edwards, Trinity & -- & -39.0 & 3.15 & 7.21 & gaging station (08178585), p.m., at Wilderness Rd \\
\hline 357 & $29^{\circ} 36^{\prime} 06^{\prime \prime}$ & $98^{\circ} 32^{\prime} 22^{\prime \prime}$ & Edwards & -- & -21.7 & 4.06 & 4.06 & gaging station (08178592), p.m., FM 1604 \\
\hline 358 & $29^{\circ} 37^{\prime} 49^{\prime \prime}$ & $98^{\circ} 33^{\prime} 42^{\prime \prime}$ & Edwards, Trinity & -- & -29.4 & 3.15 & 7.21 & gaging station (08178585), p.m., at Wilderness Rd \\
\hline 359 & $29^{\circ} 37^{\prime} 49^{\prime \prime}$ & $98^{\circ} 33^{\prime} 42^{\prime \prime}$ & Edwards, Trinity & -- & -25.5 & 3.15 & 7.21 & gaging station (08178585), a.m., at Wilderness Rd \\
\hline 359 & $29^{\circ} 36^{\prime} 06^{\prime \prime}$ & $98^{\circ} 32^{\prime} 22^{\prime \prime}$ & Edwards & -- & -19.5 & 4.06 & 4.06 & gaging station (08178592), a.m., FM 1604 \\
\hline 360 & $29^{\circ} 27^{\prime} 51^{\prime \prime}$ & $98^{\circ} 28^{\prime} 03^{\prime \prime}$ & -- & -- & 1.51 & .9 & 0 & $200 \mathrm{ft}$ above Hildebrand Ave \\
\hline 360 & $29^{\circ} 27^{\prime} 37^{\prime \prime}$ & $98^{\circ} 28^{\prime} 21^{\prime \prime}$ & -- & -- & .46 & 1.1 & .9 & \\
\hline 360 & $29^{\circ} 27^{\prime} 05^{\prime \prime}$ & $98^{\circ} 28^{\prime} 42^{\prime \prime}$ & -- & -- & 1.44 & .3 & 2 & \\
\hline 360 & $29^{\circ} 26^{\prime} 56^{\prime \prime}$ & $98^{\circ} 28^{\prime} 45^{\prime \prime}$ & -- & -- & .31 & .7 & 2.3 & \\
\hline 360 & $29^{\circ} 26^{\prime} 29^{\prime \prime}$ & $98^{\circ} 28^{\prime} 52^{\prime \prime}$ & -- & -- & .53 & .7 & 3 & \\
\hline 360 & $29^{\circ} 26^{\prime} 04^{\prime \prime}$ & $98^{\circ} 29^{\prime} 00^{\prime \prime}$ & -- & -- & -.82 & 2.1 & 3.7 & \\
\hline 360 & $29^{\circ} 24^{\prime} 34^{\prime \prime}$ & $98^{\circ} 29^{\prime} 39^{\prime \prime}$ & -- & -- & 6.2 & .9 & 5.8 & \\
\hline 360 & $29^{\circ} 24^{\prime} 15^{\prime \prime}$ & $98^{\circ} 29^{\prime} 24^{\prime \prime}$ & -- & -- & 2.2 & .5 & 6.7 & \\
\hline 360 & $29^{\circ} 23^{\prime} 58^{\prime \prime}$ & $98^{\circ} 29^{\prime} 20^{\prime \prime}$ & -- & -- & .3 & .8 & 7.2 & \\
\hline 361 & $29^{\circ} 23^{\prime} 24^{\prime \prime}$ & $98^{\circ} 29^{\prime} 52^{\prime \prime}$ & -- & -- & 12.1 & .42 & 228.7 & gaging station (08178050) \\
\hline 361 & $29^{\circ} 23^{\prime} 15^{\prime \prime}$ & $98^{\circ} 29^{\prime} 55^{\prime \prime}$ & -- & -- & 2.4 & 2.8 & 228.28 & gaging station (08178505) \\
\hline 361 & $29^{\circ} 22^{\prime} 33^{\prime \prime}$ & $98^{\circ} 28^{\prime} 51^{\prime \prime}$ & -- & -- & -3.0 & 1.97 & 225.48 & \\
\hline
\end{tabular}


Table 4. Gains and losses from gain-loss studies in Texas-Continued

\begin{tabular}{|c|c|c|c|c|c|c|c|c|}
\hline $\begin{array}{l}\text { Stream- } \\
\text { flow } \\
\text { study } \\
\text { no. }\end{array}$ & $\begin{array}{l}\text { Latitude of } \\
\text { upstream } \\
\text { end of } \\
\text { subreach }\end{array}$ & $\begin{array}{l}\text { Longitude } \\
\text { of upstream } \\
\text { end of } \\
\text { subreach }\end{array}$ & $\begin{array}{c}\text { Major aquifer } \\
\text { outcrop } \\
\text { intersected } \\
\text { by subreach }\end{array}$ & $\begin{array}{c}\text { Minor aquifer } \\
\text { outcrop } \\
\text { intercepted } \\
\text { by subreach }\end{array}$ & $\begin{array}{c}\text { Gain or } \\
\text { loss }(-) \text { in } \\
\text { subreach } \\
\left(\mathrm{ft}^{3} / \mathbf{s}\right)\end{array}$ & $\begin{array}{l}\text { Length } \\
\text { of subreach } \\
\text { (river mi) }\end{array}$ & $\begin{array}{l}\text { Location of } \\
\text { upstream } \\
\text { end of } \\
\text { subreach } \\
\text { (river mi) }\end{array}$ & $\begin{array}{l}\text { Descriptive location } \\
\text { of upstream end of } \\
\text { selected subreaches }\end{array}$ \\
\hline 361 & $29^{\circ} 21^{\prime} 31^{\prime \prime}$ & $98^{\circ} 28^{\prime} 06^{\prime \prime}$ & Carrizo-Wilcox & -- & -2.1 & 12.35 & 223.51 & \\
\hline 362 & $30^{\circ} 20^{\prime} 11^{\prime \prime}$ & $95^{\circ} 06^{\prime} 14^{\prime \prime}$ & Gulf Coast & -- & -1.5 & 3.7 & 19.9 & gaging station near Cleveland (08070000) \\
\hline 362 & $30^{\circ} 17^{\prime} 39^{\prime \prime}$ & $95^{\circ} 07^{\prime} 05^{\prime \prime}$ & Gulf Coast & -- & 3.0 & 4.2 & 16.2 & \\
\hline 362 & $30^{\circ} 15^{\prime} 16^{\prime \prime}$ & $95^{\circ} 06^{\prime} 41^{\prime \prime}$ & Gulf Coast & -- & 1.8 & 3.7 & 12 & \\
\hline 362 & $30^{\circ} 12^{\prime} 51^{\prime \prime}$ & $95^{\circ} 06^{\prime} 06^{\prime \prime}$ & Gulf Coast & -- & .9 & 6.4 & 8.3 & \\
\hline 363 & $30^{\circ} 20^{\prime} 57^{\prime \prime}$ & $95^{\circ} 33^{\prime} 36^{\prime \prime}$ & Gulf Coast & -- & 4.17 & 6.4 & 37.9 & $3,200 \mathrm{ft}$ above mouth of Base Creek \\
\hline 363 & $30^{\circ} 18^{\prime} 51^{\prime \prime}$ & $95^{\circ} 30^{\prime} 41^{\prime \prime}$ & Gulf Coast & -- & 1.56 & 4.9 & 31.5 & \\
\hline 363 & $30^{\circ} 16^{\prime} 05^{\prime \prime}$ & $95^{\circ} 29^{\prime} 36^{\prime \prime}$ & Gulf Coast & -- & -.7 & 3.2 & 26.6 & \\
\hline 363 & $30^{\circ} 14^{\prime} 40^{\prime \prime}$ & $95^{\circ} 27^{\prime} 25^{\prime \prime}$ & Gulf Coast & -- & -.75 & 2.7 & 23.4 & \\
\hline 363 & $30^{\circ} 13^{\prime} 58^{\prime \prime}$ & $95^{\circ} 25^{\prime} 57^{\prime \prime}$ & Gulf Coast & -- & 1.55 & 4.3 & 20.7 & \\
\hline 363 & $30^{\circ} 12^{\prime} 20^{\prime \prime}$ & $95^{\circ} 23^{\prime} 31^{\prime \prime}$ & Gulf Coast & -- & -.99 & 3.9 & 16.4 & \\
\hline 363 & $30^{\circ} 09^{\prime} 47^{\prime \prime}$ & $95^{\circ} 22^{\prime} 35^{\prime \prime}$ & Gulf Coast & -- & .18 & 3.3 & 12.5 & \\
\hline 363 & $30^{\circ} 08^{\prime} 44^{\prime \prime}$ & $95^{\circ} 20^{\prime} 40^{\prime \prime}$ & Gulf Coast & -- & 3.9 & 5.9 & 9.2 & \\
\hline 363 & $30^{\circ} 05^{\prime} 09^{\prime \prime}$ & $95^{\circ} 18^{\prime} 28^{\prime \prime}$ & Gulf Coast & -- & .9 & 2.7 & 3.3 & \\
\hline 364 & $31^{\circ} 31^{\prime} 49^{\prime \prime}$ & $95^{\circ} 31^{\prime} 00^{\prime \prime}$ & -- & Queen City & -3.44 & 1.6 & 26 & $\begin{array}{l}\text { at bridge on county road } 3.5 \text { mi northwest of } \\
\text { Grapeland }\end{array}$ \\
\hline 364 & $31^{\circ} 30^{\prime} 57^{\prime \prime}$ & $95^{\circ} 32^{\prime} 08^{\prime \prime}$ & -- & Queen City & 4.38 & 2.4 & 24.4 & at FM 1272 \\
\hline 364 & $31^{\circ} 29^{\prime} 21^{\prime \prime}$ & $95^{\circ} 33^{\prime} 27^{\prime \prime}$ & -- & Queen City & 1.2 & 10.3 & 22 & \\
\hline 364 & $31^{\circ} 27^{\prime} 52^{\prime \prime}$ & $95^{\circ} 39^{\prime} 36^{\prime \prime}$ & -- & Queen City & .13 & 6.5 & 11.7 & at FM 277 \\
\hline 364 & $31^{\circ} 24^{\prime} 36^{\prime \prime}$ & $95^{\circ} 39^{\prime} 21 "$ & -- & Queen City & .34 & 3.9 & 5.2 & at FM 2544 \\
\hline 364 & $31^{\circ} 22^{\prime} 42^{\prime \prime}$ & $95^{\circ} 39^{\prime} 55^{\prime \prime}$ & -- & Queen City & 2.57 & 1.0 & 1.3 & \\
\hline 365 & $31^{\circ} 25^{\prime} 51^{\prime \prime}$ & $95^{\circ} 29^{\prime} 35^{\prime \prime}$ & -- & Sparta & -.39 & 2.8 & 17.5 & at bridge on old Grapeland-Crockett Hwy \\
\hline 365 & $31^{\circ} 25^{\prime} 22^{\prime \prime}$ & $95^{\circ} 31^{\prime} 45^{\prime \prime}$ & -- & Sparta & -.93 & 4.7 & 14.7 & \\
\hline 365 & $31^{\circ} 24^{\prime} 47^{\prime \prime}$ & $95^{\circ} 35^{\prime} 00^{\prime \prime}$ & -- & Queen City & -.1 & 3.2 & 10 & \\
\hline 365 & $31^{\circ} 23^{\prime} 57^{\prime \prime}$ & $95^{\circ} 36^{\prime} 52^{\prime \prime}$ & -- & Queen City & -.17 & 6.8 & 6.8 & FM 229 \\
\hline 366 & $30^{\circ} 51^{\prime} 33^{\prime \prime}$ & $95^{\circ} 23^{\prime} 54^{\prime \prime}$ & Gulf Coast & -- & 2.1 & 25.9 & 0 & at IH-45 at Riverside gaging station $(08066000)$ \\
\hline 366 & $30^{\circ} 47^{\prime} 37^{\prime \prime}$ & $95^{\circ} 09^{\prime} 00^{\prime \prime}$ & Gulf Coast & -- & 5.6 & 39.8 & 25.9 & \\
\hline 366 & $30^{\circ} 33^{\prime} 59^{\prime \prime}$ & $94^{\circ} 56^{\prime} 46^{\prime \prime}$ & Gulf Coast & -- & 3.46 & 22.5 & 65.7 & \\
\hline 366 & $30^{\circ} 25^{\prime} 28^{\prime \prime}$ & $94^{\circ} 51^{\prime} 02^{\prime \prime}$ & Gulf Coast & -- & 26.0 & 45.3 & 88.2 & gaging station (0866500) \\
\hline
\end{tabular}


Slade, Bentley, and Michaud-
Results of Streamflow Gain-Loss Studies in Texas, With Emphasis on Gains From and Losses to Major and Minor Aquifers
_USGS OFR 02-068

District Chief

U.S. Geological Survey

8027 Exchange Dr.

Austin, TX 78754-4733 